

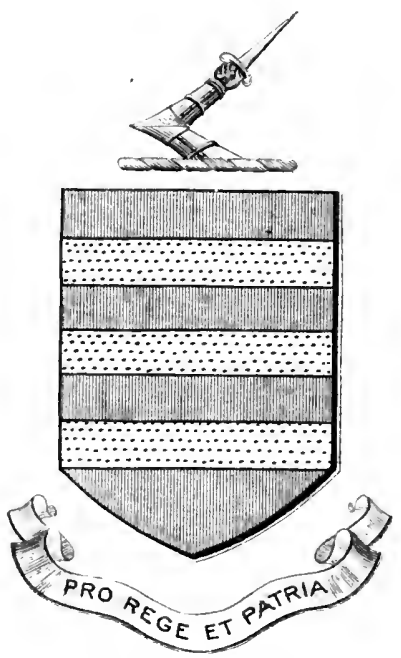

Dhe

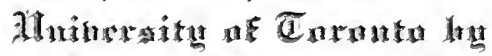

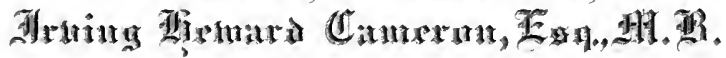

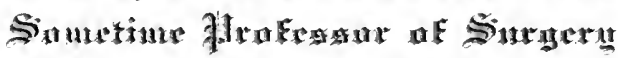
in the 3youlty of Hedixiue 


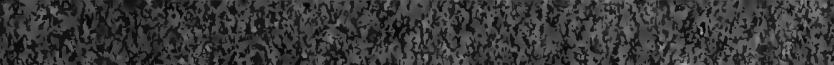

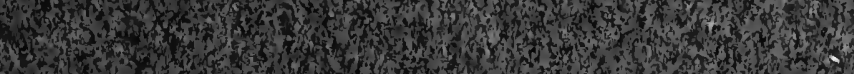
I.

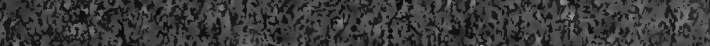

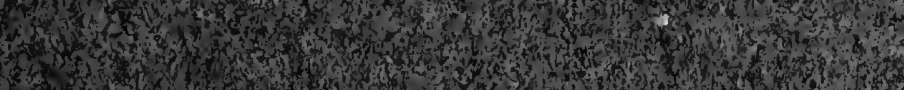

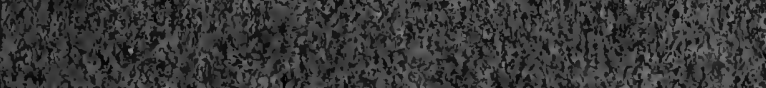
S. 7 .

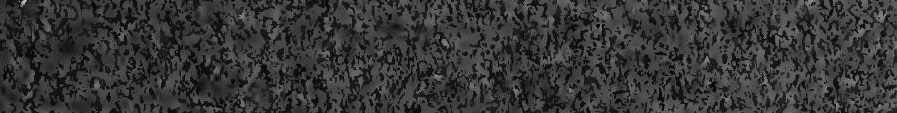
X. S. 3. W

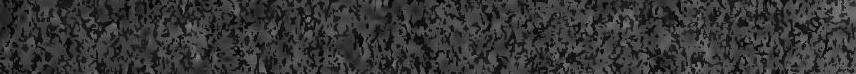

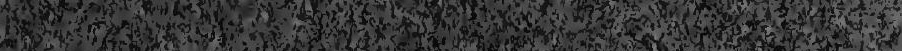

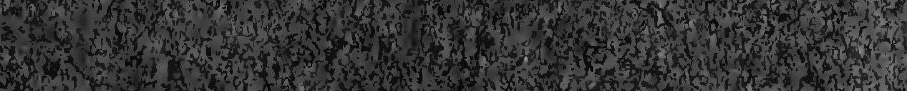

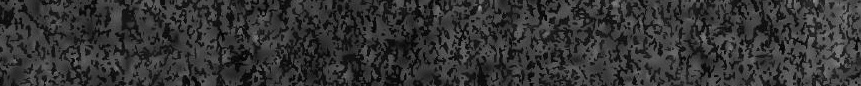
ato W.

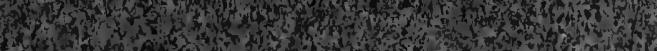
1)

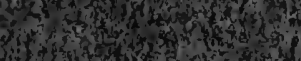

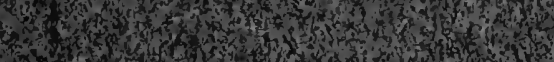

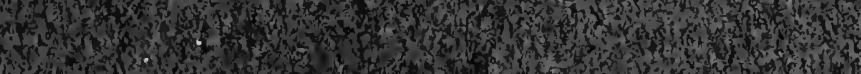

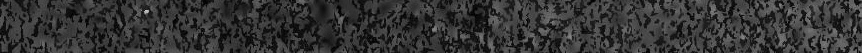

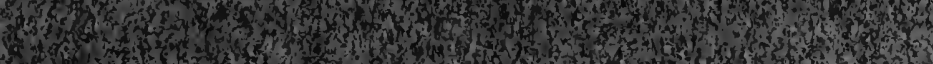
3.5
H.

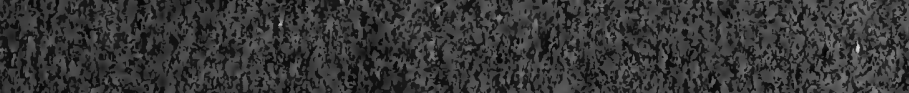

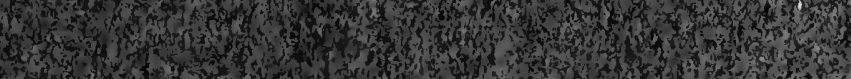

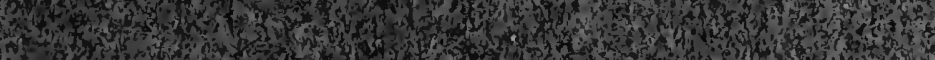
15.

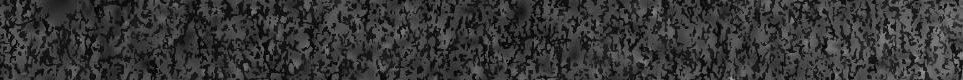

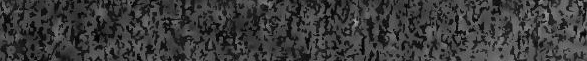

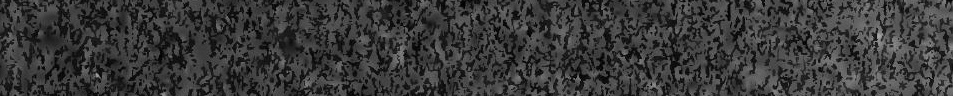

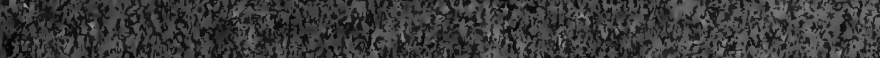

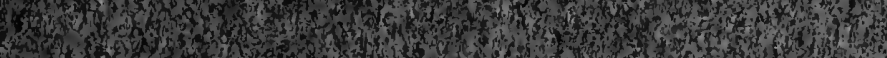

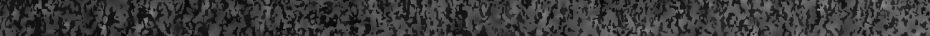

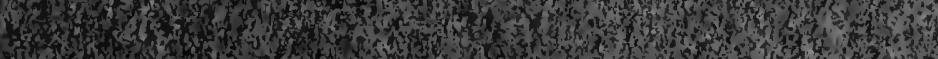

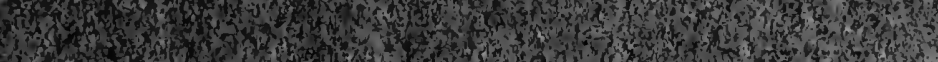

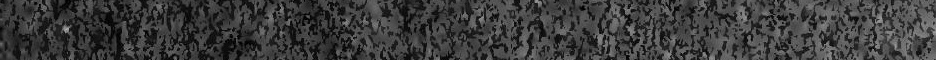
1. 
Digitized by the Internet Archive in 2007 with funding from Microsoft Corporation 


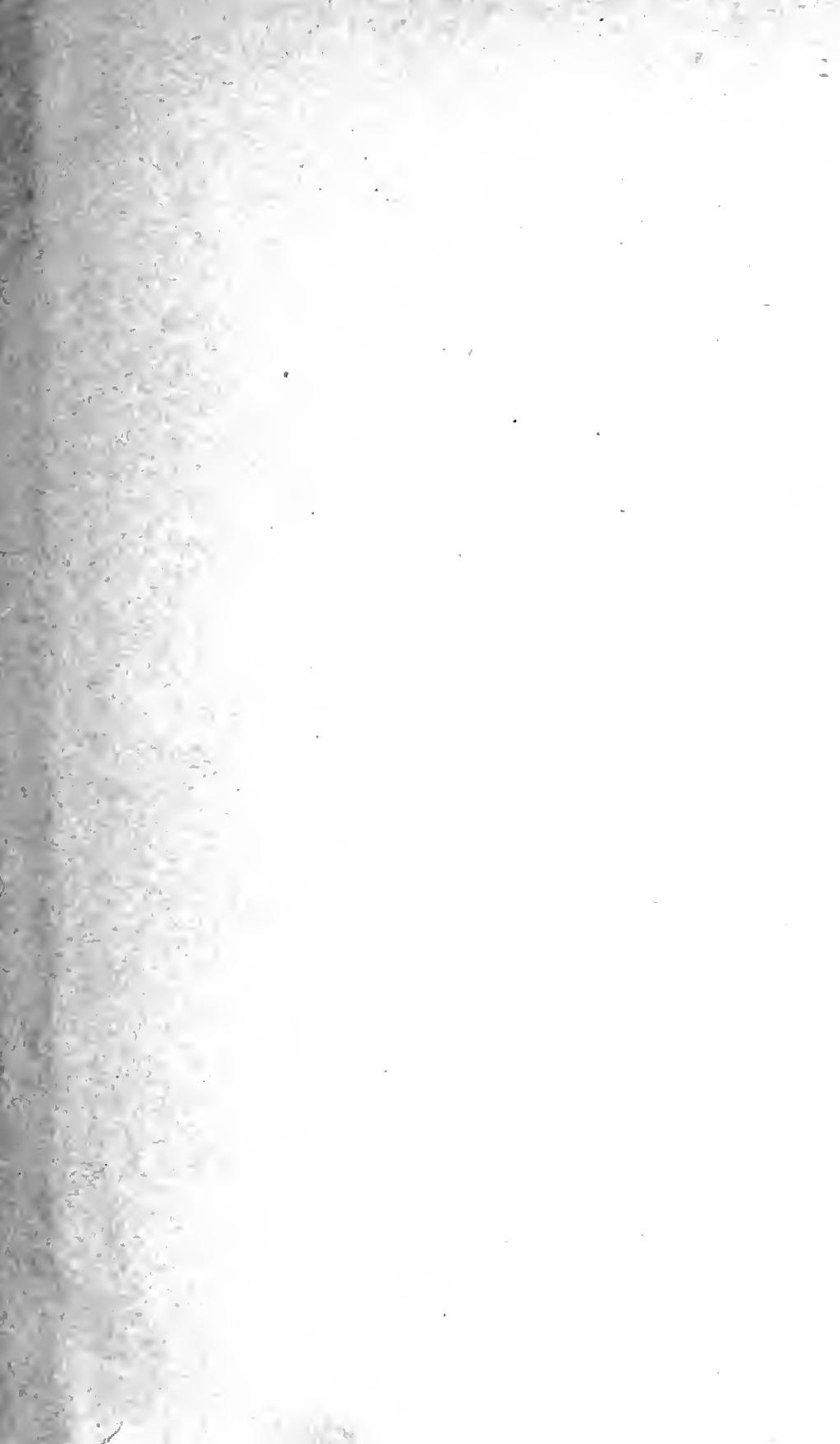





\title{
M INOR
}

A ND

\section{OPERATIVE SURGERY}

TNGI, UDING

\section{BA NDAGING}

\author{
BY \\ HENRY R. WHARTON, M.D.,
}

SURGEON TO TIIE PRESBYTERIAN IIOSPITAL, AND THE CHILDREN'S IIOSPITAL: CONSULTING SURGEON TO ST. CHRISTOPHFR'S HOSPITAL, THE BRYN

MAWR HOSPITAL, AND GIRARD COLLEGE; FELLOW OF TIE AMERICAN SURGICAL ASSOCIATION.

SEVENTH EDITION, ENLARGED AND THOROUGHLY REVISED, WITH 555 ILLUSTRATIONS.

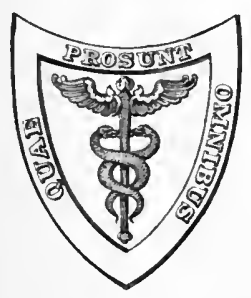

LEA \& FEBIGER,

PHILADELPHIA AND NEW YORK.

$$
1909 \text {. }
$$

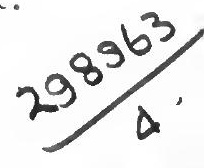


Entered according to Act of Congress, in the year 1909, by LEA \& FEBIGER,

In the Office of the Librarian of Congress. All rights reserved. 


\section{PREFACE TO THE SEVENTH EDITION.}

In revising this work for a seventh edition the author has not been unmindful of the valuable suggestions offered by reviewers of the previous issue.

The general arrangement of the volume is the same as that adopted for its predecessors; all parts have been carefully revised, and matter which has become obsolete has been omitted and a large amount of new material has been added, as well as a number of new illustrations. Some recent minor surgical procedures have been incorporated, and in that portion of the work devoted to operations a number that are new has been described.

In the present revision the author has endeavored to render it worthy of a continuance of the favor with which it has hitherto been received, and hopes that it will be found to be even more closely adapted to the requirements of the student and practitioner.

The author desires to express his thanks to Dr. Francis O. Allen, Jr., for assistance in revising the proof sheets.

1725 Spruce Street,

H. R. W.

Philadelphia. 



\section{CONTENTS.}

PART I.

BANDAGING.

Varieties of Bandages

PAGES

$23-42$

Bandages of the Head and Neck . . . . . . . . . . . . 42-54

Bandages of the Upper Extremity . . . . . . . . . . 55-72

Bandages of the Trunk . . . . . . . . . . $72-76$

Bandages of the Lower Extremity . . . . . . . . . . . 76-91

Special Bandages. . . . . . . . . . . . . . 91-102

Fixed Dressings or Hardening Bandages . . . . . 103-120

\section{PART II.}

\section{MINOR SURGERY.}

Surgical Dressings . . . . . . . . . . . . 121-126

Minor Surgical Procedures . . . . . . . . . . . 127-18т

Röntgen Rays or $x$-rays . . . . . . . . . . . 187-192

Anæsthetics . . . . . . . . . . . . . 193-217

Trusses for Hernia . . . . . . . . . . . . . . . . . 218-221

Catheters and Bougies . . . . . . . . . . . . 221-231

Sutures and Ligatures . . . . . . . . . . . . . . . . . 231-245

Treatment of Hemorrhage . . . . . . . . . 246-268

Abscess . . . . . . . . . . . . . . 265-274

Shock . . . . . . . . . . . . . 274-277

Wounds, Burns and Scalds, Sprains . . . . . . . . 277-291

Removal of Foreign Bodies . . . . . . . 291-296

\section{PART III.}

\section{ASEPSIS AND ANTISEPSIS.}

Surgical Bacteriology .

$297-312$

Theory of Asepsis and Antisepsis in Wound Treatment . . . 312-317 Agents Employed to Secure Asepsis 
Preparation of Materials Used in Aseptic Operations . . . 325-338 Methods and Dressings Employed to Secure Asepsis in Wounds 338-355

\section{PAR'T IV.}

FRACTURES.

General Considerations of Fractures . . . . . . . 357-870 Separation of Epiphyses . . . . . . . . . . . 370-372 Treatment of Special Fractures . . . . . . . . . . 372-428 Compound and Ununited Fractures . . . . . . . . . . 428-433

\section{P.ART V.}

\section{DISLOCATIONS.}

General Considerations of Dislocations . . . . . . . . . 435-437 Speeial Dislocations . . . . . . . . . . . . . 437-465

Old Dislocations, Compound Dislocations . . . . . . . 465-466

\section{PART VI.}

OPERATIONS.

Ligation of Arteries . . . . . . . . . . . 467-469

Ligation of Special Arterres . . . . . . . . . . . 469-496

\section{PART VII.}

AMPUTATIONS.

General Considerations of Amputations . . . . . . . . 497-510 Special Amputations . . . . . . . . . . . 510-550

\section{PART VIII.}

EXCISIONS AND RESECTIONS AND SPECIAL OPERATIONS.

General Considerations of Excisions and Reseetions . . . 551-556 Special Excisions and Resections. . . . . . . . . 556-569 Osteotomy . . . . . . . . . . . . . . . . 569-571 
Trephining the Skull . . . . . . . . . 571-576

Laminectomy . . . . . . . . . . . . . . . 576

Operations upon Nerves . . . . . . . . . 576-582

Operations upon Tendons . . . . . . . . . . 582-5ss

Excision of the Breast . . . . . . . . . 585-590

Tracheotomy . . . . . . . . . . . . . . . 590-ฮ97

Intubation of Larynx . . . . . . . . . . . . 597-603

Paracentís is Thoracis

603-604

Paracentisis Abdominalis . . . . . . . . 604-605

Paracentisis Pericardii . . . . . . . . . . . 605

Paracentisis Vesicæ . . . . . . . . . . . . 605

Ingrown Toenail . . . . . . . . . . . . . 605-606

Operations upon the Kidney . . . . . . . . 607-609

Operations upon the Ureter . . . . . . . . . 609-611

Operations upon the Colon . . . . . . . . . . 611-612

Operations for Appendicitis . . . . . . . . . 613-617

Lithotomy . . . . . . . . . . . . . . 617-619

Circumcision . . . . . . . . . . . . . . 619-620

Excision of the Testicle . . . . . . . . . . . 620

Operation for Varicocele . . . . . . . . . . . 620

Operation for Hydrocele . . . . . . . . . . . 620-622

Operations upon the Gall-bladder . . . . . . 622-624

Oesophagotomy . . . . . . . . . . . . . . . 624

Operations upon the Stomach . . . . . . . . . 625-637

Operations upon the Intestines . . . . . . . . 637-649

Intestinal Anastomosis . . . . . . . . . . . 642

Operation for Strangulated Hernia . . . . . . 649

Operations for the Radical Cure of Hernia . . . . 650-657

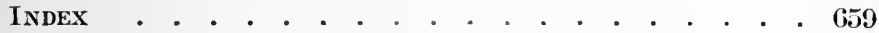





\section{PART I. \\ B A ND A G I N G.}

Bandages.-These constitute one of the most widely used and important surgical dressings; they are employed to hold dressings in contact with the surface of the body, to make pressure, to hold splints in place in the treatment of fractures and dislocations, and to maintain in their natural position parts which may have become displaced.

Bandages may be prepared of various materials, such as linen, crinoline, flannel, gauze or cheese-cloth, rubbersheeting, or muslin, bleached or unbleached; the latter material is the most commonly employed, by reason of its cheapness ; flannel, from its elasticity, is sometimes used, but its employment for bandages is now generally limited to its use in dressings for operative work in connection with the eye and abdomen, and for a primary roller in the application of plaster-of-Paris dressings.

Bandages are either simple, when composed of one piece of material, such as the ordinary roller-bandage, or compound, when prepared of one or more pieces adapted by size and shape to particular objects.

The importance of being familiar with the general rules of bandaging and proficient in the application of the roller-bandage cannot be overestimated, and both the student and the general practitioner will never have cause to regret the time occupied in learning to apply neatly this form of surgical dressing. 
A well-applied bandage adds to the security of the dressing and the comfort of the patient, and the method of application often secures for the physician the confidence both of the patient and of his friends; while, on the other hand, a badly applied bandage is apt to be uncomfortable and insecure, and to meet with their adverse criticism.

The Roller-bandage.-The roller-bandage consists of a strip of woven material, prepared from sume one of the materials previously mentioned, of variable length and width according to the portion of the body to which it is

\section{FIG. 1.}

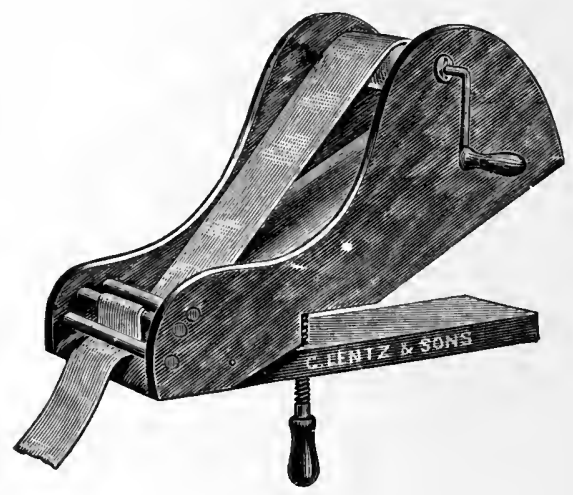

Bandage-winder.

to be applied ; this, for ease of application, is rolled into a cylindrical form.

The material commonly employed for the roller-bandage is unbleached muslin, although, for special purposes, linen, flannel, rubber-sheeting, crinoline, gauze or cheesecloth may be used. It is important that the rollerbandage should consist of one picce, free from seams and selvage, for if made of a number of pieces sewed together, or if it contains creases or selvage, it cannot be so neatly applied, and it is not so comfortable to the patient, as it is apt to leave creases upon the skin. 
In preparing the ordinary muslin bandage, the material is torn in strips varying in length and width according to the part of the body to which it is to be applied, and it is then rolled into a eylinder, either by the hand or by a machine constructed for the purpose (Fig. 1).

It is important that every student and practitioner should be able to roll a bandage by hand, for in practice the medical attendant may at any moment be called upon to prepare a bandage, in order to apply a dressing, and the art of preparing a bandage is easily acquired by a little

FIG. 2.

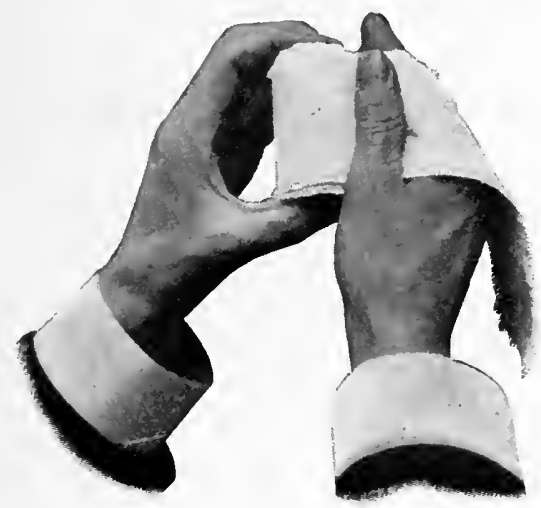

Rolling a bandage by hand.

praetice. To roll a bandage by hand, the strip of muslin should be folded at one extremity several tines until a small eylinder is formed; this is then grasped by its extremities by the thumb and index finger of the left hand; the free extremity of the strip is then grasped between the thumb and index finger of the right hand, and by alternate pronation and supination of the right hand the cylinder is revolved and the roller is formed; the firmness of the roller will depend upon the amount of tension which is kept upon the free extremity of the strip during the revolution of the cylinder (Fig. 2). A bandage rolled 
in the form of a eylinder is called a single or single-headed roller (Fig. 3); if rolled from each extremity toward the centre, so that two cylinders are formed joined by the

FIG. 3.

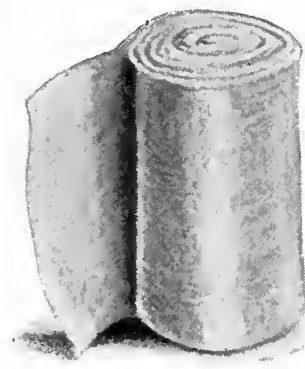

Single roller.
Fig. 4.

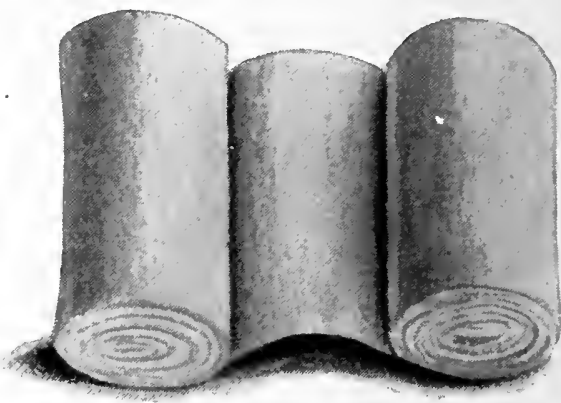

Double roller.

central portion of the strip, the double or double-headed roller is formed (Fig. 4).

Double rollers are not much used, and in practice the single roller will be found to be amply sufficient for the application of almost all the bandages employed in surgical dressings.

The free end of the roller-bandage is called the initial extremity; the end which is enclosed in the centre of the cylinder is its terminal extremity; and the portion between the extremities the body; a roller has also two surfaces, external and internal.

Dimensions of Bandages.-Bandages vary in length and width according to the purposes for which they are employed, and in practice it will be found that a small variety of bandages will be amply sufficient for the applieation of the ordinary surgical dressings.

The following list, comprising those most frequently used, will show their dimensions :

Bandages one inch wide, three yards in length, for bandages for the hand, fingers, and toes. 
Bandages two inches wide, six yards in length, for headbandages and for the extremities in children.

Bandages two and a half inches wide, seven yards in length, for bandages of the extremities in adults; a roller of this size is the one most generally used.

Bandages three inches wide, nine yards in length, for bandages of the thigh, groin, and trunk.

Bandages four inches wide, ten yards in length, for bandages of the trunk.

General Rules for Bandaging.--In applying a rollerbandage, the operator should place the external surface of the free extremity of the roller upon the part, holding it in position with the fingers of the left hand until fixed by a few turns of the roller, the cylinder being held in the right hand by the thumb and fingers; for thus as the bandage is unwound it rolls into the operator's hand, thereby giving him more control of it; care should also be taken that the turns are applied smoothly to the surface, and that the pressure exerted by each turn is miform.

When a bandage is applied to a limb, the surgeon should see that the part is in the position it is to oeenpy as regards flexion and extension when the dressing is completed, for a bandage applied when the limb is flexed will exert too much pressure when the limb is extended, and then may, by the pressure it exerts, become a matter of discomfort or even of danger to the patient, or if applied to an extended limb it will become uneonifortable upon flexion.

My experience has been that, as a rule, those who have had little experience with the application of the rollerbandage are apt to apply the bandages too tightly, and this may lead to disastrous consequences, gangrene of the extremities having resulted from the too tight application of handages, especially in the dressing of fractures. Professor Ashhurst, in his elinical teaching, advised students to make use of a larger number of turns of a bandage in securing fracture-dressings rather than to depend upon a few turns too firmly applied-arlvice which certainly conduces to the safety and comfort of the patient. When the 
bandage has been completed, the terminal extremity should be seeured by a pin or safety-pin applied transversely to the bandage, and if a pin be used its point shonld be

Fig. 5.

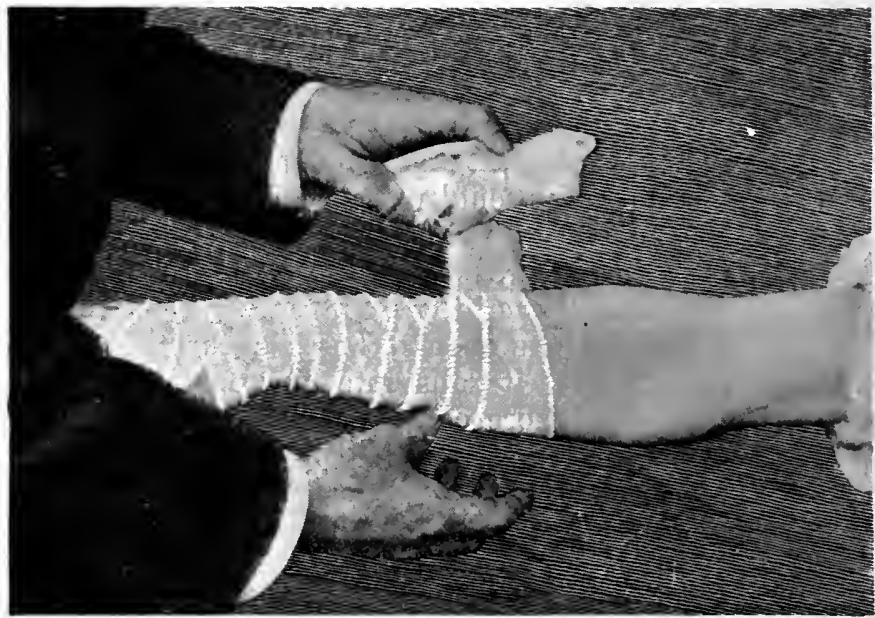

Method of removing a bandage.

buried in the folds of the bandage; if the bandage be a narrow one, the end may be split and the two tails resulting secured around the part by tying.

FIG. 6.

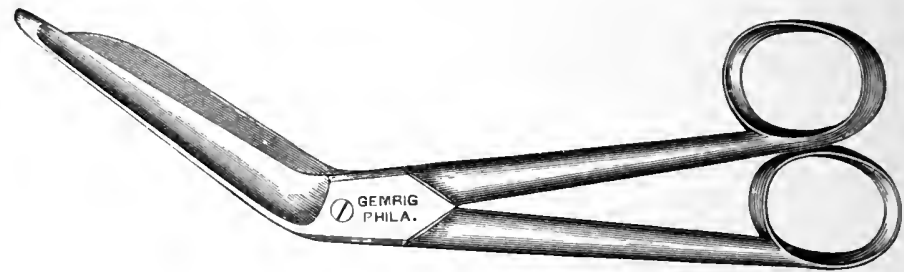

Bandage-scissors.

Removal of Bandages.--In removing a bandage, the folds should be earefully gathered $u p$ in a loose mass as 
the bandage is unwound, the mass being transferred rapidly from one hand to the other, thus facilitating its removal and preventing the part from becoming entangled in its loops (Fig. 5). If it is desirable to cut the bandage to remove it, the use of scissors made for this purpose will be found most satisfactory (Fig. 6).

\section{VARIETIES OF BANDAGES.}

Circular Bandage.-This bandage consists of a few circular turns around a part, each turn covering accurately the preceding turn. This variety of bandage may be used to retain a dressing to a limited portion of the head, neck, or limbs, to make compression upon the veins of the arm before performing venesection, or to secure a compress to control venous hemorrhage (Fig. 11).

Oblique Bandage.--In this form of bandage the turns are carried obliquely over the part, leaving uncovered spaces between the successive turns (Fig. 7). It cannot

FIG. 7.

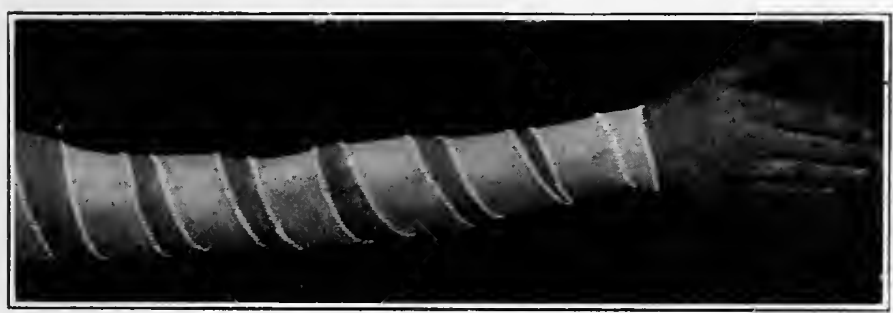

Oblique bandage.

be applied with much firmness on account of the swelling of the uncovered portions of skin between the turns of the bandage, and its principal use is for the application of temporary dressings, such as wet dressings which may require frequent removal.

Spiral Bandage.-In this bandage the turns are carried 
around the part in a spiral direction, each turn overlapping a portion of the preceding one, usually one-third or one-half; it may be applied as an ascending spiral (Fig. 8)

FI's. 8.

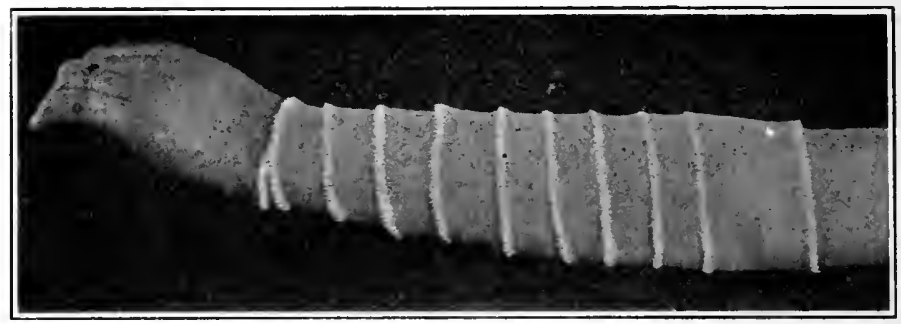

Ascending spiral bandage.

or as a deseending spiral (Fig. 9). This bandage may be used to cover a part which does not increase rapidly in diameter ; for instance, the abdomen, chest, or arm.

Fıg. 9.

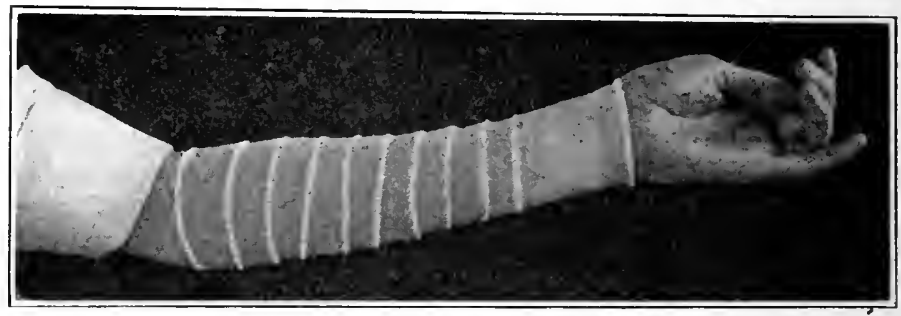

Deseending spiral bandage.

Spiral Reversed Bandage.-This bandage is a spiral bandage, but differs from the ordinary spiral bandage in having its turns folded back or reversed as it ascends a part the diameter of which gradually inereases. By its use, it is possible to cover hy spiral turns a part conical in shape, so as to make equalile pressure upon all parts of the surface. The reverses are made as follows: After 
fixing the initial extremity of the roller, as the part increases in diameter the bandage is carried off a little obliquely to the axis of the limb for from four to six inches; the index finger or thumb of the disengaged hand is placed upon the bodly of the bandage to keep it seeurely in place upon the limb, the hand holding the roller is carried a little toward the limb to slacken the unwound portion of the bandage, and by changing the position of the hand holding the bandage from extreme supination to pronation the reverse is made (Fig. 10). Care should be

FIG. 10.

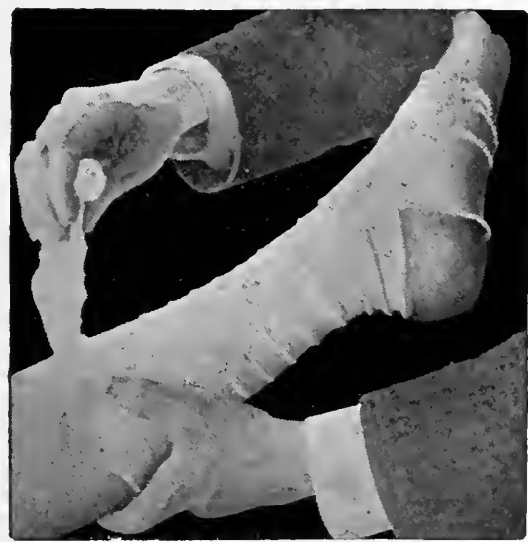

Method of making reverses.

taken not to attempt to make the reverse while the bandage is tense, for by so doing the bandage is twisted into a cord which is unsightly and uneonfortable to the patient, instead of forming a elosely fitting reverse.

The reverse should be completed before the bandage is carried around the limb, and when it has been eompleted it may be slightly tightened so as to conform to the part aecurately.

The reverses should be in line to have the bandare present a good appearance, and care should be taken that the 
reverses should not be made over prominent bony parts of the limb, for if they occupy such positions they cause creases in the skin and become nucomfortable to the patient.

To make reverses neatly and to have them in line, require skill and practice; a well-applied spiral reversed bandage is a test of a competent bandager.

Spica-bandage.-When the turns of the roller cross each other in the form of the Greek letter lambda, leaving the previous turn about one-third uncovered, the bandage is known as a spica-bandage (Fig. 12). These spica-

FIG. 11.

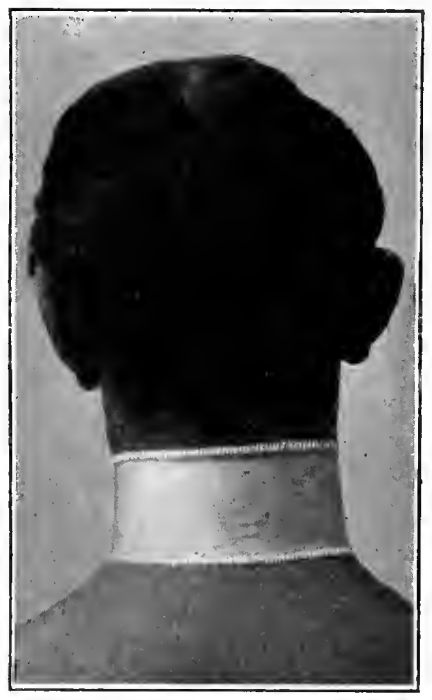

Circular bandage.
FIG. 12.

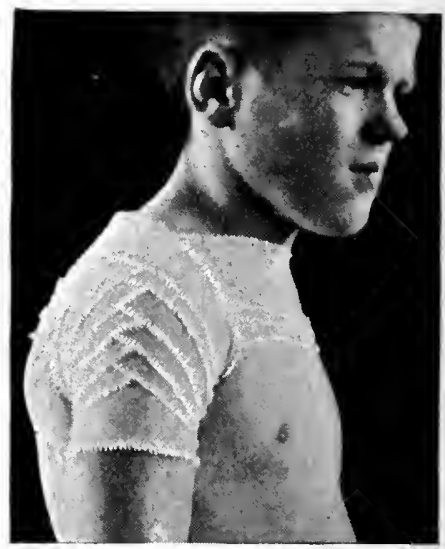

Spica-banđage.

bandages are especially serviceable as a means of retaining surgical dressings upon particular portions of the surface of the body, such as the shoulder, groin, or foot.

Figure-of-eight Bandage - This bandage receives its 
name from the turns being applied so as to form a figureof-eight. This method of applieation is made use of in the Barton's bandage, the bandages of the knee and elbow, and many other bandages.

Fig. 13.

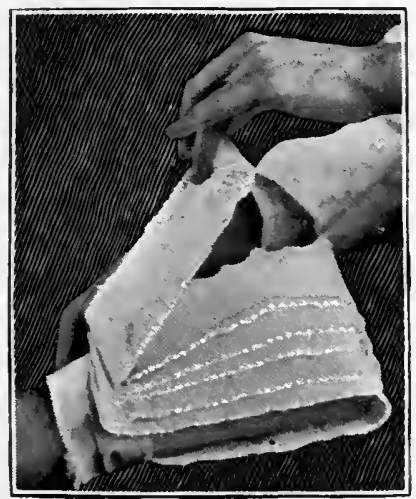

Recurrent bandage.

Recurrent Bandage.-This bandage derives its name from the fact that the roller after eovering a certain part of the surface is refleeted and brought back to the point of starting; it is then reversed and carried toward the opposite point, and this manipulation is eontinued until the part is covered by these recurrent turns, which are then secured by a few eireular turns (Fig. 13). This is the bandage usually employed in the dressing of stumps after amputation.

\section{Compound Bandages.}

These bandages are usually formed of several pieces of muslin or other material, sewed or pinned together, and are employed to fulfil some speeial indication in the application of dressings to particular parts of the body. 'The most useful of the compound bandages are the ' $T$-bandages and the many-tailed bandages. 
T-bandage.-The single $\mathrm{T}$-bandage consists of a horizontal band to which is attached, abont its middle, another having a vertical direction; the horizontal pieee should be about twice the length of the vertical piece (Fig. 14). The

Fig. 14.

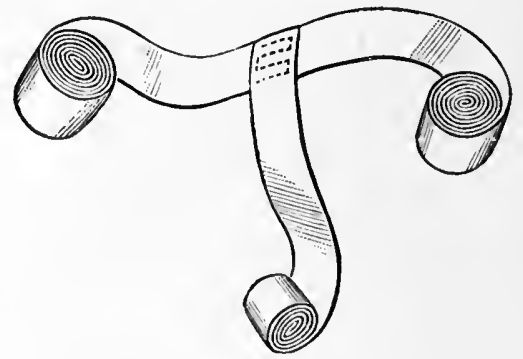

Single T-bandage.

single ' $T$-bandage may be used to retain dressings to the head, the horizontal piece being passed around the head from the oceiput to the forehead, the vertical piece being

FII. 15.

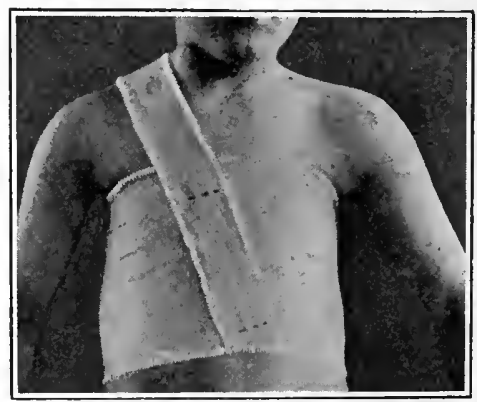

Single T-bandage for chest.

passed over the head and seeurer to the horizontal piece, the shape and width of the two pieces being varied aceording to the indications. In applying dressings to the anal 
region or perineum, or in securing a catheter in a perineal wound, the single 'T-bandage will be found most useful. In applying a T-bandage for this purpose, the body of the bandage is placed over the spine, just above the pelvis, and the horizontal portion is tied around the abdomen. The free extremity is split into two tails for about two-thirds of its length, and is carried over the anal region and brought up between the thighs, the terminal strips passing one on each side of the scrotum and being secured to the horizontal strip in front. The single 'T-bandage may be

Fig. 16.

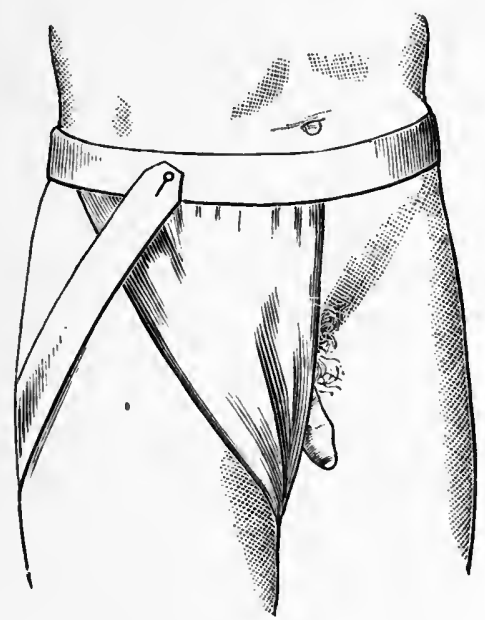

T-bandage of groin.

variously modified according to the indications which are to be met; for instance, in applying a dressing to the breasts the horizontal strip passing around the chest may be made ten or twelve inches in width; the vertical strip, two inches in width, passes from the back over the shoulder and is secured to the horizontal strip in front (Fig. 15). For the groin, a piece of muslin six inches 
wide at its base and thirty inches long is sewed to a horizontal strip of muslin one and a half yards long and two inches in width. It may be applied as in Fig. 16 to hold a dressing to this part.

Double T-bandage. - The double T-bandage differs from the single bandage in having two vertical strips attached to the horizontal strip, and it may be used for much the same purposes as the single T-bandage (Fig. 17).

FIG. 17.

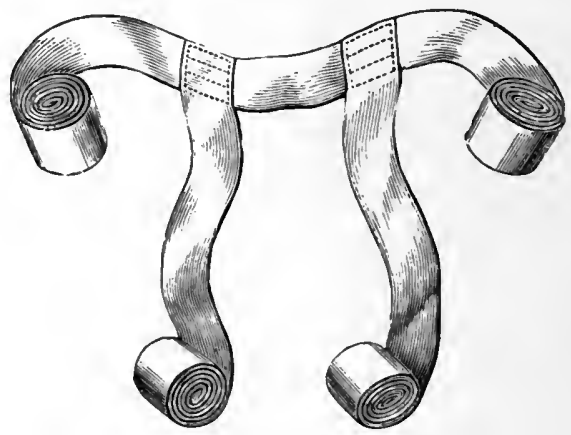

Double T-bandage.

It may be conveniently used for retaining dressings to the chest, breast, or abdomen; when used for this purpose the horizontal portion should be from eight to twelve inches wide and long enough to pass one and a quarter times about the chest; two vertical strips, two inches wide and twenty inches long, should be attached to the horizontal strip a short distance apart near its middle. In applying this bandage to the chest, the horizontal strip is placed around the chest so that the vertical strips occupy a position on either side of the spine; the overlapping end of the horizontal portion is secured by pins or safety-pins, and the vertical strips are next carried one over either shoulder and secured to the other portion of the bandage in front of the chest (Fig. 18).

The double T-bandage may also be used to secure dress- 
ings to the nose, in which event the strips should be quite narrow, about one inch in width, and should be applied as shown in Fig. 19.

Fig. 18.

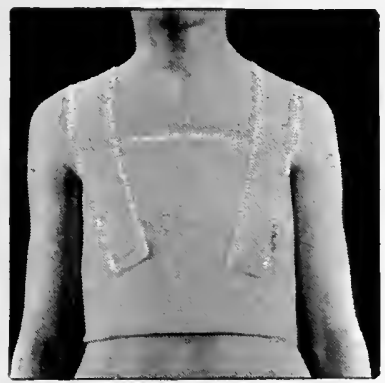

Double T-bandage of chest.
FIG. 19.

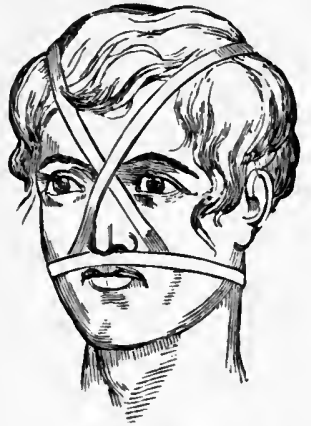

Double T-bandage of nose.

Many-tailed Bandages or Slings._-These bandages are prepared from pieces of muslin of various lengths and breadths, which are split at each extremity into two, three, or more tails up to within a few inches of their centres, their width and length being regulated by the part of the body to which they are to be applied.

The four-tailed bandage may be found useful as a tenıporary dressing in cases of fracture of the jaw, or to hold dressings to the chin. It may be prepared by taking a portion of a roller-bandage three inches wide and one yard in length, and splitting each extremity up to within two inches of the centre; it is then applied as seen in Fig. 20.

The four-tailed bandage may also be used to retain dressings to the scalp, and may be prepared by taking a piece of muslin one yard and a quarter long and six or eight inches in width, splitting it at each extremity into two tails within six inches of the centre; it may then be applied as seen in Fig. 21.

The four-tailed bandage may also be used in the tem- 
porary dressing of fractures of the clavicle, the body of the bandage being placed upon the elbow of the injured

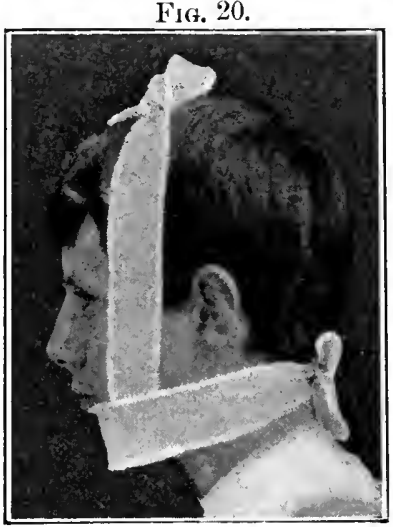

Four-tailed bandage of chin.
Fig. 21.

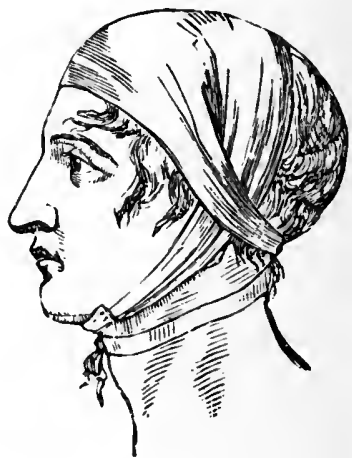

Four-tailed bandage of head.

side, two tails passing around the body, fixing the arm to the side, and two tails passing over the sound shoulder.

Fig. 22.

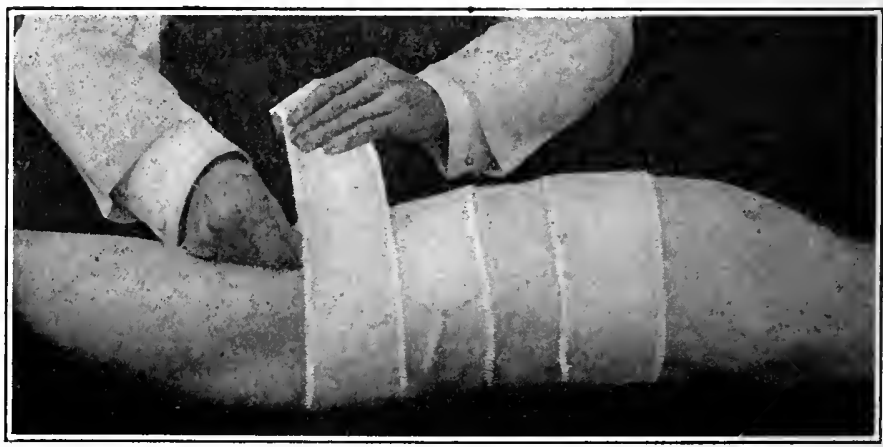

Many-tailed bandage of abdomen.

Many-tailed Bandage of Abdomen.-This bandage may also be used for holding dressings in contact with the abdomen or trunk, and is the bandage which most surgeons 
employ to hold the dressings to a laparotomy wound, and to give support to the abdominal walls after this operation. In preparing this bandage, a strip of muslin or flannel, one and a half yards in length and eighteen to twenty inches in width, is required; the extremities may be split on each side to within six inches of the centre so as to form a four- or six-tailed bandage. In applying this bandage to the abdomen, the body is placed upon the patient's back and the tails are brought around the abdomen and overlap each other, and when sufficiently firmly drawn to make the desired amount of pressure they are secured by means of safety-pins (Fig. 22).

\section{Handkerchief-bandages.}

The use of handkerchiefs or square pieces of muslin for the temporary or permanent dressing of wounds, fractures, or dislocations was advocated many years ago by M. Mayor, a Swiss surgeon, who wrote an extensive work

FIG. 23.

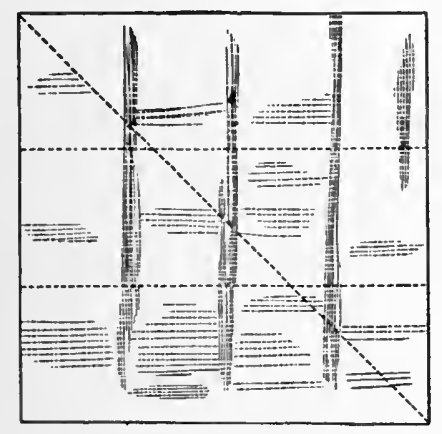

The square.
FIG. 24.

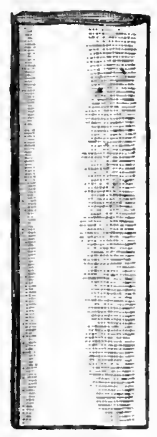

The oblong.

upon this subject, in which he reduced their application to a system. He employed a handkerchief or a square piece of muslin, and by various modifications in the application of these developed a number of very ingenious bandages.

The various forms which the handkercliief or square (Fig. 23) is made to assume are as follows: The oblong, 
made by folding the square once or twice on itself (Fig. 24 ). The triangle, made by bringing together the diagonal angles of the square (Fig. 25). The line of the folding is known as the base, the angle opposite the base the apex, and the other angles the extremities.

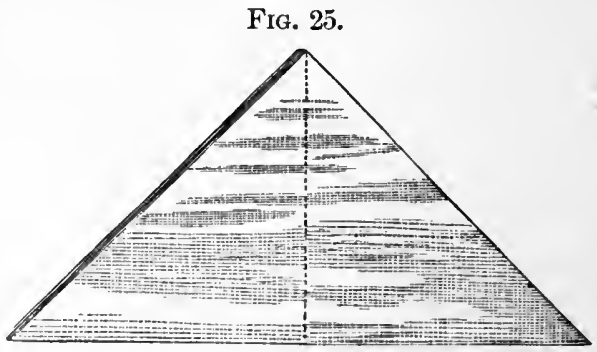

The triangle.

The cravat is prepared from the triangle by bringing the apex to its base, and folding it a number of times upon itself until the desired width is obtained (Fig. 26).

FIG. 26.

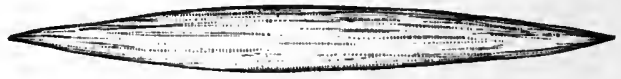

The cravat.

The cord is formed from the cravat twisted upon itself (Fig. 27). The names of the various handkerchief-bandages are derived from the shape of the handkerchiefs used and the parts to which they are applied; the names

Fig. 27.

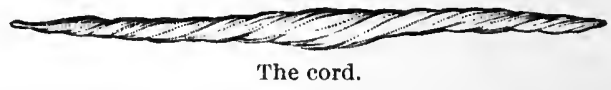

serve as guides in their application. It is to be remembered that the base of the triangle or the body of the cravat is to be placed upon the portion, the designation of which forms the first portion of the name of the bandage; thus, 
in the occipito-frontal triangle, the shape of the handkerchief is given, and we know that the base of the triangle is to be applied to the occiput and the apex carried to the forehead. In using the cravats the same rule applies; thus, in the bis-axillary cravat the body of the cravat is to be placed in the axilla of the affected side, the extremities crossed over the corresponding shoulder and carried over the chest, one before, the other behind, to the axilla of the opposite side, where they are secured.

FIG. 28.

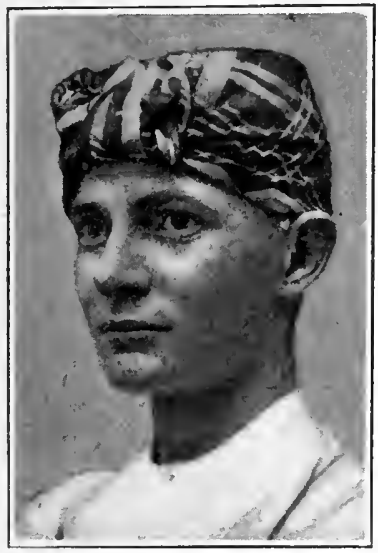

Occipito-frontal triangle.
Fig. 29.

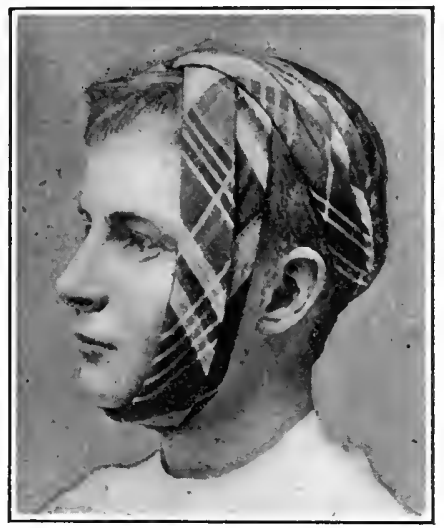

Mento-vertico-occipital cravat.

The Occipito-frontal Triangle.-To apply this handkerehief, place the base of the triangle upon or a little below the occiput, and bring the apex forward over the head, allowing it to drop over the forehead; next bring the extremities of the handkerchief forward and tie them in a knot over the forehead; finally turn up the apex over the knotted ends and pin it to the body of the handkerchief (Fig. 28).

The Mento-vertico-occipital Cravat.-To apply this handkerchief, the middle of the batse of the cravat is placed under the chin; the extremities are then carried in front 
of the ear sn each side to the vertex of the skull, and are crossed at that point; the ends are then carried downward over the parietal region to the occiput, and are secured by a knot at this point (Fig. 29). Another method of applying this handkerchief consists in placing the base of the

FIg. 30.

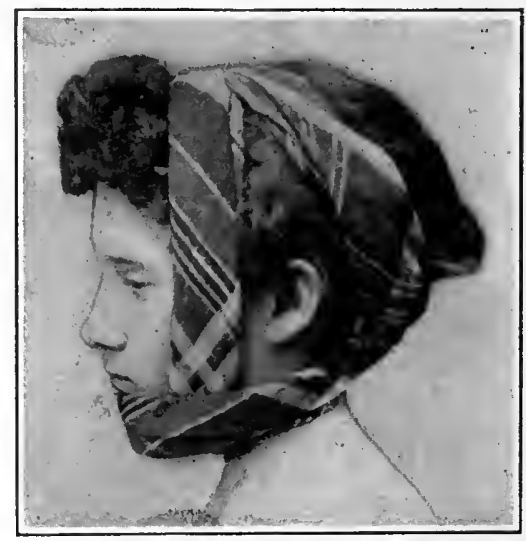

Mento-vertico-occipital cravat (modified).

eravat under the chin and carrying the extremities over the vertex of the skull, erossing them at that point; then carrying them downward to the occiput, and crossing them again here and passing them forward around the chin, and finally securing the ends by a knot (Fig. 30). The turns of the latter handkerchief eorrespond exactly to the turns of the Barton's bandage of the head.

These handkerchief-bandayes may be used to secure dressings to the chin or scalp, or may be employed as temporary dressings to seeure fixation of the parts in eases of fracture or dislocation of the jaw.

The Bis-axillary Cravat.-To apply this handkerchief, the body of the cravat is placed in the axilla, and the ends are brought up, one in front of, the other behind, the axilla, and are made to cross over the top of the 
shoulder; the extremities are then earried across the back and chest respectively to the opposite axilla, where they are secured by tying (Fig. 31). This handkerchief may be employed to secure dressings in the axilla, or to hold dressings in contact with the shoulder.

FIG. 31.

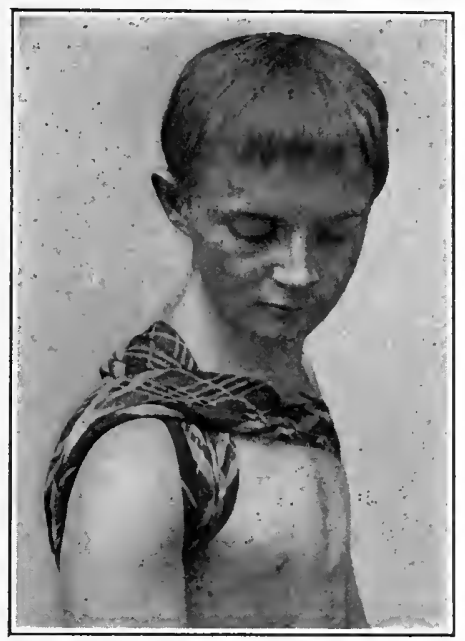

Bis-axillary cravat.

The Dorso-axillary Cravat.-This handkerehief is applied by placing the body of the cravat over the spine between the scapula, and then carrying one extremity over the shoulder and through the axilla backward to meet the other extremity, which has been earried through the axilla and over the other shoulder to the back, where the ends are seeured by a knot (Fig. 32). This liandkerchief may be used to hold dressings to the axilla or upper portion of the back of the chest.

The Compound Dorso-bis-axillary Cravat.-To apply this handkerchief, two eravats are required. The base of one cravat is placed over the front of one shoulder, and 
the ends are passed, one over the top of the shoulder, the other through the axilla, and they are then secured by a single knot over the scapula; the ends are next secured by tying them in a loop. The second cravat is next placed in front of the shoulder on the opposite side, and the ends

Fig. 32.

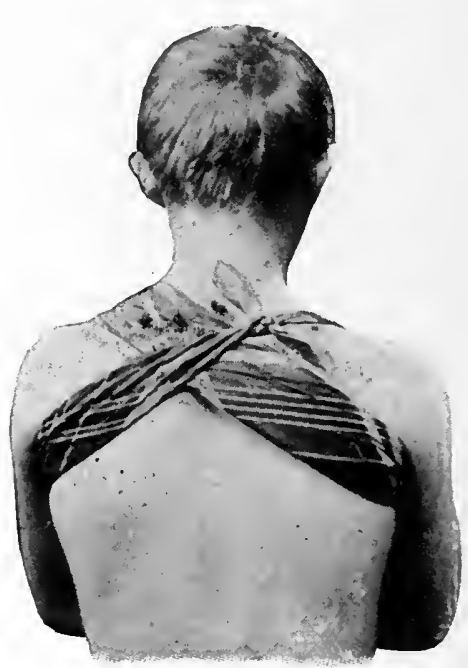

Dorso-axillary cravat.

are respectively earried over the shonlder and through the axilla to the back, where they are seenred by a single knot; the ends of the handkerchief are then passed through the loop of the first handkerehief and secured by a knot (Fig. 33). This handkerehief may be used to draw the shoulders backward in cases of dislocation or fracture of the elavicle.

Triangular Cap or Suspensory of the Breast.-To apply this handkerehief, the base of the triangle is placed under the affected breast, and one extremity is carried beneath the axilla of the same side, and the other extremity is carried around the opposite side of the neck, and they 
are secured together upon the back by a knot; the apex should then be brought up over the breast and shoulder of the affected side, and pinned to the bandage over the scapula (Fig. 34). 'This handkerchief may be employed to sling the breast in nursing-women, or to hold a dressing to the breast.

Fig. 33.

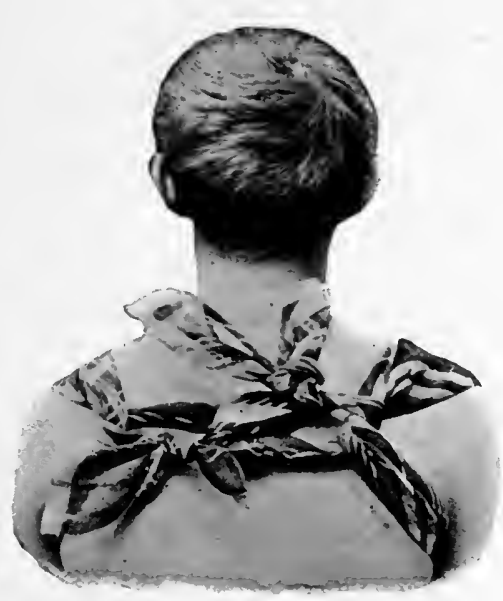

Compound dorso-bis-axillary cravat.

The Gluteo-femoral Triangle.-In applying this handkerchief, a cravat is first fastened around the waist, and a second handkerchief folded into a triangle has its base placed in the gluteo-femoral fold, and its extremities carried around the thigh and secured in front by a knot; the apex of the handkerchief is then carried upward and passed beneath the cravat around the waist, and is turned down and pinned to the body of the triangle (Fig. 35). This handkerchief may be used to retain dressings to the region of the buttock or hip ; by unpinning the apex and turning it downward, ready access can be had to the parts beneath. 
Gluteo-inguinal Cravat.-In applying this handkerchief, the base of the cravat is placed just over the gluteofemoral fold, and the extremities are carried forward, one

FIG. 34.

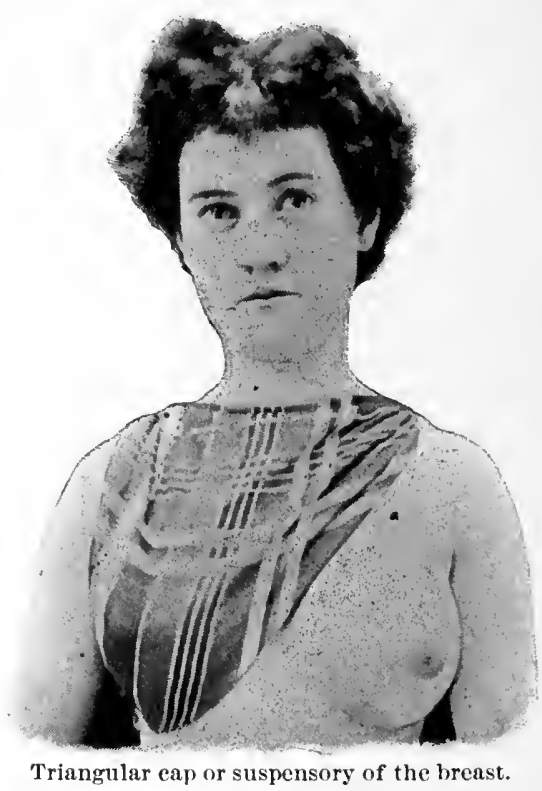

around the inner, the other around the outer portion of the thigh, and they are made to cross in the groin; the ends are next passed around the pelvis and secured together upon the back by a knot (Fig. 36). This handkerchief may be employed to hold dressings to the region of the groin.

By employing two cravats, a double gluteo-inguinal cravat may be applied, which may be used to hold dressings to both groins. The turns of these cravats correspond to the turns of the single and double spica-bandages of the groin.

I have described a few of the many ingenious bandages 
devised by Mayor to substitute the use of the rollerbandage, which will give the student some idea of their

FIG. 35.

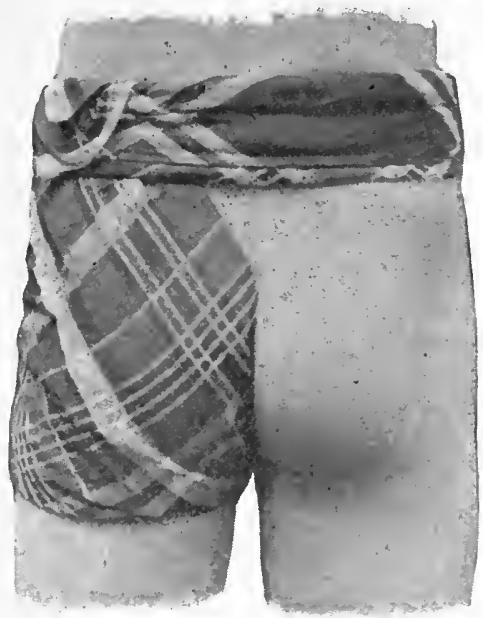

Gluteo-femoral triangle.

FIG. 36.

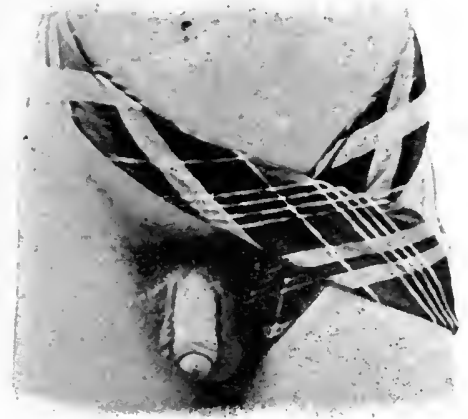

Gluteo-inguinal cravat.

design and application. It is well to bear in mind this system of dressing, for the occasion might oceur in which 
the ordinary means of bandaging could not be obtained, and the use of handkerchiefs might answer a useful purpose as temporary dressings. I think their prineipal use is for temporary dressings, and I do not believe they will ever take the place of the roller-bandage, which can be applied with greater nicety and exactness, and certainly presents a much neater appearance.

\section{BANDAGES OF THE HEAD.}

Barton's Bandage. Roller Two Inches in Width, Six Yards in Length.- The initial extremity of the roller should be placed on the head just behind the mastoid process, and the bandage shonld then be carried under the oceipital protuberance obliquely upward under and in front of the parietal eminence across the vertex of the skull, then downward over the zygomatic arch, under the ehin, thenee upward over the opposite zygomatic arch and over the top of the head, crossing the first turn which was made, as nearly as possible in the median line of the skull, and earrying the turns of the roller under the parietal eminence to the point of commencement. The bandage is then passed obliquely around under the oeeipital protuberance and forward minder the ear to the front of the chin, thence back to the point from which the roller started. These figure-of-eight turns over the head and the eireular turns from the occiput to the ehin should be repeated, each turn exactly overlapping the preeeding one until the bandage is exhausted (Fig. 37). The extremity should then be secured by a pin; and pins should be introduced at the points where the turns cross each other, to give additional fixation to the bandage. In applying the bandage, care should be taken to see that the turns overlap each other exactly, and that the turns passing over the vertex cross as nearly as possible in the median line of the skull (Fig. 38).

Modified Barton's Bandage.-To obtain additional security in the applieation of the Barton's bandage, a turn 
of the bandage passing from the occiput to the forehead may be made, this turn being interposed between the turns of the bandage as ordinarily applied (Fig. 39). In applying this bandage, after the first set of turns has been completed-that is, after the handage has been brought back to the occiput-the bandage is carried forward upon the head just over the ear, around the forehead and backward above the ear on the opposite side to the occiput; this being done, the ordinary figure-of-eight and circular

FIG. 37.

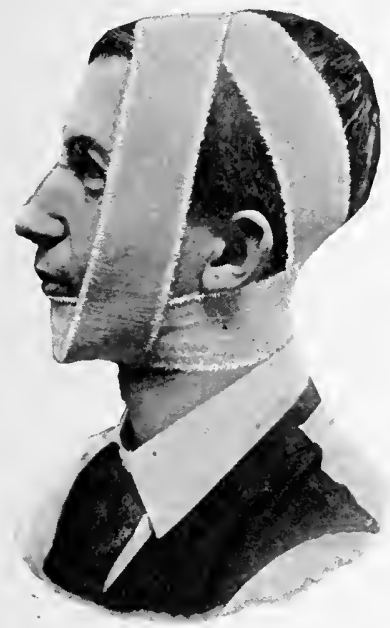

Burton's bandage.
Fit. 38.

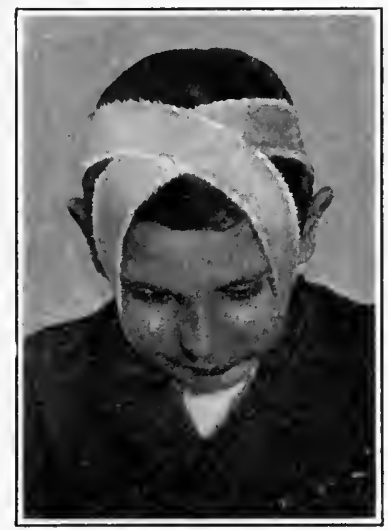

Barton's bandage, showing crossing of tulns at vertex.

turns are made, and when these have been completed another occipito-frontal turn may be made as described above, and this may be repeated as often as is desired until the bandage is exhausted, when the extremity is fastened with a pin, and pins are introduced also at all points at which the turns cross.

Use.-This bandage is one of the most useful of the 
bandages of the head, being employed to secure fixation of the jaw in cases of fracture or dislocation, and for the application of dressings to the chin. I have also employed it in place of the head-gear in slinging patients for the application of the plaster-of-Paris jacket in cases of disease of the spine, a stout cord or a piece of bandage about three inches wide and one yard long being passed under the turns crossing over the vertex ; this cord is then seenred to the cross-bar of the extension apparatus (Fig. 40). 'This will be found quite as comfortable to the patient as

Fra. 39.

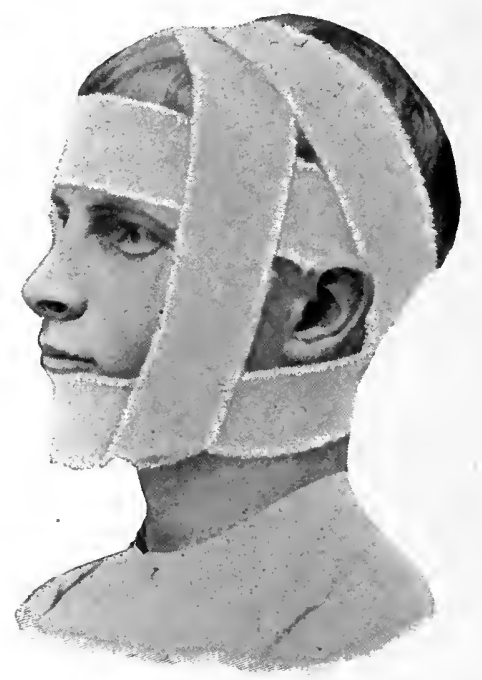

Modified Barton's bandage.

the ordinary head-gear employed, and much less likely to slip out of place and interfere with the breathing of the patient.

A firmly applied Barton's bandage holds the jaws so elosely together that care should be taken in applying it to patients who are uncler the influence of an anæsthetic, 
for if vomiting oeeurs the material may not eseape from the mouth, and suffocation might occur unless the bandage were promptly removed. This accident I once saw occur, and the patient's condition was alarming until the bandage was cut, allowing the jaw to be opened and the contents of the mouth to escape.

Gibson's Bandage. Roller Two Inches in Width, Six Yards in Lenyth.-The initial extremity of the roller should be placed upon the vertex of the skull in a line with the anterior portion of the ear; the bandage is then carried downward in front of the car to the chin, and passed under the chin, and is carried upward on the same line until it reaches the point of starting. The turns are repeated until three complete tums have been made; the bandage is then contimned until it reaches a point just above the ear, when it is reversed and is carried backward around the oeciput, and is eontinued around the head and forehead until it reaches its point of origin ; these cilcular turns are applied until three have been made. When the bandage reaches the occiput, having completed the third turn, it is allowed to drop down Fig. 40.

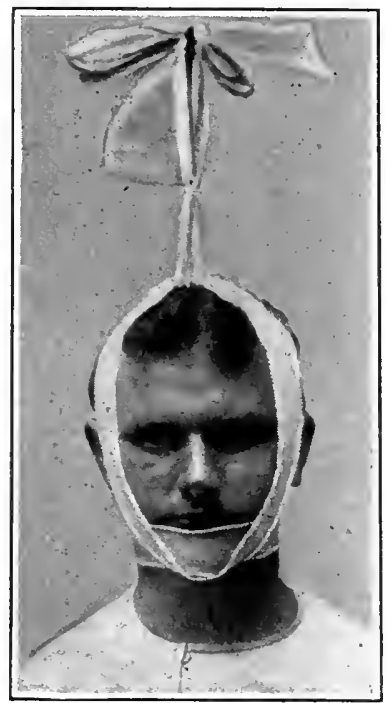

Barton's head-bandage, employed for suspension. to the base of the skull, and it is then carried forward below the ear and around the ehin, being brought back upon the opposite side of the head and neck to the point of origin; these turns are repeated until three complete turns have been made, and upon the completion of the third turn the bandage is reversed and carried forward over the occiput and vertex to the forehead, and its 
extremity is here secured with a pin. Pins should also be applied at the points where the turns of the bandage cross each other (Fig. 41).

Use.-This bandage may be used to fix the lower jaw in cases of fracture or dislocation of the jaw, but is very apt to change its position, and is, therefore, not so satisfactory as the Barton's bandage for this purpose.

FIG. 41.

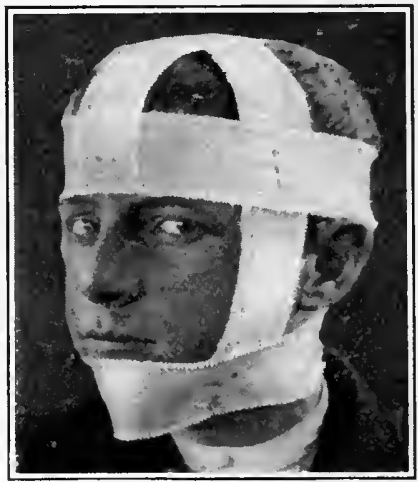

Gibson's bandage

Oblique Bandage of the Angle of the Jaw. Roller Two Inches in Width, Six Yards in Length.-The initial extremity of the roller is placed just in front of and above the left ear, and if the left angle of the lower jaw is to be covered in, the bandage is then carried from left to right, making two complete turns around the cranium from the occiput to the forehead; if, however, the right angle of the lower jaw is to be covered in, the turns should be made in the opposite direction. Having made two turns from the occiput to the forehead, the bandage is allowed to drop down upon the neck, and is carried forward under the ear and under the chin to the angle of the jaw; it is next carried upward close to the edge of the orbit, and obliquely over the vertex of the skull, then down behind the riglit ear, continuing this oblique turn under the chin to the left 
angle of the jaw, where it ascends in the same direction as the previous turn. Three or four of these oblique turns are made, each turn overlapping the preceding one and passing from the colge of the orbit toward the ear until the space is covered in; the bandage is then carried to a point just above the ear on the opposite side, is reversed, and finished with one or two circular turns from the occiput to the forehead, the extremity being secured by a pin (Fig. 42).

Use. - This will be found one of the most useful of the headbandages ; it may be used with a compress in treating fractures of the angle of the lower jaw, for holding dressings to the lower part of the chin and to the vault of the cranium, and is especially useful in retaining dressings to the sides of the face and the parotid region. As before stated, it may be applior to cover either the right or loft side of the face, and, by reason of the oblique turns, holds its

Fig. 42.

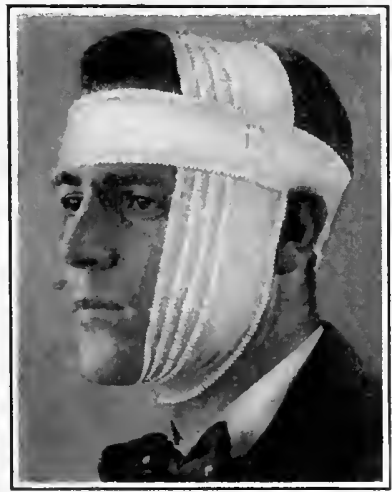

Oblique bandage of the angle o: the jaw.

position most securely, having little tendency to become displaced.

Recurrent Bandage of the Head. Roller Two Inehes in Wirlth, Six Yards in Length.-The initial extremity of the roller is placed upon the lower part of the forehead and the bandage is carried twice around the head from the forehead to the occiput to secure it. When the bandage is brought back to the median line of the forehead it is reversed, and the reversed turn is hold by the finger of the left hand while the roller is carried over the top of the head along the sagittal suture to a point just below the occipital protuberance; here it is reversed again, and the reverse is held by an assistant while the roller is carried back to the forehead in an elliptical course, each turn cov- 
ering in two-thirds of the preceding turn. These turns are repeated with snecessive reverses at the forehead and occiput until one side of the head is completely covered in, and when this is accomplished a cireular turn is made from the forehead to the occiput to hold the reverses in place.

The opposite side of the hearl is next covered in by elliptical reversed turns made in the same manner, and when this has been accomplished two or three circular turns are carried around the head from the forehead to the occiput, to fix the preceding turns. Pins should be applied at the

FIG. 43.

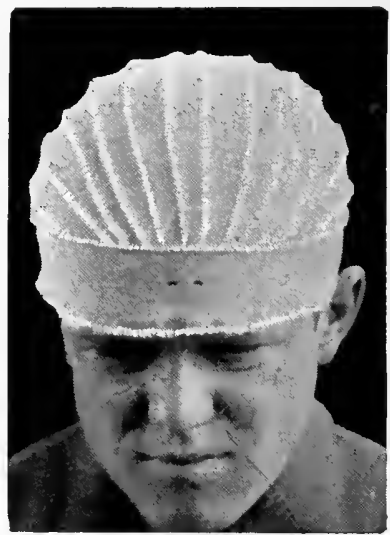

Recurrent bandage of the head.

forehead and oceiput at the points where the reversed turus concentrate (Fig. 43).

Use.-This bandage when well applied is one of the neatest of the head-bandages, and it will be found useful to retain dressings to the vault of the cranium in the treatment of wounds of the scalp in this region. It will also be found of service in holding dressings to fractures of the cranium and to wounds after the operation of trephining. In restless patients it will sometimes become displaced, and it may be rendered more secure by piuning a strip of bandage to the circular turn in front of the ear and earrying 
it down under the chin and $\mu$ to a corresponding point on the opposite side, where it is pinned to the circular turn ; or one or two oblique turns passing from the circular turn over the vertex of the skull downward behind the ear, under the chin and up to the circular turn in front of the ear, may be applied. The course of these turns is the same as those employed in the oblique bandage of the angle of the jaw, the extremity being secured by a pin.

Transverse Recurrent Bandage of the Head. Roller Two Inches in Width, Six Yards in Length.-The initial extremity of the roller is placed upon the lower part of the forehead and the bandage is carried twice around the head from the forehead to the occiput to secure it. 'The hear is then eovered in by transverse turns of the bandage; the first turn, starting from a point behind the ear on one side, is carried below the occiput to $\mathrm{a}^{\text {. }}$ corresponding point behind the opposite ear, and aseending transverse turns are then made and carried over the head, each turn covering in about twothirds of the preceding turn, until the forehead is reached, and when this has been reached two or three circular turns are

FIG. 44.

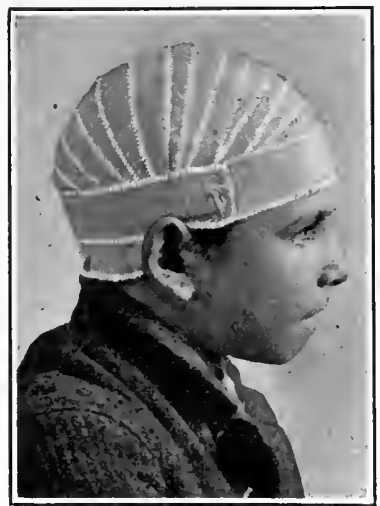

Transverse recurrent bandage of the head. carried around the head from the forehead to the oceiput to fix the recurrent turns. Pins shonld be applied at the points of starting and finishing of the reversed turns behind the ears, and at the occiput and forehead (Fig. 44).

Use.-This bandage may be employed to secure dressings to the sealp in cases of wounds or in injuries to the skull, and is used for the same purposes as the recurrent bandage of the head.

V-bandage of the Head. Roller Troo Inches in Width, Four Yards in Length.-The initial extremity of the roller 
is secured by two turns of the bandage around the cranium from the forchead to the occiput, and when the roller reaches the occipital protuberance it is allowed to drop a little below this, and is carried forward below the ear around the front of the chin and lower lip, then backward to the point of starting. 'These turns passing from the occiput to the forchear and from the occiput to the chin are alternately made until a sufficient number have been applied, and the extremity is secured by a pin over the occiput (Fig. 45).

FIG. 45.

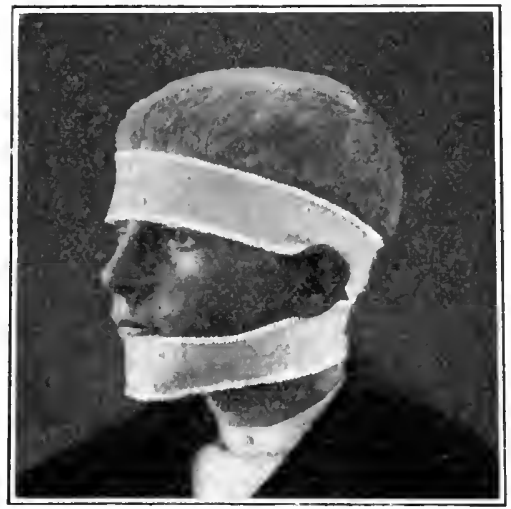

v-bandage of the hend.
FIf. 46.

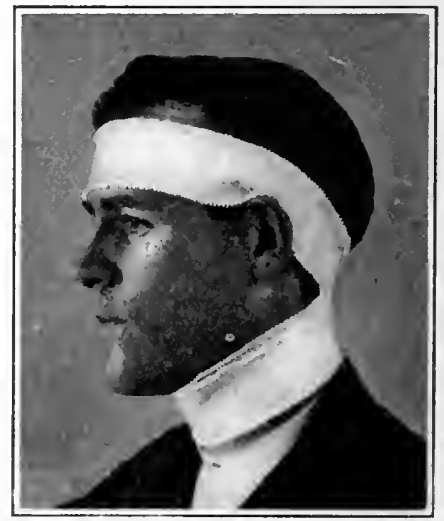

Ifead-and-neck bandage.

This bandage mav be modified by carrving the turns from the occiput forward under the ear and around the spper lip and back to the occiput, and alternating these turns with the occipito-frontal turns; if employed in this way, a bandage of one and one-half inches in width should be used.

Use.-This bandage may be employed to hold dressings to the front of the chin, to the upper and lower lips in cases of wounds, or to give support to these parts after plastic operations.

Head-and-neck Bandage. Roller Two Inches in Width, Four Yards in Length.-The initial extremity of the roller is placed upon the forehead and carried backward just 
above the ear to the occiput, and is then brought forward around the opposite side of the head to the point of starting. Two of these circular turns are made to fix the bandage, and when it is carried back to the occiput it is allowed to drop down slightly upon the neck, and is then carried around the neck, the turns around the head alternating with the neck-turns until a sufficient number of these have been applied, when the extremity of the bandigre is secured by a pin at the point of crossing of the turns at the back of the head (Fig. 46).

Use.-This bandage may be found useful in securing dressings to the anterior or posterior portion of the neck or to the region of the occiput. Care should be taken to apply it in snch a manner that too much pressure is not made by the turns around the neck, which would be uncomfortable to the patient, and might seriously interfere with respiration.

Crossed Bandage of One Eye. Roller Two Inches in Width, Four Yards in Length.-The initial extremity of the bandage is placed upon the forehear and fixed by two circular turns passing around the head from the occiput to tho forehead; the roller is then carried back to the occiput and passed around this and brought forward below the ear, and passing over the outer portion of the cheek is earried upward to the junction of the nose with the forehead, and is then conducted over the parietal eminence downward to the occiput; a circular fronto-occipital turn is next made, and when the bandage is brought back to the occiput it is brought forward again

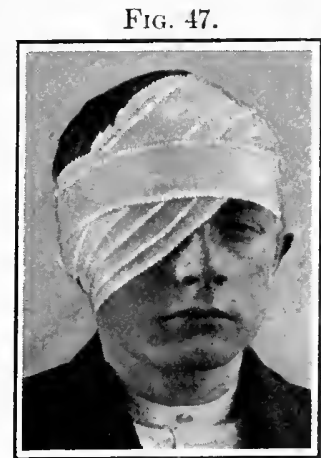

Crossed bandage of one eye. to the cheek. It should then ascend to the forehead, covering in two-thirds of the preceding turn, and again be conducted back to the occiput; these turns are repeated, the oblique turns covering the eye alternating with circu- 
lar turns around the head until the eye is completely enclosed (Fig. 47). and the bandage is finished by making a circular turn about the head and introducing a pin to secure its extremity. It will be found more comfortable to the patient to include in the turns of the bandage the ear on the same side on which the eye is covered.

Use.-This bandage will be found useful in retaining dressings to one eye. It will be more comfortable to the patient if a flamnel roller be used to apply this bandage, as well as the bandage which includes both eyes.

Crossed Bandage of Both Eyes. Roller. Two Inches in Width, Six Yards in Length.- The initial extremity of the roller is placed upon the forehead and secured by two circular turns of the bandage passing around the head from the foreheal to the occiput ; the roller is then carried downward behind the oceiput and brought forward below the ear to the upper portion of the cheek; it is then carried upward to the junction of the nose with the forehead and conducted over the parietal eminence to the occiput;

Fi(i. 48.

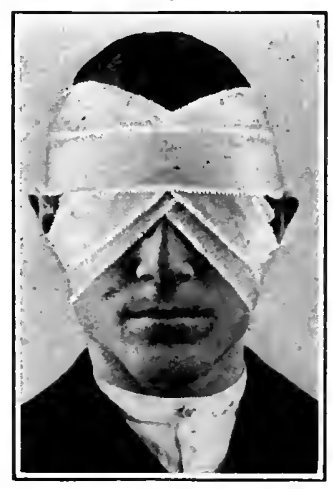

Crossed bandage of both eyes. a circular turn is now made around the head from the occiput to the forehead, and the roller is carried from the occiput over the parietal eminence of the opposite side forward to the junction of the- nose with the forehead, then downward over the eye and outer portion of the cheek below the ear and back to the occiput; a circular turn around the head is next made, and this is followed by a repetition of the previous turns, ascending over one eye, descending over the other eye, each turn alternating with a circular turn around the head. These turns - are repeated until both eyes are covered in, and the bandage is finished by making a circular turn around the head, the extremity being secured by a pin (Fig. 48). In this bandage both ears may be covered in or left-uncovered. 
Use.-This bandage may be used to apply dressings to both eyes, and both of these bandages covering the eyes are used where it is desired to make pressure; but for the simple application of a light dressing or of a bandage for the exclusion of light, the Liebreich's bandage (Fig. 91) will be found more comfortable to the patient.

Occipito-facial Bandage. Roller. Two Inches in Width, Four Yards in Length.- The initial extremity of the roller is placed upon the vertex of the head and the bandage is carried downward in front of the ear, under the jaw, and upward upon the opposite side in the same line to the vertex ; two or three of these turns are made, one turn accurately covering in the other. A reverse should be made just above and in front of the ear, and two or three turns are then made around the head from the occipnt to the forehead, which completes the bandage (Fig. 49). Pins should be inserted at the points where the turns of the bimdage cross each other.

Use.-This bandage is employed to secure dressings to the vertex, temporal, occipital, or frontal region.

FIG. 49.

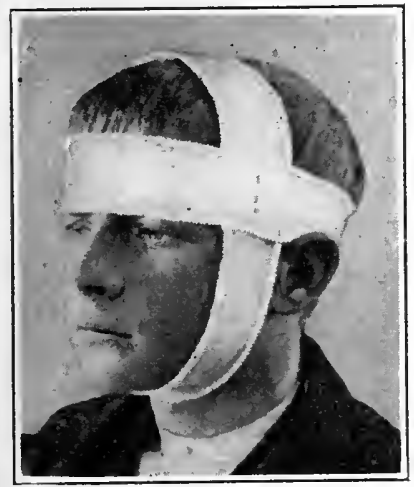

Occipito-facial bandage.
FIG. 50.

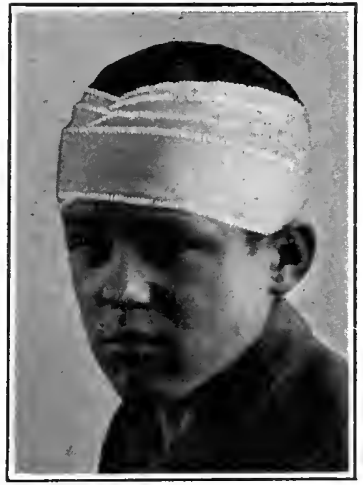

Oblique bandage of the head.

Oblique Bandage of the Head. Roller Two Inches in Width, Six Yards in Length. - The initial extremity of the bandage is placed upon the forehead, and is secured by 
two circular turns passing around the head from the forehead to the occiput. From the occiput the bandage is carried obliquely over the highest part of the lateral aspect of the head, which is to be covered in, and is passed over the forehead and back to the occiput. It is then carried to the forehead by a circular turn, which is conducted obliquely over the other side of the head and back to the occiput. A circular turn from the occiput to the forehead should be made between the oblique turns. These turns are repeated, so that each sueceeding turn cover's in threefourths of the preceding turn until the sides of the head are coverel in by descending turns, and the bandage is eompleted by a circular turn passing around the head from

FiG. 51.

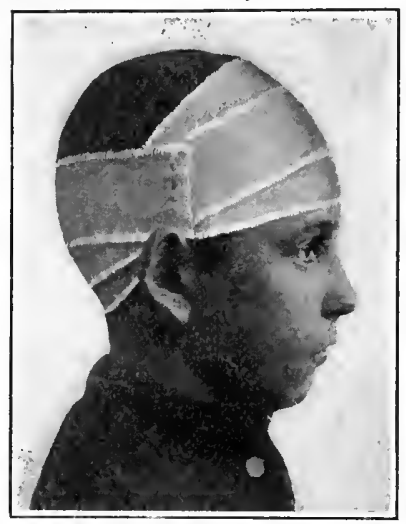

Occipito-frontal bandage. the forehead to the occiput (Fig. 50). This bandage may be applied with descending or ascending turns.

Use.-This bandage is employed to make pressure upon or to hold a dressing to the lateral aspects of the head.

Occipito-frontal Bandage. Roller Two Inches in Width, Four Yards in Length.-The initial extremity of the roller is placed upon the forchead, and a circular turn is made around the forehead and oceiput to fix it. A circular turn is then made, passing around the head from a point below the occiput to a point just above the forchead; the next circular turn is made around the head aseending posteriorly and descending anteriorly, and after a sufficient number of these turns have been made to cover in the front and back of the head the end of the bandage is secured with a pin (Fig. 51).

Use.-This bandage will be found useful in securing dressings to the forehead and anterior and posterior portions of the sealp. 


\section{BANDAGES OF THE UPPER EXTREMITY.}

Spiral Bandage of the Finger. Roller One Inch in Width, One and a Half Yards in Length-The initial ex-

FIG. 52.

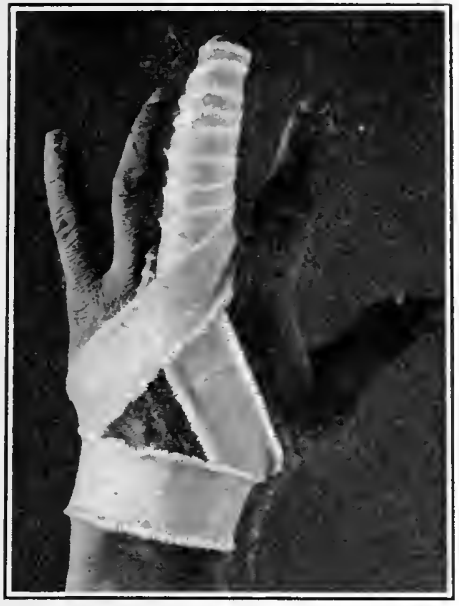

Spiral bandage of the finger.
Fig. 53.

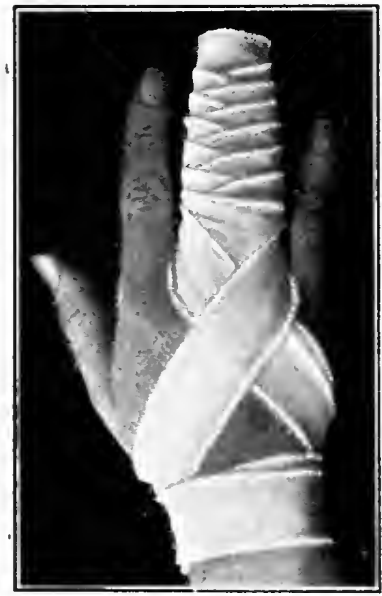

Spiral reversed bandage of fingers.

tremity of the roller is secured by two or three turns around the wrist; the bandage is then carried obliquely across the back of the hand to the base of the finger to be covered in, then to its tip by oblique turus; a circular turn is next made, and the finger is covered by ascending spiral or spiral reversed turns until its base is reached; the bandage is then carried obliquely across the back of the hand and finished by one or two circular turns around the wrist; the extremity may be pinned or may be split into two tails which are tied around the wrist (Fig. 52).

Use.-This bandage is employed to retain dressings to injuries or wounds upon the finger, and to secure splints in the treatment of fractures or dislocations of the phalanges. 
Spiral Reversed Bandage of Fingers. Roller One and a Half Inches in Width, One and a Half Yards in Length.- The initial extremity of the roller may be secured by two or three turns around the wrist; the bandage is then carried obliquely across the back of the hand to the base of the fingers to be covered in, then to their tips by oblique turns; a circular turn is next made and the fingers are covered in spiral reversed turns until their bases are reached; the bandage is then carried obliquely across the back of the hand and finished by one or two eircular turns around the wrist; the end of the bandage may be secured by a pin or split into two tails which are tied around the wrist (Fig. 53).

This bandage may also be applied so as to cover the fingers alone by making one or two circnlar turns around the base of the fingers and then carrying the bandage obliquely down to the tips of the fingers, there making a circular turn and then conducting it back to the base of the fingers by spiral reversed turns, and at this point it is secured by a pin or ly tying. This bandage does not hold its position as securely as the one first described.

Use.-This handage is employed to secure dressings to two or more fingers or to retain a splint in contact with them.

Gauntlet Bandage. Roller One Inch in Width, Three Yards in Length.- The initial extremity of the roller is fixed at the wrist by one or two circular turns of the bandage; it is then carried down to the tip of the thumb by an oblique turn of the roller, and this is covered in by spiral or spiral reversed turns to the metacarpo-phalangeal artieulations; the roller is then earried back to the wrist and a circular turn is made around it. The bandage is then carried down to the tip of the index finger by an oblique turn, which is covered in the same manner. When all the fingers have been eovered in, the bandage is finished by circular turns around the hand and wrist (Fig. 54).

Use.-This bandage nay be employed to apply dressings to the fingers and hand in eases of wounds or fractures. It was formerly much employed in the treatment of burns of the fingers to prevent the opposed ulcerated surfaces 
from adhering, but its use for this purpose has been supplanted by wrapping each finger in a separate dressing and applying a bandage over all the fingers and the hand with a few reeurrent and spiral turns of a wide roller, the application of this dressing being mueh less painful to the patient,

FIG. 54.

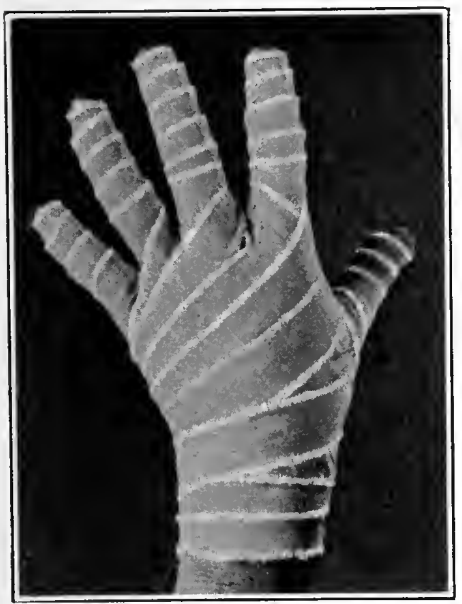

Gauntlet bandage.
FIr. 55.

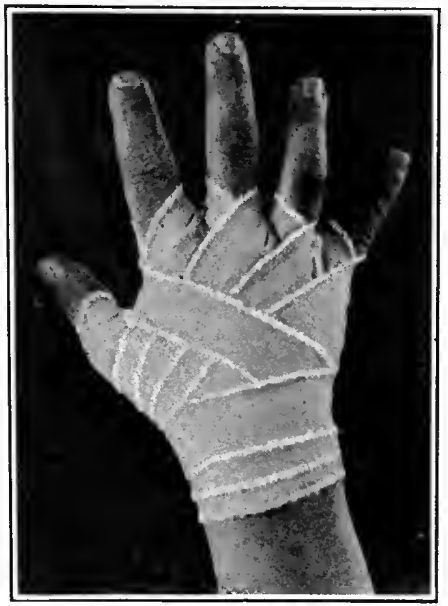

Demi-gauntlet bandage.

and being at the same time equally satisfaetory in its results.

Demi-gauntlet Bandage. Roller One Inch in Width, Four Yards in Length.-The initial extremity of the bandage should be placed upon the wrist and fixed by two cireular turns passing from the radial to the ulnar side; then carry the roller obliquely across the back of the hand to the base of the little finger, pass the bandage around this and carry the roller back to the wrist, making a eircular turn ; it should then be carried obliquely aeross the hand to the base of the ring finger, and so suecessively until the base of each of the fingers and of the thumb has been included; the bandage is then completed by an oblique turn across the baek of the hand, passing between the index finger and the thumb, and a cireular turn around the wrist (Fig. 55). 
The demi-gauntlet bandage may also be applied in such a manner as to cover over the palm and leave the dorsum of the hand uncovered.

Use.-This bandage may be employed to retain light dressings to the dorsal or palmar surface of the hand.

FIG. 56.

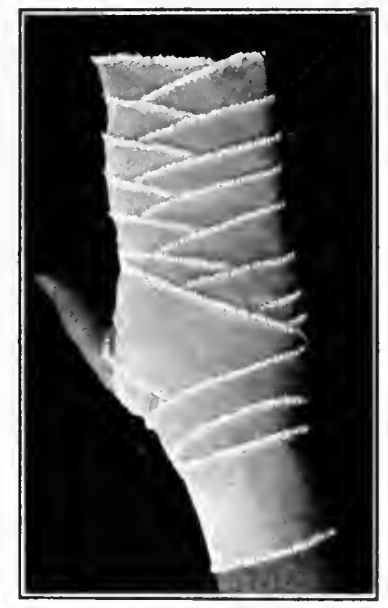

Complete bandage of hand.
FIG. 57.

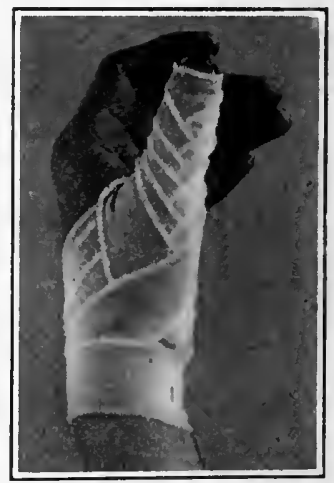

Spica-bandinge of the thumb.

Complete Bandage of Hand. Roller Tuo Inches in Width, Tuo Yards in Length.-The initial extremity of the bandaga is placed upon the wrist and the hand is covered in by three or four recurrent turns, and these are secured by a circular turn around the wrist. The bandage is then carried obliquely across the back of the hand to the tip of the index finger, when a eircular turn is made around the tip of the fingers; the bandage is then carried upward by spiral or spiral reversed turns covering in all of the fingers and the thumb as well as the body of the hand, and is completed by one or two circular turns around the wrist. This bandage may also be applied so as to leave the thumb uncovered (Fig. 56).

Use.-This bandage may be employed to secure dressings to the hand. 
Spica-bandage of the Thumb. Roller One Inch in Width, Three Yards in Length.-The initial extremity of the roller is placed upon the wrist and fixed by two circular turns ; then carry the roller obliquely over the dorsal surface of the thumb to its distal extremity; next make a circular or spiral turn around the thumb, and carry the bandage upward over the back of the thumb to the wrist, around which a circular turn should be made. The roller is then carried around the thumb and wrist, making figureof-eight turns, each turn overlapping the previous one two-thirds as it ascends the thumb, and each figure-ofeight turn alternating with a circular turn around the wrist. These turns are repeated until the thumb is completely covered in with spica-turns; a circular turn around the wrist finishing the bandage (Fig. 57 ).

FIG. 58.

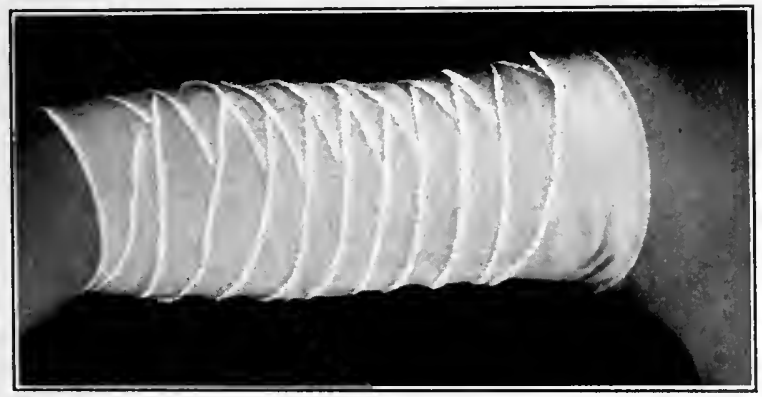

Spiral reversed bandage of arm.

Use.-This bandage is employed to apply dressings to the dorsal surface of the thumb and for the retention of splints in the dressing of fractures or dislocations of the bones of the thumb.

Spiral Reversed Bandage of Arm. Roller Two and a Half Inches in Width, Five Yards in Length.-The initial extremity of the bandage is sceured by one or two cireular turns around the arm just above the elbow and is then carried up the arm by spiral reversed turns until the axilla is reached where the extremity is secured. Care 
should be nsed in applying this bandage that it is not applied so firmly that it eanses venous obstruction and swelling of the forearm (Fig. 58).

Use.- This bandage is employed to secure dressings or splints to the arm.

Spiral Reversed Bandage of the Upper Extremity. Roller Tuo and a Half Inches in Width, Seven Yards in Length.-The initial extremity of the roller is placed upon the wrist, and secured by two turns around the wrist; the bandage is then earried obliquely across the back of the hand to the second joint of the fingers, where a circular turn should be made; the hand is covered in by two or

Fis. 59.

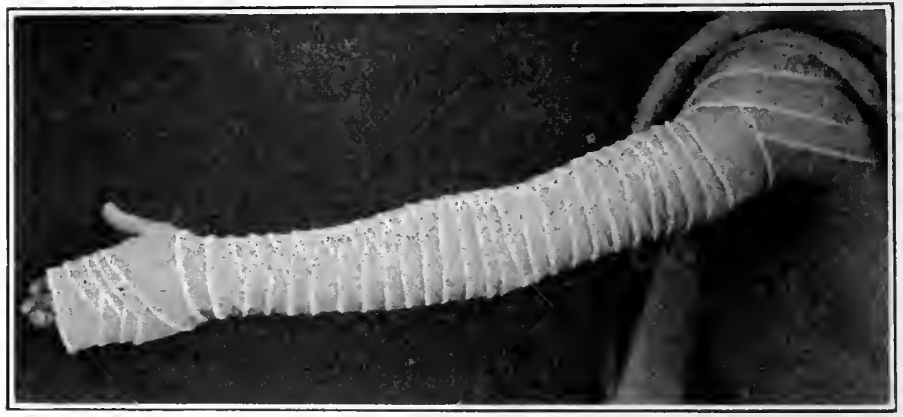

Spiral reversed bandage of the upper extremity.

three ascending spiral or spiral reversed turns. When the thumb has been reached, its base and the wrist are covered in by two figure-of-eight turns ; the bandage is then carried up the forearm by spiral and spiral reversed turns until the elbow is reaehed; this may be covered in with spiral reversed turns, and the bandage is next carried up the arm with spiral reversed turns to the axilla (Fig. 59). If, on reaching the elbow, the arm is bent, or is to be flexed in the subsequent dressing, the elbow should be eovered in with figure-of-eight turns, and when this has been done the arm may be covered in with spiral reversed turns. When properly applied, the reverses should be in line, and shoukl not be made over the prominent ridge of the ulna. 
Use.-This is one of the most generally employed of all the roller-bandages; it constitutes the primary roller which is applied in the dressing of fractures of the humerus, and it is also the bandage employed in holding dressings to the arm and forearm and in seeuring splints to these parts in the treatment of fiactures and dislocations.

Figure-of-eight Bandage of the Elbow. Roller Two Inches in Width, Four Yards in Length.-The initial extremity of the bandage is placed upon the forearm a short distance below the elbow-joint, and fixed by one or two

FIf* 60.

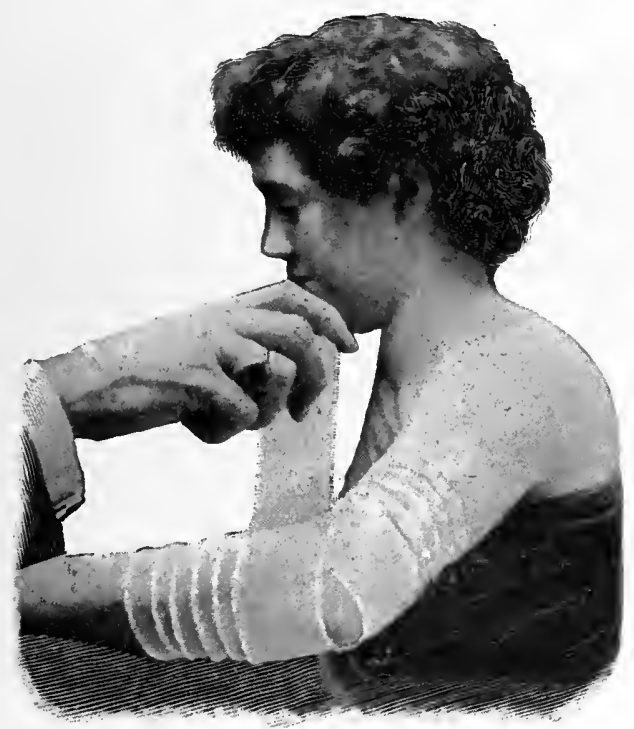

Flgure-of-eight bandage of the elbow.

circular turns, the arm being flexed. The bandage is then carried by an oblique turn across the flexure of the ellowjoint, and passed around the arm a few inehes above the elbow; a circular turn is then made, and the roller is next earried across the flexure of the elbow and passed around the forearm. These turns are repeated, the turns from the 
forearm ascending and those from the arm descending, each set of turns crossing in the flexure of the elbow until it is covered in, and a final turn is passed circularly around the elbow joint (Fig. 60). This bandage is sometimes applied by first making one or two circular turns around the elbow and then applying the figure-of-eight turns as previously described (Fig. 61).

Use. - This bandage is often employed as a part of the spiral reversed bandage of the upper extremity when the arm is to be flexed, and is also used to hold dressings to the region of the elbow-joint. It was formerly much used

Fig. 61.

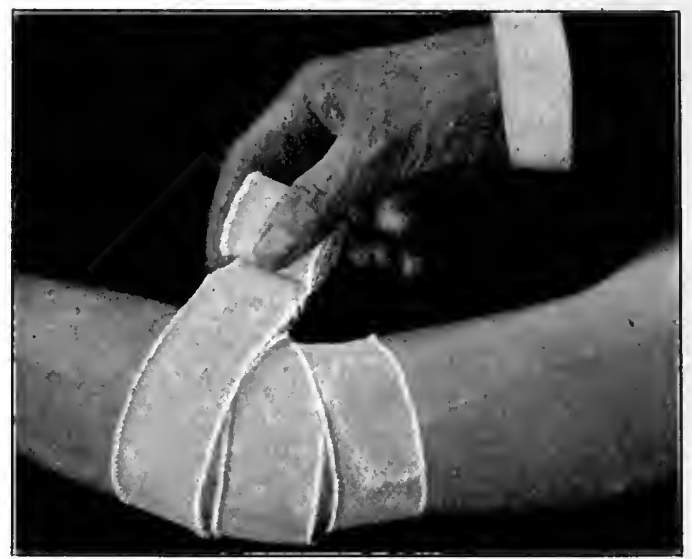

Figure-of eight bandage with primary turns around the elbow.

to hold the compress upon the wound resulting from veneseetion at the elbow.

Spica-bandage of the Shoulder (Ascending). Roller Two and a Half Inches in Width, Seven Yards in Length. -The initial extremity of the roller is placed obliquely upon the outer surface of the arm opposite the axillary fold, and fixed by one or two circular turns. If the right shoulder is to be covered, the bandage is next carried across the front of the chest to the axilla of the opposite side, 
then around the back of the chest to the point of starting upon the arm; then the roller should be conducted around the arm of this side up over the shoulder, across the front of the chest, through the opposite axilla, and back over the posterior surface of the chest to the point of starting ; continue to make these ascending turns, each turn overlapping the preceding one about two-thirds until the shoulder is covered in (Fig. 62), when the extremity of the bandage may be secured by a pin at the point of ending, or the last turn may be carried from the shoulder around the back of the neck and brought forward over the opposite shoulder

HIG. 62.

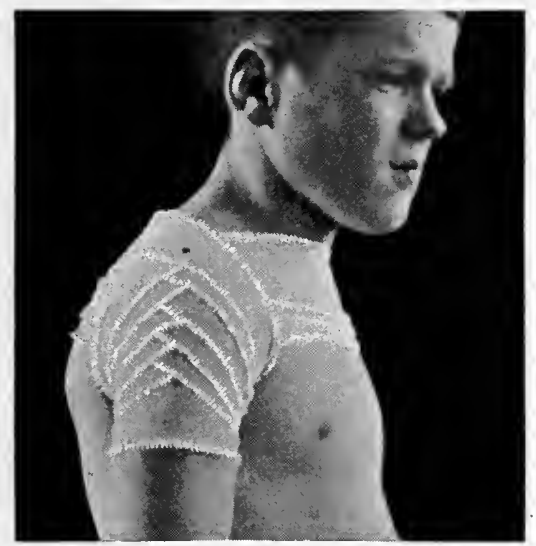

spıca-bandage of the shoulder (ascending).

and pinned to the turus which pass around the axilla. It should be remembered that the turns of the roller overlap each other exactly in the opposite axilla, and it will be found more comfortable to the patient to place a little cotton-wadding in the axilla to prevent the bandage from excoriating the skin of this part. Care should be taken to see that the turns are made in such a manner that the spica-turns occupy as nearly as possible the median line of the shoulder. When this bandage is applied to the left shoulder, after fixing the initial extremity by circular turns around the arm, the roller should be carried over 
the back of the ehest to the axilla of the opposite side and then brought back to the point of starting; the succeeding turns are then applied in the same manner.

Spica-bandage of the Shoulder (Descending). Roller Two and a Half Inches in Width, Seven Yards in Length.The initial extremity of the roller should be fixed upon the arm as near as possible to the axillary fold by one or two circular turns ; and if it is applied to the right shoulder, the bandage should be passed under tlec axilla and carried obliquely over the shoulder to the base of the neck,

\section{FIG. 63.}

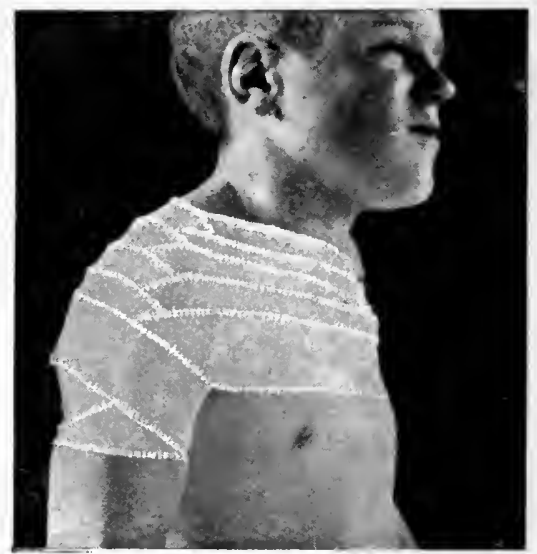

Spica-bandage of the shoulder (descending).

then downward across the front of the chest to the axilla of the opposite side; from the axilla the roller is carried over the back of the chest to the base of the neck, so as to cross the first turn at this point; it is then carried through the axillary space, then back to the neck, the turns descending toward the shoulder. These turns, taking the same course, are repeated, each turn overlapping twothirds of the previous one until the shoulder is covered in and the circular turn around the arm is reached, at which point the extremity is secured by a pin (Fig. 63).

Use.-The spica-bandages of the shoulder are employed 
to hold dressings to the shoulder, to hold compresses over the acromial end of the clavicle in dislocation of that portion of the bone, to retain the shoulder-cap used in the treatment of fractures of the upper portion of the humerus, and to retain dressings to the axilla.

Fig. 64 .

\section{Figure-of-eight Bandage} of the Neck and Axilla. Roller Two Inches in Width, Five Yards in Length.-The initial extremity of the roller is fixed upon the side of the neck and secured by one or two loosely applied circular turns; if applied to the right axilla, carry the bandage from left to right over the right shoulder to the posterior part

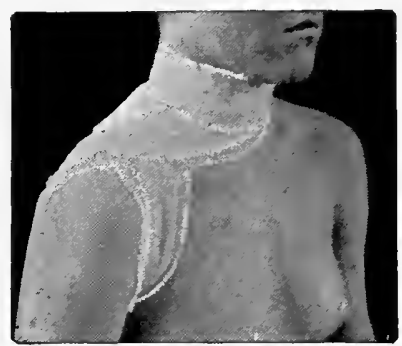

Figure-of-eight bandage of the neck and axilla. of the axilla under which it passes, to ascend in front over the same shoulder to the back of the neck; these figureof-eight turns around the neck and axilla, each turn overlapping two-thirds of the preceding turn, are repeated until the desired space is covered and the bandage is completed by a circular turn around the neck (Fig. 64).

Use.-This will be found a useful bandage to secure dressings to the base of the neck, the upper part of the shoulder, and to the axilla, as it does not restrict the motions of the arm unless drawn too tight.

Velpeau's Bandage. Two Rollers Two and a Half Inches in Width, Seven Yards in Length.-The patient should place the fingers of the hand of the affected side on the opposite shoulder; the initial end of the roller should be placed on the body of the scapula of the sound side and secured by a turn made by carrying the bandage over the shoulder of the affected side, near its outer portion, then conducting it downward over the onter and posterior surface of the arm of the same side, behind the point of the elbow, and oblicuely across the front of the ehest to the axilla of the opposite side, thence to the point of ;tart- 
ing. This turn should be repeated, to fix the initial extremity of the bandage. Having completed the second turn, carry the roller transversely around the thorax, passing over the flexed elbow of the affected side, from this point to the axilla, and through this to the back. From this point the roller is carried over the shoulder and down the outer and posterior surface of the arm behind the elbow, and obliquely across the front of the chest through the axilla to the back, and, continuing, passes transversely

Fig. 65.

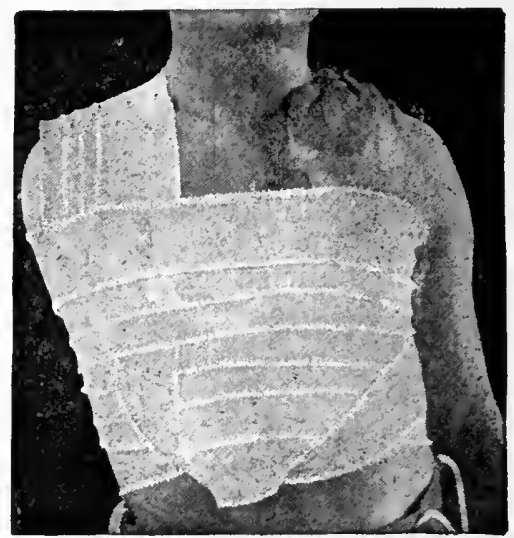

Velpeau's bandage.

across the back of the chest to the elbow, which it encircles, and then passes to the axilla. These alternating turns are repeated until the arm and forearm are bound firmly to the side and chest. The vertical turns over the shoulder, each turn covering in two-thirds of the previous turn and ascending from the point of the shoulder toward the neck and from the posterior surface of the arm toward the elbow, are applied until the point of the elbow is reached. The transverse turns passing around the chest and arm are so applied that they ascend from the point of the elbow toward the shoulder, each turn covering in 
one-third of the previous one, and the last turn should pass transversely around the shoulder and chest, covering the wrist (Fig. 65).

The extremity of the bandage should be secured by a pin where it ends, and additional fixation will be secured by introducing a number of pins at the points where the turns of the bandage cross each other.

Use.-This bandage is employed to fix the arm in the treatment of certain fractures of the clavicle and scapula ; also to secure fixation of the humerus after the reduction of dislocations of the shoulder-joint.

Desault's Bandage. Three Rollers Two and a Half Inches in Width, Seven Yards in Length.-A wedge-shaped pad to fit in the axilla is also required. These rollers are known as the first, second, and third rollers.

First Roller of Desault's Bandage.-Before applying the first roller the arm of the patient on the injured side should

Fig. 66.

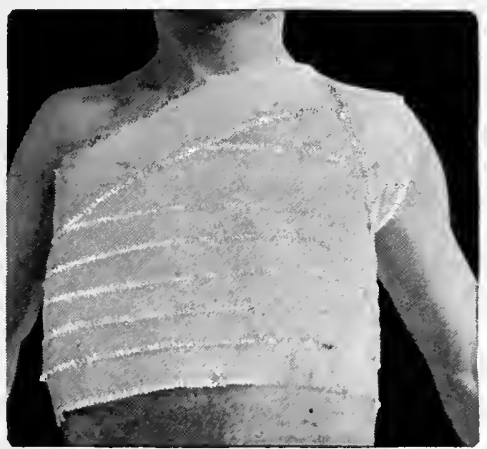

First roller of Desault's bandage.

be elevated and carried off at right angles to the body ; the wedge-shaped pad with its base in the axilla should next be applied to the side of the chest, and the initial extremity of the roller should be placed upon the middle of the pad, which may be fixed by two or three circular turns around the chest; the bandage is then carried down the 
chest by oblique circular turns until the lower extremity of the pad is reaehed, and it is then carried up the ehest by spiral turns until the npper extremity of the pad is reached, when it is conducted obliquely across the front of the chest to the sound shoulder and passed under the axilla, brought over the shoulder and condueted around the ehest, where it is secured (Fig. 66).

Second Roller of Desault's Bandage.-The arm should be brought down against the side so as to press upon the pad previously applied, and the forearm should be flexed upon the arm and brought across the lower portion of the chest.

FIG. 67.

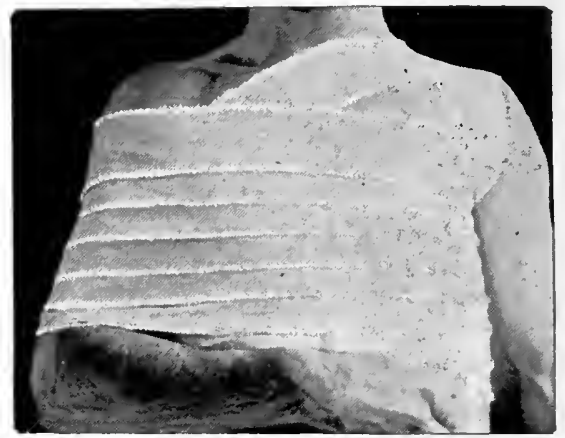

Second roller of Desault's bandage.

The initial extremity of the roller is placed in the axilla of the sound side, and the bandage is carried around the chest and over the arm of the injured side, making a eircular turn around the chest to fix it; then spiral turns are made around the chest from above downward until the elbow is reached, the turns being more firmly applied as they descend, and when this point is reached the end of the bandage is secured. Or the initial extremity of the bandage may be placed upon the chest of the sound side and a eircular turn may be made to fix it, and then spiral turns, including the chest and arm, may be made from below upward until the axilla is reached (Fig. 67). 
Third Roller of Desault's Bandage.-The initial extremity of the roller is placed in the axilla of the sound side, and the bandage is carried obliquely over the front of the ehest to the shoulder of the injured side, passed over this, and conducted down the back of the arm to the elbow, thence obliquely upward over the upper fifth of the forearm to the axilla of the sound side. From this point it is carried backward obliquely over the back of the chest to the shoulder; crossing the previous shoulder-turn, it is conducted lown the front of the arm to the elbow, then around this and backward obliquely over the hack of the chest to the

Fig. 68.

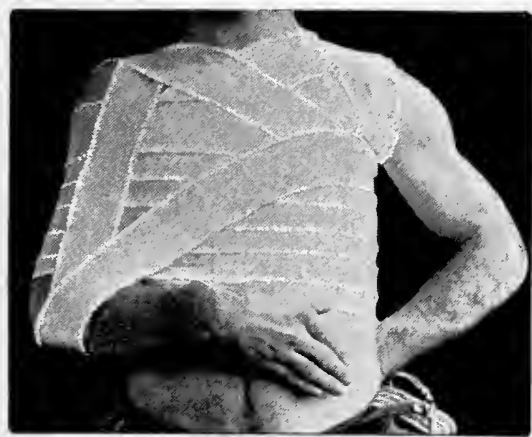

Third roller bf Desault's bandage.

axilla of the sound side. These turns are repeated until three sets of turns have been applied, which should overlie each other exactly (Fig. 68). The course of the turns of the third roller is considered the most difficult to remember, and the student may be assisted in its correct application by remembering that all the turns start at the axilla, pass to the shoulder, and then to the elhow, and from the elbow always return to the starting-point-the axilla. The turns of the third roller make two triangles, one on the anterior surface of the chest (Fig. 69), the other upon the back (Fig. 70).

After the application of the three rollers the hand and 
uncovered portion of the forearm should be supported in a sling suspended from the neck.

Use.-This bandage, applied completely, or some one of its various rollers, is employed in the treatment of fractures of the clavicle.

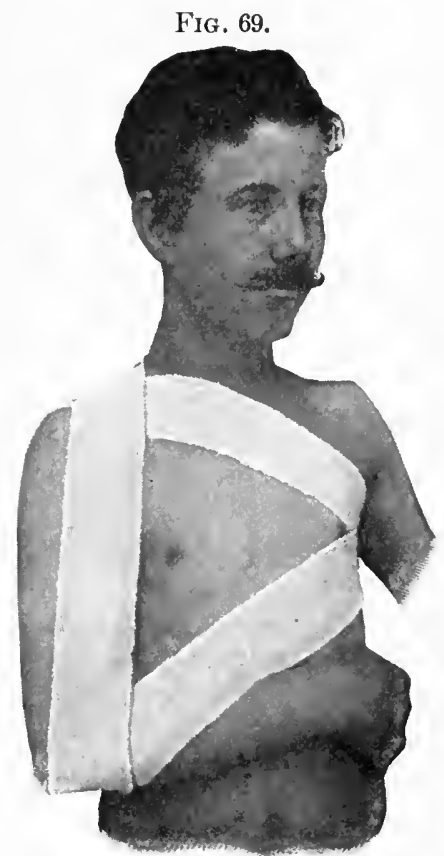

Anterior view of the turns of third roller of Desault's bandage.

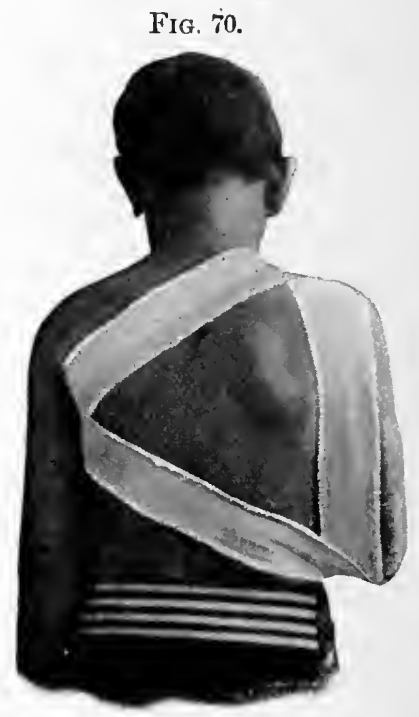

Posterior view of the turns of third roller of Desault's bandage.

Arm-and-chest Bandage. Roller Two and a Half Inches in Width, Seven Yards in Length.-Before applying this bandage, the arm should be placed against the side of the chest and a folded towel or a pad of cotton should be placed in the axilla and allowed to extend from the axilla to the elbow; the latter is used to prevent the opposing surfaces of skin from becoming excoriated by contact. 
The initial extremity of the bandage is placed upon the spine at a point opposite the elbow-joint, and it is fixed by a turn or two passing around the arm and chest; the bandage is then continued by making ascending spiral turns, covering in the arm and chest until the axilla is reached; at this point the bandage is carried through the axilla of the sound side and over the back of the chest to the top of

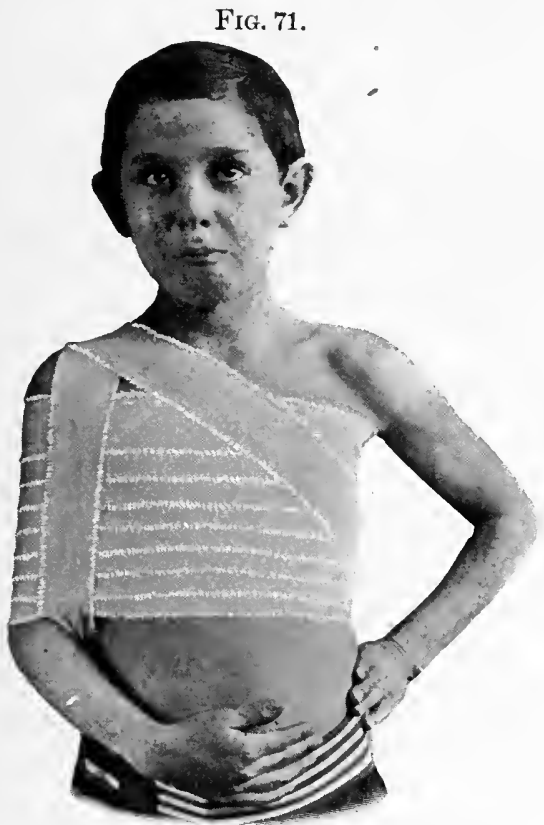

Arm-and-chest bandage.

the opposite shoulder, and it is then conducted down the front of the arm to the elbow, is passed between the arm and chest, and carried up the back of the arm to the shoulder. It is then passed obliquely across the front of the chest, and is secured upon the back of the chest. Pins should be introduced at the points of crossing of the bandage (Fig. 71). 
Use.-This bandage will be found useful in fixing the arm to the body and in fixing the shoulder-joint where it is desirable to allow the forearm to be free. It is employed in the treatment of fractures of the shaft and neek of the humerus to fix the arm and hold splints in position.

\section{BANDAGES OF THE TRUNK.}

Spiral Bandage of the Chest. Roller Three Inches in Width, Nine Yards in Length.-The initial extremity of the roller is applied to the anterior portion of the waist, and fixed by one or two cireular turns; the bandage is then earried npward, encircling the chest by ascending spiral turns, eael turn eovering in one-half of the previous turn until the axillary fold is reaehed; the roller is sext earried around the axilla to the back, and obliquely over this to the base of the neck of the opposite side, and then it may be passed down over the ehest and pinned to the spiral turus at several points; a pin should also be inserted at the point where the last turn of the roller leaves the spiral turn upon the back of the cliest (Fig. 72).

Use.-This bandage is employed to hold dressings to the chest, and may be used as a temporary dressing in fractures of the ribs or sternum. Care should be taken that the bandage be not so tightly applied as to interfere witl respiration.

Anterior Figure-of-eight Bandage of the Chest. Roller Two and a Half Inches in Width, Seven Yards in Length.-The initial extromity of the roller should be placed in the axilla of one side, and the bandage is then

FIti. 72.

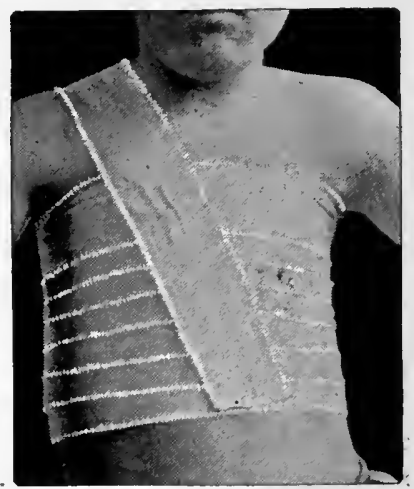

Spiral bandage of the ehest. earried obliquely across the anterior portion of the chest 
to the shoulder of the opposite side; it is then carried baekward around the shoulder and through the axilla, and is next eondueted obliquely over the anterior portion of the ehest to the opposite shoulder, through the axilla, and again back to the anterior portion of the ehest, the turns crossing in the median line over the sternum. These turns should be repeated, aseending from the shonlder toward the neck, each turn overlapping three-fourths of the preeeding one, until five or six turns have been applied, the end of the bandage being secured by a pin

FIG. 73.

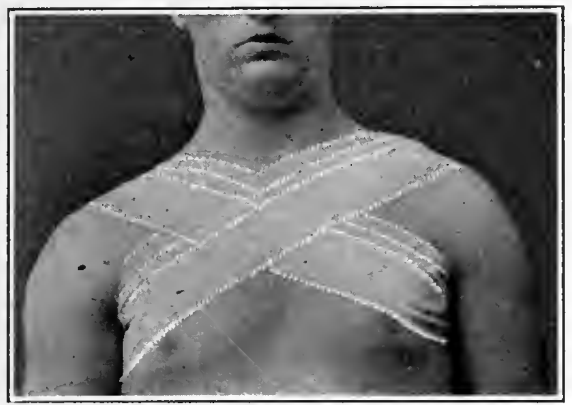

Anterior figure-of-eight bandage of the chest.

(Fig. 73), or it may be completed by a cireular turn around the chest.

Use.-This bandage may be employed to bring the shoulders forward, and to hold dressings to the anterior portion of the elest.

Posterior Figure-of-eight Bandage of the Chest. Roller Two and a Half Inches in Width, Seven Yards in Length.-The initial extremity of the roller should be placed in the axilla of the left side, and the bandage should then be carried obliquely across the back of the chest to the top of the opposite shoulder; it is next carried through the axilla and conducted across the posterior portion of the ehest to the top of the opposite shoulder, and passed through the axilla to the point of starting. These turns are re- 
peated, descending from the neck toward the shoulder, until five or six have been applied, the end of the bandage being secured by a pin (Fig. 74). In applying both of these bandages the crosses of the bandage, either anterior or posterior, should be made in the median line of the chest.

Use.-This bandage may be employed to hold dressings to the posterior portion of the chest and to draw the shoukders backward.

FIG 74.

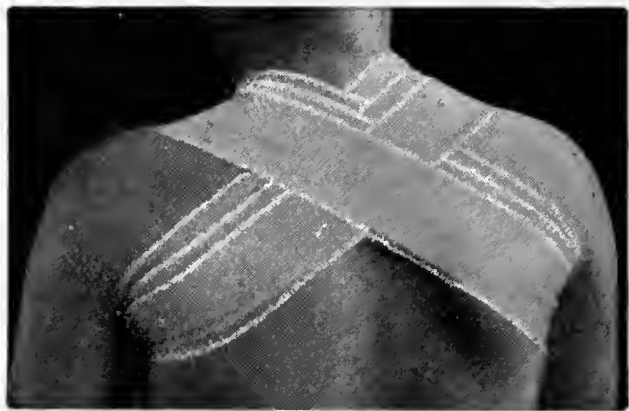

Posterior figure-of-eight bandage of the chest.

Suspensory and Compressor Bandage of the Breast. Roller Two and a Half Inches in Width, Seven Yards in Length.-The initial extremity of the roller should be placed upon the scapula of the affected side, and secured by two oblique turns carried over the opposite shoulder and conducted downward under the breast to be covered in, and then carried to the axilla of the same side. Next carry the roller transversely around the chest, covering in the lowest portion of the affected breast. These turns should be repeated, the oblique turns from the axilla over the shoulder alternating with the transverse turns around the chest, until the breast is covered in, each series of turns ascending and covering two-thirds of the preceding turns (Fig. 75 ). 
Use.-This bandage is employed to support the breast and to make compression at the same time; it may also be employed to hold dressings to the breast.

FIG. 75.

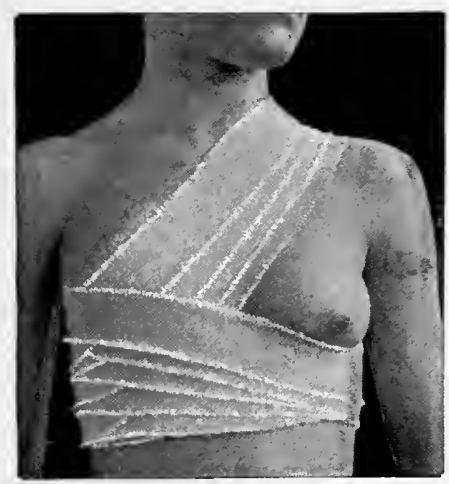

Suspensory and compressor bandage of the breast.

Suspensory and Compressor Bandage of Both Breasts. Two Rollers Two and a Half Inches in Width, Seven Yards in Length.-The initial extremity of the bandage should be secured by oblique turns of the axilla and shoulder, passing under one breast, as in the preceding bandage; the roller should next be carried transversely around the back to the other breast, then under the breast and upward over the opposite shoulder, then obliquely downward around the chest to the other side, being carried transversely over the lower portion of both breasts to the point of starting upon the back. Repeat these oblique turns from the shoulder to the breast and from the breast to the shoulder, and alternate them with a transverse turn around the chest and over both breasts. Both series of turns should ascend, and each turn should overlap two-thirds of the preceding one (Fig. 76).

Use.-This bandage is employed to support and compress both breasts and to retain dressings to them. 
FIG. 76.

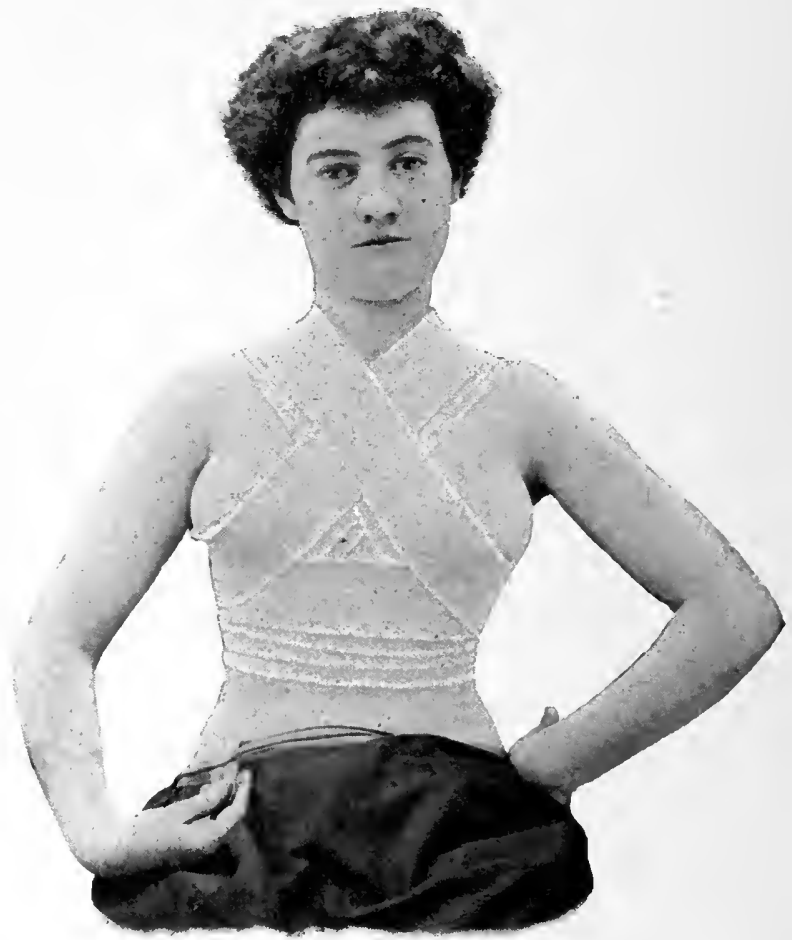

Suspensory and compressor bandage of both breasts.

\section{BANDAGES OF THE LOWER EXTREMITY.}

Single Spica-bandage of the Groin (Ascending). Roller Two and a Half Inches in Width, Seven Yards in Length.-Place the initial extrenity of the bandage upon the anterior portion of the right thigh just below the groin, and secure it by one or two circular turns around the thigh, or place the initial extremity of the roller obliquely upon the upper part of the thigh and carry it behind the limb and upward around the outer side of the thigh to the abdomen, omitting the circular turns; then carry the bandage 
obliquely across the lower part of the abdomen to a point just below the crest of the left ilium, and conduct it transversely around the back of the pelvis to a corresponding point on the opposite side; then bring it obliquely downward to the groin and over to the inner portion of the thigh, carrying it around the limb, erossing the starting-turn in the middle line of the thigh. These turns are repeated, each turn ascending and covering in two-thirds of the preceding turn, until six or eight complete turns have been made, and the bandage is then secured at any point where it ends (Fig. 77). This bandage may also be applied by

FIG. 77.

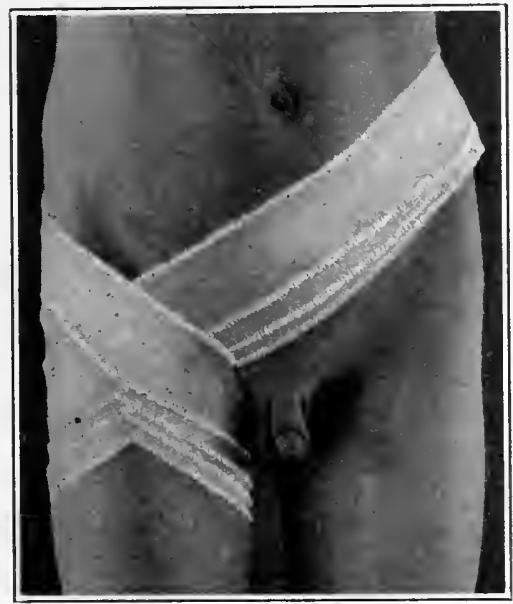

Ascending spica-bandage of the groin.

placing the initial extremity of the bandage just below the anterior superior spinous process of the ilium, and making two turns around the pelvis and then earrying the bandage to the thigh below the groin, passing it hehind the thigh, bringing it up on the opposite sile of the thigh to eross the first turn in the middle line of the groin ; ascending spica-turns are then made until a sufficient number have 
been applied to cover in the groin to the desired extent (Fig. 78). This bandage possesses the advantage that it is less likely to become displaced than the one previously described.

Single Spica-bandage of the Groin (Descending). Roller Two and a Half Inches in Width, Seven Yards in Length.-Place the initial extremity of the roller obliquely upon the anterior surface of the right thigh and secure it by one or two circular turns around the limb, or start the bandage with an oblique turn, as previously described; then carry the bandage obliquely across the abdomen to a point just below the erest of the ilium, and conduct it transversely around the back of the pelvis to a corresponding point on the opposite side; then bring it obliquely

FIG. 78 .

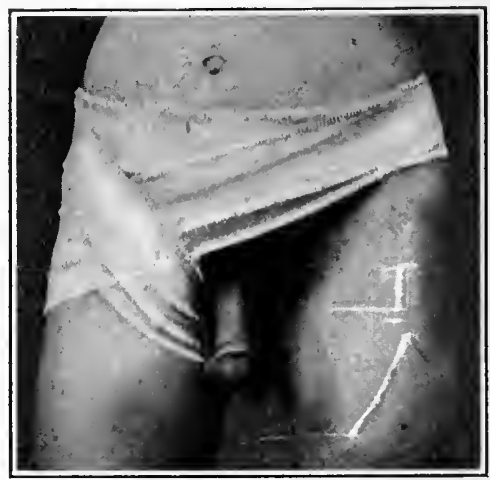

Aseending spica-bandage of the groin applied with pelvic turns.

down over the lower portion of the abdomen, crossing the first turn, to the junction of the thigh with the scrotum, pass it under the thigh and bring it up over the lower part of the abdomen, and let it follow the course of the first turn. These turns are repeated, each turn descending and overlapping two-thirds of the preceding turn until the groin is covered (Fig. 79). When either of these bandages is applied to the left groin, after the initial extremity of the 
roller is fixed, it is carried first to the crest of the ilium of the same side, then around the back of the pelvis to a corresponding point on the opposite side, then obliquely across the lower part of the abdomen to the outer aspect of the thigh, being conveyed around this and brought up between the thigh and the scrotum, passing obliquely over the groin to follow the course of the original turn. This handage may also be applied by making one or two circular turns around the pelvis, and the groin is next covered in by descendirrg spica-turns.

Double Spica-bandage of the Groins. Roller Three Inches in Width, Nine Yards in Length.-The initial extremity of the roller is placed upon the abdomen just above

FIG. 79.

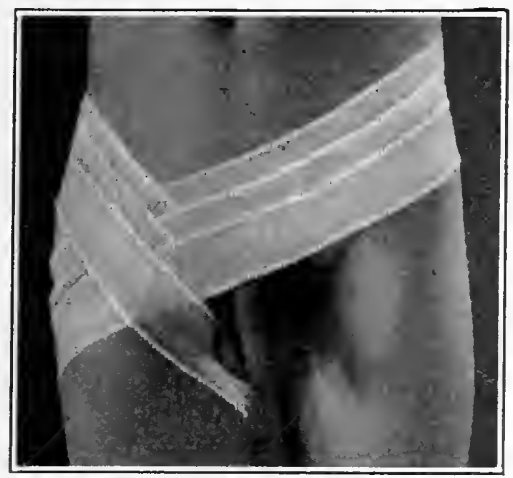

Descending spica-bandage of the groin.

the iliac crests and secured by one or two circular turns: the bandage is then carried from a point just below the crest of the right ilium obliquely across the lower portion of the abdomen to the outer portion of the left thigh, is carried around this and brought up between the scrotum and the thigh, and is passed obliquely over the groin, crossing the previous turn in the median line, and is conducted to a point just below the crest of the ilium on the same side. The bandage is then continued 
around the pelvis to the same point on the opposite side, and from this point is marle to pass obliquely over the groin to the inner side of the right thigh, passing around this and coming up on its outer side, crossing the preceding turn at the middle line of the groin, to be carried obliquely across the groin and lower part of the abdomen to the crest of the ilium on the opposite side. These turns are repeated, each turn covering in two-thirds of the previous turn, until both groins have been covered (Fig. 80). The turns may be so applied as to ascend or descend, forning the ascending or descending double spica-bandage of the

Fig. 80.

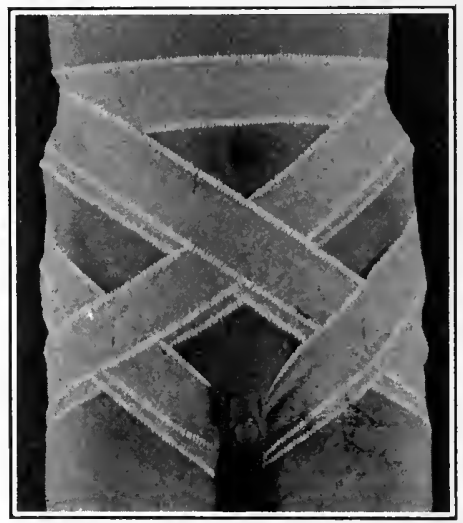

Double spica-bandage of the groins.

groins. When properly applied, this bandage presents three sets of crossing-turns, one in each groin and one in the median line of the abdomen.

Use.-The spica-bandages of the groin, either single or double, are employed to hold dressings to wounds in the inguinal region - for instance, those resulting from herniotomy, or from operations upon the glands of the groin. They are also employed to make pressure upon this region, and will often prove of use in the securing of compresses applied for the temporary retention of herniæ. 
Spica-bandage of Buttock. Roller Two and a Half Inches in Width, Seven Yards in Length.-The initial ex-

Fia. 81.

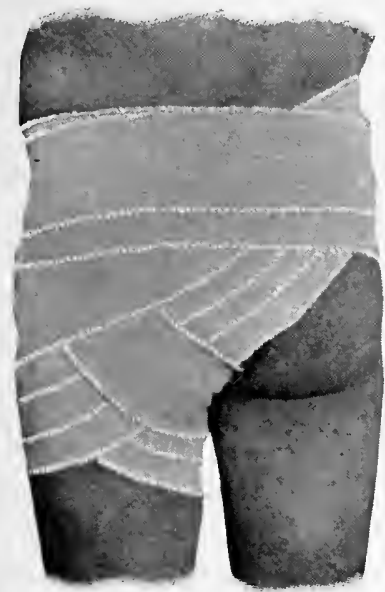

Spica-bandage of buttock.
FIG. 82.

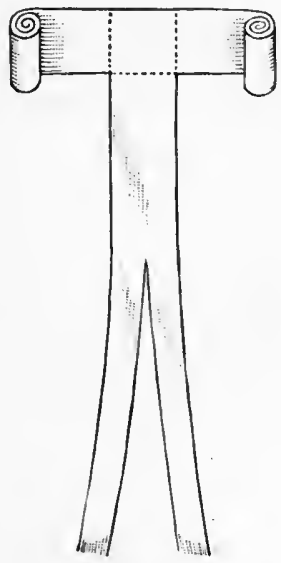

T-bandage of perineum.

tremity of the bandage is placed upon the back of the thigh just below the gluteal fold, and is carried around the thigh and brought back to the posterior aspect of the limb, so as to fix and cross the starting-turn near the middle of the thigh. It is next conducted obliquely across the thigh and buttocks and carried to the brim of the pelvis of the opposite side, when it is brought obliquely over the abdomen and back to the posterior surface of the thigh. These ascending turns are applied, each turn covering in about three-fourths of the preceding one, until the buttock is covered, and the bandage is then finished by one or two circular turns around the pelvis and abdomen (Fig. 81).

Use.-This bandage is employed to hold dressings to the upper posterior portion of the thigh, or the buttock. 
T-Bandage of the Perineum.-This bandage, which consists of a strip of muslin three inehes in width and four feet in length, has pinned or sewed to its centre a strip of similar material about forty inches in length. The horizontal strip is tied around the abdomen just above the pelvis with the attached strip on the lower end of the spine; this strip is brought between the nates and over the perineum, and its extremity is split into two tails to a point where the scrotum joins the perineum. :The tails are next carried up on each side of the scrotum and tied to the horizontal strip around the abdomen. When used in the female the perineal strip need not be split. (Fig. 82).

Use.-This bandage is employed to hold dressings to the anus and perineum. A very satisfactory substitute for this bandage consists in the use of a pair of swimming tights which may be employed to hold dressings to the anus, perineum, or scrotum.

FIG. 83.

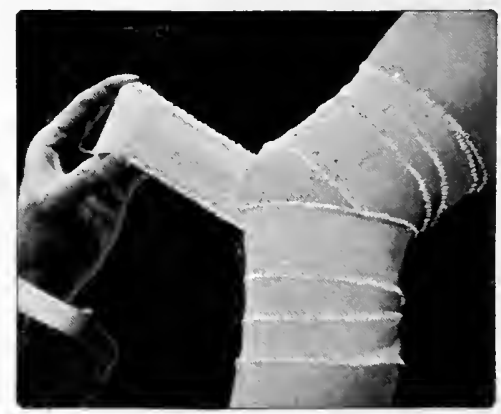

Figure-ofeight bandage of the knee.

Figure-of-eight Bandage of the Knee. Roller 's $c$. and a Half Inches in Widlh, Five Yards in Length.-The initial extremity of the roller is placed upon the left thigh three inches above the patella and secured by two or three cireular turns; then eonduct the bandage over the outer condyle of the femur across the popliteal space to the inner 
border of the tibia and around the anterior surface below the tubercle and head of the fibula, and make one circular turn ; the roller should then be carried obliquely across the popliteal space to the inner condyle of the femur, crossing the previous turn; then carry it around the front of the thigh to the outer condyle; repeat these turns, ascending toward the knee from the leg and descending from the thigh toward the knee, and finish the bandage by a circular turn over the patella (Fig. 83).

This bandage may also be applied by making two circu-

Fia. 84.

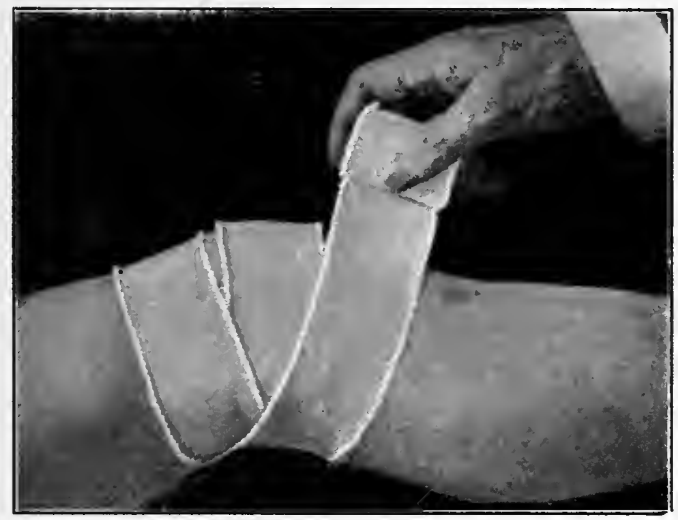

Figure-of-eight bandage of the knee.

lar turns around the patella and popliteal space, and then earrying the bandage to the thigh three inches above the patella, and finishing it with descending turns from the thigh and ascending turns from the head of the tibia, making all turns cross in the popliteal space (Fig. 84).

Use.-This bandaige is employed to hold dressings to the knee-joint either anteriorly or posteriorly. These figureof-eight turns are often employed in covering the knee in applying the spiral reversed bandage of the lower extremity when it is desired that the patient be allowed to bend the knee. 
Figure-of-eight Bandage of Both Knees. Roller Two and a Half Inches in Width, Seven Yards in Length.-Place the knees of the patient together with a compress between them; then place the initial extremity of the roller upon one thigh, about three inches above the patella, and secure it by one or two eircular turns around both thighs;

FIG. 86.

Fig. 85.

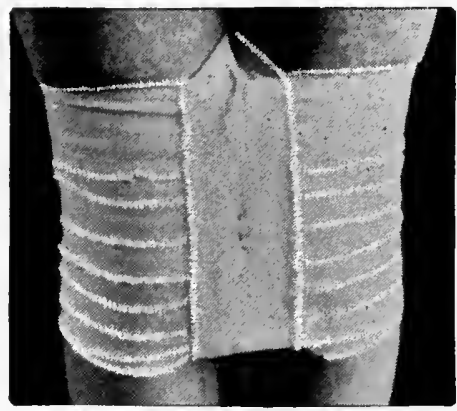

Figure-of-eight bandages of both knees.

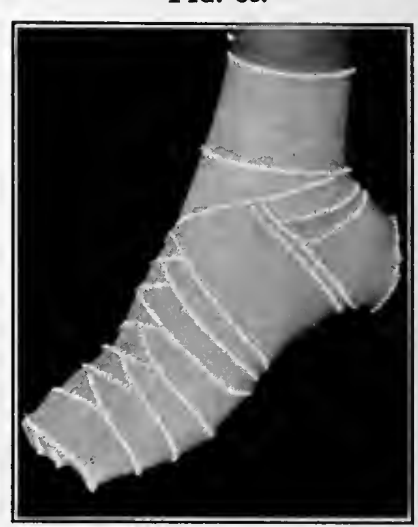

Complete bandage of the foot.

then eonduct the roller from the outer condyle of the left femur obliquely across the popliteal spaces of both legs to the head of the fibula on the opposite side, making a circular turn around both legs; pass the roller from the head of the fibula on the opposite side aeross the popliteal spaoe to the external condyle opposite the point of starting.

Repeat these turns, deseending from the thighs and ascending from the legs, until the knees are covered, and finish the bandage by carrying a turn of the bandage at right angles to the preceding turns between the thighs and the legs (Fig. 85).

Use.-This bandage is employed to seeure fixation of the limbs after operations upon the perineum, and may also be employed to obtain temporary fixation of the limbs in transporting eases of fraeture of the femur, and after the reduetion of dislocations of the head of that bone. 
Complete Bandage of the Foot. Roller. Two Inches in Width, Three Yards in Length.-The initial extremity of the bandage is placed upon the sole of the foot near the heel, and the fout is covered in by three or four recurrent turus; these are fixed by one or two circular turns around the instep; the heel is then covered in by a cirenlar and figure-of-eight turn, which passes around the ankle. The bandage is then carried obliquely over the dorsum of the foot to the tip of the toes, when a cireular turn should be made; the foot is then covered in by ascending spiral reversed turns until the ankle is reached, when it is secured by one or two cireular turns. This bandage may also be applied by first making two or three recurrent turns, covering in the toes, the plantar, and dorsal surface of the foot, and the bandage is then completed by spiral reversed turns as described above (Fig. 86).

\section{Spica-bandage of the Foot. Roller 'lwo and a Half}

Fig. 87.

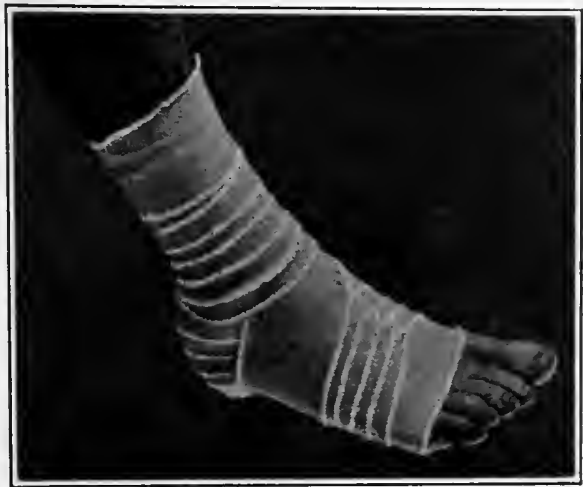

Spica-bandage of the foot.

Inches in Width, Five Yards in Length.-Fix the initial extremity of the roller upon the ankle and secure it by two circular turns; then carry the bandage obliquely over 
the dorsum of the foot to the metatarso-phalangeal articulation, and make a eircular turn around the foot at this point; then continue it upward over the metatarsus by making two or three spiral reversed turns; next carry the bandage parallel with the inner or outer margin of the sole of the foot, according to whether it is applied to the right or left foot, direetly across the posterior surface of the heel; thenee along the opposite border of the foot and over the dorsum, crossing the original turn in the median line of the foot. This completes the first spiea-turn. These spica-turus are repeated, gradually ascending by allowing each turn to cover in three-fourths of the preceding turn, until the foot is covered in with the exception of the posterior portion of the sole of the heel (Fig. 87). Care should be taken to see that the turns cross each other in the median line, and that they are kept parallel to each other throughout their course.

Use.-This bandage will be found very useful when it is desired to make firm compression upon the foot or to retain dressings to it; it is especially useful in the treatment of sprains of the ankle or the anterior tarsus.

Bandage of Foot Covering the Heel (American). Roller Two and a Half Inches in Width, Seven Yards in Length.-The initial extremity of the roller is placed upon the leg just above the malleoli and fixed by two circular turns around the leg; the bandage is then earried obliquely across the dorsum of the foot to the metatarso-phalangeal articulation, at which point a circular turn is made; two or three spiral or spiral reversed turns are then made, ascending the foot; the roller is next carried direetly over the point of the heel and continued back to the dorsum of the foot; thence beneath the instep around one side of the heel and up over the instep ; from this point it is earried beneath the instep around the other side of the heel (Fig. 89 ), and up in front of the ankle, from which point it may be continued up the leg (Fig. 88).

Use.-This bandage is employed to cover in the foot, and retain dressings to the foot and heel. 
FIG. 88.

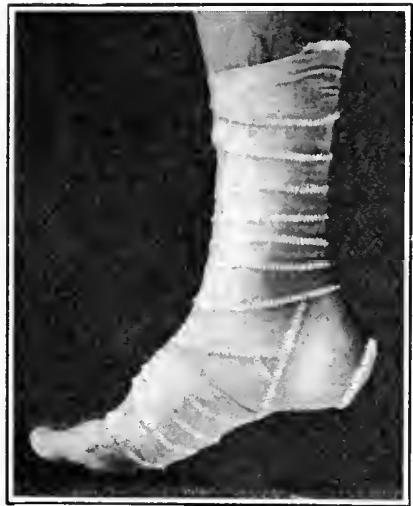

Bandage of foot covering the heel.
FIG. 89.

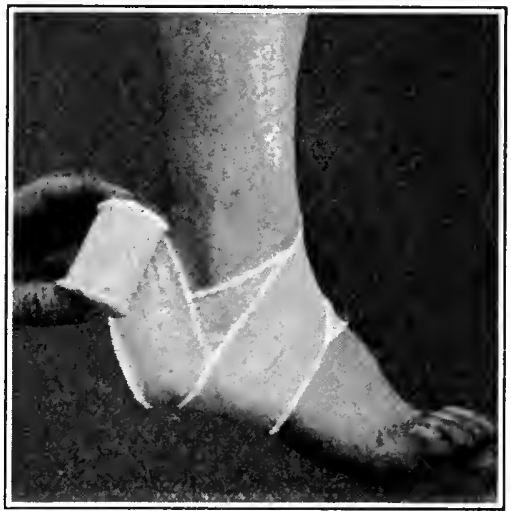

Turns covering heel in American bandage.

Bandage of Foot Not Covering the Heel (French). Roller Two and a Half Inches in Width, Seven Yards in Length.-Fix the initial extremity of the roller upon the leg just above the malleoli and secure it by two circular

FIG. 91.

FIG. 90.

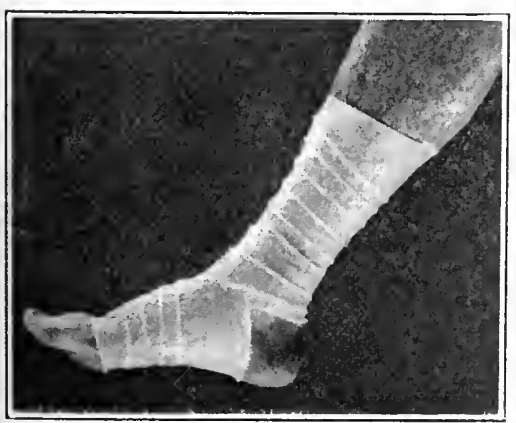

Bandage of foot not covering the heel.

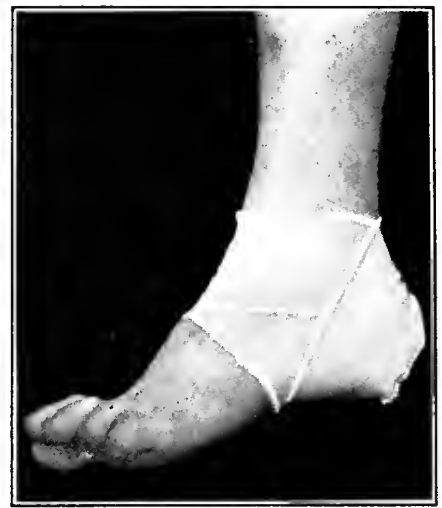

Bandage of the heel.

turns around the leg; the bandage is then carried obliquely across the dorsum of the foot to the metatarso-phalangeal 
articulation, and at this point a eireular turn should be made. The roller is now carried up the foot, covering it in with two or three spiral reversed turns, and at this point a figure-of-eight turn is made around the ankle and instep; this should be repeated onee, which will cover in the foot with the exeeption of the heel ; the bandage may then be continued up the leg with spiral reversed turns (Fig. 90).

Use.-This bandage may be employed to serure dressings to the foot, and is the one generally used to cover this part in applying the spiral reversed handage of the lower extremitr.

Bandage of the Heel. Roller Two Inches in Width, Three Yards in Length.-The initial extremity of the bandage is placed over the anterior surface of the ankle and is fixed by two circular turns passing over the point of the heel. The bandage is then earried obliquely over the dorsum of the foot to the tarso-metatarsal articulations, at which point a eireular turn is made; the bandage is then earried beneath the instep around one side of the heel and upward over the instep ; from this point it is carried beneath the instep around the other side of the heel. Several layers of these turns should be made, and the bandage may be finished by a cireular turn around the leg just above the ankle (Fig. 91).

Use.-This bandage may be employed to retain dressings to the heel.

Spiral Reversed Bandage of the Lower Extremity. Roller Two and a Half Inches in Width, Seven Yards in Length.-The initial extremity of the roller is placed upon the leg just above the malleoli and secured by two cireular turns. It is then carried obliquely over the foot to the metatarso-phalangeal artieulation, where a circular turn is made around the foot. Two or three spiral reversed and two figure-of-eight turns of the ankle and instep should be made, while just above the ankle one or two circular or spiral turns are made around the leg, and as the bandage is earried up the leg, as it increases in diameter, spiral reversed turns are made until it approaches the knee; at this point, if the limb is to be 
kept straight, the spiral reversed turns may be eontinued over this region and up upon the thigh. If the knee is to be bent, figure-of-eight turns may be applied until the knee is covered, and then the thigh may be covered with spiral reversed turns (Fig. 92). To cover in the thigh as

FIG. 92.

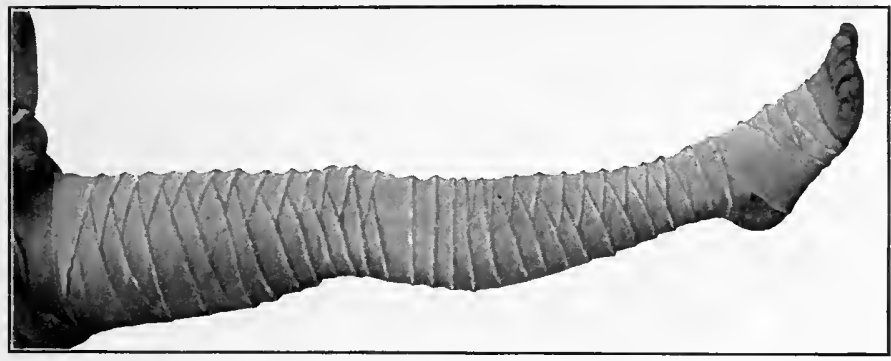

Spiral reversed bandage of the lower extremity.

well as the leg, two bandages of the dimensions before given will be required. Care should be taken to keep the reverses in a line, and not to make them over the spine of the tibia, as they may thus become painful to the patient.

Use.-This is one of the most frequently employed of the roller-bandages; it is used to apply pressure to the lower extremity, to retain dressings, and to secure splints in the treatment of fractures and dislocations.

Spiral Reversed Bandage of the Thigh. Roller Three Inches in Width, Six Yards in Length.-The initial extremity of the bandage is seeured around the thigh just above the knee-joint by two or three circular turns, and the bandage is earried up the thigh by spiral reversed turns until the groin has been reached, when it is secured by one or two circular turns around the thigh. Care should be taken that it is not applied too firmly, that the superfieial veins are not obstructed, eausing swelling of the leg below the knee (Fig. 93).

Use.-This bandage is employed to retain dressings or splints to the thigh. 
Figure-of-eight Bandage of the Leg. Roller Two and a Half Inches in Width, Seven Yards in Length.-This bandage differs from the spiral reversed bandages of the

Fia. 93.

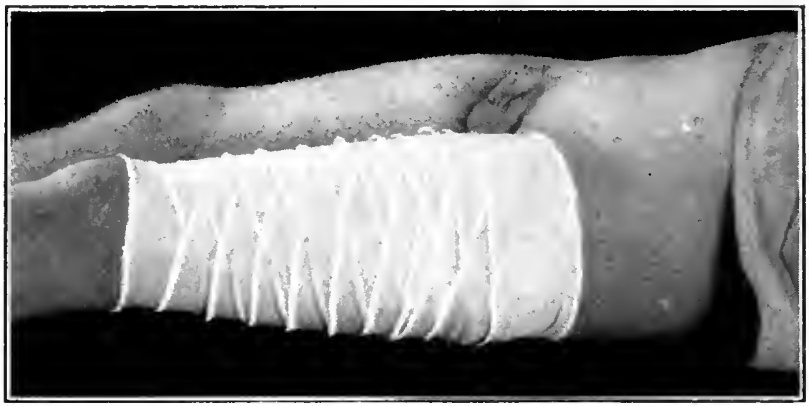

Spiral reversed bandage of the thigh.

lower extremity only in the fact that when the swell of the ealf is reached figure-of-eight turns are made around

FIG. 94.

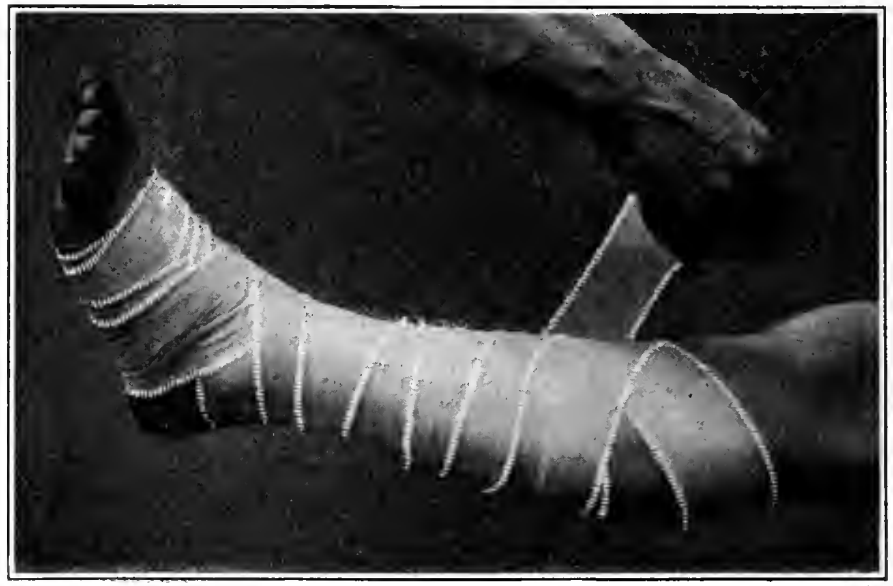

Figure-of-eight bandage of the leg. 
the leg instead of spiral reversed turns. In applying the roller, when the calf of the leg is reached the bandage is carried obliquely around the leg to the crest of the tibia and then made to cross the starting-turn in the median line; these descending and ascending turns are repeated until the calf of the leg has been covered in, and the bandage is finished with one or two circular turns just below the knee (Fig. 94).

Use.-This bandage holds its place more firmly than the ordinary spiral reversed bandage of the leg, and may be enployed in the treatment of ulcers of the leg in eonjunction with strapping, where it is desirable to change the dressings at infrequent intervals and to allow the patient to walk about during the course of treatment.

\section{SPECLAL BANDAGES.}

Spiral Reversed Bandage of the Penis. Roller Threequarters of an Inch in Width, Thirty Inches in Length.Fix the initial extremity of the roller by two circular turns around the penis elose to the pubis; then carry the bandage obliquely down to the corona glandis; from this point ascend the body of the penis by spiral reversed turns to the pubis, and finish the bandage by two figure-of-eight turns around the neck of the scrotum and root of the penis, or split the end of the bandage so as to form two tails, and secure it by tying these around the root of the penis (Fig. 95).

Bandage of Perineum.-To hold dressing to the perineum or anal region a single T-bandage is usually employed. (Fig. 82). To secure dressings to this region the use of swimming tights will be found very satisfactory in holding the dressings in place if the patient is compelled to be on his feet (Fig. 96).

Recurrent Bandage of a Stump. Roller Two and a Half Inches in. Width, Five to Seven Yards in Length. -Place the initial extremity of the roller upon the anterior or posterior surface of the limb a few inches above the 
extremity of the stump, and carry the bandage to the end of the stump, and then conduct it upward or downward on the limb, as the case may be, to a point directly opposite the point of starting; then bring the bandage back

Fig. 95.

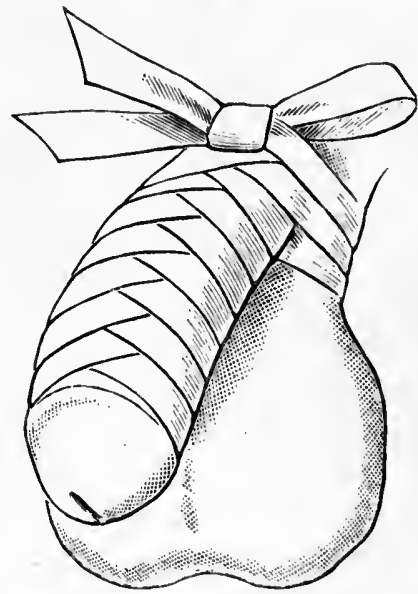

Epiral reversed bandage of the penis.
FIG. 96.

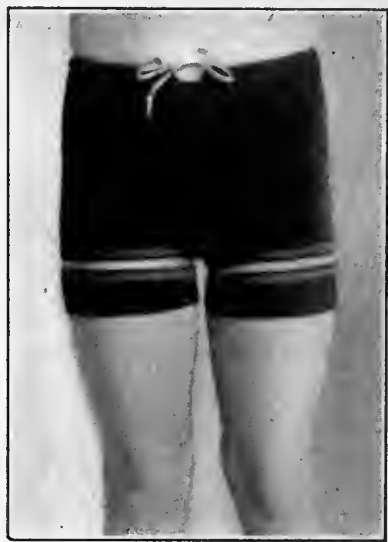

Swimming tights employed to seeure dressing to perineum or anal region or groin.

over the face of the stump to the point of starting, and continue these recurrent turns, each turn overlapping twothirds of the preceding one, until the face of the stump is covered; then reverse the bandage and secure the recurrent turns at their points of origin by two or three circular turns. The roller should next be carried obliquely down to the end of the stump, and a circular turn should be made around this. The bandage should then be carried up the limb by spiral or spiral reversed turns beyond the point at which the recurrent turns terminated, and secured by one or two circular turns (Fig. 97).

In applying this bandage to very short stumps resulting from amputations at or near the shoulder- or hip-joint, after making the recurrent and spiral turns, it will be 
found necessary to carry the bandage, in the case of amputations of the shoulder, across the chest to the opposite axilla and back, and apply several of these turns; so in case of hip amputations it will be found best to finish the bandage with a few turns about the pelvis.

Fig. 97.

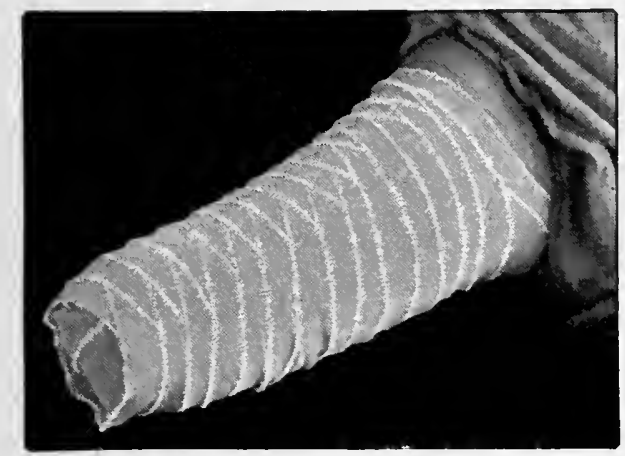

Recurrent banduge of a stump.

Bandage for Securing the Hands and Feet in the Lithotomy Position.- The hand of the patient should be brought down and made to grasp the outer side of the foot; the initial extremity of the roller is fixed by two circular turns around the wrist and ankle, and the bandage is then passed around the foot and hand, and these turns are alternated with turns around the wrist and ankle until the hand and foot are firmly secured. The same procedure is adopted with the hand and foot of the opposite side (Fig. 98).

Liebreich's Eye-bandage.-This bandage consists of a strip of flannel two and a half inches in width and from six to ten inches in length, to the extremities of which tapes are sewed. It may be applied transversely so as to cover both eyes, or obliquely so as to cover only one eye ; it is secured by the tapes carried around the head and tied over the forehead (Fig. 99). 
Use.-This bandage is used to hold compressed or dressings to the eye or eyes; the elasticity of the flannel permits of its being applied so as to make a variable amount of pressure.

Borsch's Eye-bandage.-This bandage is employed for bolding a dressing to one eye, and consists in a strip of Hanuel, two or two and a half inches in widtl, which is passed around the head from the occiput and covers both

Fig. 98.

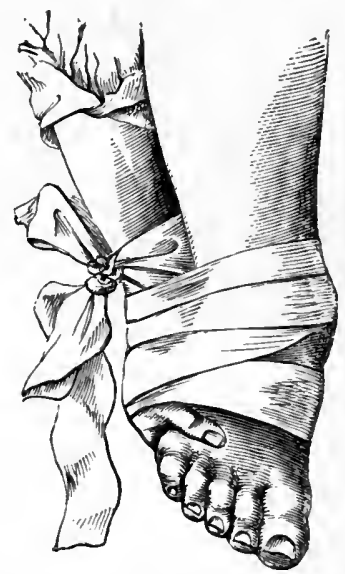

Bandage for securing the hands and feet for lithotomy.
FIG. 99.

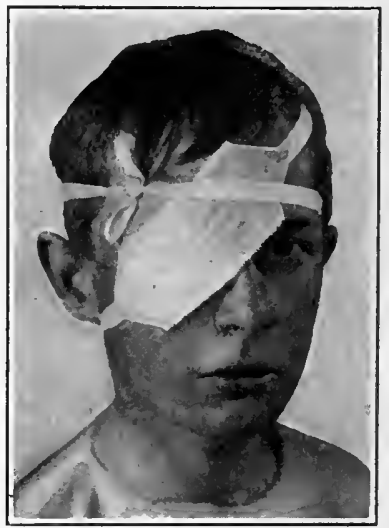

Liebreich's eye-bandage.

eyes (Fig. 100). A narrow strip of flannel is attached to the posterior portion, which is carried over the head and passed under the horizontal strip in front of the eye which is to be left uncovered, and is then folded back so as to raise the horizontal strip from the eye, and secured (Fig. 101).

Bandages of Scultetus.-This is a compound bandage, consisting of a number of pieces of muslin, and may be prepared from a two and a lalf or three inch roller by cutting off strips sufficiently long to encircle the part abont one and one-third times. The strips are placed under the 
part in such a manner that the first piece shall be overlapped by the second, the second by the third, and so on from below upward; the pieces are then brought around the limb, and the extremities of the last piece are secured by pins (Fig. 102). This bandage was formerly much employed in the treatment of compound fractures to secure dressings to the wound, and possessed the advantage that

Fig. 100.

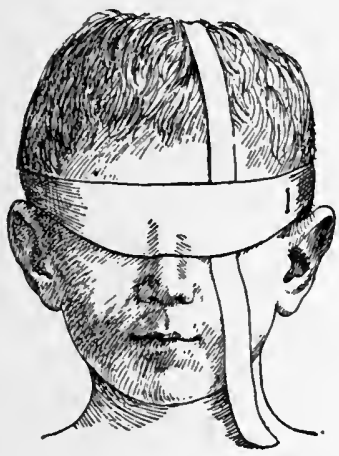

Fig. 101.

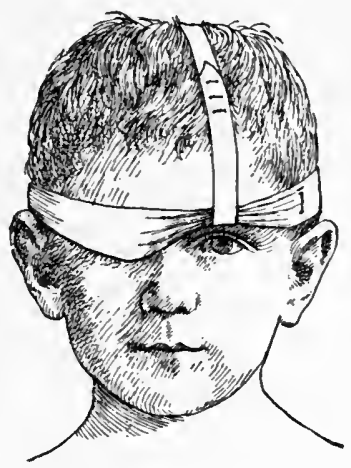

Application of Borsch's eye-bandage.

when a single strip became soiled it eould be removed without disturbing the whole dressing, the new strip to be introduced being pinned to the extremity of the soiled piece to be removed, and then being drawn through by its removal. This bandage will often be found convenient in applying dressings to cases of excision of the joints, where as little disturbance of the parts as possible is important in dressing the wound. When the strips are attached to each other by a thread passed through the centre of each strip, the bandage is known as Pott's bandage. It is applied and secured in the same manner, but it possesses no advantages over the bandage of Scultetus.

Gauze Bandages.-Bandages may be prepared from gauze, the same material that is used for gauze dressings, and are now very extensively used in surgical practice. 
The gauze bandages are prepared by cutting or tearing the material into strips varying in width from two inches to three inches, and in length from five yards to eight yards; these strips are then wound so as to form rollerbandages. Gauze bandlages are sometimes employed in the dressing of fractures, but do not furnish as substantial a dressing as the ordinary muslin bandages. They, how-

FIG. 102.

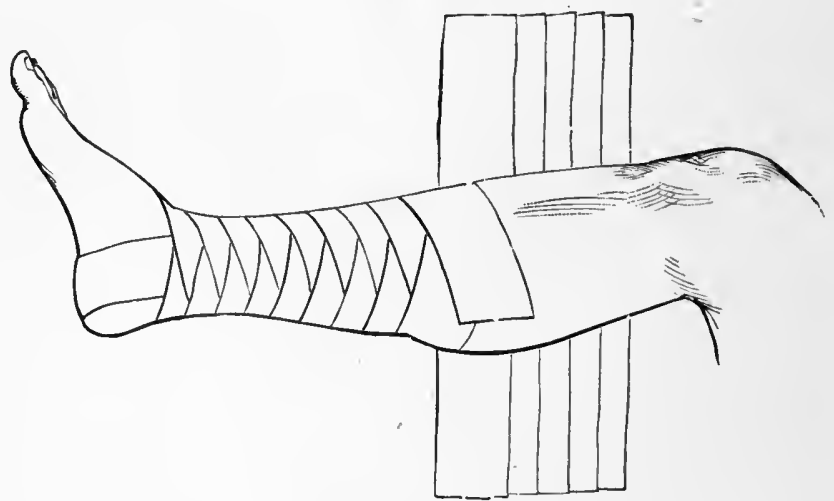

Bandage of Scultetus.

ever, constitute a soft and comfortable material for holding dressings to wounds. They are applied in the same manner as the ordinary muslin roller, with the exception that in their application reverses are seldom required, as the open mesh of the bandage gives it considerable elasticity, so that the bandage can be made to adapt itself to the part without making reverses. Any of the ordinary bandages which have been previously described may be applied by means of the gauze bandages, such as those of the head, extremities, and trunk.

Gauze Bandage of Head and Neck.-In applying dressings to wounds of the head and neek, it is advisable to cover in both the head and neck, and also to make a few turns over the upper part of the chest and around each 
shoulder, whieh prevents the turns of the bandage from slipping and holds the dressing in place, so that it eannot be disarranged by movements of the patient (Fig. 103).

Gauze Bandage of Upper Extremity.-The initial extremity of the bandage is seeured by two or three turns around the wrist, and the bandage is then carried obliquely over the dorsum of the hand to the tip of the little finger,

FIG. 103.

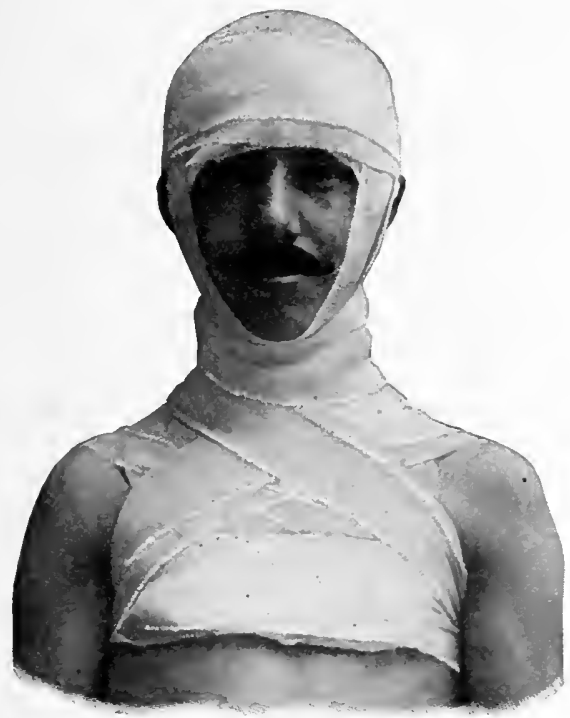

Gauze bandage of head and neck.

when a eireular turn is made; the hand is then eovered in by eireular turns. The region of the wrist is next covered by figure-of-eight turns and the bandage is carried up the forearm by circular turns. Figure-of-eight turns are made at the elbow and the bandage is continued with cireular turns around the arm. No reverses need be made in applying this bandage. 
Use.-This bandage is used for the same purposes as the, spiral reversed bandage of the upper extremity.

Gauze Bandage of Chest and Shoulder.-In applying this bandage, a gauze roller, three or four inches in width, is employed. The initial extremity of the bandage is secured around the lower portion of the chest by one or two turns and it is carried up to the axillary lines by circular turns; at this point it is secured obliquely across the chest to the base of the neck and then behind the shoulder to the posterior portion of the axilla. From this point the turn is

FrG. 104.

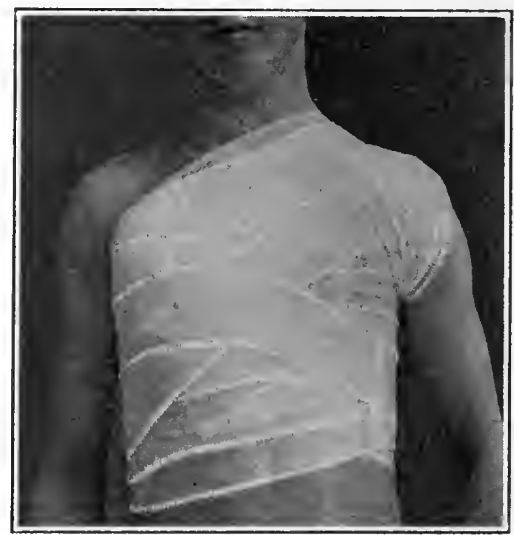

Gauze bandage of chest and shoulder.

carried through the axilla and over the shoulder and is con . dueted over the back of the chest to the posterior aspect of the opposite axilla, through which it passes to be carried over the anterior portion of the chest and shoulder to the opposite axilla. Then turns are repeated until a dressing of sufficient firmness is applied (Fig. 104). This bandage is used to secure dressings to the chest, axilla, and shoulder.

Flannel Bandage.-These bandages are prepared from flannel, which is cut into strips from two to four inches in width and from five to seven yards in length. These 
strips are formed into rollers either by hand or by means of the bandage-winder. Flannel bandages, by reason of the elasticity which they possess, can be applied without

FIG. 105.

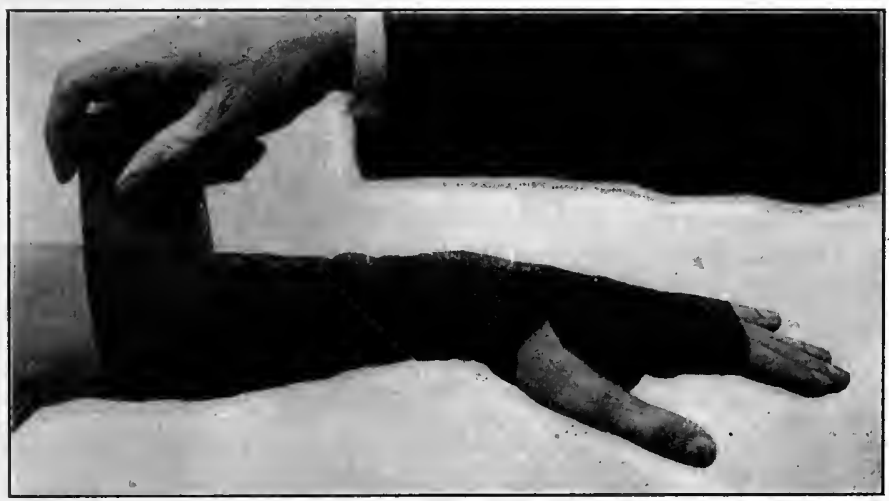

Black muslin bandage of hand and arm.

reverses, and are used to make a moderate amount of elastie pressure. They are often employed in applying dressings

Fig. 106.

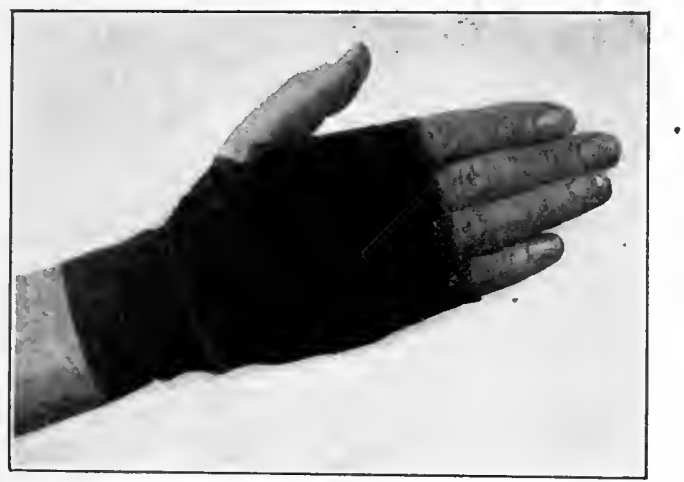

Black muslin bandage of the hand.

to the head, especially after operations upon the eyes, and are generally applied as a primary roller before the appli- 
cation of plaster-of-Paris dressings, and may also be used in subacute joint-affections, both to protect the parts and to make a moderate amount of elastic pressure.

Black Muslin Bandages.-From the fact that darkcolored bandages are less conspicuous than white ones, they are sometimes prepared from black or brown muslin. They are applied in the same manner as the ordinary muslin or gauze bandage. For this reason they may be used for bandages of the head, hand, or arm in patients who are treated as walking cases, and who object to the conspicuousness of a white bandage (Figs. 105, 106 and 107).

The Rubber Bandage.-This bandage, which was introdueed to the profession by Dr. Martin, of Boston, is made from a strip of rubber-sheeting, from one inch to four inches in width and from three to five yards in

FIG. 107.

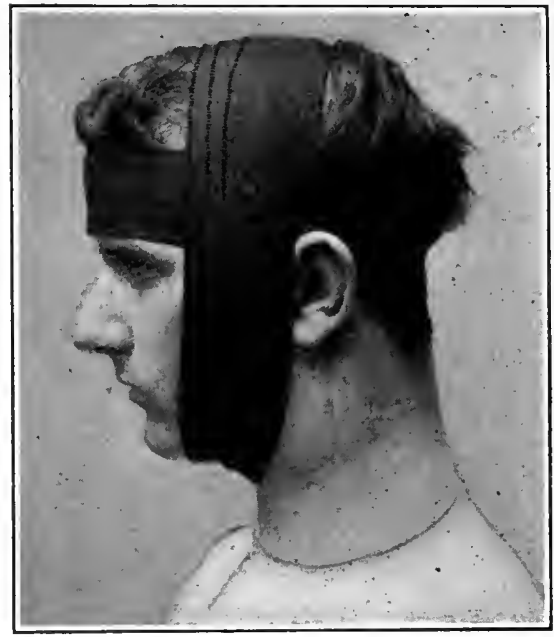

Black muslin bandage of head. length, which, for convenience of application, is rolled into a cylinder. It will be found a useful form of dressing where it is considcred desirable to apply elastic pressure to a part (Fig. 108). It may be employed in the treatment of varicose veins of the legs, in chrouic ulcers of those parts where pressure is an inportant elenent in the treatment, and may be used as a substitute for strapping to secure this object. Its application has also been recommended in the treatment of swelled testicle in that stage of the affection in which pressure is indicated. 
For applicatin to the leg, a rubber bandage two and a half inches in width and three yards in length is required.

The initial extremity of the roller is fixed upon the foot near the toes and secured by a circular turn; the foot is then covered in by spiral turns overlapping each other about two-thirds, and a figure-of-eight turn is made from the ankle to the instep. The bandage is then carried up the limb to the knee with spiral turns, where it is secured by two tapes sewed to the terminal extremity of the bandage, which are passed around the leg and tied. The bandage need not be reversed, as its elasticity allows it to conform to the shape of the limb. Care should be taken not to apply the turns with too much firmness; the bandage should be stretched very slightly; if this precaution is not taken, it soon becomes uncomfortable

to the patient. A patient using one of these bandages will soon learn to apply it himself, making just the requisite amount of tensiou to secure its holding its place, and to insure a comfortable

Fig. 108.

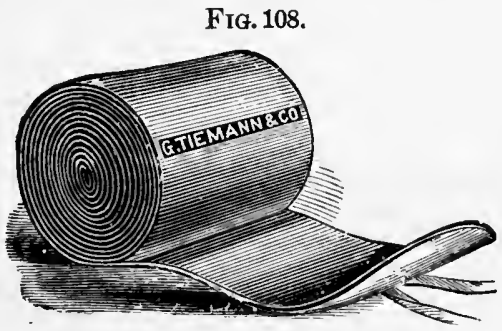

Martin's rubber bandage. degree of pressure upon the part. A well-fitting stocking may be placed upon the limb before the bandage is applied, or it may be applied directly to the skin.

The bandage should be removed at night when the patient goes to bed and hung up to dry, as its inner surface becomes moist from the secretions from the skin; it should be reapplied as soon as the patient rises in the morning.

In using it in the treatment of ulcers of the leg no ointment should be applied to the ulcer, as oily dressings soon destroy the rubber; applications may be made to the ulcer by means of dry powders, such as oxide of zinc, iodoform, or aristol, before the bandage is applied.

In the treatment of swelled testicle the bandage is ap- 
plied to the testicle by means of recurrent turns not too firmly made, and secured in place by spiral turns, until the whole surface of the organ is covered in ; the end of the bandage is secured with tapes tied around the root of the scrotum. The same precaution to apply the bandage so as to make only moderate pressure should also be observed here.

Elastic-webbing Bandage.-This bandage, which is woven from threads of rubber covered with cotton or silk, has recently been introduced, and possesses all the advantages of the rubber bandage as regards elasticity, and has the additional advantage that air can circulate through the meshes of the bandage and moisture can evaporate from the surface covered by the bandage, so that the skin covered by it does not become bathed in perspiration, as is the case with the rubber bandage. It is applied in the same manner and for the same purposes as the rubber

FIG. 109.

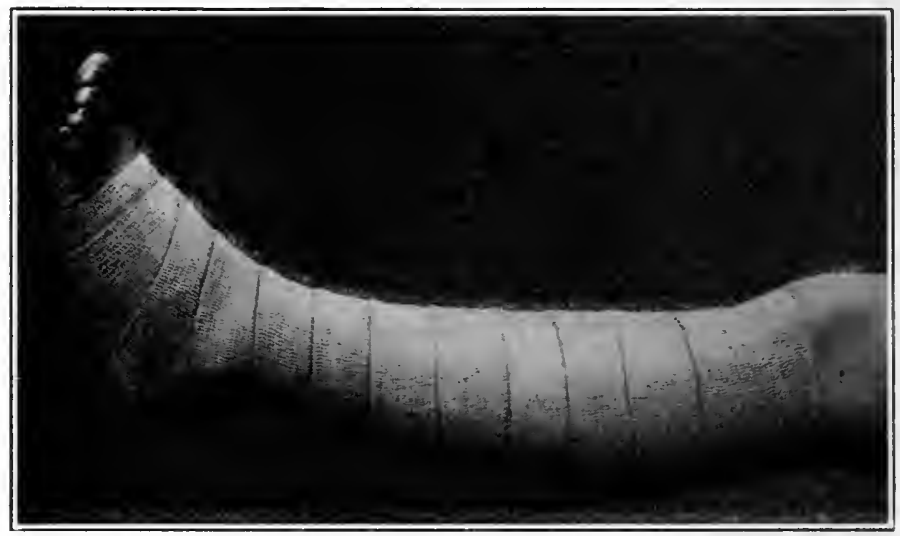

Elastic webbing bandage applied to leg.

bandage (Fig. 109). The patient soon learns to apply it himself, so as to make the requisite amount of pressure. In the treatment of varicose veins and odema of the legs we have found it a most satisfactory dressing. 


\section{FIXED DRESSINGS, OR HARDENIN}

For the application of these dressings a variety of substances are used which are incorporated in the meshes of some fabric, such as crinoline or cheese-cloth, or painted over its surface to give fixity or solidity to the bandage.

The materials most commonly used in the preparation of fixed dressings are plaster-of-Paris, starch, silicate of sodium or potassium, and paraffin.

\section{Plaster-of-Paris Dressings.}

The plaster-of-Paris used for the application of surgical dressings should be of the same quality as that which dental surgeons employ in taking casts for teeth-that is, the extra-calcined variety. If moist or of inferior quality, it will not set rapidly or firmly, and will fail to give sufficient fixation to the dressing.

Methods of Applying Plaster-of-Paris Dressings.The plaster-of-Paris dressing may be applied in several ways, either by covering the part to be enclosed with some loose fabric, and rubbing the moist plaster into it, alternating the layers of the fabric with layers of moist plaster, or it may be applied by means of a roller which has been prepared by incorporating plaster-of-Paris in its meshes.

It may also be applied in the form of the Bavarian. dressing (page 111), or in the form of moulded plaster-ofParis splints (page 112).

To apply a plaster-of-Paris dressing according to the first method, the part to be enclosed-the leg, for instanceshould first be covered by a neatly applied flannel bandage, or a muslin bandage which has been shrunken by being washed; new muslin is not satisfactory as a primary application to a limb in applying a plaster-of-Paris dressing, as the moisture from the plaster wets it and causes it to shrink, so that it may exert injurious pressure after the bandage becomes dry.

The limb having been covered by the bandage, and any 
bony prominences, such as the malleoli, having been padded with small wads of cotton to prevent undue pressure upon them, the part is next covered by a layer of turns of a crinoline bandage or by strips of cheesecloth or any other loose material. A small quantity of plaster-of-Paris is next mixed with water until it has the consistence of thick cream, when it is smeared evenly over the whole surface of the previously applied bandage. Another layer of the bandage or of strips is next applied, and the plaster is smeared over this in the same manner, and so alternate layers of plaster-of-Paris and bandage are applied until a casing of the desired thickness is obtained. If plaster-of-Paris of the quality previously described be used, it will set or become hard in a few minutes.

The most eonvenient method of applying the plasterof-Paris dressing is that introduced by the late Professor Sayre, which consists in the use of bandages which have been previously prepared with plaster-of-Paris; these are moistened and applied while moist to the part to be encased.

Preparation of Plaster-of-Paris Bandages.-These bandlages are prepared by taking cheese-cloth, mosquitonetting, or crinoline, which latter is by far the best fabric, and cutting or tearing it into strips two and a half to three inches in width and five yards in length. These are laid on a table, and plaster-of-Paris of the quality before mentioned is dusted over them and rubbed into the meshes of the fabric ; the material when impregnated with plaster is loosely rolled into a cylinder, and these bandages when prepared should be placed in air-tight jars or tin cans until required.

Bandages thus prepared, which have been exposed to the air or have been kept for a long time, are not apt to set well when applied; hut if such bandages are placed in a hot oven and baked for half an hour before being used, they will be found to set as satisfactorily as those freshly prepared.

These bandlages may be prepared by a machine made for this purpose; but $\dot{I}$ do not think that they are apt to have the plaster as evenly distributed through them, and, 
therefore, are not as satisfactory as those prepared by hand.

Application of the Plaster-of-Paris Bandage.-Before applying this dressing, the part to be encased-the leg, for instance-should be covered by a flannel roller, the bony prominences being protected by pads of cotton, or a closely fitting stocking may be applied to the part. Cotton wadding cut into strips of the desired wilth and formed into a roller may be used instead of the flannel roller.

The bandage should be dipped in warm water and kept completely immersed for a few minutes; it should then be squeezed with the hand, and as soon as bubbles of air cease to escape it is a sign that it is thoroughly soaked

FIG. 110.

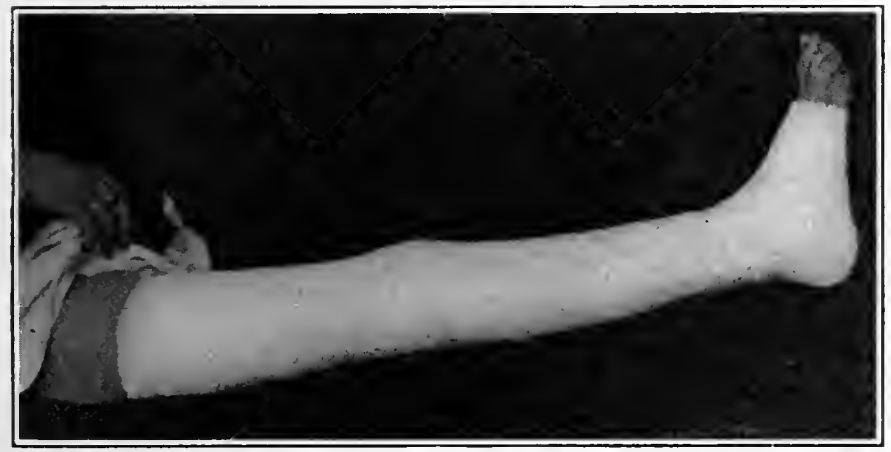

Leg encased in plaster-of-Paris dressing.

and is ready for application. On removing it from the water the excess of water should be squeezed out by the hand, and the bandage should then be applied evenly to the limb with just sufficient firmness to make it fit the part nicely, and as few reverses as possible should be made. A sufficient number of bandages are applied to make a dressing as firm as may be required; three rollers of the above dimensions are usually ample for a dressing for the leg, and when the last roller has been applied dry plaster should be moistened with water until it has the consistency of thick cream, and rubbed evenly over the 
surface of the bandage to give it a finish (Fig. 110). If a good quality of plaster has been used, the bandage should be quite firm in from ten to fifteen minutes, but the patient should not for a few hours be allowed to bear any weight upon the bandage.

An equally firm dressing may be applied with the use of a less number of bandages, if the surgeon rubs over the surface of each layer of bandage applied a little moist plaster, then applying another layer and repeating the procedure; finishing the dressing by an external coating of moist plaster, as above described.

In applying these dressings a fewer number of bandages will be required if narrow strips of tin, zinc, or binders' board are incorporated in the layers of the bandage, which increase the strength of the dressing.

Application of the Plaster-of-Paris Bandage to the Thigh and Pelvis.-Where it is desirable to apply a

FIG. 111.

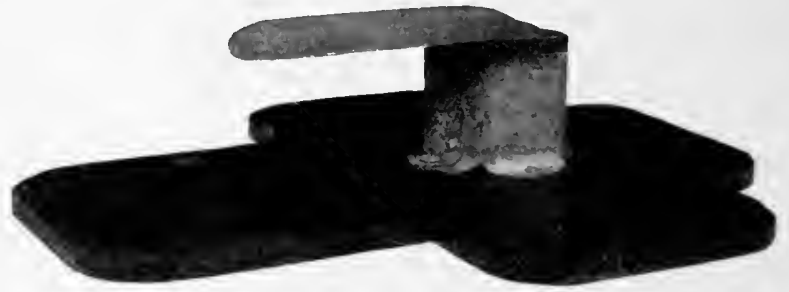

Pelvic supporter.

plaster-of-Paris bandage to the thigh, and at the same time fix the hip-joint by including the pelvis in the bandage, the use of a pelvic supporter (Fig. 111) is most satisfactory. The patient is placed upon the supporter so that the lumbar spine rests upon the body of the supporter, while the pelvis rests upon the metal shelf which extends from it, as seen in Fig. 112. The limb is extended and held in the required position, and the plaster bandage is applied to the thigh, and is also carried around the pelvis and passed over the metal shelf upon which the pelvis 
rests. When the bandage has become firm, the supporter is removed by slipping it upward.

FIG. 112.

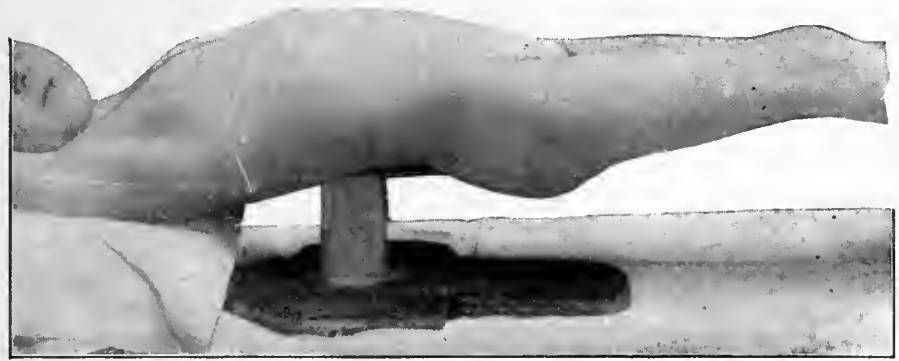

Position of patient upon pelvic supporter.

Interrupted Plaster-of-Paris Dressing.-This form of plaster-of-Paris dressing is applied by first placing a short iron rod under the extremity, opposite to and extending some distance above and below the point at which the

FIG. 113.

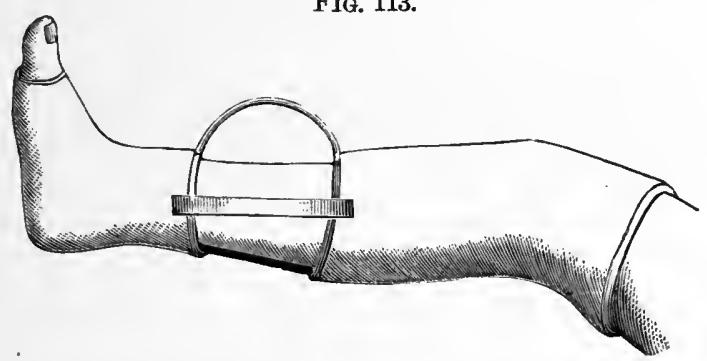

Interrupted plaster-of-Paris dressing. (STIMson.)

dressing is to be interrupted ; this is fixed by a few turns of the plaster bandage above and below the portion of the limb which is to be left exposed; stout wire is next bent into loops, the extremities of which are incorporated in the subsequent turns of the plaster bandage; three loops thus placed in addition to the posterior iron bar 
will usually make the dressing sufficiently firm (Fig. 113). A number of turns of the bandage are applied to fix the metal loops firmly, and the limb is held in the desired position until the plaster has set.

Application of the Plaster-of-Paris Jacket.-The patient's body should be covered with a soft, closely fitting woven shirt without arms, but with shoulder-straps to hold it in position, or an ordinary woven undershirt may be employed; one or two folded towels, or a pad of cotton wrapped in a towel, are next placed over the abdomen between the shirt and the skin-this was called, by Professor Sayre, the dinner pad, and is intended to leave space for distention of the abdomen after eating. Small pads of raw cotton may also be plaeed over the anterior iliae spines, and, in the case of females, a pad of cotton wrapped in a handkerchief may be placed over each mammary gland.

The patient should next be suspended by the apparatus, consisting of a collar and arm-pieces attached to a crossbar (Fig. 114), which is attached by a cord and pulley to a tripod. If this apparatus is not at hand, a very satisfaetory substitute may be made by folding two towels into cravats and tying together the ends, so as to make two loops, one of which is placed in each axilla; a bar of wood two and a half feet in length is next taken, and the loops are secured to the ends of this by stont cords or handkerchiefs ; a Barton's bandage is next applied to the head, and a strip of bandage is passed under the turns which cross the vertex and is seeured to the middle of the cross-bar. The bar is next suspended by a eord passed through a pulley or ring, which may be attached to the top frame of a door if the ordinary tripod cannot be obtained.

The patient should be raised slowly by the apparatus until the toes only are in contact with the floor, and the extension should not be carried to the point which makes it uncomfortable to the patient (Fig. 115). The shirt should be drawn downward over the hips by an assistant and held in place until a few turns of the bandage have been applied.

The plaster-bandage having been soaked and squeezed, a turn should be made around the body above the pelvis, and it should then be carried downward below the iliac 
spines, and from this point made to ascend gradually by spiral turns until it reaches the axillary line. The turns should be applied smoothly and not too tightly. After two or three layers of turns have been applied, the surgeon may rub some moist plaster upon their surface if
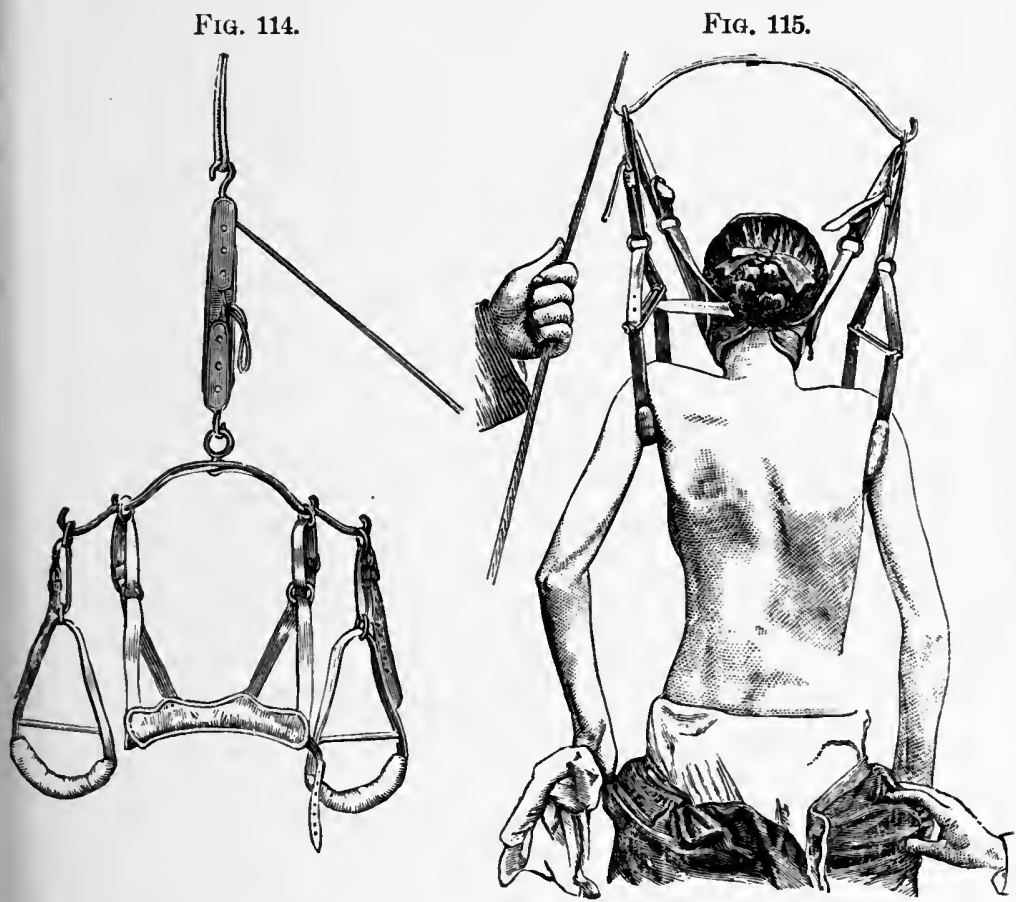

Suspensory apparatus.

Patient suspended for application of plaster jacket.

he desires to use fewer bandages. These turns are repeated until a bandage of the desired thickness is applied, and the surface of the dressing may be finished by rubbing it over with moistened plaster. This jacket for a child will generally require the use of three or four bandages of the dimensions given; for an adult, from six to eight bandages. 
The patient should be kept suspended until the bandage has set, usually from ten to fifteen minutes, and then should be lifted earefully so as not to bend the spine, and placed on his back upon a mattress, until the dressing becomes perfectly hardened. The dinner pad and mammary pads,

Fig. 116.

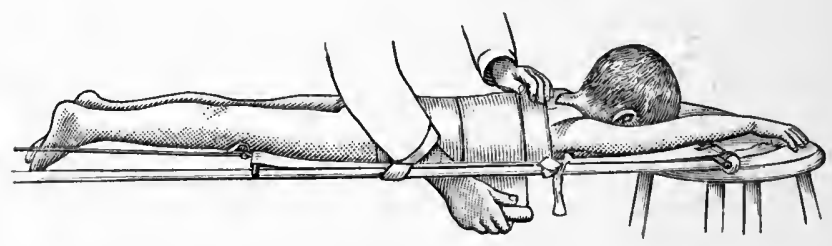

Application of plaster jacket in the recumbent posture. (Lovett.)

if they have been used, should next be removed. In applying this dressing, strips of zinc or tin may be placed between the layers of bandage if it is desired to give more strength to the jacket.

\section{FIG. 117.}

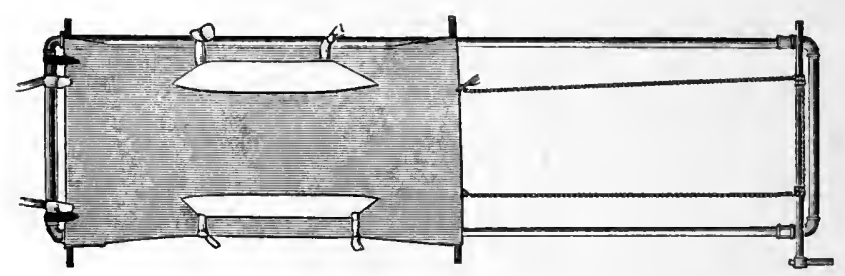

Frame for the application of plaster jacket in the recumbent posture. (Lovett.)

The plaster jacket may also be applied in the reeumbent posture, the patient being placed upon a frame (Figs. 116 and 117).

Application of the Jury-mast by Means of Plasterof-Paris.-In disease of the spine involving the cervical or upper dorsal region the ordinary plaster-of-Paris jacket 
is not satisfactory, and in such eases the "jury-mast" is employed in connection with the plaster jacket. In applying the "jurymast," the same steps are taken in the preparation of the patient as in applying the plaster-of-Paris jacket, with the exception of extension, which need not be used.

After three or four layers of the plaster-bandage bave been applied to the body, an apparatus made of two bars of metal having two perforated strips of zinc attached to them a few inches apart, which partly encircle the body, is applied and held in position by turns of the plaster-bandage. The perpendicular bars have at their upper part a slot, into which the lower end of the "jury-mast" fits, and is secured by a serew ; to the upper part of this is attached a morable

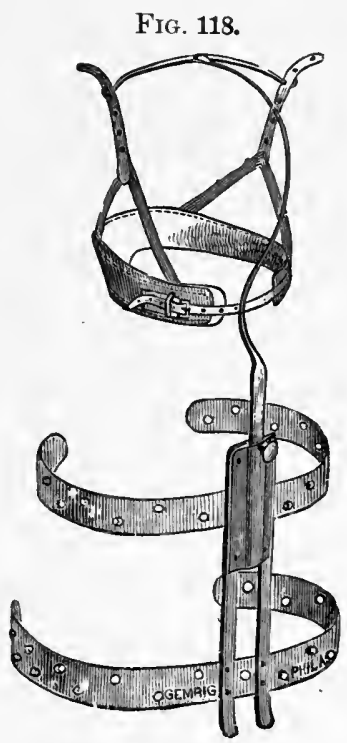

Head-support and jury-mast. cross-bar, to which are fastened the straps of the collar from which the head is suspended (Fig. 118).

The Bavarian Dressing.-To apply this dressing, which is sometimes employed in the treatment of fractures of the extremities, take two pieces of Canton flannel the length of the part to be enclosed, and more than wille enough to envelop its circumference. In applying it to the leg, these pieces should be eut so as to eorrespond to the outline of the leg and posterior portion of the foot. These pieces should be placed one over the other and sewed together in the middle line, the seam eorresponding to the back of the leg. This dressing is then placed under the foot and leg, and the inner layer of flannel is brought up in front of the leg and over the dorsum of the foot, and made fast with pins or a few stitches (Fig. 119.) Plasterof-Paris is next mixed with water to form a paste, which 
is rubbed thickly and evenly over the flannel next the limb until a sufficient thickness is obtained; the outer layer of flannel is then brought up about the leg and monlded to its surface by the hands. A loosely applied roller may be used to hold the dressing in place until the plaster has set.

When it is necessary to inspect the parts, the turns of the bandage are cut, and upon separating the layers of flannel the two halves can be turned aside, the seam at the back acting as a hinge. Upon reapplying the splints to

FIG. 119.

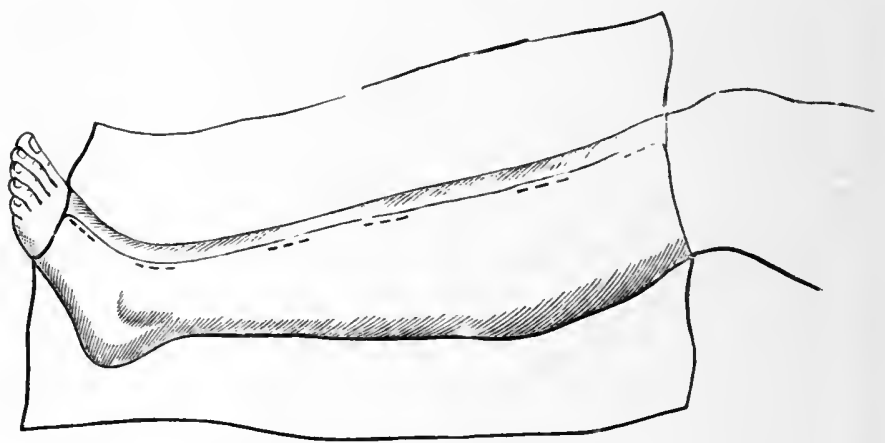

Bavarian dressing.

the leg they may be retained in position by a roller or by one or two strips of bandage.

Moulded Plaster Splints.-It is sometimes found difficult to apply the ordinary plaster dressings to parts irregular in shape, and at the same time to have a splint which can be removed with ease. 'To aceomplish this purpose, moulded splints of plaster may be made by cutting a paper pattern of the part to be covered in, and then cutting pieces of erimoline to conform to this pattern ; eight or ten pieces will usually form a splint of sufficient thickness. One of these pieces of crinoline is laid upon a table and dry plaster is rubbed into its meshes; another is laid 
upon this and plaster is applied to it in the same way, and so on until all the piees have been placed in position, one over the other, with plaster rubbed well into the meshes. The dressing is then folded up and dipped into water, squeezed out, and moulded to the part and held in position, until it sets, by the turns of a bandage. The edges should overlap slightly, and in applying it a strip of waxed paper may be plaeed under the overlapping edge to prevent its adhesion to the dressing below, and thus faeilitate its removal. Splints prepared in this way ean be removed with ease, and are often of service in cases where it is desirable to inspect the parts frequently. I have employed with advantage sueh splints in making fixation of the hip-joint in cases of coxalgia, and also for the same purpose in diseases of other joints. The splints upon being reapplied are seeured by a few strips of adhesive plaster or by a roller-bandage.

Trapping Plaster-of-Paris Bandages.-In applying the plaster-of-Paris dressing to a part where there is a wound whieh is covered by the plaster-bandage, it is well to make some provision whereby the plaster dressing over the site of the wound may be eut away, making a trap or window through which the wound may be inspected, or dressed if neeessary (Fig. 120). To aceomplish this, before applying the plaster-bandage, a compress of lint or gauze or a small pasteboard box should be placed over the wound, which, when the dressing is completed, forms a projection on its surface, indieating the position of the wound, and also allows the surgeon to cut away the dressing without injuring the skin below. These traps may be eut out after the bandage has partially set, or after it has become hard. In applying the plaster-of-Paris dressing in eases of eompound fracture, I always make provision for trapping of the bandage if it should beeome neeessary, althongh in the vast majority of eases if the wound remain aseptie it does not liave to be done.

Removing Plaster-of-Paris from the Hands.-One objection to the use of plaster-of-Paris dressings is the difficulty of removing it from the hands of the surgeon, 
and the harsh condition in which the skin is left after its removal. If, however, the hands are washed in a solution of carbonate of sodium-a tablespoonful to a basin of water-the plaster will readily be removed and the skin will be left in a soft and comfortable condition. Rubbing the hands with glycerine, moist brown sugar or cornmeal accomplishes the same object.

Fig. 120.

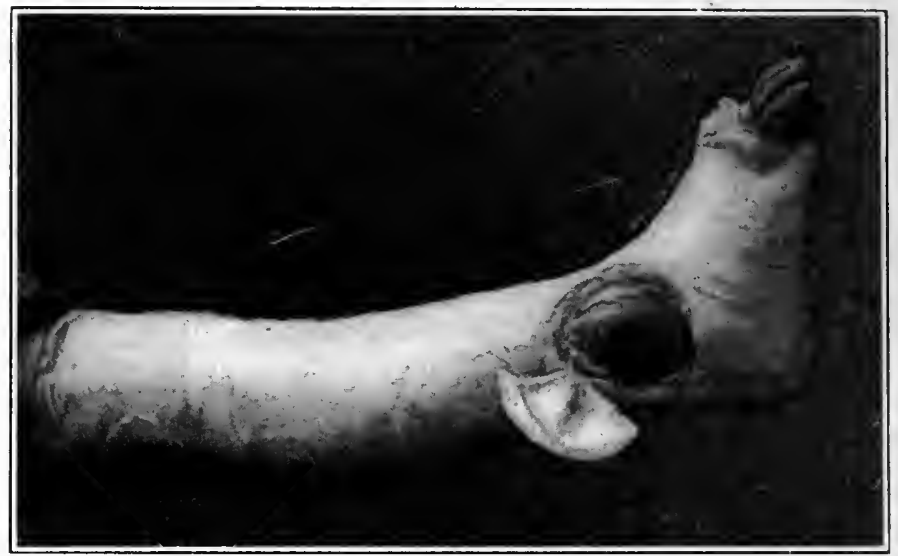

Plaster-of-Paris bandage trapped.

Removal of the Plaster-of-Paris Bandage.-The removal of the plaster-handage is sometimes a matter of difficulty, particularly, in the case of fractures, if it has to be removed before the fragments below it are consolilated, as it may disarrange them and cause the patient pain if it is not accomplished without much force.

When the bandage is applied to get a cast of a part, or in the treatment of fractures where it may be necessary to remove the bandage in a few days to inspect the parts, a strip of sheet-lead one-half of an inch in width is first placed over the flannel bandage and is allowed to project at each end beyond the dressing; the plaster can then be 
readily cut through upon the strip of lead with a knife without injury to the parts below (Fig. 121). As soon as the

FIG. 121.

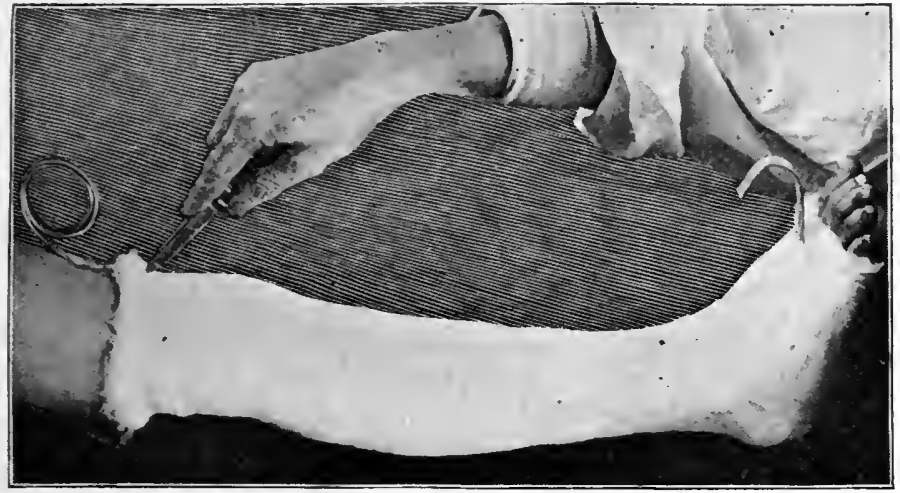

Cutting plaster-bandage upon lead strip.

bandage has become firm, the lead strip is removed by tractim upon one end of it; and, if the bandage has been entirely divided, it ean be removed at any time without difficulty.

In applying plaster dressings to the extremities, even if their removal is not likely to be immediately required, I usually employ the lead strip, cutting the bandage upon it, but leaving three or four bridges of undivided band-

FIG. 122.

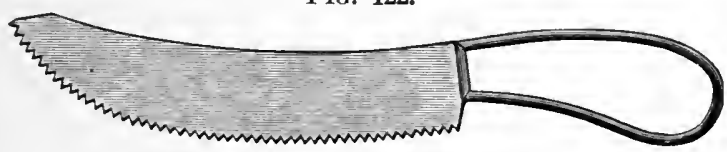

Hunter's saw for removing plaster bandages.

age, which can easily be divided when the removal of the bandage is finally required.

Plaster-bandages may also be removed by means of a saw devised for this purpose (Fig. 122); by Gigli's wire saw drawn under the bandage by a string, which euts 
rapidly and does not endanger the skin ; by strong cuttingshears of various kinds (Fig. 123); or a line may be painted over the dressing with hydrochloric acid or vinegar, which softens the plaster, so that it can readily be cut through with a knife. The incision of the bandage upon the lead strip or the use of the saw or shear's is, I think, most satisfactory in removing these dressings. They should

FIG. 123.

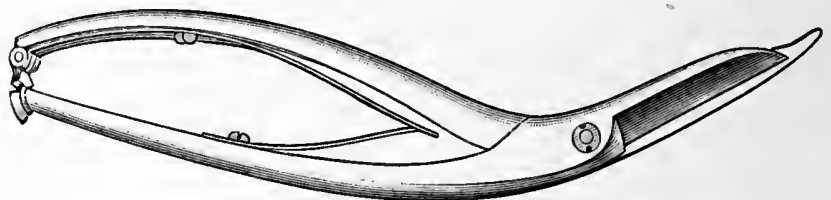

Shears for cutting plaster-bandages.

be used carefully, as the final layers of the bandage are divided, to avoid wounding the skin.

Uses of Plaster-of-Paris Dressings.-These dressings are employed to secure fixation as primary or secondary dressings in the treatment of fractures, and in the ambulant treatment of fractures, and for a like purpose in injuries and diseases of the jo:nts. They are also largely enployed in the treatment of discases and deformities of the spinal column, and will be found most satisfactory applications after osteotomy and tenotomy, to secure immobility and to hold the parts in their corrected positions; when employed in the dressing of cases after tenotomy, they are generally used for a few weeks until the appropriate mechanical apparatus is applied.

The Starched Bandage.-To apply this bandage, starch is first mixed with cold water until a thin, creamy mixture results, and this is heated until it is converted into a clear mucilaginous liquid. The part to be dressed is first covered with a flannel roller, and over this a few layers of a cheese-cloth or crinoline bandage, which has been shrunken, are applied; the starch is then smeared or 
rubbed with the hand evenly into the meshes of the material, and the part is again covered with a layer of turns of the bandage, and the starch is again applied; this manipulation is continued until a dressing of the desired thickness is produced. Strips of pasteboard may be applied between the layers of the bandage, to give additional strength to the dressing, if desired.

It requires from twenty-four to thirty-six hours for the starehed bandage to become dry and thoroughly set. It may be removed in the same way in which the plaster-ofParis dressing is removed.

Use.-Before the introduction of the plaster-of-Paris dressing, it was frequently employed in the treatment of fractures, and in injuries and diseases of the joints. It may be used in such cases, but possesses no advantage over the plaster-of-Paris dressing, and has the disadvantage of setting much less promptly.

Silicate of Potassium or Sodium Bandage.-In applying this bandage, after a flamel roller and several layers of a cheese-cloth or crinoline bandage have been applied to the part, the surface of the latter is coated with silicate of sodium or potassium applied by means of a brush, then a second layer of bandage is applied and treated in the same manner, and this manipulation is continned until a bandage of the desired thickness is produced. This dressing may also be applied by soaking loosely wound rollers of crinoline in silicate of potassium or sodium and applying them to the part as the plaster-ofParis bandage is applied. It requires twenty-four hours for this dressing to become firm. As it is irksome for a patient to keep a part quiet while the silicate bandage is becoming firm, I often cover it as soon as applied with a layer of tissue-paper, and apply over it a light plaster-ofParis bandage, which sets in a few minutes; this is removed at the end of twenty-four hours, when the silicate bandage is usually firm. In removing the silicate bandage, it may first be softened by soaking it in warm water, and then it can readily be cut with scissors, or it may be eut with bandagc-shears. 
In applying either the starched bandage or the silicate of potassium bandage, care should be taken to use cheese-cloth or crinoline which has been shrunken by being moistened and allowed to dry before being employed; otherwise, dangerous compression of the part may occur if the bandage has been firmly applied and shrinks after its application.

The Paraffin-bandage.-Paraffin, which melts at from $105^{\circ}$ to $120^{\circ} \mathrm{F}$., is used in the application of this bandage. The limb being covered by a flannel roller, a vessel containing paraffin is placed in a basin of boiling water. As the roller, which may be either of flannel, cheese-cloth, or crinoline, is unwound, it is passed through the melted paraffin and applied to the part, and the turns are repeated until a dressing of sufficient thickness results, when the surface may be brushed over with melted paraffin. This dressing sets very rapidly, being quite firm in from five to ten minutes.

\section{Moulded Splints.}

Raw-hide or Leather Splints.-In moulding rawhicle or leather splints, it is necessary, first, to apply a plaster-of-Paris bandage to the part to which the rawhide splint is to be fitted; and as soon as the plaster has set, it is removed, and a solid plaster cast is next made by pouring liquid plaster-of-Paris into this mould. When this has become dry, a piece of raw-hide, which has been soaked for a time in warm water, is moulded to the cast and held firmly in contact with it by tacks or a bandage until it has become perfectly dry. It is then removed, and its surface is covered with several coats of shellac, to prevent its absorbing moisture from the skin when applied, and clanging its shape. Eyelets or hooks are fastened to the edges of the splint, through which tapes are passed to secure it in place.

Made in this manner, raw-hide splints fit the part very accurately, and constitute a very satisfactory dressing for cases of joint-disease, and in the form of leather jackets are often employed in the treatment of disease of the spine in place of the plaster-of-Paris jacket (Fig. 124).

In the treatment of high clorsal or cervical caries a 
FrG. 124.

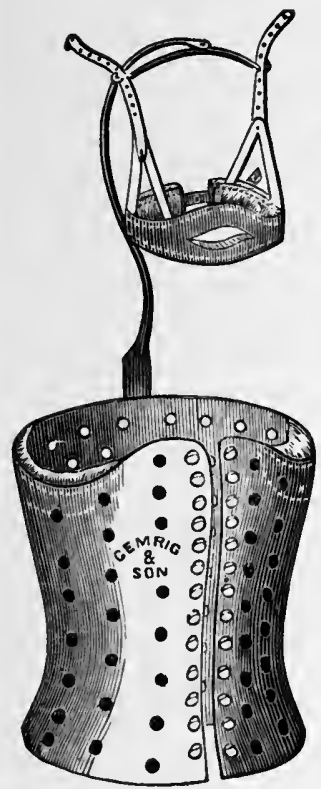

Leather jacket. with jury mast.

leather splint in two sections, which rests upon the shoulders and supports the head, is often used with good results (Fig. 125).

Binders' Board or Pasteboard Splints.-This material, which can be obtained in sheets of different thicknesses, is frequently employed for the manufacture of splints. In moulding these splints, a portion of the board of the requisite size and

FIG. 125.

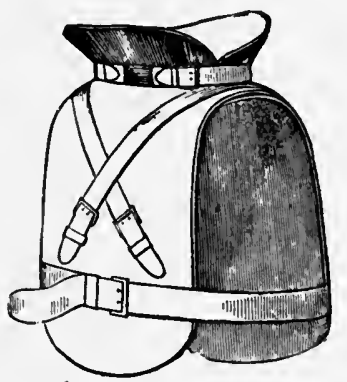

Leather splint for cervical caries. (OWEN.)

thickness is dipped in boiling water for a short time, and when it has become softened it is removed and allowed to cool ; a thick layer of cotton-batting is next applied over it and it is then moulded to the part and held firmly in place by the turns of a roller-bandage; in a few hours it becomes dry and hard.

This material, from its cheapness and the ease with which it is obtained, is frequently employed to mould splints in the treatment of fractures. Moulded splints of this kind on account of the ease of their application and removal are often useful in the temporary treatment of compound fractures and excision of joints to fix the ends of the bones. In such cases it is often desirable to inspect and dress the 
wounds until healing las occurred, when a fixed dressing of plaster-of-Paris may be employed.

Porous Felt Splints.-This material is also employed for the manufacture of splints, and is applied by dipping the material in hot water and then moulding it to the part: and securing it by a bandage; as it dries, it becomes hard.

Hatters'-felt Splints.-Hatters'-felt may also be employed for the manufacture of splints or dressings. It is softened by dipping it in boiling water or heating it in the flame of an alcohol lamp, and when soft and pliable it is moulded to the part, and as it cools it again becomes hard. These splints are employed for the same purpose as those made of plaster-of-Paris, leather, or pasteboard. 


\section{PA R T I I. M I NOR SURGERY.}

Antiseptic and Aseptic Surgical Dressings,-This subject is considered on page 325 .

\section{MATERIALS USED IN SURGICAL DRESSINGS.}

Lint.-This material is employed in surgical dressings, and is of two varieties: the domestic lint, which consists of pieces of old linen or muslin which have been thoroughly washed or boiled and then dried, or the surgical lint, which resembles Canton flannel in appearance; the latter is the best material, as it has a greater absorbing capacity.

Lint is used as a material on which unctuous preparations are spread in the dressing of wounds, and is employed also as a material for saturating with the various solutions which are used in wet dressings, such as leadwater and laudanum; the lint, after being saturated with the solution, is covered with rubber-tissue or oiled silk when applied, to prevent too rapid evaporation of the solution. It is also one of the best materials from which to construct the compresses employed in the treatment of fractures.

Paper-lint.- This is made from old rags or wood-pulp, has great absorbing power for fluids, and may be used as a substitute for surgical lint in the application of wet dressings to surfaces when the skin is unbroken.

Oakum.-This material, made from old tarred rope, was formerly much employed in the dressing of wounds, before the introluction of the antiseptic method of wound treatment. From its elasticity it is found to be an excellent material for padding splints or other surgical appliances. It is employed also in the form of pads to place under patients to relieve portions of the body from pressure, or to absorb discharges which soak through the 
dressings. A mass of oakum which has been well teased out and wrapped in a towel forms an excellent pillow on which to support a stump.

Cotton.-Cotton is now employed in surgical dressings principally as a material to pad splints or to relieve salient parts of the skeleton from pressure in the application of splints or bandages; for instance, in the application of the plaster-of-Paris bandage, the bony prominences are generally covered with small masses of cotton. It possesses but little absorbent power unless used in the form of absorbent cotton, and is not much employed in surgical dressings except for the purposes mentioned above.

Absorbent Cotton.-This material is prepared from ordinary cotton, which is boiled with a strong alkali to remove the oily matter which it contains. When so prepared, it absorbs liquids freely, and by reason of its great absorbing eapacity it is employed largely in surgical dressings. A small mass of sterilized absorbent cotton wrapped upon the end of a probe is now generally employed to make applications to wounds, and has taken the place of the sponge or brush which formerly was enployed for this purpose. On account of its cheapness, after one application it ean be thrown away and a new piece used, and thus the danger of carrying infection from one wound to another by the applicator is abulished. It is largely employed in gynecologieal practice for making applications to the female genital organs.

Wood-wool. - Wood-wool made from wood-pulp, such as is employed in the manufacture of paper, is also furnished in the shape of lint, sponges, and pads, and may be used for the same purposes as the ordinary surgical lint.

Oiled Silk or Muslin.-These materials are employed as an external covering for moist dressings to prevent rapid evaporation from the dressings; they form excellent materials for this purpose, but as they are quite expensive their use is limited.

Waxed or Paraffin-paper.-This dressing is prepared by passing sheets of tissue-paper through melted wax or paraffin, and then allowing them to dry. Paper thus treated 
forms an excellent and cheap substitute for oiled silk or muslin, and may be employed for the same purpose for which the latter materials are user.

Rubber-tissue.-This material, which is prepared by rubber manufacturers, consists of rubber run out into very thin sheets. It has a glazed surface, is very pliable, and at the same time strong, forming, therefore, a cheap and satisfactory substitute for oiled silk, and is employed for the same purposes. A tent made of rubber-tissue may be used as a drain for wounds; it is also used in making the cigarette drain.

Parchment-paper.-This paper is prepared so as to render it water-proof; it is employed in surgical dressings for the same purposes as oiled silk and rubber-tissue.

Compresses. - Compresses are prepared by folding pieces of lint, muslin, linen, or gauze upon themselves, so as to form firm masses of variable size; oakum or cotton may also be used to form compresses. Compresses are employed to make pressure over localized portions of the body, as in the treatment of fractures, or to inake pressure upon vessels for the control of hemorrhage.

Tampon.-A tampon is a form of compress which is employed in cavities to make pressure, to control hemorrhage, or to apply various solutions or powders to the surface of the cavity. Tampons used to control hemorrhage are generally made of strips of bichloride, iodoform or sterilized gauze. In applying these, the strips of gauze are packed into the cavity, and when the latter is full a compress is applied superficially and held in place by a bandage. The application of a tampon to the vagina is a favorite method of controlling uterine hemorrhage.

Glycerin Tampon.-This is made by pouring half an ounce of glycerin on a piece of cotton or wool, and then turning up the ends and securing them by a string, one end of which is allowed to remain long enough to hang from the vagina, to facilitate its removal; it is a favorite application to the os uteri.

Tent.-This consists of a small portion of lint, oakum, muslin, or sterilizerl or antiseptic ganze rolled into a coni- 
cal shape, which is employed to keep wounds open and to facilitate the eseape of discharges.

Retractors.-Retractors are made by taking a piece of muslin four inches wide and twelve to eighteen inches in length, and splitting it as far as the eentre, thus making a two-tailed retractor (Fig. 126). A three-tailed retractor is made in the same way, except that the muslin is slit twice instead of once (Fig. 127). Retractors are used to retract the soft parts in amputations, to prevent their injury by

FIg. 126.

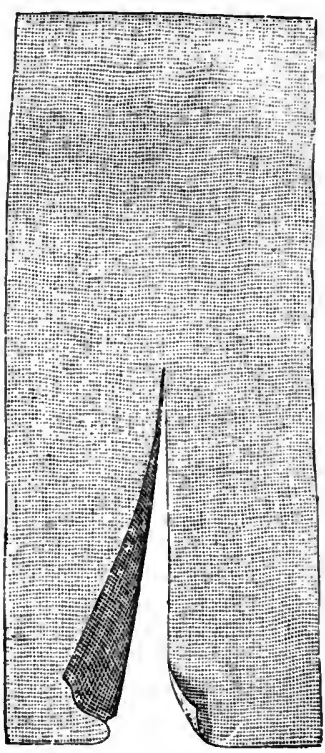

Two-tailed retractor.
FI(., 127.

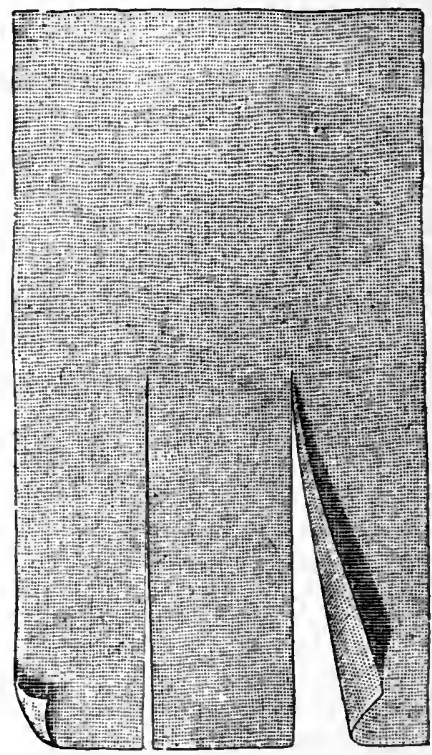

Three-tailed retractor.

the saw in the division of the bones. When one bone is sawed a two-tailed retractor is used, and when two bones are sawed a three-tailed retractor is employed.

Plasters. - The varieties of plaster which are most commonly employed in surgical dressings are adhesive or resin plaster, isinglass plaste; and rubber adhesive plaster. 
Before using any of these plasters upon parts which are eovered by hairs, the latter shonld be removed by shaving, otherwise traction upon them, if the plaster be used for the purpose of extension, will eause the patient diseomfort, and unneeessary pain will also be inflicted at the time of its removal.

Resin Plaster.-This plaster, which is machine-spread, is employed frequently in surgical dressings; the spread surface is eovered with a layer of tissue-paper, which should be removed before it is used; it is eut into strips of the required width and length, and the strips should be cut length wise from the roll of plaster, as the eloth upon which it is spread stretches more transversely than in a longitudinal direction. When heated and applied to the surface it holds firmly; it is prepared for applieation by applying the unspread side to a vessel containing hot water, or it may be passed rapidly through the flame of an alcohol lamp.

This is the variety of plaster which is generally used in making the extension-apparatus for the treatment of fractures, for strapping the chest in fiactures of the ribs and sternum, for strapping the pelvis in eases of fractures of the pelvie bones, and for strapping the breast, the testicle, ulcers, or joints.

Swans'-down Plaster.-This plaster is much the same as resin plaster, but is spread upon a heavier material, and is an excellent plaster to use for an extension-apparatus, where it is to be worn for a long time.

Ichthyol Plaster.-This plaster is prepared by ineorporating iehthyol and the ordinary rubber plaster, it is much less irritating to the skin and possesses the same adhesive properties and is used for the same purposes as the resin or zine oxide plasters.

Rubber Adhesive Plaster.- $I$ 'his plaster is made by spreading a preparation of Inclia-rubber on muslin, and has the advantage over the ordinary resin plaster that it adheres without the application of heat. It is employed for the same purpose as resin plaster, but when applied continnonsly to the skin it is apt to produce a eertain amount of irritation, and for this reason when it is to be 
applied for some time, as in the case of an extensionapparatus, it is not so comfortable a dressing as that made from resin plaster.

Zinc Oxide Adhesive Plaster.-This plaster is prepared by incorporating with rubber adhesive plaster oxide of zinc. It is equally as adhesive as the rubber plaster, and possesses the advantage that it is not apt to produce irritation of the skin. It is used for the same purposes as the rubber adhesive plaster.

Isinglass Plaster.-This plaster is made by spreading a solution of isinglass upon silk or muslin, and it has been found a most useful dressing in the treatment of superficial wounds. It is cansed to adhere to the surface by moistening it, and when used in the treatment of wounds it should be moistened with an antiseptic solution. The best variety is spread on muslin, and when properly applied adheres as firmly and possesses as much strength as the ordinary resin plaster.

Soap Plaster.-Soap plaster for surgical purposes is prepared by spreading emplastrum saponis upon kid or chamois skin. It is not employed for the same purposes as the resin or rubber plaster, as it has little adhesive power, and is used simply to give support to parts or to protect salient portions of the skeleton from pressure. It is found to be a most useful dressing when applied over the sacrum in cases of threatened bedsores, and may be applied for the same purpose to other parts of the body where pressure-sores are apt to occur.

In the treatment of sprains of joints, a well-moulded soap-plaster splint secured by a bandage will often be found a most efficient dressing, and in the treatment of fractures the comfort of the patient is often materially increased by applying small pieces of soap plaster over the bony prominences, upon which the splints, even when well parded, are apt to make an undue amount of pressure. 


\section{STRAPPING.}

This consists in applying pressure to parts by means of of strips of plaster firmly applied; it is a procedure often employed in surgical practice.

Strapping the Testicle.-In strapping the testicle, strips of resin plaster are usually employed; a dozen or more strips one-half an inch wide and twelve inches in length will be required.

The scrotum should first be washed and shaved, and the surgeon next draws the skin over the affected organ tense by passing the thumb and finger around the scrotum at its upper portion, making circular constrietion ; a strip of muslin is passed in a circular manner around the skin of the scrotum above the organ, and is tightly drawn and secured by passing around it a strap of plaster which has been lieated; this isolates the part and prevents the other straps from slipping. Straps are now applied in a longitudinal direction, the first strap being fastened to the circular strap and carried over the most prominent part of the testicle, and then carried back to the circular strap

Fig. 128.
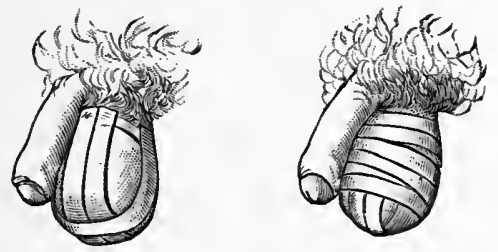

Strapping the testicle. (SмIтн.)

and fastened. A number of these straps are applied in an imbricated manner until the skin is covered (Fig. 128), and the dressing is completed by passing transverse straps around the testicle from its lowest portion to the circular strap; care should be taken to see that no portion of the skin is left uneovered. 
Strapping the testiele is enployed with advantage in the subacute stage of orchitis or epididymitis; as the swelling of the testicle diminishes the straps become loose, and the part will require re-strapping. It will also be found a useful means of applying pressure to the scrotum after the injection-treatment of hydrocele.

Strapping of the Chest.-To strap one-half of the chest, strips of resin plaster two and a half inches wide, and sufficiently long to extend from the spine to the median line of the sternun, are required-eighteen to twenty inches in length. The first strap is lieated, and one extremity is placed upon the spine opposite the lower portion of the ehest; it is then earried over the clrest, and its other extremity is fixed upon the skin in the median line of the sternum. Straps are next applied from below upward in the same manner, each strap overlapping onethird of the preceding one, until the axillary fold is reached (Fig. 129); a seeond layer of straps may be applied over the first, if additional fixation is desired, or a few oblique straps may be employed.

Adhesive straps applied in this manner very materially limit the motion of the ehest-wall npon the affeeted side,

FIG. 129.

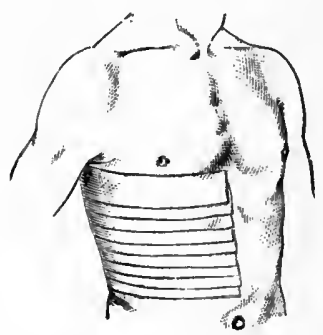

Strapping over the chest. and are frequently employed in the treatment of fractures and dislocations of the ribs, in contusions of the ehest, and in cases of plastic pleurisy when the motions of the chest-wall are extremely painful to the patient.

Strapping of Ulcers.-To strap ulcers of the leg, strips of resin plaster one and a half inches wide, and suffieiently long to extend twothirls of the distance around the limb, are required. The ulcer should be thoronghly eleansed, and the skin surrounding it well dried; the first strap, after being heated, is applied transversely to the long axis of the leg about two inches below the ulcer. and is carried two-thirds of the 
aistance around the limb; another strap is applied to a corresponding point of the skin above this one, so that it overlaps one-third of the strap first applied, and it is carried two-thirds of the way around the limb. Addi-

\section{FIG. 130.}

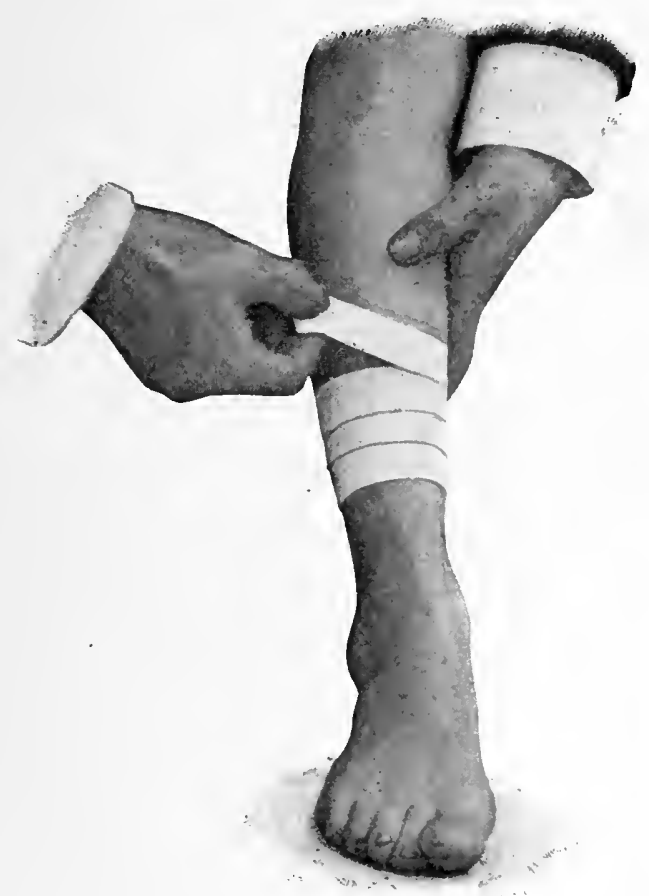

Strapping an ulcer of the leg.

tional straps are thus applied until the ulcer is covered in, and the straps are earried several inches above the ulcer (Fig. 130). Strapping of ulcers may also be accomplished by using narrow straps of plaster one and a half inches in width. The ends of two straps are placed upon the limb 
some distance below the ulcer, and the straps are brought up and made to cross each other so as to draw the tissues toward the point of crossing; a number of imbricated

FIG. 131.

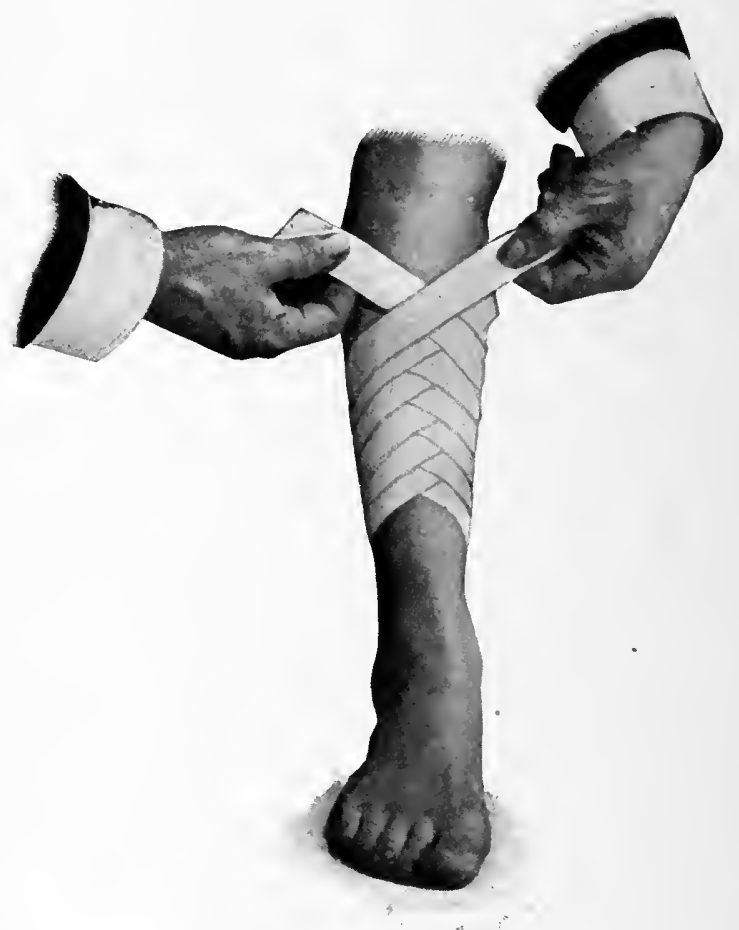

Strapping an ulcer of the leg.

straps are applied in this way until the parts are sufficiently covered in and supported (Fig. 131). Care should be taken to see that the straps are so applied as not to meet or cover the entire circumference of the limb, as by so doing injurious circular compression might result. 
Chronic ulcers upon other portions of the body may be strapped in the same manner.

Strapping of leg ulcers is usually reinforced by the application of a firmly applied spiral reversed or spicabandage of the lower extremity.

Strapping of ulcers of the leg applied in the manner described will be found a most satisfactory method of treating chronic ulcers in this location in patients who have to work during the course of treatment; the straps need be removed only at intervals of a week, and if well applied, the dressing is generally a comfortable one to the patient.

Strapping of Joints.-Strips of resin plaster two inches in width and sufficiently long to extend two-thirds

FIG. 132.

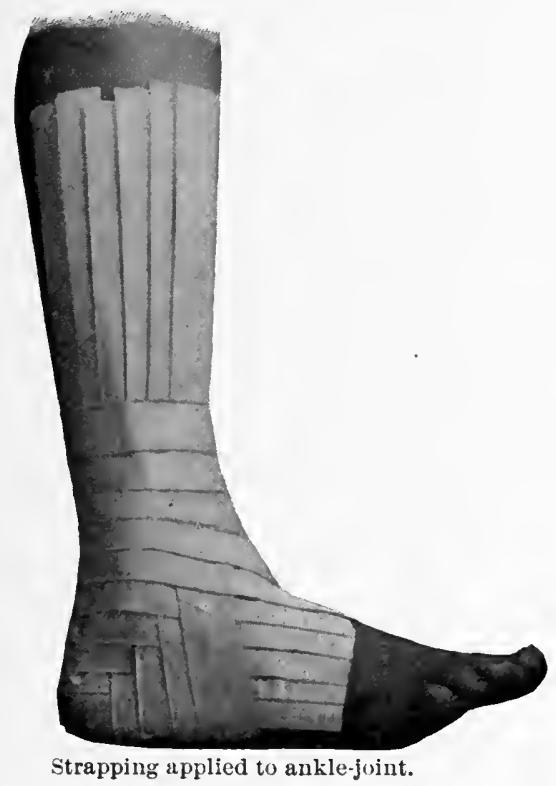


around the joint are required. The first strap is applied a fow inches below the joint, and straps are then applied over this, each strap covering in two-thirds of the preceding one until the joint is covered in and the dressing extends a few inches above the joint.

Strapping will be found a satisfactory dressing in the treatment of sprains of joints in their acute or chronic state.

Strapping the Ankle-joint.-In applying strapping in a sprains of the ankle- or tarsal joints, strips of rubber adhesive plaster one and a half inches in width and eighteen inches in length are required. The first strap is started at the junction of the middle and upper part of the leg, either upon the inner or the outer side, and applied closely to the edge of the tendo Achillis, and carried across the sole of the foot to the base of the great or little toe; several of these straps are applied, covering in the inner or outer surface of the ankle. A strap is next placed with its middle at the point of the heel, the ends being carried to a point on the foot at the junction of the metatarsal bones and the tarsus; a number of these ascending straps are applied, alternating with the vertical straps, until the ankle-joint is covered in. These straps should not be applied so as to meet in front of the foot or ankle and make circular constriction (Fig. 132). After the ankle has been strapped as above described, the foot and ankle are covered with a gauze bandage, and the patient is allowed to walk upon the injured foot.

Strapping the Back.-After contusions or sprains of the lumbar region of the back the firm application of straps gives fixation and support to the parts, and relieves pain. The patient should be placed in such a position that the spine is moderately extended, and strips of plaster two and a half inches in width should be applied, one slightly overlapping the other, from the upper part of the sacrum to the lower ribs. The straps should be long enough to include the posterior half of the trunk, and several layers should be applied. 


\section{POULTICES.}

This form of dressing was formerly much employed in the treatment of inflammatory conditions as a means of applying heat and moisture to the part at the same time, and although the use of poultices is now much restricted since the introduction of the antiseptic method of wound treatment, yet $I$ think there are still conditions in which their employment is both useful and judicious. They are often employed with advantage in inflammatory affections of the chest and of the abdominal organs; and in inflammatory affections of the joints and of bone, combined with rest, their action is often most satisfactory. They constitute a form of dressing which is conducive to the comfort of the patient in cases of deep suppuration by their relaxing effect upon the tissues, and their previous use does not prevent the surgeon from using all aseptic precantions in the opening and drainage of these abscesses, and the employment of aseptic or antiseptic dressings in their subsequent treatment.

Flaxseed Poultice. - This poultice is prepared by adding first a little cold water to ground flaxseed, and then boiling and stirring it until the resulting mixture is of the consistency of thick mush. A piece of ganze or muslin is next taken which is a little larger than the intended poultice, and this is laid upon the surface of a table, and with a spatula or knife the poultice-mass is spread evenly upon it from one-quarter to one-half an inch in thickness; a margin of the muslin of one or one and a half inches is left, which is turned over after the poultice is spread, and serves to prevent it from escaping around the edges when applied. The surface of the poultice may be thinly spread over with a little olive oil, or may be covered with a layer of thin gauze, to prevent the mass from adhering to the skin. It is next applied to the surface of the skin, and is covered with a piece of oiled silk, rubber-tissue, or waxed paper, and held in position by a bandage or a binder. 
Soap Poultice.-This is made by saturating a number of layers of gauze in a mixture of 1 part of green soap to 6 parts of water. It is then applied to the surface and covered with oiled muslin or waxed paper. It may be employed as a primary dressing for some hours to the feet or other parts of the body where the epidermis is thick, before sterilizing these parts previous to operation.

Starch Poultice.-This poultice is prepared by mixing starch with cold water until a smooth, creamy fuid results; boiling water is then added, and it is heated until it becomes clear and attains about the same consistency as the starch used for laundry purposes. When sufficiently cool, it is spread upon gauze or muslin, applied to the part, and covered with oiled silk or waxed paper. This variety of poultice is principally useful in the treatment of diseases of the skin, especially those of the scalp accompanied by the formation of scals or crusts, to facilitate their removal and to afford a clean surface for the application of ointments or wet dressings.

Fermenting Poultice.-This poultice may be prepared by adding yeast (two tablespoonfuls) to a mixture of flaxseed with hot water, making a thin poultice-mass, and allowing it to stand for a few hours in a warm place; it rises and becomes light, and is then spread upon ganze or mnsin and applied as required. A few ounces of porter or a piece of yeast-cake may be used as a substitute for the yeast in preparing this poultice; animal charcoal may also be added to it to increase its disinfectant power.

Antiseptic Poultice.-This is prepared by soaking a pad of sterilized ganze in hot bichloride or carbolic solution and wringing it out to remove the excess of fluid. It is next applied to the part and covered with oiled silk or rubber-tissue, which may be heid in place by a bandage. Such a dressing will absorb a considerable amount of discharge.

Hot Fomentations.-Hot fomentations are employed to keep up the vitality of parts which have bcen subjected to injury, as seen in severe contusions resulting from railway or machinery accidents; also to combat inflammatory 
action. Gauze (several layers in thickness) or surgical lint should be soaked in sterilized water having a temperature of $120^{\circ} \mathrm{F}$.; these are wrung out, placed over the part, and covered with waxed paper or rubber-tissue; a second pad should be placed in the hot water, and applied as soon as the first-applied cloth begins to cool, and so by continuously reapplying them the part is kept constantly covered by a hot dressing. The use of these hot fomentations may in many cases require to be continued for hours before the desired result is obtained. Hot compresses applied in this manner are frequently employed in treating inflammatory conditions of the eye, and are also of the greatest service in keeping up the vitality of parts which have been subjected to severe injury interfering with their blood-supply. I have seen contused limbs, which were cold and seemed doomed to gangrene by reason of diminished blood-supply, have their temperature and circulation restored by the patient and persistent use of this dressing. After the vitality of such a part is restored, it should be covered with cotton and a flannel bandage and surrounded by hotwater bags or hot-water cans.

Lead Water and Laudanum.-This consists of a mixture of Liquor Plumbi Subacetatis, $\tilde{z}$ ss; Tr. Opii,

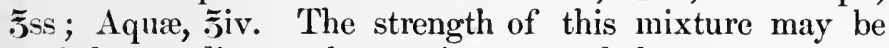
varied according to the requirements of the case.

This is used as a local application, being applied on lint saturated with the solution.

It has long been a popular application in the early treatment of fractures, contusions and sprains and in certain forms of dermatitis. It should not be applied to open wounds or a broken skin surface.

Magnesium Sulphate Solution.-A saturated solution of magnesium sulphate is frequently employed as a local application in the treatment of inflammatory affections of the joints, cellulitis, epididymitis and orchitis. Lint saturated with the solution is applied to the affected part and covered with waxed paper. It is a cleanly dressing and seems to relieve pain and swelling and is frequently employed as a substitute for lead water and laudanum. 
Ichthyol.-This substance combined with lard or lanolin is frequently used in the treatment of inflammatory affections. The ointment generally employed consists of Ichthyol Ammoniacal, כ̈ii.; Adipis or Lanolin, „̋i. This may be rubbed over, or spread upon lint and applied to the part. It is used in the treatnent of sprains, inflamed joints, erysipelas, enlarged lymphatic glands, frost-bites chilblains and burns.

\section{IRRIGATION.}

This may be accomplished by allowing the irrigating fluid to come in contact with the wound or inflamed part -immediate irrigation; or by allowing the cold or warm fluid to pass through rubber tubes which are in contact with or surround the part-mediate irrigation.

Immediate Irrigation.- In employing immediate irrigation in the treatment of wounds or inflammatory conditions, a funnel-shaped can with a stop-cock at the bottom, or a bucket, is suspended over the part at a distance of a few inches (Fig. 133), or a jar with a skein of thread or lamp-wick arranged to act as a siphon may be employed (Fig. 134). The can or jar is filled with water, and this is allowed to fall drop by drop upon the part to be irrigated, which should be placed upon a piece of rubber sheeting so arranged as to allow the water to run off into a receptacle, to prevent wetting the patient's bed. The water employed may be either cold or warm, in accordance with the indications in special cases. If it is desired to make use of antiseptic irrigation, the water is impregnated with carbolic acid or bichloride of mercury; a $1: 5000$ to $1: 10,000$ bichloride solution, or a $1: 60$ carbolic acid or acetate of aluminum solution, being frequently employed with good results.

Antiseptic irrigation employed in this manner will be found a most useful method of treating lacerated and contused wounds of the extremities in which the vitality of 
the tissues is much impaired; in such cases water at a temperature of $100^{\circ}$ to $110^{\circ} \mathrm{F}$. should be preferred to cool water.

Under the use of warm irrigation it is sometimes sur-

FrG. 133.

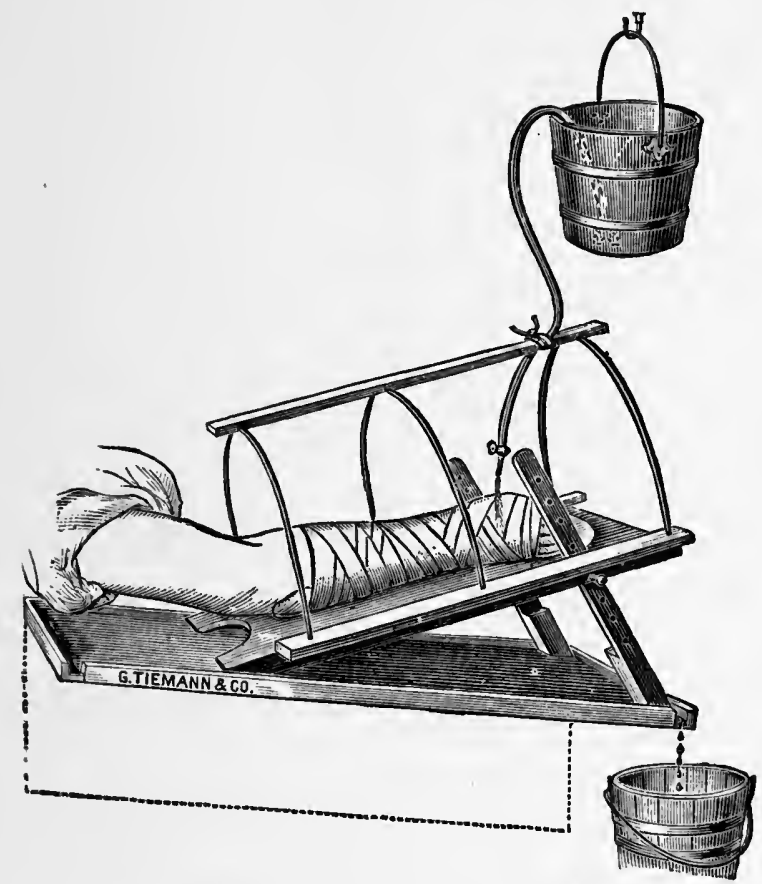

Apparatus for continuous irrigation. (EsMarCH.)

prising to see tissnes apparently devitalized regain their vitality in a short time; the absence of tension from the non-introduction of sutures and firm dressings, and the warmth and moisture kept constantly in contaet with the wound by this method of irrigation, are the important factors in the attainment of this favorable result.

Mediate Irrigation.-In this method of irrigation cold 
or warmth is applied to the surface by means of eold or warm water passing tlurough a rubber tube in contact with

FIg. 134.

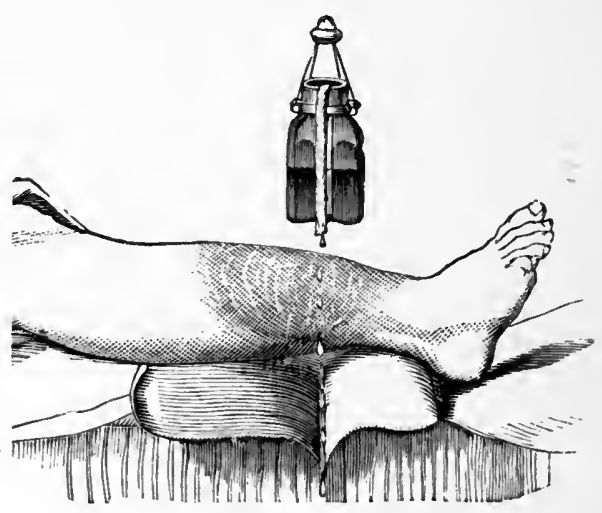

Irrigating-apparatus. (ERICHSEN.)

the part. A flexible tube of India-rubber half an inch in diameter, with thin walls, and sixteen or twenty feet in length, is applied to the limb like a spiral bandage, or is applied in a eoil to the head, breast, or joints, and held in place by a few turns of a bandage; the end of the tube is attached to a reservoir filled with eold or warm water above the level of the patient's body, and the water is allowed to flow constantly through the tubing and eseape into a receptacle arranged to receive it (Fig. 135). Coils of rubber tubing adapted to fit different portions of the body, known as Leiter's coils, are frequently employed in this method of irrigation.

Cold-water Dressings.-These dressings are applied by bringing the cold water either directly in contaet with the part or by applying it by means of a rubber bag or bladder. The temperature of the water may vary from cool water to that of ice-water.

These dressings are employed in local inflammatory conditions. A favorite method for the employment of this 
dressing is by means of cold compresses, which are made of a few layers of gauze or surgical lint, dipped in water of the desired temperature and applied to the part; they are renewed as soon as they become warm. When it is desirable to have the compresses very cold, they may be laid upon a block of ice or in a basin with broken ice; to obtain the best results from their employment, they should be renewed at very short intervals.

FIG. 135.

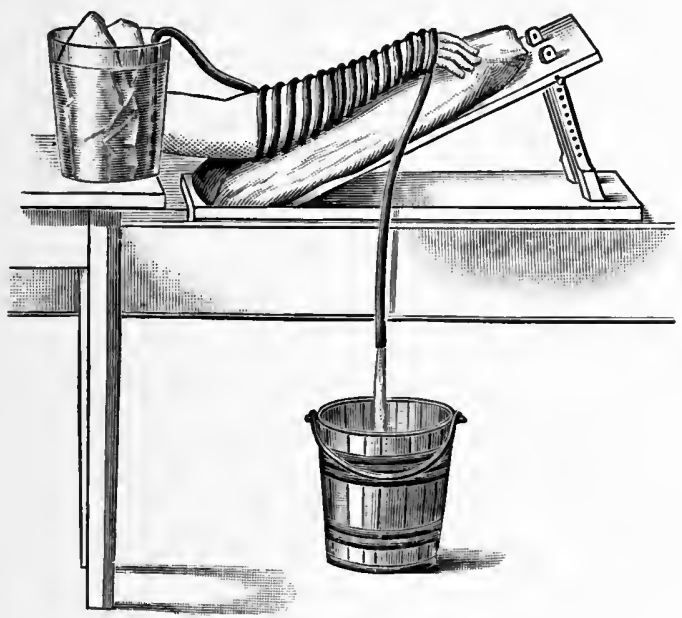

Cold coil applied to arm. (EsMa RCH.)

Ice-bag.-A convenient method of applying cold without moisture is by the use of the ice-bag. 'This is either a rubber bag or bladder, which is filled with broken ice and applied to the part. In using an ice-bag, it is better to cover the part first with a towel or a few layers of lint or gauze, which prevent the surface from becoming wet by absorbing the moisture which condenses upon the surface of the bag or bladder, and thus renders the dressing more comfortable to the patient. The ice-bag is often employed as an application to the head in inflammatory 
conditions of the brain or membranes; to the abdomen in cases of appendicitis or peritonitis, and is used also upon the surface of the body to control internal hemorrhage.

\section{COUNTER-IRRITATION.}

Counter-irritants are substances employed to excite external irritation, and the extent of their aation varies according to the material used and the duration of their application; superficial redness or complete destruction of the vitality of the parts to which they are applied may result.

The use of counter-irritants under favorable circumstances is found to have a decided effect in modifying morbid processes, and they are widely employed as local revulsants in cases of congestion or inflammation, and in cases of collapse for their stimulating effect.

Caution should be exercised in applying counter-irritants to patients who are comatose or under the influence of a narcotic, for here the sensations of a patient cannet be used as a guide to their removal, and their too longcontinued application when the vitality of the tissues is impaired may result in their superficial destruction.

Rubefacients.-These agents, by reason of their irritating properties when applied to the skin, produce intense redness and congestion.

Hot Water.-When it is clesired to make a prompt impression upon the skin, the application of gauze, muslin, or flannel cloths, wrung out in lot water and renewed as rapidly as they become cool, will soon produce a superficial redness of the integument.

Spirit of Turpentine.-This drug applied to the skin is a very active counter-irritant; it may be rubbed upon the surface until redness results. When used upon patients whose skin is very delicate, its action may be modified by mixing it with an equal part of olive oil before applying it; this combination will be found nseful as a rubefacient to the tender skin of young children. 
When redness of the skin has resulted from the application the skin should be wiped dry by means of a soft towel or absorbent cotton, to remove any turpentine from the surface, which by its continued contact may cause vesication.

Turpentine Stupe.-This is prepared by sprinkling spirit of turpentine over flannel cloths which have been wrung out in hot water, or by dipping hot flannel in warm spirit of turpentine: prepared in either way, the stupe should be squeezed as dry as possible to remove the excess of turpentine before being applied to the surface of the body. A turpentine stupe may cause vesication if allowed to remain for too long a time in contact with the skin; its application for from five to ten minutes will usually produce the desired effect; it should be removed after this time, and it may be reapplied if desired.

If the patient complains of severe burning of the skin after the use of turpentine, the painful surface should be smeared freely with vaseline or lard, which will relieve the uncomfortable sensation.

Tincture of Iodine.-This drug is frequently used as a counter-irritant in chronic inflammation. It is painted upon the part at intervals until irritation of the skin is observed, when its use is discontinued for a few days before reapplying the application.

Chloroform.-A few drops of chloroform applied to the surface of the body by means of a piece of lint, muslin, or flannel, and covered by oiled silk or rubber-tissue, will exeite a rapid rubefacient effect.

Mustard.-Ground mustard or mustard flour, prepared from either Sinapis alba or Sinapis nigra, is one of the most commonly used substances to produce rubefacient action. It is generally employed in the form of the mustard plaster or sinapism, which is prepared by mixing equal parts of mustard flour with wheat flour or flaxseed meal, and adding to this sufficient warm water to make a thick paste; this is spread upon a piece of old muslin, and the surface of the paste covered with some thin material, such as gauze, to prevent the paste from adhering to the 
skin. In making a mustard plaster for application to the skin of a child, 1 part of mustard flour should be mixed with 3 parts of wheat flour or flaxseed meal.

A mustard plaster or sinapism may be allowed to remain in eontaet with the skin for a period varying from fifteen to thirty minutes, the time being governed by the sensations of the patient; if it is allowed to remain longer, it may cause resication, which is to be avoided, as uleers prodiced by mustard are very painful and extremely slow in healing. After removing a sinapism, the irritated surface of the skin should be dressed with a piece of muslin or lint spread with vaseline, borie acid or oxide of zine ointment.

To excite a rapid revulsive action, the mustard foot-bath is often employed; it is prepared by adding two or three tablespoonfuls of mustard flowr to a bucket or foot-tub of water at a temperature of $100^{\circ}$ to $110^{\circ} \mathrm{F}$.; in this the patient is allowed to soak his feet for a few minutes.

Mustard Papers.-Chartce Sinapis, which can be obtained in the shops ready for use, are a convenient means of obtaining the rubefacient action of mustard. They are dipped in warm water, and as they are generally very strong, it is well to place a layer of muslin between the surface of the plaster and the skin before applying it to the latter.

Capsicum.-This is also sometimes employed alone as a rubefacient, but it is generally used in combination with spices, forming the well-known spice plaster; this is prepared by taking equal parts of ground ginger, eloves, cinnamon, and allspice, and adding to them one-fourth part of Cayenne pepper; these are thoroughly mixed, enclosed in a flannel bag, and evenly distributed; a few stitches should be passed through the bag at different points, to prevent the powder from shifting its position; before applying it, one side of the bag should be wet with warm whiskey or alcohol. Capsine plasters are employed also to obtain the rubefacient effect of Cayenne pepper.

Aqua Ammonia.-This may also be employed for its 
rubefacient action. A piece of lint saturated with the stronger water of ammonia, placed upon the skin and covered with waxed paper, and allowed to remain for one or two minutes, will produce a marked rubefacient effect.

Vesicants.-Where it is desirable to make a more permanent counter-irritant effect than that produced by rubefacients, substances are employed which by their action on the skin cause an effusion of serum, or of serum and lymph, beneath the cuticle, thus giving rise to vesicles or blisters; they are known as vesicants. The substance most commonly employed to produce vesication is Cantharis, or Spanish fly, and the preparation commonly used is the Ceratum cantharidis.

Fly Blister.-This is prepared by spreading ceratum cantharidis upon adhesive plaster, leaving a margin onehalf an inch in width uncovered, which will adhere to the skin and hold the blister in position. The time required for a fly blister to produce vesication is from four to six hours; it should then be removed, and the surface covered with a flaxseed-meal poultice or with a warmwater dressing. When the blister or vesicle is well developed, it may be punctured at its most dependent part to allow the serum to escape, and it should be dressed with vaseline or boric ointment. If for any reason it is desired to keep up continued irritation after allowing the serum to escape, the cuticle should be cut away and the raw surface should be dressed with some stimulating material, such as the compound resin cerate.

Cantharidal Collodion. - This may be employed to produce vesication; it is applied by painting several layers upon the skin with a brush over the part on which the blister is to be produced. It is a convenient preparation to use when the patient wonld disturb the ordinary blister, as in the case of a child or an insane patient, or where the surface is so irregular that the ordinary blister cannot well be applied. The after-treatment of blisters produced by cantharidal collodion is similar to that described above.

Caution should be observed in using blisters upon the tender skins of children; if employed, they should be 
allowed to remain in eontact with the skin for a short time only. They are contraindicated in patients in whom the vitality of the tissues is depressed by adynamic diseases, and in aged persons.

Strangury, which is shown by frequent and painful micturition, the urine often containing blood, sometimes occurs from the use of cantharidal preparations as blisters. This eondition should be treated by the use of opium and belladonna by suppository, demulcent drinks, and warm sitzbaths, and by leeches to the perineum if the symptoms are very severe.

To avoid the development of strangury, small blisters should be employed, and they should not be allowed to remain ton long in contact with the surface; eantharidal preparations should not be employed in cases where renal or vesical irritation has existed or is present. Strangury may also be avoided by ineorporating opium and camphor with the cantharidal eerate.

Aqua Ammonia Fortior and Chloroform.-These drugs may be employed to produce rapid vesieation, a few drops being placed upon the surface of the body and covered by an inverted watch-glass for a few minutes; or lint saturated with aqua ammonia or chloroform may be placed mpon the skin and covered with waxed paper or oiled silk. Either of these agents applied in this manner, and allowed to remain in contact with the skin for fifteen minutes, will produce marked vesication. The blisters resulting from these agents are painful, and they are only to be used where a rapid result is desired.

Seguin's Method of Counter-irritation.-This consists in stroking the surfaee of the skin lightly and rapidly with the point of a Paquelin cautery ; the lines of stroking may be made at right angles; the application is practically painless, but a very decided counter-irritant effect is produced. It is employed with advantage in neuralgic affections of the spine and joints, and in cases of neuritis of superficial nerves.

Actual Cautery.- This method of counter-irritation is accomplished by bringing in eontact with the skin some 
metallie substance brought to a high degree of temperature. This constitutes one of the most powerful means of comnter-irritation and revulsion; it is rapid in its action, and is not more painful than some of the slower methods. The cauteries generally employed are made of iron, and are fixed in handles of wood or other non-conducting material, and have their extremities fashioned in a variety of shapes (Fig. 136). 'The irons are heated by placing their extremities in an ordinary fire, or by holding them in the flame of a spirit-lamp until they are heated to the desired point, either a white or a dull-red heat. They are then applied to the surface of the skin at one point, or drawn over it in lines either parallel to or crossing one another. The intense burning which follows the use of

FIG. 136.

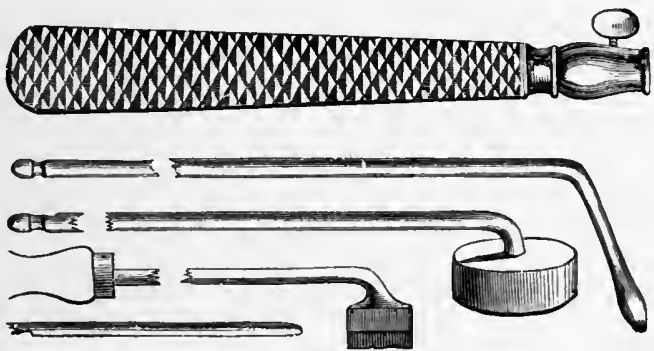

Cautery irons,

the cautery may be allayed by placing upon the cauterymarks compresses wrung out in ice-water or saturated with equal parts of lime-water and sweet oil.

Where the ordinary eautery irons are not at hand, a steel knitting-needle or iron poker heated in the flame of a spirit-lamp or in a fire may be employed with equally satisfactory results. Where the cautery iron is held in eontact with the surface for some time to make a deep burn, the pain of its applieation may be allayed by placing a mixture of salt and cracked ice upon the spot to be cauterized, for a few ninutes immediately before its applica- 
tion. The eautery iron should not be placed over the skin covering salient parts of the skeleton or over important organs.

The actual cautery, in addition to its use in producing counter-irritation and revulsion, is often employed to control hemorrhage and to destroy morbid growths.

Paquelin's Thermo-cautery. - A very convenient and efficient means of using the thermo-cautery is the apparatus of Paquelin, which utilizes the property of heated platinum-sponge. to become incandlescent when exposed

FIG. 137.

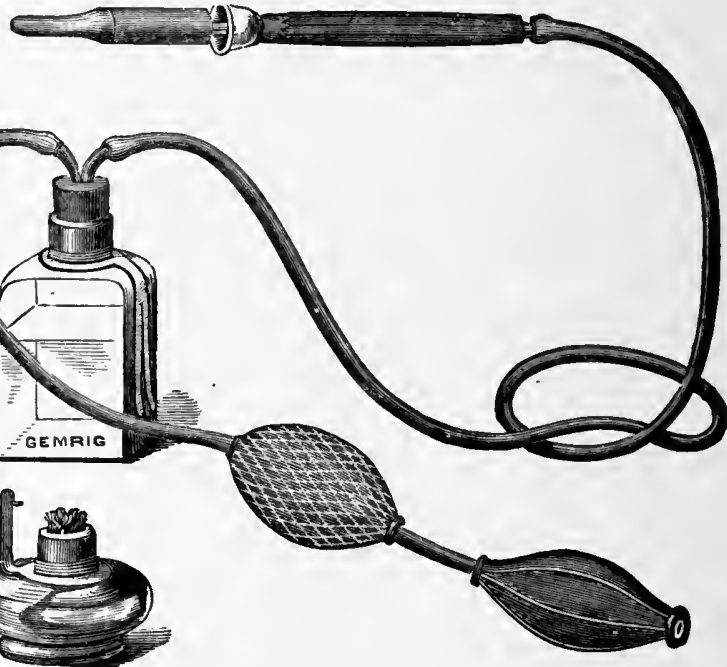

Paquelin's cautery.

to the vapor of benzole or rhigolene (Fig. 137). The cautery is prepared for use by attaching the gum tube to the receiver containing benzole, and heating the platinum knife or button, which also is attached to the benzole receiver by a rubber tube, in the flame of the alcohol lamp for a few moments, and then passing the vapor of benzole 
through the platinum-sponge, which is enclosed in the knife or button, by compressing the rubber bulb. The point may be brought to a white heat or only to a dullred heat.

This form of eautery may be employed for the same purposes as is that previously mentioned; its great advantage consists in the ease with which it can be prepared for use. The knives heated to a dull-red heat will be foun? of great service in operating upon vascular tumors, where the use of an ordinary knife would be accompanied by profuse or even dangerous hemorrhage. Wounds made by the actual cautery are aseptic wounds, and when dusted with an antiseptic powder generally heal promptly under the scab without suppuration.

\section{BLOODLETTING.}

This procedure is often resorted to, to obtain both the local and the general effects following the withdrawal of blood from the circulation. Local depletion is accomplished by means of some one of the following procedures: scarification, puncturation, cupping, and leeching; and general depletion is effected by means of venesection or by arteriotomy.

Scarification.-Scarification is performed by making small and not too deep incisions into an inflamed or congested part with a sharp-pointed bistoury; the incisions should be in parallel lines, and should be made to correspond to the long axis of the part, and care should be taken in making them to avoid wounding superficial veins and nerves. Incisions thus made relieve tension by allowing blood and serum to escape from the engorged capillaries of the infiltrated tissne of the part. Warm fomentations applied over the incisions will increase and keep up the flow of blood and serum. Scarification is employed with advantage in inflammatory conditions of the skin and subcutaneous cellular tissue and in acute inflammatory swelling or cedema of the mucous membrane, for instance, of the conjunctiva, and in acute inflammation of the ton- 
sils, tongue, and epiglottis it is an especially valuable procedure.

A modification of scarification, known as deep incisions, is practised in urinary infiltration to establish drainage and to relieve the tissues of the contained urine, and to prevent sloughing; in threatened gangrene and phlegmonous erysipelas the same procedure is adopted to relieve tension by permitting of the escape of blood and serum, and its employment is often followed by most satisfactory results.

Puncturation.-This procedure consists in making punctures into inflamed tissues with the point of a sharppointed bistoury, which should not extend deeper than the subcutaneous tissue; it is an operation similar in character to that just described, its object being to relieve tension and bring about depletion. It is employed in cases similar to those in which scarification is indicated, and is resorted to in cases of diffuse areolar inflammation or erysipelas.

Cupping.-Cupping is a convenient method of employing local depletion by inviting the blood from the deeper parts to the surface of the body. Cupping is accomplished by the use of dry or wet cups. When the former are used, no blood is abstracted, and the derivative action only is obtained; when wet cups are employed, there is an actual abstraction of blood or local depletion as well as the derivative action.

Dry Cupping.-Dry cups as ordinarily applied consist of small cup-shaped glasses, which have a valve and stopcock at their summit; these are placed upon the skin and an air-pump is attached, and as the air is exhansted in the cup the congested integument is seen to bulge into the cavity of the cup. When the exhaustion is complete the stop-cock is turned and the air-pump is disconnected, the cup being allowed to remain in position for a few minutes, and is then removed by turning the stop-cock and allowing air again to enter the cup. This procedure is repeated until a sufficient number of cups have been applied (Fig. 138). 
In cases of emergency, when the ordinary cuppingglasses and air-pump are not available, a very satisfactory substitute may be obtained by taking a wineglass and burning in it a little roll of paper, or a small piece of lint or paper wet with alcohol, and before the flame is

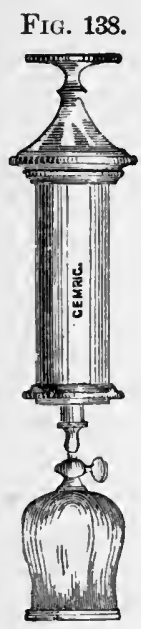

Cupping-glass and air-pump. extinguished rapidly inverting it upon the skin; or the air may be exhausted by the introduction, for a moment or two, of the flame of a spirit-lamp into the cup. Applicd in this manner, cups will draw as well as when the more complicated

Fig. 139.

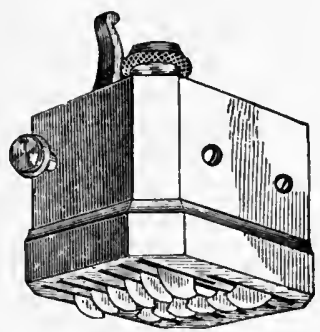

Scarificator.

apparatus is used; and when they are to be removed, it is only necessary to press the finger on the skin close to the edge of the cup until air enters it, when it will fall off. Although dry cups do not remove blood directly, there is often an escape of blood from the capillaries into the skin and cellular tissue, as is evidenced by the ecchymosis which frequently remains for some days at the seat of the cup-marks.

Wet Cupping.-When the abstraction of blood as well as the derivative action is desired, wet cups are resorted to, and here it is necessary to have a scarificator as well as the cups and air-pump (Fig. 139).

Before applying wet cups, the skin should be washed carefully with bichloride or carbolic solution, and the 
scarificator should also be sterilized by boiling. A cup is first applied to produce superficial congestion of the skin ; this is removed, and the scarificator is applied and the skin is cut by springing the blades. The cups are immediately reapplied and exhausted, and they are kept in place as long as blood continues to flow. When the vacuum is exhansted and blood ceases to flow, they should be removed and emptied, and may be reapplied if it is desirable to remove more blood. A sharp-pointed bistoury which has been sterilized may be employed to make a few incisions into the skin instead of the scarificator, and improvised cups may be employed if the ordinary cuppingapparatus cannot be oltained.

After the removal of wet cups the skin should be washed carefully with a bichloride or carbolic solution, and an antiseptic dressing should be placed over the wounds and held in place by a roller-bandage.

Leeching.- The abstraction of blood by leeching is not much employed at the present time. Two varieties of leeches are used - the American leech, which draws about a teaspoonful of blood, and the Swedish leech, which draws three or four teaspoonfuls.

Before applying leeches the skin should be carefully washed, and the leech should be placed upon the part from which the blood is to be drawn, and confined to this place by inverting a tumbler or glass jar over it ; if it does not bite or take hold, a little milk or blood should be smeared upon the surface, which will generally secure the desired result. As soon as the leech has ceased to draw blood it is apt to let go its hold and fall off; if, however, it is desired to remove leeches, they may be made to let go their hold by sprinkling them with a little salt. After the removal of leeches bleeding from the bites may be encouraged, if desirable, by the application of warm fomentations. Leech-bites should be washed with a bichloride or carbolic solution, and a compress of bichloride or iodoform gauze placed over them and secured by a bandage.

It sometimes happens that free bleeding continues from the leech-bite after the removal of the leeches; in this 
event, if a compress does not control the hemorrhage, the bleeding point should be touched with the point of a steel knitting-needle heated to a dull-red heat, and if this fails to control the bleeding a delicate harelip pin should be passed through the skin under the bite and a twisted suture thrown around this; the wound should then be washed and dressed as previously described.

In applying leeches in or near the mucous cavities care should be taken to see that they do not escape into the eavities and pass ont of reach. Leeches should not be employed directly over inflamed tissue, but should be applied to parts surrounding it; they should not be allowed to take hold directly over a superficial artery, vein, or nerve, and should never be applied to a part where there are delicate skin and a large amount of loose cellular tissue, as in the eyelid or scrotum, as unsightly ecchymoses will result, which persist for some time. Leeches should not be used a second time.

The Mechanical Leech.-The mechanical leech is an apparatus which has been constructed to take the place of the leech; it consists of a scarificator, cup, and exhausting syringe or air-pump (Fig. 140). In using this apparatus, after the scarificator has been used the piston of the exhaustinginstrument should be drawn out slowly, which secures a better flow of blood than if a sudden vacuum is created.

Venesection.-Venesection, as its name implies, consists in the division of a vein, and it is the ordinary operation by which FIG. 140.

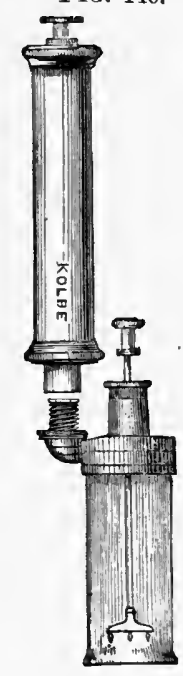

Mechanica! leech. general depletion or bleeding is accomplished. Venesection at the bend of the elbow is the operation which is now usually resorted to for general bloodletting; the vein selected is the median cephalic, which is further from the line of the brachial artery than the median basilic vein (Fig. 141). 
To perform venesection, the surgeon requires a bistoury or lancet-the spring lancet was formerly much used, but it is not employed at the present time-several bandages, a small antiseptic dressing, and a basin to receive the blood.

The patient's arm should carefully be cleansed, washed over with a bichloride solution, and a few turns of a roller-bandage placed around the middle of the arm, being applied tightly enough to obstruct the venous circulation and make the veins below become prominent, but not tight enough to obstruct the arterial circulation. The

Fig. 141.

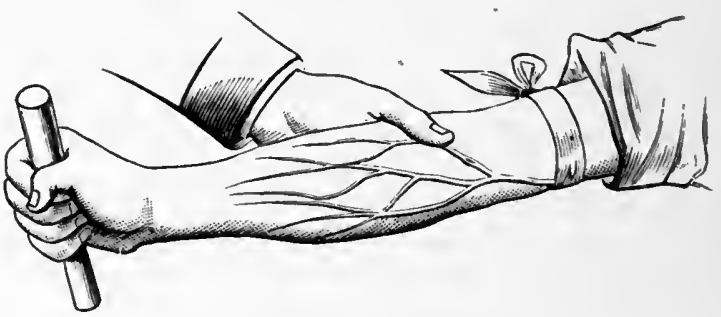

Venesection. (HEATH )

patient at the same time should be instructed to grasp a stick or a roller-bandage and work his fingers upon it. The surgeon should next assure himself that there is no abnormal artery beneath the skin, and having selected the vein, the median cephalic by preference, he steadies it with the thumb and passes the point of the bistoury or lancet beneath it and cuts quickly outward, making a free skin opening. The blood usually escapes freely, and the amount withdrawn is regulated by the condition of the pulse and the appearance of the patient. For this reason it is better to have the patient sitting up or semi-reclining when venesection is performed, as the surgeon can appreciate better the constitutional effects of the loss of blood while the patient is in this position.

When a sufficient quantity of blood has been removed, the thumb is placed over the wound of the vein and the 
bandage removed from the arm above. The wound is next washed with a bichloride solution, and a compress of antiseptic ganze is applied over the wound and held in position by a bandage, which should be so applied as to envelop the limb from the fingers to the axilla. The dressing need not be disturbed for five or six days, at which time the wound is usually found to be healed.

Wounds of the brachial artery have occurred in opening the veins at the bend of the elbow, but if care is taken, this accident should not take place.

Venesection may be practised on the external jugular vein when, from excess of fat or in the case of children, the veins at the bend of the elbow cannot be easily found. The veiu is rendered prominent by placing the thumb or a pad over the vein at the outer edge of the sterno-cleidomastoid muscle just above the clavicle. The vein is next opened over this muscle by an incision parallel to its fibres. After a sufficient quantity of blood has escaped, the wound is washed with an antiseptic solution and closed by a compress of antiseptic gauze held in position by a bandage carried around the neck.

The internal saphenous vein is also sometimes selected for venesection, and here care should be taken not to wound the accompanying nerve which lies directly behind the vein.

Arteriotomy.-This operation is now scarcely ever performed; but if done, the vessel generally selected is the anterior branch of the temporal artery. The position of the vessel is fixed by the finger and thumb, and it is opened by a transverse incision with a bistoury. After a sufficient quantity of blood has escaped, the wound is inspected, and if the vessel is not completely divided, its division is completed and the ends of the vessel should be secured with ligatures, and the wound irrigated with an antiseptic solution and closed with sutures. A gauze compress should next be applied and held in position by a firmly applied bandage.

Transfusion of Blood.-This operation may be employed to introduce a certain quantity of blood into the circulation of a patient who has suffered from profuse 
hemorrhage; it is rarely employed at the present time, being almost entirely superseded by the intravenous injection or infusion of normal saline solution. There are two methods by which transfusion may be effected : the direct, by which the blood is conveyed directly and without exposure to the air from the bloodvessel of one person to that of another; and the indirect, in which the blood is first drawn from one person and is then injected into the veins of another, being deprived of its fibrin before being injected. The latter method involves some risk of embolism and is not now often employed.

Direct Transfusion of Blood.-This procedure as praticed by Crile consists in anastomosing an artery of one person with a vein or artery in another person. It has been found by experiment that the blood of an individual of one species cannot with safety be introduced into one of another speeies. The proximal end of the radial artery of one individual has been anastomosed into the basilie vein of another. The lumen of each vessel during the application of sutures securing the anastomosis is closed by special elamps. After the anastomosis is completed the clamps are removed and a sufficient amount of arterial blood is allowed to flow from the donor into the vessel of the donee. If the proximal end of the artery of the donor be anastomosed with the proximal end of a corresponding artery of the donee there is practically no risk of excessive blood transference, as the two pressures will tend to balance at some point lower than the normal. This method of transfusion has been suceessfully employed in the treatment of cholemic, hemophilic and other forms of pathological hemorrhage.

Auto-transfusion.-This proeedure is recommended in eases of excessive hemorrhage to support a moribund patient until other means of resuscitation can be adopted. It consists in the application of rubber or muslin bandages to the extremities for the purpose of forcing the blood toward the vascular and nervous centres. 


\section{INTRAVENOUS INJECTION OF SALINE SOLUTION.}

It has been proved by experiments and by clinical experience that human blood is not more efficacious in supplying volume to and restoring a rapidly failing circulation than normal salt solution, and as the latter can be obtained with much more ease than blood, its use has largely superseded the former. The solution should be at a temperature of $110^{\circ}$ or $120^{\circ} \mathrm{F}$.

The saline solution is 0.6 per cent. The following solution is highly rcommended for intravenous injection : Calcium chloride 2 parts, potassium chloride 3 parts, sodium chloride 9 parts, sterile water 1000 parts.

Frg. 142.

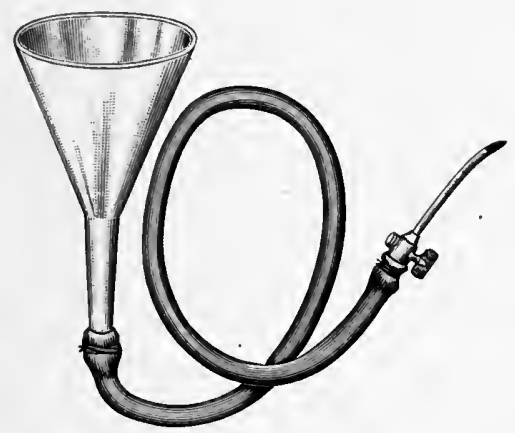

Funnel and tube for intravenous injection.

A vein of the patient, at the elbow, should be exposed, and should have placed under it, about one-half inch apart, two eatgut ligatures; the distal ligature is then tied and an opening is made into the vein between the ligatures; a canula is next inserted into the opening in the vein, and is secured in position by tying the proximal ligature. The canula is first filled with the saline solution, and is then connected with a funnel by means of a 
rubber tube (Fig. 142), which is filled with saline solution to displace the air, and upon raising the funnel above the part the solution enters the vein; care should be taken to see that the fumnel is kept well supplied with the solution until a sufficient quantity has been introduced. The quantity introduced is regulated by the condition of the patient's pulse.

Saline solution may also be introduced into a vein by means of a syringe when the apparatus described cannot be obtained.

Infusion of Saline Solution-Hypodermoclysis.The introduction of saline solution into the cellular tissue has been followed by results equally as satisfactory as those obtained by intravenous injection, and this procedure is now very frequently employed.

The saline solution is conveyed into the cellular tissue through a large hypodermic needle, which should be sterilized by boiling, and is then introduced into the connective tissue, being previously connected by a rubber tube with a reservoir containing warm sterilized salt solution. The usual situations for the introduction of the solution are the external portions of the thighs and the anterior and lateral portions of the abdominal walls. As much as two or three pints of the solution are often introduced in this manner, with good results. Infusion of saline solution may be used with most satisfactory results in cases who have suffered from profuse hemorrhage, and has also proved of great service in cases of shock, and has a distinct value in the treatment of septicæmia and uræmia.

\section{ARTIFICIAL RESPIRATION.}

This procedure is resorted to in cases of threatened death from apnoa consequent upon drowning, profound anæsthetization, eleetric shock, or the inhalation of irrespirable gases, or when from any cause there is interference with the function of breathing. Before resorting to artificial respiration, care should be taken to see that nothing is present in the month or air-passages which will obstruet the entrance of air into the lungs, such as mucus, 
foreign bodies, or liquids, and also that all tight clothing interfering with the free expansion of the chest-walls is removed from the chest.

In cases where the apnœa is due to the presence of a foreign body in the larynx or trachea, it is evident that no efforts at respiration can be suceessful until the air-passages are freed from the oceluding body ; and if it cannot be removed through the mouth, tracheotomy should be performed before artificial respiration is attempted; the tracheal wound should be held open by retractors, which in a case of emergency can be made from bent hairpins, or by a dressing-forceps or a tracheotomy-tube, if one be at hand.

When artificial respiration is resorted to, the operator should persevere with it for some time, even when no apparent spontaneous respiratory movements are excited; for resuscitation has been accomplished in seemingly hopeless cases by patient perseverance with the manipulations. When the first natural respiratory movement is detected, the operator should not cease making artificial respiration, but should continue these movements in such a way as to coincide with the spontaneous inspiratory and expiratory movements until the breathing has assumed its regular character.

The temperature of the body should also be restored by friction to the surface by the hands or by rough towels and hot-water bottles, and warm coverings should be applied for the same object.

Mouth-to-mouth Inflation.- This method of artificial respiration has been resorted to in cases of great emergency, especially in very young children. The operator draws the tongue forward, closes the nostrils, and applies his mouth directly to the mouth of the patient, and by a deep expiratory effort endeavors to force air into the chest; when this is accomplished, the air can be expelled from the lungs by pressure upon the walls of the chest, and the procedure should be repeated about sixteen times in a minute. The same object may be accomplished by passing a flexible catheter into the trachea through the mouth, 
and the lungs can be inflated by the operator blowing into the catheter.

Direct Method of Artificial Respiration (Howard's). -This method of artificial respiration is at the present time considered the most efficacious, and is the one adopted by the United States Life-saving Service; and although the rules given are for the resuscitation of cases of apparent drowning, the same procedures may be adopted in cases of apnoa arising from other causes.

The rules laid down by Dr. Howard are as follows :

Rule I.- "To expel water from the stomach and lungs,

FIG. 143.

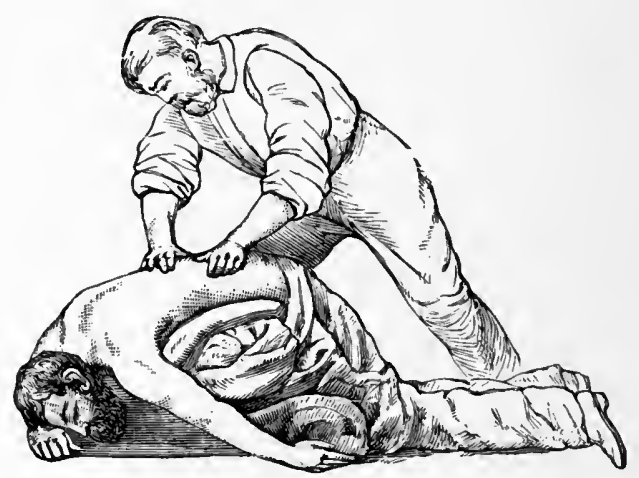

First munipulation in Howard's method.

strip the patient to the waist, and if the jaws are clenched separate them and keep them apart by placing between the teeth a cork or a small piece of wood. Place the patient face downward, the pit of the stomach being raised above the level of the mouth by a roll of clothing placed beneath it (Fig. 143). Throw your weight forcibly two or three times upon the patient's back over the roll of clothing, so as to press all fluids in the stomach out of the mouth."

The first rule applies only to cases of drowning, and in using Howard's method in apnoca from other causes it is to be omitted.

Rule II.- "To perform artificial respiration, quickly 
turn the patient upon his back, plaeing the roll of clothing beneath it so as to make the breast-bone the highest point of the body. Kneel heside or astride of the patient's hips. Grasp the front part of the chest on either side of the pit of the stomach, resting the fingers along the spaces between the short ribs. Brace your elbows against your sides, and steadily grasping and pressing forward and upward throw your whole weight upon the chest, gradually increasing the pressure while you count one-two-three. Then suddenly let go with a final push which springs you

FIG. 144.

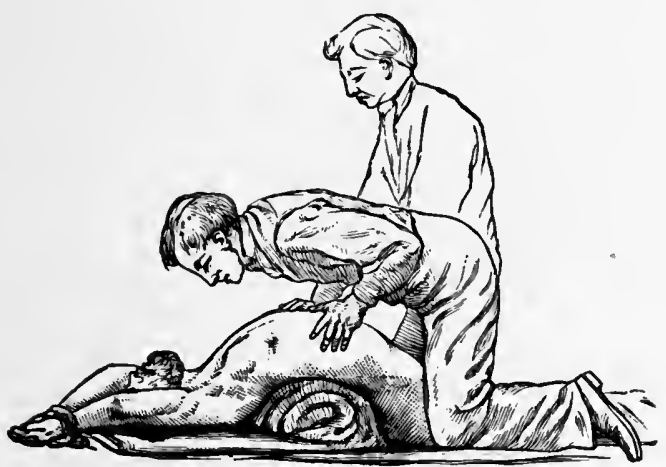

Direct method of artificial respiration.

back to your first position (Fig. 144). Rest erect upon your knees while you eount one-two; then make pressure as before, repeating the entire motions at first about four or five times a minute, gradually increasing them to about ten or twelve times. Use the same regularity as in blowing bellows and as seen in the natural breathing which you are imitating. If another person is present, let him with one hand, by means of a dry piece of linen, hold the tip of the tongue out of one corner of the mouth, and with the other hand grasp both wrists and pin them to the ground above the patient's heal." This method may be employed in cases of stillbirth, or in 
young children, the operator holding the body of the child in his left hand and compressing it with the right hand.

Silvester's Method of Artificial Respiration.-In employing this method of artificial respiration the patient should be placed on his back upon a firm flat surface; a cushion of clothing is placed under the shonlders, and the head should be dropped lower than the body by tilting the surface on which he is laid. The mouth being cleared

FIG. 145.

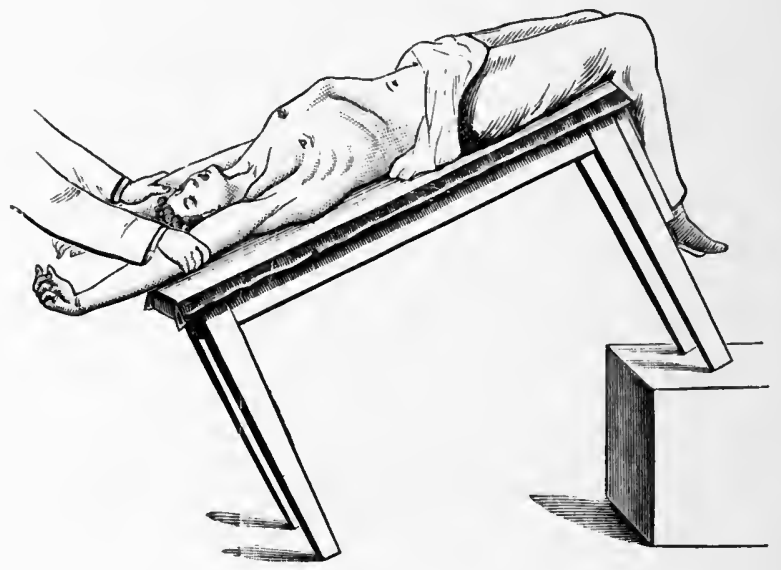

Silvester's method-inspiration. (ЕsмaRCH.)

of mucus or foreign substances, the tongue is drawn forward and secured to the chin by a piece of tape tied around it and the lower jaw, or may be pulled out of the mouth and held by an assistant. 'The operator, standing at the patient's head, grasps the arms at the elbows and carries them first outward and then upward until the hands are brought together above the head; this represents inspiration (Fig. 145); they should be kept in this position for two seconds, after which time they are brought slowly back to the sides of the thorax and pressed against it for two seconds; this represents expiration (Fig. 146). 
These movements are repeated fifteen times in a minute until the breathing is restored or it is evident that the case is a hopeless one.

FIG. 146.

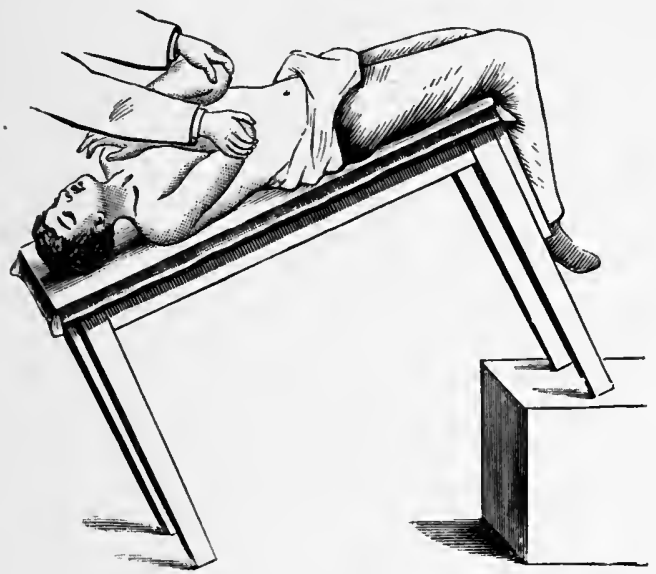

Silvester's method-expiration. (EsMarch.)

Laborde's Method of Artificial Respiration.Laborde has shown that systematic and rhythmic traction upon the tongue is a powerful means of restoring the respiratory reflex, and consequently the function of respiration. The procedure is accomplished as follows: The body of the tongue is seized between the thumb and fingers, and traction is made upon it with alternate relaxation, fifteen or twenty times a minute, imitating the function of respiration, taking care to draw well on the tongue. When a certain amount of resistance is felt, it is a sign that the respiratory function is being restored. Noisy respiration first occurs, termed by Laborde hoquet inspirateur (inspiratory hiccough). Tongue forceps or dressing or hæmostatic forceps may be used in place of the fingers to grasp the tongue. It is important to persist in the manipulations for half an hour to an hour, unless the case is absolutely hopeless. 'This procedure, which cannot be 
employed with advantage when there is fixation of the tongue from inflammation or malignant disease, has been employed with success in cases of drowning, toxic asphyxia, asphyxia during anæsthesia, and arrest of respiration from electric shock.

Forced Respiration.-By this method of artificial respiration air is forcibly passed into the lungs. This procedure is strongly advocated by Fell, who has devised an apparatus by which it may be satisfactorily accomplished. Professor H. C. Wood has also made use of forced respiration in the resuscitation of animals with an apparatus somewhat similar to that devised by Fell, with good results. Wood's apparatus consists of a pair of bellows, a few feet of rubber tubing and a face-mask of rubber, and one or two intubation-tubes; the mask or intubationtube is attached to one end of the rubber tube and the bellows to the other extremity. The mask is applied over the mouth, or, if this is not used, the intubation-tube is introduced into the larynx, and air is forced into the lungs by working the bellows. He also advises that in the tubing a double metal tube be introduced, with the openings so placed that their size can be so regulated by turning the outer tube that the operator can allow any excess of air thrown by the bellows to escape.

The apparatus of Fell, which he has used in a number of cases with good results, consists of a mouth-mask or tracheotomy-tube, and a tube connected with the air-control valve, which is attached to an air-warming apparatus, which in turn is connected with a bellows by another tube. By means of this apparatus air is forced into the lungs, and allowed to escape, when the lungs have been expanded, by the elasticity of the lung tissue and the chest walls.

Forced respiration has proved of value in cases of nar-cotic poisoning and other accidents, in which death is produced by paralysis of the respiratory centres.

Aspiration.-This procedure is adopted to remove fluid from a closed cavity without the admission of air, and the instrument which is employed to accomplish this object is 
known as an aspirator. The form of aspirator most generally employed is that of Potain.

Potain's Aspirator.- This consists of a glass bottle, into the stopper of which is introduced a metallic tube, which is connected with two rubber tubes, one of which is connected with an exhausting-pump, and the other with a delicate canula carrying a fine trocar; the apparatus is provided with stop-cocks to prevent the admission of air (Fig. 147). In using this aspirator, the air is exhausted from the bottle by using the air-pump; the canula enclosing the trocar is next pushed through the tissues into the cavity containing the fluid to be removed; the trocar is then removed, and upon opening the stop-cock the fluid is forced out of the cavity by atmospheric pressure and passes into the bottle or receiver. If the fluid contains masses of lymph or clots which block the canula, inter-

FIG. 147.

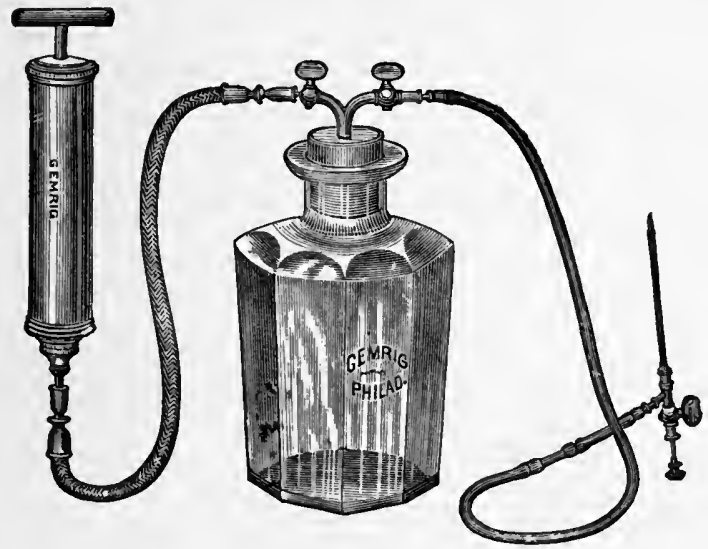

Potain's aspirator.

rupting the flow of fluid, a stylet may be passed through the canula to free it from the obstruction.

To diminish the pain produced in introducing the trocar and canula, the skin at the point to be punctured may be 
rendered less sensitive by holding in contact with it for a few minutes a piece of ice wrapped in a towel, or a towel containing broken ice and salt. Care should also be taken to see that the trocar and canula have been perfectly sterilized; to accomplish this, they should be carefully washed and placed in boiling water or a 5 per cent. carbolic solution before being used. In introducing the trocar and canula, the operator should be careful to avoid injuring important veins, arteries, or nerves.

After removing the canula the small puncture should be dressed with a compress of antiseptic or iodoform gauze, held in place by a bandage or adhesive straps.

The aspirator is frequently employed in cases of hydrothorax, empyema, and ascites, to evacuate the contents of cold abscesses in diseases of the hip and spine, and to remove the contents of a distended bladder until a more radical operation can be performed. It is also a valuable instrument for diagnostic purposes, being frequently used to ascertain the character of the contents of deep-seated tumors containing fluid.

The Stomach-tube.-This consists of a partially flexible tube about twenty-eight inches in length and threeeighths of an inch in diameter, which is introduced while the patient is in the sitting posture, the head being thrown backward so as to bring the month and gullet as nearly as possible in the same line (Fig. 148). The tube being

Fig. 148.

G.TIEMAMN-T.O.

The stomach-tube.

warmed and oiled, the surgeon standing in front of the patient passes it directly back to the pharynx, at the same time introducing the index-finger of the left hand to guide its point over the epiglottis; it is then passed gently downward into the stomach. If any obstruction is met with in its passage, it should be withdrawn a little and then pushed gently downward; all manipulations should 
be made without much force, to avoid perforating the wall of the esophagus.

The introduction of the stomach-tube may be required for the evacuation of poisons from the stomach or to wash out the eavity of this viscus. It may also be used to introduce liquid nourishment into the stomach of patients who are unable or unwilling to swallow food. In introducing liquid nourishment a syringe or funnel is fitted to the free end of the tube, which has been passed into the stomach; the syringe or funnel having been filled with milk or beeftea or broth, the contents are injected gently or allowed to run into the stomach.

In cases of poisoning, where it is desirable to withdraw the contents of the stomach and to wash out the organ, a stomach-tube and syringe may be employed; several syringefuls of warm water are first thrown into the stomach and then withdrawn by suction, but in such cases the use of the stomach-pump will be found more satisfuctory.

Lavage.-In the recently introduced method of treating disorders of the stomach by irrigation, the introduction of a flexible rubber stomach-tube is required; the tube here employed is from twenty-four to thirty inches in length, and the fluid is introduced by means of a funnel attached to its free extremity, or it may be attached to a stomachpump.

The Stomach-pump.-This consists of a brass syringe, the nozzle of which is connected with two tubes, one at the end, the other at the side. The passage of fluid through the nozzle is regnlated by a valve controlled by a lever. The nozzle of the pump is attached to a stomach-tube, and the end of the lateral tube is placer in a pan of warm water. By withdrawing the piston and opening the valve, water may be drawn from the basin, and by closing the valve and depressing the piston it is forced through the stomachtube into the stomach; when a sufficient quantity has been injected in this manner, by reversing the action of the valve the fluid is drawn out of the stomach and discharged through the lateral tube into a basin. This manpulation is continued until the water returns clear 
and the stomach has been completely washed out. The stomach-pump shown in Fig. 149 may also be employed.

Esophageal Bougie.-This instrument-which may be passed through the œsophagus into the stomach for the purpose of diagnosis or for the purpose of dilating strietures of the cesophagus-is employed in exactly the same manner as the stomach-tube, and, as in the case of the latter instrument, it should be introduced without the use of much force. as perforations of the osophagus have followed the forcible introduction of such instruments.

The Rectal Tube.-The introduction of the rectal tube is best accomplished by placing the patient upon his left side, and the surgeon should introduce his index finger well oiled into the rectum and guide the tube upon this through the anus, when by gentle pressure it is gradually passed into the rectum; if a stricture exists in the rectum

FIG. 149.

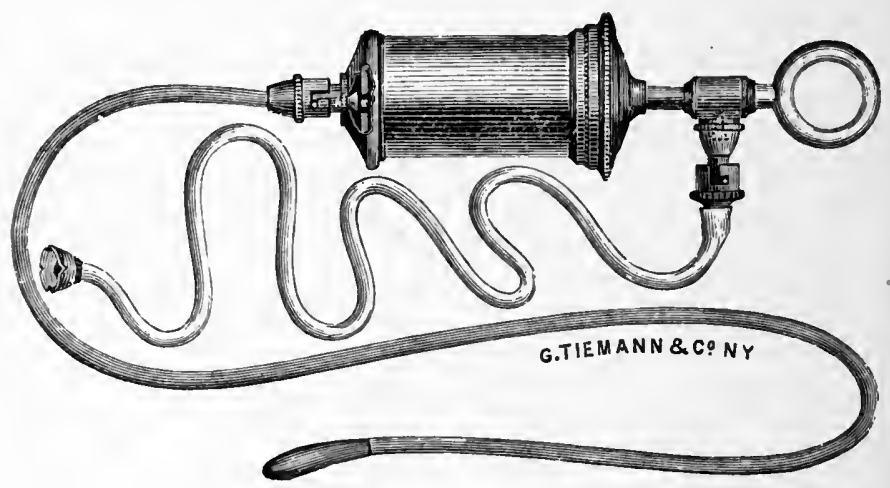

Stomach-pump.

within reach of the finger, the latter should be used to guide the tube throngh the opening in this; if the tube becomes canght in a transverse fold of the mucous mentbrane and doubles upon itself, it should be withdrawn and a fresh attempt made to pass it. In passing a rectal tube all manipulations should be made with extreme 
gentleness, as it has been shown that its passage is not without danger, perforations of the intestine having followed its use in some cases. In cases of stricture of the rectum high up, the operator has to depend upon the sense of resistance experienced in passing the tube, and in such cases the manipulations should be most carefully made. When the rectal tube is employed to introduce fluid into the large intestine, the fluid may be introduced by means of a syringe, or by pouring it into a funnel attached to the free end of the tube, or by attaching the tube to a fountain syringe, thus allowing the liquid to pass slowly into the intestine.

The rectal tube is often employed with good results in irrigating the large intestine, relieving the intestine of flatus, and in introducing water or oil into the intestine in cases of intestinal obstruction, and in those cases where the obstruction results from intussusception or fecal accumulations its use will often prove satisfactory.

Rectal Bougies.-These instruments are made of Indiarubber or the same material as the English flexible catheter, and are of varions sizes. They shonld first be oiled, and are introduced in the same manner as the rectal tube. They are generally employed in cases of stricture of the rectum, and should be introduced with great care to avoid perforating the wall of the rectum; this accident has occurred in the hands of skilful surgeons. A very satisfactory substitute for a rectal bougie is a tallow candle, one end of which is melted or rubbed down to a conical shape.

Enemata.-These may be administered by means of an ordinary syringe, or by means of a gravity or fountain syringe; the precautions which should be observed are to introduce the nozzle of the syringe gently and in the right direction, as perforation of the lower portion of the rectum has taken place from careless and forcible intruduction of the nozzle of the enema-syringe; the fluid should also be injected slowly, as by so doing there is less resistance and less tendency for the patient to pass the fluid before the desired quantity has been introduced. 
The enema most commonly employed to empty the lower bowel is made by adding a tablespoonful of sweet oil and two teaspoonfuls of spirit of turpentine to one or two pints of warm water in which a little Castile soap has been dissolved; warm water and sweet oil are also frequently used for the same purpose.

Glycerin Enema.-One or two teaspoonfuls of glycerin injected into the rectum, or a suppository made of glycerin, will often be found an efficient substitute for the larger enemata of water.

Nutritious Enema.-When it is found neeessary to resort to feeding by the rectum, the substanees employed should be injected into the reetum by means of a syringe, and care should be taken that the quantity is not too large, and that it is of such a nature as not to eause irritation of the walls of the rectnm, or it will not be retained; two to four ounces in the case of an adult is generally a sufficient quantity to inject at one time.

Peptonized milk or beef-juice, or the yolk of an egg beaten up with milk, is often employed, and any unirritating drugs may be mixed with the enema and administered at the same time.

Vaccination.-This is a minor surgieal procedure which every physician is called upon to perform. The surface may be prepared for the reception of the lymph by abrading the skin at one or two points with a dull lancet, or by making several superficial incisions with a knife, or by seratehing the surface of the skin with the ivory point charged with lymph, in lines with crossing lines, crossscratch, until a little serum exudes. It is not advisable to draw blood, which washes away the lymph, and for this reason we prefer the abraded surface made by the dull knife or the ivory point.

The lymph used may be the humanized or the bovine.

Bovine lymph or virus, which is now most generally employed, is taken from the vaccine vesicles upon the udders and teats of heifers. The lymph may be mixed with sterilized glycerin and placed in fine glass tubes, which are sealed; or ivory points or quills are dipped in the lymph and allowed to dry, and in using these they are dipped in 
water for a moment, to moisten the lymph, before being applied to the abraded surface. The ivory-point is one of the most convenient means of vaccination, as the surface may be abraded with it before the lymph is applied.

It has recently been advised that antiseptic precautions be exercised in performing vaccination, and although all of the details cannot be carried out, we have found that the exercise of care as regards cleanliness of the surface has been followed by much fewer inflammatory complications in vaccination wounds.

The surface to be abraded, usually the left arm below the deltoid, is first washed with soap and water, then with a 1 : 2000 bichloride solution, or with alcohol, and finally washed with sterilized water. Two points of this surface, an inch apart, are then abraded by using a knife which has been washed or dipped in boiling water, or by using the ivory-point which has been dipped in water that has been boiled and cooled. When the surface has been prepared in the manner lescribed, the moistened virus is rubbed upon it and allowed to dry. Vaccination upon the leg, which is practised by some physicians to prevent the scar from showing, I think is not to be recommended, and I never practise it in this situation, as it is more difficult to keep this part at rest.

Hypodermic Injections.-The syringe used to make hypodermic injections is provided with a perforated needle, which is passed into the cellular tissue (Fig. 150), Care should be taken to see that the instrument and needle are perfectly clean before being used; they should be rendered aseptic by soaking them for a few minutes in boiling water or in a 5 per cent. carbolic solution. Hypodermic injections are generally made into parts in which the cellular tissue is abundant, and great care should be observed to avoid introducing the needle into a large vein or artery, as by neglect of this precaution serious symptoms have resulted, from the drug being thrown rapidly into the circulation instead of being slowly absorbed from the subcutaneous cellular tissue; injury of superficial nerves should also be avoided. Care should also be taken 
to see that the solutions employed are sterilized if possible, and freshly made solutions should be preferred.

To avoid using solutions for hypodermic use which undergo change in keeping, it will be found convenient to use the compressed pellets which are prepared by manufacturing chemists, the alkaloids being compressed with a little sulphate of sodium, which increases their solubility,

FIG. 150.

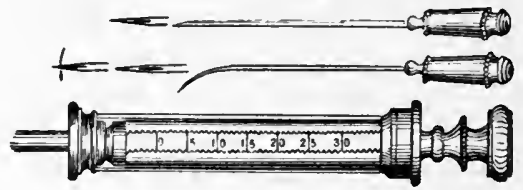

Hypodermic syringe and needles.

the solution being prepared with boiled water just before being used.

The portions of the body usually seleeted for hypodermic injection are the outer surface of the thighs or arms and the anterior surface of the forearms. In making a hypodermic injection, the syringe is charged and the needle is fastened to the nozzle of the syringe; the skin is next pinched up and the needle is quickly thrust through this into the cellular tissue (Fig. 151); the syringe is then emptied by pressing down the piston, and when the cylinder is empty the needle is withdrawn.

FIG. 151.

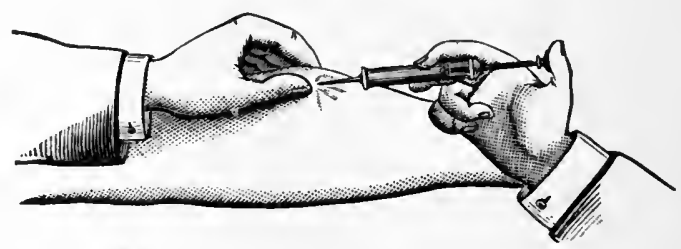

Method of giving a hypodermic injection.

Injection of Antitoxins. - In the treatment of diseases such as diphtheria, anthrax, septicæmia, pneumonia, and 
tetanus by the injection of serum, the pypodermic method is made use of ; in using antitoxin injections in diphtheria, the dose of the antitoxin is proportionate to the age and weight of the patient as well as to the severity and duration of the disease. A child three years old should be given 2000 units; an adult, not less than 3000 units; and the injection should be repeated in twelve to twenty-four hours. Before employing the injection the skin should be sterilized, and the best variety of syringe to employ is one holding about 20 c.c. (Fig. 152).

It is well to have the needle connected with the syringe by a short rubber tube, so that the needle will not be broken if the patient struggles. The injections are usually made below the angle of the scapula or in the

FIG. 152.

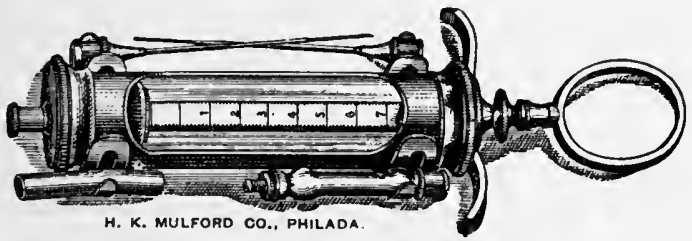

Syringe for serum-injection.

lumbar region, and the serum is introduced slowly to avoid local reaction.

Coley's Fluid.-This fluid, which consists of the mixed toxins of streptococcus erysipelatis and bacillus prodigiosus, has been employed in the treatment of sarcoma with some success. It has been especially used in inoperable sarcomata of the abdomen and recurrent and secondary sarcomata of bone. Five to ten minims are injected hypodermically at intervals of a few days, depending upon the amount of constitutional reaction produced, and the dose is gradually increased if the patient bears it well. The treatment should be continued for some weeks until it is shown that no results are obtained or the growths are diminishing.

Injections of Mercury in Syphilis.-Injections of mercury may be made into the subcutaneous tissue of the loins, buttocks, or scapular regions in the treatment of 
syphilis. Injections may also be into the veins. The solution most commonly used is a 1 per cent. solution of the cyanide of mercury, 20 minims being injected every day or on alternate days.

Exploring-needle.-This consists of a fine-grooved needle fitted into a handle (Fig. 153), which is introduced into tumors or swellings to ascertain the nature of their contents, and its use is often of service for purposes of

FIG. 153.

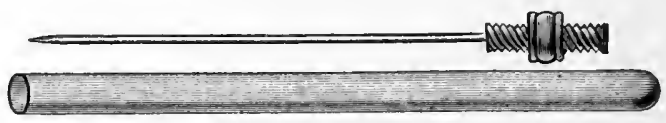

Exploring-needle.

diagnosis. The exploring-trocar (Fig. 154) is employed for the same purpose, or the needle of the hypodernic syringe or a fine needle attached to an aspirator may be used for a like purpose. When either the exploring-needle or trocar is employed, care shonld be taken to see that it is rendered perfectly aseptic before being used; otherwise its employment is not without danger, for we have seen the introduction of an exploring-ncerlle into an effusion in a joint for diagnostic purposes followed by infection

FIG. 154.

Exploring-trocar.

and destruction of the joint, which subsequently necessitated its excision.

Skin-grafting.-This is a surgical procedure which may be employed to fill a gap in the tissues or to hasten cicatrization where large granulating surfaces are exposed, such as result from extensive operations and from burns.

The operation consists in applying shavings of the epidermis, or of the epidermis and cutis together, to the 
granulating surface and holding them in contact with it for a few days; the grafts often seem to disappear, but at the end of a few days, if the part is closely inspected, bluish-white points will be seen to occupy the positions at which the grafts were applied, which become converted into isolated cicatrices from which the healing process rapidly extends. To have a successful result follow the use of skin-grafts, the surface of the ulcer should be healthy, and its surface as well as the surrounding skin rendered aseptic, and the grafts should be applied at a number of points.

The surface from which the grafts are to be taken should also be rendered aseptic, and the skin should be removed by scissors or by a sharp razor, or by raising the epidermis with a needle or with forceps, and cutting out a small portion with a sharp scalpel. The graft is next applied to the granulating surface with its raw surface in contact with the granulations; after a sufficient number of grafts have been applied, a piece of sterilizerl protective is laid over them and is held in place by means of a few strips of isinglass plaster. A sterilized gauze dressing is next applied, and the dressing is not disturbed for a week or ten days, at which time, if the grafts have taken, isolated cicatrices at the points where the grafts were applied will be found.

Thiersch's Method.-In skin-grafting according to this method, the surface of the ulcer is rendered aseptic, and all antiseptics are washed away with sterilized salt solution. The surface of the ulcer is next curetted to remove suft granulations, and it is then irrigated and covered with protective, and a compress applied to control bleeding. Shavings of skin are then removed from a surfacewhich has been rendered aseptic-by means of a razor or section knife; the use of McBurney's hooks (Fig. 155) will facilitate the removal of the grafts. Each graft should be as long and broad as possible, and, when cut, it should be floated from the section knife upon the prepared surface of the ulcer by a stream of salt solution and gently pressed into place. After a sufficient number of grafts have been 
applied, strips of protective are laid over the surface of the grafts, and over these is placed a compress moistened with salt solution and covered by protective, and a few layers of sterilized gauze and cotton are next applied over this, and the dressing is held in position by a bandage.

Fra. 155.

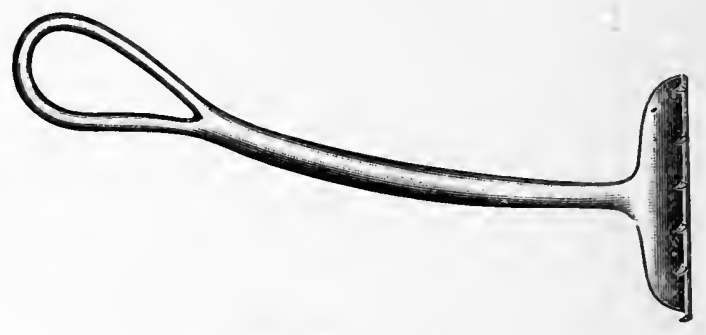

McBurney's hook.

The dressings need not be removed for a week or ten days, and a second dressing should be applied in the same manner until the grafts have become thoroughly vitalized. The skin of the bellies or backs of frogs, or the hairless skin of young animals may be used in place of human skin.

Krause's Method.-Skin-grafting is sometimes accomplished by immediately applying an isolated piece of skin to a raw surface to fill a gap; the graft in such cases includes the. whole thickness of the skin, but has all of the cellular tissue removed from it, and should be cut one-third larger than the gap to be filled, to allow for the shrinking after its removal, and is secured in position by sutures.

Bone-grafting.-This procedure is resorted to to replace portions of bone which have been separated, to fill up cavities in bone, or to restore the continuity of the long bones. The bone to be introduced should be rendered thoroughly aseptic, and shonld be placed in a sterilized salt solution at a temperature of $100^{\circ}$ to $105^{\circ} \mathrm{F}$; it 
may be inserted in one piece or broken into fragments and laid over the surface.

When it is desired to restore the continuity of one of the long bones, after the surfaces of the bone have been exposed and rendered aseptic a bone is removed from a freshly killed animal, is rendered aseptic, and fitted into the gap and secured to the ends of the bone by sutures. Or a portion of the bone may be partially separated by a chisel and fitted into the gap, or is split into strips and packed into the cavity.

In the case of parallel bones, such as the tibia and fibula, where there has been a loss in substance of the tibia, the fibula has been divided on a line with the lower end of the tibia, and after freshening the end of the tibia the upper end of the lower fragment of the fibula is shifted over to the tibia and secured to it by sutures.

Decalcified Bone Plates or Chips.-These will be found useful in filling up the cavities resulting from extensive removals of bone by injuries or in the operation for necrosis or caries. In such cases, after the cavity has been sterilized, it is dusted with iodoform and is then packed with bone chips; iodoform is next dusted over them and they are covered by a piece of protective. A compress of iodoform or sterilized gauze and bichloride cotton is next applied, and the dressing is held in position by a bandage.

When bone plates are employed, they are cut to fit the cavity, and provision should be made for drainage.

Bone Wax.-This material, devised by Mosetig-Moorhof, is employed to fill up cavities in bone remaining after the removal of diseased bone or tumors of bone. The wax is prepared by melting together iodoform, 20 parts; spermaceti, 40 parts, and oil of sesame, 40 parts. This preparation is heated to $50^{\circ} \mathrm{C}$. and is poured into the cavity, which has been rendered sterile and dry, and when the wax has become firm the soft tissues are sutured over it. Very good results have been obtained by the use of this preparation. 
Moore has employed a bone wax containing iodine which he considers more satisfactory than that of Moorhof. It consists of iodine, 1 per cent.; olive oil, 2 parts, and spermaceti, 8 parts. The iodine is added after the spermaceti and olive oil have been mixed in a water-bath, and heat should not be applied after the iodine has been added. The mass becomes solid at the body temperature. The bismuth emulsion recommended by Beck may also be used in the same manner as bone wax in filling cavities in bone. This consists of bismuth subnit., 30.0 g.m.; white wax, 5.0 g.m.; vaseline, 60.0 g.m.

Thiersch flaps have also been employed with success in the treatment of open surfaces in bone cavities. The surface of the bone cavity is covered with Thiersch flaps, and they are held in contact with the bone by a dry gauze tampon.

Muscle-grafting and nerve-grafting are also occasionally resorted to, to supply deficiencies in muscles or nerves, fresh muscle or nerve tissue being employed to fill up the gap, but the results as a rule have not been satisfactory.

Electrolysis.-Electrolysis, or the chemical decomposition induced by electricity, is employed in surgery to destroy morbid products, tumors, or exudations. For this procedure, a galvanic or continuous-current battery is required, which is provided with electrodes and needles of suitable shapes. In applying electrolysis to a tumor, for instance, the needle connected with one of the poles of the battery is inserted into the tumor, and the other rheophore is applied to the surface of the body, or two fine needles, carefully insulated nearly to their extremities, are connected with both poles of the battery by conducting cords; these are introduced into the tumor and a weak current is allowed to pass. The strength of the current is gradually increased as the operation advances; the current is passed for fifteen or twenty minutes, and the procedure is repeated at intervals of several days until some decided change occurs in the tumor. 
some forms of club-foot, and in lateral curvature of the spine the judicious use of the faradic current will often be found to be followed by the most satisfactory results. The current is applied in such a manner as to bring about contraction of the afiected or wasted muscles, and thus improve their nutrition.

Franklinization.-The earliest application of electricity in the treatment of disease was in the form of statical electricity, and although it fell into disuse, it has recently, with the perfection of modern machines, been widely revived. In applying statical electricity the patient may be treated by insulation, or the so-called dry electric bath.

FIG. 157.

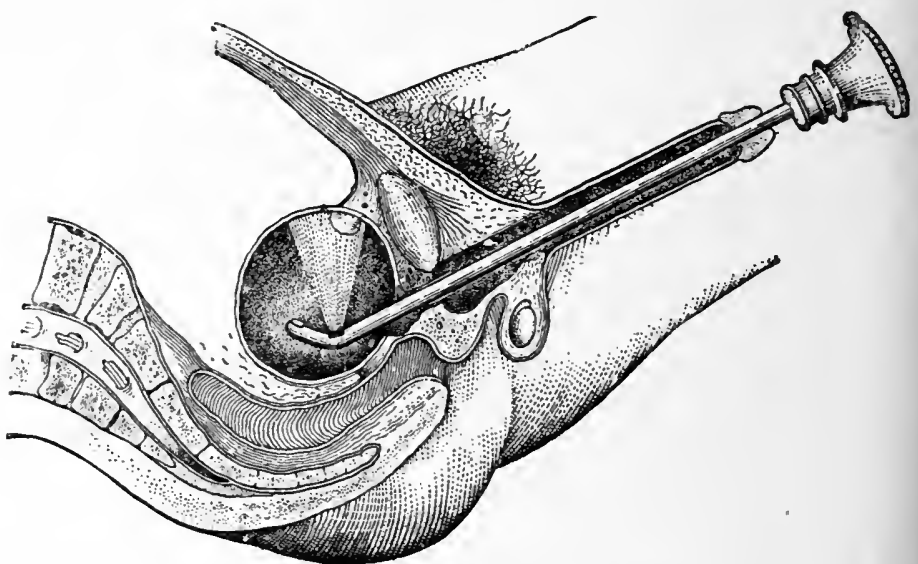

Illumination of the wall of bladder by cystoscope. (PARK.)

The second method of using statical electricity is by sparks or shocks from a Leyden jar, which is charged from the prime conductor of an electrical machine in motion, or by the electric brush. McClure states that in the static induced current we have a means of producing muscular contractions when failure results from the strongest faradic currents that can be borne by the patient.

The Cystoscope.-This is an instrument employed for 
ocular examination of the walls of the bladder, and is one of the most important and useful of the electric-lamp instruments. A cystoseope eonsists of a beaked sound in which there is a telescopic arrangement, by which the inner surface of the bladder is viewed through a small window of rock erystal. The lamp is enclosed in the beak of the instrument and throws its light through another window, also of erystal, upon any part of the bladder wall. The bladder should contain six or eight ounces of clear urine or clear water if a proper view of the walls is to be obtained. If the fluid is turbid or contains blood, the view is very much obscured; if too little fluid be present in the bladder, the beak of the instrument containing the lamp is likely to become buried in the folds of mucons membrane and the light will be cut off, and the mucous membrane may be burned. The bladder may be emptied of urine and distended with air which accomplishes the same purpose. A certain amount of practice is required to use the cystoscope properly and to recognize the appearance of the mueous membrane of the bladder in health and in its varied morbid conditions (Fig. 157).

The Urethroscope.-The urethroscope consists of a straight metal tube provided with an obturator of hard rubber, which projects slightly beyond the end of the tube. This tube is introduced into the urethra until the bladder is reached, when it is slightly withdrawn and the obturator removed. The instrument is then attached to the mirror of an clectric lamp, by which a strong light is thrown into the tube, and as the tube is withdrawn the urethra is exposed to view. By means of the urethroscope a very accurate inspection of all portions of the urethra can be obtained.

The Panelectroscope.-This instrument, introduced by Leiter, consists of an electric lantern, with tubes and a mirror. The light from a small incandescent lamp is projected by the mirror along the tube, which is inserted into the part to be examined. Tubes of various sizes are adapted to the instrument. It is employed for endoscopy of the urethra, ear, pharynx, and stomach. 
The Bronchoscope.-Chevalier Jaekson has combined the tube of Killian with the lighting principle of the œsophagoseope of Einhom and produced a bronchoseope by means of which inspection of the larynx, trachea aud larger bronchial tubes may satisfuctorily be made. The asophagoscope, devised by the same person, also secures a satisfactory inspection of the œesophagus and stomach. By the use of these instruments, with the aid of delicate forceps adopted to them, it is possible to remove foreign bodies or growths from the trachea, bronchi, œesophagus and stomach. Such bodies as pins, tacks, pieces of bone, safety-pins and needles have been removed from these localities. The patient is usually anæsthetized before their employment. To use these instruments with satisfaetion a considerable amount of practice is required.

\section{MA_SSAGE.}

Massage consists in a variety of manipulations, such as pinching up the integument and muscles, and rolling them between the thumb and fingers; in stroking or rubbing the surface with the palm of the hand from the periphery toward the centre, to empty the distended reins and lymphatics; rubbing the parts circularly with the extremities of the fingers and thumbs or the palms of the hands. Kneading of the parts is another method of practising massage. Massage may also be practised by tapping the surface of the affected part more or less forcibly with the tips of the fingers held in a row, or with the ulnar border of the hand or the palm of the hand. Before applying massage to an affected part, if there be a heavy growth of hair, it should be shaved off; otherwise the manipulation may give the patient pain, and irritation of the hair-follicles resulting in abscesses will be apt to occur. The part should also be rubbed over with olive oil, vaseline, or eacao-butter before and during the manipulations.

Massage is employed often with advantage in the treat- 
ment of sprains and strains in their subacute and chronic stages. Lncas-Championnière advocates and practises immediate and continuous massage in the treatment of fiactures. It will also be found of great service in the later treatment of fractures involving the joints or their vicinity, in restoring the motion of the parts as well as in improving the nutrition of muscles which have become wasted from disuse.

Passive Motion.-This manipulation consists in alternately flexing and extending or rotating the limb, to imitate the normal joint-movements. The motions should be carefully practised, and in cases of fracture they should not be undertaken, as a rule, until there is firm union at the seat of fracture; if for any reason passive motion is made use of before this time, the fragments should be firmly supported while it is being employed. Other forms of massage, such as stroking and kneading, may be employed in conjunction with passive motion in the treatment of the stiffness of joints resulting from fractures, dislocations, and sprains; passive motion applied in this manner will often restore the function of a stiff joint more satisfactorily and with loss pain to the patient than the forcible manipulations which are sometimes practised under an anæsthetic.

Compression.--This is a valuable means of diminishing swelling in the early stages of inflammation, and of bringing about absorption of the effusion in the later stages. It may be applied by means of compresses, bandages, or strapping. Pressure applied in this manner is often employed in the treatment of injuries of the joints and bursi, and in chronic inflammatory swellings. It should be used with caution when the circulation in the tissues is impaired.

Application of Hot Air.-The employment of a continnous hot-air bath has recently been advocated in the treatment of painful and partially anchylosed joints, synovitis, teno-synovitis, and chronic rheumatism. In applying this method of treatment, the limb is wrapped loosely in lint and introduced into a metallic cylinder 
(Fig. 158), the temperature of which is raised to about $300^{\circ} \mathrm{F}$. The part is exposed to this temperature for three-quarters of an hour to one hour, and at intervals of twenty minutes the door is opened for a short time to allow the ingress of fresh air; if the part is perspiring, it is wiped dry, for if moisture is present upon the limb burns are likely to result. Under this form of treatment pain is often temporarily or permanently relieved, synovial effiusions absorbed, and adhesions are softened and disappear. Clinically it has been found that the best

FIG. 158.

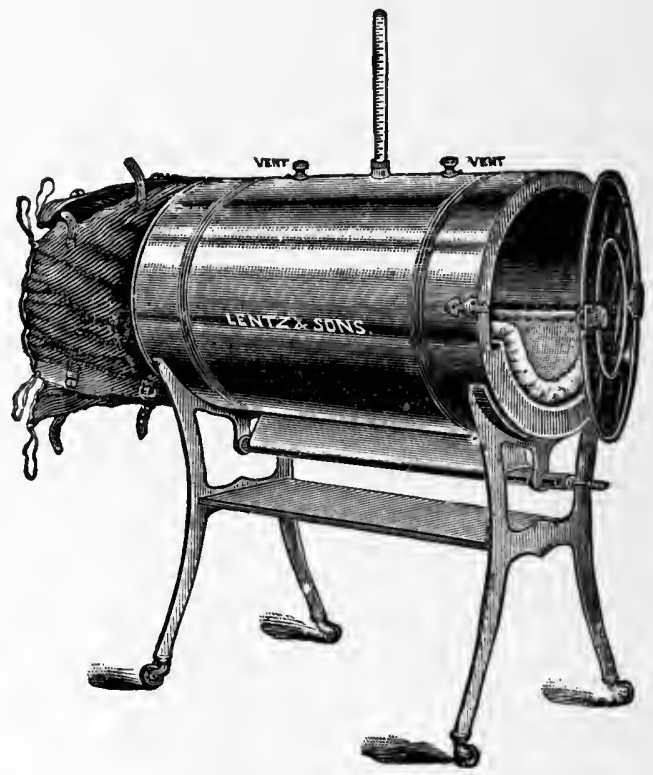

Apparatus for hot-air treatment.

results following this method of treatment have occurred in painful and anchylosed joints following traumatisms; and although temporary improvement has occurred in rheumatic, gonty, tuberculous, and gonorrhœal affections 
of joints, permanent improvement is not so likely to result. Bier has also recommended the use of hot-air to produce arterial hyperæmia in the treatment of certain joint and neuralgic affections, the part to be treated being enclosed in specially devised hot-air boxes.

\section{BIER'S HYPER无MIC TREATMENT.}

Bier has called attention to the value of an artificial congestive hyperæmia in the treatment of acute and chronic inflammations. He recognizes two kinds of congestion (1), arterial or active, produced by him with hot air ; (2) venous or passive congestion.

FIG. 159.

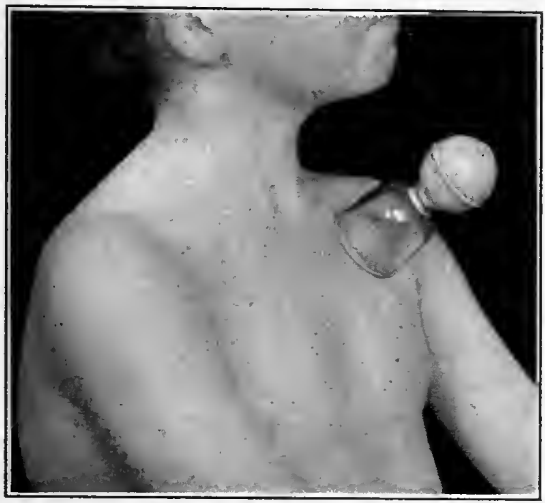

Suction cup applied.

Passive hyperæmia may be caused ( $a$ ) by suction and (b) by constriction. To produce passive hyperæmia by suction a glass vessel or cup is applied to the skin with a rubber bulb or suction pump attached to rarify the air within. The cups are now made of various sizes and shapes to adapt themselves to all surfaces of the body (Fig. 159). In applying this methor the procedure should 
not cause pain and the suction should be intermittent. Thepatient is the best judge as to the amount of suction. If the patient complains of pain from the suction, it is excessive and a lesser degree should be employed. If the suction is not intermittent but little benefit is derived. The cup is applied for three or four minutes, then renewed for one or two minutes, and this procedure is repeated at intervals for at least half an hour.

FIG. 160.

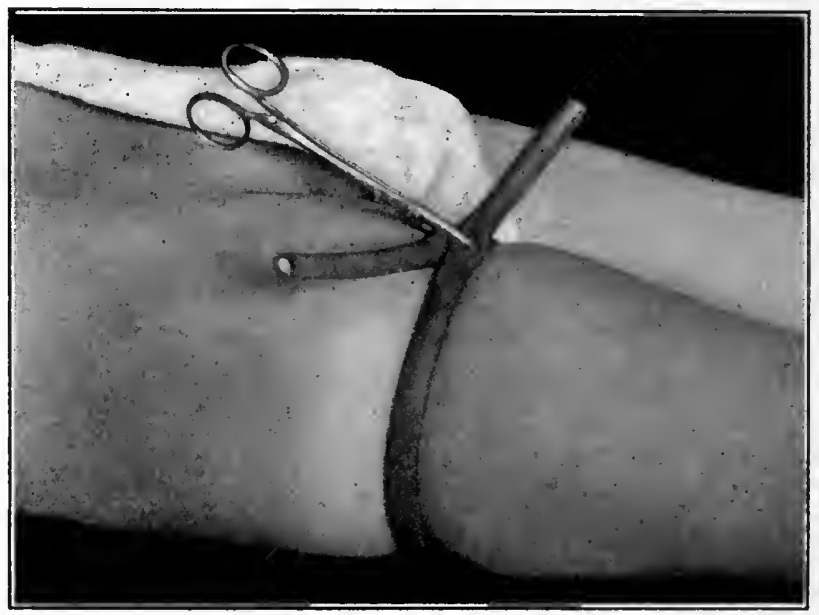

Rubber tube applied to the thigh.

This method of artificial hyperæmia is employed with good results in abscesses, acute or chronic, enlarged or inflamed lymphatic glands, furuncles, carbuncles, and many other localized inflammatory conditions.

If pus is present, a small incision should be made and the subsequent suction favors its escape.

Passive hyperæmia by constriction is produced by placing a thin rubber band or tube around a part and thus producing a congestion of the parts distal to it (Figs. 160, 161,162 and 163). The compression should be just sufficient to produce a reddish blue coloration of the skin 
beyond the point of eonstriction. It is especially applicable to the extremities. The arterial pulse should not be interfered with and should be as free as that upon the opposite limb. The temperature of the constricted part should be the same as the corresponding part on the other limb.

The constriction should not cause pain in the eongested area. It should be noted that in acute inflammatory affections slight pain and throbbing is experienced even if the constriction is not too thoroughly applied. This usually disappears in from five to ten minutes.

By rubbing the parts active hyperæmia should be produced.

When applied above wounds serum is poured out which improves the drainage and escape of pus.

When odema appears the treatment should be suspended and not resumed until it disappears.

If the part is odematous before the treatment is begun,

Frg. 161.

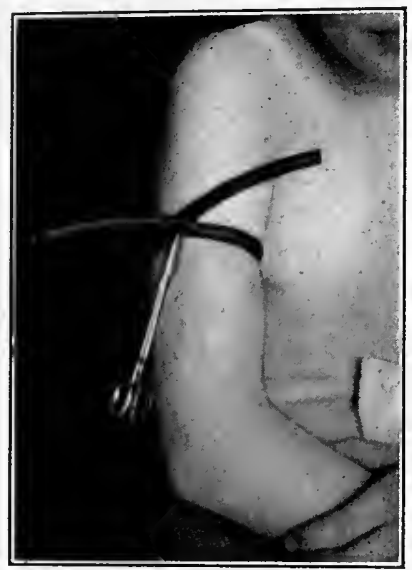

Rubber tube applied to the arm. multiple incisions should be made before this is instituted.

The ease should be under eareful observation during the course of treatment, and if any of the following signs appear it is evident that the compression is not accurate:

1. If the parts become very cyanotic.

2. Marked pain and discomfort.

3. Coldness of the part beyond the point of constriction.

4. Pulse diminished in force or lost.

5. Development of œedema.

6. Non-appearance of active hyperæmia upon rubbing the part.

The duration of treatment in acute cases should be short. Half an hour to an hour three or four times a 
day at intervals for twenty-four hours. It has been followed by good results in acute pyogenie infections. It

FIG. 162.

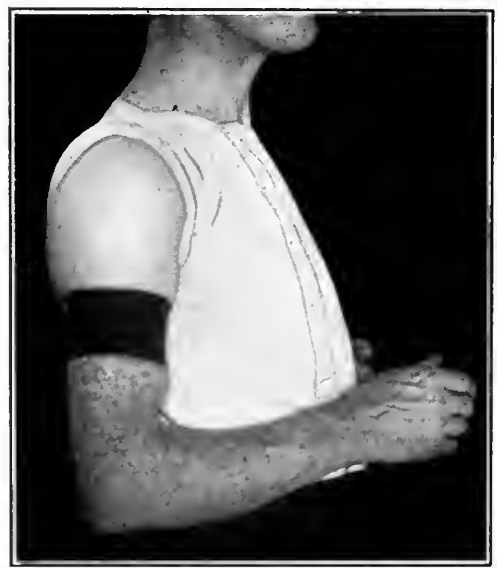

Rubber tube applied to the arm to produce passive hyperæmia.

FIG. 163.

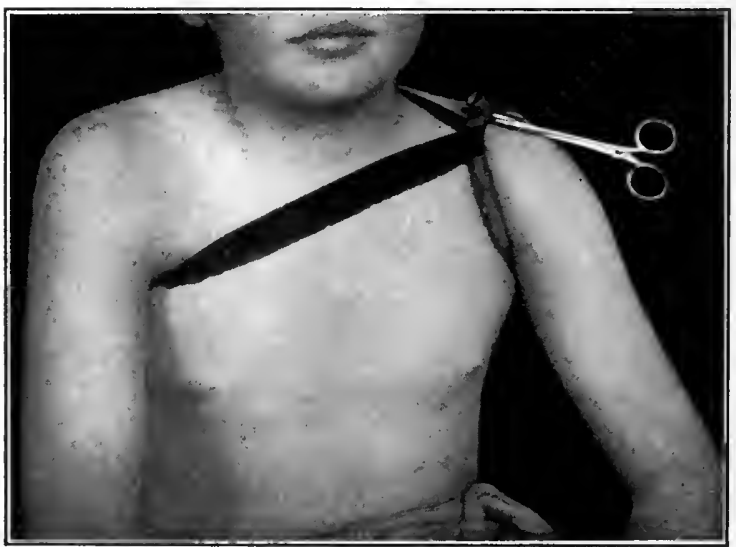

Rubber tube applied to the axilla and shoulder to produce passive hyperæmia of arm.

has been emploved in wounds of the extremities and inflammation of joints with most satisfactory results. Ab- 
scesses, if present, should be incised, but drainage should not be introduced. Baidages should be removed before the constrietion is applied to permit of swelling of the congested part and not interfere with drainage.

In chronic cases the constriction should be applied for a longer period-from two to four hours a day for a number of days. Applied in this manner it has been employed with marked success in tuberculous affections of the bones and joints, chronic ulcers and in ununited fractures.

Patients can be taught how to apply the constrietion to produce the proper amount of compression, and when so instrueted can use this method of treatment at their own homes.

Active or Arterial Hyperæmia.-This is produced by surrounding the affected area by hot air. This is accomplished by an apparatus which encloses the part, leaving an air space, which is connected with an aleohol lamp. Various forms of hot-air boxes have been devised to fit different parts of the body. This form of treatment has been found especially serviceable in rheumatic joints, neuritis, and neuralgia.

\section{SKIAGRAPHY, OR EMPLOYMENT OF THE RÖNTGEN RAYS.}

Röntgen, in 1895, while investigating the eathode rays as developed in Crookes's tubes, discovered the energy which he named $x$-rays. The rays are invisible, but have great power of penetration, and pass through many substances whieh are opaque to sunlight and ordinary electric light. If the rays are intercepted by a body not readily permeable, which is placed between the Crookes's tube and a dry photographic plate, a shadow will be formed, and an impression of this shadow will be formed upon the plate. Such a shadow is known as a skiagraph. The fluoroscope consists of a fluorescent screen, which is so placed that the rays emanating from the Crookes's tube aud passing through any intercepted substance to be studied are refleeted direetly upon it. If the body is more or less 
resistant, the observer ean see it clearly through the skin and subcutaneous tissue.

The time of exposure to the rays varies with the strength of the current and the thickness of the tissues.

FIG. 164.

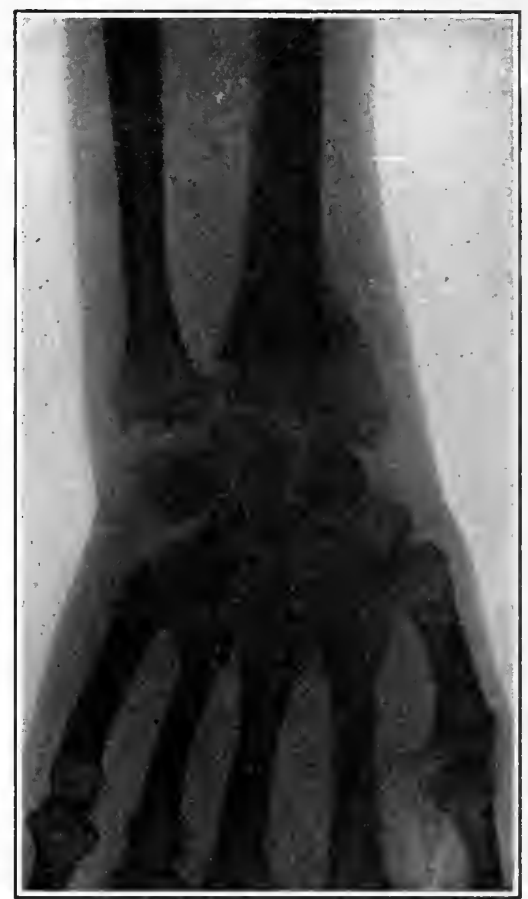

Skiagraph of fracture of the lower end of the radius. (NEwCONET.)

The exposure is usually from three to fifteen minutes. The tube should not be placed too near the surface of the body, and the exposure should be as short as possible. Much shorter exposures are now generally made. 
There occasioually develops after the use of the $x$-rays a peculiar disturbance of the tissues, probably trophic in nature, which is known as an $x$-ray burn. The skin, several weeks after exposure to the rays, may become

FIG. 165.

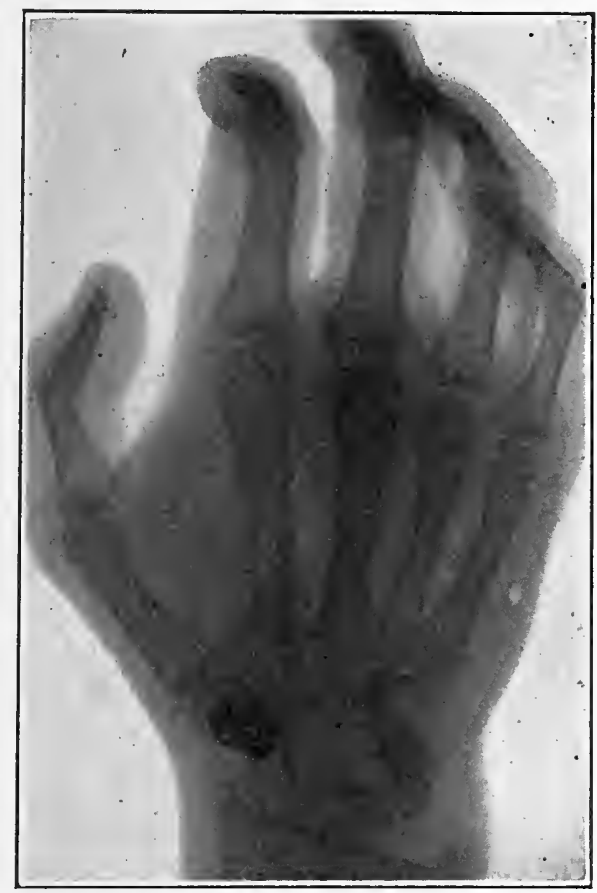

Skiagraph of bullet in wrist-joint. (Newcomer).

ulcerated, the nails may be lost, and a very iutractable form of ulceration or gangrene develop.

'The $x$-rays are of great value in locating foreign bodies, such as needles, pius, bullets and pieces of 
glass. They are employed also with advantage in locating mineral calculi in the bladder, ureter, and kidney. They are also useful in detecting the presence of frac-

FIG. 166.

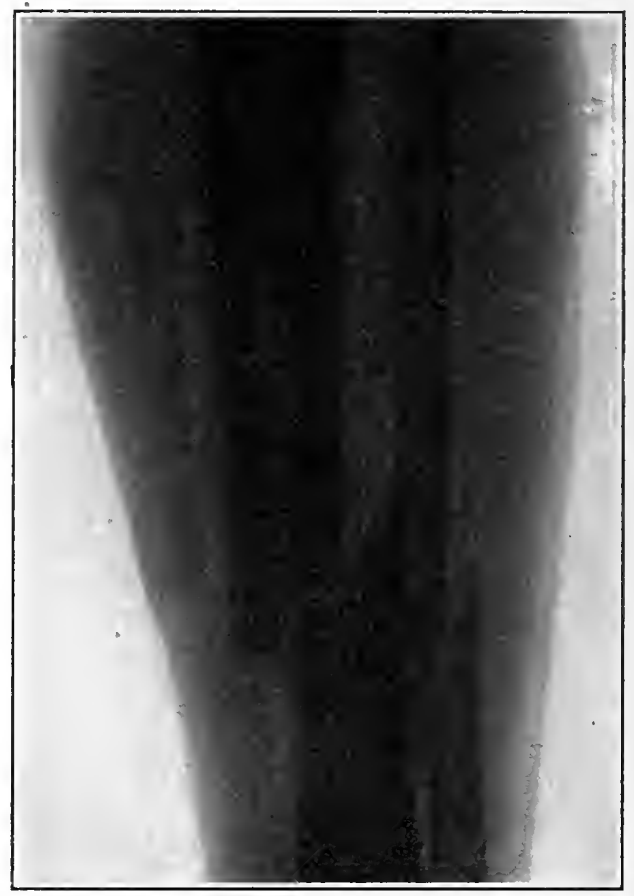

Skiagraph of fracture of tibia and fibula.

tures and dislocations. In fractures about the joints, epiphyseal separations, and ununited fractures, their use has proved most satisfactory. Very satisfactory results have recently been obtained in the treatment of superficial malignant growths and lupus and inoperable forms 
of carcinoma and sarcoma by frequent short exposures to the $x$-rays. Skiagraphs of fractures are shown in Figs. 164, 166, and 167; of a bullet in the wrist-joint in

FrG. 167.

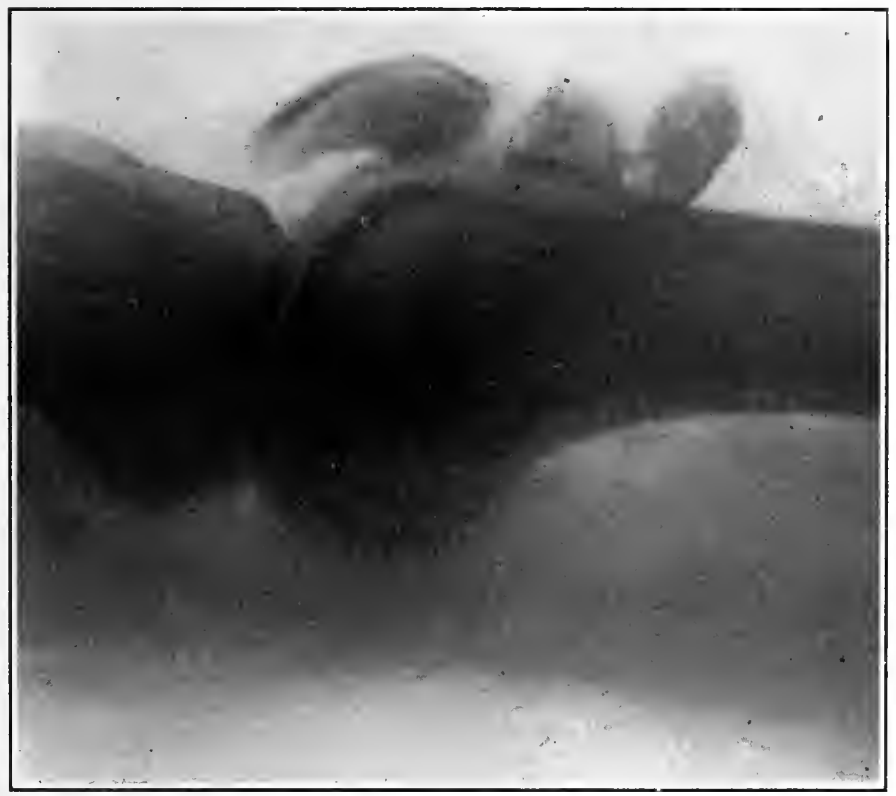

Multiple fracture of patella. (Newcomet.)

Fig. 165; and of an epiphyseal separation of the humerus in Fig. 168.

Radium.-Under certain conditions the use of radium in malignant ulcerations has proved to be useful and is to be recommended, the indications for its use are about the same as those ordinarily for the Roentgen rays. The salt that is usually employed is radium bromide and should be of fairly ligh activity, the quantity of this salt need not 
be very large, one milligramme being ample. It must be placed in a suitable container the best being made of aluminum. The technical name given to this container is the "radiode." When the radiode is suitably protected

Fig. 168.

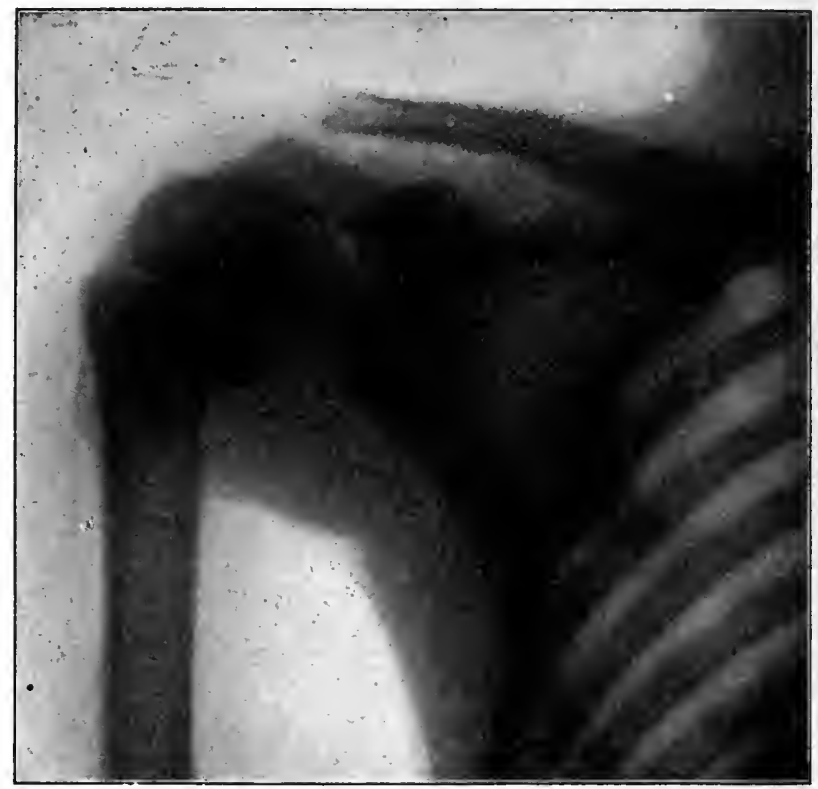

Skiagraph of separation of upper epiphysis of the humerus.

by a capsule of celluloid it can be introduced into the cavities of the body, and at the same time small lead shields may be employed to protect the surrounding parts that are not diseased. The value of radium as a therapeutic agent is due to emanation of the $a$ and $b$ rays. The $a$ rays possess very little power of penetration, and, therefore, are not able to penetrate the walls of the radiode. 


\section{AN 压HETICS.}

Anæsthesia may be locate, regional, or general.

Local Anæsthesia.-This results from the direct application of anæsthetic agents to nerve-terminations, and causes analgesia of the tissues to a limited extent only. It may be produced by the use of cold, a spray of ether, rhigolene, ethyl chloride, or kelene, cocaine or eucaine hydrochlorate, novocaine, holocaine hydrochlorate, stovaine hydrochloride, magnesium sulphate, guaiacol, or by Schleich's method of infiltration.

Regional Anæsthesia. - This is also sometimes described as neural anæsthesia, and results from the application of anæsthetic agents to the nerve-roots, nerve-trunks, or the spinal cord. The analgesia in this form of anæsthesia extends from the point of application to the tissues supplied by the nerve or nerves, and therefore is not limited in extent.

General Anæsthesia.-This is characterized by unconsciousness, as well as abolition of sensation, and may be induced by the administration of nitrous oxide gas, ether, chloroform, bromide of ethyl, chloride of ethyl or kelene, A.-C.-E. mixture, C.-E. mixture, Schleich's anæsthetic mixture, or scopolamine. It may also be induced by a combination of these substances with nitrous oxide gas or oxygen. Hypnotism may also be employed to produce general anæsthesia.

\section{Local Anaesthesia.}

Cold.-Local anæsthesia may be produced by the application of cold, or freezing mixtures, either by a piece of ice or a mixture of ice and salt held in contact with the part for one or two minutes, or by directing a spray of rhigolene or sulphuric ether upon the surface of the part whose sensibility is to be obtunded (Fig. 169).

Chloride of Ethyl or Kelene.-This substance is used also to produce local anæsthesia, and is conveniently fur- 
nished in glass tubes, one end of which is drawn out into a fine point and hermetically sealed. When used, the end of the tube is broken off and a fine jet of ethyl is projected upon the part to be anæsthetized, the warmth of the hand being sufficient to force the fluid from the tube. This form of local anæsthesia is made use of in minor surgical procedures, such as aspiration, the opening of abscesses, and the removal of superficial tumors.

The objection to these methods of anæsthesia consists in the pain which accompanies the thawing process, and the interference with the vitality of the tissues by the freezing, which prevents prompt healing.

Rapid Respiration.-Rapidly repeated deep inspirations kept up for a few minutes will produce insensibility to pain, but sensibility to contact is not obliterated. This form of anæsthesia may be made use of in a slight operation, such as opening an abscess.

FIG. 169.

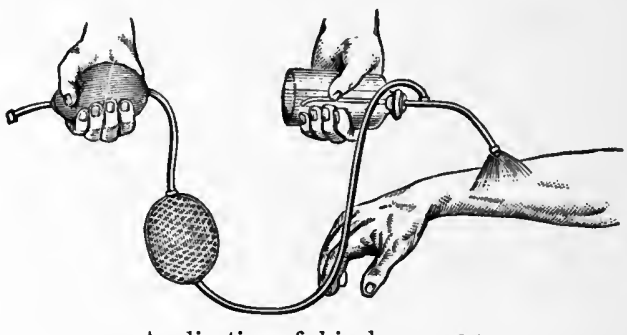

Application of rhigolene spray.

Cocaine.-Local anæsthesia produced by the employment of an aqueous solution of the hydrochlorate of cocaine, in strength from 1 to 2 per cent., is often made use of in minor surgical procedures. Solutions as strong as 10 or 12 per cent. were formerly employed, but experience has proved that there is always danger in the use of the stronger solutions of cocaine, so that it is now considered wise not to use one stronger than 1 or 2 per cent., 
as the full analgesic effect can be obtained by a solution of this strength. When mucous membrane is to be operated upon or growths removed from it, analgesia is produced by brushing over the surface with the solution of cocaine, or by applying to the part for a few minutes a compress of absorbent cotton saturated with it; in mucous cavities the latter method of application will be found most convenient. In using a solution of cocaine to produce anæsthesia in operations upon the eye, a 2 per cent. solution is dropped into the eye, and is repeated until analgesia is complete.

In applying cocaine to the urethra, a 1 to 2 per cent. solution is injected, and is allowed to remain for two or three minutes; more than 1 or 2 grains should not be injected at one time, as fatal results have followed the injection of larger quantities; this is especially the case in using cocaine in the urethra and the rectum, and in these situations great caution should be exercised in its employment.

When it is desired to produce local anæsthesia of the skin or deeper tissues, the application of cocaine to the surface is not satisfactory, and it should in such cases be injected hypodermically into the deeper layers of the skin and into the cellular tissue of the parts to be operated upon; to avoid multiple punctures, the needle is not completely withdrawn from the wound, but its direction is changed and the solution is thrown into different portions of the tissues. It is not safe to inject more than $\frac{1}{4}$ grain of this drug. It is well, in situations where it can be accomplished, to cut off the circulation from the part to be operated upon by placing around it a lubber strap or tube, which prevents rapid absorption of the cocaine into the general blood-current.

In employing any of these drugs hypodermically to avoid the pain following the puncture of the skin by the needle a minute drop of carbolic acid may be applied to the skin at the point of puncture which anæsthetizes the skin so that the patient does not feel the insertion.

Some persons have an idiosyncrasy for cocaine, and 
children seem more susceptible to its constitutional effects than adults. I have seen several instances in children in which marked symptoms of cocaine poisoning resulted from the application of a 4 per cent. solution to the nasal mucous membrane.

The treatment of cocaine poisoning consists in placing the patient in the recumbent position and the hypodermic injection of morphine, strychnine, or ether.

Cocaine ancesthesia may he employed with advantage in minor surgical operations, such as amputations of the fingers, circumcision, opening of abscesses, and removal of superficial tumors, but its utility is most marked in operations upon the eye and upon the mucous membranes of the nose, throat, rectum, vagina, and urethra. Applied for a few minutes to the surface of an ulcer which is to be cauterized, it will render the operation almost painless.

Eucaine Hydrochlorate.-This drug, which possesses the same properties as cocaine, as regards the production of analgesia, is employed as a local application to mucous surfaces, and hypodermically in the deeper tissues to produce local and regional anæsthesia. It has the advantage over cocaine that it can be used with safety in much larger quantities, as it is apparently free from toxic action. Kiessel states that 2 grammes have been injected without the production of toxic symptoms. It may be used in solutions varying in strength from 2 to 10 per cent., which may be sterilized by heating; a 2 per cent. solution is that most usually employed hypodermically.

Novocaine.-This drug has been extensively used by Braun and has a low toxicity. The solution recommended is as follows: Novocaine, 0.125 gram, suprarenin borate, 0.00016 gram ; sodium chloride, 0.225 gram; sterile water, $25 \mathrm{c.cm}$.

Holocaine Hydrochlorate.-This drug, used in a 1 per cent. solution, possesses as decided analgesic action as cocaine; it is also strongly bactericidal in its action. It may be used locally without producing constitutional symptoms, but cannot be used internally or injected into the tissues, on account of its marked toxic action. 
Stovaine Hydrochloride.-This drug is employed in the same manner as cocaine and eucaine to produce local anæsthesia. The solution used is 0.7 per cent.

Magnesium Sulphate.-This drug in a 25 per cent. solution has been employed to anæsthetize either by direct application to nerve trunks or by intraspinal injection. Meltzer advises 1 c.c. of the solution be employed for every twenty-five pounds of the body weight. Sufficient experience has not been accumulated to estimate to the value of this drug as an anæsthetic.

Guaiacol.-This drug may be used for its analgesic effect, and is employed in a solution of guaiacol, grains $\mathrm{xv}$; alcohol, $\mathrm{zv}$; or in the form of an ointment of guaiacol, 5 parts, to vaseline, 30 parts ; or it mav be used hypodermically in a one-tenth or one-twentieth solution in olive oil. Its hypodermic use is not unattended with danger.

Infiltration Anæsthesia.-It has been shown by Liebreich that the injection of simple water into the tissues in such a way as to produce an artificial odema induces a transitory anæsthesia.

Schleich found that the combination of a minute quantity of cocaine and morphine with a weak salt solution, when injected hypodermically, produced a local anæsthesia of longer duration.

The anæsthesia is produced by the artificial ischæmia, by the pressure of the injected fluids upon the nerves, and by the direct action of the anæsthetic substances on the nerves.

A solution of 1 part of cocaine to 1000 parts of sterilized water may be used, or the following solution may be employed :

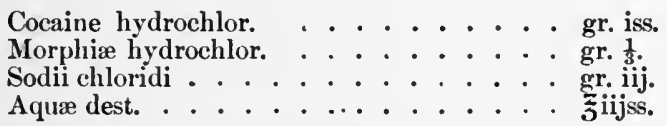

The injection should first be made into the substance of the skin itself, and then into the cellular tissues and deeper structures, as desired. 
Infiltration anæsthesia hạs becn widely employed in minor surgieal operations, and also may be employed in major operations, such as herniotomy and amputations, when for any reason a general anæsthesia is not desirable. In ehildren and nervous subjeets it cannot be employed with advantage. It also has the disadvantage of causing swelling and odema of the tissues at the seat of operation, which often interfere with the satisfaetory recognition of the various anatomieal structures.

\section{Regional Anæsthesia.}

This method, sometimes described as neural ancesthesia, consists in bringing anæsthetic drugs in contact with nerve-trnnks at some distance from the field of operation, with the view of causing analgesia in the tissues supplied by them. For instanee, in a proposed operation upon the leg, the injection would be made near or into the anterior crural and seiatic nerves. Coeaine, eueaine, or Schleieh's solution may be employed for the purpose. The nerves may be anæsthetized by the paraneural method, which consists in injecting the solution in the vicinity of the nerve-trunk as near as possible to the nerve; or by the direct intraneural method, whieh consists in producing anesthesia of the skin and cellular tissue, and then exposing the nerve-trunks by dissection and injecting the solution directly into them by passing the needle into their substanee.

This method of anæsthesia has been employed with suceess both in minor and major surgieal operations, sueh as the reduetion of hernix, amputations, and the removal of tumors, and is espeeially applieable in operations upon the extremities.

Spinal Subarachnoid Injection.-As the result of the work of Corning, Bier, and Tuffier, anæsthesia by means of spinal subaraehnoid injection of eoeaine or eucaine has recently been employed with satisfactory results. At the present time this method of anæsthesia is resorted to only 
in operations upon that portion of the body below the diaphragm, and injections are made into the spinal canal in the lumbar region. Fifteen to 20 minims of a 2 per cent. cocaine or eucaine solution are usually sufficient to produce satisfactory anæsthesia. Stovanin solution has recently been more frequently employed than cocaine solution. The solution is made by dissolving $1 \mathrm{gr}$. of stovaine hydrochloride in $15 \mathrm{~m}$. of sterilized normal salt solution. The technique of the operation is as follows: The entire lumbar and sacral regions should carefully be sterilized, and the position of the third lumbar interspace-that is, the space between the third and fourth lumbar vertebrælocated. The patient next sits astride of the operatingtable and bends forward in the position of ventral flexion, with his elbows resting upon his knees, which widens the space between the third and fourth lumbar vertebræ. A few drops of cocaine or stovanin are next injecterl into the skin over the centre of this space. A needle between 1 and 2 millimetres in circumference, and about 8 centimetres in length, attached to a syringe, is next inserted through the skin midway between the spinous processes, or a puncture by a tenotome may be made through the skin, and the needle inserted through this. The needle and syringe should be thoroughly sterilized by boiling before being used. The needle should be pushed forward and a little to the left, to cause it to enter the spinal canal in the median line, and as soon as resistance disappears and fluid appears in the syringe it is evident that the canal has been entered. In no case should the analgesia solution be injected unless the fluid escapes satisfactorily. After a few drops of fluid have escaped, the syringe is removed from the needle and replaced by one containing the anæsthetic solution, and 15 to 20 minims of the solution are injected into the spinal canal. The needle is then removed and the puncture sealed with a small piece of gauze and collodion, and the patient placed in the recumbent posture. In a few minutes anæsthesia is usually sufficiently advanced for the operation. Sec also p. 575 . 
Subarachnoid spinal injection should not be employed in children, nor in nervous and excitable patients, but may be employed in eases where for any cause a general anæsthetic is contraindicated.

This method of anæsthesia has been employed successfully in a great variety of operations, and up to the present time few fatalities have been reported as the result of its use ; but sufficient time has not elapsed to show the ultimate result of the injections. A more extended use of the method alone can prove that it is safer than the general anæsthetics now employed. The restriction in its use to operations in certain parts of the body also renders it difficult to estimate its comparative safety. The proeedure is now recognized as a valuable method of securing analgesia in certain cases when a general anæsthetic cannot be employed. It should not be used indiscriminately, and should be given by one who has had experience in its administration.

\section{General Anæsthesia.}

General anæsthesia may be produced by the administration of nitrous oxide gas, ether, chloroform, A.-C.-E. mixture, C.-E. mixture, Schleich's mixture, ethyl bromide, tehyl chloride or kelene, or scopolamine hydrobromate.

Choice of Anæsthetic.-In selecting an anæsthetic, the most important considerations are its safety and its suitability to the individual case. In point of safety, nitrous oxide gas holds the first place; but, unfortunately, its use is restricted to cases in which only a few minutes' anresthesia is required. Statisties show that the mortality following the administration of nitrous oxide is about 1 to $5,250,000$; of ether, 1 to 16,675 ; of chloroform, 1 to 3749 . Gardner's statistics show that in 22,219 chloroform administrations there were 14 deaths; while in 17,067 administrations of ether or nitrous oxide gas and ether, there was 1 death. It should be remembered, however, that both ether and chloroform are employed in the most serious surgical procedures, while nitrous oxide gas 
is used only in trivial operations, so that many of the deaths attributed to ether and chloroform may have been due to conditions resulting from the operations. General anæsthesia seems to be accompanied by especial danger in subjects presenting the condition known as the status lymphaticus.

Nitrous Oxide Gas.-Nitrous oxide causes anæsthesia by arresting the oxygenation of the blood while it is in contact with it, and, in addition, the gas produces anæsthesia by direct action on the cerebral cortex. Nitrous oxide gas is contraindicated in alcoholic subjects, or in those having marked atheroma of the arteries, as apoplexy may occur, or in any condition of obstructed respiration. It should never be administered in cases of angina Ludovici, sublingual abscess or enlargement of the thyroid gland. The apparatus best suited for its administration consists of a cylinder of metal in which the gas is compressed, which is attached to a rubber bag, which has a mouthpiece fastened to it; this is provided with a double valve, which prevents the expired air from passing back into the bag. The mouthpicce is adjusted over the mouth, and after removing any false teeth or foreign borlies from the mouth, the patient is instructed to take deep, full breaths, and in from one-half to one minute the face becomes congested and dusky and the breathing becomes stertorous, indicating that the patient is fully under the influence of the gas. The anæsthesia from nitrous oxide cannot be prolonged for more than a few minutes, so that it can only be employed in operations which take a short time for their performance, such as the extraction of teeth and the opening of abscesses. Unfortunately, it eannot be used in the reduction of fractures or dislocations, as it does not produce complete muscular relaxation. Nitrous oxide is frequently used to produce anæsthesia, and when this result is accomplished the anæsthesia is kept up by the administration of ether. Nitrous oxide gas is commonly employed in dental surgery to produce anæsthesia for the removal of teeth, but is also occasionally employed in minor surgical operations; but from the fact 
that the apparatus for its adninistration is a bulky one, its use is not so convenient as ether or chloroform.

Nitrous oxide gas may also be administered by the open method, or by an open inhaler resembling that of Allis. The gas, being heavier than the air, is introduced into the inhaler and falls to the bottom. Flux, who has employed this method of administration in a number of cases, clains that by its employment excitement, stertor, lividity, struggling, and convulsive movements are done away with.

Nitrous Oxide Gas and Oxygen.-The administration of nitrous oxide gas with oxygen has been found by Hewitt. to diminish the asphyxial symptoms, so that a more prolonged and tranquil anæsthesia can be safely obtained. 'The anæsthetic state is not produced as rapidly as by nitrous oxide gas alone, but it may be prolonged by a skilful anæsthetizer for an hour or more. It is administered by a special apparatus, by which the administrator ean increase or diminish the amount of oxygen, according to the symptoms presented. Cyanosis, stertor, and muscular twitching call for an increase in the oxygen, whereas symptoms of excitement call for its diminution. In children and in aged and anæmic subjects the amount of oxygen may be increased rapidly; whereas in strong, full-blooded subjects the quantity of oxygen should be increased cautiously.

Ether.-Sulphuric ether is one of the most widely employed substances in surgery to produce anæsthesia; it is probably the safest of all anæsthetics, except nitrous oxide gas, and for this reason should be preferred to all others. Its effects, according to Hare, result from the action of the drug, first, on the brain, then on the sensory tracts of the spinal cord, then on the motor tracts, then on the sensory side of the medulla oblongata, and finally upon the motor side of the medulla, and thereby produces death from respiratory fiulure if given to excess. Its administration is attended with risk in the following cases: (1) In infants, in whom it eauses irritation of the bronchial mucous membrane, with profuse secretion of mucus, and may cause also bronchopneumonia. (2) In aged persons a profuse secretion of mucus and bronchopneumonia may follow its use; 
it is also contraindicated in these subjects in whom there are rigidity of the chest and lessened respiratory power. (3) In advanced organic disease of the kidneys, and especially in nephritis of the interstitial form with urine of a low specific gravity and in diabetic subjects. (4) In disease of the heart its administration is more dangerous in myocardial than in valvulur lesions. (5) In cases of obstructed respiration from swelling of the pharynx, fixation of the tongne in cancer and cellulitis of the neck, and in emphysema and abdominal distention. (6) In cases in which examination of the blood shows that the hæmoglobin is diminisherl to less than 50 per cent. (7) When the bronchial irritation following its use may impair the result in operations for hernia and in laparotomy.

Preparation of Patient.-A patient should be prepared for the administration of ether by withholding all solid food for at least six hours before its inhalation; he shonll be in the reeumbent postnre, and any garments about the chest or neck should be loosened, so that the respiratory movements are not interfered with. The surgeon shonlil see also that any false teeth or foreign bodies which may be present in the mouth are removed before the administration of the drug is begun. As the vapor of ether often causes irritation of the nucous membrane of the lips and nasal passages, it is well to anoint these parts with a little vaseline or cold-cream before administering the ether.

The administration of morphia grs. $\frac{1}{12}$ to grs. $\frac{1}{8}$ hypodermically immediately before the use of ether, which diminishes the secretion of mucus, is considered by many operators to be a valuable aid to the satisfactory production of anæsthesia.

The stomach, if it contains food, should be washed out by means of the stomach-pump. This washing out of the stomach, before operation in cases of intestinal obstruction, is of the greatest importance, as the stomach may contain stercoraceous matter, which may be drawn into the respiratory passages if vomiting occurs, 
and produce death by suffocation or later cause aspirationpneumonia.

It should also be borne in mind that the vapor of ether is very inflammable, and that it is heavier than the air, so that lights brought near the patient while being etherized should be held at a higher level than the ether-can or inhaler.

The anæsthetizer should always listen to the patient's heart before giving an anæsthetic; this enables him to detect any irregularity in its action, and at the same time has a good moral effect upon the patient, especially if he can assure him that he is in good eondition to take the anæsthetic.

It is well also to have another physician present during the administration of a general anæsthetic, as unforeseen difficulties occasionally arise. There should always be at hand tongue-forceps and instruments with which tracheotomy may be performed if necessary ; also nitrite of amyl, digitalis, strychnine, and a hypodermic syringe.

In debilitated patients or in those who are weak from loss of blood the administration of half an ounce to an ounce of whiskey from fifteen to thirty minutes before the anæsthetic is given is often advisable.

The person intrusted with the administration of the anæsthetic should watch the patient closely, and should not have his attention diverted by the operation; he should carefully observe the pulse, respiration, and color of the patient's face, and be prepared to withdraw the anæsthetic upon the development of any symptom of danger, and to treat such symptoms should they arise.

The anæsthetizer should be careful that no ether comes in contact with the conjunctiva, as severe ether conjunctivitis has resulted from this accident. He should also be careful in testing the sensibility of the cornea not to touch it with the finger, but should press upon it through the eyelid, for infection of the cornea, resulting in sloughing, has resulted from neglect of this precaution.

An anæsthetic should never be given to a woman without the presence of a third person, as in some cases these 
agents give rise to erotic dreams, and it may be difficult to disabuse the patient's mind of the idea that an assault has been committed unless the evidence of eye-witnesses at the time of the anæsthetization can be brought forward to prove that such was not the case.

Ether produces more irritation of the respiratory tract than chloroform, and its administration is sometimes followed by the derelopment of bronchitis, pulmonary congestion, or pneumonia. These complications are less likely to occur if care is taken to avoid the administration of ether in patients who are suffering from bronchial irritation, and to see that a patient who has taken ether is not exposed to draughts and is not allowed to go out into cold or moist air immediately after recovering from the anæsthetic.

Administration of Ether.-In the administration of ether, a towel folded into a cone, a pad composed of a number of layers of gauze, or one of the various ether inhalers may be employed. The best of these is Allis's inhaler, which consists of a metallic framework covered with leather or a nickel-plated case, which carries a number of folds of a roller-bandage, giving a large surface for the rapid evaporation of the drug (Fig. 170).

Whether a gauze pad or

FIG. 170.

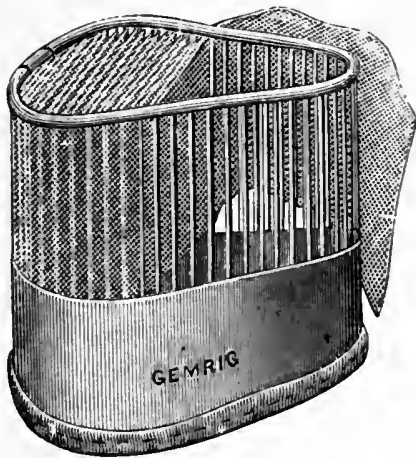

Allis's ether inhaler. inhaler be employed the continuous drop method of supplying the ether is considered the best. The point of saturation of the gauze or mask should be constantly changed.

If a towel folded into a cone is used, a few layers of stiff paper interposed between the outer layers of the towel will keep the cone in shape and prevent evaporation of the ether from its exterual surface. 
For the administration of an anæsthetic, the patient should be. in the recumbent posture and the head turned to one side, as in this position mucus is less apt to collect in the pharynx and interfere with the breathing.

In administering ether the drug is continuously dropped upon the gauze or inhaler placed over the nose and mouth of the patient. He is then requested to take deep breaths, or to blow the ether away, which latter procedure causes him to take deep inspirations. In the beginning of etherization the patient will resist the inhalation much less vigorously if the ether is given slowly with a plentiful admixture of air. "The first effect of the inhalation of ether is to produce acceleration of the pulse and respiration ; the mucous membrane of the air-passages is irritated, and coughing often oceurs; there is also in this stage a disposition to muscular movements, and it is frequently necessary to restrain the patient; the brain also is excited, and the patient is apt to ery out. These symptoms call for a continuance of the administration of the ether, and not for its withdrawal. Succeeding the stage of excitement, if the ether be pushed, profound anæsthesia takes place, as is evidenced by the loss of consciousness, relaxation of the muscular system, moist skin, loss of special senses, contracted pupils, and slow and deep respiration, tending to become stertorous. When the conjunctiva is insensitive to the touch of the finger, anæsthesia is usually profound. When the anæsthesia is complete, the amount of ether inhaled should be diminished, and the patient given only so much as will keep him well under its influence. It is surprising how small a quantity a careful and watchful anæsthetizer will require to keep the patient fully under its effects for a considerable time. The time required to produce anæsthesia varies in different cases: it is produced in children in a few minutes; in adults from ten to twenty minutes are usually required; drunkards and those who have taken ether frequently require a larger amount and a longer time to come under its influence. After the administration of the drug is stopped, the patient may continue for some time in an unconscious 
condition, resembling a quict sleep, or he may awake and exhibit more or less symptoms of cerebral excitement.

First Insensibility from Ether.-There often exists in the early course of the administration of ether a stage of primary anæsthesia, which lasts for a minute or more, and which may be taken advantage of to perform such a minor surgical operation as opening an abscess, reduction of a dislocation or a fracture, or extraction of a tooth. The recovery from this condition is usually very prompt, and is not followed by nausea or the after-effects which attend the prolonged administration of ether.

Accidents during Etherization.-During the administration of ether, particularly in the early stage, the patient may suddenly stop breathing, the face at the same time becoming cyanosed. This condition calls for withdrawal of the ether; and if an inspiratory effort does not quickly follow, pressure should be made upon the front of the chest, and when this is relaxed a deep inspiration usually takes place, and no further difficulty is experienced. This condition should not be confounded with the very common effort of holding the breath, the latter occurring with the chest fully expanded, the former with the chest empty.

Vomiting may occur during etherization, and the vomited matter may accumulate in the pharynx or the mouth, and obstruct the breathing, or may enter the larynx or trachea and cause a like result. Vomiting is more apt to take place if solid food has been taken shortly before the administration of the anæsthetic. If this acciclent occurs and interferes with breathing, the jaws should be opened and the head turned to one side, when the vomited matter will usually escape without difficulty. If, however, food has entered the larynx, and is not ejected by coughing, it will be necessary to perform tracheotomy promptly and hold the tracheal wound open, or to introduce a tube and practise artificial respiration. The breathing may also be obstructed by the accumulation of mucus and salive in the pharynx. This is less likely to occur if the head is kept to one side during the administration of the drug; if it occurs, the head shonld be turned to one side, the 
jaws opened, and the material removed with small sponges or pieces of gauze fixed to sponge-holders.

The tongue may fall backward and obstruct the breathing when muscular relaxation is complete during anæsthesia; this accident is also less likely to occur if the head is kept to one side during etherization. If asphyxia results from falling back of the tongue, it should be bronght forward by placing the fingers on each side

FIG. 171.

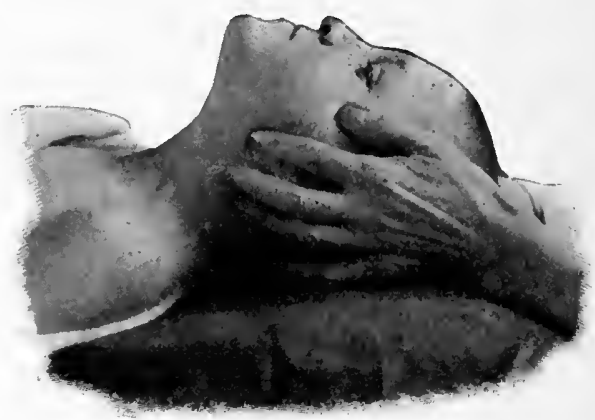

Pushing the jaw forward.

beneath the angles of the inferior maxillary bone, and pushing the jaw forward, at the same time over-extending the neck by bending the head backward (Fig. 171), or the month should be opened and the tongue drawn forward with tongue forceps. Either of these manipulations is usually sufficient to re-establish the respiratory movements.

If, however, in any of these forms of mechanical asphyxia respiratory action is not promptly restored, some form of artificial respiration should promptly be resorted to, either Laborde's or Silvester's ; and of these, Laborde's method, by rhythmical traction of the tongue, and Silvester's have yielded the most satisfactory results. Efforts at resuscitation in these cases should be persevered in for at least half an hour, as apparently hopeless cases have been saved by persistent use of these means.

Failure of respiration may occur also from paralysis of 
the respiratory centres, or spasm of the respiratory muscles; the former may occur from an overdose of the anæsthetic, or from intercurrent asphyxia, syncope, or morbid states of the respiratory system.

Spasmodic respiratory failure may occur before complete anrsthesia, and is liable to arise in muscular and emphysematous subjects. Respiratory failure from either of these causes should promptly be treated by artificial respiration and the hypodermic use of strychnine, atropine, or digitalis.

After-effects of Ether.-After complete anæsthesia from ether, nausea and vomiting are very common, and both are more apt to follow in case the patient has taken food shortly before the administration of the anæsthetic. 'They may last for only a short time, or may persist for hours. If persistent, the swallowing of a few mouthfuls of hot water will of ten relieve the condition; or the administration of cocaine hydrochlorate, grain one-quarter, with crushed ice, repeated two or three times, or the use of crushed ice with champagne or brandy, may be followed by satisfactory results. 'The inllalation of oxygen, begun as soon as the ether has been suspended and continued for some time, is now frequently employed with good results. Inhalation of the fumes of vinegar will often prevent nausea and vomiting, the vinegar being poured upon a towel or a piece of gauze, which is being held over the mouth and nose of the patient, and it should be applied as soon as the administration of the ether is stopped; it should be used continuously for some time to be followed by the best results.

Administration of Ether by the Rectum.-A few years ago prompt anæsthesia was induced by the administration of the vapor of ether by the rectum; a can of ether was placed in warm water to which a tube and nozzle were attached. The nozzle was introduced into the rectum. This method was abandoned by reason of the irritation of the bowel resulting in hemorrhage, which frequently occurred. Recently the method has been revived in Boston, with the modification that the ether is not warmed and the 
vapor is driven into the rectum by forcing air through the ether. Satisfactory results as to the prompt production of anæsthesia, and non-irritation of the rectum, have been recorded in a number of cases. This method has been used in so limited a number of cases that its value cannot yet be estimated.

Ether and Nitrous Oxide Gas.-The production of anæsthesia by the combined use of nitrous oxide gas and ether has been quite extensively employed both in England and this country. Hewitt considers this method of producing anæsthesia far superior to any other method which we possess at the present time. A special apparatus is required, which controls definitely the amount of nitrous oxide, ether, and air. Anæsthesia is produced first by the use of nitrous oxide gas, and, as soon as this is developed, the anæsthetic state is maintained by substituting the vapor of ether for the nitrous oxide gas. No air is given with the gas until anæsthesia is complete, which should be in from two to three minutes. Breathing at this time is stertorons, and cyanosis is well marked. After this time air is administered with the ether vapor. Anæsthesia by this method is rapidly induced, there is less struggling and spasm, the quantity of ether employed is smaller, and the after-effects are less marked, especially vomiting, and recovery from the anæsthetic state is more rapid than when ether is used alone.

Ether and Oxygen.-The administration of ether with oxygen gas has been employed to a considerable extent. In the employment of this combination to produce anæsthesia the patient is first allowed to inhale a small amount of ether from an inhaler, and a tube connected with the oxygen receiver is then introduced into the inhaler and the oxygen gas tumed on, so that the patient is allowed at the same time to inhale the vapor of ether and oxygen gas. A special apparatus may also be employed which regulates definitely the amount of ether and oxygen furnished. Anæsthesia produced by this combination is accompanied by less cyanosis, vomiting is rare, and the patient recover's very promptly from the anæsthetic state. 
As the ether vapor and oxygen form a highly explosive mixture, care should be exercised not to bring a flame near the patient during its administration.

Chloroform.-This drug according to Hare, first affects the brain, then the sensory part of the spinal cord, then the motor area of the cord, then the sensory paths of the medulla oblongata, and finally the motor portions of the medulla, and produces death from failure of the vasomotor centre and of the respiratory centre unless, as rarely occurs, the heart has succumbed to the drug.

Chloroform is certainly a much more dangerous anæsthetic than ether, and although it is widely used in the British Islands and upon the Continent, it is not extensively used in this country except in certain districts-as in the southern and southwestern districts of the United States, and here its use is followed by fewer fatalities than in the northern districts, so that it is possible that its use is safer in warm climates. Clinical experience has demonstrated that chloroform may be used in aged and very young subjects and in puerperal patients with comparative safety ; deaths from chloroform are more common in the middle period of life. It is also to be preferred to ether in patients suffering from emphysema of the lungs, bronchitis, and vascular degeneration of the kidneys. It is also employed by some surgeons instead of ether in operations upon the mouth when the actual cautery is used, on account of its less inflammable character.

Considerable diversity of opinion exists among different observers as to whether death resulting from chloroform is due to failure of the heart or failure of the respiration, and each has brought forward a large amount of evidence to prove his views correct. Although it has been demonstrated that chloroform is a direct depressant and paralyzant to the heart-muscle or its contained ganglia, and that cardiac dilatation of varying degrees may be brought about by the administration of chloroform, yet clinical experience shows that paralysis of the respiratory centres is probably the most important factor in causing death during chloroform anæsthesia, for circulatory failure in 
these cases is due to embarrassed or suspended breathing. and the only method of treatment which has been found of value is that which tends to bring about respiratory action, namely, some one of the various forms of artificial respiration.

Chloroform is more dangerous in the earlier stages of the administration, and the gravity of the operation appears to have little effect in increasing its danger, as statistics show that the greatest number of fatalities have occurred in minor surgical procedures, such as extracting

FIG. 172.

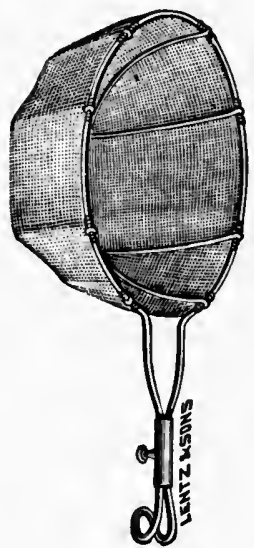

Esmarch's inhaler. teeth, amputation of fingers, reduction of dislocations, and opening abscesses.

Preparation of Patient.-A patient is prepared for the administration of chloroform as in the case of ether, the same precautions being taken as regards the removal of false teeth or foreign bodies from the mouth, and to see that the clothing about the chest and neck does not restrict the circulation or respiratory movements.

Administration of Chloroform.-Chloroform is administered by pouring a drachm of the drug upon a folded towel, which is first held a few inches from the mouth, and nose, and gradually brought nearer, but is not allowed to come in contact with the face, as from its local irritating action it will blister the surface; the lips and anterior nares should be anointed with vaseline.

The anæsthetizer should remember that one of the dangers in the administration of chloroform is the risk of too great concentration of its vapor, so that he should see that a sufficient admixture of atmospheric air takes place.

Chloroform may also be administered with Esmarch's inhaler, which consists of a wire frame covered with gauze (Fig. 172). 
Various inhalers have been devised to regulate the amount of chloroform administered and to secure the proper admixture of atmospheric air, and the best of these probably is Mr. Clover's apparatus.

Profound chloroform anæsthesia is manifested by insensibility of the conjunctiva to the touch, absence of the reflexes, complete muscular relaxation, and, usually, contracted pupils. When this stage is reached, the inhalation should be stopped, and after this time only so much chloroform should be administered as is sufficient to keep the patient fully under its influence.

Complete anæsthesia should be produced before any operation is hegun; if undertaken before that time, syncope nay be produced by reflex inhibition of the heart. If convulsive movements take place before the patient is fully anæsthetized, and the face becomes cyanosed, the inhalation should be discontinued until these symptoms disappear. The pupils should also be watched carefully, to see if they respond to light or are contracted. If the anæsthesia is not complete, insensibility to light or wide dilatation is a sign of danger which calls for removal of the anæsthetic and active treatment to stimulate the circulation and respiration. If the inhalation has been stopped and is again in a short time resorted to, it should be given very carefully and slowly, for syncope may suddenly develop from the fact that the heart or the respiration may feel the effect of the previous use of the drug.

Accidents during Chloroform Anæsthesia._Mechanical asphyxia may occur during anæsthesia produced by chloroform, as well as that by ether, by obstruction of the respiratory passages by blood, mucus, foreign bodies, or the tongue falling backward over the epiglottis. These accidents should be treated in the same manner as when occurring during etherization.

Death during the administration of chloroform may result from cardiac syncope or from respiratory arrest, and the dangerous symptoms develop so rapidly that the greatest promptness is required to meet them. The per- 
son administering chloroform should constantly watch both the pulse and the respiration, and should not for a moment have his attention diverted from the patient; great vigilance is here, if possible, more important than during the administration of ether.

Respiratory Arrest.-During chloroform anæsthesia paralysis of the respiratory centres may occur, giving rise to respiratory arrest. If this dangerous symptom appears, the patient's head should be lowered and artificial respiration promptly employed to re-establish the respiratory function.

Cardiac syncope developing during the administration of chloroform, manifested by pallor, fluttering or arrested pulse, and cessation of respiration, should be treated by lowering the patient's head or inverting the patient, the use of a rapidly interrupted electric current, the hypodermic injection of digitalis, atropine, or strychnine, and the employment of artificial respiration, either Silvester's, the direct method, or Laborde's method; and, as in cases of threatened death from ether, the treatment should not be desisted from for some time, as by persistent employment of these means apparently hopeless cases have been resuscitated.

Chloroform and Oxygen.- The combined use of chloroform and oxygen is sometimes employed to produce anæsthesia. A small amount of chloroform is first administered, and then the oxygen gas is introduced into the inhaler, and the two gases are inhaled at the same time; or a special apparatus may be employed, by means of which a definite amount of each drug may be administered.

C.-E. Mixture.-This consists of 2 parts of chloroform to 3 parts of ether and is highly recommended by $\mathrm{F}$. W. Hewitt for general anæsthesia. He considers that in general surgical cases it produces better results than any other anæsthetic. And he considers it especially satisfactory in subjects over sixty years of age. Hewitt prefers to administer it by an inhaler, but it may be given by the open method.

The A.-C.-E. Mixture.-I'his mixture, which consists of 3 parts of chloroform, 1 part of ether, and 1 part of alcohol, has been employed by some surgeons in place 
of ether or chloroform, with the idea that the dangers of chloroform are diminished by its combination with ether and alcohol. Clinical experience, however, has not proved this view to be correct. If administered with as much care as chloroform, its administration is accompanied with the same safety. It should be administered upon a gauze pad or inhaler in the same manner as chloroform, and the patient should be watched as carefully during its inhalation as during the administration of the latter drug, and any complications occurring should be treated in the same manner as those arising during the use of chloroform.

Bromide of Ethyl.--This drug was introduced as an anæsthetic some years ago, but as a number of deaths followed its use, it was abandoned. The time required to produce anæsthesia is shorter than for ether, but there is often induced violent muscular spasm, which renders it an unsuitable anæsthetic in many cases.

Bromide of ethyl has again been revived as an anæsthetic, but clinical experience has proved that its use is not devoid of danger, that it is not as safe as ether, and that it possesses no advantages in point of safety over chloroform. When used, it should be administered by pouring a drachm or two upon an inhaler or a towel, and the patient should be watched with the same care as during the administration of chloroform.

Chloride of Ethyl or Kelene.-This drug has recently been employed by inhalation to produce general anæsthesia. It is principally used to produce anæsthesia for short operations, or may be used to bring about anæsthesia, which is afterwards continued by the use of ether. The advantages of its use are that anæsthesia can be produced in a few minutes and that recovery is rapid and unaccompanied by nausea or other unpleasant symptoms. It is administered in the same manner as ether, the spray being directed into a cone or inhaler.

Scopolamine.-This drug, an alkaloid closely resembling hyoscine, has been extensively used in recent years. It is often given to produce rapid anæsthesia and the anæsthetic state is continued by the administration of ether. It 
has not proved as safe an anæsthesia as ether as a number of deaths have been reported from its use. It is usually employed in combination with morphine, either in repeated doses without any other anæsthetic, or in a single dose preliminary to inhalation anæsthesia, ether or chloroform being employed. In the former method, the usual procedure is to give three hypodermic injections of scopolamine hydrobromate, gr. I $^{\frac{1}{8}}(0.0004$ gramme $)$, and morphine sulphate, gr. $\frac{1}{8}(0.008$ gramme), at intervals of a half-hour to an hour before the operation. The solution of scopolamine should be freshly made, as it decomposes rapidly if kept for more than a few days. If the drug acts well, the patient becomes sleepy after the first injection, is fast asleep after the second, and unconscious and insensible to pain after the third. The third injection is not usually felt by the patient. After the operation is finished the patient is returned to bed unconscions, and continues to sleep for five hours after the last injection. In the latter method, a hypodermic injection of scopolamine hydrobromate, $\mathrm{gr} \cdot \frac{1}{100}$ (0.00065 gramme), and morphine sulphate, gr. $\frac{1}{6}-\frac{1}{4}(0.01-$ 0.016 gramme), is given abont half an hour before general anæsthesia is begun. The advocates of each method report very favorable results. It is probable that the second is, on the whole, the safer and more satisfactory. It certainly reduces the amount of ether or other anæsthetic employed, and almost entirely abolishes the disagreeable after-effects. In point of safety and satisfactory anæsthesia scopolamine-morphine anæsthesia compares very unfavorably with the other anæsthetics. H. C. Wood Jr. has collected 1988 cases of anæsthesia by this compound and finds that there was a mortality of 4 per 1000 , and that anæsthesia was unsatisfactory in a large number of cases.

After-effects of Anæsthetics.-Nausea is not common after chloroform anæsthesia. The treatment of this condition following etherization has been previously described. The temperature is usually notably lowered by anæsthetics, so that it is always well to apply artificial heat and keep the patient well covered. A form of mental disturbance known as confusional insanity is often 
attributed to the use of anæsthetics, but, as it does not usually develop until some time, often two or three weeks, after their employment, H. C. Wood is of the opinion that the relation between the mental synptoms and the anæsthetic has not been elearly proved in these cases, and that it is rather the outcome of a peculiar depression of the cerebral cortex produced by the shock of the operation itself, or by the emotional strain due to the surgical illness. This view seems to be confirmed by the fact that many of the cases of emotional insanity which are observed follow injuries in which no anæsthetic has been given. Albuminuria and glycosuria may follow the administration of ether or chloroform, but are usually only temporary conditions.

Patients who have been subjected to prolonged anæsthesia should be carefully watched until consciousness returns; it is well to have such patients turned from one side to the other at intervals to favor free pulmonary expansion. If there is any cyanosis due to the accumulation of mucus or blood in the fauces this should be removel and oxygen should be inhaled.

Paralysis of the nerves of the brachial plexus may follow prolonged anæsthesia when the arm is drawn high above the head; it is not due to the anæsthetic, but results from stretching of the nerves over the head of the humerus or their compression between the clavicle and the first rib. Paralysis of the musculo-spiral nerve may also occur from prolonged pressure of the arm upon the edge of the table.

Hypnotism. -The anæsthetic state of hypnotism has been utilized for the performance of surgical operations. Schmeltz and others have recorded operations done under this influence, the patient apparently suffering no pain. While there is no doubt that the anæsthetic state can be obtained by hypnotism, which might be serviceable in surgical operations, yet we do not believe that it will be of general utility. 


\section{TRU S S E S.}

A truss for the palliative treatment of hernia is a mechanical contrivance with one or more pads and a strap: these are held in position by a spring to which they are attached, which holds the pad in contact with the skin over the hernial opening.

Trusses are usually applied in cases of reducible and sometimes in irreducible herniæ, and are used in the treatment of herniæ at all ages ; in infants and young children the continued use of a properly fitting truss is often followed by a radical cure of the hernia. They are made with steel or rubber springs and with pads of wood, rubber, celluloid, or horsehair, covered witl chamois skin; their shape and the pressure which they should exert vary with the variety of hernia for which they are applied.

A firm compress applied over the inguinal canal or crural ring, secured in position by a firmly applied spicaof-the-groin bandage, forms a very satisfactory temporary means of preventing the descent of a hernia.

A properly fitting triss should be worn without discomfort to the patient-that is, should not make too much pressure upon the skin at the points where the pads are applied, and should absolutely prevent the descent of the hernia. In testing the adequacy of a truss, after application, to prevent the escape of the hernia, the patient should be instructed to separate his legs, bend forward over the back of a chair, and cough or strain forcibly; if this does not bring the hernia down, control of the rupture may be considered satisfactory.

Trusses should be applied after the complete reduction of the hernia, while the patient is in the recumbent posture. When first applied, the truss should be worn both during the night and day; and if the skin becomes tender at the points of pressure, it should be sponged with alcohol and alum, then dried and dusted with powdered starch or lycopodium. Patients at first sometimes complain of discomfort in wearing a truss, but they soon become accustomed to its presence. After a truss has been 
worn for some time, its use at night, while the patient is in bed, may be dispensed with, but the patient should not remove it until he is in bed in the recumbent posture, and he should reapply it before he rises in the morning. In children it is better to have the truss worn continuously; and if it is removed for bathing, the nurse should be instructed to place her finger over the ring to prevent descent of the hernia until the truss is applied. In applying trusses to male children care should be taken not to make pressure upon an undescended testicle.

Worsted Truss. - This appliance may be used in the treatment of inguinal hernia in infants, and is made from an ordinary skein of worsted, one part of which is made to pass around the body just

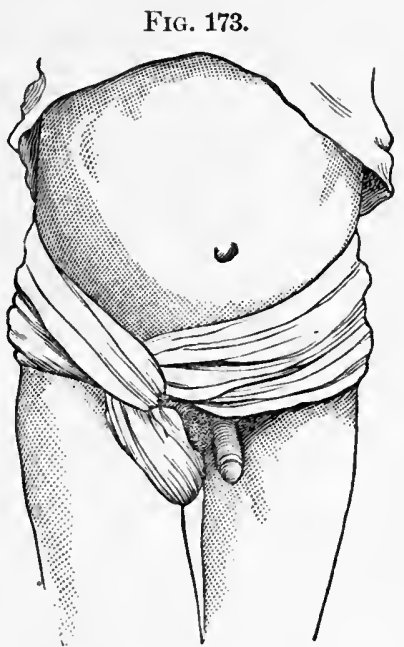

Worsted truss. (BREWER.) above the iliac crests, the other joins this in front and behind, forming a perineal band. 'The knot made by the two bands in front should be directly over the inguinal ring (Fig. 173).

Trusses for Inguinal Hernia.-In measuring a patient for this form of truss, the circumference of the body midway between the crest of the ilium and the great trochanter should be taken, and the distance from the symphysis pubis to the anterior superior spinous process of the ilium may also be given, as half of this distance corresponds to the position of the internal abdominal ring. In redueible inguinal hernia the truss-pressure should be exerted upon the inguinal canal and directly backward. To eontrol this variety of hernia, a single-spring truss (Fig. 174) may be employed, or the use of a truss having a double spring with flat pads on each side of the spine attached to the 
springs, and a smaller pad over the inguinal eanal on the unaffected side, with a full pad on the side of the hernia, will often be efficient. This, which is known as Hood's truss, is one which will be found a very satisfactory instrument, both in inguinal and femoral hernia (Fig. 175).

FIG. 174.

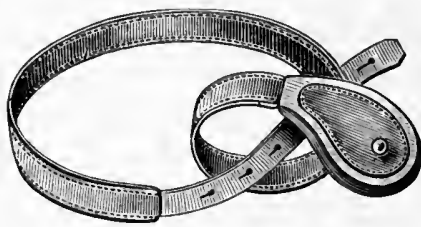

Truss for inguinal hernia.
FIG. 175.

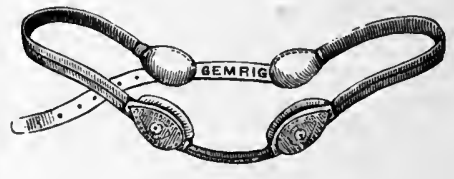

Hood's truss.

Trusses for Femoral Hernia.-In measuring a patient for this variety of truss, the eircumference of the body midway between the crest of the ilium and the great trochanter should be taken-; the distance of the saphenous opening from the symphysis pubis, as well as from the anterior iliac spine, shonld also be taken. In reducible

FIG. 176.

FIG. 177.

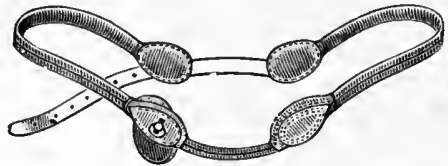

Hood's truss for femoral hernia.

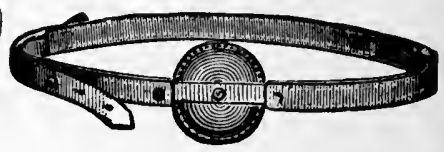

Truss for umbilical bernia.

femoral hernia the truss-pressure should be directed backward against the femoral canal, and the pad should be large enough to make pressure upon the adjacent tissues through which the hernia passes, as well as upon the relaxed tissues covering the femoral canal. As in inguinal hernia, either a single or a double spring truss may be employed (Fig. 176)

In applying a truss for femoral hernia, care should be taken to see that the pad does not rest upon the pubes, 
and thus remove the pressure from the crural ring and adjacent tissues and prevent the proper control of the hernia.

Trusses for Umbilical Hernia.-In measuring a patient for this variety of truss, the circumference of the body over the umbilicus shoul, be taken. In reducible umbilical hernia the truss-pressure should be directed backward, and the pad should bear rather on the tendinous margins of the ring than on the hernial opening. A truss for this variety of hernia should have a flat or slightly convex pad, which is held in position over the umbilical ring by means of springs having counter-pads on either side of the spine attached to their extremities; these are fastened together by a strap (Fig. 177).

A simple and satisfactory truss for umbilical hernia in infants consists of a penny covered by adhesive plaster, or a small flat compress of linen, held over the umbilical ring by one or two strips of adhesive or rubber plaster about two inches in width, or by a broad strip of perforated rubber adhesive plaster, which should be applied so as to cover in about the anterior two-thirds of the circumference of the body. A penny, or a small flat compress of linen, will be found much more satisfactory than the conical rubber or cork pad often recommended.

Trusses for Irreducible Hernia.-The application of a truss to this variety of hernia protects it from injury and prevents its further protrusion. Such trusses are secured in the same way as those for reducible hernia, but the pads are made concave or cup-shaped, or may have an air-cushion or water-cushion attached to the pad.

\section{CATHETERS AND BOUGIES.}

Catheters are hollow tubes, made either of metal, Indiarubber, or other flexible substances.

Sterilization of Catheters and Bougies.-To avoid infection of the urethra and bladder, it is important that catheters and bougies should be sterilized thoroughly before being introduced (see page 345 ). 
Infection of the bladder may occur from matter contained in the urethra, so that this canal should also be sterilized (see page 341 ). If it is possible, the patient should pass the urine to wash out the urethra, and a solution of boric acid or a borosalicylic solution should be injected before the instrument is passed.

To lubricate the instrument, sterilized liquid vaseline, olive oil, glycerin, or lubrichondrin should be employed.

Metallic Catheters.-These are made of silver, or, if constructed of other metals, they should be plated with silver or nickel, to give them a smooth, bright surface which can easily be kept perfectly clean ; and their shape should conform to that of the normal urethra (Fig. 178). The shape of the metallic catheter is sometimes changed to meet certain indications ; for instance, for use in cases of enlarged prostate it is longer and has a larger curve than the ordinary instrument (Fig. 179). The metallic female catheter is shorter and has a much smaller curve than the instrument used for the male urethra. A female catheter made of glass is now frequently employed, and has the advantage of easy sterilization.

Flexible Catheters. - The most commonly used variety of flexible catheter is that known as the English catheter, which is made of linen and shellac, and is provided with a stylet; it can be moulded into any shape desired by dipping it into hot water, which renders it flexible, and, after moulding it to the proper curve, this can be fixed by immersing it in cold water, which hardens it again.

The French flexible catheter is made of India-rubber, or a combination of this material with other substances. These instruments are conical toward their extremities, and terminate in an olive-shaped point; they are provided with one or two smoothly finished eyes near the vesical extremity (Fig. 180).

Another form of flexible catheter, known as the elbowed catheter, or Mercier's catheter (Fig. 181), has an angle or elbow near its vesical extremity; this is often found a satisfactory instrument to use in cases of enlarged 
prostate. A variety of flexible catheter made of soft Indiarubber is also sometimes employed (Fig. 182).

Catheters and bongies are made according to a certain

FIG. 178.

Fig. 179.

FIG. 180.

Fig. 181.

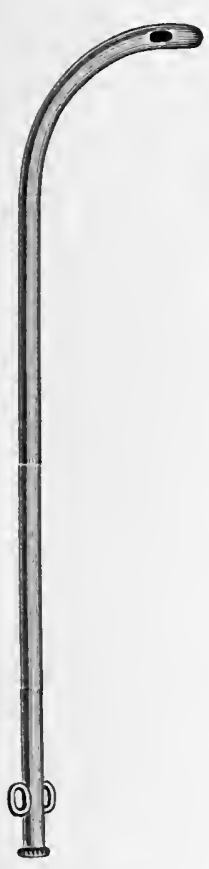

Metallic catheter.

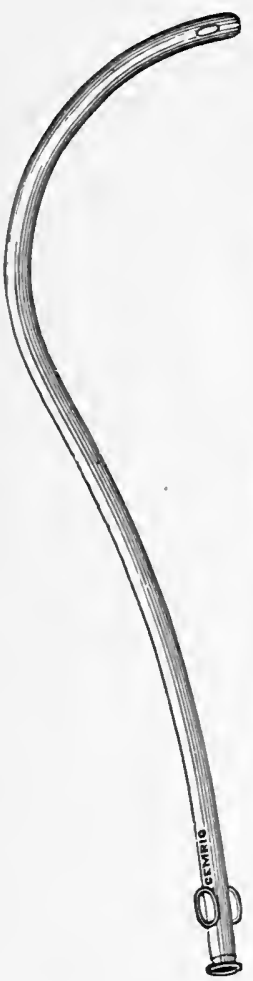

Prostatic catheter.

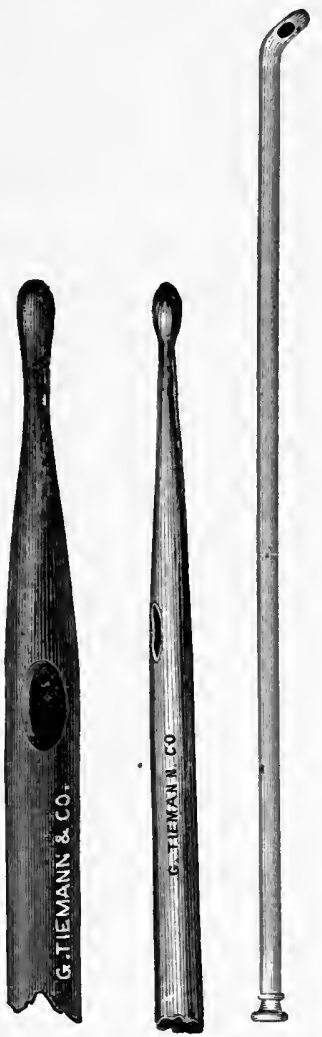

$\begin{array}{cc}\begin{array}{c}\text { French flexible } \\ \text { catheters. }\end{array} & \begin{array}{c}\text { Mercier's } \\ \text { elbowed }\end{array}\end{array}$ catheter.

scale. The English scale runs from No. 1 to No. 12 ; the American, from No. 1 to No. 20; and the French, from No. 1 to No. 40. 
Bougies and Sounds.-Bougies.-These are flexible instruments which rorrespond in size and shape to the Euglish and French eatheters; and besides there are the acorn-pointed bougie (Fig. 183) and the filiform bougie, which latter is made of whalebone or of the same material as the ordinary French bougie and eatheter. These instruments are of very small Fir. 182.

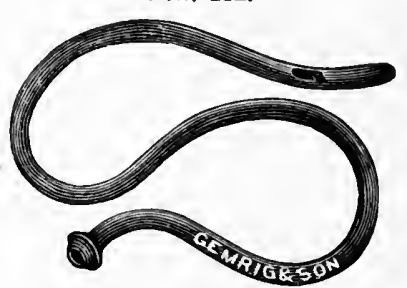

diameter, and may often be passed through strictures which will admit no other form of instrument (Fig. 184).

Sounds. - These are solid instruments, usually of steel, with a smooth surface and plated with nickel ; they correSoft-rubber catheter. spond in size and have the same eurve as the metallic catheter; the handle is flattened, to allow the operator to grasp them firmly; they are employed in the treatment of strictures by dilatation (Fig. 185). The sound used in dilating strietures of the meatus is straight, and is shorter than the sound employed in the treatment of urethral strictures (Fig. 186). A metallic sound with a shorter curve than the ordinary sound is used for exploration of the bladder for ealculus or tumor.

Introduction of a Catheter.-For the introduction of a catheter, the patient may be in the standing, sitting. or recumbent posture-the latter is the best in most cases; he should rest squarely on his baek, and have the thighs a little flexed and separated.

Before passing a metallic catheter, the surgeon should see that it has been sterilized, and after warming and oiling it he stands upon the left side of the patient and grasps the penis with the left hand, and turns it over the pubis and introdnces the beak of the catheter into the meatus, and gently passes it along the urethra until its point passes beneath the symphysis pubis; at this point the handle is elevated and gently depressed between the thighs, when the beak will pass into the blarlder (Fig. 187). 
In passing a catheter in case of enlarged prostate, when the prostatic region is reached difficulty is sometimes experienced in the further passage of the instrument; this

Fig. 183.

Fra. 184.

FIa. 185.

FIG. 186.

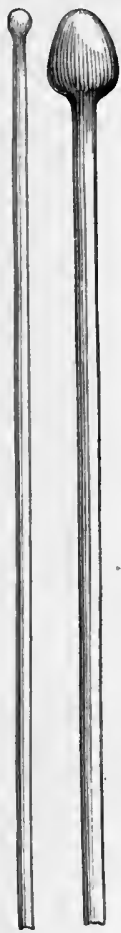

Bulbous or acornpointed bougies.

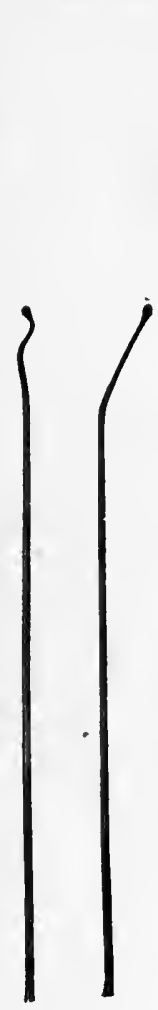

Filiform bougies.

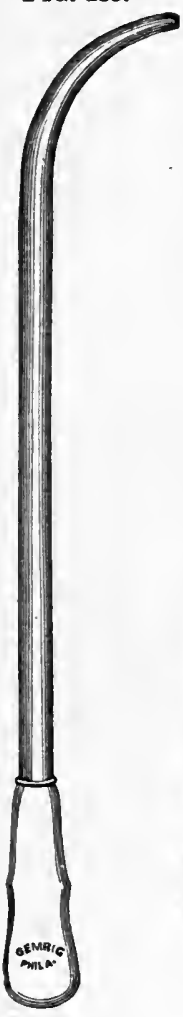

Steel sound.

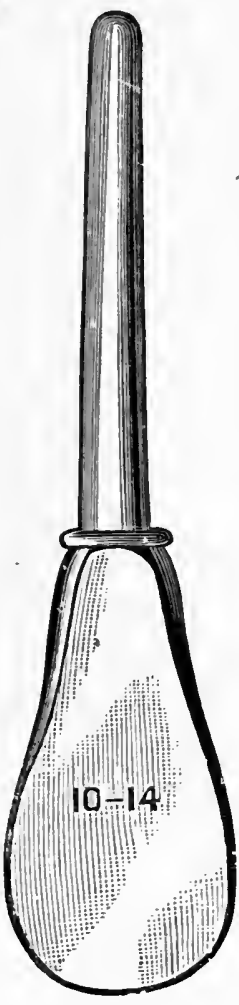

Sound for dilating meatus.

may be overcome by introducing the finger into the rectum and guiding the catheter through the prostatic urethra; or if the prostate is found much enlarged, the catheter should be withdrawn, and a prostatic catheter (Fig. 179) substi- 
tuted. The same manipulation is practised in passing metallic sounds.

Flexible catheters and bougies are passed by grasping the penis and holding it in such a position that it is at a right angle to the axis of the body, and the catheter or bougie is introduced into the meatus and conducted

FIt. 187.

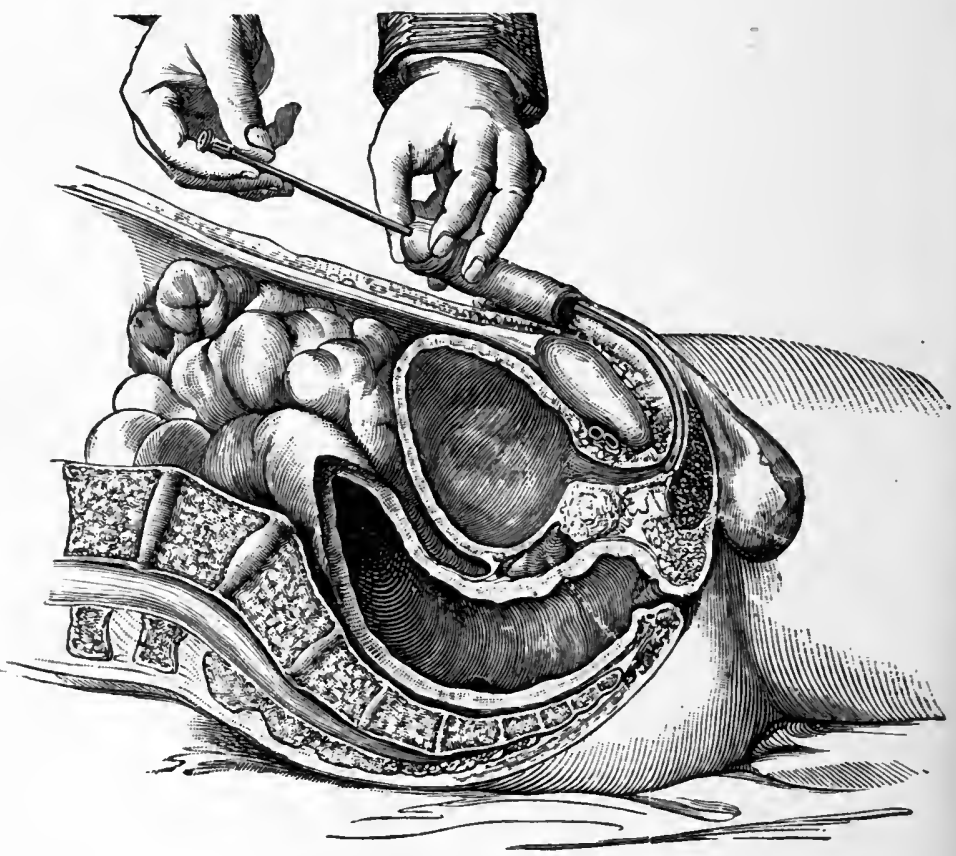

Introduction of a catherer. (VollLEMrier.)

through the urethra into the bladder by gently pushing the instrument downward. In this variety of instrument, which has no curve, the surgeon has no means of guiding the point of the instrument, and if an obstruction is met, he should withdraw the instrument slightly and make another attempt; all manipulations should be extremely gentle. 
Passing the Female Catheter.-It was formerly considered important to pass the female catheter without exposing the patient. At the present time it is rarely done, as it is considered more important to sterilize the vulva and region of the orifice of the urethra to avoid infection of the bladder. After washing the vulva with soap and water, and irrigating it with boric solution or normal salt solution, the orifice of the urethra is exposed, by separating the nymphæ, and the catheter is introduced into the bladder.

Catheterization of the Ureters.-In performing this operation in the female by the direct or Kelly's method, the patient is placed in the dorsal position with the pelvis elevated or in the genu-pectoral position, the external meatus is anæstletized by cocaine, and the urethra is dilated to admit a cylindrical speculum 12 to 15 millimetres in diameter. With the aid of a head-mirror the interior of the bladder can be directly inspected. The opening of the ureter may be exposed by turning the speculum thirty degrees to one side, and is recogniized as a small depression, the mucous membrane being of a darker color than elsewhere. A delicate elastic or silver catheter can be introduced into this opening, and by careful manipulation may be passed to the pelvis of the kidney. By this procedure, unilateral or bilateral disease of the kidneys may be clearly demonstrated, as well as the condition of the ureters themselves. Delicate bougies passed into the ureters may be used to locate their position in the operation of hysterectomy.

Catheterization of the male ureters can also be practised, but is much more difficult than in the female. The bladder should be moderately distended with sterile water, and a specially devised cystoscope is required, which carries the ureteral catheters or bougies. When the orifices of the ureters are located, the bougies or catheters are introduced into them and the cystocope is removed.

Segregation of the Urine.-To obtain the urine escaping from each ureter, so that the condition of each kidney may be ascertained, an instrument devised by Harris, 
of Chicago, may be employed. The device is known as a segregator, which separates the bladder into two compartments by a lever in the vagina or rectum, the urine being drawn from each compartment by a small catheter.

Tying the Male Catheter in the Bladder.-When it is desirable to retain a catheter for some time in the male bladder, it is necessary to secure it, to prevent its slipping out. Either a metallic or flexible catheter may be employed; but, as a rule, the flexible instrument is the most comfortable to the patient, and is to be preferred; there are several methods of securing it in the bladder.

By one method, two narrow strips of tape or two or three strong silk ligatures are attached to the rings at the end of a metallic catheter, or are securely fastened around the end of the flexible instrument; these are next brought backward, one on each side of the penis, and the skin is drawn forward and a strip of adhesive plaster half an inch in willth is passed over the strings or tapes and carried three or four times around the body of the penis just behind the glans. If the skin has been brought well forward before the strips have been applied, the ligatures are tightened

Fig. 188.

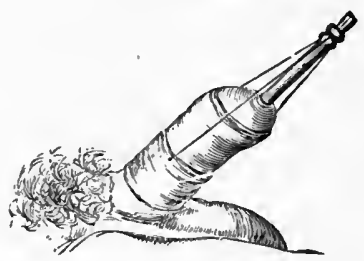

Tying in catheter. (BRYANT.) as it slips back, and the catheter has not too much play (Fig. 188).

Another method consists in fastening a strong silk ligature around the catheter just in advance of the meatus; the two ends are next brought backward and tied in a knot behind the corona glandis; the ends are then carried around the penis behind the corona and tied on one side of the frænum; the foreskin is slipped forward and covers the ligatures.

A catheter may also be secured in the bladder by tying the ends of the silk ligatures, which are attached to the instrument in advance of the meatus, to tufts of pubic hair.

Another method of securing the eatheter is to perforate the free end with a needle armed with a double ligature of silk or hemp; the needle being removed, two loops are 
made of the proper length, and these are passed through the ends of a T-bandage, which is secured around the waist, the tails being brought up on either side of the scrotum and secured to the body of the bandage passing around the waist.

In the female, when it is desirable to keep the bladder empty, the self-retaining catheter is usually employed, which eonsists of a catheter with a bulb at its vesical extremity, or an ordinary catheter with silk loops, and a T-bandage may be employed in the same manner as in securing a male catheter.

Irrigation of the Bladder.-This procedure may be required in the treatment of cystitis or in sterilizing the bladder, and is accomplished by passing a flexible catheter with a large eye into the bladder, or a double or two-way catheter may be employed. A syringe, or, better, a rubber bulb holding about a pint, having a nozzle and stopcock (Fig. 189), is filled with warm water, or with any medicated solution which is desired, and it is then attached to the free end of the catheter and the contents are gently injected into the bladder; care should be taken that the bladder is not too much distended. A small metallic or glass funnel with a rubber tube attached, which is connected with the catheter in the bladder may also be used to irrigate the bladder. When the desired amount of fluid has been injected, it is al-

FIG. 189.

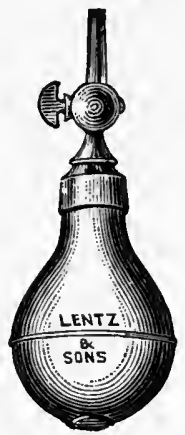

Rubber bag with stopcock, for irriga. tion of the bladder.

lowed to run out of the catheter, and the procedure may be repeated until the solution comes away perfectly clear.

The bladder may also be irrigated without using a catheter, the resistance of the compressor muscle of the urethra being overcome by the pressure of a column of water. The patient sits in a chair and a rubber or glass nozzle with a large bulbous tip, which closely fits the meatus, is inserted into it; the nozzle is connected by a rubber tube with a reservoir containing the fluid for irrigation. The 
reservoir is raised to a height of three to six feet above the patient. He is directed to take deep inspirations, and soon the bladder becomes filled with water, when the nozzle is removed, and the patient empties the bladder naturally. In some cases a little time is required before the column of water overcomes the resistance of the compressor muscle, or its entrance into the bladder may be hastened by directing the patient to attempt to urinate.

Care should be taken to see that the bladder is perfectly emptied of the solution, and in cases of paralysis of the viscus gentle pressure should be made upon the abdomen over the pubis to accomplish this object. Solutions of borie acid and permanganate of potassium, and weak solutions of carbolic acid and of nitrate of silver or argyrol are often employed in washing out the bladder in cystitis.

Urethral Injections. - In the treatment of urethral infiammations, the injection of medicated solutions is gen-

FIG. 190.

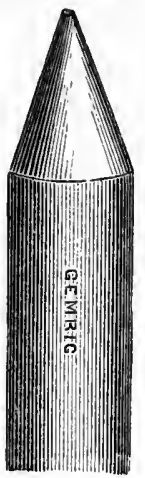
erally made use of ; and as these injections are usually made by the patient himself, he should be shown or instructed how to employ them. A rubber syringe having a conical nozzle, and holding about two or three drachms, is the best instrument to employ for this purpose (Fig. 190.) The s,ringe having been filled with the solution, the patient sits upon the edge of a hard chair, with the thighs separated, grasps the syringe between the thumb and middle finger of the right hand, the tip of the index finger resting upon the end of the piston, and inserts its conical end from a quarter to half an inch within the meatus, Shape of nozzle of urethral syringe.

which is held open by the thumb and finger of the left hand. After the introduction of the nozzle of the syringe the tissues should be pressed tightly around it, the pressure being made laterally, so as to narrow the urethral opening instead of broadening it, as is the case when compression is made in an antero-posterior direction. After the fluid has been thrown into the urethra in this manner, the syringe is removed, and the 
patient is instructed to hold the lips of the meatus together for one or two minutes, to prevent escape of the fluid.

Urethral irrigation may also be practised by means of gravity, a short rubber or glass tube, or a glass urethral nozzle being connected by a rubber tube with a reservoir containing the fluid to be used, the reservoir being placed slightly above the patient.

\section{SUTURES.}

A variety of materials are employed for sutures, such as silk, catgut, silver or iron wire, silkworm-gut, kangaroo-tail tendon, and horsehair. The materials most frequently employed at the present time are either catgut, silk, or silkworm-gut, although some surgcons prefer silver wire. Catgut and kangaroo-tail tendon are practically the only substances employed which are absorbable; the other varieties of suture require removal after their application, although some sutures, such as the silk, if absolutely sterile, when buried in wounds may be cut short, as they are apt to become encysted and remain indefinitely in the tissues. It matters little what variety of material be employed for suturing if the surgeon is careful to see that it is rendered thoroughly aseptic before being brought in contact with the wound.

Sutures of Relaxation.-These sutures are entered and brought out at some distance from the edges of the wound, and are employed to prevent dangerous tension upon the sutures which approximate the edges of the skin. This form of suture is employed in the quilled, button, or plate suture.

Sutures of Coaptation.-These are superficial sutures applied closely together, and include only the skin; they are employed to secure accurate apposition of the cutaneous surface of wounds.

Sutures of Approximation.-These sutures are applied deeply into the tissue to secure approximation of the deep portions of a wound this object is accomplished by the use of the quilled, buried, button, or plate suture.

Secondary Sutures. - These sutures are applied when the 
surfaces of the wounds are covered by granulations, when the primary sutures have failed to secure apposition of the edges of the wound, in cases of secondary hemorrhage where the opening of the wound has been necessitated to turn out the blood-clot and secure the bleeding vessel, and in plastic operations where the primary sutures have failed to secure adhesions of the edges of the flaps. They are also employed with advantage in closing wounds in cases in which it was necessary to pack the wound with antiseptic gauze, or to allow hremostatic forceps "to remain elamped upon bleeding tissues in the wound at the time of operation. The sutures should in such a case be introduced and loosely tied at this time, and when the packing or forceps is removed at the end of two or three days the sutures are tightened so as to secure apposition of the edges of the wound.

Surgical Needles.-Needles for surgical use are of different sizes and shapes (Fig. 191); straight needles are the oncs eommonly employed, but curved need-

FIG. 191.

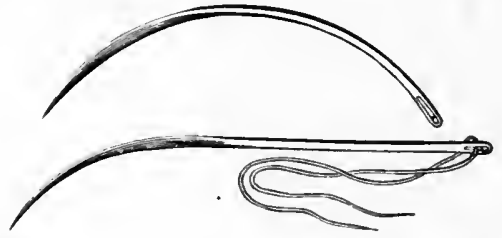

Surgical needles. les will be found most convenient for the introduction of sutures in wounds in certain locations. Hagedorn needles, which are flat and have sharp eutting edges, make a narrow linear wound in the tissues, and are useful in some cases. For the introduetion of sutures in the intestines or hollow viscera, the ordinary sewing-needle is generally employed, as it does not eut the tissues, but merely separates them, and its puncture is not likely to bleed. Tubular needles, are often employed in introducing sutures in wounds in which the use of an ordinary needle is difficult: for instance, in the operation for eleft palate, and for the introduction of sutures in deep wounds, a mounted needle will often be found very useful (Fig. 192). Reverdin's 
needle, which consists of a handled needle with an eye which is closed with a slide, is useful in passing deep sutures. The needle is first passed through the tissues, then threaded and withdrawn, carrying the suture with it. Needles should be sharp and clean, and should be rendered thoroughly aseptic before being used. Needles should be sterilized by boiling, and may be preserved in a saturated solution of carbonate of sodium or albolene to prevent rusting. A needle-holder is often required for

FIG. 192.

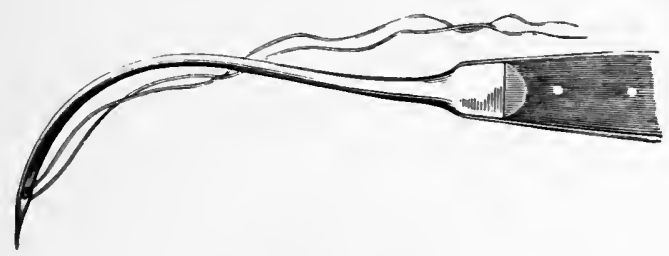

Mounted needle.

the satisfactory introduction of sutures in wounds in certain localities (Fig. 193); if this is not at hand, the needle may be held by a pair of dressing-forceps or a pair of hæmostatic forceps.

\section{FIG. 193.}

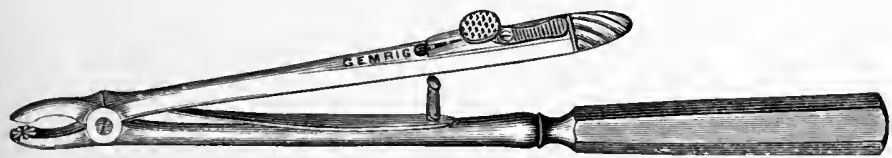

Needle-holder.

Method of Securing Sutures and Ligatures.Metallic sutures are usually secured by twisting the ends together or by passing the ends throngh a perforated shot and clamping the shot with a shot-compressor, which securely fixes them.

Sutures and ligatures of catgut, silk, silkworm-gut, kangaroo-tail tendon, or horsehair are secured by tying, and several different knots are employed to secure them. 
Reef or Flat Knot.-This is one of the best forms of knot to use in securing sutures or ligatures, and it is made by passing one end of the thread over and around

FIG. 194.

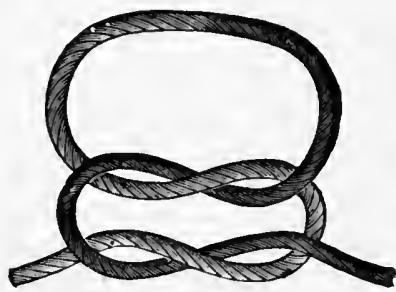

Reef or flat knot. the other end, and the knot this formed tightened; the ends of the thread are next carried toward each other and the same end is again carried over and around the other, and when the loop is drawn tight we have formed the reef or flat knot (Fig. 194).

Surgeon's Knot.- This knot is formed by carrying one end of the thread twice around the other end (Fig. 195); and after tightening this loop the same is carried over and.

FIG. 195.

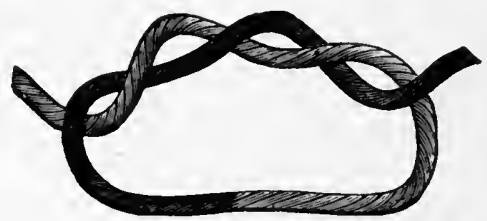

Surgeon's knot.

around the other end as in the case of the final knot of the reef or flat knot. The surgeon's knot and reef knot combined is one of the best methods of securing sutures or ligatures of catgut or silk, as the first knot is not apt to relax before the second knot is applied (Fig. 196).

Granny Knot.- This method of tying the ligature or suture should not be employed, as the resulting knot is not as secure as the reef knot and is apt to relax : it differs from the latter in the fact that one end of the thread having been carried across and around the other end, the knot is completed by carrying the same end under and around the other end of the thread (Fig. 197).

Staffordshire Knot.-This is much used to secure the pedicle in the removal of abdominal tumors, and is applied 
as follows: A handled needle armed with a stout silk ligature is passed through the pedicle, and then withdrawn so as to leave a loop on the distal side; this loop is drawn over the tumor, and one of the free ends is passed through it so that one end is above while the other end is below the retracted loop (Fig. 198). The ends are then seized and

FIG. 196.

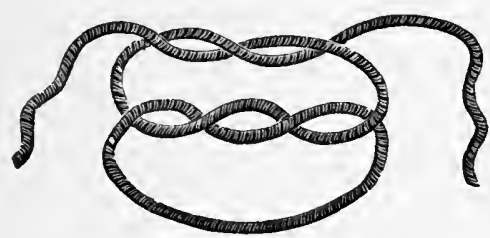

Surgeon's knot and reef knot combined.

drawn through the pedicle; at the same time the thumb and forefinger are pressed against it until sufficient constriction is made, and the ends are finally secured by tying as in the securing of an ordinary ligature.

FIG. 197.

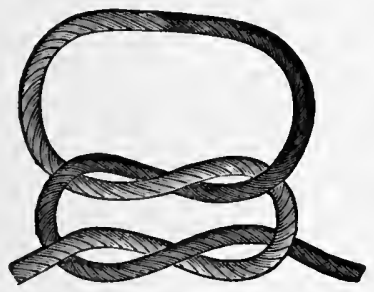

Granny knot.
Fig. 198.

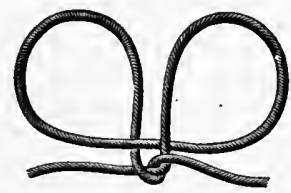

Staffordshire knot.

Varieties of Sutures.

The Interrupted Suture.-This variety of suture, which is the one most usually employed in the apposition of wounds, consists of a number of single stitches, each of which is entirely independent of those on either side. In applying this suture, the surgeon holds the edge 
of the wound with the fingers or forceps and thrusts the needle, previously threaded, through the skin three or four lines from the edge of the wound. $\mathrm{He}$ FIt. 199. then passes the needle from within outward

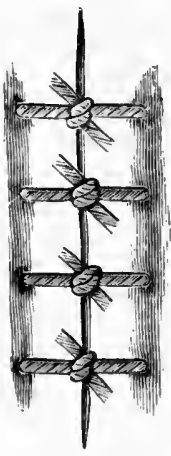

Interrupted su. ture. ('PARK.) through the tissues of the opposite flap at the same distance from the edge of the wound (Fig. 199). Each stitch is secured as soon as it is passed-by tying if a silk, catgut, or silkworm-gut suture be used, or by twisting if a silver wire suture is employed.

A suture may be used with a needle threaded on each end, in which case both needles are passed from within outward. The sutures may be secured as soon as applied, or they may be left unsecured until a sufficient number have been introduced, and then they may be secured by tying or twisting. Care should be taken to see that they make no tension on the edges of the wound, and that they are so introduced as to make the best possible apposition of the parts.

Buried Sutures.-In extensive and deep wounds it may be found necessary to introduce both deep and superficial sutures, the former bringing about apposition of the muscles and deep fascia, the superficial layer bringing together the superficial fascia and skin.

Deep or buried sutures are often employed to unite fascia, muscles, or tendons, and the best material for this variety of suture is either catgut, silk, silkworm-gut, or kangaroo-tail tendon.

Continued Suture.-This variety of suture is applied in the same manner as the interrupted suture, but the stitches are not cut apart and tied; it is made with silk or catgut, and is secured by drawing it double through the last stitch and using the free end to make a knot with the double portion attached to the needle (Fig. 200). This suture may be used in intestinal wounds, but may also be employed in obtaining apposition of the edges of wounds in tissues of loose strucure. 
Chain-Stitch Suture.-This is a variety of continued suture which differs from the ordinary continued suture,

FIG. 200.

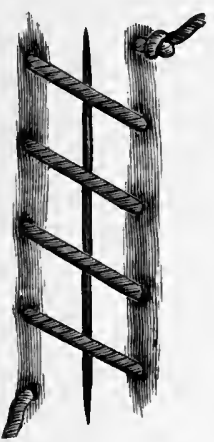

Continued or glovers' suture. (PARK.)
FIG. 201.

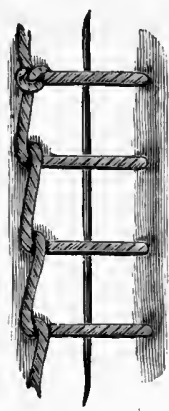

Chain-stitch suture. (BREwFr.)

in that the loop is made on one side of the wound as soon as the suture has been passed (Fig. 201).

FIf. 202.

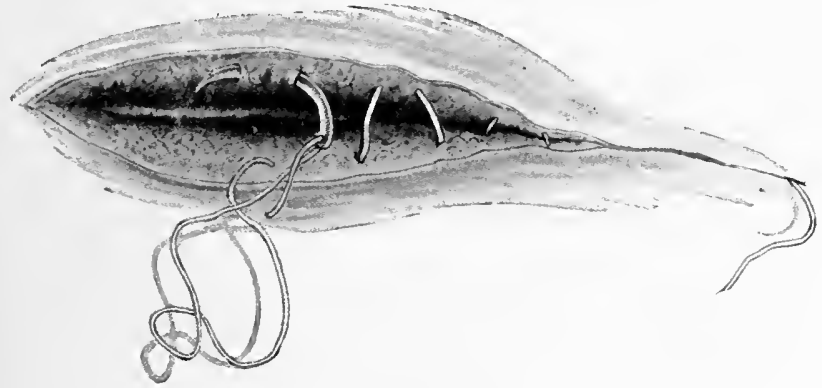

Subcuticular suture.

Subcuticular Suture.-Halsted has introduced a suture in which the needle is introduced on the under surface of 
the skin on one side, and brought out just beneath the cut edge; it is then entered in the reverse direction below the epidermic surface opposite; when tied, it will lie wholly out of sight. The object of this variety of suture is to avoid infection of the wound by the skin coccus, which may be introduced by the suture if passed from without inward. Fine silk or catgut should be used for this variety of suture, which may become encysted, absorbed, or gradually cast off after a few weeks. If employed as a continuous suture, the free ends may be tied together, and the suture subsequently removed by cutting the loop and drawing out the suture from one end of the wound (Fig. 202).

The Twisted or Hare-lip Suture.-This is a very. useful form of suture where great accuracy and firmness of apposition of the edges of the wound Fig. 203. are desired. It is applied by thrusting

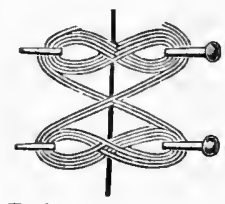

Twisted or hare-lip suture. pins or needles deeply through both lips of the wound, the edges being kept in contact over the wound by figure-of-eight turns with silk or wire (Fig. 203). The ends of the pins should be cut off with pincutters after the sutures are applied, or should be protected by pieces of cork or plaster to prevent them from injuring the skin of the patient and causing him pain. The twisted or hare-lip suture is frequently employed in plastic operations about the face and in other parts of the body where accurate apposition of the flaps is important.

Mattress or Quilt Suture.-This suture is applied by carrying the needle through the two flaps and then back again, so that a loop is left on one side and the two ends of the suture project from the opposite flap (Fig. 204). This variety of suture may be applied as an interrupted or as a continuous suture; in the latter, loops are made through the flaps on each side of the wound.

Continuous Mattress Suture.-This variety of suture, known as the Cushing suture, is often employed as an intestinal suture, but does not result in as secure or close 
apposition of the edges of the wound as the interrupted mattress suture. It is applied as shown in Fig. 205.

Fig. 204.

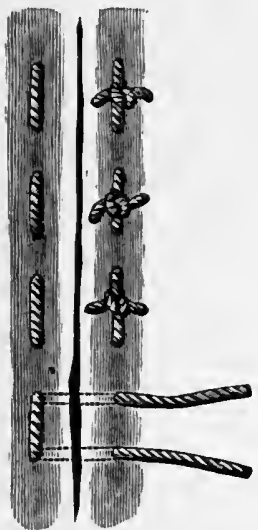

Fig, 205.

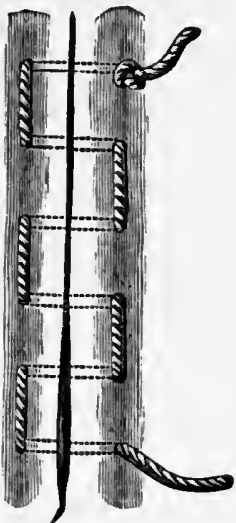

Mattress suture, interrupted. (Brewer.) Cushing suture. (BREwer.)

The Quilled Suture.-In making use of this suture, a needle armed with a double thread of wire or silk is

FIG. 206.
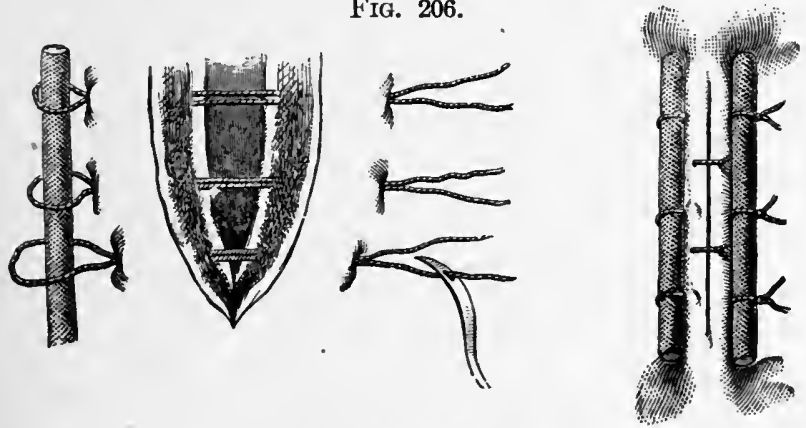

The quilled suture. (SMirH.)

passed through the tissues as in applying the interrupted suture, but at a greater distance from the edges of the 
wound. Into the loops on one side of the wound is inserted a quill or piece of a flexible eatheter or bougie, and on the opposite side the free ends of the sutures are tied around a similar object after being tightened (Fig. 206). This form of suture makes deep equable pressure along the whole line of the wound. In applying this suture, it may be found advisable in some eases to introduce a few superficial interrupted sutures along the line of the wound to

FIG. 207.

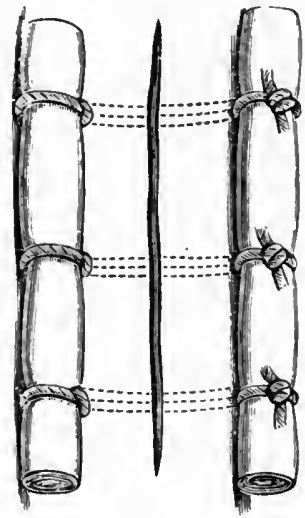

Modified quilfed suture.

(PARK.) secure accurate approximation of the skin. Two small rolls of sterilized or antiseptic ganze may be used as a substitute for the quills or pieces of catheter, as shown in Fig. 207).

Button or Plate Suture.-This suture is applied by passing a needle armed with a double thread as in the case of the quilled suture, the ends of the suture being passed through the eyes of a button or throngh perforations in a lead plate before being threaded in the eye of the needle. After the suture prepared in this way has been passed throngh both sides of the wound, the needle is removed and the free ends of the suture are passed through the eyes of a button or the perforations in a lead plate on the opposite side of the wound, and are tightened and secured (Fig. 208). In applying this forn of suture, small rolls of antiseptic ganze may be used instead of buttons, as shown in Fig. 209. This form of suture may be employed in deep wounds to accomplish the same purpose as the quilled suture. It allows the cutaneous margins of the wound to remain free from compression, and here, as in the ease of the quilled suture, a few interrupted sutures may be introduced between the button or plate sutures to secure aceurate apposition of the skin surfaces if desired.

Shotted Suture.-This suture receives its name not from any special method of application, but solely from 
the way in which it is secured; any of the previously mentioned varieties of sutures may be employed. The material used in applyiıg this suture may be eatgut, silver wire, silkworm-gut, silk, or horsehair, and after the suture has been passed the needle is removed, and the ends are passed through a perforated shot; the ends are then drawn upon to bring the edges of the wound in eontact, and the shot is pressed down to the skin and clamped by means of a shot-compressor. The suture is then cut off flush with the surface of the shot.

This method of securing sutures is especially useful in

FIG. 208.

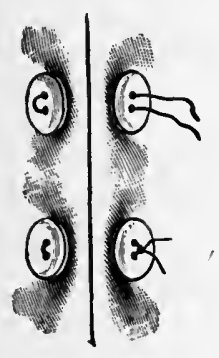

Button suture. (SмIтH.)
FIG. 209.

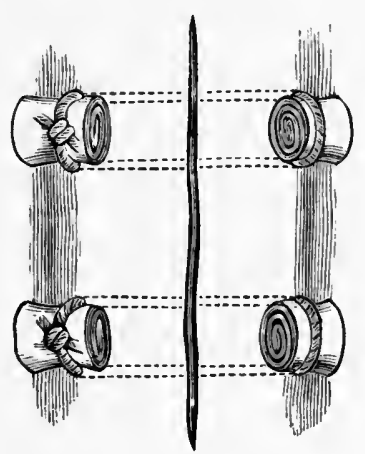

Modified plate suture, using gauze pledgets.

(PARK.)

closing wounds in the mucous cavities, such as the vagina, rectum, and mouth, where the knot or twist of the wire might cause irritation of the surface or pain to the patient; it is also a useful method of securing sutures in plastic operations; it also facilitates the removal of the sutures, as the shot is not apt to be obseured by the swollen tissue, and is easily seized by forceps when the loop is divided.

Removal of Sutures. - Where sutures are buried in the tissues or used to approxiniate parts in eavities which are subsequently closed, sueh materials should be used for sutures as will be absorbed in a few days, or will become encysted, and remain harmless in the tissues-such as cat- 
gut, silkworm-gut, or. silk-and it is needless to state that sutures used with this end in view should be rendered perfectly aseptic before being enployed.

Catgut sutures, when well prepared and used for sutures in external wounds, usually undergo absorption in from ten to fifteen days; the loop buried in the tissues is absorbed, and the knot may be removed from the surface with forceps or it may come off with the dressings.

The other substances, such as silk, silkworm-gut, silver wire, and horsehair, are removed by cutting one side of the loop and making traction upon the knot of the suture with forceps, or in the case of the wire suture, after dividing the loop and straightening out one end of it, the wire should be withdrawn in a curved direction.

Sutures which are not causing irritation should be allowed to remain until the wound is solidly healed. The time usually required for their return in case of aseptic wounds is from eight to twelve days.

\section{LIGATURES USED IN THE TREATMENT OF VAS- CULAR GROWTHS.}

Various forms of ligatures are used for the strangulation of vascular growths; the material employed is usually strong silk or hemp thread, catgut, or silver wire.

FIG. 210.

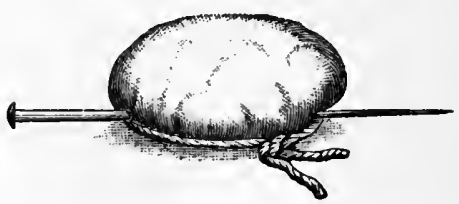

Vascular tumor strangulated with pin and ligature.

The Single Ligature with a Pin.-This is applied by first inserting a hair-lip pin through the skin near the edge of the growth, passing it under the growth and bringing it out through the skin at a point opposite the place 
of entry; a strong silk or hemp ligature is passed under the ends of the pin surrounding the base of the tumor, and is drawn tight enough to strangulate the growth, and is secured by two knots (Fig. 210). If the growth is of

FIG. 211.

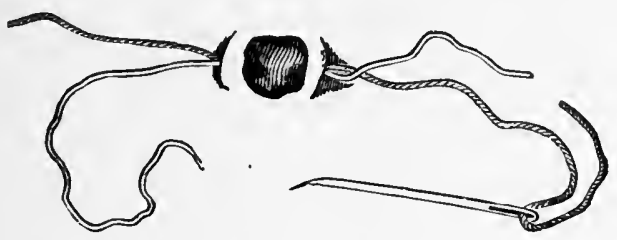

Method of applying double ligature. (RoBerts.)

considerable size, it is better before applying this ligature to introduce a second pin at right angles to the first one, and then secure the ligature under the pins. In applying these forms of ligature to healthy skin, the patient is saved much pain, and the separation of the mass is hastened, by cutting a groove in the skin with a sharp knife at the point where the ligature is to be applied; the ligature when tied is buried in the groove thus made.

Double Ligature-This ligature is applied by passing a needle or a needle with a handle, armed with a double ligature, through the skin near the growth, and then passing it under the tumor and bringing it out through

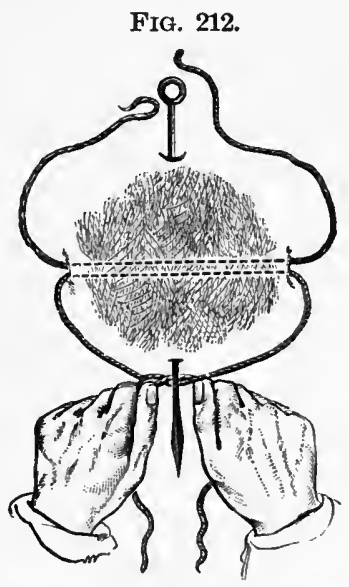

Method of applying double liga. ture and pin. (BRYANT.) the skin at a point directly opposite the point of insertion; the ligature is then divided and the needle 
removed. The tumor is strangulated by tying firmly the corresponding ends of the ligature on each side of the tumor, each ligature including one-half of the growth (Fig. 211).

The double ligature may also be applied by first passing a pin under the growth and then passing a needle armed with a double thread under the tumor at right angles to the pin, and after removing the needle the ends of the ligature are tied and the tumor is strangulated in iwo sections (Fig. 212).

Quadruple Ligature.-In applying this ligature, two needles carrying a double thread are passed under the growth at right angles to each other; or if the handled needles be used, they may first be passed in this manner, and then threaded with double ligatures, which are carried under the growth as they are withdrawn. The needles being removed, the surgeon ties two ends of the ligature together, and repeats this procedure until the growth has been strangulated in four sections.

Subcutaneous Ligature.-This is applied by introducing a needle armed with a ligature through the skin near the growth, and carrying it through the subcutaneous

FIt. 213.

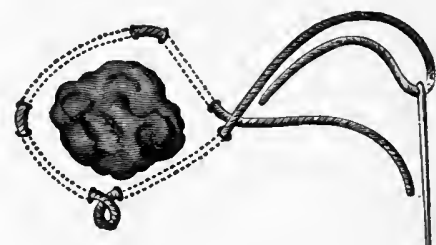

Method of applying subeutaneous ligature. (HoLMEs.)

tissues around the part to be constricted for a short distance, then bringing it ont through the skin. The needle is again introduced through the same puncture, and is again brought 
out through the skin at some distance from the first point of exit. It is next introduced through this puncture and brought out at a more distant point. In this way the growth is completely encircled by a subcutaneous ligature, which is finally brought out at the point of entrance; the tumor is strangulated by firmly tying together the ends of the ligature (Fig. 213).

If a needle armed with a double ligature is first passed under the growth, the ligature is divided, and by passing each end of the divided ligature subentaneously around the growth it may be strangulated subeutaneously in two scetions.

Elastic Ligatures.-Ligatures made of India-rubber varying from half a line to several lines in thickness are often made use of in surgery. They may be employed to strangulate growths such as moles or nævi, or in the treatment of fistulæ, and are especially useful in the treatment of those cases of fistula in ano in which the internal opening into the bowel is situated high up, as the division of such fistulæ by this means is accomplished without hemorrliage and with less risk than by the employment of the knife. In applying elastic ligatures in such cases, the ligature, after being passed through the fistula by means of a probe, is carried out through the internal opening; the sphineter is next well stretched, and the elastic ligature is then firmly tied with two or three knots; the greater the tension made before the ligature is tied the nore rapidly will it cut its way ont. The smaller sizes of rubber drainage-tubes muy be substituted for the solid rubber ligatures. 


\section{TREATMENT OF HEMORRHAGE.}

The surgeon may be called upon to treat the following varieties of hemorrhage: arterial, venous, or capillary; and these again are classified according to the time of their occurrence, as primary-that is, bleeding which oceurs at the time the wound is inflicted; intermediary or consecutive, that which occurs within twenty-four or fortyeight hours after the reception of the injury, and which generally takes place during the period of reaction; and secondary, which usually results from a septic condition of the wound, eausing a septic arteritis, and oceurs usually after forty-eight liours, but may occur at any time subsequent to this period until the wound is healed. "The treatment of hemorrhage is both constitutional and local.

Constitutional Treatment.- This consists in keeping the patient in the recumbent posture and avoiding any sudden clevation of the head or arms which might induce fatal syncope. Opium is a valuable remedy and should be freely used. Ergot, gallic acid, acetate of lead, and tincture of iron may also be employed, and stimulants and food should be carefully administered; in extreme cases the intravenous injection or infusion of normal salt solution should be resorted to. The hæmostatic properties of gelatin have led to its use by subcutaneous injection in varions forms of internal hemorrhage. A sterilized aqueous solution, containing 2 per cent. of gelatin in normal salt solution, is injected into the loose cellular tissue of the abdominal walls or buttock, about 200 c.c. being employed. It has been used in hæmoptysis, epistaxis, and in intestinal hemorrhage in typhoid fever.

Local Treatment.-This consists in the adoption of various local measures to control the bleeding, which may be either temporary or permanent in their action. 


\section{Temporary Control of Arterial Hemorrhage.}

This may be effected by pressure applied directly to the bleeding vessel in the wound or by pressure applied indirectly to the main artery between the point of its injury and the centre of the circulation, and this pressure may be made by the fingers-digital compression-by compresses, or by means of tourniquets.

Digital Compression.-This constitutes one of the most valuable means employed in the temporary control of hemorrhage: the finger is pressed directly upon the

FIG. 214.

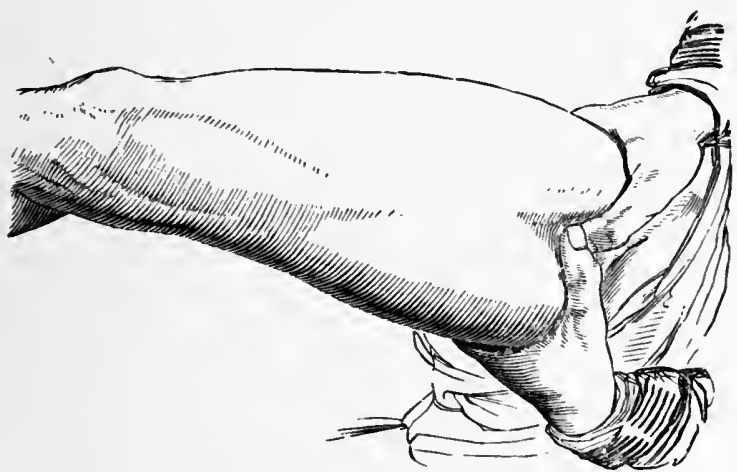

Digital compression of the femoral artery.

bleeding vessel, in the wound, or is used to make pressure upon the artery from which the bleeding arises at some point between the wound and the centre of the circulation (Fig. 214). Control of hemorrhage by digital pressure can be maintained only for a few minutes, for the fingers of the surgeon or assistant soon become tired, so that it is employed only until means are adopted for permanent arrest of the bleeding. Digital compression of the radial and ulnar arteries may be resorted to for the control of hemorrhage during amputations of the fingers, of the axillary and femoral arteries in amputations at the shoulder-joint and the hip-joint. It is also used to control 
hemorrhage from wounds either the result of accident or those made by the knife of the surgeon, in which case the finger is placed directly upon the divided ressel or is employed to hold a sponge or compress firmly in the wound.

Compresses.-By the use of compresses placed directly in the wound or applied to the vessel between the wound and the centre of the circulation, the temporary control of hemorrhage may be very satisfactorily accomplished. The compress which is applied in the wound should be made of antiseptic or aseptic gauze, thereby diminishing the chanees of wound-infection. The compress shonld be held in position by a bandage firmly applied, and is generally employed only as a temporary expedient until a more permanent means of controlling the bleeding is adopted.

FIG. 215.

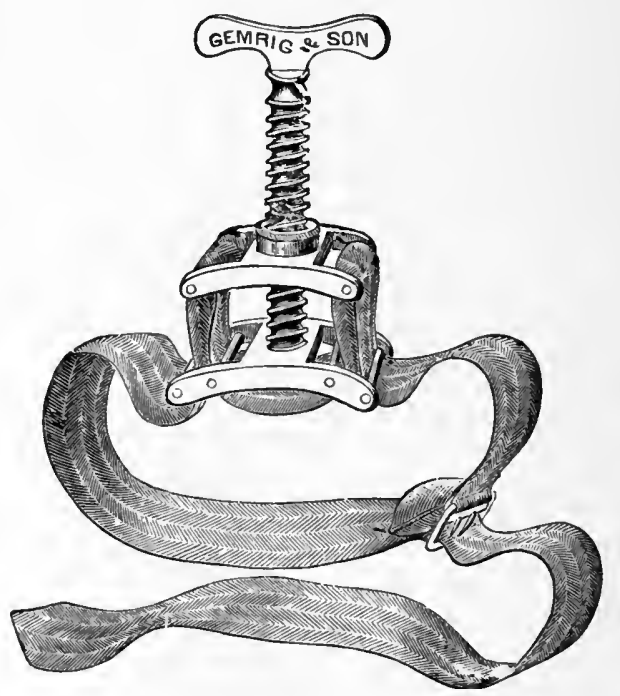

Petit's tourniquet.

Tourniquets.-These instruments, which are employed for the temporary control of hemorrhage from wounds, are of many different kinds. 
Petit's Tourniquet.-This consists of two metal plates connected by a strong linen or silk strap, with a buckle, the distance between the plates being regulated by a screw (Fig. 215). In applying this tourniquet, a compress or roller-bandage is placed directly over the artery to be compressed, and may be held in position by a few turns of the bandage. The lower plate of the tourniquet is placerl directly over this pad, and the strap is tightly secured around the limb to keep the instrument in place. The screw is then turned so as to separate the plates and tighten the strap, thus forcing the compress or pad upon the artery and controlling its circulation. This instrument is very generally employed for the control of hemorrhage in wounds of the extremities, and is especially useful in amputation of these parts, being placed over the main artery some distance above the seat of operation.

The Spanish Windlass.-An improvised tourniquet, known as the Spanish windlass, may be employed in eases of emergency; it is prepared by folding a handkerchief or piece of muslin into a cravat and placing a compress or smooth pebble on the body of the cravat; this is placed over the artery to be controlled, and the ends of the handkerchief are tied loosely around the limb; a short stick is passed through this loop, and by twisting the stick the loop is tightened and the compress is forced down upon the artery (Fig. 216).

Many other forms of tourniquet have been devised which have the pad and counter-pad arranged to make pressure upon the vessel, such as Lister's aorta compressor (Fig. 217 , which is employed in the treatment of aneurism of the iliac vessels and for the control of hemorrhage in amputation at the hip-joint. Signorini's tourniquet (Fig. 218) is constructed upon the same principle, and is frequently employed to eontrol the circulation in the femoral artery in cases of operations on the thigh and leg and in the treatment of femoral or popliteal aneurism.

Elastic Constriction.-The elastic tube, or the strap of Esmarch's apparatus (Fig. 219), may also be employed 
for the temporary control of arterial hemorrhage, being applied above the wound; and if it is not at hand, any

FIG. 216.

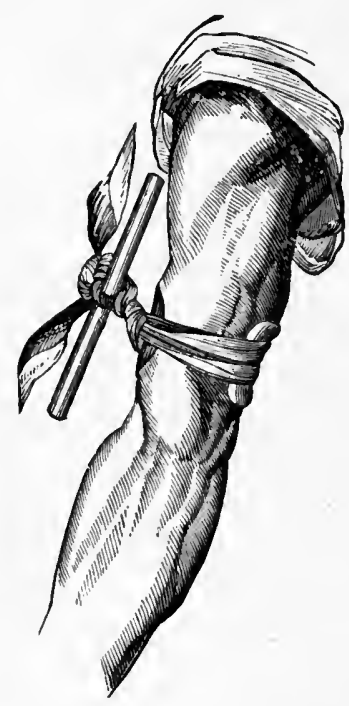

The Spanish windlass. strong rubber cord or a piece of large-sized drainage-tube may be used as a substitute. Elastic suspenders or garters may also be employed in an emergency. In hemorrhage from wounds of the hands and feet, especially in chil-

FIG. 217.

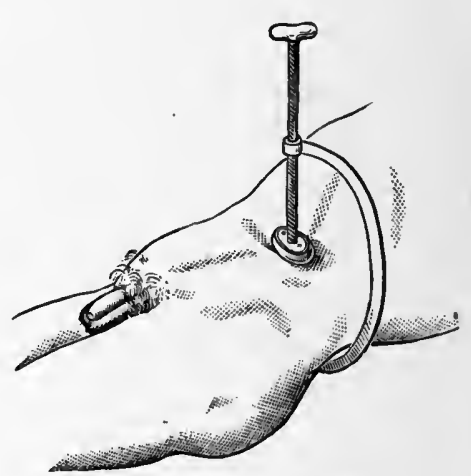

Lister's aorta compressor.

dren, and in controlling hemorrhage from wounds of the penis, a piece of drainage-tube, firmly applied above the wound, may be employed with advantage. Care should be observed in applying elastic constriction, for if the elastic tube be applied too tightly, the subcutaneous tissues may be divided or nerves may be so compressed that their function is destroyed. The tube or strap, although generally employed to control hemorrhage from vessels of the extremities, may be used to control the femoral artery as it crosses the brim of the pelvis, by placing a compress over the artery in this position, and then applying the elastic band to secure it by making a figure-of-eight turn, passing under the thigh, crossing over the pad, and 
then carrying the ends around the pelvis, and securing them.

To make pressure on the axillary artery, a compress should be placed in the axilla, and the middle of the tube

FIG. 218.

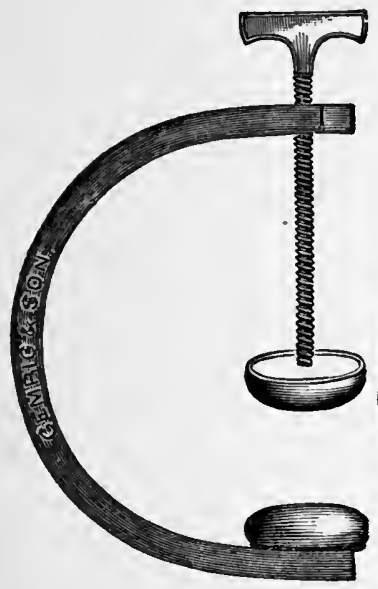

Signorini's tourniquet. placed over this to hold it in position ; the ends of the tube are then carried over the shoulder, where they are crossed, and then carried to the opposite axilla and secured.

FIG. 219.

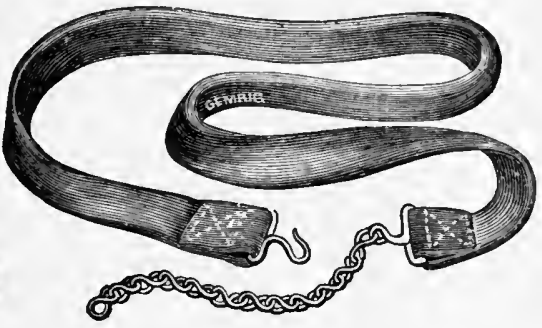

Elastic strap of Esmarch's apparatus.

Hæmostatic Forceps.-The temporary control of arterial hemorrhage by the use of hæmostatic forceps is now very generally employed in surgical operations, and their use has done much to diminish the shock following operations from the loss of blood. The hæmostatic forceps in general use is self-retaining ; it is clamped upon the bleeding vessel, and is allowed to remain until the operation is completed, when the vessel is secured permanently by the application of a ligature, and the forceps is removed. The use of these forceps will be found very satisfactory in controlling hemorrhage during the removal of tumors; in amputations, and for the temporary control of bleeding during the operation of tracheotomy, they will be found most efficient, as also in abdominal operations, in which their utility was first demonstrated (Fig. 220).

\section{Esmarch's Bandage and Tube.-This apparatus,}


which is applied to the limbs to render them blood-

FIG. 220.
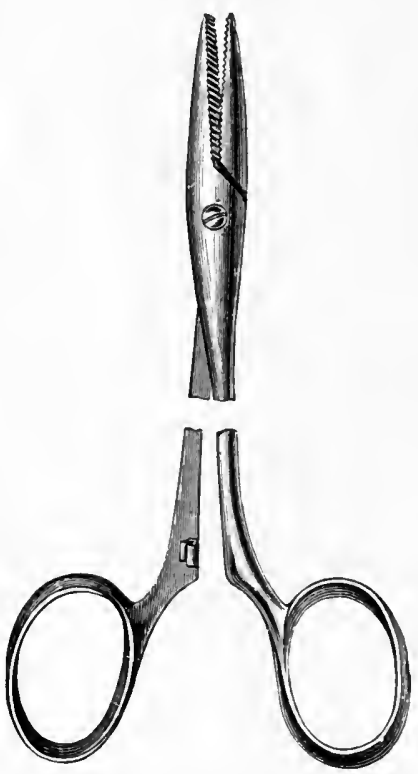

Hæmostatic forceps. less during operations, consists of a rubber bandage two and a half inehes in width and three or four yards in length, and a rubber tube two yards in length, to one end of which is attached a chain and to the other a hook, or, better, a rubber strap, one inch in width and one and a half yards in length, with a hook and chain. The bandage is applied to the extremity of the limb, and is earried up the limb to a point some distance above the seat of proposed operation; the bandage is applied firmly, each turn overlapping onefourth of the preceding one, and when the last turn has been made the rubber tube or strap is wound firmly around the limb and secured

by fastening the hook into one of the links of the chain (Fig. 221). After securing the tube or strap, the rubber bandage is removed from the limb; and if the tube has been sufficiently firmly applied, the limb will be found blanehed, and should be free from blood during the operation. Care should be taken not to apply the tube or strap too tightly upon poorly developed limbs, or on parts of the limb where large nerve-trunks approach the surface, as they may be subjected to an amount of pressure which will interfere with their functions subsequently. I have knowledge of one case of this nature in which permanent paralysis of the limb followed the use of Esmarch's apparatus; the tube should be applied with 
just sufficient firmness to control the circulation. As the strap, when firmly applied, completely cuts off' the circulation of the parts below, it should be applied for as short a time as possible, as gangrene has resulted from its prolonged use. After removal of the tube or strap there is generally free capillary hemorrhage, due to paralysis of the vasomotor nerves from pressure, but this in a short time stops. This appliance is of the greatest service in

FIG. 221.

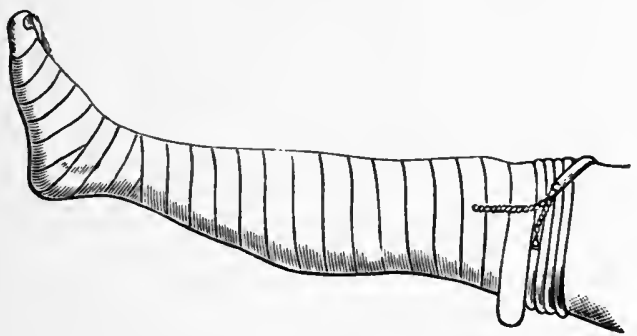

Esmarch's bandage and tube applied.

controlling hemorrhage at the time of operation, and in amputations and for removal of vascular tumors from the limbs will be found most satisfactory. In operations upon bones, such as resection or sequestrotomy, it is especially useful, as it allows the surgeon to inspect the parts unobscured by hemorrhage. I have found its use most satisfactory in operations for the removal of foreign bodies, such as needles embedded in extremities.

\section{Permanent Control of Arterial Hemorrhage.}

To secure this end, the surgeon may resort to the use of position, cold, heat, styptics, pressure, cauterization, ligation, torsion, suture of the artery, or acupressure.

Position.-In arterial hemorrhage from wounds of the extremities, elevation of the part will be found to materially diminish the amount of bleeding; in hemorrhage from wounds of the arteries of the hand, forearm, foot, or 
leg, forcible flexion of the forearm on the arm or of the leg on the thigh will be found useful in diminishing the force of the blood-current.

Cold.-The application of cold by means of a stream of cold water or an ice-bag or pieces of ice will often be found an efficient means of controlling hemorrhage from vessels of small calibre ; it is especially applicable to hemorrhage from wounds of the vessels of the mouth, nostrils, vagina, or rectum.

Hot Water.-Hot water will be found a very efficient means of controlling hemorrhage from small vessels, and it may be used in the form of a hot antiseptic solution. It is of especial value in capillary or parenchymatous hemorrhage, and is employed in the form of a douche or by means of sponges or gauze pads dipped in the hot solution and packed into the wound. The injection of hot water is a most satisfactory method of controlling uterine hemorrhage.

Styptics.-These agents are sometimes employed to control capillary bleeding or hemorrhage from small vessels, and althongh their use is often satisfactory as regards the control of the bleeding, they have the disadvantage of interfering with primary union in wounds, and since the value of asepsis in wound treatment has been demonstrated they are now very seldom employed. The most valuable styptics are alcohol, alum, oil of turpentine, perchloride of iron, persulphate of iron or Monsel's solution, acetic acid, vinegar, adrenalin chloride, antipyrin, and gelatin.

Adrenalin Chloride.-A solution of adrenalin chloride, in normal salt solution 1-1000 to 1-10,000, has been recently employed for the control of hemorrhage. It seems to be most serviceable in capillary hemorrhage. Adrenalin extract, in the form of powder, may also be dusted upon a bleeding surface to secure hæmostasis.

Antipyrin.-A solution of antipyrin, 5 per cent., in sterilized water possesses marked styptic action. As it also possesses antiseptic properties and is not toxic, it may be used to control capillary bleeding from the surface of the brain, the intestines and peritoneum, and from the bone-cavities.

Gelatin.-This may be used as a styptic where it can 
be applied locally in a 5 to 10 per cent. solution in normal salt solution. It may be applied by injecting, irrigating, or tamponing the bleeding area. It has been employed successfully in epistaxis, hæmatemesis, vesical and uterine hemorrhage, and in superficial wounds in patients the subjects of hæmophilia.

Pressure.-For the permanent control of arterial hemorrhage, pressure may be applied directly to the bleeding point or surface by means of a compress of antiseptic gauze or by strips of gauze packed firmly into the cavity from whose surface the bleeding arises.

Compresses are used with the best results where the proximity of a bone gives a firm substance upon which the vessel may be compressed, as is the case in the vessels of the scalp. Pressure applied by means of packing with strips of gauze will be found most efficient in controlling hemorrhage from cavities, such as the nose, vagina, or rectum, and in the cavities resulting from the removal of necrosed or carious bone. Pressure may be indirectly applied to an artery by flexing the joint over a compress or by firm bandaging of the limb.

In controlling bleeding from a divided artery in a bony cavity, such as the inferior dental, a piece of catgut ligature may be forced into the canal, and will control the bleeding in a most satisfactory manner, or it may be controlled by foreing a small piece of Horsley's wax into the opening in the bone; this wax is composed of wax, 7 parts ; oil, 2 parts; and carbolic acid, 1 part.

Halsted has introduced a material known as gut wool, which is prepared from the same material from which catgut is made. This is cut into fine shreds, and is used to control hemorrhage from bone, being pressed into the opening or eavity in the bone from which the bleeding arises.

The troublesome hemorrhage sometimes occurring after the removal of a tooth may be controlled by packing the alveolar cavity with a strip of iodoform ganze, or by introducing a wedge-shaped piece of cork and holding it in place by fastening the jaws together by means of a bandage. 
Cauterization.-The use of cauterization by means of a hot iron is a satisfactory method of arresting hemorrhage. Care should be taken to have the iron only of a dull-red or black heat, as the result desired is not the destruction of the tissues, but the coagulating effect of heat upon them. The form of cautery-iron employed will depend upon the size and position of the vessel. Paquelin's cautery is also a satisfactory apparatus to use for the control of hemorrhage.

The control of arterial bleeding by cauterization is often resorted to in operations upon the jaws and in the removal of tumors from the mouth or pharynx or of the tonsils; it is also frequently employed to control hemorrhage in operations upon the uterus and the rectum, and also that resulting from the removal of abdominal tumors, where the application of a ligature is difficult and of ten impossible.

Torsion.-This method of controlling arterial hemorrhage consists in seizing the end of the artery, drawing it slightly out of its sheath and twisting it ; it may be accomplished with a single pair of forceps or hæmostatic forceps, or by two pairs of forceps. In the latter method the vessel is held by one pair of forceps and is twisted by the second pair.

Torsion of arteries in accidental wounds is quite common, and in many cases controls the hemorrhage until surgical aid is rendered. I have seen hemorrhage from the femoral artery in Scarpa's triangle completely controlled in this manner in a case of avulsion of the thigh from a railway injury.

FIG. 222.

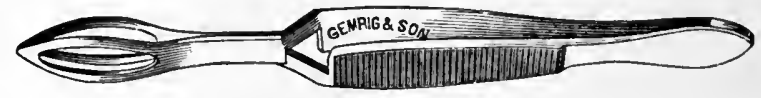

Double-spring artery forceps.

In vessels of moderate size it may be practised with one pair of forceps, and the ordinary double-spring artery forceps (Fig. 222) or hæmostatic forceps will be found satis- 
fictory for such eases. In larger arteries two forceps should be employed, or some of the numerous forms of torsion forceps which have been devised for this purpose.

The Ligature.-The use of the ligature is by far the most generally employed method of eontrolling arterial hemorrhage. The materials used are silk, hemp thread, or catgut. Catgut or silk is the material generally employed.

FIG. 223.

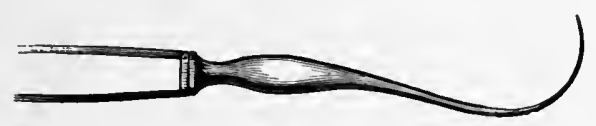

Tenaculum.

The vessel is seized with a pair of artery or hæmostatic forceps or a tenaculum (Fig. 223) and drawn out of its sheath, and a ligature of sterilized catgut or silk is thrown around it and secured by a surgeon's kuot, or by a reef knot and a surgeon's knot eombined, and when firmly tied the ends of the ligature are cut short in the wound.

FIG. 224.

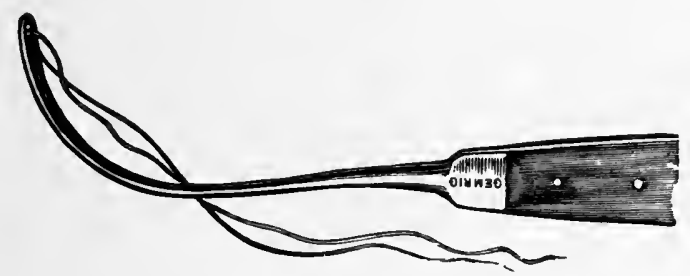

Aneurism needle armed with ligature.

When ligatures are applied to vessels in their continuity, they may be threaded into an eyed probe or aneurism needle (Fig. 224) and carried around the vessel and secured.

Temporary or Provisional Ligation of Arteries.This procedure is employed when it is desired to control for a time the arterial circulation during an operation, or as a precaution in case where free hemorrlage may occur.

The artery to be temporarily occluded is exposed by a 17 
careful dissection. The sheath is not opened and a flat ligature or tape is passed beneath the sheath and is closely tied or elamped with forceps. When it is desired to occlude the vessel, an assistant lifts the vessel from its bed by the ligature, which arrests the flow of blood through the vessel. When the neeessity for the control of the circulation has passed the ligature is removed and the wound is closed.

Special forceps or clamps with blades covered by rubber so that they cause no injury to the walls of the vessel may also be employed to secure temporary control of the circulation through the artery.

Ligature En Masse or Deep Suture.-A convenient method of applying a ligature to a bleeding point, or a limited area of hemorrhage in a deep wound, or to a vessel in tissues which are of such a nature as not to permit of the isolation of the vessel, is to use a curved needle threaded with a catgut ligature, which is passed deeply into the tissues

FIG. 225.

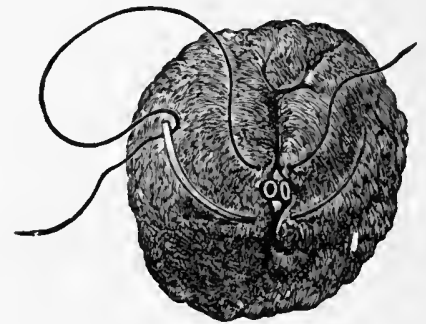

Artery occluded by suture. (EsMarCH.)

near the vessel and brought ont on the opposite side ; the ligature thus placed is then tied firmly enough to control the bleeding, but not so tight as to produce strangulation of the tissues; the ends are eut short in the wound (Fig. 225).

Arteriorraphy. Suture of Arteries.-Wounds of arteries, both longitudinal, oblique and transverse, have been successfully closed by sutures both in man and the lower animals. It is recommended in the larger arteries, where more than two-thirds of the eircumference has been di- 
vided, to resect the injured portion of the vessel, where it can be done without removing more than three-fourths of an inch of the vessel, and invaginate one end into the other, and to secure their fixation by fine silk or catgut sutures, which include all the coats of the vessel (Fig. 226). The distal end of the vessel is slit for a short distance to aid in the

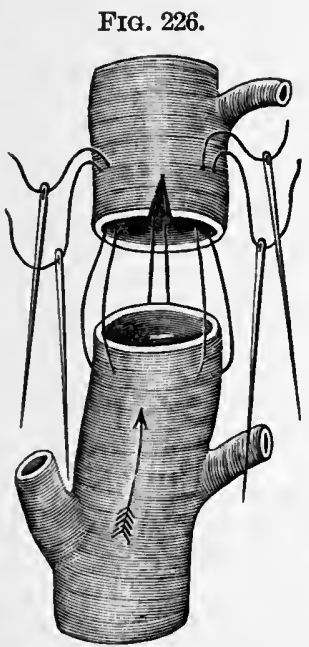

Invagination of wounded artery. (After BICKHAM.)
FiG. 227.

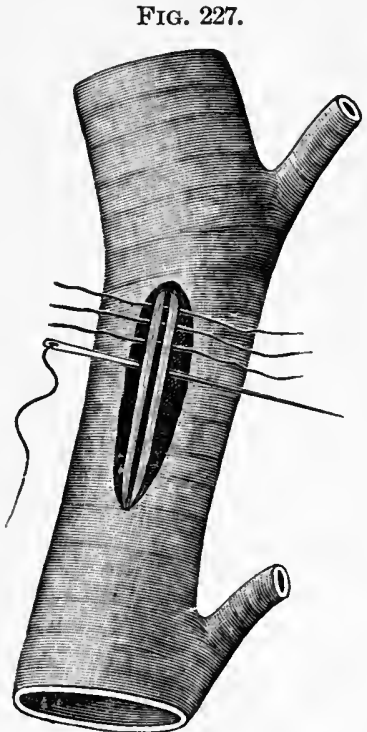

Suture of longitudinal wound of an artery. (After Bickнам.)

invagination, which is accomplished by traction upon the sutures. When this is accomplished the sutures are tied tightly with reef knots. The line of juncture is reinforced by sutures uniting the edges of the slit formed so as not to include the intima or the invaginated vessel. In longitudinal wounds the edges may be brought together by fine silk sutures, introduced by means of a fine cambric needle. The sutures should be inserted from one-sixteenth to onetwentieth of an inch apart, and one-sixteenth of an inch from the edges of the wound, and should include only the 
adventitia and media, not perforating the intima (Fig. 22i), During the operation the circulation in the vessel should be controlled both above and below the wound by forceps covered with rubber tubing or temporary ligatures. Where a distinct sheath is present, it should be sutured over the wound; and if this is not present, muscle or fascia should be sutured over the closed wound in the vessel. The application of sutures to wounds of arteries has been advocated, to secure permeability of the vessel at the seat of the wound, but it is still a mooted question whether the vessel remains ultimately permeable at the seat of the wound.

Closure by Plaster Tape.-Brewer, in experiments upon wounds of the arteries in animals, has secured control of the bleeding and closure of the wound by wrapping around the wounded vessel a strip of specially preparcd adhesive plaster. The plaster is a variety of rubber plaster which has been thorcughly sterilized, so that it ean remain indefinitely in the tissues. He has suggested that this procedure may be employed in wounds of the larger arteries in man, where for any reason sutures camnot be applicd.

Acupressure.- In this method of controlling arterial hemorrhage a needle or pin is used, which is thrust

Fig. 228.

FIG. 229.

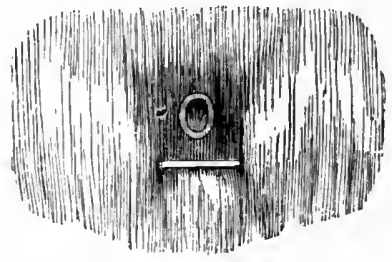

Acupressure-first method ; raw surface. (ERICHSEN.)

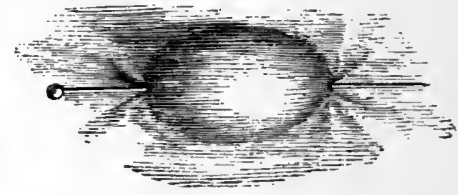

Acupressure-first method; cutaneous surface. (ERICHSEN.)

through the tissues in such a way as to compress the artery. In the first method of acupressure the surgeon places a finger of his left hand upon the mouth of the bleeding vessel, and with his right hand introduces the needle from the cutaneous surface and passes it throngh the thickness 
of the flap until its point projects for a couple of lines or so from the surface of the wound a little to the right side of the end of the vessel. By forcibly inclining the head of the needle toward his right, he brings the projecting portion of its point firmly down on the side of the vessel, and after seeing that it oceludes the artery he nakes it re-enter the flesh as near as possible to the left side of the wound and pushes the needle through the flesh until its point comes ont again at the cutaneous surface (Figs. 228 and 229).

There are a number of methods of using the needle or pin in acupressure to produce occlusion of the vessel, but as this method of arresting hemorrhage is not often employed at the present time ther need not be described.

Rules for Ligating Wounded Arteries.-The following rules for the application of ligatures to wounded arteries have been recommended by Ashhurst:

1. In eases of primary hemorrhage, no operation should be performed upon an artery unless it is at the moment actually bleeding. The exception to this rule is in the cases where the vessel is seen to pulsate in the wound, or where the wound involves the region of a large artery and the patient has to be transported or may be in a position not to receive surgical aid subsequently if needed; under these circumstances, the vessel shonld be tied or the wound should be explored to ascertain the fact that no important vessel has been injured.

2. In applying a ligature to a wounded artery, the surgeon should cut down directly upon it at the point from which it bleeds and secure it in the wound. This rule holds good for both primary and secondary hemorrhage.

3. 'Two ligatures should be applied, one to each end of the artery if it be completely divided, and one on each side of the wound if the latter has not severed all the coats of the artery. This procedure is adopted for the reason that arterial anastomosis is so free that the proximal ligature will not always, even temporarily, arrest the bleeding ; and if it does accomplish this object at the time, after the collateral circulation is established bleeding is apt to occur from the distal extremity of the divided vessel. If the 
coats of the artery are not completely severed, their division should be completed, either before or after the application of the proximal and distal ligatures, thereby favoring contraction and retraction of the ends of the divided vessel.

\section{Treatment of Venous Hemorrhage.}

Bleeding from small veins often stops spontaneously unless there is pressure upon the wounded veins on the eardiac side of the wound. It is, however, very satisfactorily controlled by position or by the application of a compress and bandage. The free bleeding arising from ruptured varicose veins of the leg is easily eontrolled by the applieation of a compress and a bandage.

The Ligature.- When large veins have been divided both ends should be seeured by ligatures, as in the

FIG. 230.

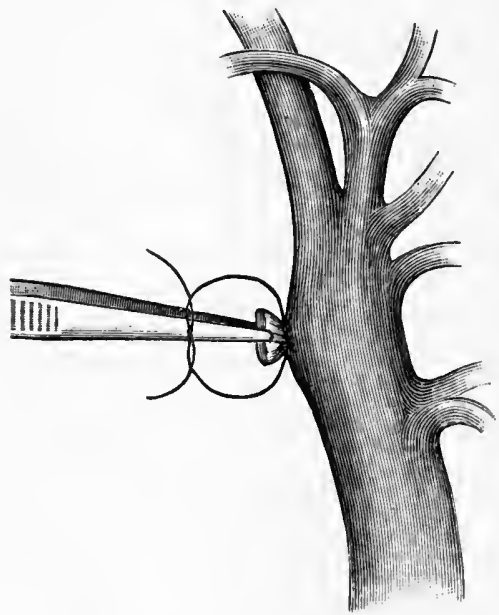

Lateral ligature. (After BICKнAм.) ease of divided arteries, small rounds should be treated by the lateral ligature or suture.

The Lateral Ligature. -The application of the lateral ligature to small wounds of large veins, such as the femoral,or to wounds of venous sinuses, has been recommended and enployed with good results ; this procedure consists in pinching up the wall of the vein so as to include the orifice of the wound with forceps and throwing a delicate silk or catgut ligature around it (Fig. 230).

Suture of Veins.-This procedure has also been employed with suceess in the ease of the larger veins. The bleeding should be controlled by pressure upon the vein on both sides of the wound, and the wound in the vessel should be closed by fine silk or catgut sutures applied closely 
together by means of a fine eambric needle (Fig. 231). The employment of sutures and lateral ligatures in wounds or veins possesses the advantage of controlling the bleeding and at the same time not causing obliterition of the vessel at the seat of injury.

The actual cautery may also be employed for the control of venous hemorrhage in situations in which its arrest by pressure or the ligature is not feasible.

Compression by means of strips of sterilized gauze is often employed to control venous hemorrhage from cavities. This is the most satisfactory manner of controlling hemorrhage for the venous sinuses of the brain.

\section{Treatment of Capillary}

\section{Hemorrhage.}

Capillary or parenchymatous hemorrhage is often arrested spontaneously on exposure of the surface of the wound to the air, but the bleeding may not be controlled and may be so profuse that its arrest becomes a matter of importance. To control this form of bleeding, pressure may be applied to the bleeding sur-

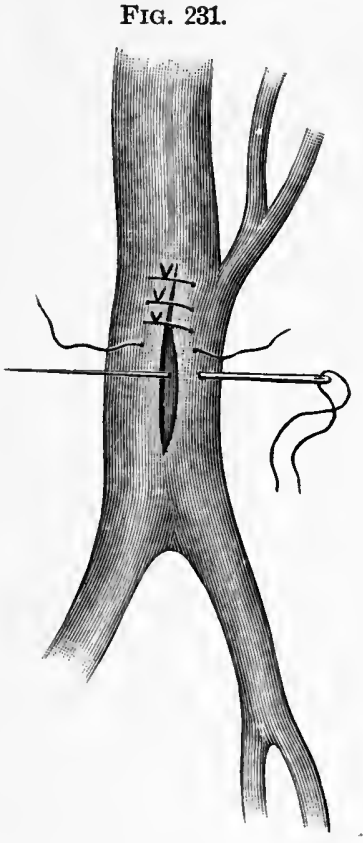

Suture of a vein. (After BICKHAM.) face for a short time, and if this fails to arrest it, sponging the surface with dilute alcohol will sometimes prove satisfactory; but the best application to arrest hemorrhage of this nature is hot water, which may be used in the form of a hot bichloride solution or antipyrin solution.

Adrenalin ehloride solution, 1 to 1000 to 1 to 10,000 , may also be employed with good results.

In eases where the means mentioned above fail to control the bleeding, it may be necessary to pack the wound 
with strips of sterilized gauze; this dressing is most serviceable when the hemorrhage comes from cavities such as result from the removal of tumors or excisions of joints, and for the control of bleeding following the removal of necrosed or carious bone. To control hemorrhage from mucous cavities, such as the nose, rectum, and vaginia, this method of treatment is also frequently resorted to.

\section{Treatment of Secondary Hemorrhage.}

Secondary hemorrhage following the use of the ligature or other means of controlling bleeding, usually results from a septic condition of the wound, and is due to a septic arteritis. Since the adoption of the antiseptic and aseptic methods of wound treatment it is a much less frequent complication of wounds.

The treatment of this complication is both constitutional and local. The constitutional treatment consists in the use of those remedies which were mentioned as serviceable in primary hemorrhage, and the drugs upon which most reliance should be placed are opium and ergot.

The local treatment of this form of hemorrhage consists in the use of the various means of controlling hemorrhage which have been mentioned, such as the ligature, hot water, pressure, or the actual cautery. If possible, it is well to secure the vessel from which the bleeding arises in the wound; if for any reason this cannot be done, the main artery should be ligated above the wound if the hemorrhage be arterial.

\section{Control of Hemorrhage from Special Parts.}

Epistaxis, or hemorrhage from the nose, may be so profuse as to require surgical interference. To control this form of hemorrhage, the application of iced compresses to the surface of the nose may first be made use of, or the injection of cold sterile water or adrenalin solution. If this fails to control the bleeding, the surgeon or the patient should grasp the cartilaginous portion of the nose with his thumb and forefinger in such a manuer as to keep the nos- 
trils tightly closed, which will prevent the passage of air throngh the nose and thus permit elots to form, arresting the flow of blood. Bleeding from the nose often arises from the erosion of a small artery low down upon the septum, which can be freely exposed by introducing a nasal speculum, and the bleeding point may be tonehed with a cautery-iron, thus avoiding the necessity of plugging the nares. If these simple means fail to arrest the bleeding, the nasal cavity or cavities may be packed with strips of sterilized gauze introduced into the anterior nares, and pushed backward by a director or probe; this will often be found a satisfactory means of arresting the bleeding. This method may be supplemented by a plug of sterilized cotton introduced into the posterior nares with the finger. The use of a rubber tampon, consisting of a rubber bag, introduced into the nares in a collapsed state and afterward inflated, has also been recommended for the control of this variety of hemorrhage.

Another method of eontrolling hemorrhage from the nose consists in introdueing a small piece of sponge or pledget of sterilized ganze, tied to a strong silk ligature, into the anterior nares and pushing it back along the floor of the nose to the posterior nares; a piece of sponge or gauze about the size of a marble, with a hole in the centre, is threaded on the ligature and pushed baek until it comes in contaet witl the first piece introduced, and thus by introducing a number of pieces of sponge or gauze in this way the nasal eavity may be completely filled and the bleeding arrested. Care should be taken to see that the sponge has been rendered aseptic before being introducerl, and the nasal cavity should be washed ont with an antiseptic solution before its introduction. The sponges or ganze may be allowed to remain for twenty-four to forty-eight hours (Fig. 232).

Plugging the nares by means of Bellocq's canula is also employed to arrest hemorrhage from the nasal cavities; the canula, armed with a strong ligature, is passed along the floor of the nose until it reaches the pharynx, when the spring being protruded, the ligature is seized and 
FIG. 232.

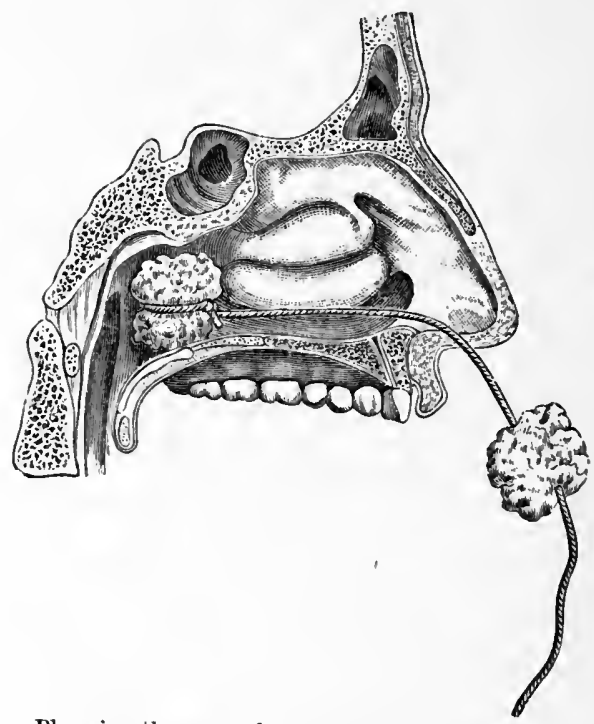

Plugging the nares from the front. (RoBerts.)

FIG. 233.

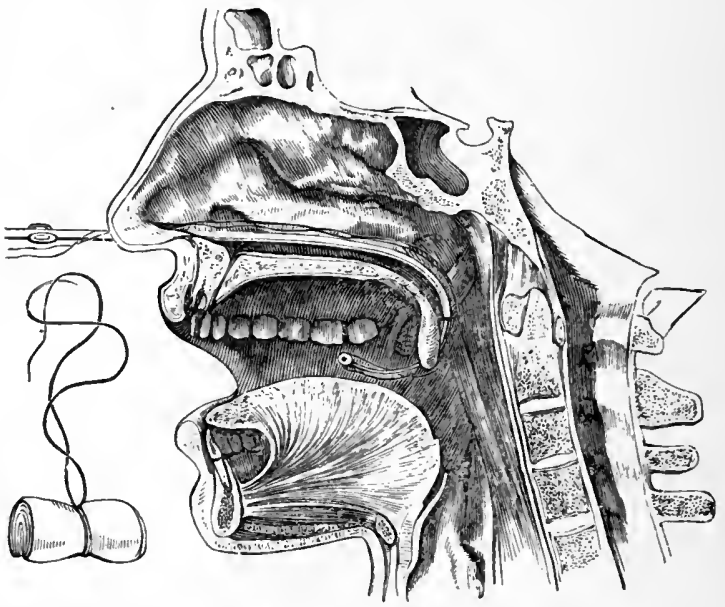

Plugging the nares with Bellocq's eanula. (FERGUsson.) 
brought out of the mouth and secured to a plug of lint or of antiseptic gauze of the required size, and upon withdrawing the instrument the plug is bronght into position in the posterior nares and the end of the ligature allowed to protrude from the mouth to facilitate its removal (Fig. 233.) An ordinary flexible catheter may be employed instead of Belloeq's canula for the introduction of the ligature.

Hemorrhage from the Urethra.-In hemorrhage from the urethra, if profuse, the blood will trickle from the meatus ; or if efforts at micturition are made, the first portion of urine will contain blood, but afterward will be elear, and the last portion will contain a few drops of pure blood.

This variety of bleeding, if it proeeeds from the anterior portion of the urethra, may be controlled by the introduction of a catheter and the application of a bandage around the penis applied so as to make only moderate pressure.

If the bleeding eomes from the posterior portion of the urethra, it will of ten be eontrolled by the application of cold or pressure to the perineum, or by the introduction of a cold steel bougie, or by the injection of a weak solution of antipyrin or of adrenalin solution.

Hemorrhage from the Bladder.- In this variety of hemorrhage the first portion of the urine may be bloodstained, and the last portion will eontain more blood and clots as the organ contracts, which distinguishes it from hemorrhage from the kidneys, in which admixture of bloorl with the urine renders it of a smoky color, or dark red if the bleeding is profuse.

To control bleeding from the bladder, a catheter should be introduced and the urine and elots withdrawn; the bladder should next be washed out with a warm or eold boric aeid solution. In severe eases a weak solution of tannic acid, antipyrin, alum, or adrenalin solution may be employed. The application of ice to the perineum and suprapubic regions may also be employed with advantage.

Hemorrhage from the Rectum. - This variety of bleeding may be controlled by the injection of cold or as- 
tringent enemata. If the bleeding be profuse, a speculum should be introdnced, and when the source of the bleeding has been discovered the actual cautery or a ligature should be applied. If $t$ lis is not feasible, the rectum may be-plugged with strips of antiseptic gauze, or a piece of a rubber catheter of large calibre may be wrapped with gauze and introduced into the rectum, the end of the catheter being allowed to protrude; by using this tube flatus can escape, and if the bleeding is not controlled blood will escape throngh the tube, preventing the risk of concealed hemorrhage. If the bleeding arises from hemorrhoids or polypas of the rectum, operative treatment of these conditions should be undertaken to remove the cause of bleeding.

\section{TREATMENT OF ABSCESS.}

In operations for evacuation of the contents of abseesses care should be taken to observe every precaution to prevent a new infection of the wound or abscess cavity; the skin over the abscess should be carefully cleaned to make it aseptic, the hands of the surgeon and the instruments to be bronght in contact with it should also be aseptic. These precautions should be especially observed in the opening of ehronic abscesses when a new variety of infection is liable to be introduced if aseptic precautions are not rigidly observed.

Acute Abscess.-This variety of abscess should be opened by incision, and this is best done with a straight, narrow, sharp-pointed bistonry. The incision should be deep enough to expose freely the cavity of the abscess, and should be parallel with and not across important structures, and it should also be made at as dependent a portion as possible. Abscesses of the limbs are opened by a longitudinal incision, and those in the region of the anus and breast hy an incision radiating from the anus or nipple.

Hilton's Method.-In deep-seated abscesses in the region of important struetures the method of opening suggested by Mr. Hilton may be employed with advantage: it con- 
sists in making a small ineision through the skin and eellular tissue; a director is next pushed through the tissues into the abscess cavity, which will be shown to have been reached by the eseape of pus along the director; a dressing-foreeps with the blades elosed is now pushed along the director into the absecss cavity, and when this has been accomplished the director is withdrawn and the forceps removed with the blades expanded so as to dilate the wound and allow the pus to escape. Pressure should not be made upon the walls of the abscess to empty it, as by so doing delieate vessels may be ruptured and eause hemorrhage, and the spread of the infection may be facilitated.

The eavity of the abseess having been emptied of pus, it may be irrigated with carbolic or normal salt solution, or the irrigation of the eavity may be omitted, and if the cavity is not very large or deep, no drainage-tube need be introdueed, and a small piece of protective may be placed between the lips of the wound to prevent their adhesion; but if, on the other hand, the cavity is extensive and deeply situated, a rubber drainage-tube or a strip of iodoform or sterilized gauze should be introduced to the bottom of the cavity to seeure free drainage, and if a tube be used, it shonld be fixed at the surface of the skin by a safety-pin. A ganze dressing, consisting of a number of layers, which has been moistened in carbolie or bichloride solution, is next placed over the wound, and is covered by a number of layers of dry gauze, which are in turn covered by a piece of rubber tissue. The latter may be substituted by a few layers of sterilized or bichloride eotton, and the dressing is finally secured by a rollerbandage. The dressing is removed at the end of two or three days, the eavity being washed out with one of the antiseptic solutions previously mentioned. The drainage-tube may then be shortened or removed, and the dressings reapplied, as at the primary dressing. Under this method of treatment aente abseesses usually heal promptly.

Chronic or Tuberculous Abscess.-This variety of 
abscess, which occurs chiefly in connection with diseases of the bones or joints or of the lymphatic system, is tubercular in origin, and may be opened in various ways, the time at which this should be done depending upon the size and situation of the abscess and the amount of constitutional and local disturbance which the patient experiences from its presence.

Aspiration.-A tuberculous abscess may be evacuated by means of the aspirator ; the pus being withdrawn as far as possible, the puncture is sealed with a small piece of gauze covered with iodoform collodion. Reaccumulation of pus often takes place, and the aspiration has to be repeated a number of times. The greatest difficulty in the successful removal of the contents of tuberculous abscesses by means of aspiration is the presence of cheesy masses in the pus, which occlude the canula and often prevent complete emptying of the cavity.

Puncture and Injection.-This variety of abscess may also be evacuated by making a puncture through the skin and overlying tissues with a narrow bistoury, the surface having been previously thoroughly washed with soap and water and with a carbolic or bichloride solution; a director is next pushed through this small wound into the cavity of the abscess, and the pus is allowed to escape by stretching the wound with the director; when the cavity is emptied of pus it is washed out with a carbolic or bichloride solution or sterile water introduced into it by pushing the nozzle of a syringe into the cavity, and this is allowed to escape in the same way as the pus previously did. When all the irrigating solution has escaped, the cavity may be injected with an emulsion composed of iodoform 1 part, glycerin or olive oil 10 parts; after this has been introduced the small wound is closed by a compress of aniseptic gauze held in place by a compress of bichloride cotton and a bandage or by strips of adhesive plaster. The injection of the iodoform emulsion need not be repeated as long as iodoform continues to be excreted in the urine.

In evacuating tuberculous abscesses by means of the as- 
pirator or by a small puncture, there is absence of shock, and the loss of blood is insignificant, so that these procedures should generally be first employed, and the more radical operation of incision and curetting of the cavity of the abscess, which is accompanied with a certain amount of shock and hemorrhage, should be reserved for those cases in which the less severe operations have not been followed by a satisfactory result.

Incision.-Tuberculous abscesses are also treated by making a free incision into the abscess cavity with full antiseptic precautions, and after the escape of the purulent matter the walls of the abscess should be thoroughly scraped with a curette; after the cavity has been freely washed out with a carbolic or bichloride solution or sterile water, large drainage-tubes are introduced and an antiseptic dressing is applied to the wound. The edges of the incision may be brought together by sutures without the introduction of drainage, or the cavity may be packed with jodoform gauze and allowed to heal by granulation. The dressings are removed as soon as they become soaked, and the drainage-tubes are shortened or removed as the discharge diminishes and the cavity contracts.

Diffuse Suppuration.-This form of suppuration is treated by numerous punctures or incisions, which allow the purulent matter to escape; and where sloughs are present, free incisious may be required to give exit to the necrosed tissues; the introduction of drainage-tubes may also be required. The wounds and the cavities, as far as possible, should be washed out with sterile water or bichloride solution and an antiseptic gauze dressing applied.

Sinuses.-These are suppurating tracts which result from abscesses or wounds. If superficial, they should be laid open freely and their surfaces scraped with a curette, and then lightly packed with strips of bichloride or iodoform gauze and covered by an antiseptic dressing. If they are too deep to be treated by incision, their healing may be facilitated by the injection of stimulating solutions introduced by means of a syringe; the employment of solutions of chloride of zinc, nitrate of silver, and sul- 
phate of copper, varying in strength from 5 to 20 grains to the ounce of water, will often prove satisfactory.

Beck's Bismuth Emulsion has recently been used with good results in the treatment of deep sinuses and fistulous tracts. Two different emulsions are employed known as No. 1 and No. 2. No. 1 eonsists of bismuthi subnit, 30.0 gm., vaseline, $60.0 \mathrm{gm}$. No. 2 consists of bismuthi subnit, $30.0 \mathrm{gm}$., white wax $5.0 \mathrm{gm}$. Liquid paraffin, $5.0 \mathrm{gm}$., vaseline, $60.0 \mathrm{gm}$. These should be mixed while boiling.

No. 1 emulsion is not so consistent as No. 2, and is used in superficial sinuses and is apt to escape from the sinuses in a short time, while No. 2 emulsion becomes more firm upon cooling and may remain in the tissues for a long time, and healing may occur with the emulsion in the tissues.

These emulsions should be warmed in hot water until they become liquid and they can then be injected into the sinuses or fistulous tracts with a syringe, and soon beeome consolidated at the temperature of the body. They seem to exert a stimulating action on the walls of the sinuses favoring the growth of granulations and healing. These injections are also very useful in showing the depth and extent of sinuses conneeted with diseases of the bones and joints, when after the injection of the emulsion and $x$-ray examination will show the location in which the emulsion has been deposited.

Cases of bismuth poisoning and death have been reported for the use of this emulsion.

Felon or Whitlow.-This may consist 1. The subeute cular felon, which consists of a collection of pus under the epidermis in which after incision and evacuation of the pus and the removil of the dead skin, pain is relieved and a prompt recovery takes place.

2. The subcutaneous form which consists in a cellulitis of the pulp of the finger or thumb over the last phalanx, resulting from infection through a puncture or abrasion. The inflammatory condition results in severe throbbing pain and swelling of the parts. Unless the condition is 
relieved by incision the bone may become involved or the process may extend to the tendon sheath.

The treatment consists in early and free incision and the subsequent use of hot antiseptic dressings.

3. Suppurative Thecitis.-This may follow the variety just described or may arise from infection of the tendon sheath from wounds. Pain, swelling and disability of the fingers are prominent symptoms. If the condition is not arrested by treatment, suppuration may extend along the tendon sheaths to the palm of the hand and give rise to palmar abscess. Sloughing of the tendon is apt to occur, and may result in a useless finger. This condition is more likely to occur when either the little finger or thumb is involved because their tendon sheaths communicate with the common palmar sac.

Treatment.-The patient should be anæsthetized and a careful dissection should be made through the inflamed tissues parallel to the sheath of the tendon to evacuate the purulent matter, if pus is found external to the sheath, this should not be opened, but if the sheath is found distended it should be opened at one or more points to secure free drainage. Hot antiseptic dressings should be applied and the hand should be put at rest upon a splint.

Palmar Abscess.-This may develop from an infection arising in the tendon sheaths of the fingers or from wounds, contusions or abrasions of the palm of the hand, as the pus accumulates under the palmar fascia swelling and œdema of the back of the hand may occur. Severe pain, throbbing in character and swelling of the hand are prominent symptoms. If the condition is not relieved promptly by operation, the purulent matter is apt to follow the tendon sheaths and find its way into the forearm.

Treatment.-The patient should be anæsthetized and a careful incision should be made into the tissues of the palm, the line of incision should correspond to the middle of the metacarpal bones and should be distal to a line crossing the palm at the level of the web of the thumb to avoid the superficial palmar arch. When the palmar fascia 
has been opened the pus usually escapes freely. If there is much swelling of the back of the hand incisions may be required at this point. Gauze drains should be introduced into the incisions and a wet gauze dressing applied, and the hand should be placed upon a palmar splint.

\section{SHOCK.}

Shock is a condition of physical depression or prostration which often develops after severe injuries or operations. Paralysis of the vascular tone in the arteries, with coincident feebleness of the action of the heart, causes an unequal distribution of the blood, and the balance of the circulation is disturbed ; the abdominal veins become distended and the right side of the heart becomes engorged, the amount of blood in the arteries being correspondingly lessened ; the brain and the lungs become anæmic, and if the condition persists the action of the heart is arrested. The essential condition of shock is inhibition of nerve force and reflex paralysis. Shock may develop immediately upon or some time after the reception of the injury. Every traumatism is probably followed by a certain amount of shock, and, as a rule, its degree is proportionate to the severity of the injury received. Yet this rule is not without exception; certain classes of injuries are attended with marked shock, and the part of the body sustaining the injury will have an important influence upon the degree of development of shock. Contusions of the viscera, wounds of the testicle, contused and lacerated wounds of the trunk and extremities, if extensive and accompanied by free hemorrhage, are usually followed by marked and often fatal shock. Gunshot wounds causing perforation of important cavities of the body, injuries of the viscera, and shattering of the bones are also well recognized as giving rise to shock in a marked degree. Burns and scalds, if they involve a considerable surface of the body, are attended with severe shock.

Diagnosis.-The condition of shock resulting from purely emotional causes is usually not profound or prolonged, and can be differentiated from that resulting from corporeal injuries by the history of the case. The con- 
dition arising from excessive hemorrhage presents many symptoms common to shock, but here the nature of the injury will often assist in the diagnosis, and in doubtful cases examination of the blood may be of service, for if such an examination shows that the red blood-cells are considerably diminished, being $3,500,000$ or less, it is probable that the condition is due to hemorrhage rather than shock. Fat embolism may also be confounded with shock, but it should be remembered in differentiating the conditions that shock usually appears promptly, and the symptoms of fat embolism from thirty-six hours to three days after the injury. The experimental researches of Crile have largely confirmed our clinical observations as regards the development of shock in injuries and operations in different regions of the body.

A patient suffering from shock presents pallor of the surface, paleness of the lips, dilated pupils, clammy moisture of the skin, muscular debility, occasionally relaxation of the sphincters, frequent, feeble, irregular pulse, subnormal temperature, and feeble, short, sighing respiration ; in many cases extreme thirst is a prominent symptom. The senses are often perfectly retained. The temperature is always subnormal, and may vary from a point a little below the normal to a point below $90^{\circ} \mathrm{F} .\left(32^{\circ} \mathrm{C}\right.$.). A depression below $97^{\circ} \mathrm{F}$. $\left(36^{\circ} \mathrm{C}\right.$.), if it persists for a few hours, usually indicates a grave condition of shock, and reaction may not occur, although it has been observed in cases where the temperature was as low as $90^{\circ} \mathrm{F} .\left(32^{\circ} \mathrm{C}\right.$. $)$.

Prophylaxis.-Unfortunately, many of the worst cases of shock are due to accidents, and here treatment can be directed only to the condition of shock itself; but the surgeon is often able to diminish to some extent the amount of shock following operations by judicious prophylactic treatment. In patients in whom shock is apt to be markedly developed, as in children or feeble or aged subjects, or in certain classes of operations, he may give stimulants before the operation, and see that the surface of the body is not unnecessarily exposed to chilling during the operation, that the operation is not needlessly prolonged, and 
that as little blood as possible is lost during its performance. The electro-thermic mattress may be used with advantage, but care should be exercised in its employment, as serious burns have followed its use. The previous administration of an ounce of whiskey and the hypodermic injection of from $\frac{1}{30}$ to $\frac{1}{20}$ of a grain of sulphate of strychnine, and the use of a small dose of morphine, in feeble and aged patients, will be followed by good results. A full close of quinine given an hour or tro before the operation is also said to arrest the development of shock.

Treatment.-The first indication in the treatment of shock is to establish reaction. The patient should be covered with woollen blankets, the head should be kept low, and dry heat should be applied to the surface of the body by means of hot-water bags, hot bottles, or hot bricks; these should be wrapped in towels to prevent them from coming directly in contact with the surface; neglect of this precaution, which is most important if the patient is unconscious, often produces burns which may be followed by extensive sloughing. If the patient can swallow, he should be given small quantities of whiskey or brandy, with 30 minim doses of aromatic spirit of ammonia, and, as absorption by the stomach is probably very slow in these cases, stimulants should be administered hypodermically ; in onr judgment, strychnine is the most valuable stimulant that can be employed. From $\frac{1}{30}$ to $\frac{1}{20}$ of a grain should, therefore, be injected, and the injection repeated every hour or half-hour until several doses have been given. Caffeine citrate in doses of grs. ij may also be used with good results. Sulphuric ether, 30 minims, may also be injected into the cellular tissues at intervals, as well as digitalin or tincture of digitalis.

If shock develops during an operation under ether anæsthesia, the use of ether hypodermically is contraindicated. A stimulating enema of whiskey and warm water may be employed. In cases of shock where there is profuse sweating, the use of $\frac{1}{60}$ of a grain of atropine, repeated as required, is often followed by good results. A large enema of warm saline solution may also be employed. As 
patients often complain of urgent thirst, it is well to let them take a little black coffee, but not large quantities of water; free indulgence in water does not seem to quench the thirst, and is apt to be followed by vomiting. Intravenous injection of saline solution is likely to be of most service when the condition has been preceded by the loss of a large quantity of blood. Infusion of saline solution also has been employed with good results.

\section{DRESSING OF WOUNDS.}

Incised Wounds.-These wounds present the conditions favorable for prompt healing, and after sterilizing the surrounding skin they should first be carefully irrigated with saline solution or sterilized water, to remove any blood-clots or foreign bodies, or wiped with a sterilized gauze pledget; and after any hemorrhage which is present is controlled by the use of ligatures, if the wound be an extensive or deep one, provision should be made for drainage by introducing a drainage-tube or a f'ew strands of sterilized catgut at the bottom of the wound, allowing the ends to project from its most dependent portion. Irrigation of the wound with a $1: 2000$ or $1: 4000$ bichloride solution may be employed if there is reason to suppose the wound has been infected before coming under treatment. In superficial incised wounds, after the hemorrhage has been controlled, it is not usually found necessary to make provision for drainage. If the wound be a deep one, involving the muscles and deep fascia, buried sutures of catgut or silk should be applied to approximate the muscles and fascia ; and if important nerves or tendons have been divided, their ends should be bronght into apposition by sutures of catgut or sterilized silk; the superficial portions of the wound should next be brought together by the introduction of a number of interrupted sutures, catgut, silkworm-gut, silver wire, or silk being employed for this purpose; the accurate apposition of the edges of wounds of this variety is secured by 
the introduction of a number of sutures placed closely together.

After a wound of this variety has been closed, the subsequent dressing is accomplished by covering the surface of the wound with a number of layers of sterilized gauze and a pad of sterilized cotton, which are held in place by a gauze bandage. Or a few layers of ganze, which have been soaked in a 1:2000 bichloride solution, may be applied to the wound, and over this is laid a pad of dry bichloride gauze of the same thickness, overlapping the wet gauze by a few inches in all directions; a few layers of bichloride cotton are next applied over the gauze dressings, and the whole dressing is secured in position by the application of a gauze bandage. Under this form of dressing prompt healing of incised wounds is the rule, and the wound need not be redressed for a week or ten days unless some indications exist for change of dressing at an earlier period. At the time of the first dressing the catgut drain or the drainage-tube is nsually removed, and if adhesion of the edges of the wound is firm the sutures may also be removed. A sterilized or bichloride gauze dressing is usually next applied, and allowed to remain for a few days longer.

In superficial incised wounds involving only the skin and cellular tissue if limited in extent, after cleansing the wonnd and controlling the bleeding the edges should be approximated with sutures. The wound should then be covered with strips of sterilized ganze, over which is painted a mixture of: tr. benzoin, $3 \mathbf{j}$; collodion, $3 \mathrm{vij}$. This forms a firm antiseptic scab which need not be removed until the wound has healed.

Lacerated Wounds.- These present edges which are torn and not sharply cut, and the vitality of the injured parts is often so seriously impaired that prompt union in this variety of wounds is not, as a rule, to be looked for. Wounds of this nature should first be irrigated with saline solution, sterilized water, or a 1:2000 bichloride solution, and blood-clots and foreign bodies removed. If the wounds be deep, drainage-tubes should be intro- 
duced; on the other hand, if they be superficial, or if the erlges are not closely approximated, provision for drainage may be omitted. The torn or irregular edges of the wound should next be brought into apposition at a few points, by the introduction of catgut or silkworm-gut sutures, applied not very closely together; and if the edges are discolored and their vitality seems markedly. impaired, it is better not to use sutures. If the edges of the wound are so much crushed that their vitality is destroyed, they may be trimmed away with scissors until a surface possessing a fair vitality is secured. The evil results arising from the introduction of sutures into this variety of wounds, with the idea of closely approximating their edges, are so common that the surgeon who dispenses with the use of sutures entirely errs upon the safe side. The use of many sutures in wounds of this nature often causes marked tension, which is frequently followed by impairment of the vitality of the injured tissues, and sloughing results. The wound should next be dressed with sterilized ganze and cotton, or a bichloride gauze dressing may be employed, and if it runs a farorable course it need not be redressed for a week or ten days; the time required for repair of a wound of this nature is longer than that for an incised wound, and more frequent dressing may be required.

In lacerated wounds of the extremities continuous irrigation of the wound by a warm bichloride or carbolic solution, applied as described (p. 137), is often followed by the most satisfactory results; wounds produced by machinery and railway accidents, in which the vitality of the tissues is much impaired, are particularly suitable cases for this method of treatment, and here the same caution should be exercised as regards the introduction of sutures.

Contused Wounds.-This variety of wounds possesses many characteristics in common with lacerated wounds: the edges are bruised and the injury of the subcutaneous tissue is often more extensive than the external wound would lead one to suspect. They are dressed in the same inanner as lacerated wounds, and the same objection here 
exists to the use of sutures as in the latter class of injuries.

Punctured Wounds.-These wounds are inflicted by sharp-pointed instruments, and it may happen that a portion of the vulnerating body remains in the wound, as is frequently the case in wounds produced by needles, splinters of wood, metal, or glass; another complication in this variety of wound is the injury of vessels, giving rise to concealed hemorrhage, or of nerves, resulting in neuritis or neuralgia. Simple punctured wounds should be irrigated with 1:2000 bichloride solution and covered by a sterilized or bichloride gauze dressing, and if no complication exists their healing is usually very rapid.

A very serious form of punetured wounds arises from the impaling of a portion of the body by pieces of wood or metal, the part being transfixed or simply penetrated ; the penetrating object may break off, leaving a portion of it in the wound, or may retain its position in the body, so that it is difficult to separate the body from it. This accident usually results from persons falling upon sharp sticks, wooden or iron palings.

When a foreign body remains in the wound, as often happens in punctured wounds produced by needles and splinters, the punctured wound should be converted into an incised wound, and the body should be searched for and removed; in doing this in the case of wounds of the extremities the operation is much facilitated by the employment of Esmarch's bandage. The Röntgen or $x$-rays may be employed with advantage in locating foreign borlies, such as pieces of glass or metal, in punctured wounds. After the removal of the foreign body the wound is treated as an incised wound, and an antiseptic or aseptic gauze dressing should be applied. When concealed hemorrhage occurs after a punctured wound, the wound should be laid open and the bleeding vessel searched for and ligated if possible, and the wound should afterward be dressed as an incised wound.

Poisoned Wounds.-These wounds are caused by the absorption, by means of a cut or abrasion in the skin, or 
by the sweat or sebaceous glands, of fluids from a dead body in making dissections or post-mortem examinations, or in operating upon living subjects, and often result in scrious consequences. Infection occurring from a living subject in operating is apt to give rise to a similar specific infection, or a mixed infection may result; whereas infection occurring from dead bodies is usually caused by the bacteria of putrefaction, as infective micro-organisms retain their virulence for only a short time after death. Such wounds, as soon as possible after their reception, should be carefully washed out with a solution of bichloride of mercury, $1: 2000$, and the surface touched with a 30-grain solution of chloride of zinc, and then dressed with an antiseptic dressing. If, however, this precaution is not taken, or the wound has escaped notice, and in a few hours becomes inflamed and painful, and evidences of lympliatic involvement show themselves, the wound should be opened and its surface should be thoroughly sponged with a 2 per cent. solution of formalin or with a 30-grain solution of chloride of zinc, and finally with a $1: 2000$ bichloride solution, and it should then be dressed with an antiseptic gauze dressing. Under this method of dressing, the poisoned wound is often converted into a healthy one, even after the lymphatic involvement is well marked, and it usually heals promptly without further constitutional disturbance.

Gunshot Wounds.-These wounds are produced by small shot, or fragments of shells, and are of the nature of contused and lacerated wounds, and the vulnerating body as well as portions of the clothing is often imbedded in the tissues.

The modern small arms ball has much greater velocity than the leaden ball formerly employed; it has great penetrating power, and is more apt to pass through the bones withont eomminuting them. Primary hemorrhage is also more common in injuries produced by this ball. Within a certain range it also possesses marked explosive action, producing great destruction of the tissues with which it comes in contact, which has been recently 
explained upon the theory of hydrodynamic pressure or vibratory action. The explosive effect of a small calibre ball depends upon its velocity, striking energy, area of impact, and the resistance to be overcome, so that the damage to the tissues in gunshot injuries is always greater at short range, and decreases with the increase of distance. Stevenson now maintains that the conclusions drawn from experiments upon dead animals and men are not borne out by what is observed when living men are wounded by small calibre projectiles. In dressing these wounds any foreign bodies, if they can be located, should be removed, and in the search for and removal of balls from the extremities the application of the Esmarch bandage will be found most useful. The $x$-rays may also be satisfactorily employed in locating balls or fragments of metal in gunshot wounds. The wound should next be thoronghly washed ont with a 1:2000 bichloride solution, and an antiseptic dressing applied as in the case of other contused and lacerated wounds.

Powder-burns.-These result from the explosion of gunpowder, and, in addition to the burning and laceration of the tissues, are accompanied by the introduction of grains of unburnt powder into the skin, which, if not removed, leave permanent points of pigmentation. These wounds should first be washed with a $1: 2000$ bichloride solution, and upon the face, to avoid unsightly pigmentation of the skin, care should be taken to pick out the small masses of powder with a needle or the sharp point of a tenotony knife. The surface should then be dressed with antiseptic ganze or with lint spread with an ointment of boric acid or an ointment of aristol, consisting of half a drachm or a drachm of aristol to an ounce of vaseline, this dressing. being covered by a few layers of gauze and cotton, held in place by a roller-bandage.

In pigmented scars following powder-burns, the powder grains may be removed by electrolysis.

Contusions or Bruises.-These wounds differ from contused wounds in the fact that the skin is not broken, 
although in spite of this faet there may exist very extensive laceration of the subcutaneous tissues, aecompanied by more or less extravasation of blood from the injured vessels. When not sufficiently severe to require operative treatment, they should be dressed by applying over them several layers of lint saturated with lead-water and laudanum, and over this dressing is placed a layer of waxed paper or rubber-tissue, and the dressing is secured by a roller-bandage. A solution which I find most atisfaetory in the dressing of contusions is as follows: ammonii chloridi, grs. $x x$; tr. opii et alcoholis, āā $f z j$; aquæ, föj. Several layers of lint saturated with this solution are laid over the contused tissues, and are covered with waxed paper, oiled silk, or rubber-tissue.

Extensive collections of blood following contusions often remain in the tissues for some time, but usually are absorbed. If this result does not follow, or an abscess forms, the blood or pus should be removed by incision with full antiseptic precautions.

Brush-burn.-This is a form of contused and lacerated wound which is produeed by violent friction applied to the surface of the body, and is often caused by coming in contact with rapidly revolving wheels or the belting of machinery, or by the body being rapidly propelled over an uneven surface, or by a rope being rapidly drawn through the closed hands. The injury may vary from a superfieial abrasion to absolute destruction of the skin. The surface of the brush-burn should be cleansed by a stream of normal salt solution, sterilized water, or 1:2000 bichloride solution, and then dressed with a powder of acetanilid and boric acid, equal parts, and a sterilized gauze dressing applied ; if suppuration occurs, a moist bichloride or acetate of aluminum dressing or boric acid ointment should be applied.

Burns and Scalds.-The dressings employed in the treatment of burns and scalds are similar, as the injury to the tissnes is practically the same in both classes of injuries. Superficial burns or scalds, in which the effect of the heat has extended only to the siperficial layer of the skin, may 
be treated by the application of lint saturated with a solution of cerbonate of sodium, a drachm to an ounce of water; this dressing rapidly relieves the pain, and is a satisfactory application in this variety of burns and scalds. If blisters are present they should be punctured with an aseptic needle, allowing the epidermis to remain. In cases in which the effects of heat have extended to the deeper tissues, the affected surface may be dressed with Carron oil, which is prepared by rubbing together lime-waier and linseed oil until a thick, creamy paste results ; lint is saturated with this mixture and laid over the surface of the burn or scald. This dressing is a comfortable one to the patient, but possesses no antiseptic qualities and soon becomes offensive, and for this reason requires frequent renewal.

White-lead Dressing.-This application, which con-

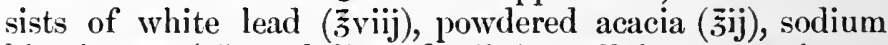
bicarbonate (3j), and linseed oil (a sufficient quantity to make a mixture of the consistency of thick cream), is extensively used in the coal regions of Pennsylvania, where severe burns are very frequent. It is spread upon lint or gauze and applied to the burned surfaces; it does not require frequent renewal, and repair of the injured surfaces is rapid under its use.

Picric Acid Dressing.-Picric acid solution has recently been very extensively used in the treatment of burns and scalds. This dressing should not be used in extensive burns or those which involve the tissues deeply as poisoning may result. After the surface has been cleaned and blebs have been opened, strips of sterilized lint or gauze are soaked in the solution and applied to the surfiace and covered with a layer of dry absorbent cotton, and the dressing is held in place by a bandage. The dressing soon dries and may be left in place for several days, when it is reapplied in the same manner. This application relieves pain and seems to diminish suppuration and leaves a healthy scar. The solution employed is as follows: Picric acid, gr. lxxv; alcohol, כ̋iiss; aquæ dest., Oij. 
The disadvantage met with in the antiseptic method of dressing burns and scalds is the fact that the raw surface presented offers most favorable conditions for absorption of the antiseptic substances employed in the dressings, and for this reason the use of bichloride of mercury, carbolic acid, and iodoform is not to be recommended in burns or scalds involving a large extent of surface, on account of the toxic symptoms which may result from their absorption.

In Germany the treatment of extensive burns by continuous immersion of the patient in a warm bath has been followed by good results.

As a recent burn or scald, by reason of the heat employed in its production, is practically an aseptic wound, a simple sterilized dressing may be employed. It may he dressed by covering it with a number of layers of sterilized gauze and cotton, or with powdered boric acid, aristol, or acetanilid, and placing over this a number of layers of sterilized cotton, holding the dressings in position by a bandage.

A satisfactory dressing for extensive burns, consists in covering them with strips of sterilized protective or rubber tissue, several inches in width, the strips should be so placed that there is a gap between the edges of each strip. Sterile gauze wrung out of warm salt solution is next placed over the strips and is held in place by a bandage. The ganze should be changed as soon as it becomes saturated and the discharges which escape between the edges of the strips. Fresh gauze should be applied without removing the strips which renders the dressing much more comfortable to the patient.

A recent method of treatment of burns, which it is claimed has been followed by very satisfactory results consists in exposing the burus to the air and simply dusting them with sterate of zinc, removing scabs when pus collects beneath them.

Ulcers resulting from separation of the dead tissues should be touched with a solution of nitrate of silver, 5 grains to the ounce of water, and dressed with lint spread 
with an ointment of boric acid, aristol, or ichthyol. In the dressing of extensive burns or scalds of the neck, face, and region of the joints, the possibility of serious deformity from contraction of the tissues in healing should not be lost sight of, and position, splints, and bandages should be employed to prevent, as far as possible, this complication.

Injuries from Electricity.-Since the extensive introduction of electricity in the arts, injuries from contact with heavily charged wires are of frequent occurrence. If the current be a strong one, death may be instantaneous, or the patient may be knocked down, become unconscious, and present severe burns at the point of contact, then regain consciousness, and subsequently suffer from numbness in the extremities, traumatic neuroses, and in rare cases true paralysis. If the skin be dry at the time the current is received, there will be more burning, less penetration and less shock, and less danger of death. The burns are not painful, but are apt to be followed by extensive sloughing. Alternating currents are more dangerous than continuous currents; a continuous current of one thousand volts is not apt to be followed by serious consequences, whereas an alternating current of the same strength is likely to produce death.

Death from exposure to strong alternating currents is considered by Hedley to be caused by destruction of the tissnes or by arrest of respiration producing asphyxia. Exposure to a strong electric current may prodnce burns or ecchymoses, and occasionally wounds; the latter bleed freely and are apt to slough. A burn from electricity presents a dry blackened surface and is surrounded by an area of pale skin. They are not as painful as ordinary burns, but healing in electric burns is usually slow. Inflammation and suppuration of the tissues usually develop in a few days, and are often followed by the development of an extensive area of moist gangrene, a small burn being followed by extensive and deep destruction of the surrounding tissnes.

Treatment.-The treatment of a person who has been 
exposed to a strong electric current, even if apparently lifeless, consists in practising artificial respiration, Laborde's or Silvester's method being employed; also friction to the surface of the body and enemata of hot saline solution; in some cases venesection has been employed with advantage. Hedley records a case of apparent death in a man who received an alternating current of four thousand five hundred volts short-circuited through his body for many minutes, who showed no signs of life for thirty minutes. In this case, after the employment of Laborde's method of artificial respiration for some time, normal respiratory action was restored, and the patient recovered. Artificial respiration should he practised in all cases, and should be continued until it is certain that the patient is dead. At the same time strychnine should be used hypodermically.

The burns shonld be treated by the application of antiseptic dressings, but these often fail to arrest the sloughing. DaCosta recommends in the early stage of these burns the use of fomentations of hot saline solution, which facilitates separation of the sloughs, and in the subsequent dressing of the wounds peroxide of hydrogen followed by irrigation with saline solution. After the sloughs have scparated, dry sterilized dressings should be employed.

Lightning-stroke.-In this form of electric injury a person may be struck directly or may be shocked by an induced current, the lightning having struck some object near at hand. The results of lightning-stroke upon the body differ according as the electrical or the burning action predominates. 'There may be present severe burns or extensive lacerations, involving the muscles, bloodvessels, and bones; or sudden death may result from paralysis of the respiration and circulation. Upon regaining consciousness, the patient may complain of disturbance of vision, and may suffer from paralysis of the nerves of motion or sensation; paralysis of the lower limbs is said to be more common than that of the upper limbs.

Treatment.-The treatment of the stage of shock following lightning-stroke consists in the application of external heat, the employment of artificial respiration, and the 
administration of stimulants. If burns exist upon the surface of the body, they should be treated like burns arising from artificial currents. If paralysis persists for some time after recovery from the immediate effects of the shock, the use of galvanism and the administration of strychnine may be followed by good results.

$X$-Ray Burns.-A peculiar lesion of the skin and subjacent tissues, following prolonged exposure to the $x$-rays, resulting in ulceration of the skin and loss of the nails and hair in the damaged area, is described as an $x$-ray burn. This lesion differs from an ordinary burn in that it may not appear for several days or weeks after the exposure, and that the inflammatory or gangrenous process arises in the tissues and finally involves the skin. These lesions are very painful and slow in healing; and if an extensive surface be involved, they may result in serious consequences : amputation of the limb has been demanded by reason of a burn of this nature. The lesion is probably due to trophic changes.

Treatment.- The dressings employed in ordinary burns have not proved satisfactory in these injuries. Dry sterilized dressings may be employed, and skin-grafting when the ulcerated surface is extensive may be of service. When a small area only is involved, and healing fails to occur, Powell recommends excision of the ulcerated tissues.

Bedsores.-These sores usually occur over the sacrum or hips in patients who are confined to bed for a considerable time, as the result of long-continued pressure, or in cases where the vital powers are depressed by adynamic diseases, and are also a frequent and troublesome complication in spinal injuries, in which cases they result from trophic disturbances. Their formation may be prevented in many cases by the use of air-cushions or of a water mattress, and by keeping the parts exposed to pressure scrupulously clean and frequently bathing them with stimulating lotions, such as alcohol, olive oil and alcohol (equal parts), or soap liniment. The parts should also be protected from pressure by the application of adhesive 
plaster, or, still better, soap plaster spread upon chamois skin. When a bedsore has actually formed-and in many cases its formation is very rapid and the slough will be found to involve a large surface of the skin over the sacrum, and to extend down to the bone-we have present a very serious complication, and one which requires most careful treatment.

The dressing of a bedsore before separation of the slough consists in relieving the part from pressure by the use of an air-cushion placed under the buttocks, and the application of a moist antiseptic dressing until the slough has separated. When the slough has become detached, the ulcer remaining should be well irrigated with a $1: 2000$ bichloride solution, and the granulations touched with a 5-grain solution of nitrate of silver; and aristol, or boric acid ointment spread upon lint, should be applied to the surface of the ulcer, and a piece of soap plaster a little larger than the ulcer should be placed over this dressing and held in place by broad strips of adhesive plaster. This dressing should be renewed every day or every other day, and means should be adopted to protect the parts from further pressure, and the constitutional condition of the patient should be improved by the administration of a nutritious diet, tonics, and stimulants. The application of the galvanic current has been employed to promote healing of the ulcer in obstinate cases.

Sprains.-Sprains of the joints from twists or other external violence resulting in the stretching or laceration of the ligaments are injuries which require careful dressing.

Sprains may be first treated by the application of coldwater or hot-water dressings for a few hours, or by the application of lead-water and laudanum, the joint being kept at rest by the use of a splint or by confining the patient in the recumbent posture in the case of sprains of the joints of the lower extremities.

After a few days' use of the lead-water and laudanum dressing the swelling usually subsides, and the joint may be fixed by the application of a moulded soap-plaster splint or felt splint held in place by a firmly applied roller-band19 
age, which should be worn for a week or ten days; in ordinary cases after this time the splint may be removed and the patient should be encouraged to use the joint. In cases of severe sprains, on the other hand, the pain and swelling persist for some time, and here the fixation of the joint by a plaster-of-Paris bandage will be found useful for a few weeks.

In the chronic stage of a sprain, after all dressings have been removed, the methodical use of massage is often most beneficial; and after the parts have been thoroughly manipulated a flannel bandage should be applied, which, by its elasticity, gives a certain amount of support to the parts.

Strapping.-The treatment of sprains which I have found the most satisfactory; both in the acute and chronic stage, consists in the use of strapping. Strips of adhesive or rubber adhesive plaster one and $a$ half inches in width are applied around the joint, and are made to extend some distance above and below it; a gauze bandage is next applied over the straps, and the patient is allowed to use the part as soon as he can do so without discomfort (see page 131).

Sprain-fracture.-Under this name Mr. Callender has described an injury which consists in the separation of a ligament or tendon from its point of insertion into a bone, with the detachment of a thin shell of the bone; this injury is apt to occur about the ankle-joint, knee-joint, elbow-joint, and wrist-joint, and the treatment is the same as that of an ordinary fracture in the same locality. This injury is probably much more common than is generally supposed in connection with sprains of the joints, and is, I think, in many cases the canse of tardy restoration of the function of sprained joints, this injury being overlooked, simply being treated as a sprain, and the patient being encouraged to use the part before union of the bone has been accomplished.

Strains of Muscles and Fascia.--These vary in severity from simple stretching of the fibres to absolute rupture, and should be treated by putting the parts at rest and by the application of pressure by means of adhesive straps 
or of a bandage; in strains of the muscles and fascia of the back the use of broad strips of adhesive plaster, applied as in cases of fracture of the ribs, will be found most satisfactory. In the treatment of the later stages of these injuries the employment of massage will often be followed by good results.

\section{REMOVAL OF FOREIGN BODIES.}

Foreign bodies may enter the body through the normal openings or by means of wounds. The location of these bodies may be made by palpation, a probe or by an $x$-ray examination.

Foreign Bodies in the Urethra.-These are generally introduced into the urethra for purposes of sexual excitement and often slip from the grasp of the individual and pass within the meatus or into the bladder. If lodged in the urethra they may be removed with delicate forceps, firm pressure being made upon the base of the penis to prevent it slipping into the bladder. A pin lodged in the urethra with its point toward the meatus may have its point pushed through the urethra and skin, and being reversed the head may be made to present toward the meatus when it can be removed with forceps or by manipulation. Pins, needles, pencils, hair-pins, and many other objects are often removed from the urethra.

Foreign Bodies in the Bladder.-If there is doubt as to the nature of the foreign body in the bladder a cystocopic or $x$-ray examination should be made. In the male a small body may be grasped with a lithotrite and crushed and removed, or may be removed without crushing. In the female dilitation of the urethra will often permit the introduction of the female urethroscope and inspection and removal of the body with forceps. If the body is irregular in shape or is encrusted with urinary salts it should be removed through a suprapubic incision into the bladder.

Metallic Rings are sometimes slipped over the penis, or strings may be tied lightly around the organ, and if they 
fit tightly the resulting eongestion quickly swells the glans and tissues beyond the ring so that it becomes buried and it is impossible to remove it. If the constrietion is not promptly removed gangrene results. A flat divider should be carefully passed under the ring to prevent injury of the skin, and it should be divided at one or two points by a file or small steel saw, when it can be removed without difficulty. Strings can be removed by cutting them.

Foreign Bodies in the Rectum.-These may consist of objects introduced into the rectum to excite sexual feeling or to assist in defecation, or masses of feeal matter may be allowed to remain in the rectum until they become so firm that they require the same treatment for their removal as foreign bodies. Hard and fecal masses may sometimes be grasped with foreeps and removed or be broken up with the finger, and removed by the finger, or by a stream of water. It is usually well after locating the position of the foreign body to anæsthetize the patient and dilatate the sphineter musele. The body can then be removed with the finger or foreeps. If the body is irregular in shape a speeulum may be introduced or the body may have to be broken or cut into fragments before it ean be removed. Care should be taken to do the least possible damage to the rectal mucus membrane that is possible, to avoid infection.

Foreign Bodies in the Vagina.-Foreign bodies are frequently introduced into the vagina to produce sexual exeitement, or they may consist of bodies introduced to prevent uterine displacement. Pessaries often are found whose presence had been forgotten by the patient. If the body becomes buried in the vaginal walls diffienlty in urination and purulent discharge are the prominent symptoms. The vagina should first be irrigated and an antiseptic solution and a speculum introduced to locate the position of the body. Negleeted pessaries can usually be removed without difficulty, but if embedded for a long time it may be neeessary to divide them at one or two points with eutting foreeps. 'They can then be removed with forceps, care being taken to do the least possible damage to the vaginal walls. 
Foreign Bodies in the Pharynx. - Small foreign bodies such as pins, fish-bones, pieces of bone, pieces of straw, jack-stones, etc., may become lodged in the pharynx and soon become embedded by swelling of the mueus membrane. The sensation of the patient will often assist in locating the position of the body.

The pharynx should be inspected with the aid of reflected light and explored by the finger. When the body is located it can usually be removed with forceps. I have seen a jackstone embedded behind the larynx, which was removed with great difficulty.

Foreign Bodies in the Oesophagus._Foreign bodies may be arrested at any point of the œsophagus ; but are apt to lodge opposite the cricoid cartilage or near the cardiac orifice of the stomach. A great variety of objects have been found lodged in the resophagus, coins, buttons, pieces of bone or meat, pins, safety-pins, and plates with artificial teeth, etc. The body should be removed as soon as possible, to prevent ulceration and perforation of the osophagus. A soft, smooth body may be dislodged and pressed downward into the stomach with a bougie. The body may be located by the use of Jackson's œsophagoscope and forceps. If, however, the body is of considerable size and irregular in shape, like a tooth plate, it is better to remove it by operation, external œsophagotomy, if low down in the œesophogus, it may be necessary to perform thoracic œesophagotomy.

Foreign Bodies in the Stomach.-Bodies which have entered the stomach often pass into the intestines and escape from the rectum. If the body is of such a shape that it is apt to be arrested in the stomach or intestines, it should be located by an $x$-ray examination, and should be removed by gastrotony.

Foreign Bodies in the Intestines.-Bodies usually pass with great facility through the intestinal tract and escape from the rectum. Purgatives should not be given, rather a dict which produces inereased fecal matter. Intubation tubes are frequently swallowed and pass without difficulty. An $x$-ray examination taken at intervals will 
show the change in the position of the body in the intestinal tract.

If arrested at any point and symptoms of obstruction are present, it should be removed by abdominal seetion and enterotomy.

Foreign Bodies in the Trachea and Larynx.-Bodies lodge in the larynx and trachea produce violent eoughing effects and soon dyspnœa develops. The bodies most commonly found are, grains of corn; seeds, pins, safetypins and fragments of bone. They are often dislodged by eoughing and expelled. They may be removed by using the laryngeal mirror and forceps or by the use of Jackson's laryngoseope and forceps. If the dyspnoea is urgent tracheotomy should be performed and the body removed through the tracheal wound or later by tracheal forceps.

Foreign Bodies in the Bronchi.-A foreign body which has passed below the trachea usually lodges in one of the larger divisions of either bronchus, usually the right bronchus. If the breathing is much embarrassed a low tracheotomy should be performed and the body may be grasped with forceps through the tracheal wound and removed. Brouchoscopy by Jackson's method has been used successfully for the removal of bodies impacted in the bronchi.

Foreign Bodies in the Eye.-Bodies such as particles of sand, cinders, fragments of steel, often lodge in the eye, and may be adherent to the conjunctiva or embedded in the cornea. The eye should be inspected with a good light, and the lids turned if necessary.

The body may be removed if in the conjunctiva or cornea by touching it a whisp of cotton twisted on a probe or match stick, if embedded in the eornea a few drops of a 2 per cent. solution of cocaine should be dropped into the eye and the body removed with an eye spud.

If the body has penetrated the cornea the case should be referred to a specialist.

After removing the foreign body the eye should be frequently douched with boric acid solution. 
Foreign Bodies in the Nose.-Foreign bodies, such as peas, grains of corn, beans, buttons, beads and pencils are often introduced into the nasal canals, especially by children. Their presence often causes no marked symptons, but if ulceration, purulent and bloodstained discharges occurs; a persistent unilateral nasal discharge in a child should always suggest the possibility of an impacted foreign body. The body may pass backward and lodge in the nasopharynx.

A dry body, like a pea or bean, may absorb moisture and swell so that it becomes very firmly imbedded, the impaction, in all cases, becomes firmer from the swelling of the surrounding mucous membrane. It is well to give an anæsthetic before attempting to remove foreign bodics from the nose.

The nasal cavity should be inspected through a speculum and the body grasped with forceps and removed, a small curette is sometimes usefin. When the body is in the nasopharynx the removal is very difficult. A month gag should be used, and the finger should be passed into the nasopharyux from the mouth when by manipulation and forceps it may easily be removed by way of the nasal cavity, or by the nasopharynx. An antispetic spray should be used after the removal of the body.

Foreign Bodies in the Ear.-Hardened wax, beads, seeds, and insects are often found in the ear. These may be removed by syringing the ear with warm water, and if this fails to remove them their removal may be accomplished by delicate angular forceps, with which the body is grasped. Live insects may be killed by dropping a little olive oil in the ear, and they then may be removed by syringing the ear with warm water. 



\section{PART III.}

\section{ASEPSIS AND ANTISEPSIS.}

\section{SURGICAL BACTERIOLOGY.}

Bacteria (Schizomycetes).-These are minute cellular organisms of microscopic size, elassified as belonging to the vegetable kingdom, among the fungi. They play an active part in the causation of the processes of fermentation and putrefaction, and are the causal agents of many varieties of diseases. The word germ is often used as synonymous with bacterium in speaking of the organisms that cause disease, but we must remember that certain pathogenic germs, as the hamatozoon malarice, the amoba coli, and the coccidia, are members of the animal kingdom and are not bacteria.

Bacteria may be divided into the lower and the higher bacteria. The lower forms are always unicellular, although in the process of growth cells may remain attached to each other; while the higher forms are filamentous, often branched, are made up of numbers of simple cells joined together, and the cells sometimes show a tendency to specialization. To this class belong the organism which causes actinomycosis, the actinomyces bovis seu hominis, and also the streptothrix madurce, the organism of Madura foot or mycetoma. The lower bacteria, with which we are mainly concerned, are unicellular and exceedingly minute, the round forms measuring not more than 1 micromillimetre $\left(\frac{1}{25000}\right.$ inch) in diameter, and, therefore, only capable of investigation under the highest powers of the 
nicroscope. When unstained they appear to be homogeneous, but by staining they can be seen to possess a cellwall or limiting membrane, not always well defined, called the ectoderm, enclosing the protoplasmic contents or endoderm, which contains no nucleus. The cell-wall is probably of a gelatinous nature, and when it is well defined the bacteria are said to be capsulated. In the protoplasm of the cell-body certain bodies, metachromic granules, are sometimes seen by staining, as well as other rotind or oval unstained spaces, which, when situated at the ends of a bacillus, are known as polar granules. Both of these are probably either the results of degenerative changes, or are artificially produced in drying.

Certain bacteria produce coloring-matters-red, yellow, and blue-many of which are allied to the lipochromes, a class of coloring-matters found in certain animal and vegetable organisms.

Unicellular bacteria are classified according to their shape into cocei, or round cells, bacilli, or rod-shaped cells, and spirilla, which are cylindrical cells of curved or spiral ontline. Motility in those bacteria which possess it is due to the presence of cilia or flagella. The ordinary mode of growth of bacteria is by division or splitting. Under circumstances unfavorable to growth they may also produce spores, but not as a means of multiplication, as one bacterium usually produces but one spore.

Spores.-These may be of endogenous or arthrogenous origin. Endogenous spores arise especially in the bacilli. They appear in the protoplasm of the cell as granules, which develop into round, oval, or short rod-shaped bodies, the remaining portion of the bacterium either persisting for a time or disappearing very soon. Arthrogenous spores appear to be cocci which have swollen, become more refractive, and are more resistant to unfavorable surroundings than the original coccus. Spores are highly refractive, and consist of a protoplasmic body with a dense surrounding membrane. They are very resistant to unfavorable surroundings, and are much more difficult to destroy by heat, chemical reagents, or drying, than are 
adult bacteria. When placed under circumstances favorable to their growth, the capsule splits, and a little bud appears and develops into an adult bacterium.

The ordinary method of multiplication of bacteria is by division or fission, one individual dividing into two, and these again into two more, the process sometimes taking place with great rapidity. 'The new cells may remain attached or separate, according to the nature of their limiting membrane. In the case of cocci, when forming pairs, they are called diplococci. They may also be tetragenous, or form chains, as in the streptococci and streptobacilli; or bunches, as in the case of the staphylococci. A zoöglea mass is formed by the cohesion of a large number of bacteria, where, owing to the gelatinous nature of their envelopes, they adhere to each other and appear to be imbedded in jelly.

Bacteria are found widely distributed in the air, the water, the earth, and wherever there is organic substance from which they can obtain their nutrition. They live by breaking up into simpler forms the complex organic compounds on which they are dependent for their carbon and nitrogen, being unable to extract the same from inorganic material. They also require moisture, being destroyed in time by drying. Those which require oxygen are called aërobic, while those which only grow when it is excluded are called anaërobic. Facultative aërobic and facultative anaërobic are terms used to designate those bacteria which can grow in its presence or absence; the first, however, growing best with and the latter best without it. Another division of bacteria is into saprophytic, or those living on dead organic matter, and parasitic, or those depending on living organisms, the latter embracing the pathogenic bacteria. The boundary line between these two classes is not well defined, however. A certain amount of heat is necessary to bacterial existence, the pathogenic germs growing best at the body temperature; they are destroyed by high temperatures, most of the pathogenic bacteria being killed between $122^{\circ}$ and $140^{\circ}$ F. $\left(50^{\circ}\right.$ and $60^{\circ}$ C. $)$. The spores are, as a rule, much 
more resistant to heat. Low temperatures tend to inhibit the growth of bacteria rather than to destroy their life. Direct sunlight also has an injurious action upon them.

Cultivation.-Bacteria are studied outside of the body by growing them on culture-media, which may be liquid or solid, proteid or carbohydrate-containing material. The media are sterilized and kept in tubes or dishes (Petri's dishes). A little of the culture or material to be studied is transferred to the culture-medium by a steriliced platinum wire (called an $\ddot{s} s$ ), and spread on the surface of the solid medium (stroke-culture), or plunged into it (stab-culture), or mixed with the fluid medium. The tubes or plates are then placed in an oven heated to the required temperature. The germs form colonies of characteristic size, shape, and coloring, and the different species may thus be isolated and studied. The liquid media include bouillon, peptone solution, and extracts of vegetable substances, as potato. Solid media include mixtures of beef-extracts with gelatin or agar-agar, coagulated blood-serum, and slices of potato or other vegetables.

Inoculation.-The action of bacteria and their toxins is studied experimentally by the injection of cultures, or of the body fluids or the juice of bacterially infected tissues into some of the lower animals. The animals usually employed are the guinea-pig, rabbit, mouse, rat, and pigeon. Injections are made with a sterile hypodermic syringe under the skin, into the peritoneal cavity, intravenously, and into the anterior chamber of the eye, or the skin may merely be scarified. The animal is carefully watched afterward, its symptoms noted, and when dead of the disease, or killed, cultures are made from the organs and the tissue-changes studied.

Staining.-In order to detect bacteria in the tissues, or to study and differentiate them from each other, it is necessary to stain them, and this is accomplished by the use of dilute aqueous or alcoholic solutions of the aniline dyes, counter-staining the tissues to make their detection easier. Bacteria differ widely in the facility with which they take the stains, some staining readily, while others require the 
action of heat or of a mordant; and they differ also in the tenacity with which they retain the stains in the presence of various reagents, as alcohol and the mineral acids. We are thus able to separate different bacteria by the use of special methods of staining and decolorizing. For example, the gonococcus, the bacillus coli communis, and the typhoid bacillus are decolorized by the use of Gram's method; while the bacilli of anthrax, tuberculosis, diphtheria, and tetanus are stained by it. The aniline stains most frequently employed are methylene-blue, gentianviolet, thionin, fuchsin, dahlia, and vesuvin.

Koch's Law.-To prove that a certain bacterium is the cause of a disease, the following rules have been laid down by Koch: The bacterium must first be found in the diseased person or animal. It must be cultivated outside of the body. When inoculated in pure culture in a healthy animal it must produce the original disease. From the body of the animal the original microbe must be capable of again being isolated.

Entrance of Bacteria into the Tissues.-Bacteria may gain entrance into the tissues of the body through the skin or mucous membrane, or by means of wounds or other primary surgical affections. The direct entrance of bacteria into the blood-current sometimes occurs, but is rare. When entrance occurs through apparently intact skin it is likely that small lesions of the skin are present and have been overlooked. The mucous membranes are less resistant to the entrance of bacteria. Fresh wounds present conditions most favorable for the entrance of bacteria into the system.

The relation of insects to the spread of infections agents has recently attracted much attention. It has been shown that insects not only transport disease-producing microorganisms, but may also be the agents of inoculation of pathogenic micro-organisms.

It has been shown that the location of entrance of bacteria bears a more or less direct relation to the severity of the infection. It has been demonstrated in animals that direct inoculation into the peritoneal cavity and general 
vascular system are followed by much more severe symptoms than subcutaneous inoculations.

Schemmelbusch has shown that the absorption of bacteria in fresh wounds by lymph and blood-vessels is very rapid, and that the absorption is markedly interfered with by the presence of a blood-clot. The surface of a healthy granulating wound also offers great resistance to the entrance of bacteria.

Intoxication and Infection.-Bacteria vsually gain entrance into the body through some break in the continuity of the skin or mucous membrane, especially the latter, owing to its being easier of penetration. They often enter through an open wound. Favoring elements are a weakened or diseased state of health of the individual, or an unusual virulence of the germ. If the gerns remain localized, and only their products are absorbed, the process is spoken of as intoxication. If the germs themselves enter the circulation, we have infection, although the term infection is used also by surgeons to denote the presence of bacteria in a wound, without necessarily or even usually implying their presence in the circulation. If the germ be pyogenic-that is, one that excites suppuration-the symptoms produced by the absorption of its products constitute sapromia; if the germ enters the circulation, we have septicamia; and if it finds lodgement in the tissues or organs and gives rise to secondary abscesses, we have pyoemia.

Elimination.-Bacteria are eliminated by the kidneys, the intestine, the salivary glands, in the bile and milk, and probably also by the sweat-glands. They frequently cause lesions in the eliminating organ.

Pathogenic Action.-The pathogenic action of bacteria is due to the formation of certain poisonous products secreted by them, or produced by their action upon the tissues. From the bacteria themselves, by their degeneration, we have also formed the proteins. The bacteria by their secretion produce the ferments, and, perhaps, the toxins; and by their action upon the tissues we have produced the ptomaïnes, amines, peptones, albumoses, fatty acids, etc. 
Toxins.-The toxins are produced by the pathogenic bacteria. They are poisonous when injected, even in very minute doses, acting after a period of incubation, and are looked upon by many observers as being of the nature of ferments. Others have classified them as toxalbumins or toxalbumoses. The different pathogenic bacteria elaborate their own specific toxins. Some of them have a local as well as a general action, producing inflammation, necrosis, etc., when injected into living animals.

Resistance of the Tissues to Bacteria.-That the introduction of bacteria into the body is not always followed by the development of disease is due to a number of circumstances, one of the most important being the resistance offered by the tissues. Certain of the lencocytes have what is known as a phagoeytic action-that is, the power to take into themselves and destroy by intracellular action the invading germs. The leucocytes appear to be attracted to the germs by a power residing in the bacteria, known as positive chemotaxis, their migration being accompanied by the nutritive changes constituting the process of inflammation, and in the case of pyogenic germs of suppuration. Inflammation seems to be a limiting and protecting process. The bacteria if very virulent may overcome the leucocytes, or repel them by the production of toxins, which are negatively chemotactic - that is, they repel the lencocytes and interfere with their phagocytic action, and we have in consequence a general invasion of the organism by the bacteria, often without any local inflammation. In addition to the phagocytic action of the leucocytes, the blood and fluids of the body have a certain germicidal power, said to be due to the presence of albuminous bodies-alexins. The presence in a wound of a foreign body favors the growth of bacteria, as does, to a certain extent, the presence of blood-clot or other material which may act as a culture-medium for the germs.

Immunity.-This consists in the freedom from liability to a disease, and may be natural or acquired. In natural immunity the person or animal is immune from birth; 
while acquired immunity may be the result of a previous attack of the disease or may be produced artificially. As examples of natural immunity we have that shown by the lower animals to syphilis and leprosy, and of man to certain diseases of the lower animals. One attack of smallpox, scarlet fever, or typhoid fever confers an acquired immunity on the patient which is usually permanent; while an attack of pneumonia, influenza, or diphtheria is followed by a period of temporary immunity.: Immunity may also be absolute or relative; the first being rare, the latter common, being overcome by unusual conditions. Artificial immunity is active or passive. Active immunity is obtained by the injection into animals of increasing doses of a pathogenic organism, or of its toxins, the dose being gradually increased until a high degree of immunity is obtained. This method is preventive of future attacks, but owing to its slowness is not useful against an existing disease. Passive immunity, which is less lasting than active immunity, is conferred by the injection into an animal of the serum of an animal that has been highly immunized by the previous method. The serum will destroy existing toxins and organisms, and confer temporary immunity against further infection.

Antitoxin.- The mechanism of the production of immunity is largely, if not altogether, dependent upon the formation, by the reaction of the tissnes to the toxins, of an albuminous body known as antitoxin. To the presence of this substance in the serum of an actively immunized animal is due its curative power when injected into an animal suffering from the sane disease. The antitoxin of diphtheria has been widely employed of late years with beneficial results, and the investigations now being carried on in tetanus, hydrophobia, antbrax, and other diseases, afford foundation for the hope that similar good results may be obtained with their antitoxins. A distinction is made between antitoxic serum and antimicrobic serum: the former being produced by the injection of toxins, and the latter by the injection of living bacteria. The antimicrobic serums tend to the destruction or 
paralysis of the miero-organisms, but not neeessarily of their toxins.

Opsonic Treatment.-The opsonic method of treatment is based upon the theory that the blood contains substances known as opsonins, whose function is to act upon bacteria in such a way as to make them more easily destroyed by the phagocytes.

The treatment consists of the injeetion into the subeutancous tissue of the patient of a sterilized emulsion of the bacteria with which the patient is infeeted. The injection of such an emulsion increases the opsonic power of the patient's blood, presumably by stimulating the opsoninforming elements of the body.

This emulsion is called a vaccine, and is preferably prepared, in most instances, from a culture isolated from the patient's lesion. Stock vaccines may be used when the isolation is difficult or prolonged, as in tuberculosis and chronic gleet; when the infection is so acute that treatment must be instituted at once, as in gonorrhœal conjunctivitis; when the virulence of the infecting organism is greatly reduced, as in chronic osteomyelitis; or when the organism has invariably the same characteristics, like the pneumocnecus.

The dosage and the intervals between doses are regulated by the opsonic index of the patient. The opsonic index is the relation between the opsonic power of the patient's blood serum toward the infeeting organism and the opsonic power of a normal person's blood serum toward the same organism. This index is determined by exposing a portion of the culture of the organism to the action of the patient's serum, in the presence of specially prepared polymorphonuclear leucocytes, and a sinilar portion to the action of a normal person's serum under the same conditions, and then counting the bacteria ingested by a definite number of the leucocytes in each preparation. The relation between the two counts is the opsonic index, and is expressed by the number obtained by dividing the patient's count by the normal count. For example, if 
200 bacteria were found in the normal count and 150 in the patient's, the index would be 150 divided by 200 , or .75 .

The more important surgical conditions in which this treatment may be of value are local infections due to the tubercle bacillus, the bacillus coli communis, the staphylococcus, streptococcus, pneumococcus, or gonococcus, such as arthritis, periostitis, osteomyelitis, peritonitis, appendicitis, cholecystitis, salpingitis, carbundle, furuncle, and ulcer.

Leucocytosis.-This consists in a marked increase in the number of the polynuclear leucocytes over the normal about 8000 in the circulating blood which is frequently observed in local inflammatory processes, in most acute infectious diseases, and in many toxic conditions. This increase occurs physiologically to the amount of 10,000 to 12,000 during the digestion of proteid foods, and the same increase is as frequently observed during the latter months of pregnancy. The leucocytosis of inflammation is caused by chemistoxis which is an attraction exerted by the products of bacterial activity upon the leucocytes in the bloodmaking organs which are probably stimulated to an increased rate of production.

The number of lencocytes per c.cm. of blood may be increased in inflammation from 8000 , the normal, to 15,000 or 50,000, the amount is, however, independent of the extent of the focus of inflammation. It is found in suppurative and gangrenous inflammations, in acute articular rheumatism, scarlet fever and pneumonia, after ether anresthesia, and is frequently observed after copious hemorrhage, and cases of sarcoma often show this condition. It is absent in typhoid fever and tuberculosis.

If a sudden overwhelming septicæmia accompanies the beginning of an inflammation, as in peritonitis, caused by intestinal perforation leucocytosis may fail to develop, and may be absent when an abscess exists, which is well encapsulated.

From these facts it is evident that leucocytosis is a 
symptom which may be very useful in diagnosis, but it must be interpreted with care.

A slight leucocytosis is of little value, but a rise above 15,000 if examinations at intervals show that the count is increasing, may prove of great diagnostic value.

\section{Varieties of Bacteria.}

The bacteria of importance surgically are those giving rise to ordinary suppuration, the gonococcus, the tubercle bacillus, the bacillus of malignant cedema, of glanders, of anthrax, of tetanus, of infectious emphysema, and the organisms causing actinomycosis and mycetoma.

Bacteria of Suppuration.-A large number of bacteria are capable of giving rise to suppurative inflammation, but the most important are the staphylococcus, especially the staphylococcus pyogenes anreus, and the streptococcus pyogenes or streptococcus erysipelatis, they

FIG. 234.

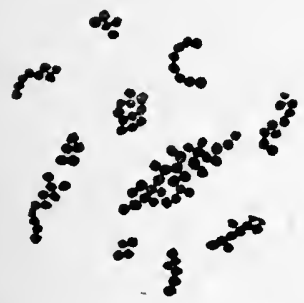

Staphylococcus pyogenes aureus.
FIG. 235.

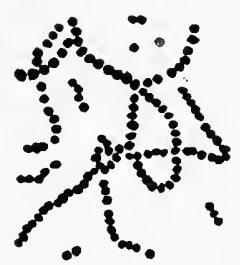

Streptococeus pyogenes.

being identical. Besides these, as rarer causes, we have the bacillus pyocyaneus, the bacillus coli communis, the typhoid bacillus, the gonococcus, the diplococcus pncumoniæ and the bacillus pneumoniæ (Friedländer).

Staphylococcus Pyogenes Aureus.-This bacillus, which causes 80 per cent. of suppurative inflammations, and is almost always the cause of osteomyclitis, grows in clusters (Fig. 234), can be cultivated on ordinary media, but best 
on agar, and forms small round colonies, at first whitish, later of an orange-yellow color. It is found in health on the skin, in the pharynx, and in the external extremities.

The staphylococcus pyogenes albus, or epidermis albus, as it is called, from being found in the epiderm, is less virulent than the preceding, and forms white colonies. It not infrequently is the cause of stitch abscesses.

Streptococcus Pyogenes. -This is a small round organism which forms chains (Fig. 235). It is found occasionally on mucous surfaces in health, and cause's dangerous phlegmonous inflammations. It also causes erysipelas, being identical with the streptococcus erysipelatis.

Bacillus Coli Communis.-This is a rod-shaped bacillus, and may be long and slender or short and rounded. It strongly resembles the typhoid bacillus. It is provided with flagella. It is found in the intestines in health, and seems to acquire virulent properties from inflammation or strangulation of the bowel, giving rise to appendicitis and peritonitis by migration through the diseased wall of the bowel or by escape through a rupture; it may also be

FIG. 236.

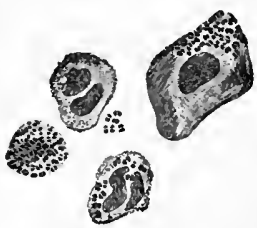

Gonococcus. (After Busm.) the cause of cystitis, pyelitis, pyelonephritis, and occasionally of localized abscesses.

Gonococcus.-This, the germ of gonorrhœe, is a kidney-shaped coccus, arranged in pairs, with the concave edges toward each other; the diplow cocci usually inhabit the pus-cells, but are occasionally free (Fig. 236). Besides specific urethritis, it causes salpingitis, oöphoritis, arthritis, endocarditis, conjunctivitis, proctitis, and other lesions.

Tubercle Bacillus.-This, the cause of tuberculosis, is a rod-shaped bacillus, sometimes slightly curved, 1.5 to 3.5 micromillimetres in length and 0.2 to 0.5 micromillimetre thick. It is not motile, and occurs singly, in pairs, and in groups; spore-production has not as yet been demonstrated (Fig. 237). Inoculation may be directly through a wound, or by inhalation, ingestion, or placental trans- 
mission, the last being rare. It may infect any organ of the body. It causes tuberculosis in many of the lower animals, cattle being especially liable to its infection.

Bacillus Mallei._Glanders is caused by this bacillus, which resembles the tubercle bacillus, but is shorter and thicker (Fig. 238). Infection of the mucous membranes of the respiratory tract and through the skin is not un-

FIG. 237.

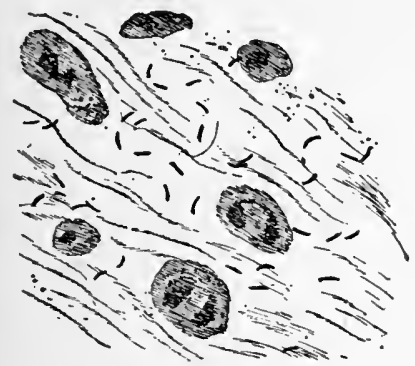

Tubercle bacilli.
Fig. 238.

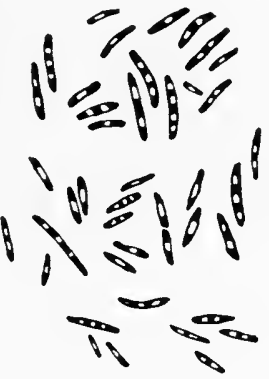

Bacillus mallel.

common in men who are exposed to infection from horses.

Bacillus Anthracis.-This, the cause of anthrax, is a very large, straight bacillus, usually from 5 to 20 micromillimetres in length, sometimes, however, attaining a length of 50 micromillimetres. It forms long chains and produces spores, which are very resistant (Fig. 239). Infection in man usually arises from handling infected skins and hides, and causes a local inflammation, with general septicæmia. Infection may also take place through the lungs or through the gastro-intestinal tract.

Bacillus of Tetanus.-This is a rod-shaped organism which, owing to the formation of a spore at one end which distends it, is often of a drumstick shape (Fig. 240). It is anaërobic, being found especially in garlen-earth, in the excrement of animals, and around stables. Infection follows wounds, especially punctured wounds by nails or 
splinters, which are liable to be contaminated from the earth; infection is also quite common in pucrperal women and in the newborn. Suppuration in a wound favors its development. The bacterium apparently remains localized, producing its characteristic symptoms by the action of very powerful toxins, of which two, tetanin and

FIG. 239.

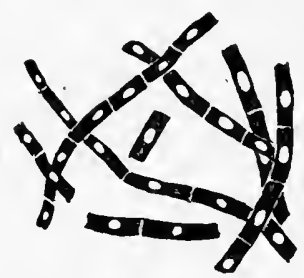

Threads of bac1llus anthracis containing spores.
FIG. 240.

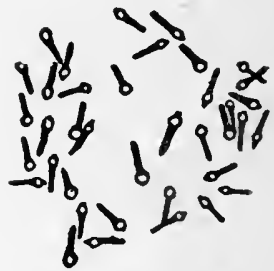

Tetanus bacillus.

tetanotoxin, have been isolated. An antitoxin has been isolated from immunized animals, and good results have been reported from its administration in individuals suffering from tetanus, but it has often proved disappointing.

Bacillus of Malignant Edema. - This resembles the

Fig. 241.

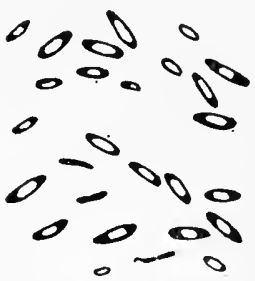

Bacillus of malignant œdema, spore stage. anthrax bacillus in appearance, being more slender, however, and, like it, has a tendency to form chains. It is motile, being provided with flagella, is anaërobic, and forms spores (Fig. 241). It occurs in the soil, in dust, and in the contents of the intestines of lower animals. In the lower animals it is the cause of the disease known as malignant œedema, which is associated with suppuration and necrosis of the subcutaneous tissues, emphysema, and gangrene. In man it has been found in certain cases of rapidly spreading traumatic gangrene and gangrenous enphysema, arising in connection with compound fractures and other deep punctured wounds. 
Bacillus Aërogenes Capsulatus.-This organism is from 3 to 6 micromillimetres in length, and may be found singly, clumped, or in chains. It is non-motile, anaërobic, and does not form spores. It finds entrance into the body through a wound or nlceration, external or internal, and its effects resemble somewhat those produced by the bacillus of malignant œedema, viz., necrosis, gangrene, and the production of gas, which in this case is found in any or all of the tissues and organs and in the blood, in the form of minute bubbles, in the walls of which the bacilli may be found. In man it produces the condition which has been described as gaseous gangrene, infectious emphysema, gas phlegmon, and emphysematous necrosis.

Actinomyces, or Ray Fungus.-This organism probably belongs to the higher order of bacteria, and occurs in yellow masses, which may be visible to the naked eye. The masses consist of organisms with diverging rays, consisting of threads with bulbous ends (Fig. 242). It

Fig. 242.
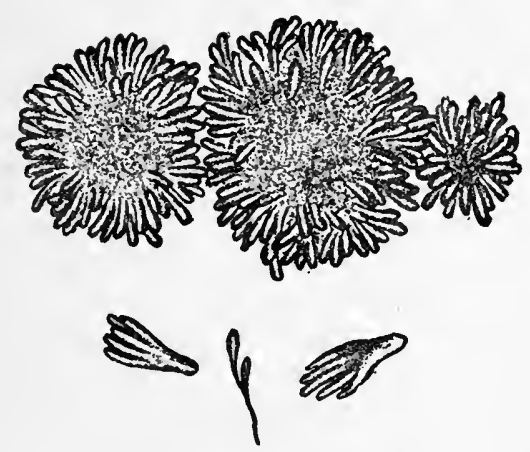

Actinomyces. (BAUMgarten.)

occurs rarely in man, commonly in the lower animals, from which it has been obtained in pure culture. When implanted in the tissnes, to which it is conveyed through a wound or carious tooth, sometimes apparently in seeds or in grains, it excites a chronic inflammation, with the 
presence of granulation-tissue, necrosis, and suppuration. In man it occurs most frequently in the mouth, tongue, and internal organs. In cattle it affects the jaws, causing "lumpy jaw."

\section{THEORY OF ASEPSIS AND ANTISEPSIS IN WOUND TREATMENT.}

Before the introduction of Lister's method of treating wounds, it was the rule in accidental and operative wounds to have profuse suppuration, fever, pain, and in many cases such wound complications as septicæmia, pyæmia, erysipelas, and hospital gangrene, and the mortality following operative and accidental wounds was very high. The mortality in eompound fractures from sepsis was formerly great, but by modern methods of wound treatment has been diminished to an insignificant percentage. The same diminished mortality has followed amputations and other wounds, accidental or operative.

Lister's method of wound treatment was largely based upon the idea that the infection of the wound occurred from contact with the air, which contained spores and germs, and his method of treatment was chiefly directed to their destruction. The air may be a medium of wound infection to a certain extent, for it has been demonstrated that dry air contains dust in which spores and bacteria are present in much larger numbers than in moist air, and such air coming in contact with an open wound deposits there numbers of bacteria, which may set up inflammatory changes. Koch later demonstrated the fact that atmospheric microbes were chiefly of an innocuous character, and that wound infection was generally caused by bacteria or spores being brought in direet contact with the wound by the clothing and skin of the patient, the instruments and the hands of the surgeon and assistants, and unclean surgical dressings.

Cheyne has shown that the relative number of bacteria entering the tissues is an important factor in producing suppuration and septic infection, for we know that bacte- 
ria may exist in an aseptic wound and yet the wound heal and remain aseptic, the antiseptic qualities of the bloodserum and the cell-activity in bealthy tissues being sufficient to destroy or remove a certain number of microorganisms, and suppuration or septic infection occurring only when the tissues are overwhelmed by the number of organisms or when their power of resistance is diminished by injury or disease. This explains the satisfactory behavior of wounds which pursue an aseptic course where very imperfect details of aseptic or antiseptic treatment have been employed. It may, therefore, be assumed that infection does not necessarily depend upon the presence of a few microbes, lout rather upon the quantity and quality of the germs which are present in the wound.

Pyogenic micro-organisms under different conditions may produce a series of different diseases, for it is now generally accepted that Fehleisen's streptococcus erysipelatis is identical with streptococcus pyogenes, which is recognized as the cause of very different inflammatory affections. The theory has been advanced by Reger that all the so-called pus-diseases are simply local expressions of a general infection caused by many different microorganisms.

Sepsis.-Sepsis is due to the entrance and multiplication of micro-organisms, or the absorption of their products in the body, and is characterized by local inflammation of the wound, and marked constitutional symptoms, such as fever, disorders of the nervous system, and inflammation of the viscera. Microbic infection represents a pathological process which causes serious wound complications, and differs materially from that process which attends the repair of wounds that run an aseptic course. Aseptic chemical irritation of the tissues may result in the production of a puruloid fluid, which is not jus, but merely a fibrinous exudation containing numerous cells, and does not produce infection if injected into animals. Acute suppuration in a wound is considered clinically to be always due to the presence of bacteria, for their exclusion will prevent its occurrence. 
Asepsis.-A sepsis aims at thorough sterilization of the field of opcration and of all objects brought in contact with the wound, and the exclusion of micro-organisms by occlusive sterilized dressings.

Antisepsis, on the other hand, has in view the destruction of micro-organisms by keeping germicidal agents constantly in contact with the wound. The object of antisepsis is, therefore, to produce asepsis.

No surgeon should undertake the performance of an operation or the treatment of an open wound without having clearly impressed upon his mind the important part that pyogenic and specific micro-organisms may play in the subsequent course of the wound.

\section{Methods of Disinfection or Sterilization.}

Since the majority of wound complications are due to the presence in the wound of micro-organisms, it is the duty of the surgeon to prevent their contact with it, or to employ means for their destruction. We must, however, employ means of disinfection or destruction of these microorganisms which will not have any injurious effect upon the tissues with which they come in contact. Mechanical disinfection or sterilization is not applicable to wounds, but is employed to remove any micro-organisms which may be present upon the objects which are to come in contact with the wound, namely, the hands of the surgeons and assistants, instruments, and the skin surrounding the wound. Mechanical disinfection is accomplished by the use of friction with a brush, soap, and water. Germicidal solutions may be used for disinfection of wounds, but are most useful in the disinfection of the hands of the operator, the skin of the patient, the instruments, and the dressings. If these have been carefully employed before the wound is made, their subsequent use in the wound is usually unnecessary.

Some forms of bacilli contain spores which resist the action of germicidal substances, while the bacilli them- 
selves are readily destroyed by these agents: the surgeon should, therefore, employ that means of disinfection which is generally applicable to the destruction of both bacilli and their spores. The bacilli of anthrax, tuberculosis, and tetanus contain spores; hence to destroy these organisms is a matter of more difficulty than to render harmless such micro-organisms as staphylococcus pyogenes aureus, albus, and citreus, streptococcus pyogenes and streptococcus erysipelatis, and the bacilli of diphtheria and glanders, which contain no spores.

Heat when used as a germicide cannot be applied to the wound itself, except in cases where a limited surface of the wound may be touched with the hot iron. Heat can, therefore, be used only for the disinfection of substances coming in contact with the wound, and for this purpose it is employed in the form of steam, dry heat, or boiling water.

Sterilization of the wound or the substances coming in contact with it may be accomplished by using either the aseptic method or the antiseptic method, and at the present time these two methods are to a certain extent combinedthat is, it is impossible to be strictly aseptic withont employing means of disinfection by the use of antiseptics. The aseptic method, which employs germicidal substances only for the purpose of sterilization of objects coming in contact with the wound when their disinfection by heat is impossible, is the method which has generally been adopted.

Antiseptic Method.-In the antiseptic method the sterilization of the field of operation, the hands of the surgeon and assistants, the instruments, ligatures, sponges, and sutures, is accomplished by the use of germicidal solutions, and, in addition, the wound is irrigated frequently during the operation with germicidal solutions, and is afterward covered with dressings impregnated with germicidal substances. The antiseptic method was that first employed, and, recognizing its value in surgical procedures, many surgeons still continue to employ this method; but it has certain disadvantages. Recent inves- 
tigations have shown that many germicidal substances have not the power which was formerly attributed to them, as they only arrest bacterial development; many chemical germicides causc the formation of a dense layer of coagulated albumin around albuminous substances, and also fail to destroy micro-organisms associated with fatty or oily substances. Chemical germicides may also form combinations in the tissues with substances with which they come in contact, seriously impairing their germicidal action. Antiseptic substances which are active as germicides often canse irritation of the surface of the wound, interfering with its repair.

It has been shown that irrigation of a fresh wound with a $1: 10,000$ solution of bichloride of mercury is followed by distinct evidence of superficial necrosis of the tissues. Antiseptic irrigation of wounds is apt to cause very free oozing of serum, which necessitates the use of drainage, and makes frequent dressing of the wound necessary. Many antiseptic substances produce marked toxic effects upon the patient, and also cause severe irritation of the skin with which they come in contact.

Aseptic Method.-In employing the aseptic method in the treatment of wounds, the field of operation, the hands of the surgeon and assistants, the instruments, ligatures, sponges, and sutures, are sterilized by the use of germicidal solutions and heat, and after this has been accomplished, relying upon the completeness of the sterilization, no germicidal substances are bronght in contact with the wonnd, sterilized water or sterilized salt solution being used if it is necessary to flush the wound, and the dressings employed are those only which have been sterilized by moist or dry heat. The advantages of the aseptic method are as follows: the method is applicable to all parts of the body; wounds treated by this method heal more promptly and do not require frequent dressing; there is no risk of toxic effects, and there is no irritation of the skin by the dressings. Dry sterilized dressings are efficient to produce absorption, and at the same time the dryness may be a factor iu the destruction of germs, 
for depriving bacteria of moisture robs them of one of the conditions necessary to their existence. The aseptic method is, therefore, to be preferred to the antiseptic method in the treatment of wounds wherever it is possible.

\section{Agents Employed to Secure Asepsis.}

A great variety of agents possessing more or less germicidal properties have been at different times employed in the practice of aseptic or antiseptic surgery; those most employed at the present time are lieat, bichloride of mercury, carbolic acid, iodoform, formalin, betanaphthol, formaldehyde, chloride of zinc, acetate of aluminum, peroxide of hydrogen, kreolin, permanganate of potassium, sulphocarbolate of zinc, salicylic and boric acids, acetanilid, aristol, and certain silver salts.

Heat.-The most reliable and universally available agent for the destruction of micro-organisms is heat, either dry or moist; many forms of bacteria are rendered inert at a temperature of $140^{\circ} \mathrm{F}$, and none can withstand the application of moist heat at $212^{\circ}$ F. continued for a short time. Spores which will resist the action of powerful germicides for a considerable time are destroyed by boiling for a few minutes. Dry heat is not as efficient for sterilization as moist heat, for some spores will resist dry heat of $284^{\circ} \mathrm{F}$. for three hours. As moist heat is the most efficient sterilizer, it should be preferred, and can always be made use of for this purpose by

FI G. 243.

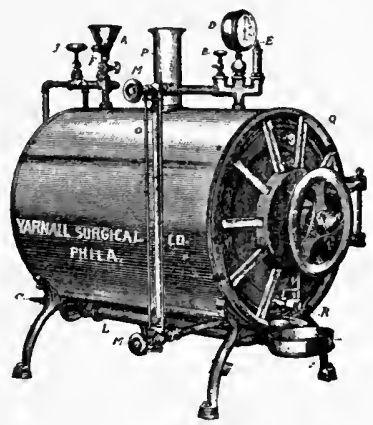

Steam sterllizer. boiling the instruments and dressings for a few minutes; and if for any reason it is thought advisable to employ dry heat as a sterilizer, this may be made use of by baking the instruments or diressings in a hot oven. The best results may be obtained by the use of one of the various dry or moist sterilizers (Fig. 243). An improvised sterilizer may be made by placing a perforated metal stand inside a large 
kettle, so that only the steam comes in eontact with the instruments and dressings.

Bichloride of Mercury.-This is employed as an antiseptic in watery solutions varying in strength from $1: 500$ to $1: 10,000$.

The solution of $1: 1000$ to $1: 1500$ is used only for the irrigation and disinfeetion of the hands and skin; for the irrigation of wounds, a solution of $1: 2000$ or $1: 4000$ may be employed. At the present time bichloride solutions are not frequently used in fresh wounds, on account of their irritating effects. Where continuous irrigation is kept up, or where it is employed in large cavities, a still weaker solution, $1: 5000$ to $1: 10,000$, should be employed.

In using bichloride solutions the surgeon should watch the patient carefully for signs of poisoning due to absorption of the bichloride of mereury ; the symptoms denoting this are vomiting, fetid breath, salivation, inflammation of the gums, diarrhoea, blood-stained stools, and bleeding from the mouth and nose. Locally the use of moist bichloride dressings may cause well-marked dermatitis. The continuous application of biehloride solution to the hands of the surgeon eauses the skin to beeome roughened and blackens the nails.

In preparing solutions of biehloride of mercury for use, it will be found convenient to have a concentrated solution of the salt in aleohol, 1 part of the bichloride to 10 parts of alcohol; this can be kept in a well-stoppered bottle, and to it should be added one teaspoonful of common salt, which prevents disintegration of the mercuric compound. One teaspoonful of this solution added to one quart of water makes a $1: 2500$ solution.

A 10 per cent. bichloride solution may be made as follows:

$$
\begin{aligned}
& \text { Bichloride of mercury . . . . 2 } 2 \text { parts. } \\
& \text { Sodium chloride . . . . . } 1 \text { part. } \\
& \text { Dilute acetic acid . . . . . . . } 1 \text { " } \\
& \text { Aquæ dest. . . . . . . . . } 16 \text { parts. }
\end{aligned}
$$

By adding water in an appropriate quantity, a $1: 1000$ or $1: 2000$ solution can be made.

Or the solution may be prepared with tartaric acid in the proportion of 5 parts of the acid to 1 part of bi- 
chloride of mercury, the following formula being employed :

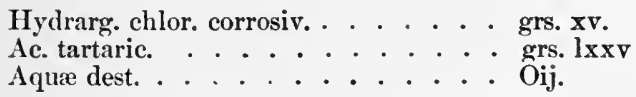

Pellets containing a definite amount of bichloride of mercury compounded with a few grains of common salt of muriate of ammonium, which, when dissolved in a definite quantity of water, make a solution of $1: 1000$ or $1: 2000$, will also be found very convenient for the preparation of solutions. The pellets should also contain a little coloringmatter, which gives a faint color to the solution and serves to distinguish it from other solutions.

Carbolic Acid.-This drug is employed in solutions of $1: 20$ or $1: 40$. The stronger solution, $1: 20$, is usually employed to sterilize instruments, the latter being allowed to remain in this solution for thirty minutes before being used. As a carbolic solution of this strength benumbs and eracks the skin of the hands of the operator, it should be diluted just before the instruments are required, by adding an equal quantity of boiled water, making it a $1: 40$ solution. The rusting of steel instruments and the lulling of the edges of knives by exposure to carbolic acid may be prevented by the addition of 5 per cent. of sodinm carbonate to the solution.

The $1: 40$ or $1: 60$ solution is used for the irrigation of wounds and the washing of sponges. As carbolic acid in strong solutions is a local caustic and coagulates albumin, it should not be used in fresh wounds. A realy method of making a 5 per cent. carbolic solution is to add one tablespoonful of carbolic acid to one pint of hot water.

In using carbolic acid solutions continuously, the surgeon should be on the watch for symptoms of poisoning, which will be manifested by dark-colored urine, headache, dizziness, vomiting, and in severe cases bloody diarrhœa, hæmoglobinuria, and death from collapse. Carbolic acid solutions should be used with great caution in young children, as they seem to be more susceptible than adults to its constitutional effects. 
The use of weak solutions of carbolic acid seems to involve more risk of toxic action than does the employment of the pure drug, the superficial layer of tissue being coagulated by the latter, so that absorption of the drug is prevented. Gangrene of the skin and subjacent tissues has frequently been observed to follow long-continued use of quite dilute solutions of carbolic acid or of ointments containing small quantities of the drug. Cases of gangrene of the fingers and toes from this cause are not infrequently seen.

Iodoform.-Iodoform has been shown by experimental research to possess little direct germicidal action, but in spite of this fact clinical experience has proved that it possesses powerful antiseptic properties, due, as shown by Behring and De Ruyter, not to the destruction of germs, but to its undergoing decomposition in their presence, and thus rendering inert the ptomaines which have resulted from the germ-growth. It may be rendered absolutely sterile by exposing it to heat, and, as it is easily decomposed, fractional sterilization may be employed, or by washing it in a 1:1000 bichloride solution; it should then be dried and kept for use in closely stoppered bottles. Iodoform is often employed in the form of a powder as an application to wounds, and is frequently used in aseptic wounds which are liable from their position to become infected, such as those about the mouth, rectum, and vagina, and is especially useful as a dressing in infected wounds and in tubercular or syphilitic ulcers and in bone cavities. In operations upon the mouth, anus, rectum, uterus, and abdominal cavity iodoform gauze packing is largely employed, and serves to keep the discharges from becoming foul, thus often preventing septic intoxication; it must, however, be used with caution in the mouth. Iodoform collodion, made by adding iodoform, gr. xlviii, to collodion, $\mathbf{f} \not \mathbf{j}$, is a useful dressing in superficial wounds. Iodoform may also be employed in the form of an ethercal solution, iodoform, gr. xv; ether, fäj, as an application to wounds or ulcers. An emulsion of iodoform in glycerin, iodoform, $3 \mathrm{j}$; sterilized glyceriu, $3 \mathbf{x}$, or an emul- 
sion of iodoform made by adding sterilized iodoform, $3 \mathrm{j}$; to boiled olive oil, $3 x$, is much employed as an injection in the treatment of tubercular abscesses and joints. For packing cavities, a 5 per cent. gauze is best; a 10 per cent. gauze is too strong except in small amounts. For large cavities a Mikulicz pack, consisting of a bag of iodoform ganze stuffed with sterilized gauze, may be employed.

Numerous cases have been reported in which toxic symptoms have followed the use of iodoform, such as urticarial eruptions, dermatitis, headache, depression, delirium, mania, debility, and sleeplessness. Elderly persons and ir. fants are very susceptible to the toxic action of iodoform.

Formaldehyde.-This is a pungent, penetrating gas, possessing valuable antiseptic properties, which is principally used for the disinfection of elothing, instruments, bedding, and rooms. The gas is generated in a lamp or generator by passing the vapor of methyl alcohol over a coil of glowing platinum wire or gauze, or over platinized asbestos.

Formalin.--This is a 40 per cent. solution of formaldehyde gas in water, and has valuable antiseptic properties. A solution of this strength is a powerful irritant, and should not be used in the treatment of wounds. It may be used in a 2 per cent. solution to disinfect wounds or instruments, or in 0.25 per cent. solution for irrigation. Brewer recommends a 1 per cent. solution applied for three minutes to disinfect the skin, a 2 per cent. solution, applied under anæsthesia, to sterilize infected tissues, and 0.3 per cent. solution for gauze.

Beta-naphthol.-Beta-naphthol, in a $1: 2500$ solution, is employed for much the same purposes as bichloride of mercury solutions ; it is not, however, so powerful a germicide. It is employed in irrigating large cavities, because it is not a poisonous agent, and is especially useful as a bath for instruments, as it does not corrode them, as do sublimate solutions. It may be employed as a dustingpowder on sloughing surfaces, and especially to wounds exposed to feces or urine. It also possesses the advantage 
over a carbolic acid solution of not irritating the skin of the surgeon's hands.

Silver Salts.-Silver lactate (actol) and silver eitrate (itrol) are two antiseptics which have been recommended by Credé, who considers their germicidal properties superior to those of bichloride of mercury. These salts may be used in a 1:4000 or 1:8000 solntion, which should be made with water free from chlorides, which precipitate the silver; distilled water should be employed. Credé speaks highly of an ointment made of metallic silver, which may be employed as an inunction in septic diseases.

Acetanilid.-This preparation possesses antiseptic properties, and is frequently used as a substitute for iodoform. It may be used in the form of powder as an application to suppurating or ulcerating tissues, but in tubercular conditions is not as satisfactory as iodoform.

Chloride of Zinc.-Chloride of zinc, in a solution of 30 to 40 grains to water $\mathrm{f} \overrightarrow{\mathrm{z}}$, is a very powerful antiseptic. When employed upon raw surfaces it produces marked blanching of the tissues; it is especially useful in wounds which are.infected or which have been exposed to infection. I have found it by all means the best application for the poisoned wounds which are received in dissecting dead bodies and in operating. In such cases the whole cavity or surface of the wound should be washer with a 30-grain solution, and then the wound should be dressed with moist bichloride ganze.

Sulphocarbolate of Zinc.-This drug has been found to possess more decided antiseptic properties than the chloride of zinc, and is much less irritating. It may be used in the same strength and for the same purposes as the latter drug.

Acetate of Aluminum.-This drug is used in solution, and is prepared as follows: aluminis, $3 \mathrm{vj}$ ( 24 grammes); plumbi acetatis, 3ixss (38 grammes); aque, Oij (1000 grammes). Mix, and filter after standing twenty-four hours. It has decided germicidal qualities, is employed for irrigation and moist dressing where carbolic or bichloride 
solutions cannot be used, and is by all means the safest and best antiseptic substance for wet dressings.

Peroxide of Hydrogen.-Peroxide of hydrogen is em- ployed in what is known as the 15-volume solution. It may be used in this strength or may be diluted. It seems to have a direct action upon pus-generation by destroying the micro-organisms of pus, and is frequently employed in the sterilization of sinuses or suppurating cavities, such as remain after the opening of abscesses or result from diseases of or operations upon the bones. It is injected into the simuses and cavities by means of a glass syringe, or may be applied to open wounds in the form of a spray. Its action is shown by the escape of bubbles of gas, which cleanse suppurating surfaces or sinuses mechanically, and it should be used as long as these continue to escape.

Pyrozone.-Pyrozone possesses the same qualities as the peroxide of hydrogen, and apparently to a somewhat higher degree, and is used for the same purposes.

Kreolin.-This substance is obtained from English coal by dry distillation, and has been found to possess powerful germicidal properties; it is non-irritating and practically non-toxic. It is insoluble in water, but forms an emulsion with it which possesses marked antiseptic properties. It is especially useful as a deodorant in offensive malignant ulcers. It may be employed for the same purposes as carbolic acid. It is used in an emulsion, in strength of from 2 to 5 per cent., and is employed in the irrigation of large wounds or cavities of the hody, and has been most favorably recommended in gynecological practice.

Boric Acid.-This drug has not very marked antiseptic qualities, and is usually unirritating even in saturated solutions; but occasionally it produces marked irritation of the skin. It is frequently employed in a 5 per cent. solution to cleanse and disinfect mucous surfaces and large cavities. It is often employed to wash out the bladder before the operation for the removal of calculi or growths from that organ. In the dressing of superficial wounds, or in wounds in which the bichloride or carbolic acid 
dressings produce irritation, an ointment of boric acid, 1 part, to petrolatum 5 parts, will be found very satisfactory.

Boro-salicylic Powder.- This powder, which ensists of 4 parts of boric acid to 1 part of salicylic acid, is used as a dusting-powder and as a dressing for wounds. It has been recommended highly by Senn in the treatment of fresh wounds.

Salicylic Acid.-Salicylic acid does not have very marked antiseptic qualities, but possesses much less toxic action than carbolic acid, and is used for somewhat the same purposes. Its antiseptic power is said to be increased by the addition of boric acid, and a boro-salicylic lotion (Thiersch's solution) is prepared by addling salicylic acid, 1 part; boric acid, 6 parts ; to hot water, 500 parts, making a bland solution, which, when reduced to 25 to 50 per cent. of the original strength, may be used for irrigation of the bladder or the peritoneal cavity.

Permanganate of Potassium. - This drug, owing to its rapid absorption of oxygen, acts as an antiseptic, and is often employed for the disinfection of foul wounds and ulcers. It is employed also in solution for washing the operator's hands and for the washing of sponges. It is practically non-irritating, and may be used in quite concentrated solutions, but is usually employed in the following strength : permanganate of potassium, $3 \mathrm{j}$; water, $f \mathbf{z j}$. One fluidrachm of this solution to a pint of water makcs a $1: 1000$ solution.

Aristol.-Aristol, which is a compound of iodine and thymol, possesses germicidal properties, and has been introduced as a substitute for iodoform. It has the advantage over iodoform of not being poisonous, and is also without disagreeable odor. It may be employed for the same purposes as iodoform, and it seems to be particularly useful as a dressing to chronic and specific ulcers.

Xeroform.-This is a combination of bromine, carbolic acid and bismuth. It possesses marked antiseptic properties and is frequently used as a drying powder in the treatment of wounds or may be combined with petrolatum and used as an ointment in the dressing of uleers. 
Sodium Chloride.-This salt has no direet antiseptie action, but is used in the preparation of normal salt or saline solution, the strength of which is 0.6 per cent.

Saline Solution.-This is prepared by adding 6 drachms of sterilized sodium chloride to 1 litre of distilled water, which is contained in a sterilized oval glass flask. The mouth of the flask should be plugged with sterilizerl cotton, and a piece of ganze fastened over the mouth and neck of the bottle. The solution should be exposed to steam sterilization one-half hour on two successive days. Saline solution is non-irritating, and is frequently used in the irrigation of fresh wounds, to remove foreign bodies or blood, and for the eleansing of mucous and serous surfaces. Its utility by intravenous injection or infusion is well recognized (see page 155). In emergeneies a solution prepared by adding a drachm of common salt to a pint of water which has been sterilized by boiling, may be employed.

Sterilization of Water.- Water may be rendered absolutely sterile by boiling from fifteen to thirty minutes. It should be distilled or filtered before being boiled, to remove any inert matter which is not desirable in wounds. After being boiled, it should be placed in sterilized glass flasks, and corked with sterilized cotton, the months of the flasks also being eovered with several layers of gauze. It is employed for the irrigation of wounds and of mucous and serous surfaces.

\section{PREPARATION OF MATERIALS USED IN ASEPTIC OPERATIONS.}

Sponges.-Marine sponges are considered by some surgeons the best materials for the purpose of sponging, but their satisfactory sterilization is often a matter of difficulty. It is better to use a cheap grade of sponges, and to use them only onee. The sterilization of sponges by boiling destroys to a certain extent their elasticity and their absorbent power. Schimmelbusch recommends the following method: The dried sponges are freed from dirt or sand by beating, and are then soaked for several days in 
cold water slightly acidulated with hydrochloric acid, being kneaded from time to time. They are next thoroughly washed in cold and in warm water, wrapped in a linen sheet, and placed in a boiling 1 per cent. soda solution; the solution should not be allowed to boil after the sponges are placed in it. They are allowed to remain in this hot solution for thirty minutes, are then washed in boiled water to remove the soda, and placed in a 0.5 per cent. carbolic acid solution until needed.

Gauze Pledgets or Pads.-On account of the difficulty in satisfactorily sterilizing sponges, as well as of their expense, folded gauze pledgets have largely superseded them.

Gauze Pledgets._Gauze pledgets are prepared by cutting a piece of gauze, composed of from twelve to sixteen layers, in pieces six inches square; the four angles of these pieces are then tied together or secured by a few stitches.

Gauze Pads.-Gauze pads are marle from a piece of gauze composed of from sixteen to twenty layers cut the desired size, the different lavers in each pad being quilted together by a few stitehes, and the edges loosely whipped with a thread to prevent them from fraying. Gauze pads are used as a substitute for the flat sponges formerly employed in abdominal surgery, and for the drying of wounds. Where pads are used in abdominal operations, to prevent them from being lost in the abdomen it is well to have sewed to each pad a piece of tape twelve inches in length, which is allowed to protrude from the wound, and to which a hemostatic forceps is attached. The pads or pledgets may be sterilized by boiling or by exposure to steam or dry heat in a sterilizer, or may be sterilized and preserved for some time in a 1:2000 bichloride solution. When so preserved, before being employed the moisture should be squeezed from them, and they should be washed in sterilized water before being brought in contact with the wound. Sterilization by steam is the safest method.

Silk Sutures and Ligatures.-Silk for sutures or ligatures, either the plaited silk or the Chinese twisted silk, should be sterilized by boiling from ten to thirty 
minutes, the time of boiling depending upon the thickness of the threads; frequent boiling renders the silk weak. It should then be placed in stoppered bottles and covered with a 5 per cent. solution of carbolic aeid or with absolute alcohol, or in $1: 1000$ biehloride and alcohol solution.

As boiling diminishes the strength of the silk, it may be rendered sterile by being wound upon a glass spool and placed in a test tube plugged with cotton; the tube should be placed under 10 pounds pressure in an autoclave for thirty minutes on three successive days. Silk ligatures and sutures are extensively used in abdominal surgery for the ligation of pedicles and suturing of the viscera.

Silkworm-gut.-Silkworm-gut is an excellent material for sutures, and may be sterilized by boiling it for fifteen minutes, or by placing it for one-half hour in a 5 per cent. carbolic solution; after being sterilized, it should be kept in 95 per eent. alcohol. There has recently been introduced an iron-dyed black silkworm-gut, which makes the sutures more prominent and thus facilitates their removal.

Silver Wire Sutures.-Fine silver wire was formerly very extensively used as a suture material. The heavier grades of silver wire are still used, as bone sutures. This material is sterilized by boiling.

Horsehair Sutures.-This material is frequently used for sutures, it is more pliable than silkworm-gut and is often used for skin sutures. It should be stcrilized by boiling.

Catgut Ligatures and Sutures.-Catgut is the ideal matcrial for ligatures and sutures, but has the disadvantages of difficulty and uncertainty in its sterilization. Raw catgut is often infected with micro-organisms, and, therefore, thorough sterilization alone can render it a safe material for ligatures and sutures.

Von Bergmann's Catgut.-This method of preparing catgut, consists in winding the catgut loosely upon glass rods or spools; these spools are placed in ether for twenty-four hours; the ether is then poured off, and they are placed in the following solution: bichloride of mercury, 10 parts; 
absolute alcohol, 800 parts; distilled water, 200 parts. Remove from this solution after twenty-four hours, and place them in a similar solution for forty-eight hours; then place in absolute alcohol. If soft catgut is desired, add 20 per cent. of glycerin to the absolute alcolrol. To make the sterilization absolutely certain, it has been found advantageous to soak the catgut for thirty minutes in a $1: 1000$ aqueous bichloride solution before placing it in the alcoholic solution of bichloride.

Dry Sterilized Catgut.-Boeckman's process for sterilizing catgut consists in cutting the gut in pieces twenty to forty inches in length, wrapping each piece in paraffinpaper and sealing in a paper envelope. The envelopes are then placed in a steam sterilizer for three hours at a temperature of $284^{\circ} \mathrm{F}$, and then for four hours longer at a temperature of $290^{\circ} \mathrm{F}$. When required for use, the envelope is opened, the paraffin-paper removed, and the gut immersed for a few minutes only in sterilized water.

Boiled Catgut.-Catgut may also be sterilized by boiling in alcohol under pressure. The most satisfactory method is that devised by Fowler, which consists in placing a number of strands of eatgut in an ordinary test-tube which is filled with 95 per cent. alcohol to within half an inch of the top; a wad of cotton is next pushed into the mouth of the tube, and a cork is introduced. The tubes thus prepared are placed inverted in a fruit-jar filled with 95 per cent. alcohol; the jar is then closed and placed in a water-bath, and kept at a boiling temperature for an hour. Or the catgut may be loosely wound upon glass rods and spools, and placed in a metallic cylinder or jar having an accurately fitting serew-top. The catgut is then covered with absolute alcohol, the top is screwed lown, and the cylinder or jar is immersed in boiling water for an hour.

Formalin Catgut.-This is prepared by winding catgut loosely on glass spools and keeping them for forty-eight hours in a vessel containing equal parts of alcohol and ether. They should next be washed for a few minutes in alcohol and placed in a jar containing equal parts of 
alcohol and formalin, and allowed to remain for several days. The excess of formalin should then be washed away with alcohol, and the catgut kept for use in 95 per cent. alcolıol.

Cumol Catgut.-The catgut is rolled lonsely on glass spools, which are placed in a glass beaker having a layer of cotton in the bottom; the beaker is covered by a piece of cardboard having a hole in the centre throngh which a thermometer-is introduced, and is placed on a sand-bath heated by a Bunsen burner. Heat is applied until the temperature is raised to $176^{\circ} \mathrm{F}$.; this is maintained for one hour, and removes all moisture from the catgut. Cumol, at a temperature of $212^{\circ} \mathrm{F}$., is next poured into the beaker, completely covering the catgut, and the temperature is then raised to $329^{\circ} \mathrm{F}$. and maintained for one hour. The cumol is next poured off, and the catgut is allowed to dry in the beaker on the sand-bath at a temperature of $212^{\circ} \mathrm{F}$. for two hours; it is then transferred to sterile jars or tubes, which shonld be air-tight.

Elsberg's Method of Sterilizing Catgut.-The catgut is immersed for forty-eight hours in a mixture of 1 part of chloroform to 2 parts of alcohol, then wound loosely upon spools and boiled for thirty minutes in a saturated solution of ammonium sulphate, and upon its removal from this solution it should be immersed in sterilized water to remove crystals of ammonium sulphate. It may then be preserved in absolute alcohol.

Iodine Catgut.-This variety of catgut has recently been extensively used with most satisfactory results. The catgut is soaked in a 1 per cent. solution of iodine for seven days, when it is ready for use. The solution is prepared by dissolving iodide of potassinm, 1 part; iodine 1 part in 100 parts of water. The solution is prepared by dissolving the iodide of potassium in a small quantity of water, to which the iodine, finely powdered, is added and the concentrated solution is diluted to a 1 per cent. solution.

The catgut may be kept in the sterilizing solution or in a mixture consisting of absolute alcohol 950, glycerine 50, iodoform, finely powdered, 100. 
Iodine Alcohol Catgut.-A single layer of eatgut should be evenly wound npon a glass spool and the end secured to prevent its kinking. This is placed in a solution of iodime, 1 part to alcohol (proof spirit) 15 parts and kept immersed for eight days. It may be kept indefinitely in this solution.

If preserved in the iodine solution the gut is washed in a solution of carbolic acid, 3 per cent., or in sterile salt solution before being used.

Silverized Catgut.-This is prepared by winding catgut upon glass slabs and placing them in a jar containing a 2 per cent. solution of collargolum. They should be kept in this solution for a week, being shaken several times during this time. The slabs are then removed and washed in stcrile water to remove the excess of collargolum, and placed in 95 per cent. alcohol for fifteen to thirty minutes. The catgut is then wound on glass spools and kept in 95 per cent. alcohol until used. According to Blake eatgut prepared in this manner is in tensile strength and resisting qualities equal to chromic acid eatgnt.

Chromic Acid or Chromicized Catgut.-Catgut, after being soaked in ether for twenty-four hours and washed in alcohol, is placed for twenty-four hours in a 4 per cent. aqueous solution of chromic acid; it is then removed and dried in a hot oven, and placed in closely stoppered jars, or may be preserved in absolute alcohol. Catgut thus prepared will resist the action of living tissues for several weeks, the time of its absorption depending upon the size of the gut. Before being used, it should be sterilized by either the cumol, alcohol, or formalin method.

Owing to the fact that it undergoes very slow solution in the tissues, chromicized catgut is often employed for sutures or for the ligation of the larger vessels in their continuity, and for bone sutures.

Celluloid Thread.--This material, recently introduced by Pagenstecher, is prepared by boiling linen thread for thirty minutes in a 1 per cent. solution of sodium carbonate. It is then dried between sterile compresses and soaked for some hours in celluloid solution. It may be kept dry or 
in an alcoholie solution of bichloride of mercury. It may be resterilized by boiling or under steam pressure. It has proved a satisfactory material for sutures and ligatures, and may be used in place of catgut or silk.

Drainage-tubes.-Thedrainage-tubes usually employed are prepared from rubber-tubing of different sizes perforated at short intervals; the black-rubber tubes are softer and more pliable than the red-or white-rubber tubes, and

FIG. 244.

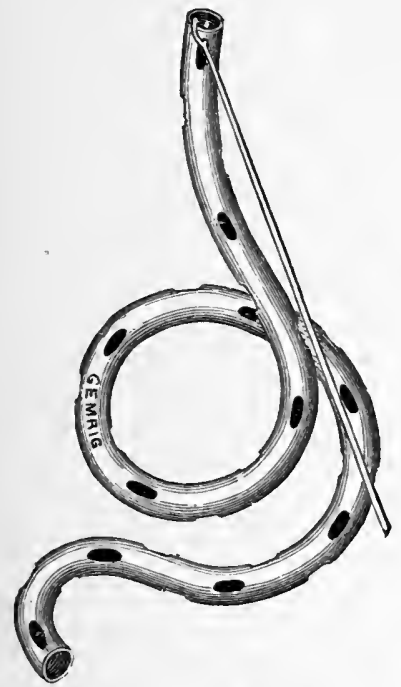

Rubber drainage-tube.
FIG. 245.

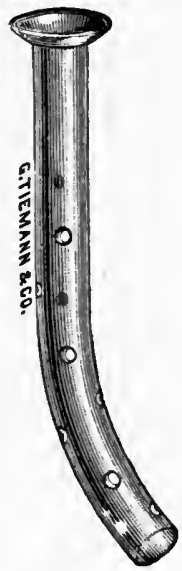

Glass drainage-tube.

are generally preferred (Fig. 244). In using rubberdrainage tubes in contact with organs on which they may exert injurious pressure it is sometimes found of advantage to split the tube for its entire length which does not alter its shape or interfere with its function, unless it is subjected to severe pressure. Drainage-tubes are also made of glass, straight or curved (Fig. 245), which are almost exclusively used in abdominal surgery, and also of decaleified bone. The tubes should be kept in a 5 per cent. solution 
of carbolic acid, or, if kept dry, they should be well washed and sterilized by boiling water for a few minutes before being used.

Gauze Drainage.-Strips of gauze, either sterilized or iodoform gauze, are often used for drainage, and are preferred by some surgeons to the various kinds of drainage tubes. This form of drainage is now very much employed; it will be found that the drainage is more prompt if the gauze is wet with saline solution or any antiseptic solution, preferred, before being introduced.

Cigarette Drain.-This consists of a roll of sterilized or iodoform gauze covered by rubber proFig. 246. tective tissue (Fig. 246). It may be pre-

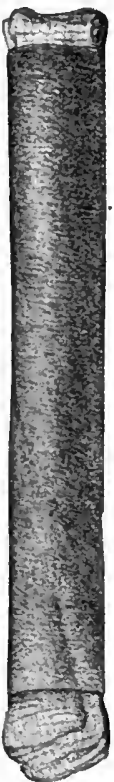

Cigarette drain. pared in lengths of twelve inches and a sufficient amount cut off as is required by the depth of the wound to be drained. It is a very satisfactory form of drain in abdominal wounds.

Catgut and Horsehair Drainage.Catgnt as ordinarily prepared for ligatures may be used to secure drainage in small and superficial wounds; a number of strands are placed in the bottom of the wound, and the ends are allowed to project from one or both extremities of the wound.

Horsehair may be employed for the same purpose, a number of strands of the hair being placed in the wound in the same manner. Before being used, it should be well washed with soap and water, and then sterilized by boiling.

Protective.-Protective is employed to prevent the wound from being irritated by the antiseptic substances with which the ganze is impregnated or by its irregular surface. The great objection to the use of protective is that it sometimes interferes with drainage, and permits of the accumulation of serum beneath it, which may become infected and cause infection of the wound. 
Various materials are employed as protectives, one of the best is rubber-tissue, the principal requirement being that they can be readily rendered aseptic and do not absorb irritating materials from the dressings.

Silver Foil.-The inhibitive action of metallic silver on the growth of micro-organisms is utilized in the employment of silver foil to cover the surface of wounds. The foil is sterilized by dry heat and placed directly on the surface of the wound after it has been closed by sutures. It is claimed that the foil prevents infection of the wound from the exterior, and also destroys micro-organisms which may come in contact with it.

Rubber-dam.-This is a thin, pure rubber-tissue, which may be cleansed and sterilized with greater facility. It is often attached to the drainage-tube in abdominal wounds to shut off the opening of the tube from the abdominal wound. Before being used, it should be washed with soap and water, rinsed, and then placed in a bichloride or carbolic solution for a time sufficient to sterilize it.

Rubber-tissue.-This consists of a very thin shcet of India-rubber with glazed surfaces, which can be obtained from the rubber-manufacturers; it is employed to cover moist dressings, and, as previously stated, may be used when properly sterilized instead of protective for covering the wound.

Cargile Membrane.-This material, in the form of a thin transparent membrane, is prepared from the peritoneum of the ox. It is employed to cover portions of the intestines or other abdominal viscera which have been denuded of peritoneum, and thus prevent adhesions. The membrane should be thoroughly sterilized, and when applied to the defect should be held in place by a few sutures of fine silk or eatgut. It has also been employed in plastic operations upon the nerves or tendons to prevent the formation of adhesions to the surrounding tissues. Some difference of opinion exists as to its utility by different observers. 


\section{Gauze Dressings.}

The most convenient and cheapest material for wound dressing is a material known to the trade as eheese-cloth or tobacco-cloth, and for surgical use should contain no sizing. From the fact that it has a very open mesh, it absorbs well either the materials with which it is prepared or the discharges from the wound, and is soft and pliable, so that it is a comfortable form of dressing to the patient.

Gauze containing various antiseptic substances was formerly much employed in surgieal dressings, but at the present time it has been largely superseded by sterilized gauze.

Bichloride or Corrosive Sublimate Gauze.-Bichloride or corrosive sublimate gauze is prepared by placing cheese-cloth in a washing-kettle and covering it with water to which is added two pounds of washing-soda or a pint of lye; the latter is added to dissolve any oily matter which the cheese-cloth contains, thus making it more absorbent. The gauze is boiled in this solution for an hour, and is then removed and washed in boiled water and passed through a sterilized clothes-wringer ; it is then immersed in a $1: 1000$ bichloride solution for twenty-four hours; the excess of fluid is then squeezed out of it, and it may be packed in air-tight jars and preserved as a moist gauze, or may be dried in a warm oven and packed in sterilized jars and kept as a dry gauze. Dry bichloride gauze, unless freshly prepared, possesses little antiseptic properties.

In using the sublimate gauze on delicate skins a dermatitis sometimes results, which is known as mercurial eczema; this is particularly apt to occur if the gauze is moistened or covered with rubber-tissue. If this condition develops, the parts covered by the gauze should be rubbed over with boric acid ointment or vaseline before it is reapplied, or a sterilized gauze dressing should be substituted.

Iodoform Gauze.-This may be prepared by soaking sterilized gauze in a mixture containing iodoform, 5 parts; glyceriu, 20 parts; and alcohol, 75 parts. This furnishes 
the 5 per cent. iodoform gauze; if 10 per cent. gauze is desired, the quantity of iodoform should be doubled. When the gauze is thoroughly saturated, it should be of a uniform yellow color. It should then be thoroughly wrung out with sterilized hands to remove the alcohol, and packed in sterilized jars with tight-fitting covers.

Iodoform gauze may also be prepared by saturating sterilized gauze with a mixture of ether and iodoform, and then allowing the ether to evaporate, the iodoform being distributed evenly through the gauze.

Carbolized Gauze.-In preparing carbolized gauze, cheese-cloth which has previously been boiled and dried is soaked for a few hours in the following solution: resin, 16 ounces ; alcohol, 5 pints ; castor oil, 24 ounces ; carbolic acid, 12 ounces. The gauze is removed from this solution and passed throngh a sterilized clothes-wringer, and is then eut into pieces from four to six yards in length, which are folded and packed in air-tight jars for use.

\section{Improvised Aseptic or Antiseptic Dressings.--} Aseptic dressings in cases of emergency may be improvised, where the ordinary gauze dressings cannot be obtained, by tearing muslin or mosquito-netting into pieces half a yard square and placing them in boiling water for a few minutes; they are then removed, the excess of moisture is wrung out, and they are applied to the wound.

If it is desirable, they nay be used as antiseptic dressings by soaking them for a few minutes in a $1: 1000$ or $1: 2000$ bichloride solution, or in a 5 per cent. carbolic solution. This dressing will keep the wound aseptic until a more elaborate dressing can be obtained.

Sterilized Bandages.-Sterilized bandages are prepared by tearing or cutting gauze into strips from two and a half to three inches in width, and forming these strips into rollers, which are sterilized by steam or dry heat. They should be used soon after being prepared, or, if kept for any time, should be resterilized before being used.

Bichloride Cotton.-This material is prepared by soaking absorbent cotton in a $1: 1000$ bichloride solution 
for twenty-four hours, and allowing it to dry, or it may be dried in a hot oven; when dry, it is packed in jars of in air-tight boxes. Several layers of bichloride cotton are usually applied over the gauze dressing, as its great absorbing power and elasticity make it, when properly prepared, a most valuable dressing. Borated, carbolized, and salicylated cotton, prepared in the same manner, are also frequently employed for similar purposes.

Sterilized Cotton.-Sterilized cotton is prepared by plaeing absorbent cotton, enclosed in perforated metal cans, in a steam sterilizer and allowing it to remain for half an hour under ten pounds pressure. It is used for the same purposes in dressings as the bichloride cotton.

Moist Sterilized Gauze Dressings.-Moist sterilized gauze dressings may be prepared by subjecting gauze which has been boiled in soda solution to the action of boiling water or of steam for thirty minutes. Gauze thus treated should be used as soon as prepared.

Sterilized Gauze.-This is prepared by cutting pieces of gauze the desired size, wrapping them in a towel, and placing them in wire baskets; or the gauze may be placed in cylindrical tin boxes, 3 inches in diameter and 8 inches in height, with perforated metal covers, covering the gauze at each end with a layer of cotton before putting on the covers. The gauze is next placed in a steam sterilizer and subjected to ten pounds pressure of live steam for half an hour. The steam is then shut off from the sterilizer and allowed to circulate in the jacket of the apparatus without pressure for half an hour, to dry the dressings. If the gauze has been sterilized in metal cases, it may be kept for some time and still remain sterile. Cotton may be sterilized in the same manner.

Dry Sterilized Gauze Dressings.-Dry sterilized ganze dressings are prepared by cutting gauze into proper lengths and packing it loosely in wire cages or perforated metal cans, which are next placed in a dry sterilizingoven for several hours, and upon removal it is placed in air-tight jars or metal boxes. In using dry sterilized gauze 
dressings, it is safer to have the dressings freshly sterilized immediately before each operation. A convenient form of sterilizing-oven is shown in Fig. 247. Towels and operating-gowns may be sterilized in the same oven.

FIG. 247.

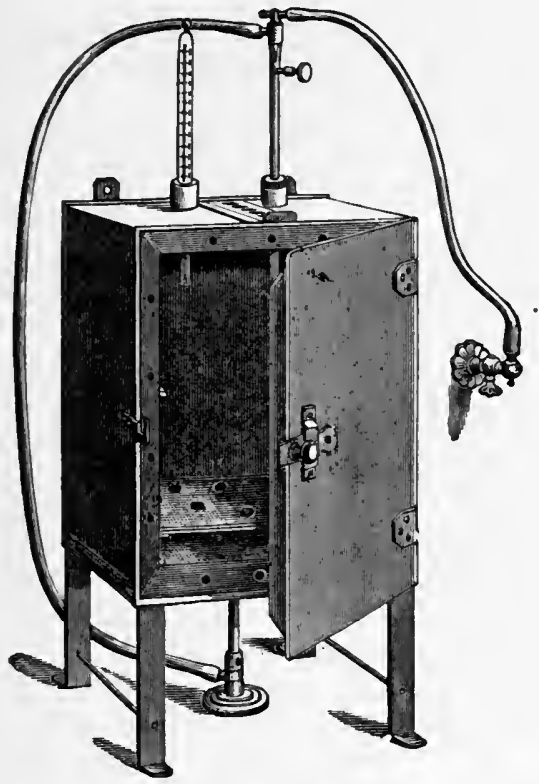

Hot-air sterilizer.

Surgical Operating-bag.-For operations in private houses, the surgeon will find it convenient to have a bag or kit containing several packages of sterilized gauze dressings, bichloride pellets, carbolic acid, alcohol, turpentine, ligatures, sutures, needles, syringes, a small instrument sterilized or a metal tray in which instruments may be boiled, a nest of small agate-ware basins, sponges, gauze pads, a sheet of rubber eloth, drainage-tubes, and operating-gown. These can all be packed in a comparatively small space, and when the surgeon is called upon to 
perform any special operation at short notice the instruments required may be selected, wrapped in a Cantonflannel scroll, and placed in the bag. Much time will be saved by having the materials required in operations always in readiness.

\section{METHODS AND DRESSINGS EMPLOYED TO SECURE ASEPSIS IN THE TREATMENT OF WOUNDS.}

To prevent infection of wounds, the various chemical sterilizers and dressings are employed in different ways, and the principal types of dressings are as follows:

Method by Simple Drying.-This method is employed in small and not very deep wounds. The edges having been brought together by sutures, the surface of the wound is dusted with powdered iodoform, the serum and blood forming with this, as it dries, a scab, which protects the wound from infection from without; repair taking place promptly under this scab. Treves employs this method of dressing in compound fractures. A pledget of gauze saturated with iodoform-collodion or tr. benzoin, $3 \mathrm{j}$; collodion, $3 \mathrm{vij}$, may be employed instead of iodoform. Dry sterilized ganze and cotton dressings may also be employed in this method of dressing.

Method by Drying and Chemical Sterilization.The object of this method of dressing is to provide a means of sterilizing the blood or serum which escapes from the wound, and at the same time to insure the sterilization of the air coming in contact with the discharges from the wound. It is employed in large or deep wounds, where there is always more or less escape of hlood or serum, and is accomplished by applying a number of layers of sublimate or jodoform-gauze and sublimated cotton over the wound. Evaporation not being interfered with, the whole dressing becomes hardened, and the wound is surronnded by a large antiseptic crust made up of the dressing and serum or blood.

Moist Dressings.-In this method of dressing, the wound is covered by layers of moist antiseptic gauze, which are kept moist and evaporation prevented by apply- 
ing over them some impervious material, such as mackintosh or rubber-tissue. This method of dressing is not at the present time often employed.

Modified Moist Dressing.-In using this method, the wonnd itself is covered by a piece of protective or rubber-tissue ; over this is placed the sublimated or iodoform-gauze dressing and some layers of bichloride cotton. In this way the wound itself is kept in a moist condition favoring particularly the organization of blood-clots; the external dressings become dry as the discharges which have eseaped into them evaporate, forming an antiseptic crust or covering over the wound.

\section{Preparation for Aseptic Operation.}

Preparation of Room.-In hospital practice, suitable operating-rooms are provided; in private practice, however, the surgeon is often called upon to select a room and give directions as to its preparation. A well-lighted room should always be selected, and all unnecessary articles of furniture, such as ornaments, pictures, and curtains, should be removed. The carpet should be taken up and the floor scrubbed. A few small tables and a large wooden table should be placed in the room, having previously been dusted and wiped off with a bichloride solution. All preparations should be made, if possible, upon the day before the operation, as the stirring up of dust incidental to the change in furniture in cleaning the room on the day of operation immediately before the time set, is more dangerous than no eleaning of the room whatever, since the principal contamination of the wound is likely to eome from germs contained in the dust. In case of emergency, the floor may be well moistened by sprinkling with water to lay the dust, or covered by sheets wrung out of bichloride solution. The preparation of the room is not, in my judgment, a matter that affects the results of operations as much as does the exercise of great eare in regard to aseptic details of the operation itself.

Preparation of the Patient.-The skin always con- 
tains micro-organisms, which develop upon it and are constantly being deposited upon it from the air. We can scarcely hope to obtain absolute sterilization of the skin under these cireumstances, but by careful preparation seek to obtain that relative sterility which enables us to obtain primary union. The patient should be given a general bath the night before the operation, and the skin surrounding the site of operation should be thoroughly scrubbed with a brush and soap and water; or a soap poultice may be applied to the part for a few hours before the final sterilization with alcohol and bichloride is made. In scrubbing the skin a soft brush should be used, since too forcible scrubbing may cause irritation or dermatitis. After this scrubbing has been continued for a few minutes the skin is washed with alcohol and ether, then donched with sterilized water, and there should be applied to the surface a folded towel or gauze dressing saturated with a $1: 1000$ bichloride solution; or if a moist dressing is uncomfortable to the patient, a few layers of sterilized gauze should be placed over the surface and held in place by a bandage. A similar washing and preparation of the seat of operation should be made the-next morning, a few hours before the time fixed for operation.

The skin may also be sterilized by formalin. It should first be scrubbed thoroughly with soap and water, and then a few layers of gauze saturated with a 1 per cent. solution of formalin should be laid over it and covered by an impermeable dressing. This solution should be kept in contact with the skin for twenty-four or thirty-six hours, the compress being changed every twelve hours.

Sterilization of the skin in the region of the operation may be accomplished by painting it when dry with a 10 to 12 per cent. tincture of iodine. The iodine is applied a short time before operation and again just before the operation is begun. It is essential that the application be made to dry skin. Grossin and other surgeons speak highly of this method of sterilization.

It is well to remember that regions of the body which contain hair and numerous sweat-glands, such as the axilla, navel, scrotum, groin, and the creases about the joints, are 
those in which micro-organisms grow with the greatest activity. All the surrounding hair should be shaved off; and if the operation be upon the skull, it is well to shave the scalp completely.

Sterilization of the Feet.-There is usually present upon the feet a large amount of thickened epidermis, which renders their sterilization difficult. The feet should be washed thoroughly with soap and water and scrubbed vigorously with a brush; or a soap poultice should be applied to the whole surface of the feet for some hours and held in position by a bandage. A moist dressing favors separation of the superficial layers of the epidermis, and after it has been worn for a few hours it is possible to remove a large amount of the latter by the use of the brush. After having been washed thoronghly with a $1: 1000$ bichloride solution they should be wrapped in a towel or a few layers of gauze saturated with bichloride of mercury solution, $1: 1000$.

Sterilization of the Vagina.-The vagina and external genitals require great care in their sterilization. According to Schimmelbusch, the best method of sterilizing the vagina is to dilate it fully with a speculum, and to scrub it thoroughly with pads of gauze saturated with green soap and water, and after this cleansing, to irrigate it with a $1: 2000$ bichloricle solution or a 1 per cent. solution of kreolin.

Sterilization of the Bladder and Urethra.-It is impossible to sterilize completely the mucous membrane of the bladder. The bladder shonld be emptied by catheter and filled with sterile water or normal salt solution; this procedure should be repeated several times. The best means we have at our disposal at the present time of sterilizing the mucous membrane of the bladder consists in irrigating the organ frequently with a 10 grain to the ounce solution of boric acid in boiled water. In operations upon the urethra the same care should be taken to render the urethra sterile by frce irrigation with normal salt solution or boric acid solution.

Sterilization of the Stomach.-The stomach should 
be sterilized by thorough lavage with normal salt solution or boric acid solution. This is important, not only in operations upon the stomach itself, but also in operations upon the pharynx, to diminish the risk of infection by vomited matter. In cases of intestinal obstruction with vomiting, lavage of the stomach should always be employed before operation.

Sterilization of the Rectum.-When an operation is to be performed upon the anus and rectum, the patient should be given a purgative and an enema some hours before the operation, to remove any fecal matter which may be in the rectum. The region of the anus should be disinfeeted with soap and water and thoroughly serubbed, and after the patient has been anæsthetized the sphincter should be well stretched and the rectum irrigated with a boric acid solution, or a 1 to 3 per cent. kreolin solution. A tampon of sterilized gauze, with a string attached, should be packed into the rectum above the seat of operation, to prevent the wound from becoming soiled with feces during the operation. 'The tampon can be removed by means of the string after the operation has been completed.

Sterilization of the Scalp.-Great care should be observed in sterilizing the scalp before operations on the scalp or brain, as the scalp is often covered by dense masses of epidermis. The entire scalp should be shaved and a soap poultice applied for twelve hours, or the application of sweet oil for twenty-four hours before the use of the soap poultice may be of use in softening the epidermis. It should be rubbed thoroughly with soap and water, and finally with a $1: 1000$ bichloride solution.

Sterilization of the Mouth and Nasal Cavities.-To render the month as far as possible sterile, the teeth should be thoronghly brushed with tooth-powder and the cavity of the month frequently rinsed with a solution of peroxide of hydrogen, 1 part to 6 parts of water, or with a saturated solution of boric acid. The nasal cavities and the post-nasal region should be sterilized by spraying them with the same solution, or with Dobell's solution.

Sterilization of the Hands.-The difficulty of com- 
pletely sterilizing the hands has been shown by bacteriological tests, for it has been demonstrated that after great care in the process complete sterility could be obtained only in abont 95 per cent. of the tests. The hands of the surgeon, unless properly sterilized, may be the most efficient agents in producing infection of the wound; the region of the finger-nails and the interdigital folds are locations where germs are particularly abundant. The hands and forearms of the surgeon, assistants, and nurses who are to take part in the operation, may be sterilized by first rubbing them with spirit of turpentine, and then thoroughly scrubbing them with Castile soap and water, using a nail-brush freely. Care should be taken that the brush is sterilized. This scrubbing should be employed for several minutes; the hands are then rinsed to remove the soap, and are soaked for two minutes in a 1:1000 bichloride of mercury solution. If turpentine has not been employed before washing with the soap, strong alcohol, benzine, or ether should be rubbed well over the hands before they are immersed in the bichloride solution. When the hands have been sterilized they should not be brought in contact with anything that is not sterile.

Harrington's Method.-Harrington after washing the hands thoroughly with green soap immerses them in the following solution for a few minutes : alcohol (94 per cent.) 640 c.c., hydrochloric acid 60 c.c., water 300 c.c., corrosive sublimate 0.8 grammes. This solution has been found to be a most efficient sterilizing agent.

Permanganate of Potassium and 0xalic Acid.-A method of sterilizing the hands which is very satisfactory is that employed by Kelly, which consists in washing the hands and forearms with soap for ten minutes, and then soaking them for a few minutes in a warm saturated solution of permanganate of potassium, which stains them a deep mahogany color; they are then, washed in a warm saturated solution of oxalic acid until all the permanganate stain is removed, and should next be washed in sterilized water to remove the oxalic acid which may adhere to the skin. 
Chloride of Lime and Carbonate of Sodium.-Weir recommends the following method of sterilizing the hands. After washing them with green soap, put a tablespoonful of commercial chloride of lime and an equal amount of carbonate of sodium (washing-soda) in the hand, with enough water to make a paste. Rub this into a thick cream, which should be rubbed into the hands until the grains of lime disappear and the skin feels cool. The hands are then rinsed in sterile water. This method of sterilization of the hands has, in my experience, been most satisfactory.

Sterilization of Instruments.-The sterilization of instruments may be accomplished by dry or moist heat; they should be placed in a hot-air sterilizer or baked for twenty minutes in a hot oven. Sterilization of instruments by dry heat or baking is not often employed, as it is apt to spoil the temper of the steel. Instruments may be sterilized by the method suggested by Schirnmelbuscl, now almost universally employed, which consists in boiling them for fifteen minutes in water to which a tablespoonful of washing-soda (carbonate of sodium) has been added for each quart of water; this prevents the rusting of the instruments, and also makes the water a better solvent for any fatty matter which may be upon the instruments, thus increasing the sterilizing effect of the heat. If wooden-handled instruments are used, which would be injured by boiling, they should first be thoroughly scrubbed with soap and water and a brush, and after laving been rinsed in sterilized water they should be placed in a tray and covered with $1: 20$ watery solution of carbolic acid, and allowed to remain in this solution for at least half an hour; before being used they should be transferred to a bath of sterilized water, which will prevent the benumbing effect of the carbolic solution upon the surgeon's hands.

A frequent boiling injures the cutting edge of knives, they may be rendered sterile by first thoroughly washing them and placing them in pure carbolic acid for from three to five minutes, then transferring them to a vessel containing alcohol. 
Instruments may also be sterilized by formalin : the latter is generated by heating pastilles of paraform with Schering's formalin lamp. The instruments are placed in racks in a metal ease, and by burning from 10 to 15 grains of paraform the instruments may be rendered sterile in fifteen minutes.

Instruments which fall upon the floor or come in contact with the elothing of the surgeon or of the patient during the operation, should again be sterilized before being brought in contact with the wound.

Sterilization of Catheters and Bougies. - These, if made of metal or glass, may be sterilized by boiling for ten minutes in a 1 per cent. solution of sodium carbonate. If constructed of gum, prolonged boiling. destroys them; these may, however, be sterilized by first washing them with soap and water and then placing them for fifteen minutes in a 1 per cent. solution of sodium carbonate, heated nearly to the boiling point; they are next placed in a $1: 1000$ bichloride solution until required. They should, on being removed from this solution for use, be soaked thoroughly in hot sterile water to remove all the bichloride solution. Rubber catheters may be sterilized by boiling, they may also be sterilized by soaking them for an hour in a 2 per cent. solution of formalin, or by placing them in an air-tight metallic case or glass jar containing pastilles or paraform. They can be kept indefinitely in such a receptacle, and when removed for use should be washed in sterilized water. For lubricating catheters and bougies, oily materials should be avoided, and sterilized glycerin, liquid vaseline or lubrichondrin, should be employed.

Rubber Gloves.-These gloves are now extensively employed in operative work, and the results following their use have been most satisfactory. They are made of very thin rubber, so that there is litile interference with tactile sensation, and from their elasticity they fit the hands accurately. They can be rendered absolutely sterile, and as they are impervious to moisture there is no risk of wound infection if the hand is not completely 
sterilized unless the gloves have been torn or punctured. To avoid this possibility of infection the hands should be sterilized as completely as possible before they are applied. They may be sterilized by first washing them with soap and water, and then wrapping them in a towel and boiling them for ten minutes. They are usually applied by filling them with sterilized water or salt solution, and then introdueing the hand; some operators prefer to apply them dry to the hand, using a dry sterilized powder, such as starch or soapstone. If properly cared for, a pair of gloves will withstand a number of sterilizations. A freshly sterilized pair of gloves should be used for each operation.

Cotton or silk gloves, which have been sterilized by boiling or by dry heat, have been recommended by Mikulicz and other surgeons, to be worn during operations. Experiments, however, have shown that cotton or silk gloves are not as safe as those made of rubber.

Clothing of Surgeon and Assistants.-The surgeon and his assistants should wear sterilized linen or muslin suits, or be provided with gowns with sleeves reaching to the elbows, for the protection both of the patient and of their clothing. The operating-gown should be made of muslin or linen, which ean easily be sterilized by boiling or heat; a variety of linen known as butchers' linen is very serviceable for this purpose. As a matter of additional precaution, many surgeons and their assistants wear during the operation closely fitting skull-caps of linen or gauze, and wear over the nose and mouth a mask or a pad composed of a number of layers of sterilized gauze to prevent infection of the wound by the expired air. The surgeon and assistants will often find it convenient to wear under their linen gowns India-rubber aprons, to prevent soiling of the clothing by blood or solutions. The nurses should wear sterilized linen or muslin operating-gowns and dresses of washable goods. An operating-apron may be improvised from a clean sheet folded so as to be one and a half yards in width and from five to six feet in length, by turning in about ten inches of one end of the sheet over the upper part of the chest and placing a strip of bandage in this 
fold, which should be secured around the neck, and tying a seeond strip of bandage over the sheet at the waist.

Details of an Aseptic Operation.-The patient being prepared for operation as described, and having been anæsthetized, is placed upon the operating-table, the surgeon, assistants, and nurses also being prepared for the operation as previously described. If the operation be one upon the face, neck, or chest, it is well, before the dressings eovering the seat of operation are removed, to cover the patient's hair with a towel or handkerehief-bandage made of several layers of sterilized or biehloride ganze. The portions of the patient's body which it is not neeessary to expose in the operation should be eovered with a woollen blanket, and this eovered with a sterilized sheet. Some surgeons prefer to have the patient wear a sterilized gown, which is ripped or cut to expose the part to be operated upon. The region of the wound and the operating-table are next proteeted with sterilized towels or eloths. The surgeon having assigned the assistants and nurses their duties, the dressing is removed from the part to be operated upon, and the operation is begun. Hemorrhage is eontrolled by the use of hæmostatic foreeps, and sterilized gauze pledgets are employed to keep the wound free from blood. When the operation is completed, the vessels are ligated, the hæmostatic foreeps are removed, and the wound is dried with gauze pledgets. If, for any reason, the surgeon deems it advisable to irrigate the wound, it may be done with hot sterilized water or with sterilized salt solution. If the surgeon decides that drainage is not necessary, the deeper parts of the wound may then be brought together with buried sutures of catgut or silk, and the edges of the superficial wound next approximated by sutures of eatgut, silk, or silkworm-gut. If the surgeon deeides to use drainage, before elosing the wound a few strands of eatgut, a strip of sterilized gauze, a tent of rubber-tissue, or a rubber or glass drainage-tube is introdueed into the deepest portion of the wound and brought out at its most dependent part. The wound is then dressed with a number of loose masses of sterilized 
gauze placed so as to cover the wound and extend beyond it in all directions, and these are covered by a number of layers of sterilized gauze, and the dressings are held in place by a gauze bandage. The bandage should be applied so as to cover the cotton at the edge of the dressing, and thus make the occlusion of air from the wound as complete as possible. Over the gauze dressing are placed a few layers of sterilized cotton, extending on all sides well beyond the gauze, and the dressings are held in place by a sterilized gauze bandage. The dressings should be voluminons; it is always a mistake to apply scanty dressings. In redressing the wound the same care should be exereised as regards asepsis as was observed at the primary dressing.

Details of an Antiseptic Operation.-The region of the wound being previously sterilized and the patient being anæsthetized and placed upon the table, the clothing is so arranged as to expose freely the part to be operated upon ; the clothing or the skin surrounding this region is next covered with towels wet with a $1: 1000$ bichloride solution. If any considerable surface of the patient's body is covered by these towels, to avoid chilling the surface and adding to the shock which naturally follows the operation, they should be wrung out in a hot bichloride solution, and should be replaced as they become cold by hot towels prepared in the same manner. The patient being ready for operation, the surgeon should assign the assistants and nurses their duties, and having previously sterilized his hands and forearms, and again immersed them in the bichloride solntion, the operation is begun.

During the operation the wound is irrigated frequently with a $1: 2000$ to $1: 4000$ bichloride solution, which may be applied to the wound by means of a syringe or irrigating apparatus (Fig. 248), and the hands of the suryeon and assistants should also be washed in this solution at not too long intervals. In prolonged operations, or in those in which a large wound is made, it is especially important that the irrigating solutions should be used as warm as can comfortably be borne by the hands of the 
surgeon; warm solutions, it has been shown by recent investigations, possess a greater germicidal power than those of the same strength when used cold, and they also possess the advantage of preventing chilling of the patient, and thus diminish the shock of the operation.

Hemorrhage during the operation is controlled by the use of hæmostatie forceps, which are applied to the bleeding vessels, or the vessels may be ligatured as they are divided. After the operation has been completed, and all hemorrhage has been controlled, the wound is thoroughly irrigated with a $1: 4000$ to $1: 2000$ bichloride solution.

The next step is to provide for drainage; this may be disregarded in small, superficial wounds, but in a wound of considerable size or depth it is safer to provide free drainage. This is accomplished by the use of perforated rubber drainage-tubes, or a number of strands of catgut, or strips of iodoform or bichloride gauze.

FIG. 248.

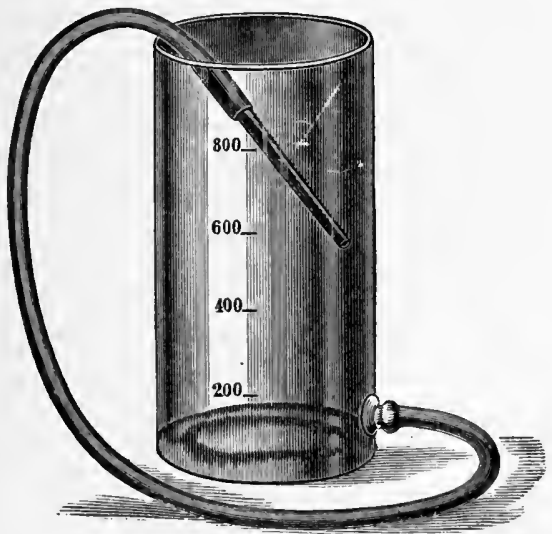

Irrigating apparatus. (EsMaRCH.)

The rubber tube may be laid in the wound, the ends being allowed to extend from the extremities of the wound, or it may be so introduced that one end of the tube rests 
in the deepest part of the wound and the other extremity is brought out of the wound at its most dependent portion; in large or irregularly shaped wounds a number of tubes may be required to secure free drainage. The ends of the drainage-tubes are transfixed with safety-pins which have been sterilized, and should next be cut off close to the pins so as to be as nearly as possible flush with the skin.

The wound being closed by sutures, a final irrigation of its deepest parts should be made, by injecting a stream of bichloride solution, $1: 4000$ to $1: 2000$, into the end of the drainage-tube. The external surface of the wound, and the skin for some distance surrounding it, should next be washed with a $1: 4000$ to $1: 2000$ bichloride solution, and a piece of protective, a little longer and wider than the wound, is dipped in a bichloride or carbolic solution and placed over it. The use of protective over the wound is important only if it is desired to keep the wound moist, in order to obtain organization of the bloodelot, otherwise it need not be employed. Over this is laid the deep dressing, which consists of a pad of bichloride gauze from eight to sixteen layers in thickness, and large enough to overlap the wound two or three inches in all directions. This should be dipped in a $1: 4000$ to $1: 2000$ bichloride solution, and wrung out as dry as possible before being applied. The superficial gauze-dressing is next applied, and consists of sixteen layers of gauze, which should be large enough to extend from three to six inches beyond the wound in all directions; this gauze is applied dry. Over the superficial gauze-dressing there is next applied a number of layers of bichloride cotton, so arranged as to extend a little beyond the margin of the superficial gauze-dressing. These dressings are next secured in position by the application of a gauze-bandage, which is prevented from slipping by the introduction of a few safety-pins. Iodoform, carbolized, or any other variety of medicated gauze, may be used in place of the bichloride gauze.

In this method of dressing, no mackintosh or rubbertissue is employed outside of the superficial gauze-dress- 
ing ; the discharges of the wound are disseminated through the dressing and become dry by evaporation, and the dressing forms an antiseptie seab which covers and surrounds the wound.

Moist Method of Dressing.-If, for any reason, it is desired to adopt the moist method of dressing, a piece of mackintosh or rubber-tissue larger than the superficial gauze-dressing is placed over it, and over this are placed a few layers of bichloride cotton, care being taken to see that the layers of cotton overlap the mackintosh or rubbertissue by a few inches; the application of an antiseptic gauze-bandage then completes the dressing. On removal of this dressing the gauze will generally be found to be soaked with the discharges from the wound, and in a moist condition. The disadvantage of this variety of dressing is that there is apt to be more irritation of the skin set up by the bichloride gauze when kept moist than when applied in the mamer of a dry dressing.

In operations involving the abdominal cavity, the greatest care should be taken to see that no instrument, sponge or ganze pad is left in the cavity before the wound is closed. The pads and instruments should be counted before the operation and fully accounted for before the wound is closed.

Redressings of the Wound.-The redressing of a wound which remains aseptic need not be made for some days; if the temperature remains normal or a little above this point, and the patient exhibits no unfavorable constitutional symptoms, and the dressing is comfortable to the patient, it need not be disturbed for a week or ten days; at the expiration of this time it is well to examine the wound and to remove the drainage-tube if drainage has been used, and to remove a portion or all of the sutures if the superficial parts of the wound are firmly healed

In redressing a wound in which the antiseptic method was employed, at the end of a week or ten days, to prevent any possible infection, as much care should be exercised as in the original dressing of the wound. The 
patient's clothes should be removed so as freely to expose the dressing, and a rubber cloth should be placed under the patient so as to protect the bed, and the clothing and skin in the region of the wound should be protected by towels wrung out in a $1: 1000$ bichloride solution. The surgeon should wash his hands and immerse them in a 1 : 1000 bichloride solution before removing the dressings. The bandage retaining the dressing shonld be divided with bandage-scissors and the gauze removed layer by layer, and when the deep dressing is removed care should be taken that the drainage-tubes are not pulled upon if they are adherent to the dressing; the protective should next be removed and the surface of the wound irrigated with a $1: 2000$ bichloride solution. If the wound is found aseptic, the drainage-tube may be removed, and the superficial wound be irrigated with bichloride solution. If the wound is healed, the sutures may be removed at this dressing; but if the wound has been an extensive or deep one, it may be well to remove only a portion of the sutures; if catgut sutures have been employed, they need not be removed. The surface of the wound is next irrigated with a $1: 2000$ bichloride solution, and deep and superficial gauze-dressings are applied as previously described, and covered with layers of bichloride cotton, and the whole dressing is secured by the application of an antiseptic bandage. If the wound remains aseptic, the dressings need not be changed for a week or ten days, and at this time the wound will usually be found healed, so that further dressings are not required.

In the redressing of a wound in which the aseptic method was employed, the use of germicidal solutions is omitted, and the wound is redressed with sterilized gauze and cotton. If, however, the wound is not rumning the typical course of an aseptic wound, constitutional symptoms will be developed, as evidenced by a rise in the temperature and pulserate and other constitutional disturbances. In this event the wound should be redresserl as soon as possible, and if the cause of the disturbance can be found, it should be removed; for instance, hemorrhage may have taken place 
into the wound, and the blood not being able to escape through the drainage-tubes may have caused so much distention of the wound that the vitality of the skin covering the wound is threatened, or the sutures may be found to be causing irritation, or suppuration may be present.

If, on exposure of the wound, it is found that it is distended with blood-clots, and that blood is escaping from the wound, the sutures should be removed, the clots turned out, and the bleeding vessel or vessels sought for and ligatured, and the wound, after a thorough irrigation with $1: 4000$ to $1: 2000$ bichloride solution, should be drained and closed with sutures, and dressed as previously described.

If, however, on exposure of the site of the operation, and upon the removal of a portion or all of the sutures, the wound is found distended with a blood-clot, and no evidence of hemorrhage at the time exists, or of suppuration in the wound, the clot may be allowed to remain in place, and the wound should be redressed as in the original dressing, trusting to the organization of the blood-clot if it has remained aseptic. If the patient's condition improves after the dressing, and the temperature and pulserate become normal, it is an indication that the wound is still aseptic, and it need not be redressed for some days.

If, on the other hand, examination of the wound shows that the drainage is insufficient, or that the drainagetubes are occluded by blood-clots, these should be removed by washing out the tubes with a $1: 4000$ to $1: 2000$ bichloride solution by means of a syringe, and introducing additional drainage-tubes, if it is deemed necessary; the wound should then be redressed.

When it is found on examination of the wound that suppuration is present, it should thoroughly be irrigated through the drainage-tubes with a $1: 2000$ bichloride solution, and after thorough irrigation it should be redressed, and, if the constitutional symptoms improve, it may be assumed that the wound has been rendered aseptic.

Aseptic or Antiseptic Treatment of Infected Wounds.-It often happens that the surgeon is called 
upon to treat a wound which is septic when it comes under his care, as evidenced by the inflamed state of the wound, inflammation of the lymphatic vessels and skin, foul discharges and slonghing of the tissues, and the coexistent constitutional symptoms of sepsis. In such a case it would at first sight appear that the surgeon or his assistants could not introduce any material of infection worse than that which already existed in the wound, but he should bear in mind the fact that it is possible to introduce a new form of infection in addition to that already existing. With this possibility in view, he shonld observe the same precautions as regards the sterilization of his - hands, the region of the wound, the instruments, and dressing, as he would employ in treating a perfectly fresh wound.

Recent investigations, however, have shown that the germs in abscesses are to a great extent dead, and that the pus-formation is largely due to the irritation caused by their products. In view of these facts, it would seem that the most important part of the treatment of infected wounds is thorough drainage. It is a question whether the micro-organisms in the walls of infected cavities or sinuses can be destroyed by antiseptic irrigation. Some surgeons recommend active treatment, both mechanically and by the use of germicidal solutions, while others are satisfied simply to secure free drainage; and if irrigation is necessary, they do not employ strong germicidal fluids but use simply sterilized water or sterilized salt solution. I prefer to employ the antiseptic method in dealing with infected wounds, and can recommend the following plan. The skin surrounding the wound for some distance should be wiped over with spirit of turpentine and carefully scrubbed with soap and water, and should next be washed with a 1:1000 bichloride solution; the wound itself should next be washed with peroxide of hydrogen and a 1:1000 bichloride solution. With forceps and curette, any dirt or sloughing tissue should be removed; then the wound again washed with peroxide of hydrogen and douched with a $1: 2000$ bichloride solution. The wound 
should then be dried with ganze pledgets and dusted with iodoform, and loosely packed with strips of iodoform gauze. If from the appearance of the tissues the surgeon has reason to think that the infection has passed beyond the reach of the curette or scissors, he may swab over the surface of the wound with a solution of chloride of zinc, 30 grains to the ounce of water, or a 2 per cent. solution of formalin. Pure carbolic acid may be used, and is recommended by some surgeons, for the same purpose as chloride of zinc, but the toxic action of carbolic acid causes its employment to be attended with some danger. Toxic effects and too extensive cauterization may be prevented by washing the part with absolute alcohol. Free drainage being secured by the introduction of a few strips of iodoform gauze, the wound is dressed with a voluminous dressing of bichloride gauze and bichloride cotton. No attempt, as a rule, should be made to bring together the edges of such a wound by the introduction of sutures. In the dressing of infected wounds, when the discharges are ropy or viscid they are not well absorbed by dry dressings, and in this class of wounds it is, therefore, often of advantage to employ moist antiseptic dressings. By this method of treatment it is often possible to convert a septic wound into an aseptic one, and have rapid improvement follow both in the local condition of the wound and in the constitutional condition of the patient. 



\section{PART IV. \\ F R A CT URES.}

In the following section the author has endeavored to confine himself simply to a description of the varieties of fracture and to their dressing and treatment, and he has tried as far as possible to avoid the multiplication of dressings, being satisfied to describe a few of the methods of dressing most frequently employed. $\mathrm{He}$ has also avoided the description of complicated splints and dressings, by the use of which in certain fractures most excellent results are obtained, but has preferred to recommend the employment of simple splints and dressings, which can be obtained by physicians practising in districts remote from large cities, where the services of an instrument-maker cannot be obtained to construct special apparatus for the treatment of these injuries.

\section{VARIETIES OF FRACTURE.}

Complete Fracture.-This is a fracture in which the line of separation completely traverses the bone, involving its entire thickness.

Incomplete Fracture.-This is a fracture in which there is only a partial separation of the bone-fibres (Fig. 249 ), under which name is included partial or "greenstick" fracture, in which some of the bone-fibres have given way, while the remaining fibres have been bent by 
the force, but have not been broken (Fig. 250). Fissured, punctured, indented, and perforating fractures are also included in the class of incomplete fractures (Fig. 251).

FIG. 249.

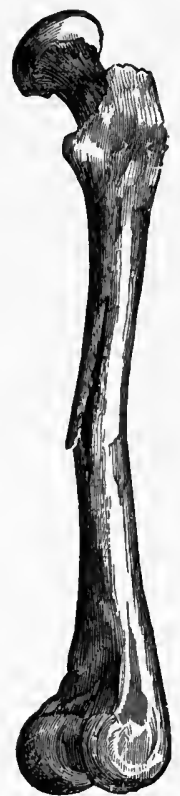

Incomplete fracture of femur.
FIG. 250.

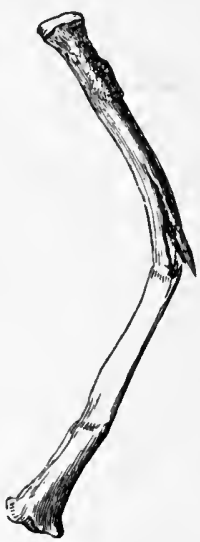

Partial or green-stick fracture of radius.
FIG. 251.

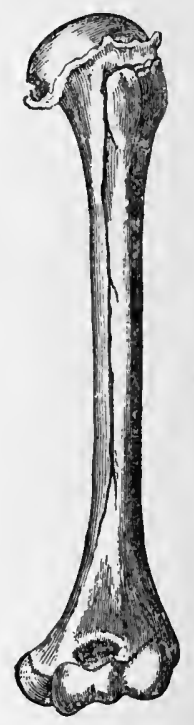

Fissured fracture of the humerus. (GURLT.)

Subperiosteal Fracture.-This is a fracture in which the fibres of the bone are ruptured but the periosteum remains untorn; it is scen in infants and young children.

Gunshot Fractures.-The nature of the injury to the bone depends upon the density of the latter, and upon the size, shape, composition, and velocity of the ball. In gunshot injury of the spongy bones the cancellated structure yields to pressure, and the striking energy is not transmitted in lateral directions, producing explosive effects; while in the dense bones, such as the submaxillary bones 
or the shafts of the long bones, extensive comminution and fissuring are apt to result. In the articular ends of the long bones clean perforations are often observed, except at close range, when more or less comminution of the cancellated structure may occur. The tissues from the wound of entrance to the bone are usually injured only in the line of perforation, but those beyond the seat of injury are often extensively lacerated and contused, not only by the ball, but also by the splinters of bone driven into the tissues, and acting as secondary missiles.

Simple or Closed Fracture.-This is a fracture in which there are but two fragments, and the seat of injury in the bone does not communicate with the external air by a wound in the soft parts.

Compound or Open Fracture.-This is a fracture in which the seat of injury in the bones communicates with the external air by a wound in the soft parts.

Comminuted Fracture.-This is a fracture in which there are more than two fragments, the lines of fracture intercommunicating with each other (Fig. 252).

Multiple Fracture.-This is a fracture in which a bone is the seat of two or more distinct fractures at different points, the lines of fracture not necessarily communicating with each other.

Complicated Fracture.-This is a fracture accompanied by some serious injury of the parts in the region of the fracture-as, for instance, the laceration of important bloodvessels or nerves, contusion or laceration of the muscles, or dislocation of a neighboring joint.

Impacted Fracture.-This is a fracture in which one fragment is driven into and fixed in the other, the impaction taking place at the time of fracture, or being, caused by a force subsequently applied (Fig. 253).

Transverse Fracture. - This is a fracture in which the general line of division of the bone is at right angles with the long axis of the bone (Fig. 254). Transverse fractures of the long bones are rarely met with, the line of fracture usually being more or less oblique.

Oblique Fracture.-This is a fracture in which the line 
FIG. 252.

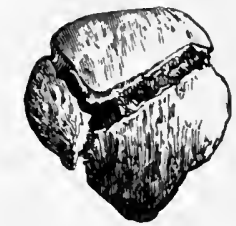

Comminuted fracture of patella.

FIG. 253.

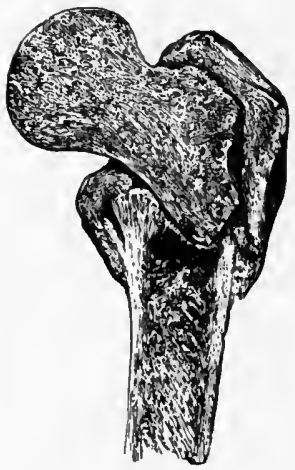

Impacted fracture.
Fig. 254.

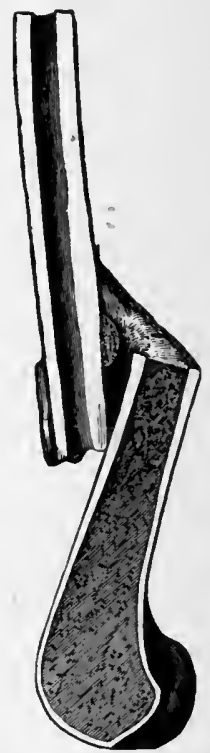

Transverse fracture of femur. (GURLT.)

of separation is oblique to the long axis of the bone. This is one of the most common directions of the line of fracture (Fig. 255).

Longitudinal Fracture.-This is a fracture in which the line of separation runs in the general direction of the long axis of the bone (Fig. 256). This form of fracture is rare, but is sometimes met with in the long bones as the result of gunshot injury.

Symptoms of Fracture.-The most prominent symptoms of fracture are loss of function, deformity, preternatural mobility, pain, crepitus, and muscular spasm. In impacted fractures, crepitus and preternatural mobility are absent. 
FIG. 255.

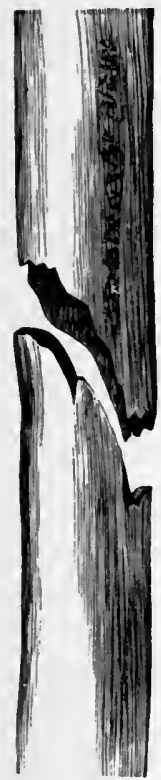

Oblique fracture of humerus. (STIMSON.)
FIG. 256.

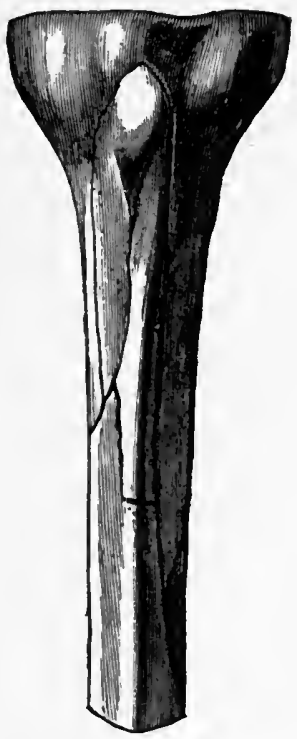

Longitudinal fracture of tibia. (STIMsoN.)

Deformity.-The deformity or displacement in fractures is either angular, transverse, longitudinal, or rotary.

Examination of Fractures.-In examining a case of fracture to locate the nature and seat of the injury, the clcthing should be removed from the part with as little disturbance as possible, and it is better, in most cases, to cut or rip the clothing rather than to attempt to remove it in the ordinary manner. The surgeon should first inspect the injured part, and, where possible, compare it with its fellow, as in the case of injuries of the extremities; much valuable information is also derived from the patient or his friends as to the manner in which the injury was produced. The part should next be carefully examined by the surgeon; if it be one of the extremities which is in- 
jured, it should be gently lifted, firm extension being made at the same time, the surgeon by his touch and by gentle movements seeking to loeate the seat of fracture; and he may, by his manipulation, at the same time develop crepitus.

All manipulations should be made with care, and with the greatest gentleness, not only to save the patient from pain, but also to prevent the soft parts in the region of the fracture from being injured by the rough or sharp fragments of the bone. Rough handling of iractures may increase the muscular spasm by the irritation caused by the sharp fragments of the bones, and may also result in the injury of important vessels and nerves, and indeed a simple fracture may readily be converted into a compound one by forcible and injudicious manipulations.

The sooner the examination is made after fracture has occurred the better, for at this time there is less swelling in the region of the injury, and the surgeon can locate the bony prominences with much more ease, and often discover the exact seat of the fracture with the least amount of manipulation of the parts. When a case of suspected fracture is not subjected to examination for several days after reception of the injury, the parts in the region of the supposed fracture are often so much swollen that it is impossible to accurately locate its seat, and in such a case it is often necessary to have an $x$-ray examination or wait until the swelling has subsided before the position of the fracture can be satisfactorily fixed, the case being treated in the meantime as one of fracture.

Anæsthetics.-These may be employed to relieve the patient from pain and to obliterate muscular spasm in the examination of fractures. Their employment is often of the greatest service in the diagnosis of obscure or complicated fractures, especially those in the neighborhood of joints ; but the surgeon should remember that all manipnlations should be made with the same gentleness as when the examination is conducted without anesthesia, for there is the same risk of injury to the surrounding structures by the fragments ; this precaution is often neglected when an anæsthetic has been given, the surgeon being inclined to 
handle the parts more roughly than he otherwise would; sueh practice cannot be too severely condemned.

X-ray Examination.--This method of examination is now very widely employed in fractures. The use of the fluoroscope or of a skiagraph taken by the $x$-rays has proved a valuable means of ascertaining the existence location, and nature of the fracture in obscure cases. It is advisable in every case of fracture where it is possible to have an $x$-ray examination and if possible to have another examination after the ease has been under treatment for a short time to ascertain if the fragments are in good position. 'This form of examination has done much to increase our knowledge of fractures. By this method of examination we have learned that comminution is much more common in fractures than was generally supposed. Distortion of the image sometimes occurs from the direction in which the rays are applied, and this fact should not be lost sight of in examining $x$-ray plates.

Provisional Dressings of Fractures.-It generally happens that fraetures occur at localities more or less distant from the point where the treatment of the fraeture is to be eonducted, and the transportation of the patient and the temporary dressing of the fracture are, therefore, matters of the first importance. In frictures of the upper extremity, if the fracture be simple, the clothing need not be removed, and the arm should be bound to the side by some article of clothing, or supported in a sling made from handkerchiefs or the clothing, and the patient can usually walk or ride for a short distance without much injury to the parts in the region of the fracture or inconvenience to himself. When the bones of the lower extremities or the trunk are the parts involved, the transportation of the patient is a matter of more difficulty. When the bones of the trunk are involved, the part should be surrounded by a binder firmly pinned or tied, made from clothing or from towels, or sheets, or other strong materials which are at hand. When the bones of the lower extremity are involved, if the fraeture be a simple one the clothing need not be 
removed, and the motion of the fragments should be prevented by applying to the sides of the limb, extending above and below the seat of fracture, strips of wood, shingles, pasteboard, bundles of straw, strips of bark

Fig. 257.

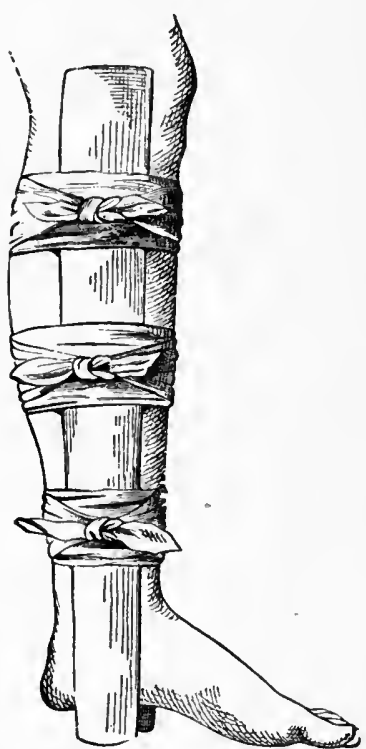

Provisional dressing for fracture of the leg. (EsmaRCH.)

taken from trees, or bundles of twigs, these being held in place by handkerchiefs or strips torn from the clothing (Fig. 257). Umbrellas or eanes; or broomsticks, applied in the same manner, may be eniployed, the object of all of these dressings being to secure temporary fixation of the fragments of bone during the transportation of the patient.

If the fragments are not fixed in some way, but are allowed to move about during the transportation of the patient, much damage may result to the soft parts surrounding the fractured bones, and simple fractures may become compounded ones by the bones being forced through the skin, the discomfort of the patient at the same time being much increased.

Having applied a dressing to bring about fixation of the fragments, the patient should next be placed upon a broad board or settee ; if a mattress cannot be obtained, the fractured limb should be laid upon a mass of clothing, or upon straw, and he should be placed in a wagon or carried to the point where the subsequent treatment of the fracture is to be conducted.

Reduction or Setting of Fractures.-This should be effeeted as soon as possible after the occurrence of the injury and as soon as the surgeon is prepared to apply the dressings to keep the parts in their proper position ; reduc- 
tion at an early period is less painful to the patient, and is aceomplished with more ease to the surgeon than at a later period, when marked inflammation and swelling are present at the seat of fracture. Reduction eonsists in bringing the fragments, by manipulation, as nearly as possible in their normal position; this is accomplished by extension and manipulation with the hands, eare being taken to use as little force as possible to attain the object. Very little force is required if the surgeon places the part in such a position as to relax the muscles which produce the displacement; when this is accomplished, the fragments can usually be pressed into position by the fingers without the applieation of considerable force. When the reduction of a fracture has been accomplished, the fragments are retained in position by the applieation of various splints or dressings which serve to prevent their displacement.

\section{MATERIALS AND APPLIANCES USED IN THE DRESSING OF FRACTURES.}

The Fracture Bed.-Many ingenious forms of beds have been devised for the use of patients suffering from fractures of the bones of the trunk and lower extremities, - with the object of permitting the patient to have fecal evacuations without disturbing his position; but a simple bedstead provided with a firm hair mattress is usually more satisfactory than the complicated form of bed.

It will be found more convenient in handling the patient to use a single bed not over thirty-two or thirty-six inches in width, and it is not essential that the mattress be perforated, as a bed-pan can usually be slipped under the patient. The use of an ordinary shallow tin pie-plate. covered with a piece of old muslin to receive the fecal evacuations may be substituted for the bed-pan, and will be found in many cases more satisfactory, especially in the case of ehildren suffering from fracture of the lower extremities.

Splints.-After the reduction or setting of the fragments in cases of fracture, they are usually retained in position until union oecurs by the use of splints held in 
position by means of bandages or strips of muslin. Splints may be made of wood, or of tin, lead, copper, or wire, binders' board, leather, felt, paper, gutta-percha, or plasterof-Paris.

Wooden Splints.-The simplest splints are made from wood-white pine, willow, or poplar being the best material to employ for their construction, being sufficiently strong to give fixation to the parts and at the same time being light. Splints made from smooth white pine, willow, or poplar boards from one-eighth to one-fourth of an inch in thickness may be employed in the form of straight or angular splints, and their preparation is a matter of little difficulty.

Wooden splints before being applied to the part should be well padded with cotton, oakum, or hair; and where lateral wooden splints are employed in the treatment of fractures of the lower extremity it is usual to place bandages or junk bags between the limb and the splint. The carved wooden splints which are sold by the instrumentmakers are not to be recommended, as a rule, for unless the surgeon has a large number to select from, it is rare that a splint can be obtained to accurately fit any individual case.

Binders' Board Splints.-Binders' board is an excellent material from which to construct splints; it is first soaked in boiling water, and when suffieiently soft is padded with cotton or a layer of lint and moulded to the part. It may be secured in position by a bandage ; as it becomes dry, it hardens and retains the shape into which it was moulded.

Undressed Leather Splint. - Undressed leather is a good material from which to construct splints; it is applied by first soaking the leather in boiling water, and after padding it with cotton or lint it is moulded to the part and retained in position by a bandage.

Felt Splints.-These are made from wool saturated with gum shellac and pressed into sheets. This material is prepared for application to the surface by heating it before a fire until it becomes pliable, or by dipping it into boiling water. 
Gutta-percha Splints.-These are made from sheets of this material from one-sixteenth to one-fifth of an inch in thickness, and may often be employed with advantage. The splint is prepared for use by immersing it in hot water; when it becomes soft it can be moulded to the surface. Care should be taken that it is not allowed to become too soft by long immersion, as it then cannot be conveniently handled.

Paper.Splints. - These are made from layers of Manila paper stiffened with starch, and constitute a very fair substitute for some of the varieties of splints previously mentioned.

Plaster-of-Paris, Starch, Chalk and Gum, Silicate of Potassium or Sodium Splints.-These may be employed for the eonstruction of splints, either movable or immovable, in the treatment of fractures; their methods of preparation and application are deseribed on page 93 et seq.; the plaster-of-Paris dressing is the one which is most generally used at the present time.

Fracture-box.-This is a form of splint used in the treatment of fractures of the lower extremity, and consists of a board eighteen to twenty inches in length, with a foot-board firmly

FIG. 258.

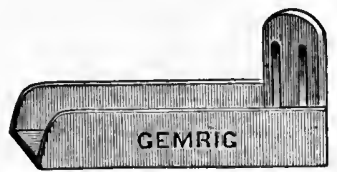

Fracture-box with movable sides. secured at its lower extremity; the sides are seeured by hinges which allow them to be raised or lowered (Fig. 258). A fracture-box of greater length is required for the treatment of fractures about the knee-joint.

Bran, Sand, or Junk Bags.-These are constructed by taking a piece of unbleached muslin five feet in length and fourteen and one-half inches in width, doubling it, and securing the free margins, except at the mouth, by stitches so as to form a bag; the bag is then inverted so that the edges of the seams are brought on the inner surface of the bag. 'The bag is next filled with dry sand, bran, hair, or straw, and the mouth of the bag is closed by stitehes or by being tied with a string. Bran bags 
with splints, or sand bags, are frequently employed in the treatment of fractures of the femur.

Bandages.-These are made of muslin, and are used to retain splints in the treatment of fractures, and are also sometimes applied directly to the injured part before the application of splints to control muscular spasm and limit the amount of swelling ; when a bandage is so used, it is known as a primary roller. The use of the primary roller is sometimes of the greatest service in the-dressing of fractures, but its use in inexperienced hands has so often been followed by unfortunate results in the early treatment of fractures, or in cases which are not under constant observation, that $I$ think it a safe rule of practice to discard entirely the use of the primary roller.

Compresses.-These are made from a number of folds of lint, or of cotton or oakum, and are often employed to

FIG. 259.

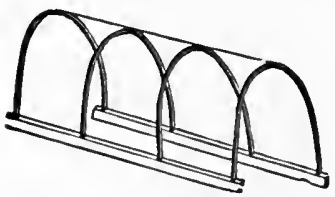

Rack for supporting bedclothes in fractures of the lower extremity. retain fragments in position or to make localized pressure upon certain points in the treatment of fractures. The compresses are held in position by strips of adhesive plaster, by a few turns of a roller-bandage, or by the splints. Compresses are sometimes employed to protect bony prominences of the skeleton from the pressure of the splints, but this purpose is often better effected by the use of small pieces of soap plaster spread on chamois skin fitted over the prominent points.

Rack or Cradle.-This is made of wire or wooden hoops, and is often employed to support the weight of the bedclothes in the treatment of fractures of the lower extremity (Fig. 259).

Evaporating Lotions in Fracture.-The employment of evaporating lotions such as lead-water and laudanum, or muriate of ammonium and laudanmm, to the skin in the region of fractures is highly recommended by many surgeons, especially in fractures involving or situated near joints. They are here employed to relieve pain, to limit 
inflammatory swelling, and to hasten absorption of the blood and serum at the seat of fracture. Many surgeons, on the other hand, think that their use causes irritation of the skin and delays the process of repair in the union of the fracture, and strongly condemn their employment. Personally, I have never seen bad results from their use, and have generally employed them in fractures near or involving the joints; but I do not consider their employment essential, and when I use them I do so for only two or three days. In cases of fractures accompanied with much pain and swelling, when the surgeon does not wish to use any of the lotions named, an ointment of ichthyol 1 part, lanoline 3 parts, spread on lint and wrapped around the limb, will often prove a satisfactory dressing, or a layer of cotton may be simply wrapped around the part before the application of the splints.

\section{Massage in the Treatment of Fracture.-Lucas-} Championnière advocates and practises immediate and continuous massage in the treatment of fractures, and holds that by its use pain is diminished, repair of the bone hastened by the profuse deposit of callus, and atrophy of muscles and stiffening of joints avoided.

Massage is employed as soon as possible after the fracture has occurred, and consists in manipulations with the thumb, the fingers, or the whole hand. The limb is held by an assistant and extension is made, or it is placed upon a firm pillow or a sand cushion. The manipulations should be made in the direction of the muscular fibres and of the blood-current, and firm pressure should not be made directly over the seat of fracture.

Massage shonld be practised for from fifteen to twenty minutes daily, and no retention apparatus should be applied in the intervals unless there is marked tendency to displacement of the fragments, when some form of retention apparatus or splint may be used. These manipulations should be continued for some weeks, until union is firm at the seat of fracture. Massage has also been combined with the ambulatory method of treatment of fractures of the lower extremity. This method of treating 
fractures by massage may be said to be still on trial, suffieient experience not yet having aceumulated to prove that it possesses marked advantage over the generally adopted method of treatment by immobilization.

\section{SEPARATION OF THE EPIPHYSES.}

This lesion consists in a separation of the epiphysis is the bone from its diaphysis. The epiphyses are entirely eartilaginous in infants, but ossification occurs later at various periods for different bones. The separation may occur at any time from birth up to the twenty-first year. The age at which traumatic separation of the epiphyses has been most observed is from the twelfth to the fifteenth year. Epiphyseal separations may be simple or compound.

Simple Separations. - Traumatie separations of the epiphyses may result from direet and indirect violence, from traction or torsion, and in rare cases from museular action. The injury is always aeeompanied by stripping of the periosteum from the end of the shaft of the bone, but it generally remains firmly attached to the epiphysis. Separation of the epiphyses in children results from the application of considerable foree; aceording to Poland, an injury which would be able to produce a dislocation in an adult will in a ehild usually result in a separation of an epiphysis. Separation of the epiphyses may result from disease, as in tuberculous and syphilitic ostitis and acute infective ostitis. Suppuration in the region of an epiphysis may result in its separation.

Compound separations of the epiphyses are frequently met with, being most common at the lower epiphysis of the femur and the upper epiphysis of the humerus. These are grave injuries, from the fact that infection is apt to occur, resnlting in suppurative osteomyelitis and necrosis, followed by arrest of growth of the limb and shortening.

Symptoms.-These are mobility, deformity, crepitus, loss of function, pain, and swelling. Mobility, which exists at a point where it should not be observed, is a most important symptom, and is most marked if the separa- 
tion of the periosteum be extensive. Deformity is also more marked than in fractures, the smoothmess of the separated surfaces permitting of displacement; this varies with the amount of displacement of the diaphysis and the amount and mode of application of the force. Crepitus is soft and muffled; loss of function is usually marked; and pain and swelling at the seat of injury are soon followed by extravasation of blood.

Diagnosis. - Separations without displacement are difficult to diagnose, and are often considered as sprains of joints. In infants this lesion is difficult to recognize, and often escapes detection, but may be followed in a few weeks by swelling, suppuration, and symptoms of chronic osteomyelitis.

Separation of the epiphyses is most apt to be confounded with fracture or dislocation; the diagnosis is made from fracture by observing the line of separation, shape of the displaced epiphyseal fragment, the deformity (which is very characteristic in certain separations), and the soft character of the crepitus. From dislocation, the diagnosis is based upon the following signs: Dislocations are rare in infants and children. In separations of the epiphyses, if the displacement is reduced, it tends to recur upon removal of the force; while in dislocation, if reduction is accomplished, it is not likely to recur when the force is removed. Rigidity is piesent in dislocation, while preternatural mobility is marked in epiphyseal separation. In many joints the epiphysis will still be found to be connected with the joint and to retain its normal relations with the surrounding articular structures. In compound separations of the epiphyses the diagnosis may be made by observing that the displaced end of the bone is not covered by articular cartilage. An $x$-ray examination is valuable in the diagnosis of this injury.

Prognosis.-Union of the separated epiphyses occurs by the same process as that of a fracture. The amount of callus, which is formed largely by the periosteum uniting the fragments, varies with the completeness of their reduction. Non-union has never been observed in this injury. 
Ankylosis of the neighboring joint may result in spite of the greatest care in the reduction of the deformity and in the treatment, yet permanent deformity may be present and interfere very little with the function of the limb. Arrest of growth of the limb after this injury in young snbjects may be observed, but is not a necessary result, for the epiphyseal cartilage may perform its function as completely as before the injury, but is more apt to occur if the separation takes place between the epiphyses and the epiphyseal cartilage, or the cartilage itself is severely injured. Arrest of growth is not marked in many cases, for the reason that the injury occurs at a period when the growth of the skeleton is almost complete.

Treatment.-This consists in reduction of the deformity, which in many cases is difficult unless an anæsthetic be administered, and fixation of the parts after reduction by the use of splints and bandages, the dressings employed being similar to those used in fracture at a corresponding portion of the bone. Muscular wasting should be prevented by the early employment of massage. Compound separations of the epiphyses are treated in the same manner as compound fractures, great care being taken to render the wound aseptic and to maintain it in this condition.

\section{DRESSING OF SPECIAL FRACTURES.}

Fracture of the Nasal Bones.-Fractures of the nasal bones are often accompanied with fractures involving the septum, the nasal process of the maxillary bone, and the nasal spine of the frontal bone.

Treatment.-This consists in replacing the fragments, if displacement exists, by manipulation with the fingers over the seat of fracture and by pressure made from within the nostrils by a probe or a steel clirector. When the displacement is once corrected, it is not apt to recur, and in the majority of cases no dressing is required. Before resorting to any manipulation within the nasal cavities the mucous membrane should be thoroughly cocainized to render the operation painless. When there is a return of the depression of the fragments or displacement of the septum 
after correcting the deformity by raising the depressed fragment, or bending the septum into place with a director, the parts may be held in position by packing the nasal cavity firmly with a strip of antiseptic gauze or by the use of Asch's tubes.

In lateral displacements of the nasal bones from fracture, after reducing the displacement a small compress held over the fragment by strips of adhesive plaster will be the only dressing required.

Mason transfixes the nose, after reduction of the fragments, with a stout needle, and steadies the pieces with a strip of plaster crossing the bridge of the nose and fastened to the ends of the needle. The needle is kept in position for eight or ten days (Fig. 260).

FIG. 260.

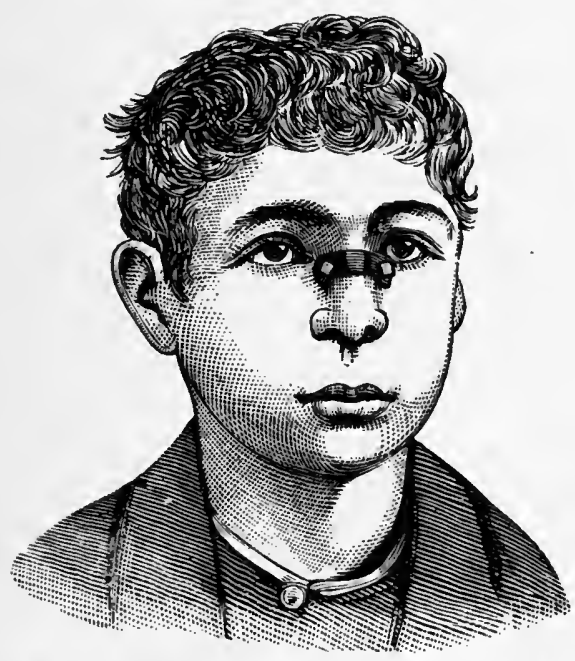

Mason's dressing for fracture of the nasal bones.

Profuse hemorrhage sometimes occurs after fracture of the nasal bones, and may require plugging of the nares to control it. Fractures of the nasal bones are usually quite 
firmly united in two weeks, and dressings may be dispensed with after this time.

Fractures of the Malar Bone and Zygoma.-These fraetures are usually the result of direet foree; the displacement is upward or backward, and when the zygomatie arch is broken the fragments from pressure upon the masseter musele or on the tendon of the temporal musele may interfere with the movements of the lower jaw in mastication. This displacement is corrected by cutting down upon the fragment and elevating it or by passing a tenaenlum into the fragment and raising it. Outward displacements may be corrected by pressure and the applieation of a eompress.

Treatment.-The dressing of these fractures after the correction of the deformity consists in the application of a compress of lint over the

FIG. 261.

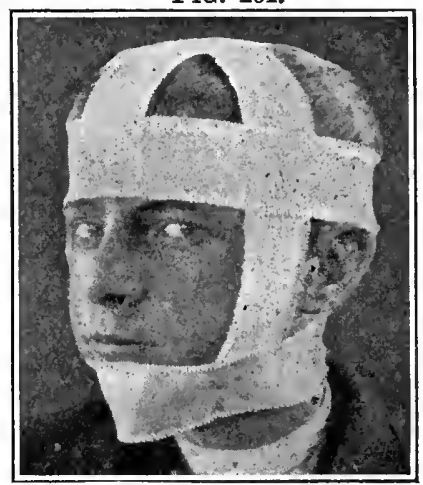

Dressing for fracture of the upper jaw. seat of fracture, held in position by strips of adhesive plaster or a bandage. There is little tendeney to recurrence of the deformity after it has been eorreeted, and union at the seat of fracture is usually firm at the end of three weeks.

\section{Fractures of the Upper} Jaw.-These fractures may involve the body, the nasal processes or the alveolar processes.

Treatment.- The deformity should be correeted, and if any teeth have been displaced they should be replaced; if there is eomminution of the alveolus, the teeth in the separate fragments may be fastened together by fine wire to fix the fragments and hold them in place; the tecth of the lower jaw should be brought up in contact with those of the upper jaw, and the jaws should be secured together by the applieation of 
a Gibson's or a Barton's bandage (Figs. 261, 262). Interdental splits, made of silver or aluminum with grooves to fit the teeth, or of gutta-percha, are also employed in the dressing of these fractures. The patient should not be allowed to move the jaw in mastication, and should be nourished by liquid and semisolid food, which may be taken without removing any teeth to give space for its introduetion. The bandage should be removed every second or third day, and it should be reapplied in the same manner. Union is usually firm at the end of four or five weeks, and dressings may be dispensed with at this time.

Fractures of the Lower Jaw.-The lower jaw may be broken at or near the symphysis, the most usual seat of fracture being near the mental foramen; it is often broken at two places at once, and the fractures are in many cases

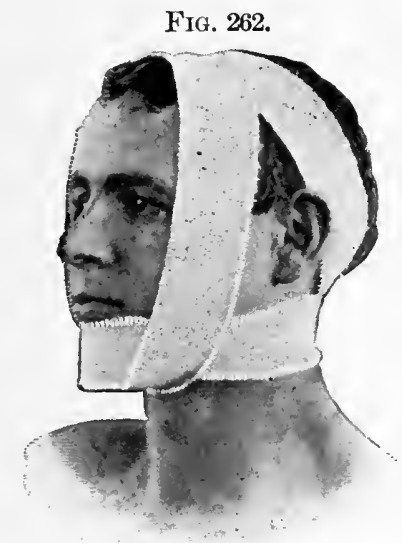

Dressing for fracture of the lower jaw.

rendered eompound by laceration of the mucous membrane, or the injury may consist in a separation of a portion of the alveolar process of the bone.

Treatment.-The dressing of a fracture of the lower 
jaw, after reducing the displacement and replacing any loosened or detached teeth, consists in applying a pad of lint under the chin and bringing the jaw up against the upper jaw, holding the compress in place, and securing the jaws firmly in contact by applying a Barton's (Fig. 262), modified Barton's, or Gibson's bandage. The bandage should be removed and reapplied at the end of the second or third day, and at like intervals during the course of treatment. The patient should be fed upon a liquid or semisolid diet, not being allowed to chew solid food until union at the seat of fracture has become firm. A very satisfactory temporary dressing for a fracture of the lower jaw consists in the application of a four-tailed sling.

Some surgeons prefer to use an external splint moulded from pasteboard or gutta-percha fitted to the chin in the dressing of this fracture (Figs. 263 and 264), this being

Fig. 263.

Frg. 264.

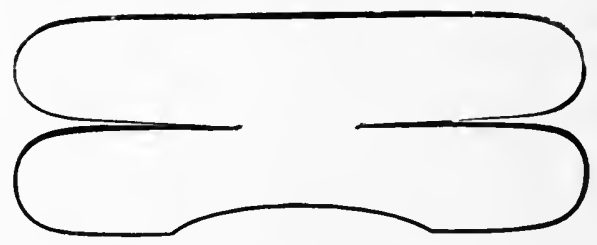

Shape of splint before being fitted to chin. (RoBERTS.)

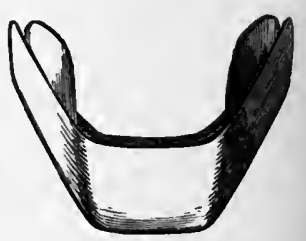

Splint moulded to fit chin. (ROBERTS.)

padded with cotton and held in place by a Barton's or Gibson's bandage. Where there is much difficulty in keeping the fragments in position wiring together of the teeth may be employed, or the fragments may be perforated with a drill and held in place by a strong silver wire suture; interdental splints of metal or gutta-percha are also sometimes used for this purpose. The best results in fractures of the jaw with marked displacement are obtained by the use of interdental splints, a plaster cast of the teeth is first made and from this is constructed a gold, silver or aluminum splint, which fits the teeth accurately. The splint may be cemented to the teeth in one jaw or may 
be worn between the jaws, being held in position by holding the jaws together by a Barton's bandage. During the course of treatment of fracture of the jaws the mouth often becomes very offensive from fermentation of the saliva and discharges, and it is well to use frequently a mouthwash of chlorate of potassium and tincture of myrrh, or boric acid solution.

The dressings for fracture of the lower jaw are applied for four or six weeks, the union usually being quite firm at the end of this time.

Fracture of the Hyoid Bone.-In fracture of the hyoid bone, if displacement exists, its reduction is facilitated by pressure made with the finger in the pharynx.

Treatment.-This consists in enforced quiet and the use of opium if cough is a prominent symptom, and the inflammatory symptoms may require the employment of active local treatment. A dressing may sometimes be employed with advantage, consisting of a splint of pasteboard or leather moulded to the anterior portion of the neck.

Fractures of the Larynx or Trachea.-In fractures of the larynx or trachea where there is little displacement and dyspnoa is not marked, the parts should be supported by the application of compresses of lint held in place by strips of adhesive plaster. If, on the other hand, the respiration is embarrassed or there is free expectoration of blood, tracheotomy should be performed, and if the injury be seated in the larynx the displacement of the fragments may be overcome by manipulation with the finger or a director through the tracheal wound, or the larynx may be packed with a strip of antiseptic gauze to control hemorrhage or hold the fragments in position, the patient in the meantime breathing through a tracheotomytube secured in the tracheal wound; the packing should be removed in a few days, the tracheotomy-tube being permanently removed as soon as the patient can breathe comfortably through the larynx with the tracheal wound closed. In fracture of the trachea the opening into the trachea should be below or at the seat of injury.

Fractures of the Ribs.-Fractures of the ribs are 
more frequent than fractures of any other bones of the trunk; the ribs most commonly broken are those from the fourth to the tenth; the most common seat of fracture is near the anterior or posterior portion; the displacement is usually not marked, unless a number of ribs be broken, being prevented by the intercostal muscles and aponeuroses.

Treatment.-The dressing of fractures of the ribs is best accomplished by enveloping the side of the chest on which the rib or ribs are broken with broad straps of adhesive or rubber plaster. The adhesive straps should be two and a half inches in width and sufficiently long to extend from the spine to the middle of the sternum. The straps are warmed, and the first strap is firmly applied at the base of the chest, extending from the spine to the mid-sternal line; a number of ascending straps are applied in this way, each strap overlapping the preceding one by about one-third of its width until half the chest is covered in (Fig. 265). This dressing usually gives the patient

FIG. 265.

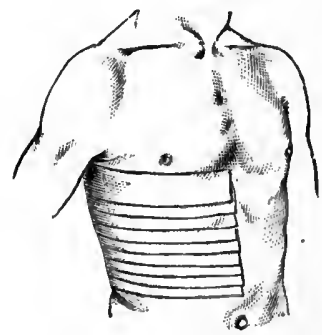

Adhesive plaster dressing for fracture of the ribs. (HAMILTON.) much comfort, and the straps need not be renewed until they become slightly loosened, usually at the end of a week or ten days; they should then be renewed in the same manner. The dressings are nsually dispensed with at the end of three or four weeks, as repair of the fracture is generally well advanced at this time.

A satisfactory temporary dressing consists in surrounding the chest by a broad binder of stout linen or muslin ; indeed, some surgeons prefer to employ this dressing during the course of treatment, but, as a rule, I think it is not as good a dressing as the adhesive plaster dressing, as the former confines the movements of both sides of the chest.

Fractures of the Costal Cartilages.-These fractures often take place at the junction of the cartilages with the ribs or in the body of the cartilages, and the union of the 
fracture usually takes place by the production of a mass of bone at the seat of fracture.

Treatment.-It consists in the application of strips of adhesive plaster applied in the same manner as for fracture of the ribs, and the dressings should be retained for about the same time.

Fractures of the Sternum.-Fractures of the sternum are rare injuries, but diastasis of the bones of the sternum is a more common accident.

Treatment.- The treatment for both fracture and diastasis is the same, and consists in the application of a compress over the seat of fracture held in place by a broad bandage, or, better, by strips of adhesive plaster (Fig. 266 ), applied so as to cover and fix the anterior portion of the chest, covering the entire length of the sternum. This dressing should be retained for at least four weeks, being. renewed if it becomes loose at the end of a week or ten days.

\section{Fractures of the Pelvis.} -These fractures may involve the ilium, ischium, pubis, or sacrum. Vertical fractures, either single or double, and separations of the pelvic bones from their

FIG. 266.

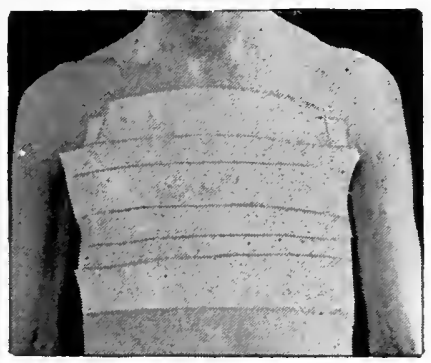

Adhesive plaster dressing for fracture junctions may also occur, and are often serious injuries from implication of the pelvic viscera.

Treatment.-The reduction of the displacement should be first accomplished as far as possible by external manipulation, together with internal manipulation by the fingers introduced into the rectum, or into the vagina in the female. The patient sbould be placed upon a firm bed on his back, with the knees slightly flexed over a pillow, and the parts should be kept at rest by surrounding the pelvis with broad straps of adhesive plaster or a stout muslin binder, or by a firmly applied padded pelvic belt. 
The hip-joints should be kept at rest by the applieation of pasteboard splints or by sand bags. Fractures and diastases of the pelvic bones may also be satisfactorily treated by the application of a plaster-of-Paris dressing. The parts being well padded with cotton, a plaster dressing is applied to include the upper portion of both thighs, the pelvis and a portion of the trunk. The dressings should be retained for a period of at least six weeks.

When these fractures are complicated by injury of the pelvic viscera various operative procedures may be required, which will compel the surgeon to modify the method of dressing.

Fractures of the Sacrum and Coccyx.-The dressing of fractures of the sucrum, after effeeting reduetion of the frag. ments as far as possible by pressure from within the rectum, consists in the application of broad adhesive straps around the pelvis, the patient at the same time being kept at rest in bed.

When the coceyx is fractured, after reduction of the displacement, which may sometimes be aceomplished by manipulation with the finger in the reetum, the patient should be confined to bed and the bowels kept at rest by the use of opium by suppository. The patient should remain in bed for two or three weeks.

Fractures of the Vertebræ.-Fractures of the vertebræ" are always most serious injuries, not only from the damage to the bones themselves, but also from that to the spinal cord, membranes, and nerves, which often accompanies them. These injuries are often assoeiated with dislocations of the vertebræ, so that the term fracture-dislocation is used to deseribe them. In transporting or turning in bed a patient suffering from fracture of the vertebræ great care should be exereised, for rough or sudden motions may cause a displacement of the fragments which might, by injury of, or pressure upon, the spinal cord, rapidly prove fatal.

Treatment.-If the deformity is marked, efforts should be made to reduce it by extension and counter-extension; and the result may be successful, especially if the fracture be associated with a dislocation of the vertebræ. In some cases the use of permanent extension by means of weights 
attached to the legs, shoulders, and chest by adhesive plaster and bandages, has been successful in reducing the deformity. Laminectomy may be practised in certain cases.

The patient should be placed upon his back upon a bed with a hair mattress, or better, if it can be obtained, a water-bed, which eonsists of a rubber mattress filled with water, which distributes the weight of the patient's body evenly over the surface. Whatever form of bed be used, the greatest eare should be exercised to keep the patient absolutely clean, and the parts of the body or limbs which are exposed to pressure should be frequently bathed with alcohol or soap liniment; and to distribute the pressure, small pads should be placed under the parts and changed at intervals. These precautions are necessary to prevent, if possible, the formation of extensive bedsores, which are a frequent and troublesome complication of these injuries.

The bowels should be carefully watched, and, if constipation is present, it shonld be relieved by the use of enemata ; and, as it is not desirable to lift the patient to slip a bed-pan under him, the discharges may be received in a flat tin plate pushed under the thighs and buttocks, or on pads of oakum or old muslin.

The care of the bladder is also a matter of the greatest importance; the retention which at first exists should be relieved by the use of a flexible catheter earefully sterilized and introduced with great gentleness, and when incontinence supervenes a catheter which has been thoroughly sterilized should also be used at intervals; the employment of a soft instrument, if used with care, is not apt to produce injury to the urethra or bladder.

The employment of a plaster-of-Paris jacket has been followed, in some cases, by good results, and it may be applied early in the case, or after the patient has been kept in the recumbent posture for some weeks; by its use it is often possible to get the patient out of bed and allow him to sit in a chair.

In fraetures involving the cervical vertebra, care should be exercised in lifting or moving the head; it is often of advantage in these cases to apply short sand bags to the 
sides of the neck and head, to give additional fixation to the parts while the patient is in the recumbent posture, or, if he is allowed to get ont of bed, to apply a moulded leather or pasteboard splint to the neck, shoulders, and back of the head, for the same purpose.

The course of treatment in cases of fractures of the vertebræ, if the patient does not succumb to the injury in a few days or weeks, often extends over many months, and recovery is often more or less incomplete as regards the function of the parts below the seat of fracture.

Fracture of the Skull.-Treatment.-This depends largely upon the nature of the injury-whether simple or compound-and the condition of the cranial contents. In simple fractures unaccompanied with cerebral symptoms no special dressing is required, but in compound fractures where loose fragments are present, these should be removed; and if there is no depression of the fragments, and if no cerebral symptoms are present, the wound should be drained, carefully closed and dressed antiseptically, the dressings being held in place by a recurrent bandage of the head. The patient should be put to bed, and the use of an ice-cap to the head is often of service. The diet should be restricted, while calonel and opium or bromide of potassium should be administered; it is well to keep the patient for a few weeks in a quiet and darkened room. Where cerebral symptoms are present, either in simple or compound fractures, and trephining is resorted to, the dressing of the wound is similar, and the same general treatment should be adopted. In all cases of fracture of the skull, whether subjected to operative treatment or not, it is well to keep the patient at rest in bed for three or four weeks, and he should be cautioned to avoid excesses afterward, and should not resume active work for some months.

Fractures of the Clavicle.-Fractures of the clavicle may be complete or incomplete, and in the latter variety of injury the deformity is not usually very marked. The indications for treatment in complete fractures of the clavicle are to relax the sterno-cleido-mastoid muscle, to pre- 
vent the weight of the arm on the injured side from dragging down the outer fragment of the clavicle, and, by fixing the scapula, to carry the attached external fragment outward and forward. A large variety of dressings have been devised and used to accomplish these objects.

Dressing by Position.-The treatment of fractures of the clavicle by position is accomplished by placing the patient in bed on his back upon a firm mattress with a low pillow under his head, and the arm on the side of injury should be fastened to the side of the chest by a few circular turns of a bandage passing around the arm and chest; the deformity is usually very satisfactorily reduced upon the patient assuming this position, and after three weeks' rest

FIG. 267

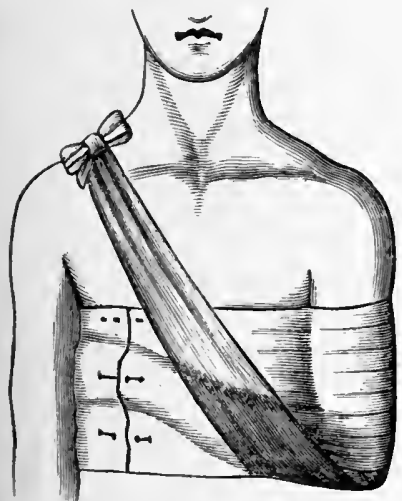

Four-tailed bandage for fracture of the clavicle. (STIMson.)
Fig. 268

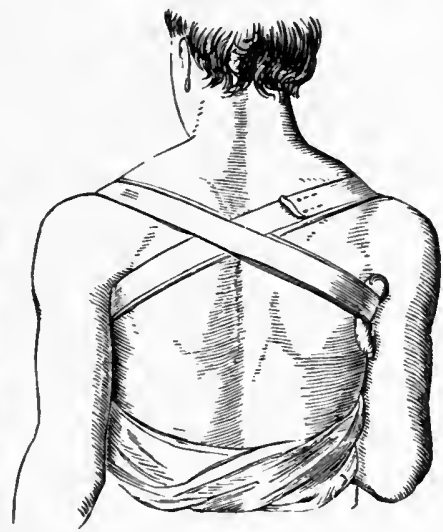

Posterior figure-of-eight dressing for fracture of the clavicle. (HAMILTON.)

in this position the union is generally sufficiently firm to allow the patient to get out of bed and be about with the arm bound to the side or carried in a sling or with a Velpeau bandage applied, without any recurrence of the deformity.

Temporary Dressing.-A satisfactory temporary dressing 
for fractures of the clavicle consists in the application of a four-tailed bandage; the bandage is made from a piece of muslin two yards in length and fourteen inches in width; a hole is cut in its centre about four inches from its margin, to receive the point of the elbow; the bandage is then split into four tails in the line of the hole and to within six inches of it; the body of the bandage should be applied so that the point of the elbow rests in the hole, and a folded towel being placed in the axilla, the lower tails should be carried, one anteriorly, the other posteriorly, diagonally across the chest and back, to the neck on the side opposite the seat of fracture, and secured; the remaining tails are next carried around the lower part of

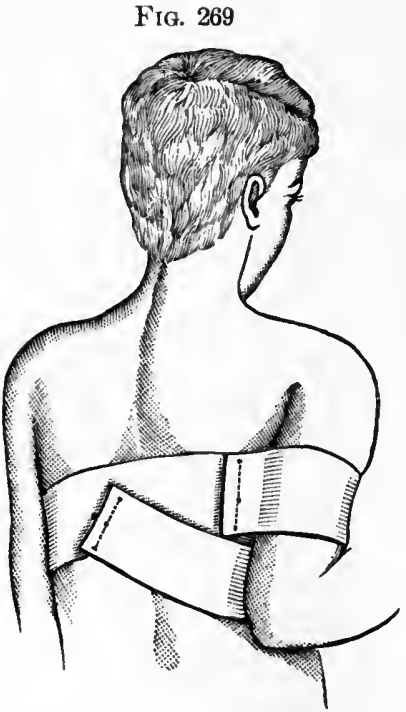

Sayre's dressing for fracture of the clavicle. First strip applied.

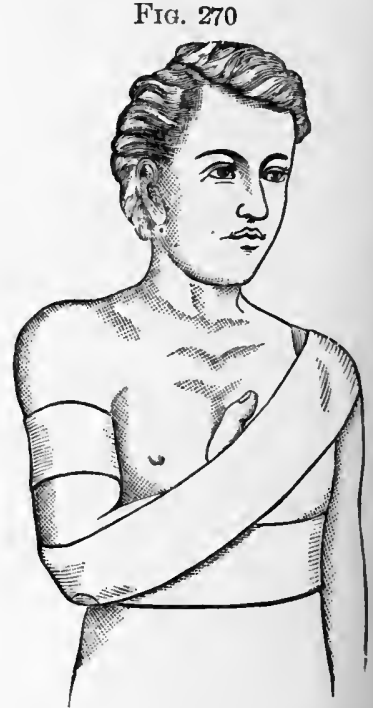

Sayre's dressing for fracture of the clavicle. Second strip applied.

the chest and secured so as to fix the arm to the side of the body (Fig. 267).

In some cases the deformity is corrected by the applica- 
tion of a posterior figure-of-eight bandage, the forearm on the side of injury being carried in a sling (Fig. 268).

Sayer's Dressing.-This consists of two strips of adhesive plaster three and a half inches wide and two yards in length. The first strip is looped around the arm just below the axillary margin, and is pinned or. sewed with the loop sufficiently open not to constrict the arm. The arm is then drawn downward and backward until the clavicular portion of the pectoralis major muscle is put sufficiently upon the stretch to overcome the action of the sterno-cleido-mastoid muscles, and in this way draws the sternal fragment of the clavicle down to its place. The strip of plaster is then carried completely around the body and pinned or stitched to itself on the back (Fig. 269). The second strip is next applied, commencing upon the front of the shoulder of the sound side; thence it is carried over the top of the shoulder diagonally across

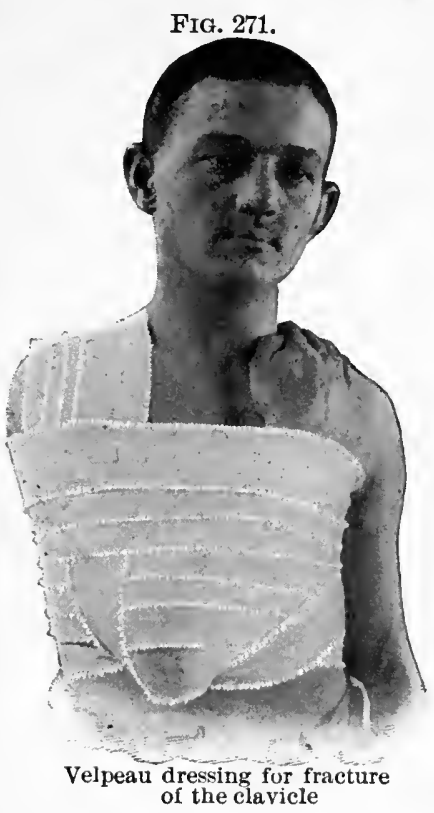
the back, under the elbow, diagonally across the front of the chest to the point of starting, where it is secured by pinning or sewing. A slit is made in this strip to receive the point of the elbow. Before the elbow is secured by the plaster it should be pressed well forward and inward (Fig. 270).

Velpeau's Dressing.- This may also be used in the treatment of fractures of the clavicle (Fig. 271). A oompress may also be secured by the vertical turns of this bandage over the seat of fracture if needed. The application of the bandage is described on page 66 . 
In any form of dressing in which the arm is held against the side of the chest it is well to apply a folded towel or piece of lint between the arm and chest to prevent the skin surfaces from becoming excoriated.

FIG. 273.

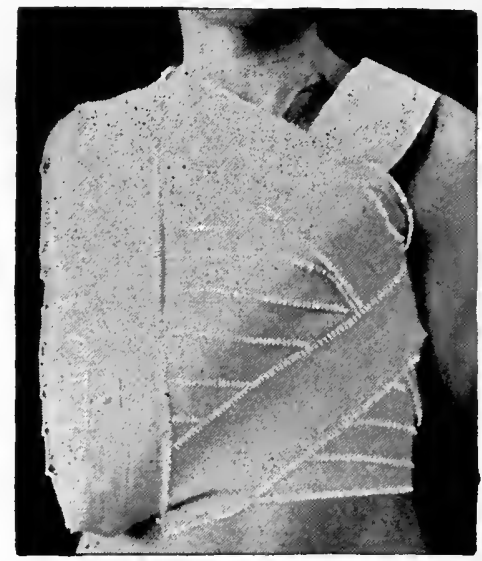

Modified Velpeau dressing for fracture of the right clavicle.

Modified Velpeau's Dressing.-A modified form of Velpeau's dressing for fracture of the clavicle is applied as follows: A soft towel or piece of lint is placed against the side of the body and over the front of the chest, and held in position by a strip of adhesive plaster; the arm is next placed in the Velpeau position, a good-sized pad of lint is next applied over the scapula, and this is held in place by a strip of adhesive plaster two and a half inches in width and one and a half yards in length; this strip is continued downward and forward so as to pass over the point of the elbow, and is carried diagonally across the chest to the shoulder of the opposite side, and is secured, a slit being cut in it to receive the point of the elbow; a compress of lint is next placed over the seat of fracture and held in place by a strip of adhesive plaster; an additional strip of plaster is next carried from the 
spine around the arm and chest and secured on the opposite side of the chest; circular turns of a roller-bandage are then passed around the chest, including the arm from below upward until the arm is securely fixed to the body, and the dressing is finished by making one or two turns of the third roller of Desault (Fig. 272). Or the turus of the third roller of Desault may be applied first, and the dressing may be finished by circular turns of a roller passing around the arm and chest, extending from the elbow to the shoulder.

The removal of dressings and their reapplication will depend upon the comfort of the patient and the manner in which they keep their position. As a rule, in fractures of the clavicle the dressings are removed at the end of the second or third day, the parts are inspected, and the skin is sponged with dilute alcohol; the dressings are then reapplied, and if the patient is comfortable and the parts are in good position, the dressings are made at less frequent intervals until union is completed at the seat of fracture.

Union in cases of fracture of the clavicle is generally quite firm at the end of four or five weeks, and at this time the dressings may be removed, and the patient should carry the arm of the affected side in a sling for several weeks, and should not undertake any work requiring forcible movements of the arm until eight or ten weeks have elapsed from the receipt of the injury.

Fractures of the Clavicle in Children.-In the treatment of fractures of the clavicle in children the Velpeau or modified Velpeau dressing will be found to be the most satisfactory dressing to employ; and as these patients are particularly apt to disarrange the dressings, it is well to render them additionally secure by applying a few broad strips of adhesive plaster over the turns of the roller-bandage, the strips following the turns of the bandage.

The time required for union in fractures of the clavicle in children is somewhat shorter than in adults ; the dressings may be removed at the end of three weeks.

Fractures of the Scapula.-Fractures of the scapula 
may involve the body, neck, acromion, or coracoid process of the bone. Fractures of this bone are rare, those of the acromion process being most common.

Fracture of the Body of the Scapula.-Treatment.-If deformity is present, it is reduced by manipulation, and compresses of lint are placed above and below the seat of fracture and held in place by adhesive strips; the arm is next fixed to the side of the body by spiral turns of a roller-bandage passing around the arm and chest, and the forearm is supported in a sling.

Fracture of the Neck, Acromion, or Coracoid Process of the Scapula.-Treatment.-The treatment of these fractures consists in placing a pad of lint or a folded towel in the axilla and binding the arm to the body by spiral turns of a roller-bandage passing around the arm and chest, and supporting the forearm in a sling. These fractures may also be dressed by first placing a pad of lint or a folded towel in the axilla and then securing the arm in the Velpeau position by the application of a Velpean bandage (Fig. 271). In fractures of the acromion or coracoid processes the union is usually fibrous. In the treatment of fractures of the scapula the

Fig. 273.

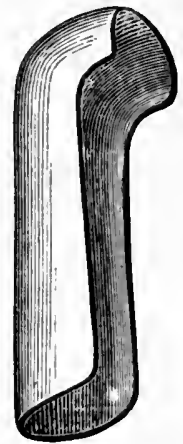

Moulded splint for shoulder and arm. dressing should be retained for about four weeks.

Fractures of the Humerus.-Fractures of the humerus may involve the upper extremity, the shaft, or the lower extremity of the bone.

Fractures of the Upper Extremity of the Humerus.-These include fractures of the head and anatomical neck of the bone, fractures through the tuberosities, fractures through the surgical neck of the humerus, and separation of the upper epiphysis of the humerus.

Treatment.-The most satisfactory dressing for all fractures of the humerus above the upper third of the bone is applied as follows: A primary roller should be evenly applied from the tips of 
the fingers to the seat of the fracture, the arm being flexed at the elbow before the bandage is carried above this point, to prevent the dangerous constriction which might result if the bandage were applied with the arm in the straight position, and it were afterward flexed at the elbow. A folded towel or a thin pad of lint should next be placed in the axilla and over the outer surface of the chest, to furnish a firm basis of support for the humerus, and also to prevent excoriation from the contact of the skin surfaces. A splint of pastehoard, felt, or leather (Fig. 273) is next moulded to the shoulder and arm; this should be long enough to extend some distance below

FIG. 274.

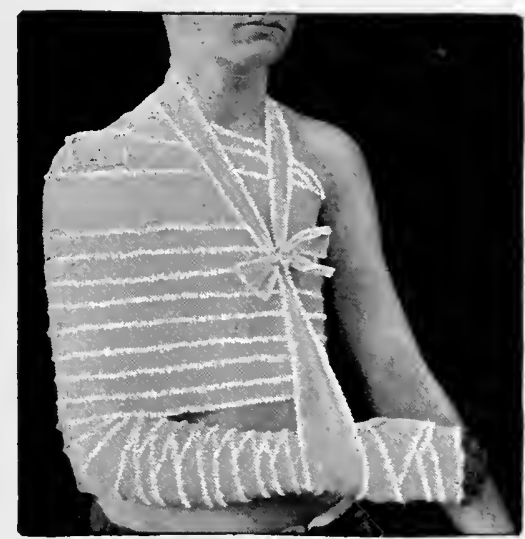

Dressing for fracture of the upper extremity of the humerus.

the seat of fracture and wide enough to cover in about one-half of the circumference of the arm, and is padded with cotton and fitted to the shoulder and arm. The splint and arm are next secured to the side of the body by spiral turns of a roller-bandage, including the arm and chest in its turns and applied from the elbow to the top of the shoulder. The forearm is carried in a narrow sling suspended from the neck (Fig. 274). This dressing should 
be removed at the end of twenty-four or forty-eight hours, and after the parts have been inspected and sponged with alcohol the dressings should be reapplied in the same manner, and if the patient is comfortable they need not be disturbed again for three or four days, subsequent dressings being made at the same intervals. Union in fractures of the upper extremity of the humerus, except in those within the capsule, in which bony union is the exception, is usually quite firm at the end of five or six weeks, and the dressings can be dispensed with at this time.

Separation of the Upper Epiphysis of the Humerus.-This accident is not unconmon in patients under twenty years

F1G. 275.

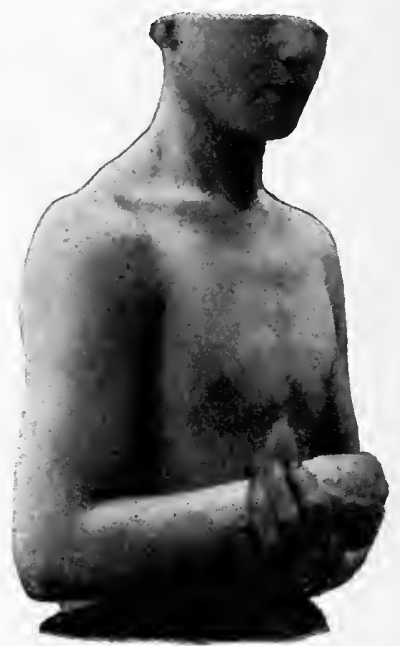

Separation of upper epiphysis of the humerus.

of age, and may be confused with fracture of the neek of the humerus. There is usually a marked projection of the npper extremity of the lower fragment in front of the shoulder (Fig. 275).

Treatment.-This consists in reducing the displacement by manipulation, and the dressing is similar to that em- 
ployed in fracture of the neck of the humerus (Fig. 274). In some cases, after the deformity has been reduced under anæsthesia, the reduction is maintained by fixing the arm in the abducted position by a plaster-of-Paris bandage for a few weeks. The functional result following this injury is usually very good.

Fracture of the Shaft of the Humerus.-This fracture may occur at any point between the surgical neck and the condyles of the humerus; the line of fracture is usually oblique.

Treatment. - This consists in the application of a primary roller from the tips of the fingers to the seat of fracture; a short, well-padded, wooden splint extending from the axilla to a point a little above the internal condyle is next placed on the inner surface of the arm and against

FIG. 276.

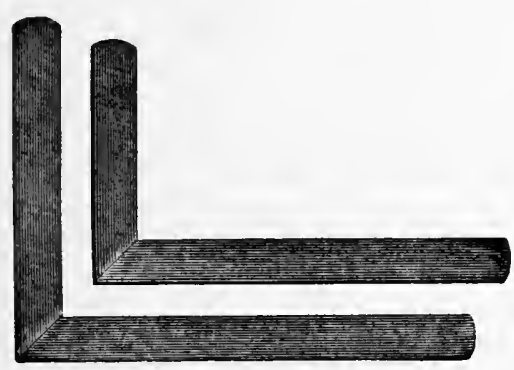

Internal angular splints.

the chest; a moulded pasteboard or felt splint, fitted to the shoulder and outer side of the arm and extending a short distance below the seat of fracture, is padded with cotton and applied to the shoulder and arm. The splints are held in position by the turns of a bandage, and the arm is secured to the body by spiral turns of a roller-bandage carried around the chest and arn, and the forearm is carried in a sling suspended from the neck. The dressing is much the same as that for fracture of the upper part of the humerus, with the addition of the short internal splint. 
Fracture of the shaft of the humerus may also be dressed by first applying a primary roller and then plaeing the forearm and arm upon a well-padded internal angular splint (Fig. 276). Care should be taken to see that the end of the splint extends only to the axilla and does not press upon the brachial vein. A pasteboard or felt moulded splint is next applied to the shoulder and outer side of the arm, and should be long enough to extend below the seat of fracture. The splints are held in position by turns of a roller-bandage heginning at the fingers and carried up to the shoulder, and finished with a few spica-of-theshoulder turns (Fig. 277). If there is great overlapping

Fİt, 277.

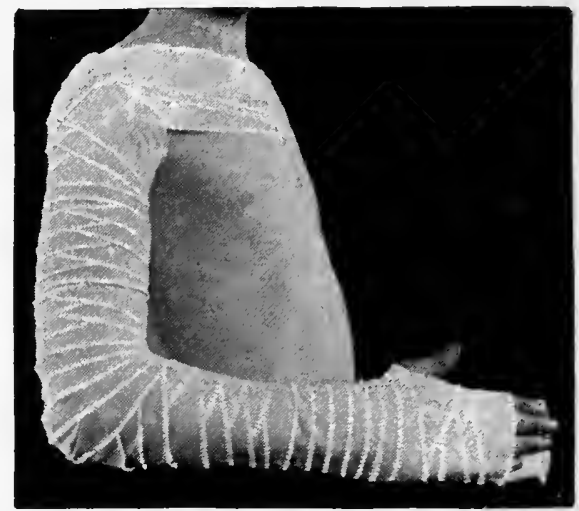

Dressing for fracture of the shaft of the humerus with internal angular splint and external splint of binders' board.

of the fragments producing marked shortening, the patient should be kept in bed and the elbow flexed, and weight or elastic extension made by adhesive strips applied to the arm, short coaptation splints also being applied. If the patient is treated as a walking ease, the same result can be accomplished with a bag of shot or weight fastened to the arm so as to hang below the elbow. The arm is supported by a sling applied at the wrist, and sometimes for additional seeurity the arm is bound to the side of the body by 
spiral turns of a bandage carried around the arm and chest. The after-treatment of these fractures as regards the removal and renewal of the dressings is the same as in cases

FIG. 278.

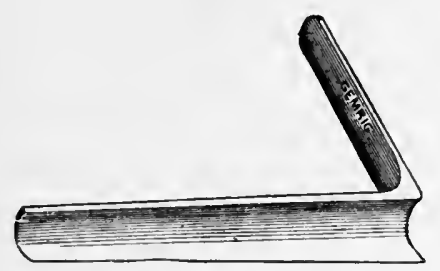

Anterior angular splint.

of fracture of the upper portion of the humerus; the dressings should be retained for five or six weeks.

Fractures of the Lower Extremity of the Humerus.-These include fractures at the base of the condyles splitting fract-

Fig. 278.

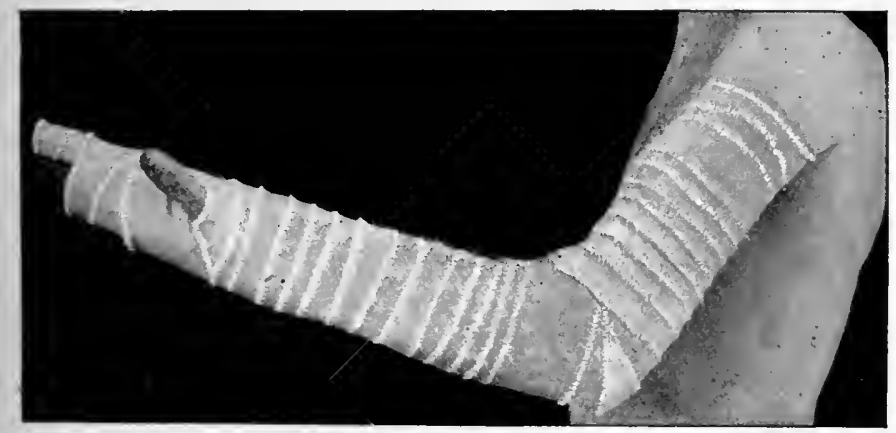

Dressing for fracture of the lower extremity of the humerus with anterior angular splint.

ures between the condyles or those of the internal or external condyle, and epiphyseal separation of the lower epiphysis of the humerus.

Treatment.-The displacement is reduced by extension and manipulation, and before applying any splint it is 
well in many cases to apply over the region of the fraeture several layers of cotton-wadding. An anterior angular splint (Fig. 278) well padded with cotton or oakum is next applied and held in position by the turns of a rollerbandage applied from the fingers to the upper portion of the splint (Fig. 279). These fractures may also be dressed with a well-padded internal angular splint, this splint being substituted by an anterior angular splint at the end of ten days or two weeks.

Some surgeons prefer to dress fractures of the condyles of the humerus with the arm in the extended position upon a straight anterior splint, or with short, narrow pasteboard splints applied around the joint, as favoring more aceurate

FIG. 280.

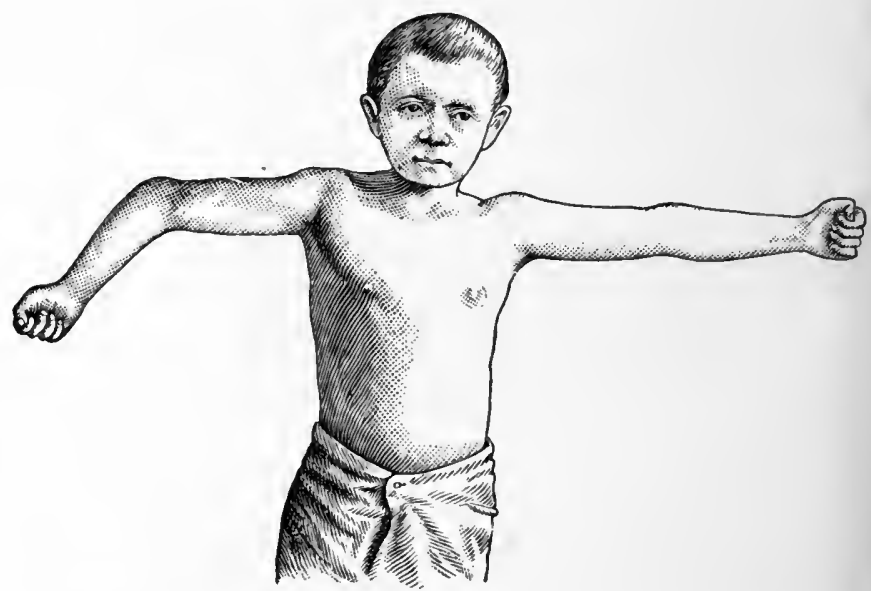

Gunstock deformity after fracture of the condyle of the humerus.

coaptation of the fragments, and diminishing the tendency to what is known as gunstock deformity and loss of the carrying angle (Figs. 280, 281). If this position is employed, a straight wooden splint is applied to the anterior surface of the arm and forearm, or moulded splints of pasteboard may be used, and after the union is moderately 
firm, at the end of two weeks, the elbow should be flexed and kept in this position during the remaining time of the treatment.

Treatment by Acute Flexion (Jones's Method).-In this dressing of fractures of the condyles of the humerus, the displacement of the fragments is corrected by manipulation and the forearm is placed in a position of acute flexion at the elbow (Figs. 282 and 283), and the hand of the injured arm is brought up and is supported by a sling carried around the neek (Fig. 284). The flexion of the forearm on the arm may also be rendered more secure by passing broad strips of adhesive plaster or bandage around the arm and forearm. The arm is kept in the position of acute flexion for about two weeks and it is then dressed in a position of less flexion, and at the end of $\begin{gathered}\text { Showing loss of carrying angle after } \\ \text { racture of the condyle of the humerus. }\end{gathered}$ three or four weeks is simply carried in a sling. After this time the arm is gradually extended. This method of dressing seems to be followed by the best results in fractures of the condyles of the humerus in children. By this method of dressing the fragments are firmly held in position, better motion is obtained, and the tendency to gunstock deformity is diminished. The more extended use of this method of dressing fractures of the condyles of the humerus has shown that the practieal results obtained are most satisfactory. 
Fig. 282.

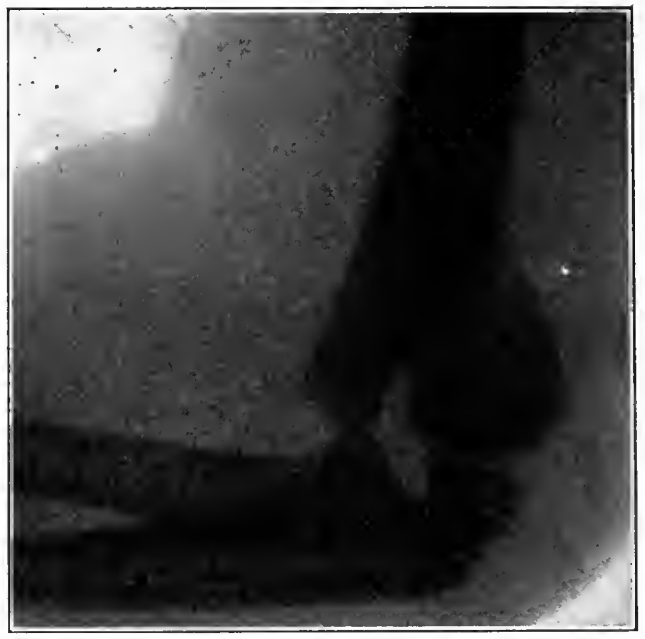

Fracture of the condyles of the humerus before reduction.

FIG. 283.

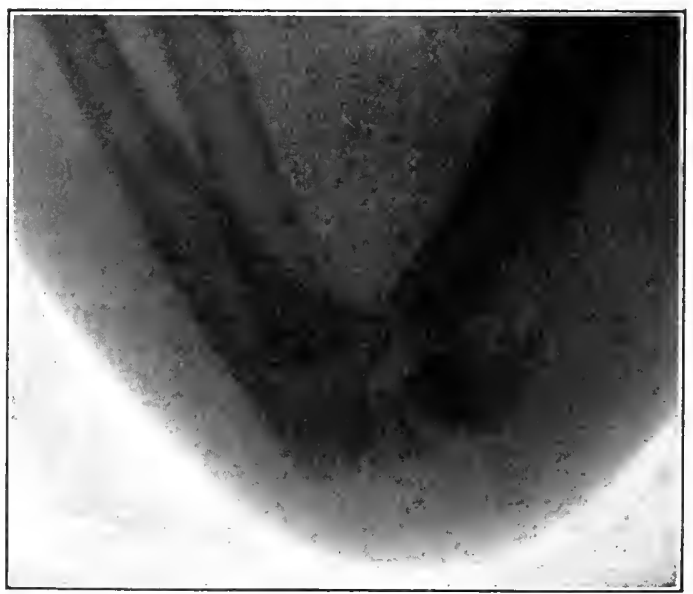

The same case after reduction wfth the elbow in acute flexion. 
When fractures of the lower extremity of the humerus involve the elbow-joint, a certain amount of impairment

Fig. 284.

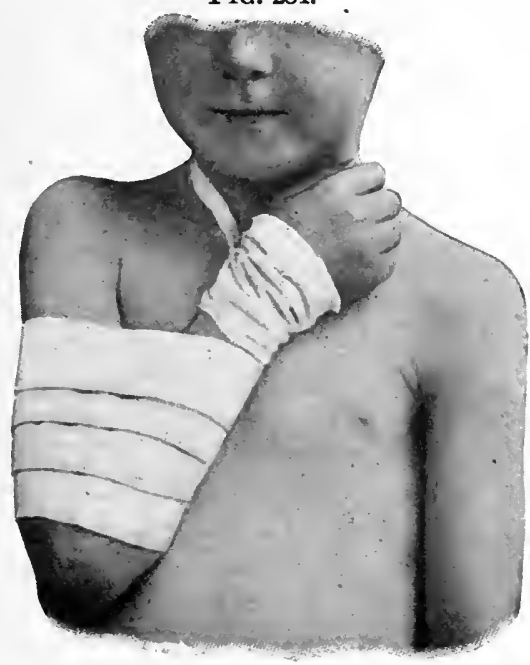

Dressing for fracture of condyles of humerus in acute flexion.

of joint-motion is apt to occur either from anchylosis or from displacement of the fragments, giving rise to gumstock deformity and loss of the carrying angle, which in many cases it is impossible to reduce completely, so that flexion and extension of the joint are restricted. Bearing these facts in mind, it is well to make passive motion in these cases as early as the second or third week. It is well to explain to the patient or his friends that impairment of jointmotion may result in these fractures in spite of the greatest skill and care in the treatment. In a case of fracture in the region of the condyles of the humerus the dressings should be removed in twenty-four hours, and should be redressed in the same manner, and if the swelling does not increase and the dressing is comfortable to the patient it should afterward be dressed at less frequent intervals: 
the union is generally quite firm at the end of four weeks, and the splint may be removed at this time. Fractures of the condyles of the humerus are very common in children, and epiphyseal separations of the lower epiphysis of the humerus are also met with; the dressing of these injuries in this class of patients is similar to that described for fractures of the condyles of the humerus.

\section{Fractures of the Olecranon Process of the Ulna.-} Fracture of the olecranon may consist in simply a separation of the cortical layer of bone over the summit of the process to which the triceps is principally attached, or the line of fracture may pass through the sigmoid fossa.

Treatment.-This fracture is dressed with the arm slightly flexed at the elbow, or with it completely extended; the former position is possibly a little less irksome to the patient. 'The separation of the fragment by the action of the triceps muscle is usually not very marked; but, if the displacement is considerable, it may in a measure be overcome by the use of a compress above the fragment, over which figure-of-eight strips of adhesive plaster are fastened to draw it down into position (Fig. 285). The ends of the strip are then attached to a well-

FIG. 285.

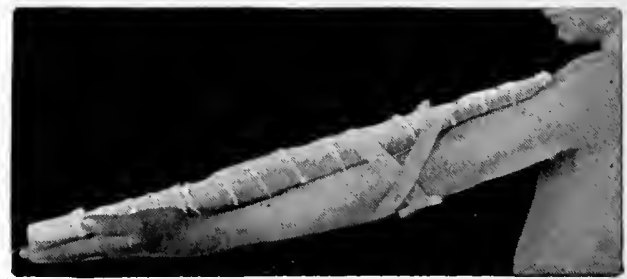

Adhesive straps applied in fracture of the olecranon.

padded straight splint which should be long enough to extend from the upper third of the arm to the ends of the fingers, and is secured in position by the turns of a roller carried from the fingers to the upper extremity of the splint, with figure-of-eight turns at the elbow to reinforce the action of the strips of plaster (Fig. 286). 
This fracture may also be dressed by first applying a primary roller to the elbow, and then placing over the arm a well-padded anterior obtuse-angled splint, or a straight splint with a good-sized pad of lint or oakum fastened at a point corresponding to the position of the flexure of the elbow. When either of these splints is placed upon the arm a position of moderate fiexion is obtained. A compress of lint is next placed above the fragment, if there is a displacement, and one or two narrow strips of adhesive plaster are fastened over this and passed obliquely downward and attached to the splint on either side. The splint is then securely fastened to the arm by the turns of a roller-bandage applied from the fingers to the upper end of the splint.

FIG. 286.

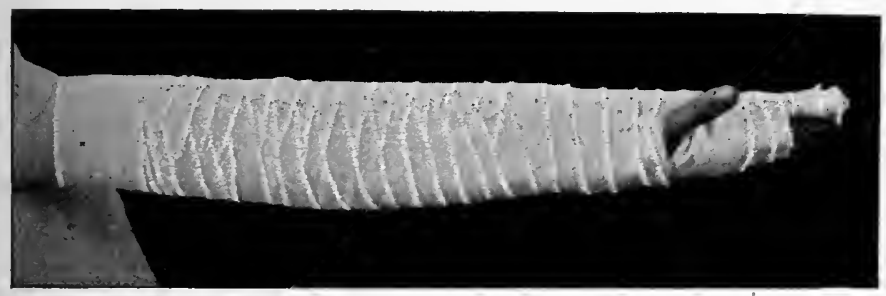

Fracture of olecranon dressed in the extended position.

The dressings should be removed at the end of twentyfour or thirty-six hours, or sooner if there is evidence of swelling of the tissues in the region of the fracture, and they should he reapplied in the same manner. If the dressing is comfortable to the patient, and there is no evidence of swelling, the subsequent dressings shonld be made at less frequent intervals; the dressings are usually retained in this fracture for five or six weeks. Passive motion should not be made until this time, as flexion of the elbow tends to separate the fragments, unless union has taken place. The union of a fracture of the olecranon is in most cases fibrous, but in a few instances bony union has been observed. 
Fracture of the Coronoid Process of the Ulna.-Fracture of the coronoid process is an extremely rare injury.

Treatment.-This is accomplished by placing the arm in a flexed position and applying a well-padded internal right-angled splint, or an anterior right-angled splint, and securing it to the arm by the turns of a roller-bandage. A moulded pasteboard or leather gutter may be substituted for the angular splint. The dressings should be changed at intervals, and after their removal, at the end of three or four weeks, passive motion should be practised.

Fractures of the Head and Neck of the Radius.These fractures are also quite rare.

Treatment.-This consists in reducing the fragments by manipulation, by flexing the elbow and keeping it in this position, and by the application of a well-padded anterior right-angled splint, the splint being firmly secured in position by the turns of a roller-bandage applied from the tips of the fingers to the upper end of the splint (Fig. 279). The splint should be changed at intervals, and should not be permanently removed for four weeks, at which time passive motion, consisting in flexion and extension at the elbow and pronation and supination of the forearm, should be made.

An internal angular splint applied to the inner surface of the forearm and arm may also be used in the treatment of these fractures (Fig. 276).

Fractures of the Ulna and Radius.-These fractures are often met with as the result of direct or indirect violence.

Treatment.-After reducing the displacement, which is always marked when both bones are broken, by making extension from the hand and by manipulation, the forearm is placed in the supine position or in a position between pronation and supination. The supine position is, as a rule, to be preferred in any fracture of the radius, as the upper fragment is supinated by the action of the biceps and supinator brevis muscles, and, therefore, unless the lower fragment be placed in the supine position, union with rotary deformity will almost inevitably ensue. 
Two straight wooden splints, well padded, a little wider than the forearm, are employed. The anterior splint should be long enongh to extend from the elbow to the tips of the fingers, and the posterior splint should extend

Fig. 287.

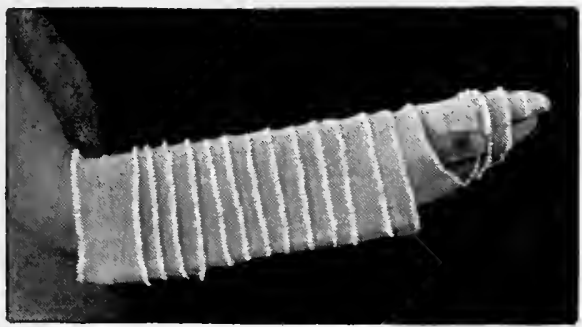

Dressing for fracture of both bones of the forearm.

from the elbow to the wrist. A primary roller should never be applied to the forearm in dressing these fractures, as its application diminishes the interosseons space, and its use has been followed by gangrene of the hand and forearm. In applying the anterior splint to the palmar surface of the forearm and hand, care should be taken that the upper end of the splint does not press upon the brachial artery and basilie vein at the elbow when the forearm is flexed ; the posterior splint is next applied from the elbow to the wrist, and the splints are beld in position by the turns of a bandage carried from the fingers to the elbow (Fig. 287).

In dressing this fracture a posterior splint equal in length to the anterior splint may be used in place of the short posterior splint extending from the elbow to the wrist.

In fracture either of the shaft of the radius or of the ulua alone, the deformity is usually not so marked as when both bones are broken at the same time, the unbroken bone acting as a splint; the rlressing for these fractures is the same as for fracture of both bones of the forearm. 
The dressing should be removed in twenty-four or thirty-six hours, and after inspeeting the parts and sponging them with dilute alcohol, the splints should be replaced in the same manner and secured. The dressings should be renewed at intervals of two or three days for two weeks at least, and after this time the dressings should be made at less frequent intervals. The time required for union in these fractures is usually five or six weeks, and the splints should be retained for this time.

Incomplete Fractures of the Ulna and Radius.In children these fractures are very eommon.

Treatment.-The deformity is redueed by bending the bones baek into place, often eonverting the ineomplete fracture into a complete one. After reduetion of the deformity, the treatment arlopted is the same as that deseribed above. In these patients there is a great tendency to displace the splints or rather to draw the forearm ont of the splints, and to prevent this I often employ an anterior angular splint, in place of the straight anterior one, the upper portion of whieh, being fastened to the arm, prevents the ehild from dragging the arm out of the dressings.

\section{Fig. 288.}

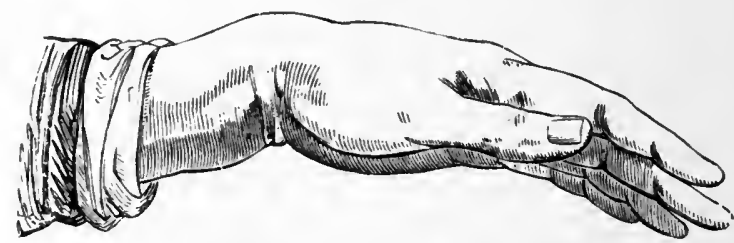

Fracture of the radius near its lower extremity,

Fracture of the Lower End of the Radius.-The most common fracture of the radius is one situated from one-half of an inch to one and one-half inches above the lower artieular surface of the bone (Colles's fracture), the line of fracture being more or less transverse, although it may in some eases be slightly oblique; the eharaeteristic deformity in this fracture is represented in Fig. 288. Numerous $x$-ray studies of this fracture have shown that it 
is a much more complicated injury than was formerly supposed, being often comminuted or impacted and associated with a fracture of the styloid process of the ulna or of the scaphoid or semilunar bones.

Treatment.-The most important point in the treatment of this fracture is to effect complete reduction of the fragments before the application of any splint; this is done by making extension from the hand, and, at the same time, by over-extending and then flexing the wrist and by manipulation, the deformity can usually be completely

FIG. 289.

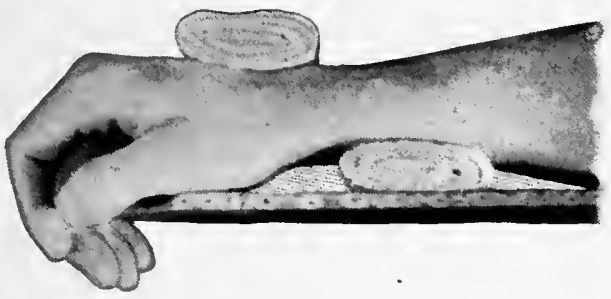

Position of compress in Colles's fracture.

reduced. The arm should then be brought into the position of supination, and a firm compress of lint is next placed over the lower end of the upper fragment on the palmar surface of the forearm ; a second compress is then

Fig. 290.

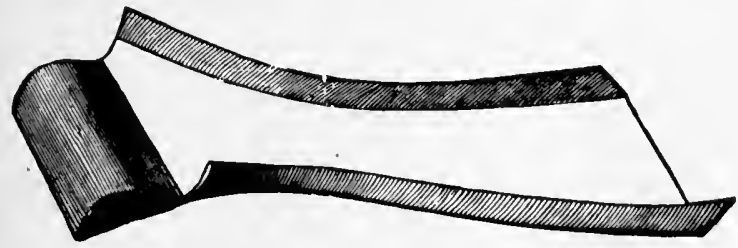

Bond's splint.

placed over the upper end of the lower fragment (Fig. 289), and a well-padded Bond's splint (Fig.290) is applied 
to the palmar surface of the arm and held in place by the turns of a roller-bandage (Fig. 291).

Many surgeons treat this fracture with the hand in a position between pronation and supination, the thumb

FIG. 291.

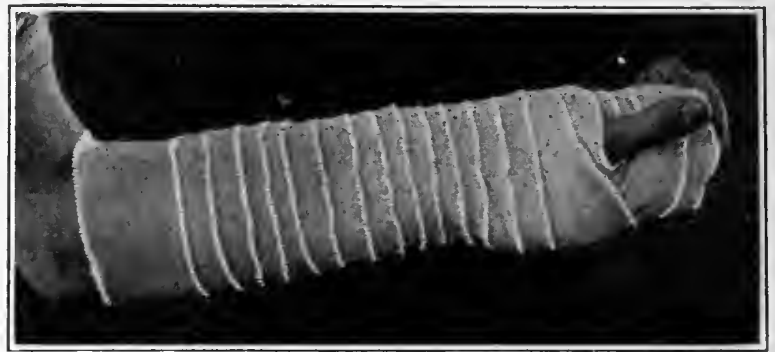

Dressing for fracture of the lower end of the radius.

pointing upward. A substitute for Bond's splint may be prepared by fastening a roller-bandage obliquely upon a

FIt. 292.

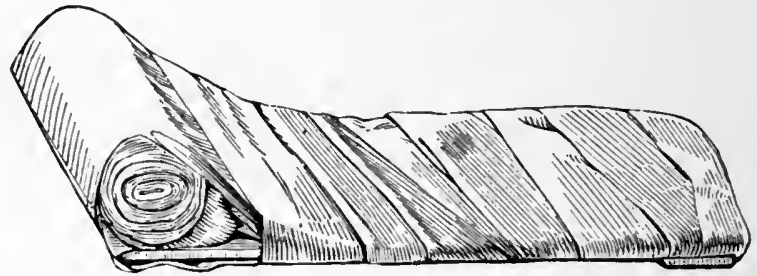

Substitute for Bond's splint.

straight wooden splint as suggested by Dr. Hays (Fig. 292).

Another method of treating Colles's fracture after the reduction of deformity consists in placing upon the dorsal surface of the forearm a padded straight splint, extending from the elbow to the tips of the fingers, and a short straight splint upon the palmar surface of the arm, ex- 
tending from the elbow to the wrist (Fig. 293). These splints are held in position by a bandage, and the forearm earried in a sling with the hand inclined to the ulnar side

FIG. 293.

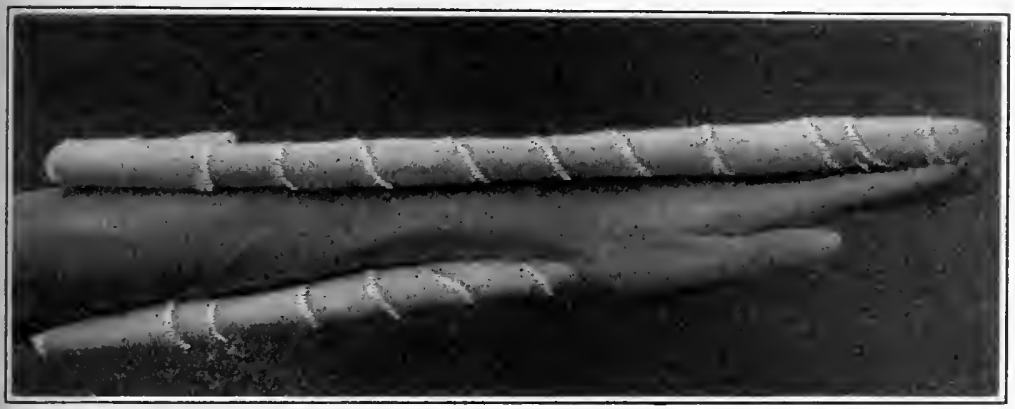

Anterior and posterior splints applied.

(Fig. 294). The hand should be bandaged to the posterior splint for about seven days and then set free. The posterior splint should be left long for another week; at the end of this time it should be shortened so as to extend only to the wrist-joint, and the patient should be encouraged to use the fingers and make motions of the wrist. At the end of three weeks both splints should be removed, and the patient should carry the forearm in a sling for a few weeks longer and be eneouraged to use the hand.

The most important point in the treatment of this fraetnre is the complete reduction of the deformity at the first dressing, and if this has been satisfactorily done almost any splint may be used with a good result, and, indeed, some surgeons use no splint, applying only a compress over the seat of fracture, held in place by a strip of plaster, the arm being carrierl in a sling.

The after-treatment of these fractures consists in removing the splint and compresses after twenty-four or thirtysix hours and in sponging the surface of the skin with 
dilute alcohol, and the compresses and splints should then be reapplied in the same manner; the fracture should be dressed every second or third day for the first two weeks, and after this time it should be dressed, at less frequent intervals. Union is usually quite firm at the end of four weeks, and the splint should be dispensed with at this time. A certain amount of stiffness of the wrist and

Fig. 294.

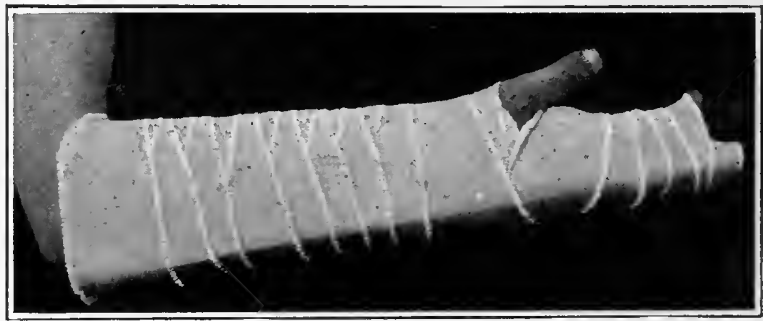

Dressing for Colles's fracture with long posterior and short anterior splint.

fingers is apt to follow this fracture, which is usually soon overeome by passive motion and physiological use of the parts.

Epiphyseal Separation.-In children separation of the lower epiphysis of the radius is often met with, and its treatment is similar to that deseribed above; a Bond splint with eompresses or two straight splints with compresses being the most satisfactory dressing to employ in this injury, the dressings being retained for three weeks.

Reversed Colles's Fracture.-This is a rare fracture of the lower end of the radius in which the lower fragment is displaced forward instead of baekward, the deformity being the reverse of that seen in Colles's fracture.

Treatment.-This consists in the reduction of the deformity, the manipulation being the reverse of that employed in Colles's fracture, and the dressings are similar to those employed in the latter, with the exception that the position of the compresses is reversed. 
Fractures of the Carpal Bones.-These fraetures are usually eompound or open fractures, and are so frequently associated with extensive laceration of the arm and hand that operative measures have to be resorted to; but if such is not the case, they are dressed, when compound, with an antiseptic dressing, and the hand and forearm are supported upon a well-padded palmar splint held in place by a roller-bandage; more or less impairment in the motion of the wrist is apt to follow these fraetures. The dressings should be retained for three or four weeks, and after their removal passive motion should be employed to overcome as far as possible the joint-stiffness resulting.

Fractures of the Metacarpal Bones.-These fraetures are often met with as the result of direct or indirect force applied to the metacarpal bones.

Treatment.-This consists in first reducing the deformity, which is usually an angular one, the projection

FIG. 295.

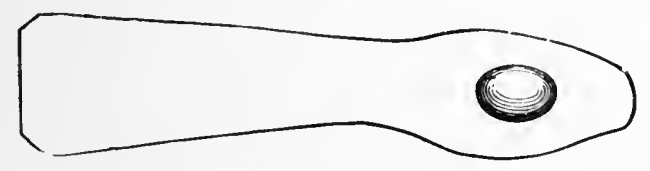

Agnew's splint for fracture of the metacarpal bones.

of the angle being toward the back of the hand; this is reduced by pressure with the fingers, and the hand and forearm should then be placed upon a palmar splint (Fig. 295) with a pad of oakum or eotton under the palm; a compress of lint is next placed over the seat of fracture, and the hand and forearm are bound to the splint by the turns of a roller-bandage (Fig. 296). At the end of three weeks union at the seat of fracture is usually quite firm, and the splint shonld be dispensed with at this time.

Fractures of the Phalanges. - These may result from direet or indireet violenee, and often present marked deformity.

Treatment.-This consists in reducing the displacement by extension and manipulation, and in placing the finger 
in a moulded gutta-percha or pasteboard splint (Fig. 297), and securing the splint in position by the turns of a rollerbandage. When the proximal phalanx is fractured, a narrow padded wooden splint extending from the end of the

FIG. 296.

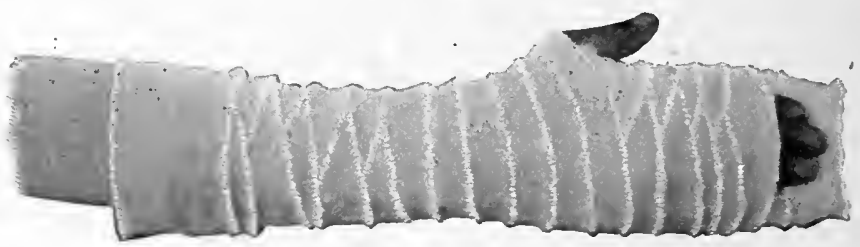

Dressing for fracture of the metacarpal bones.

finger to the wrist should be applied upon the palmar surface of the finger and hand, and a short dorsal splint should also be used ; if there is a tendency to lateral displacement, short lateral splints should also be employed, and the splints should be held in place by strips of plaster or by

FIG. 297.

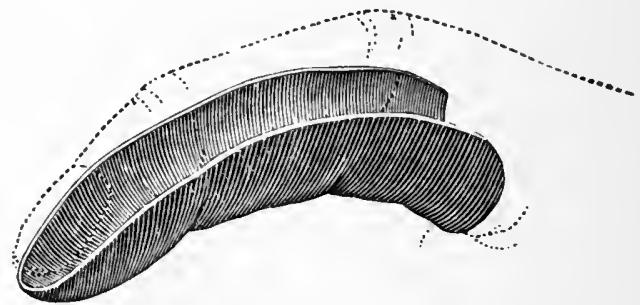

Gutta-percha splint for fracture of phalanx. (IIAMIITON.)

a roller-bandage (Fig. 298). Splints made from a piece of wire may be fitted to the finger, padded, and secured in the same manner.

Union in fractures of the phalanges is usually firm at the end of three weeks, and the splints ean be dispensed with at that time. 
FIG. 298.

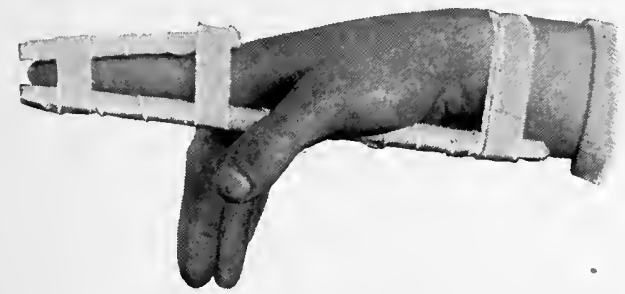

Dressing for fracture of phalanx with anterior and posterior splints.

Fractures of the Femur.-These may involve the neck, great trochanter, and upper end of the shaft, the shaft, or the lower extremity of the bone.

Fractures of the Upper Extremity of the Femur.-These may involve the head, neek, the great trochanter, and the upper portion of the shaft of the femur.

Treatment.-The patient should be placed in bed upon a firm mattress, and an extension apparatus made from adhesive plaster should be applied to the leg, extending as far as the knee-joint. The extension apparatus is constructed by taking a piece of adhesive plaster two and a half inches in width and long enough to extend from the outer side of the knee or middle of the thigh to four inches below the sole of the foot, and from this point back to the inner side of the knee or middle of the thigh; in the centre of this strip is placed a block of wood, two and a half inches wide and four inches in length, with a perforation in its centre; the block and the inner surface of the strip on each side are next faced with a similar strip of adhesive plaster to a point about an inch above each malleolus; a few straps are next wound around the wooden block to fix the previously applied straps; the strip of plaster is next warmed and applied to the sides of the leg and held in position by three or four strips of adhesive plaster carried around the leg at intervals (Fig. 299), and the plaster is made additionally secure by the application of a roller-bandage 
applied to the foot and leg and carried up to the knee. Volkmann's sliding foot-piece may be employed to make the extension more effective (Fig. 300).

Fig. 299.

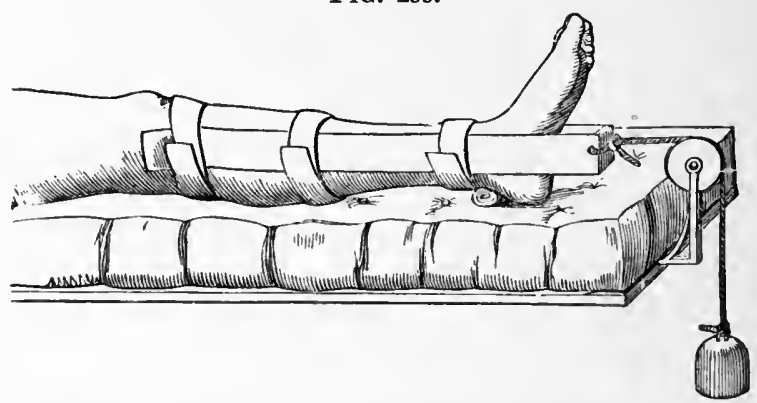

Adhesive plaster extension apparatus applied to limb. (ASHHURst.)

Through the perforation in the block or stirrup is fastened a cord which passes over a pulley attached to the bed, and to this cord is attached the extending weight.

FIG. 300 .

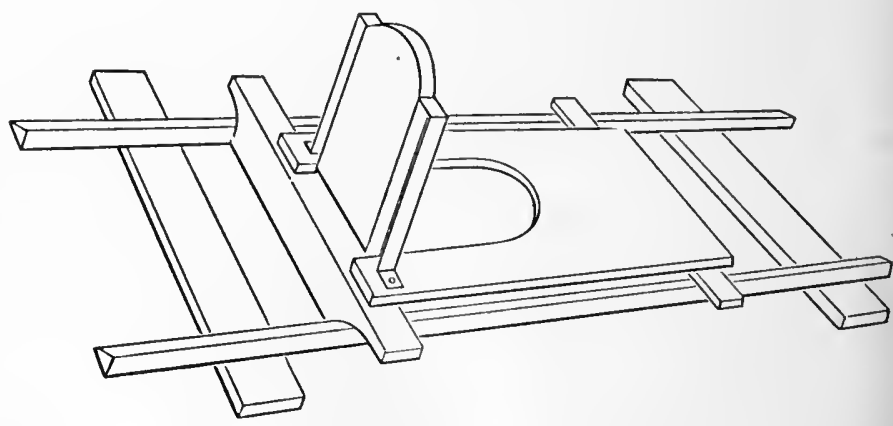

Volkmann's sliding foot-piee.

The extension apparatus being applied, lateral support is given to the leg and thigh by sand bags applied on either side; the outer sand bay should extend from the foot to 
the axilla, and the inner one from the foot to the groin. A weight of five or ten pounds is attached to the extending cord, and the lower feet of the bed should be raised on blocks a few inches high, to prevent the patient from slipping down in bed; a pad of oakum or cotton should also be placed under the tendo-Achillis, to relieve the heel from pressure. This dressing is kept in place for from four to six weeks, and if union has occurred the patient is kept in bed for a few weeks longer and is then allowed to be about, using crutches. In the majority of cases of fracture of the neek of the femur fibrous union only takes place, and after employing the dressing before described

FIG. 301.

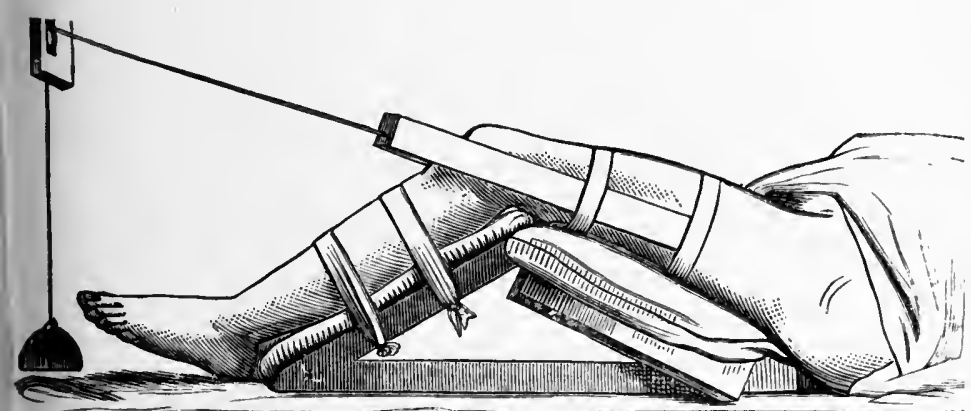

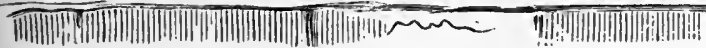

Dressing for fracture of the femur with extension upon an inclined plane. (AGNEW.)

for six weeks the patient is allowed to get up and go about on crutches. It often happens that the subjects in whom these fractures oceur are old and feeble, and if it is found that restraint in bed with the dressings here described is not well borne, under such circumstances they should be discarded and the patient allowed to sit up in bed with the limb resting on a pillow, or to get into a chair, the treatment of the local condition having to be disregarded, attention being given to the patient's constitutional condition.

In fractures in the upper portion of the femur where 
there is marked tilting forward of the upper fragment Professor Agnew employed extension made from the thigh and placed the limb upon a double inclined plane, maintaining this position during the treatment of the ease (Fig. 301). With the same object in view, in place of the double in-

Fig. 302.

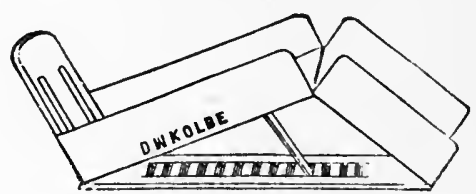

vouble inclined fracture-box. clinerl plane a double inclined fracture-box may be employed (Fig. 302), extension being made from the thigh by means of adhesive plaster strips applied above the knee, to which a weight is attached.

Fracture of the Shaft of the Femur.-This is a frequent fracture, and is usually aceompanied by marked shortening and angular or rotatory displacement of the fiagments.

Treatment.-The patient should be placed upon a fracture-bed or an ordinary bed with a firm hair mattress; an extension apparatus of adhesive plaster is applied, and extension is made by a weight attached to this, as previously deseribed. Lateral support is given to the limb by the application of two wooden splints-the outer or long one extending from the axilla to the foot, the inner or short one extending from the groin to the foot. The splints at their upper extremity should be about six inches in width and at their lower extremity abont three and a half inches. The splints are wrapped in a splint eloth, which extends from the foot to the groin, and after this has been placed under the limb the splints are fixed in their proper positions, the short one to the inner side, the long one to the outer side of the limb. Between the limb and the splints are interposed bran bags: the outer bag should be long enough to extend from the axilla to the foot, the inner one from the groin to the foot. The splints and bran bags are held in place by five or six strips of bandage passing under the limb and body and around the splints and bran bags at intervals. The heel is saved from pressure by placing a wad of oakum or 
cotton under the tendo-Achillis, and after the splints have been brought into place the strips of bandage are firmly tied to seeure them, and a weight of ten or twelve pounds is attached to the extending cord. The foot of the bed is raised, to prevent the patient from slipping downward and to allow the weight of the body to act as a counterextending force. After the application of the dressings the thigh should be slightly abdueted. During the aftertreatment of these fiactures the surgeon should see that the splints and bran bays are kept firmly in place, and that the foot does not roll outward; this is aecomplished by

FIF. 303.

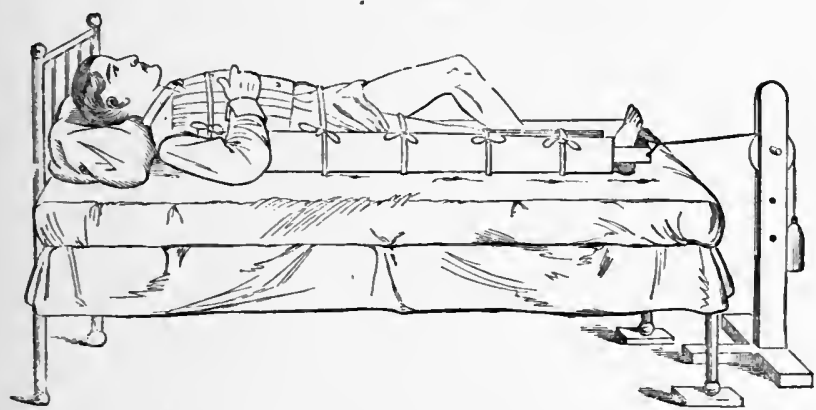

Dressing for fracture of the shaft of the femur with lateral splints and bran bags. (ASHHURst.)

untying the strips and readjusting the bags, and then bringing up the splints and securing them in position by fastening the strips (Fig. 303). The extension apparatus usually does not require renewal during the course of treatment. The extension apparatus and splints are kept in place for four or six weeks, and at this time union at the seat of fracture is usually quite firm, so that they may be removed, and the fracture is then supported by moulded pasteboard splints or by the application of a plaster-ofParis splint for several weeks longer, and at the end of eight to ten weeks it is safe to allow the patient to be up and around on crutches. 
Many surgeons, in fracture of the shaft of the femur, prefer to use a long external sand bag, and a shorter internal one in place of the eorresponding long and short splints and bran bags; if eare is observed that the sand bags are kept acenrately in eontaet with the limb and body, excellent results nay be obtained by this form of dressing. $\Lambda$ fter eonsiderable experience with both methods of furnishing lateral support in the dressing of fractures of the shaft of the femur, $I$ am well satisfied that angular deformity is less likely to result where the splints and bran bags are employed.

The plaster-of-Paris dressing, including the foot, leg, thigh, and pelvis, is employed by some surgeons in the early treatment of fracture of the shaft of the femur, the limb being kept well extended until the plaster has thoroughly set. In applying this dressing, the patient should be placed upon the pelvic supporter (see page 106).

Fracture of the Lower End of the Femur.-The fractures met with in this portion of the femur are supracondyloid fractures, those in which one condyle is separated from the other, or comminuted fractures, in which both condyles are separated; epiphyseal disjunctions of the lower end of the femur, met with in young subjeets, may also be elassed with fraetures at this portion of the bone.

Treatment.-If there is shortening, the dressing should be similar to that employed in fractures of the shaft of the femur, consisting in the application of an extension apparatus and bran bags and splints or sand bags to give lateral support ; if, however, there is no marked shortening, the dressing employed should be the same as that applied in fractures involving one or both condyles or epiphyseal separation.

The dressing employed in fracture of one or both condyles or in epiphyseal disjumetion of the lower end of the femur eonsists in placing the limb in a fracture-box extending from the foot to the upper third of the thigh, the box being well padded with a soft pillow, or a wellpadded posterior splint, or a moulder pasteboard or felt gutter may be employed; if either of these dressings is 
employed, the splint or gutter should be sufficiently long to extend from the lower part of the leg to the upper part of the thigh.

At the end of two weeks it is well to place the limb in a plaster-of-Paris dressing extending from the foot to the upper part of the thigh. This dressing should be retained for six weeks; at the end of this time the dressing should be removed, and if union is sufficiently firm to allow the patient to go abont on crutches, a fresh plaster-of-Paris splint should be applied extending from the middle of the leg to the middle of the thigh, or lateral splints of pasteboard may be substituted for the plaster dressing.

A certain amount of permanent impairment of the joint-motion is apt to follow fractures involving one condyle or both condyles of the femur.

Fracture of the Shaft of the Femur in Children.Treatment.-In infints the treatment by extension by a weight and pulley and lateral splints is often unsatisfictory on account of the difficulty in keeping the patient quiet upon his back, and from the soiling of the dressings by the feees and the urine. In children two years of age and over $I$ have never found much trouble in employing extension and lateral support by splints and bran-bags or sand-bags, and in these cases I make additional fixation at the seat of fracture, and guard against displacement of the fragments by the child sitting up in bed when not watched, by carefally moulding external and internal pasteboard or felt splints to the thigh, and holding them in place by the turns of a bandage. I have employed this form of dressing even in children under two years of age with the most satisfactory results.

In cases of fracture of the femur in children from a few months to a year or eighteen months of age, in whom it is difficult to obtain quietude, or who have to be moved to give them nourishment if they are taking the breast, the dressing which I have found most satisfaetory consists in first applying a roller-bandage from the foot to the groin, and then moulding to the outer half of the foot, leg, thigh, and also to half of the pelvis, a pasteboard or felt 
splint whieh is well padded with cotton, and held in position by the turns of a bandage carried from the foot to the pelvis and finished with circular turns about the pelvis. The splint should be so moulded as to include a little more than one-half of the cireumference of the thigh and leg. If this splint becomes soiled, it is easily replaeed by a fresh one, and its removal and renewal are much easier than the plaster-of-Paris splint which is recommended by some surgeons in these eases.

In young ehildren fraetures of the femur are often incomplete or greenstick fractures; and even when complete, the shortening is usuilly not marked, as the line of fracture is apt to be transverse, the periosteum often not being completely ruptured, which tends to hold the fragments in position.

In green-stick fractures the deformity should be reduced by manipulation, even if it is neeessary to convert the incomplete fracture into a complete one to accomplish this object.

Mr. Bryant recommends that fractures of the femur in young children be treated in the vertical position; the injured limb, together with the sound

FIG. 304.

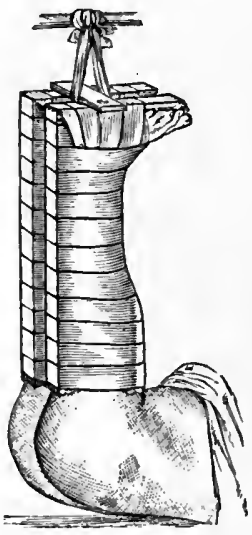

Fracture of the femur treated by vertical extension. (BRYANT.) one, is flexed at a right angle to the pelvis and fixed with a light splint, and attached to a cradle or bar above the bed (Fig. 304).

If the plaster-of-Paris dressing is used, the limb should first be enveloped from the foot to the pelvis with a flannel bandage, and extension should be made while the plaster-ofParis bandage is being applied, and should be kept up until the bandage has become fixed. The plaster bandage should extend from the toes to the pelvis, and it is well to fix the bip-joint by earrying several turns of the bandage aloout the pelvis. To prevent the splint from absorbing 
the diseharges and becoming offensive, the upper portion of it may be eoated with shellac.

The time required for union in fractures of the femur in children is about four weeks, and the dressings may be removed at this time; but the child should not be allowed to use the limb for several weeks after this period.

Ambulatory Treatment of Fractures of the Femur.-In this method of treatment in fractures of the femur the injured limb is strongly extended, and. a flannel roller is applied to the leg, thigh, and pelvis. A plaster-of-Paris bandage is then applied from the toes to the pelvis, and is made to inelude the pelvis by spica and circular turns. It should be well padded in the perineum, and the inner portion of the bandage should fit well in the region of the tuberosity of the ischium. The plaster dressing should be so applied that upon the patient standing upon the limb the weight is supported by the plaster east resting upon the tuberosity of the isehium and the expanded portion of the ilium. A Taylor hip-splint, reinforeed by plaster bandages and the use of crutches, with a high shoe on the sound foot, may be used in the ambulatory treatment of fractures of the femur.

Fractures of the Patella.-These fractures result from direet violenee and muscular action.

Treatment.-This eonsists, first, in the application of a roller-bandage from the toes to the upper part of the leg;

FIG. 305.

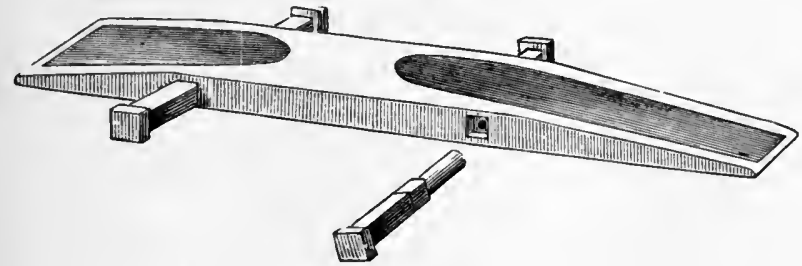

Agnew's splint for fracture of the patella.

a well-padded posterior wooden splint long enough to extend from the middle of the leg to the middle of the thigh, or an Agnew splint, which is provided with pegs 
for the attachment of strips of adhesive plaster (Fig. 305), is next placed under the limb. A small compress of lint is next placed above the upper fragment, and a similar compress is placed below the lower fragment; a strip of adhesive plaster one and a half inches in width and twenty-four inches in length has its middle portion applied over the eompress, and its ends are then brought obliquely downward and fastened to the splint, or to the pegs if Agnew's splint be used; this may be reinforced by a second or third strip. The object of these strips is to bring the upper fragment down in contact with the lower fragment. A strip of plaster with the ends passing in the opposite direction is next placed over the lower compress, and the ends are fastened to the splint or pegs; this strip serves only to steady the lower fragment, as it cannot be drawn upward to meet the npper fiagment by reason of the inextensibility of its ligamentous attachment (Fig. 306). If the Agnew splint is employed, the strips of plaster may be tightened by turning the pegs to which they are fastened without removing the splint.

FIG. 306.

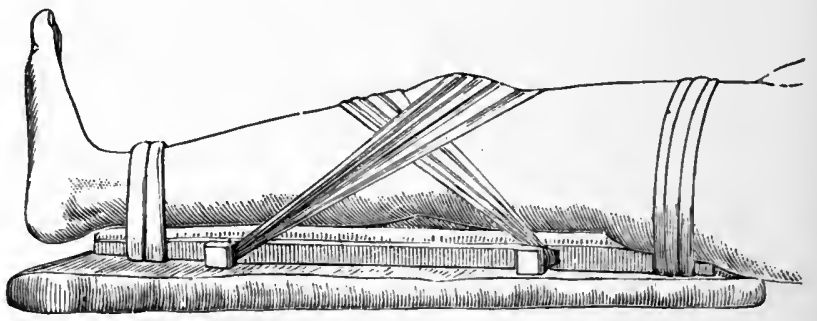

Agnew's splint applied.

The splint is next firmly fixed in contact with the limb by the turns of a roller-bandage extending from the lower to the upper end of the splint. The limb should next be plaeed upon an inclined plane or in a long fracture-box, with its foot elevated to relax the quadriceps femoris muscle. This dressing should be removed and reapplied 
in a few days, as the dressings become loose as the swelling about the seat of injury subsides, and after this disappears the dressings require renewal at less frequent intervals; and usually at the end of three weeks the splint may be removed and a plaster-of-Paris bandage may be applied, extending from the mirdle of the leg to the middle of the thigh. At the end of six weeks the patient may be allowed to walk upon the limb, the knee-joint being fixed with a plaster-of-Paris or pasteboard splint.

It is well, after removal of the splints, for the patients to wear for some months a laced muslin knee-supporter, which gives some support to the knee-joint.

A great variety of splints have been devised and used in the treatment of fractures of the patella, the main object of which is to fix the knee-joint and bring the fragments as nearly as possible in apposition.

The union in fractures of the patella is usually fibrous, although in rare cases bony union has oecurred.

In cases of rupture of the fibrous union after fracture of the patella, which is not an uncommon aceident, the treatment of the case should be the same as that for a recent fracture of the patella.

Operative Treatment.- This method, which consists in exposing the fragments by an incision and drilling and suturing them with catgut or silver-wire sutures, or in approximating the fragments by suturing the fibrous tissues with eatgut, is the most satisfactory procedure and the one often employed at the present time, the strictest antiseptic precautions being taken to prevent infection of the wound. After the external wound has been closed without drainage, the limb is put up in a plaster-of-Paris dressing extending from the foot to the groin.

Fractures of the Bones of the Leg.-In fractures of both bones of the leg the displacement is usually very marked. When only one bone is broken, the sound bone, acting as a splint, prevents much deformity, except in cases of fracture at the lower end of the fibula, when the foot inclines to the injured side.

Treatment.-The dressing of fractures of both bones of the leg, or of fracture of the tibia or the fibula alone, except in cases where the lower portion of the fibula is the 
seat of injury, is best accomplished by the use of a fracturebox. The displacement being overcome as far as possible by extension and manipulation, the leg is placed in a fracture-box, which is prepared for the reception of the limb by having the sides let down and having a soft pillow laid in it; the foot is next secured to the footboard by a

Fig. 307.

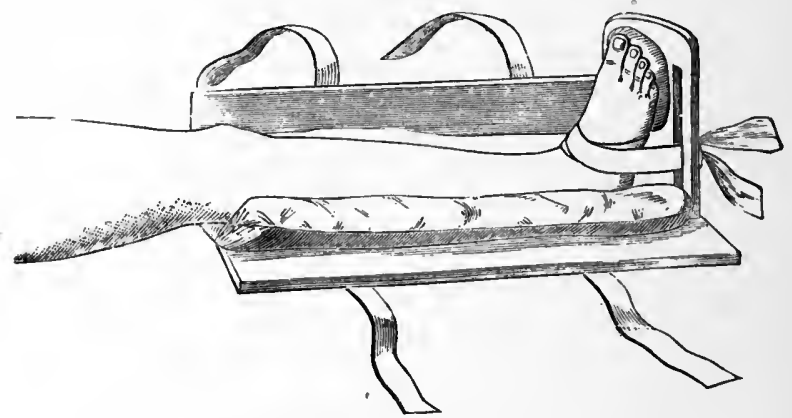

Application of the fracture-box.

loop of bandage passed around the foot, the ends being tied after passing through the slots in the footboard; a pad of oakum or cotton is placed under the tendo-Achillis, to

FiG. 308.

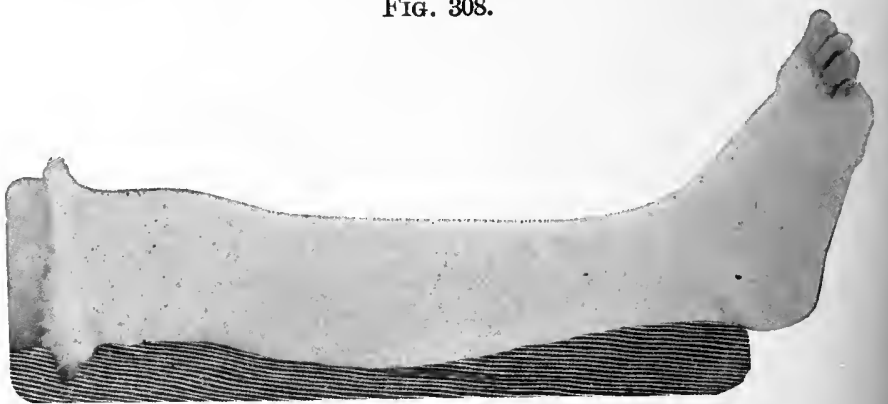

Plaster bandage applied to fracture of the leg.

relieve the heel from pressure, and a similar pad is placed between the sole of the foot and the footboard (Fig. 307). The sides of the box ale then brought up and secured by 
two or three strips of bandage tied around the box. In using a fracture-box in the treatment of fractures of the bones of the leg the surgeon should see that the foot is kept well down to the footboard and is at a right angle with the leg, that there is no eversion of the knee, and that the pillow is full enough to make equable pressure upon the leg when the sides of the box are secured, and that the heel is not subjected to undue pressure, the use of a pad of oakum or cotton under the tendo-Achillis being employed to prevent this complication. Where there is a tendency to tilting upward of the lower end of the upper fragment, the lower fragment can be brought in line with

FIG. 309.

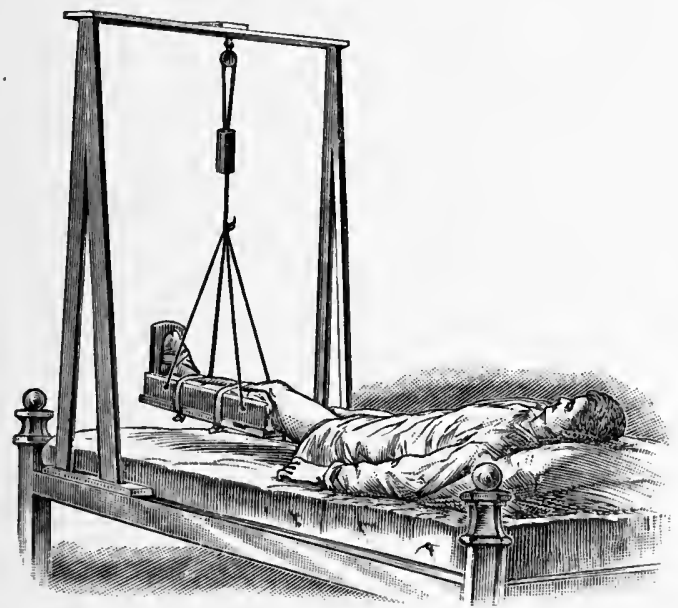

Fracture-box suspended, (AGNEw.)

this by raising the foot by a mass of oakum or cotton placed under the tendo-Achillis and heel, and so overcoming the deformity. In some cases division of the tendo-Achillis may be required before this deformity can be corrected.

The subsequent dressings of the case are conducted by letting down the sides of the box and correcting any 
displacement, if present, by adjusting the limbs and pads in their proper position, and again bringing up the sides of the box and securing them. At the end of two weeks the fracture-box may be removed and a plaster-of-Paris dressing applied to the limb, which will allow the patient more freedom of movenent in bed, or permit of his sitting up without disturbing the fragments (Fig. 308).

FIG. 310 .
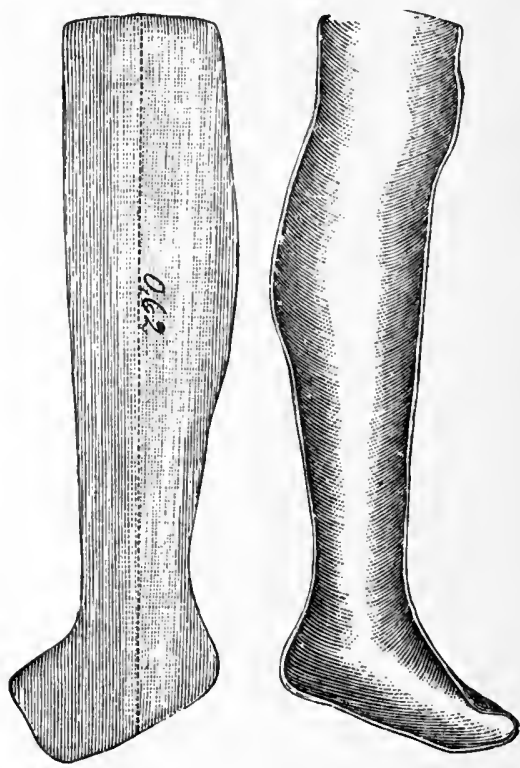

Moulded binders' board splints for fracture of the leg.

Union in fracture of the bones of the leg is usually quite firm in six weeks, but for at least eight weeks the patient should not be allowed to put his weight upon the limb in walking.

If the patient is restless, and finds his position with the fracture-box resting upon the bed irksome, the fracturebox may be swung from a frame fastened over the bed (Fig. 309). 
The application of a plaster-of-Paris dressing as a primary dressing - the ordinary plaster-of-Paris bandage or the Bavarian dressing being applied-in fractures of the bones of the leg is adopted by some surgeons, and, if employed, the case should be under constant observation for a few days, so that the dressing can be removed if a dangerous amount of swelling takes place. Moulded splints of felt or pasteboard are also sometimes applied in the treatment of these cases (Fig. 310).

This fracture may also be treated with Volkmann's splint (Fig. 311), for one or two weeks, until the swelling has subsided, and then by a plaster-of-Paris dressing.

FIG. 311.

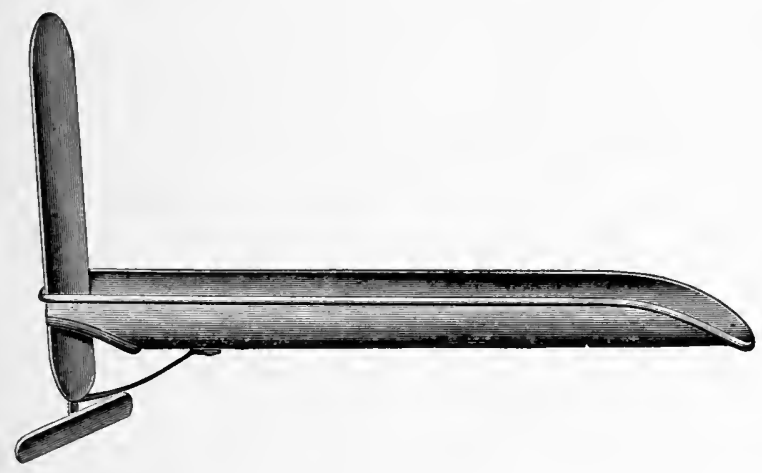

Volkmann's splint.

In patients suffering with delirium tremens, or in maniacal patients, the use of a fracture-box in the treatment of fractures of the bones of the leg is often not satisfactory, on account of the diffienlty in restraining the movements of the patient and the consequent displacement of the fragments. In such cases it is well to apply a few strips of binders' board, well padded with cotton, to the limb, extending above and below the seat of the fracture, holding them in place by a few turns of a roller, and then 
to wrap the limb and foot in a soft pillow, and hold this in place by the turns of a roller-bandage applied with moderate firmness. This dressing allows the patient to move the limb without serious disturbance of the fragments, and, after the patient recovers from his attack, the leg may be placed in the fracture-box or in a plaster-ofParis dressing.

In fractures of the bones of the leg in young children the same difficulty is often experienced in keeping them quiet, and for this reason a fracture-box cannot be used with satisfaction. In dressing these cases, two lateral splints of pasteboard, moulded to the foot and leg and well padded with cotton, may often be employed with the best results. The splints should not be wide enough to meet on the anterior or posterior surface of the leg or foot. The splints, after being carefully adjusted, are held in place by the turns of a roller-bandage; and after these splints have been applied for two weeks, and all swelling has subsided at the seat of fracture, a plaster-of-Paris bandage may be substituted for them, which should be worn for four weeks; at the expiration of this time union is usually suffieiently firm to dispense with all dressings.

Ambulatory Treatment of Fractures of the Bones of the Leg.- The application of a dressing for the ambulatory treatment of fractures of the bones of the leg is as follows: The fracture should be reduced and the skin of the leg washed with soap and water; a flannel bandage is applied from the toes to a point just above the knee. This bandage holds to the sole of the foot a number of layers of cotton-wadding, which, when moderately compressed, makes a pad three-quarters of an inch in thickness. A plaster-of-Paris bandage is applied to the foot and leg, and extends above the knee, and care should be taken to apply additional turns about the sole of the font and ankle, to give it greater strength at these points. The turns of the bandage should also be firmly applied about the expanded head of the tibia. 
In the ambulatory method of treatment, the patient, as soon as the bandage has becone firm, is allowed to walk about, first with crutches or a cane, and finally bearing his weight upon the injured limb.

Fractures of the Fibula.- In fractures of the fibula, with the exception of that fracture occurring at the lower end of the bone, the deformity is not marked, and they are usually dressed with a fracture-box applied as in the dressing of fractures of both bones of the leg, and at the end of two weeks a plaster-of-Paris dressing should be applied, and the patient allowed to get out of bed and move about on crutches. The union in a fracture of the fibula is usually quite firm at the end of five weeks, at which time all dressings may be dispensed with.

Fracture of the Lower End of the Fibula (Pott's Fracture).-This fracture usually occurs in the lower fifth of the bone, and is often associated with laceration of the internal lateral ligament of the ankle-joint or a sprainfracture of the internal malleolus, and is usually accompanied by marked eversion of the foot.

Treatment.-After reducing the displacement by extension and manipulation, the limb should be placed in a fracture-box provided with a soft pillow, the foot should be secured to the footboard, and a pad of oakum or cotton should be placed under the tendo-Achillis: before bringing up the sides of the box and securing them, two firm compresses of lint or oakum should be placed in contact with the leg and foot, one just above the inner malleolus, the other just below the outer malleolus. The sides of the box are next brought up and secured, and by the pressure of these compresses the foot is brought into an inverted position and the deformity is corrected.

The after-dressing of this fracture consists in letting down the sides of the box, and in inspecting the parts to see that the foot is kept in the proper position, and care should be taken that undue pressure is not made upon the skin by the compresses, which might result in ulceration; this may be avoided by sponging the skin with alcohol and ehanging the positions of the compresses slightly at 
each dressing. At the expiration of ten days the fracture-box and compresses may be removed and the limb put "1) in a plaster-of-Paris dressing, including the foot and leg, np to the knee. 'The patient may then be allowed to go about on crutches, and at the end of five weeks all dressings may be dispensed with.

This fracture may also be treated by the forcible correction of the deformity under ether and the immediate application of a plaster-of-Paris dressing.

Dupuytren's splint, which consists of a straight wooden splint long enough to extend from the condyles of the femur to the end of the tocs, may also be employed; this splint is provided with padding, the thickest part of which, several inches in thickness, should rest wpon the skin just above the inner malleolns when the splint is

FIG. 312.

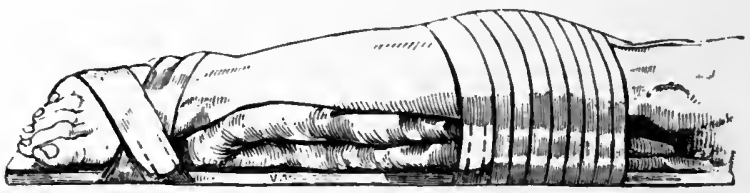

Dupuytren's splint applicd.

applied to the inner side of the leg. The splint is secured in position by the turns of a roller applied over the foot and at the upper part of the leg (Fig. 312). After using this dressing for a few days, if the displacement is satisfactorily corrceted, the splint may be removed and the leg placed in a fracture-box or in a plastel-of-Paris dressing.

Fractures of the Tarsal Bones.-The calcaneum and astragalus are the tarsal bones most frequently fractured.

Treatment. - The dressing of fractures of the calcaneum, after reducing the displacement, which is not usually marked unless the posterior portion of the bone is involved, by manipulation, consists in placing the leg and foot in a fracture-box, care being taken that the foot is kept at a right angle to the leg. When 
the fracture involves the posterior portion of the bone, and there is displacement by the action of the muscles inserted into the fragment, the leg should be flexed upon the thigh and the foot extended; this position may be maintained by applying a well-padded curved splint to the anterior portion of the leg and foot and securing it in position by a bandage.

Fractures of the astragalus, after reducing any deformity which is present by extension and manipulation, are dressed by placing the foot and leg in a fracture-box, care being taken that the foot is kept at a right angle to the leg. This precaution is important, as anchylosis not infrequently oceurs after this fraeture, and if the foot is in the proper position it is much more useful to the patient. As soon as the swelling, which is usually very marked after fracture of the calcaneum or astragalus, subsides, the foot and leg should be put up in a plaster-of-Paris bandage. The amount of tension and the inability to reduce the displacement in eases of fracture of the astragalus may be indications for excision of the fractured bone. The time required for union in fractures of the tarsal bones is from five to six weeks.

Fractures of the Metatarsal Bones.-These fractures are dressed by placing the foot upon a well-padded plantar splint, and using compresses to hold the fragments in place if there is much displacement, the splint and compresses being held in position by a bandage; or they may be treated by placing the foot and leg in a fracture-box, the footboard of the box acting as a plantar splint; the plasterof-Paris dressing may also be used in these eases. The time required for union in fracture of the metatarsal bones is from three to four weeks.

Fractures of the Phalanges of the Toes.-These fractures are often compound and attended with so much laceration of the soft parts that immediate amputation is required; when, however, the fractures are simple, or in compound fractures where amputation is not required, the dressing consists in applying a plantar splint of wood or binders' board, extending beyond the toes and securing it 
in position by the turns of a roller-bandage. When a single toe only is broken, a moulded splint of gutta-percha or binders' board nay be applied, and a portion of the splint should extend some distance upon the sole of the foot, to fix the proximal joint, and also to give the toe a firm point of fixation; the monlded splint should be held in position by a narrow roller-bandage or by strips of adhesive plaster. The time required for union in fractures of the phalanges of the toes is about three weeks.

\section{COMPOUND OR OPEN FRACTURES.}

In the dressing of compound or open fractures the same dressings and splints which are generally used in the treatment of simple or closed fractures may be employed; the wound in the soft parts requires a special dressing, and this should be so arranged as to secure free drainage and promote its prompt healing. In some cases of compound fracture the treatment of the injuries of the soft parts demands attention first, and in such eases the injury to the bones is for a time disregarded, eare being taken that the fragments are kept quiet, so as to prevent further damage to the soft parts until the wound is in such a condition that the proper manipulation to reduee the displacement and fix the fragments by splints and suitable dressings may be undertaken without interfering with the repair of the wound.

Treatment.-In the dressing of eompound or open fractures the skin surrounding the wound should first be carefully cleansed, and the wound next be thoroughly irrigated with a $1: 2000$ bichloride solution, and any foreign bodies or loose fragments of bone removed; if there is hemorrhage, it should be controlled by securing the bleeding vessels with ligatures. The reduetion of the displacement should next be aceomplished by making extension and by manipulation; if the fragments project from the wound, before this can be satisfactorily accomplished it may be necessary to enlarge the wound and 
to resect one or both ends of the fractured bones, and in some cases it may be necessary to drill the ends of the fragments and introduce a strong wire or catgut suture, or a metallic nail, serew, or plate, to hold them in their proper positions. After reduction of the displacement the wound should again be thoronghly irrigated with an antiseptic or normal salt solution, and after making provision for drainage by the introduction of a drainagetube or tubes, counter-openings being made to secure free drainage if necessary, sterilized or antiseptic gauze dressings should be applied.

The wound, if a small one, need not be closed with sutures; but if extensive, a few catgut, silk, or silkwormgut sutures may be applied to bring the edges of the wound into apposition, care being taken to avoid making undue tension; if the soft parts have been much lacerated or contused, it is better to introduce no sutures. If the limb is much swollen and the wound is a small one, free division of the deep fascia to relieve tension and secure drainage is often followed by good results. A final irrigation of the wound through the drainage-tube is next made, and the wound is covered by a bichloride gauze dressing and a number of layers of bichloride cotton, the whole dressing being held in position by a ganze bandage applied with moderate firmness.

The reduction of the fragments and the dressing of the wound having been accomplished as described, the splints appropriate for a similar fracture, if it were a simple or closed one, are next applied. If the surgeon has been able to render the wound aseptic, and has applied an antiseptic dressing, the compound fracture is often soon converted into a simple one by the prompt healing of the wound, and the patient may exhibit no more constitutional disturbance than he would have with a similar simple or closed fracture. The redressing of a compound fracture dressed in this way need not be made for a week or ten days, unless there is a rise in the patient's temperature or the dressings become soaked with discharges from the wound, or they become uncomfortable 
to the patient by reason of swelling of the soft parts in the region of the wound. When redressing of the fracture becomes necessary the dressings are removed, and the drainage-tubes may also be removed if no longer needed; the wound being redressed with an antiseptic or aseptic dressing, the splints are reapplied, and, after the wound is healed, the subsequent dressing of the fracture should be the same as that of a simple fracture. The time required. for union in a compound fracture is usually much longer than in a corresponding simple fracture.

Plaster-of-Paris Dressing.-This may be used as a primary dressing in compound fractures; the displacement being

FIG. 313.

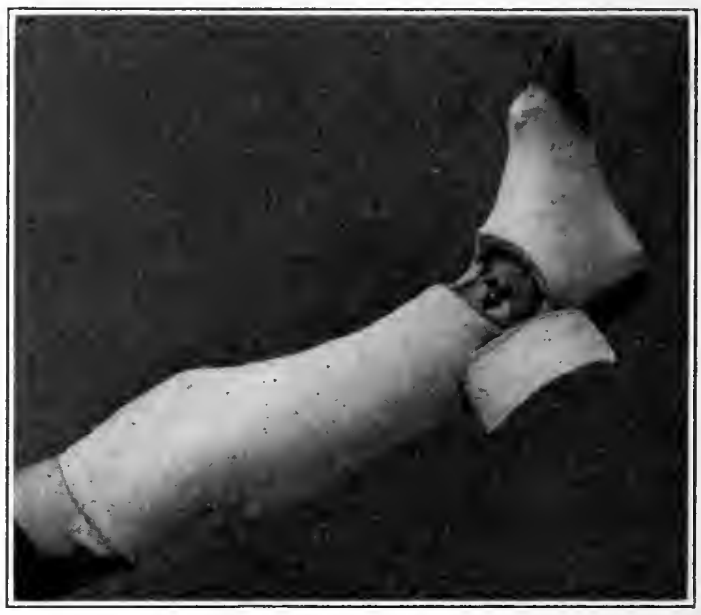

Fenestrated plaster dressing for compound fracture of the leg.

reduced and the wound dressed with an antiseptic gauze dressing, a plaster-of-Paris bandage is applied to the parts so as firmly to fix the fragments; the joints on either side of the fracture should be fixed by the bandage, and the parts should be held in position until the plaster has set firmly. After the plaster has become firm a fenestrum 
shonild be made over the position of the wound, so that it may be inspected or dressed through this when necessary (Fig. 313). The ends of a piece of stout wire, bent into a semicircle, may be incorporated in the turns of the plaster bandage above and below the position of the fenestrum, to give it additional strength after the removal of a portion of the bandage to make the fenestrum.

If the plaster-of-Paris dressing is applied as a primary dressing in compound fractures, the ease should be carefully watched for a few days, and if much swelling occurs at the seat of fracture its removal and renewal are indicated; profuse discharge of serum may also soak the dressings and bandage, so that its renewal is necessitated. Some surgeons, therefore, prefer to defer the application of the plaster-of-Paris dressing in compound fractures for a few weeks until the swelling has diminished and the wound is nearly or quite healed; the wound being covered with an antiseptic dressing, the plaster bandage is applied, and a fenestrum is made over the position of the wound if required.

Binders' Board or Felt Splints.-These may also be employed in the dressing of compound fractures, being monlded to the parts after an antiseptic dressing has been applied to the wound, and held in position by the turns of a roller-bandage.

The principal advantage in the use of these splints is the ease with which they can be removed and reapplied if frequent dressings of the fracture are necessary for any reason. They may be used during the entire course of treatment; or, after a few weeks, when the swelling has diminished at the seat of fracture and the wound is well advanced toward repair, they may be discarded and a plaster-of-Paris dressing substituted. In compound fractures of the bones of the leg, after reducing the displacement and applying an antiseptic dressing to the wound, I usually apply monlded binders' board splints to either side of the leg, including the foot, and place the leg in a fracture-box for additional seeurity. In a week or ten days I discard the binders' board splints and apply a plaster-of-Paris dressing.

A method of dressing compound fractures which has 
been introduced by Mr. Treves consists in rendering the skin in the region of the wound aseptic and removing any foreign bodies from the wound, then rendering it as far as possible aseptic; powdered iodoform is then dusted thickly over the wound at intervals, and, mixing with the blood and serum from the wound, is allowed to dry, forming an antiseptic scab, the wound being exposed to the air, and the fragments are retained in position by splints or by a fracture-box.

\section{UNUNITED FRACTURE.}

This condition usually arises from local eauses, such as imperfect coaptation of the fragments, the interposition of museular tissue, fascia, a tendon, or nerve, or

FIG. 314.

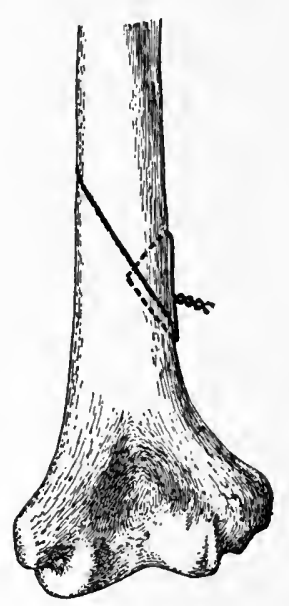

Fragments in ununited fracture seeured by silver wire.
FIG. 315.

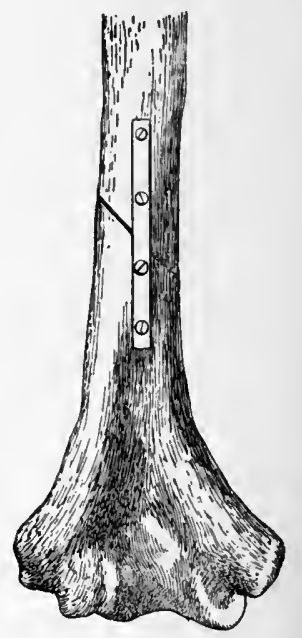

Fragments in ununited fracture seeured by silver splint.

a portion of devitalized bone between the fragments. The ends of the bones may be rounded, or may be united by 
fibrous tissue, or there may be an attempt at the formation of a false joint, the end of one fragment being rounded off and the other cupped to receive it.

In cases of fracture in which union has not occurred at the usual time care should be taken to see that fixation of the fragments is as complete as possible, and in addition to the retention of the fixation dressings a systematic use of Bier's hyperæmic treatment should be instituted as good results have often been obtained by its employment.

Treatment. - This consists in exposing the ends of the bones by incision, with full antiseptic precautions, and removing the ends of the bones to secure a healthy surface, and then fixing the bones securely together by drilling them and introducing one or more heavy silver-wire sutures (Fig. 314). In some cases the shape of the fragments is such that they can be sawed so as to form a mortise, and the bones can then be fixed by the introduction of one or more steel or silver screws. Another method of fixation is by a steel or silver splint secured to the fragments by iron or silver screws (Fig. 315). After the fixation of the bones has been accomplished, the wound should be closed and an antiseptic dressing applied; additional fixation is furnished by the application of a plaster-of-Paris dressing. 



\section{PART V. \\ DISI, OCATIONS.}

Dislocation.-This consists in displacement of the articular surfaces of the bones which enter into the formation of a joint. Dislocations may be complete, partial, simple, compound, and complicated, and they are also known as habitual, recent, and old dislocations.

Complete Dislocation.-This is a dislocation in which no portions of the articular surfaces of the bones remain in contact with each other.

Partial Dislocation.-This is a dislocation in which portions of the articular surfaces of the bones still remain in contact with each other.

Simple Dislocation.-This is a dislocation in which there exists displacement in the relation of the articular surfaces of the bones with little injury to the soft parts adjacent to the joint, and the displaced ends of the bones do not communicate with the air by a wound in the soft parts.

Compound Dislocation.-This is a dislocation in which there exists displacement of the articular surfaces of the bones which communicate with the air through a wound in the soft parts.

Complicated Dislocation.-This is a dislocation in which, in addition to the displacement of the articular surfaces of the bones, there exists a fracture, or a laceration of important bloodvessels, nerves, or muscles in connection with the dislocation. 
Habitual Dislocation.-This consists in a dislocation which constantly recurs upon slight provocation, and is usually due to a relaxed condition of the ligaments of the joint.

Recent Dislocation.-This is a dislocation in which the displacement of the articulating surfaces of the bones has existed for such a period that time has not been afforded for inflammatory changes to take place in the articular surfaces of the bones or in the adjacent tissues which would scriously interfere with their reduction.

Old Dislocation.-This is a dislocation in which the displacement of the articulating surfaces of the bones has existed for some time; in this variety of dislocation the displaced bones often form firm adhesions to the surrounding tissues, and the articulating surfaces often undergo changes.

Treatment of Dislocations.-The first indication in the treatment of dislocations is to return the displaced articular surfaces of the bones to their normal position, and to retain them in this position by the use of suitable dressings. The return of the articular surfaces of the bones to their normal position, or the reduction of the dislocation, is accomplished by manipulation, extension, and counter-extension. The reduction of dislocations should be attempted as soon as possible after they have occurred.

The principal obstacles to the reduction of dislocations are muscular resistance and the anatomical peculiarities of the joints. The former is best overcome by the use of an ancesthetic given to the point where complete muscular relaxation is produced. The resistance offered by the changed relations of the articular surfaces and the ligaments is to be overcome by the surgeon making such manipulations, founded upon his knowledge of the anatony of the parts, as will make the ligaments, muscles, and bones assist in the reduction of the dislocation.

In recent dislocations, by the use of extension and manipulation, especially if an anæsthetic be employed, the reduction is usually accomplished without the use of much force; but in old dislocations, where absolute mus- 
cular shortening has taken place, the use of extending bands is often required, and in securing these bands to the limb the clove-hitch kuot is useful (Fig. 316).

The treatment of dislocations after reduction consists in placing the joint at complete rest by the application of snitable splints and bandages, and in treating any inflammatory complications, if they arise, by the application of

FIG. 316.

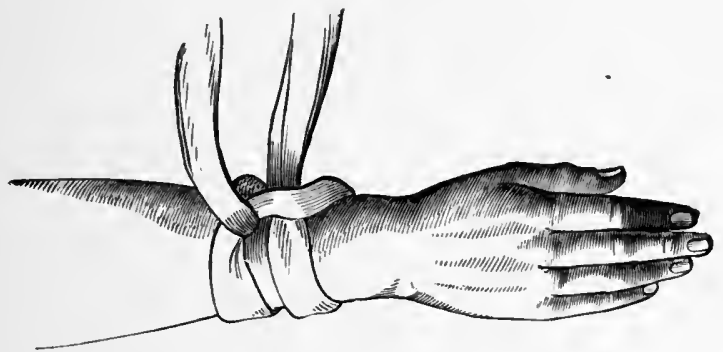

Clove-hitch knot applied. (ERICHsEN.)

evaporating lotions, and in a week or two, after the injured ligaments have been repaired, passive motion should be resorted to for restoring the function of the joint.

\section{SPECIAL DISLOCATIONS.}

Dislocations of the Vertebræ.-Dislocations of the humbar and dorsal vertebre, as simple dislocations, are extremely rare accidents ; they are occasionally met with, but are more often associated with fractures of the vertebræ in these regions. Uncomplicated dislocations of the cervical. vertebræ are more common. The treatment of dislocations of the vertebre, whether complicated with fracture or not, consists in attempting reduction by making extension and counter-extension with manipulation, and by this means, in many cases, the luxations may be reduced. If, however, the efforts at reduction are unsuccessful, permanent extension should be applied by means of a weight extension 
apparatus from both legs and from the shoulders and head. The after-treatment consists in keeping the patient at rest upon his back in bed upon a firm mattress, and if the cervical vertebra have been involved, the head and neck should be supported by short sand bags; and in case of the vertebræ below this point, the application of a plaster-of-Paris jacket may be used to give support and fixation to the part. The general management of the case as regards complications is similar to that in cases of fracture of the vertebræ.

Dislocations of the Coccyx.-These are reduced by manipulations with the finger in the rectum and external manipulation at the same time. The only after-treatment required is rest in bed for a few days and the administration of opium to keep the bowels quiet.

FIG. 317.

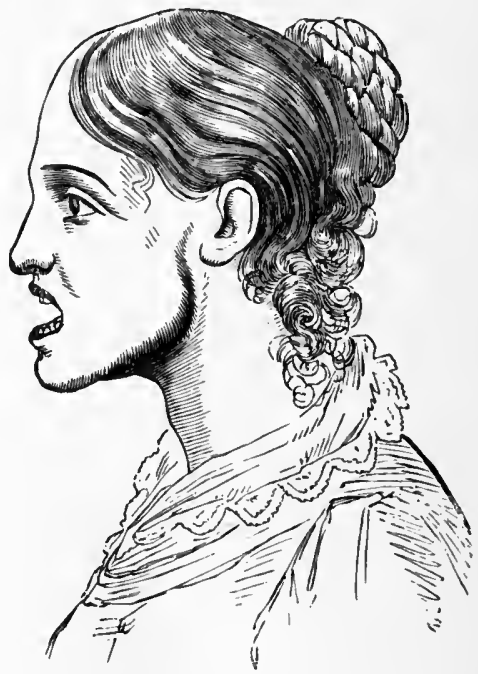

Bilateral dislocation of the lower jaw. (AshHURst.)

Dislocations of the Lower Jaw.-These dislocations may consist in the displacement of one or both condyles 
of the lower jaw from the glenoid fossæ, constituting the unilateral or bilateral dislocation of the jaw ; the latter is the more eommon form of dislocation of the jaw met with, and the deformity resulting is shown in Fig. 317.

The reduction of a dislocation of the lower jaw is aceomplished as follows: The surgeon plaeing his thumbs, well protected by strips of bandage or a towel, on the molar teeth or behind them, presses the angles of the jaw downward while he elevates the chin with his fingers, and by this manipulation the condyles of the jaw usually slip back into place with a snap (Fig. 318). After reduction of the

Fra. 318.

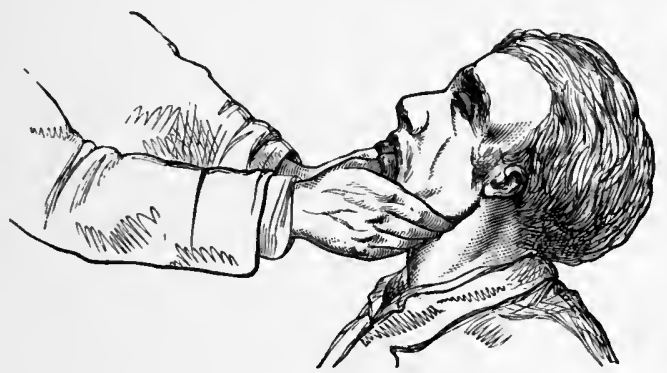

Method of redueing dislocation of the lower jaw. (Hammitow.)

dislocation the jaw should be fixed for a week or ten days by the application of a Barton's bandage or a four-tailed sling.

Dislocation of the Hyoid Bone.-A few eases of dislocation of the hyoid bone have been recorded; the treatment consists in throwing back the head as far as possible, to place the muscles of the neek upon the streteh, depressing the lower jaw, and pressing the luxated bone into position.

Dislocations of the Ribs and Costal Cartilages.The ribs may be dislocated at their vertebral articulations or at the junetion with the costal cartilages, or the cartilages may be separated from the sternum. These injuries result from the application of great force, and are often 
fatal from associated injuries of the thoracic viscera. The treatment of these dislocations consists in reducing the displacement by manipulation and pressure, and then in fixing the chest to secure immobility of the ribs by strapping the affected side with strips of adhesive plaster, the same dressing being applied as in cases of firacture of the ribs, the dressing being retained for three or four wecks.

Dislocations of the Sternum.-Dislocation or diastasis of the sternum may occur at the junction of the manubrium and the gladiolus or at the junction of the ensiform cartilage and the gladiolus. The reduction is effected by extension of the chest by bending the dorsal spine over a firm enshion placed under the back and by pressure upon the projecting bone; when the displaced bone has been reduced, a compress should be placed over the seat of injury, and held in place by broad strips of adhesive plaster, or by a bandage to keep the parts at rest. The dressing should be retained for three or four weeks.

In the few examples of dislocation of the ensiform cartilage which have been reported, the displacement of the cartilage has in some cases given rise to persistent vomiting, which was relieved by reduction of the displacement; it is, however, almost impossible to keep the bone in place after reduction.

Dislocations of the Pelvis.-Dislocation or diastasis of the bones of the pelvis may oceur at the pubic or sacro-iliac symphyses. They are generally serions injuries, as they are apt to be complicated by lesions of the pelvic viscera.

The reduction of these dislocations is effected by pressure and manipulation, and after reduction the parts should be supported by a compress held in place by a stout binder or by broad strips of adhesive plaster, the patient being kept quiet in bed and the pelvis being supported by means of sand bags. 'The dressings should be retained for from four to six weeks.

Dislocations of the Clavicle.-Dislocations of the clavicle may occur either at the sternal or acromial end the 
latter injury some writers deseribe as a dislocation of the scapula, following the general rule that the distal bone is the one dislocated.

Dislocations of the Sternal End of the Clavicle.-These may occur in a forward, backward, or upward direction, and the displacement is generally well marked (Fig. 319). The reduction of this dislocation is effected by placing the knee against the spine, and drawing the shoulders outward and backward and pressing the displaced end of the clavicle into place. The reduction is generally easy, but it is often difficult to keep the end of the bone in its proper position. 'To accomplish this, a compress should be placed over the end of the hone, and this should be secured in place by broad strips of alhesive plaster; the shoulders should be brought well backward and secured by a posterior figure-of-eight bandage of the chest, and the arm of the injured side should be fastened to the side of the chest by spiral turns of a bandage. In some cases, in addition to the compress over the end of the bone, securing the arm of the injured side in the Velpeau position will be found all that is necessary to retain the bone in position.

Dislocation of the Acromial End of the Clavicle.-This may be upward, downward, or backward (Fig. 320). The reduction is effected by manipulation of the arm and scapula and by pressure over the displaced end of the clavicle. The displacement is usnally reduced without much trouble, but it is often a matter of difficulty to keep the end of the bone in its proper place. The dressing consists in placing a compress over the acromial end of the clavicle and holding it in place by broad strips of adhesive plaster; the arm should at the same time be fixed in the Velpeau position.

Stimson's dressing consists in applying a long strip of adhesive plaster three inches wide, the centre being placed over the flexed elbow and its ends carried $\mathrm{np}$ in front of and behind the arm, crossing over the end of the clavicle and being seenred on the front and back of the chest, respectively, while the bone is held in place by pressure 
upon the clavicle and the elbow. For adlitional security, ihe forearm may be supported in a sling and the arm bound to the side of the chest.

The dressings after reduction of dislocations of the elavicle should be kept in place for at least three weeks. Although in many cases a certain amount of deformity persists, the disability resulting from the injury is not often marked.

FIG. 319.

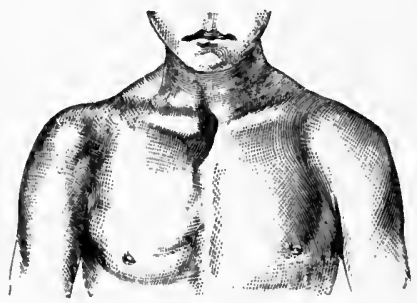

Dislocation of sternal end of clavicle forward. (BRYANT.)
FIG. 320.

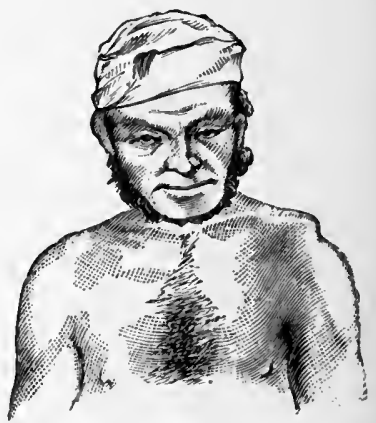

Dislocation of clavicle at acromial end. (BRYANT.)

Dislocations of the Scapula.-Dislocation of the acromion process of the seapula from the outer end of the clavicle, which has been described under dislocations of the acromial end of the clavicle, is classed by some writers as a scapular dislocation.

Dislocation of the Inferior Angle of the Scapula.-The displacement of the inferior angle of the scapula from under the latissimus dorsi muscle is due to relaxation of this muscle and of the serratus magnus, and is sometimes described as a dislocation of the inferior angle of the scapula. The reduction of this deformity consists in the employment of manipulation and pressure to overcome the displacement, and the use of a compress held in place by broad strips of adhesive plaster to secure the bone in its proper position. 
Dislocations of the Shoulder.-The head of the humerus may be dislocated downward, forward, or backward.

Subglenoid Dislocation of the Head of the Humerus. - In this variety of dislocation the head of the bone rests in the axilla (Fig. 321).

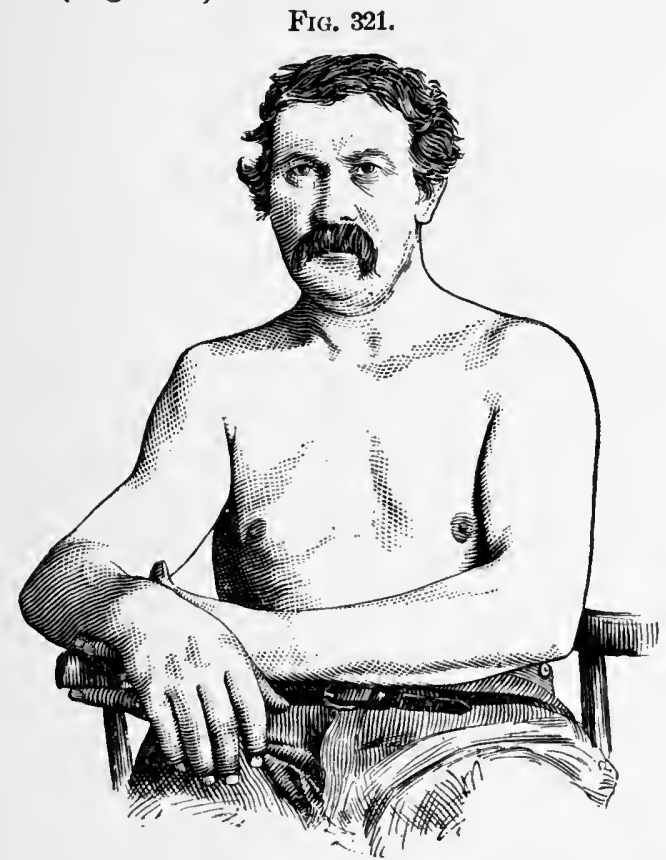

Subglenoid dislocation of the head of the humerus. (STIMson.)

Subcoracoid Dislocation of the Head of the Humerus.In this variety of dislocation the head of the humerus rests beneath the coracoid process of the scapula (Fig. 322 ).

Subclavicular Dislocation of the Head of the Humerus.This may be considered an aggravated form of the latter variety of dislocation; the head of the humerus in this dislocation rests beneath the clavicle. 
Subspinous Dislocation of the Head of the Humerus.-In this variety of dislocation the head of the humerus rests bencath the spine of the scapula (Fig. 323).

Reduction of dislocations of the humerus is effected by manipulation, by extension and counter-extension, and by a combination of these methods.

Manipulation in the reduction of subglenoid dislocation of the humerus is practised with the patient in the recumbent posture by first flexing the forearm upon the arm to

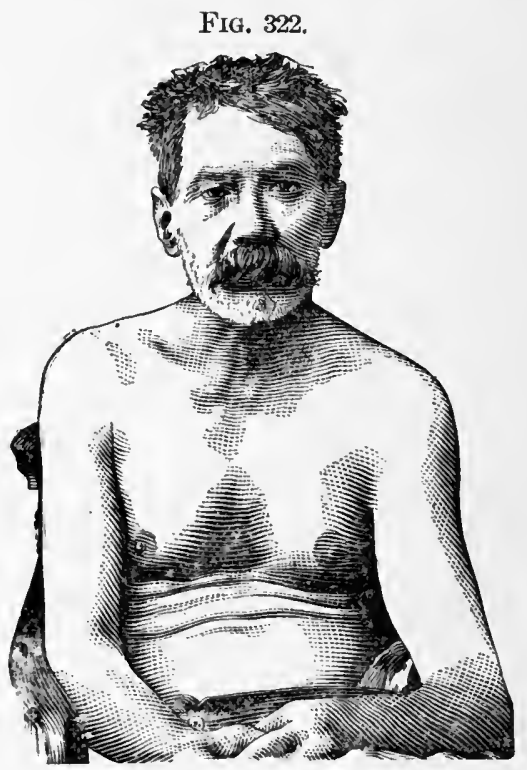

Subcoracoid dislocation of the head of the humerus. (STIMson.)

relax the long head of the biceps muscle; the elbow is next seized and abducted so as to bring it to the side of the patient's head, thus relaxing the deltoid and supraspinatus muscles; the surgeon or an assistant next places his hand upon the head of the humerus in the axilla, and, as the arm is drawn outward to a right angle with the 
FIG. 323.

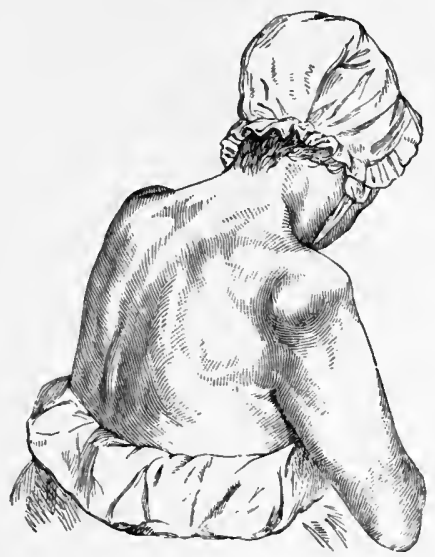

Subspinous dislocation of the head of the humerus. (ERICHsEN.)

FIG. 324.

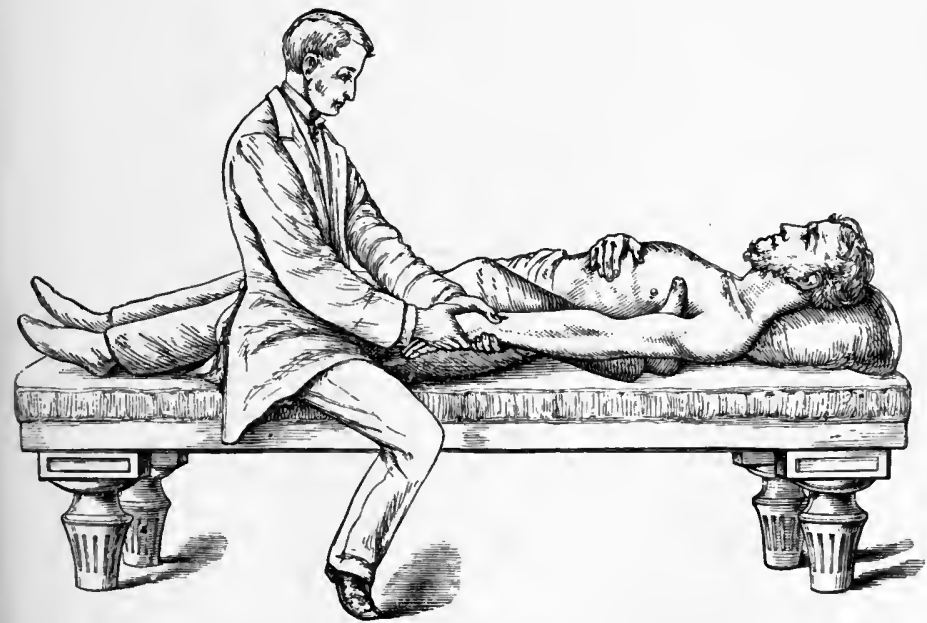

Reduction of dislocation of the humerus by heel in the axilla. (ERICHsEv.) 
body by the other hand, he pushes the head of the bone into the glenoid eavity.

In the reduction of subcoracoid and subclavicular dislo-cations the manipulations are the same, except that the arm is to be rotated outward before being carried downward.

In the reduction of subspinous dislocations, after the arm has been abducted, it should be rotated inward and direet pressure made upon the head of the bone as the arm is abducted.

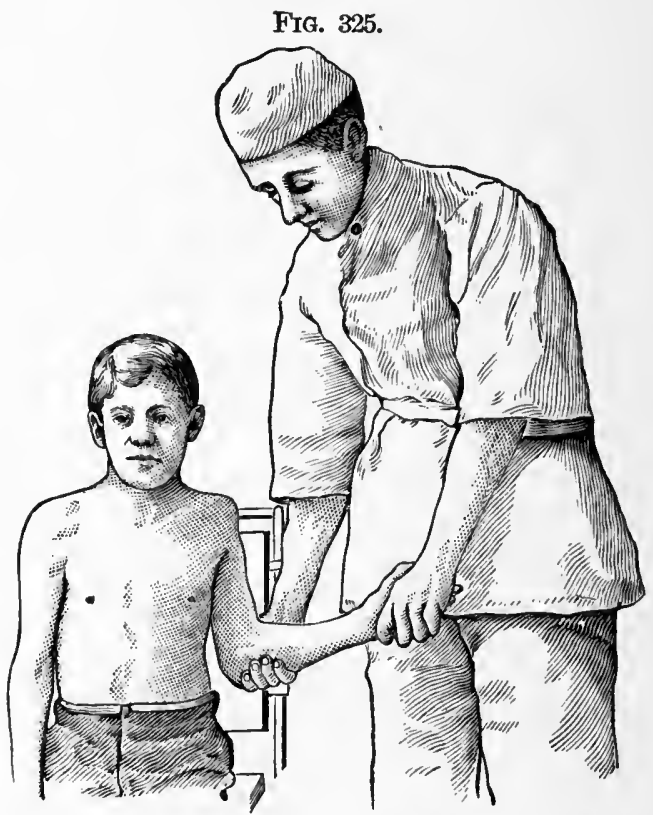

Kocher's method of reduction : first position. (BREWER.)

Reduction may also be effected by extension and counterextension, as in Cooper's method, where extension is made from the arm downward and counter-extension is made by the heel in the axilla (Fig. 324). This method is not to be recommended, on account of the damage which may occur to the axillary nerves and vessels. 
Kocher's Method.-Place the patient in the sitting posture and flex the elbow to a right angle, at the same time pressing the arm against the chest; the flexed forearm is then turned as far as possible from the trunk by rotating the humerus outward until it occupies a position parallel with the transverse plane of the body (Fig. 325). While the external rotation is being maintained the elbow is slowly carried upward along the anterior border of the chest

Fra. 326.

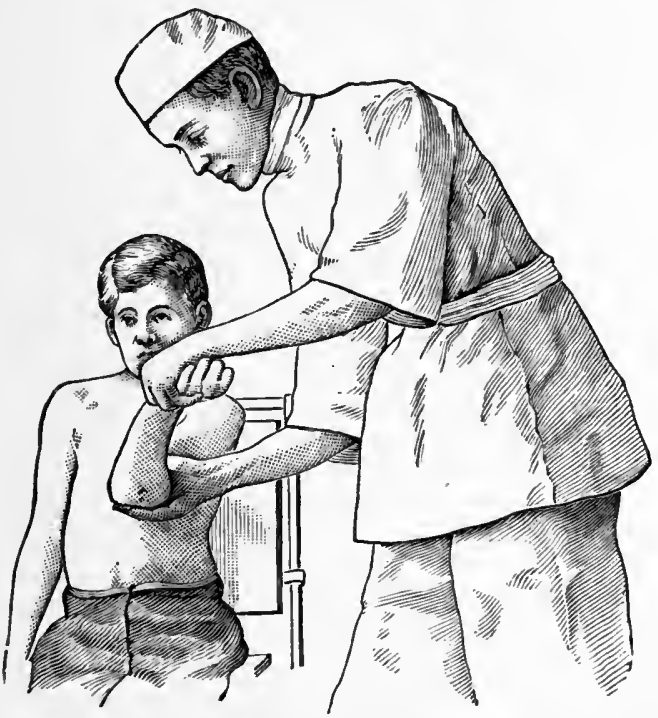

Kocher's method: second position. (BREWER.)

until it reaches a point opposite the ensiform cartilage (Fig. 326); the forearm is then quickly rotated inward until the hand touches the opposite shoulder and the elbow is lowered (Fig. 327).

Mothe's Method.-Reduction by this method may also be accomplished by extension made upward, the scapula 


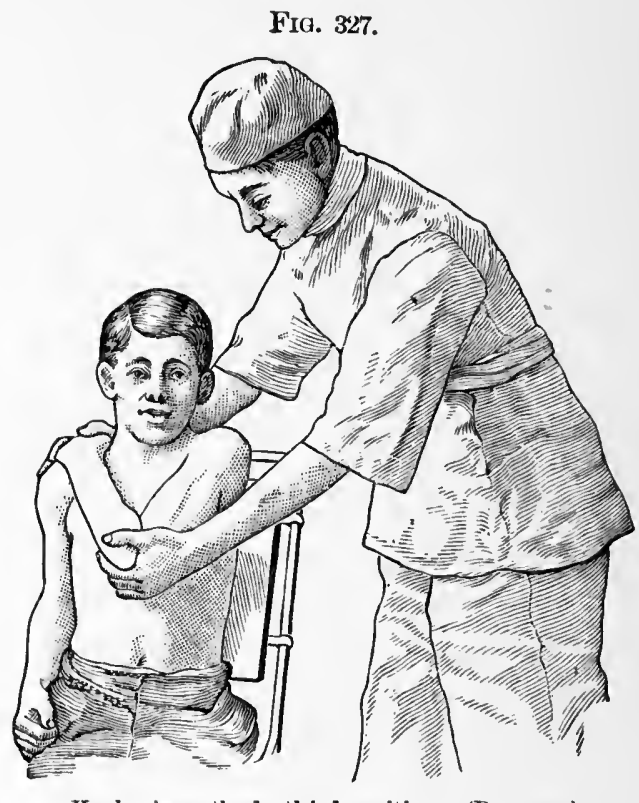

Kocher's method : third position. (BREWER.)

FiG. 328.

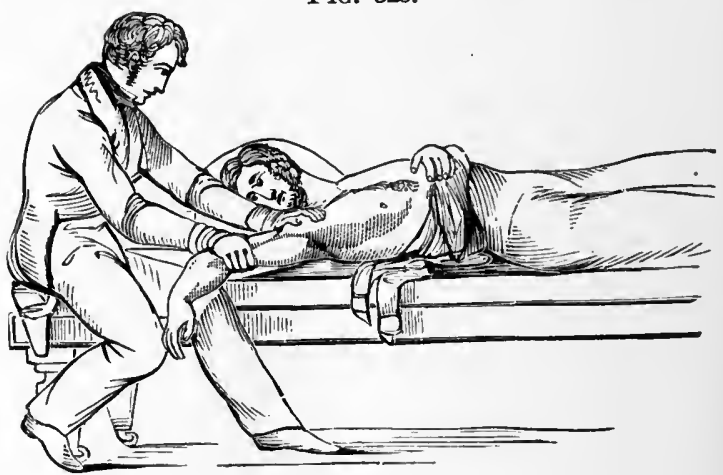

Reduction of dislocation of the humerus by extension upward. 
being fixed by the foot or hand placed over the acromion process (Fig. 328).

After reduction of dislocations of the head of the humerus the arm should be bound to the side of the body by the turns of a spiral bandage of the chest, or should be held against the side by the application of a Velpeau's bandage; this dressing should be removed at intervals of a few lays, and after ten days or two weeks all dressings should be dispensed with, passive motion should be employed, and the patient allowed to move the arm.

Dislocations of the Elbow.-Dislocations of the bones of the forearm at the elbow may be either backuard, forward, or lateral. The backward dislocation is the most common form (Fig. 329).

FIG. 329.

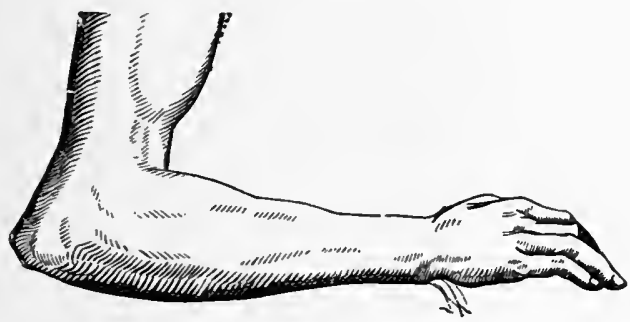

Dislocation of both bones of the forearm backward. (ListoN.)

The reduction of backward dislocations is effected by making traction upon the forearm and at the same time making pressure upon the lower end of the humerus as the forearm is flexed upon the arm.

Or the reduction may be accomplished by bending the arm slowly and forcibly over the knee placed upon the inner surface of the elbow, so as to press upon the radius and ulna, separating them from the humerus and freeing the coronoid process from its abnormal position (Fig. 330).

Lateral dislocations of the bones of the forearm at the elbow are reduced by making extension from the forearm, and at the same time making direct pressure on the dis- 
placed bones and counter-pressure on the lower end of the humerus.

Forward dislocations of the bones of the forearm at the elbow are reduced by making forced flexion at the elbow, together with extension and counter-extension, or by making forced extension of the forearm at the elbow, pressing the humerus backward, and suddenly flexing the forearm.

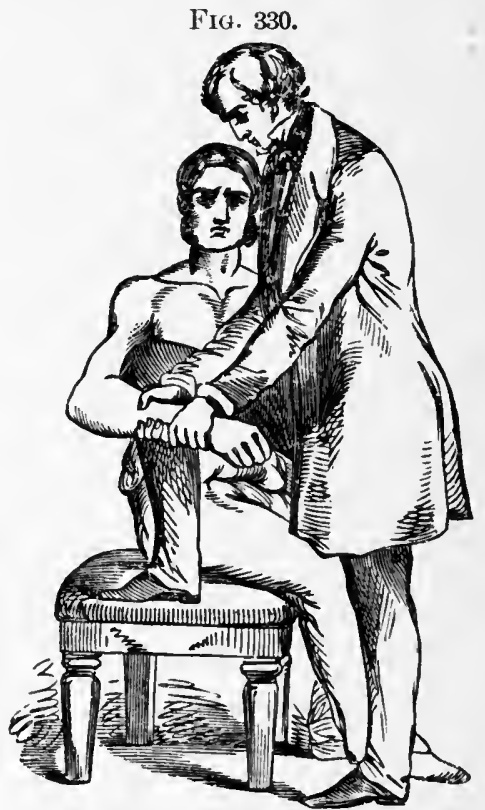

Reduction with the knee in the bend of the elibow. (HAMILTON.)

The dressing, after the reduction of dislocations at the elbow, consists in the application of a well-padded anterior right-angled or slightly obtuse-angled splint, to keep the forearm in a flexed position-the dressing being practically the same as that for fractures of the lower end of the humerus, with an anterior angular splint (Fig. 331). This dressing should be retained for two or three weeks, being removed at intervals of several days; after the re- 
moval of the splint passive motion should be practised, to prevent stiffness of the elbow-joint.

FIG. 331.

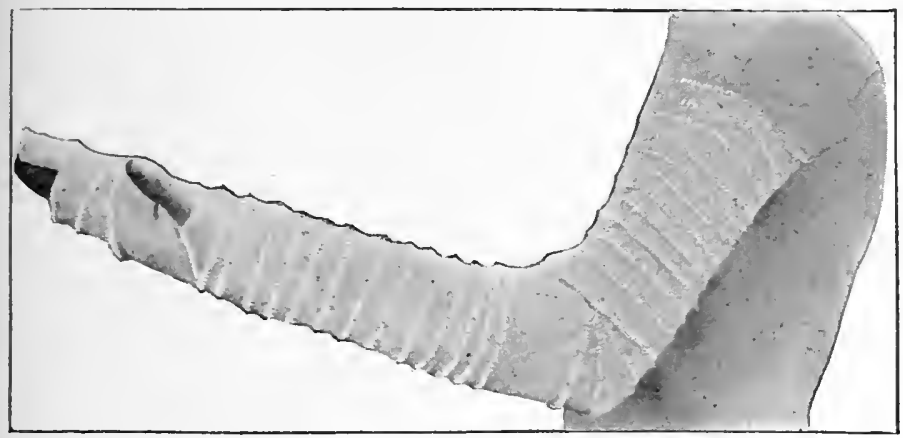

Dressing after reduction of dislocation of the elbow.

Dislocations of the Head of the Radius.-The head of the radius may be displaced forward, outward, or backward, the forward dislocation being the most frequent. The reduction of these dislocations is effected by making extension from the forearm and counter-extension from the lower end of the humerus, and at the same time the head of the bone is pressed into its proper position. The dressing after reduction of the displacement consists in the application of a compress over the head of the bone, and the arm and forearm should be placed upon a well-padded anterior angular splint, which is secured by a roller bandage. The dressing is similar to that employed after reduction of dislocations of the bones of the forearm at the elbow. Difficulty is sometimes experienced in keeping the head of the bone in position after reduction, so that the use of a compress in addition to the use of the splint is often required. The arm should be kept upon the splint for three weeks, being redressed at intervals.

Dislocation of the Upper End of the Ulna.-The upper end of the ulna may be displaced backward, the olecranon projecting beyond the condyles of the humerus, 
while the head of the radius occupies its normal position.

The reduction of this displacement is effected in the same nianner as that of both bones of the forearn backward, and the dressing after reduction is sinilar to that employed when both bones have been displaced.

Dislocations of the Wrist.-Dislocations of the carpus firom the bones of the forearm may be forward (Fig. 332) or backward (Fig. 333). The reduction in either variety

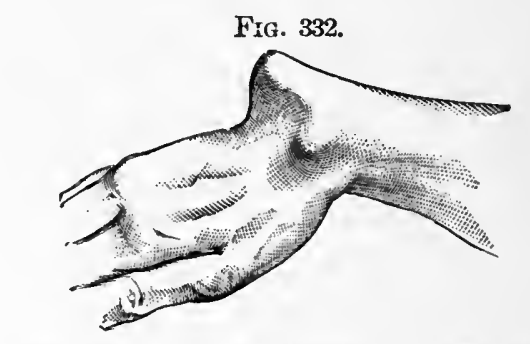

Dislocation of the carpus forward. (HaMiLToN.)

FIG. 333.

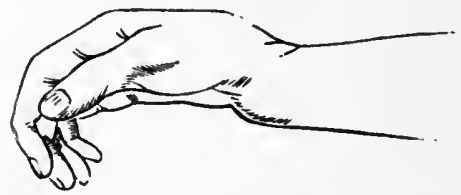

Dislocation of the carpus backward. (HAMiLton.)

of displacement is effected by extension from the hand and by pressure. After reduction of the displacement, which does not tend to recur, the hand and the forearm should be placed upon a well-padded straight splint applied to the palmar surface of the hand and forearm. The splint should be retained for ten days or two wecks.

The lower end of the ulna may be dislocated from the radius forward, backward, or inward. The reduction of these displacements is effected by fixing the radius and pressing the ulua lack into place. The dressing after reduction consists in placing the wrist-joint at rest by the 
application of well-padded anterior and posterior straight splints. The splints should be retained-for three or four weeks, dressings being made at intervals of two or three days.

Dislocations of the Bones of the Carpus.-Displacement of the individual bones of the carpus occasionally takes place, the os magnum, the semilunar, and pisiform being the bones most usually displaced, although other bones of the carpus are sometimes dislocated. Reduction is effected by means of extension and pressure, and the part should afterward be dressed with a palmar splint and compresses.

Dislocations of the Metacarpal Bones.-The metacarpal bones may be dislocated from the carpus ; the bones most commonly displaced are those of the thumb and of the index and middle fingers; the latter are usually displaced backward, while the metacarpal bone of the thumb may go either backward or forward.

Reduction is effected by extension and pressure. The dressing after reduction consists in the application of a palmar splint to the hand and forearm and a compress over the displaced bone. The dressings should be retained for two weeks.

Dislocations of the Fingers.-Dislocations of the phalanges of the fingers usually take place at the metaFIG. 334.

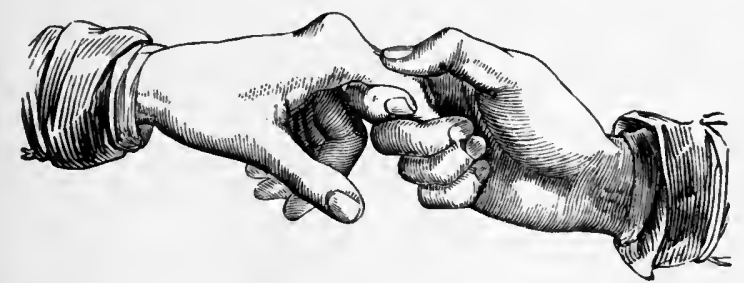

Backward dislocation of phalanx. Reduction by extension. (Hamitos.)

carpo-phalangeal junction, but sometimes occur at the interphalangeal joints. The reduction is usually easily effected by extension (Fig. 334), or by pushing the phalanx back 
until it stands perpendicularly upon the metaearpal bone, when by strong- pressure upon its base, from behind, forward, it is readily carried by flexion into its natural position.

Where difficulty is experienced in making extension in the reduction of these dislocations, the ingenious apparatus of the late Dr. Levis (Fig. 335), or the "Indian puzzle" apparatus (Fig. 336), may be employed with success.

In dislocations of the proximal phalanx of the thumb backward (Fig. 337) great difficulty in reduction is often experienced from the head of the metacarpal bone slipping between the heads of the short flexor of the thumb. The

Fra. 335.

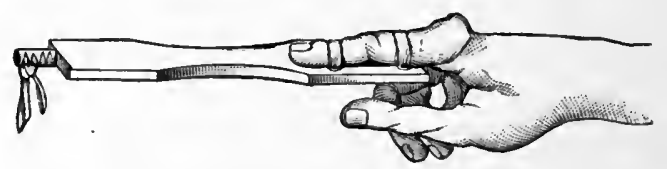

Levis's apparatus for dislocation of the phalanges applied.

interposition of the external sesamoid bone is considered by some surgeons to be the cause of difficulty in the reduction of this displacement.

In this disloeation reduction is effected by firmly pressing the metacarpal bone of the thumb strongly toward the

FIG. 336.

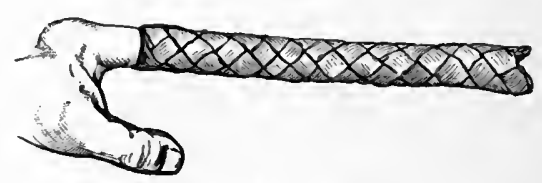

Extension by Inđian puzzle. (BRYANT.)

palm of the hand, to relax the two portions of the short flexor muscle. The thumb is next extended upon the wrist nntil its tip points to the elbow. An assistant next plaees his finger behind the proximal phalanx to prevent its slipping backward, and by bringing the thumb down to 
the flexed position the bone slips into place. It sometimes happens that all efforts at reduetion fail, and in such eases it may be necessary to divide one head of the short flexor musele subeutaneously or through an open wound before the displacement can be reduced.

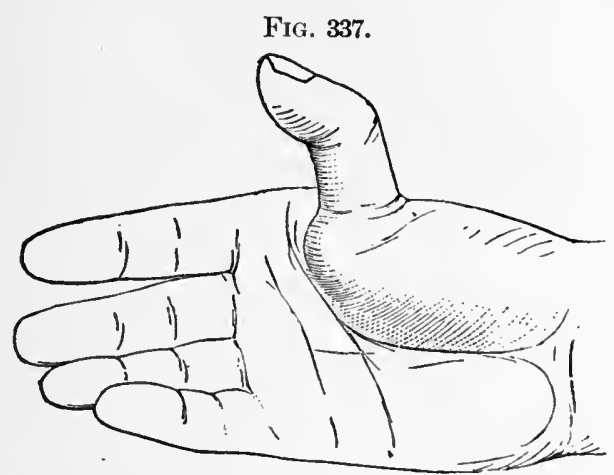

Dislocation of proximal phalanx of thumb backward. (FARABEUF.)

The dressing of disloeations of the phalanges after reduetion consists in the applieation of splints of wood, or moulded splints of binders' board, or gutta-percha, to fix the joint, which should be retained for ten days or two weeks.

Dislocations of the Hip.-The head of the femur is most frequently disloeated baekward, downward, or upward, although it may assume other positions in exeeptional eases.

Posterior or Backward Dislocations of the Head of the Femur.-These are either backward and upward, when they are described as iliac or dorsal, the bone resting upon the dorsum of the ilium (Fig. 338) ; or the dislocation may be backward, the head of the bone resting upon the isehiatie notch; these are known as ischiatic dislocations, or disloeations of the femur, dorsal below the tendon (of the obturator internus), aceording to Bigelow (Fig. $339)$. 
The reduction of the posterior dislocations of the femur can generally be effected by manipulation. The patient being anæsthetized and placed upon his back, the surgeon grasps the leg at the ankle and knee, flexes the leg upon the thigh, and the thigh upon the pelvis in the position of adduction; he then abducts the limb and rotates it out-

FIG. 338.

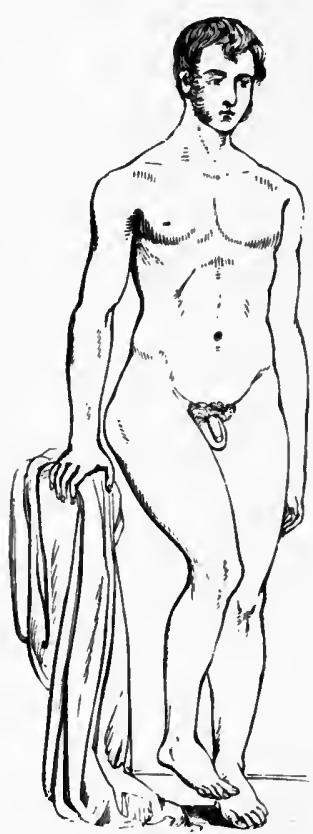

Backward and upward dislocation of femur. (COOPER.)
FIG. 339.

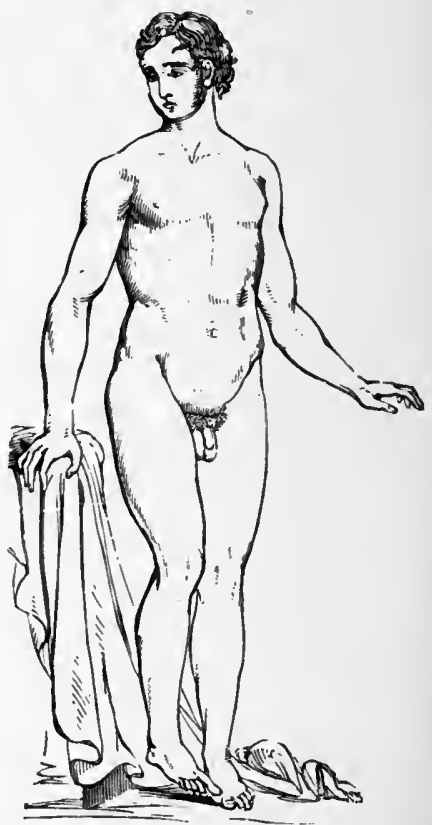

Backward dislocation of femur.

(COOPER.)

ward, bringing it in a broad swcep across the abdomen, and by bringing it down to its natural position the head of the bone will slip into the acetabulum (Fig. $340)$.

Kocher, in posterior dislocations, recommends the following manipulations: 1 . The surgeon grasps the ankle 
of the injured limb with one hand and the front of the knee with the other, and rotates the thigh inward, to relax the eapsule and lift the head of the bone from the posterior surface of the pelvis. 2. The thigh is next flexed to 90 degrees, preserving the existing adduction and inward rotation. 3. Traction is then made in the line of the femur, to make the capsule tense. 4. External rotation is then practised, which makes the posterior part of the capsule and Y-ligament tense, and returns the head of the bone to the acetabulum.

Allis, in the reduction of dorsal dislocations, recommends that, while the patient is supine, the surgeon kneel

FIG. 340.

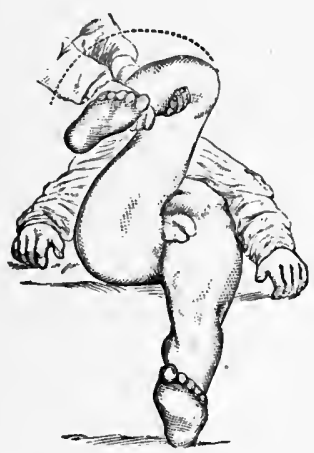

Reduction of backward dislocation of the femur. (BIGELow.)

beside him, and in the case of the right hip grasp the ankle with the right hand and place the bent elbow of the left arm beneath the knee. He then turns the bent leg outward by means of the ankle and lifts upward, and next turns the leg inward and brings the femur down in extension.

Downward and Forward Dislocation of the Head of the Femur.-In this variety of dislocation the head of the bone rests upon the thyroid foramen; this form of displacement 
is sometimes spoken of as a thyroid dislocation (Fig. $341)$.

The reduction of downward and forward dislocations of the head of the femur is effected by flexing the leg and thigh and bringing the limb into a position of abduction ; it is then adducted and rotated inward in a broad sweep

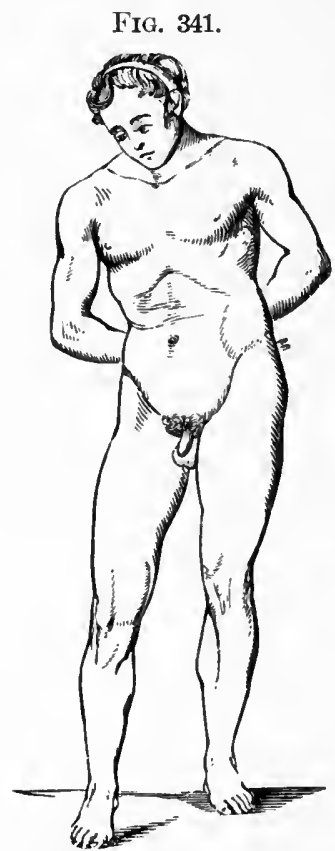

Downward and forward dislocation of femur. (COOPER.) across the abdomen and brought down to its natural position, when the head of the bone slips into the acetabulum (Fig. 342). In making these manipulations the head of the bone sometimes slips back upon the dorsum of the ilium, converting the downward dislocation into a posterior one; if this accident oceurs, the displacement should

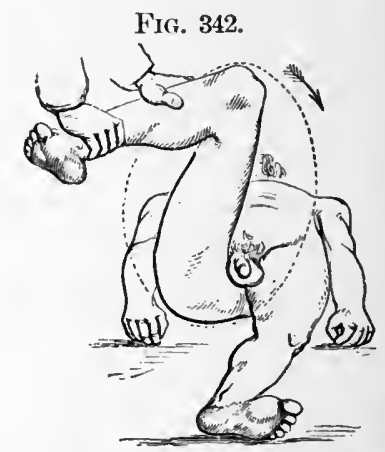

Reduction of downward and forward dislocation of femur. (BIGELOW.)

be reduced by making the manipulations appropriate for the reduction of the latter dislocation.

Kocher, in the reduction of these dislocations, recommends the following manipulations: 1 . The leg should be flexed upon the thigh and the thigh carried up to a right angle with the pelvis, maintaining abduction and external 
rotation, to relax the Y-ligament. 2. Traction should next be made in the line of the shaft of the femur, to render the posterior part of the capsule tense. 3. Outward rotation is then made, which, twisting the tense posterior portion of the capsule and the onter branch of the $\mathrm{Y}$-ligament, brings the head of the bone upward and backward into the acetabulum.

Forward and Upward Dislocation of the Head of the Femur.-In this variety of dislocation, which is very rare, the head of the bone rests upon the pubis; this form of displacement is also spoken of as a pubic dislocation (Fig. 343).

The reduction of forward and upward dislocations of the head of the femur is effected by much the same manipulation as is employed in the reduction of downward and forward dislocations, exeept that in the pubic dislocation the flexed limb should be carried across the sound thigh at a higher point. The thigh being flexed, the head of the bone is drawn down from the pubis; it is then semi-abducted and rotated inward to disengage the bone completely. While rotating inward and drawing on the thigh the knee should be carried inward and

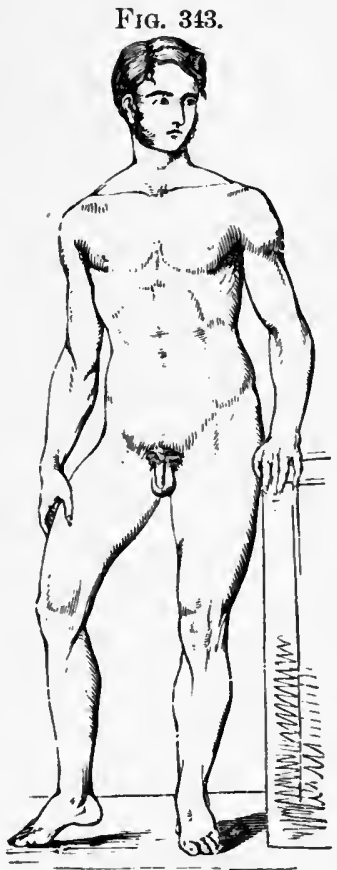

Forward and upward dislocation of the femur. (COOPER.) downward to its place by the side of its fellow, and the head of the bone will usually slip into the acetabulum.

Kocher, in the reduction of forward and upward dislocations of the femur, recommends: 1. Traction should first be made in the axis of the limb, to bring the head of the bone over the brim of the pelvis. 2. Pressure should next be made with the hand upon the head of the femur, to 
prevent its passing upward during flexion of the thigh. 3. The thigh should next be flexed to less than a right angle, to relax the $\mathrm{Y}$-ligament. 4. Inward rotation is next made, which directs the head of the bone into the acetabulum.

Anomalous Dislocations of the Head of the Femur.-These occasionally oecur; the head of the bone may pass direetly upward or downward between the sciatie notch and thyroid foramen, or downward and backward on the body of the ischium, or downward and backward into the lesser sciatic noteh, or downward, inwarl, and forward into the perineum. These anomalous displacements usually occur where there has been extensive laeeration of the capsular ligament and Y-ligament.

The dressing of cases after reduction of dislocations of the head of the femur consists in keeping the patient at rest in bed upon his back; the limb should be kept at rest by sand bags applied to either side of the limb, or the knees should be tied together. The patient should be kept at rest for two or three weeks, and at the end of this time may be allowed to get ont of bed and go about on crutches.

Dislocations of the Patella.-The patella may be dislocated outward, inward, or upuard, or it may be rotated upon its own axis. The outward dislocation is the displacement most usually scen (Fig. 344).

Upward dislocation of the patella can only result from laceration of the ligamentum patellæ, and the treatment in such cases is similar to that for fracture of the patella.

The reduction of dislocations of the patella is effected by extending the leg upon the thigh and flexing the thigh upon the pelvis, to relax the quadriccps femoris muscle, when the patella can usually be foreed back into place by manipulation with the fingers; in some cases alternate flexion and extension of the leg will accomplish the same result.

The dressing after reduction of the displacement consists in the application of a posterior straight splint or a moulded 
binders' board or felt splint to keep the joint at rest; the splint should be worn for a week or ten days.

Dislocations of the Knee.The liead of the tibia may be dislocated forward, backward, or laterally; the latter dislocations are always incomplete, forward dislocation being the variety of displacement most commonly met with (Fig. 345).

The reduction of dislocations of the knee is effected by extension and counter-extension with forced flexion of the knee with pressure, aided by rocking movements. The treatment of cases of dislocation of the knee after reduction consists in fixing the knee-joint by the application of a straight posterior splint or a moulded splint of binders' board. As there is usually marked swelling following these injuries from

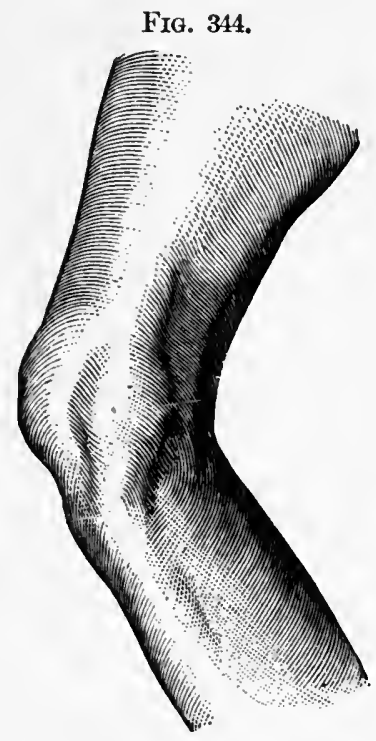

Outward dislocation of the patella. (DUPLAY.) violence to the joint-structures, the application of evaporating lotions for a few days will be found useful. As

Fig. 345.

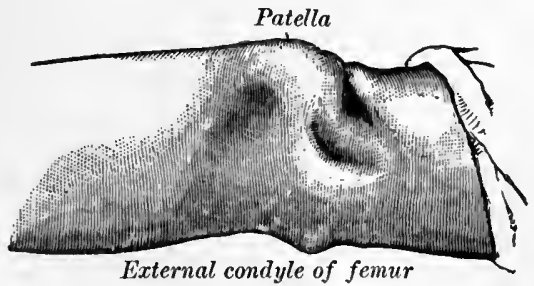

Forward dislocation of the knee. (BRYant.)

soon as the swelling has subsided, the limb should be put 
up in a plaster-of-Paris dressing, and this should be retained for four weeks.

Dislocation of the Semilunar Cartilages.-The displacement here consists in the slipping forward or backward and wedging of the semilunar cartilages between the femoral condyles and the tibia.

Reduction of the displaced cartilages ean usually be effeeted by hyperflexion of the knee, followed by sudden full extension, or by alternately flexing and extending the joint. Excision of the displaced eartilages is sometimes required in cases in which they cannot be reduced by manipulation.

The dressing of these cases after reduction of the displaced cartilages consists in the application of a posterior straight splint or a plaster-of-Paris dressing to fix the knee-joint; the splint should be worn for three or four weeks, and if there is a tendency to redisplacement, the patient should wear a brace, or a knee-cap of leather or muslin, to partially fix the joint, with compresses so arranged as to make pressure upon the edge of the joint.

Dislocations of the Fibula.-Dislocations of the fibula may oceur at either of its extremities, and the direction of the displacement may be forward, backward, or outward; dislocation of the head or upper extremity of the fibula being the most common, although all are rare forms of displacement.

The reduction of dislocations of the head of the fibula is effected by flexing the leg upon the thigh and making direct pressure and extension. Dislocations of the lower extremity of the fibula are reduced by manipulation and pressure. The dressing of cases after reduction of dislocations of the fibula consists in the application of a compress and moulded binders' board splint; the dressing should be retained for three or four weeks.

Dislocations of the Ankle.-Dislocations of the foot upon the bones of the leg result from separation of the articular surface of the astragalus from that of the tibia and fibula; the displacement may be forward, backward 
(Fig. 346), or lateral (Fig. 347), the latter variety being often associated with fractures of the malleoli.

FIG. 346.

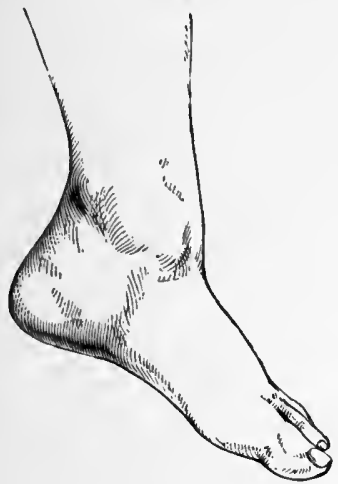

Dislocation of foot backward. (BRYANT.)
FIG. 347.

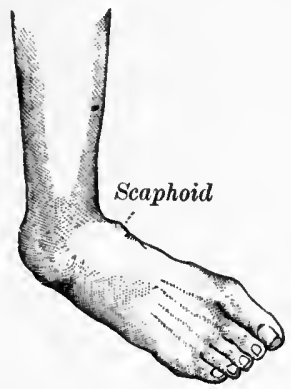

Dislocation of foot inward.

(BRYANT.)

The reduction of dislocations of the ankle is effected by traction, combined with flexion and rotation of the anklejoint, the leg being first flexed upon the thigh to relax the tendo-Achillis, and in some cases the subcutaneous division of this tendon is required before the reduction can be satisfactorily accomplished.

The dressing of dislocations of the ankle after reduction consists in the application of a fracture-box or of pasteboard splints, to fix the ankle, care being taken to see that the foot is fixed at a right angle to the leg, and in the application of evaporating lotions for a few days; after the swelling has subsided a plaster-of-Paris dressing should be applied and retained for three or four weeks.

Dislocations of the Tarsal Bones.-The astragalus may be dislocated from the bones of the leg and from the other tarsal bones, being thrust forward, backward, outuard (Fig. 348), or inward. The reduction of disloeations of the astragalus outward is effeeted by first flexing the leg upon the thigh and making extension from the foot and rotating it at the same time, direct pressure being 
made upon the displaced bone; in some cases subcutaneous section of the tendo-Achillis has assisted materially in the reduction of the displaced bone. Backward dislocation of the astragalus is usually irreducible; the patient, however, in many cases recovers with a useful foot. In cases of irreducible dislocations of the astragalus excision of the astragalus may ultimately be required.

After the reduction of dislocations of the astragalus, the foot and leg should be put at rest in a fracture-box, or by

FIG. 348.

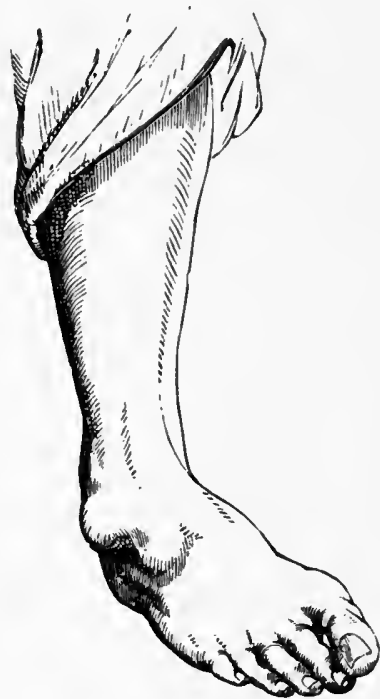

Dislocation of astragalus outward. (HAMILTON.) means of moulded splints of pasteboard or felt; evaporating lotions should also be employed over the region of the injury for a few days, and when the swelling has subsided a plaster-of-Paris dressing should be applied and retained for three or four weeks.

Dislocations of the calcaneum and scaphoid upon the astragalus, or of the calcaneum upon the astragalus and cuboid, or upon the astragalus alone; of the scaphoid and cuboid npon the calcis and astragalus; or of the cuboid, scaphoid, or cuneiform bones, are occasionally met with.

Their reduction is effected by traction and direct pressure, and after this has been accomplished the parts should be put at rest by the application of a splint and compresses.

Dislocations of the Metatarsal Bones and Phalanges of the Toes.--These dislocations usually result from crushing forces which destroy the vitality of the soft parts so completely that amputation is required. Their reduction in cases of simple or uncomplicated dislocations is effected by traction, manipulation, and pressure. After 
reduction of the displacement the parts should be kept in position by the application of splints and bandages.

old Dislocations.-The reduction of old dislocations is attended with more difficulty and dauger than that of recent dislocations, due to the permanent contraction and structural changes which occur in the museles and to the adhesions which form between the displaced bone and the parts with which it is in contact. The reduction of old dislocations may usually be accomplished by the manipulations appropriate for recent dislocations of the same variety ; but occasionally the use of more forcible extension is required, which is made by bands and pulleys. The first step in the reduction of old dislocations consists in thoroughly breaking up the adhesions which have been formed between the displaced bone and the surrounding tissues; this has, in some cases, resulted in the laceration of muscles, nerves, and bloodvessels, and in fracture of the displaced bones or neighboring bones, so that the manipulations should be made with the least force that will accomplish the object desired. After the reduction of old dislocations difficulty is sometimes experienced in maintaining the bone in its proper place, due to the changes which have occurred in the articular surfaces.

In such cases fixation of the bone in its normal position by a plaster-of-Paris dressing should be employed for some weeks, and after its removal passive motion should be practiced. If the dislocation is found to be irreducible, and the patient suffers from great pain and disability, open operation is advisable. This consists in exposing the displaced bone and dividing the soft tissues which interfere with reduction or in making a complete or incomplete excision of the joint.

Compound Dislocations.-These are always grave injuries, and amputation or excision may be required. With the modern methods of wound treatment, operative measures are not often required. The reduction is effected in the same manner as in simple dislocations of corresponding parts, the greatest care being taken to render the wound 
aseptie, and to keep it in this condition by the application of a full antiseptic dressing. After reducing the dislocation and dressing the wound some form of fixation splint should be applied to fix the joint until healing of the wound has oceurred.

\section{Complicated Dislocations.}

In dislocations complicated by fracture near the seat of displacement, the displaced bone should, if possible, be first reduced, and this in many cases is a matter of great difficulty, as the fracture prevents the surgeon from using leverage otherwise present, in the reduction, and he has often to depend entirely upon pressure and manipulation to overcome the displacement. After reduction of the dislocation the fracture should be reduced and dressed.

Dislocation complicated by rupture of the main artery of the limb may require, after reduction of the displacement, exposure and ligation of the vessel or amputation of the limb. Rupture of an important nerve-trunk complieating a dislocation may call for subsequent exposure and suturing of the divided nerve.

\section{Habitual, Pathological, and Congenital Dislocations.}

In the treatment of these varieties of dislocations after the reduction of the displacement by manipulation and pressure, much difficulty is often experieneed in maintaining the reduction. To effeet the latter object, the use of splints and bandages is employed, and also the use of many ingenious forms of apparatus adapted to particular dislocations. Operative treatment such as excision of a portion of the capsule of the joint or ligaments, or operation upon the bone to increase the capacity of the joint, may be practised.

Tenotomy or myotomy is often required to prevent recurrence of the deformity, and continuous extension is also of much value in the treatment of these displacements. 


\section{PART VI. OPERATIONS.}

Is view of the fact that at the present time in medical schools much attention is paid to practical surgery - that is, operative procedures upon the cadaver-it has been thought advisable to introduce a very brief description of a number of operations which may with advantage be performed upon the cadaver. Too much value cannot be attached to the importance of the student rendering himself familiar with the use of instruments and their manipulation in the various operative procedures, and also familiarizing himself with the appearance of the anatomieal parts exposed in operations. The introduction of sutures, the application of ligatures, the closing of wounds, the cutting and fitting of flaps in plastic operations, are procedures the practical value of which to the student cannot be overestimated.

\section{LIGATION OF ARTERIES.}

In the application of a ligature to an artery in its continuity the surgeon should make his incision in the line which corresponds to the general course of the vessel, and he should be thoroughly familiar with the anatomy and with the surgical landmarks of the part. A portion of the artery, when possible, slould be selected for the application of the ligature half an inch or an inch from any large collateral branch. The position of the incision being 
seleeted, the surgeon steadies the skin with two fingers and makes an incision of the required length through it with a sealpel; the superficial fascia is next picked up on a director, any large superficial veins which come into view being displaced, and divided to an equal length with the incision in the skin; the deep fascia being exposed, it should be nicked and divided upon a director ; the intermuscular space, or the edge of the muscle o: muscles which are the guide to the vessel, should next be sought for, and small arteries coming from the main vessel through these spaces will often serve as valuable guides to the position of the artery. The surgeon next separates the tissues with the director or handle of the knife until the sheath of the vessel is exposed ; this is recognized by its communicated pulsation and by the absence of the smooth, shining surface and pinkish-white color which the surface of the artery presents. The sheath of the artery should be pieked up with forceps and nicked with the point of the knife applied flatwise (Fig. 350, A) ; the incision into the sheath should be very limited, only sufficiently large to allow the aneurism needle to pass through it around the vessel; extensive dissections or separations of the sheath from the artery should be avoided, as the nutrition of the artery at the point of ligature may thus be impaired, and slonghing and secondary hemorrhage may result. A distinct sheath is found only about the main arterial trunks, which is replaced in the smaller arteries by a layer of loose cellular tissue. The wall of the artery being exposed, an aneurism needle (Fig. 349) is passed around the vessel, threaded with a catgut ligature, and withdrawn (Fig. $350, \mathrm{~B}$ ) ; the needle may be threaded before heing passed, in which case the ligature is grasped with forceps and drawn through while the needle is withdrawn. The best material for ligatures is silk or carefully prepared chromicized catgut. The needle should be passed away from important structures; such as accompanying veins and nerves.

Before the ligature is tied the surgeon should satisfy himself that the ligature when tied will control the circu- 
lation in the artery below its point of application, by placing the tip of his finger upon the vessel and drawing upon the ends of the ligature, so as to oeclude the vessel at the point of application. Being satisfied as to this point, the ligature is tied with a reef-knot, or a surgeon's knot and reef-knot combined, and the ends of the ligature are cut short in the wound (Fig. 350, C).

FiG. 349.

$$
\text { F10. } 350 \text {. }
$$

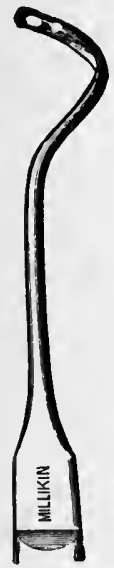

Aneurism needle.

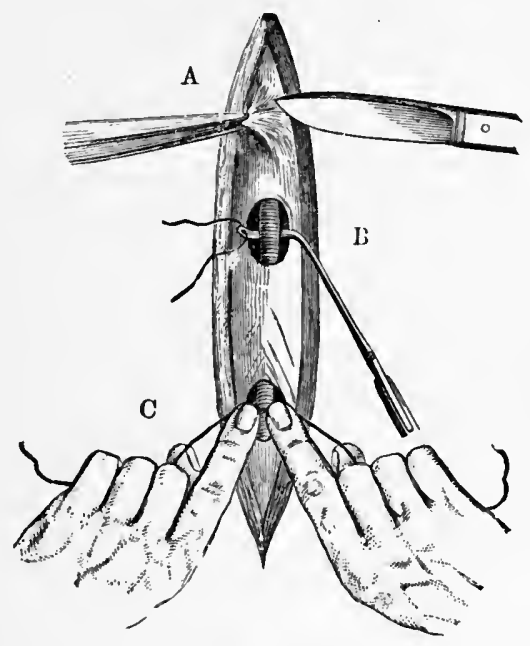

$A$, opening sheath; $\mathbf{B}$, passing ligature around the vessel; $\mathrm{C}$, tying the artery.

Some authorities recommend the application of two ligatures a short distance apart in the ligation of vessels in their continuity, and a division of the vessel between them, so that both ends may retract into the cellular sheath.

\section{Ligation of Special Arteries.}

Ligation of the Innominate Artery.-The innominate artery lies immediately behind the sterno-clavicular articulation, and is in relation in front with the innominate 
veins and pneumogastric nerve, on the inner side with the trachea, on the outer side and behind with the pleura.

The incision is a V-shaped incision, each branch of which is two and a half or three inches in length, one of which lies over the anterior edge of the sterno-cleidomastoid muscle and the other parallel to and a little above the clavicle (Fig. 351, $A$ ). The incisions are carried down to the superficial fascia and a flap is dissected up. If the anterior jugular vein is met with, it should be displaced.

FIG. 351.

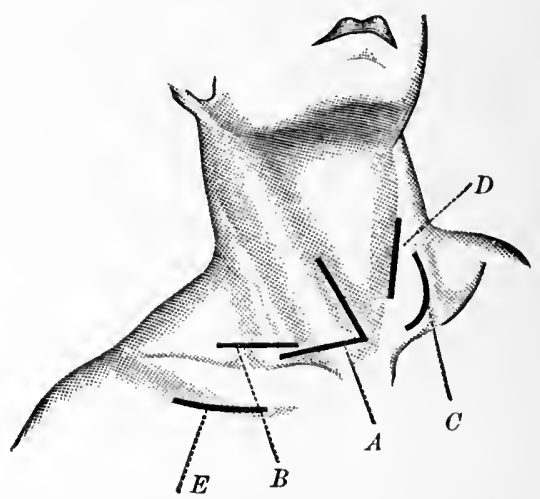

Lines of ineision for $-A$, innominate artery ; $B$, right subclavian artery; $C$, left subclavian artery; $D$, vertebral or inferior thyroid artery; $E$, axillary artery below clavicle. (STrmson.)

The sternal and clavicular attachments of the sternocleido-mastoid are next divided upon a director half an inch above the bone. The sterno-thyroid and sternohyoid muscles and the middle cervical fascia are then exposed, covered by the thyroid veins. The outer fibres of the sterno-hyoid and sterno-thyroid muscles are next divided, the thyroid rein being held aside, when upon tearing through the fiscia with a director the conmon carotid artery is exposed and traced down to the innominate artery ; the innominate veins are pressed against the sternum with the finger, and the artery is separated from 
its sheath about half an inch below its bifurcation, and the aneurism needle is passed around the vessel from the outer side, so as to avoid the vein, pneumogastric nerve, and pleura.

Ligation of the Subclavian Artery.-This artery may be tied at three points; in its first portion, between the trachea and scaleni muscles; in its second portion, behind the scaleni muscles; and in its third portion, external to the scaleni muscles.

The left subclavian artery in its first portion is larger and more vertical in its direction than the right subclavian, and is situated more posteriorly. From the difficulty in exposing this portion, and from the possibility of injuring the thoracic duct, the ligation of this artery in its first portion has been seldom attempted.

The incision for the first portion of the subclavian artery is the same as that for the innominate (Fig. 351, A), and the ligature is passed from the outer side, the pneunogastric and phrenic nerves being pressed inward toward the carotid artery.

The right and left subclavian arteries are also seldom tied in their second portions-that is, behind the sealeni muscles-but are frequently tied in their third portionsthat is, external to the scaleni muscles.

The incision for the second portion of the subclavian artery begins an inch external to the sterno-clavicular articulation, half an inch above and parallel to the clavicle, and is three or four inches in length (Fig. 351, $B$ or $C)$. The steps of the operation are the same as for ligation of the third portion, and when the scalenus anticus muscle has been exposed, it is divided upon a director; the phrenic nerve, which lies upon its anterior aspect, is to be avoided.

The incision for the third portion of the subclavian artery is the same as for the second portion (Fig. 351, $B$ or $C$ ). The skin and platysma being divided, the external jugular vein is exposed and drawn to one side or divided between two ligatures; the superficial fascia is next divided upon a director; the posterior belly of the omo-hyoid muscle is 
next found and drawn upward and outward; the outer border of the scalenus anticus is next felt for and followed down to the tubercle of the first rib-the artery lies against this, between it and the lowest bundle of the brachial plexus. 'The artery is next denuded with the direetor, and the needle is passed from below, care being taken not

FIG. 352.

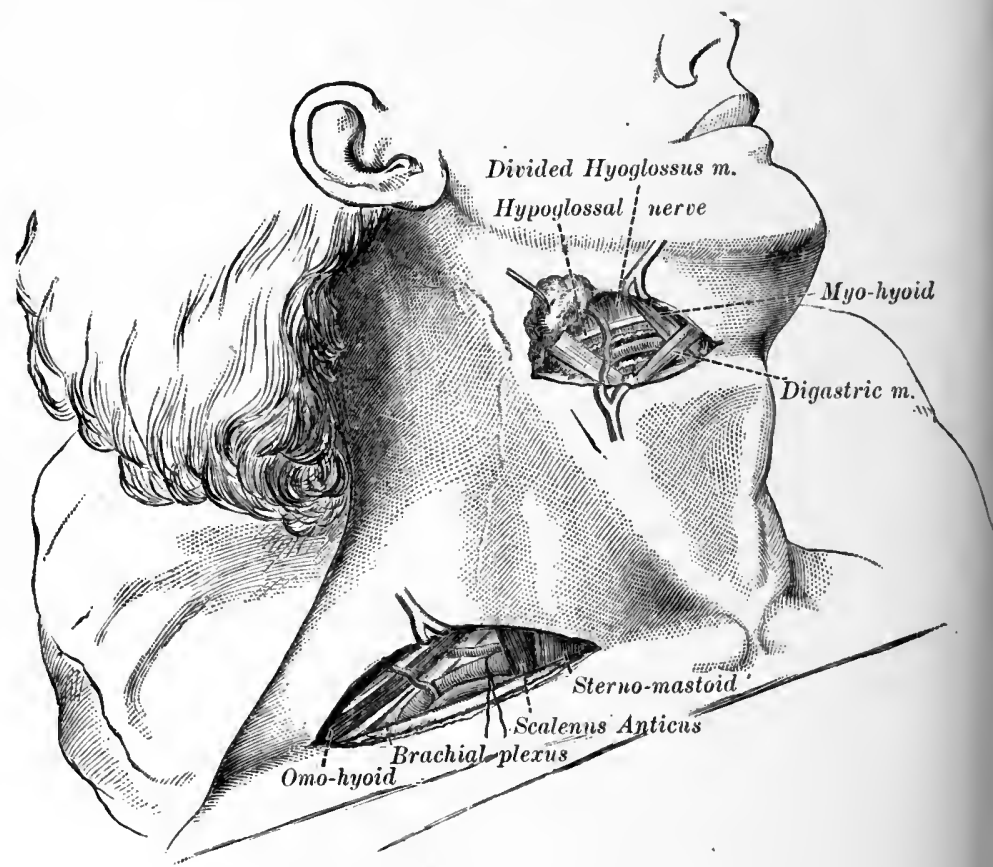

Ligation of subclavian and lingual arteries. (BRYANT.)

to include the lowest bundle of the brachial plexus in the ligature (Fig. 352).

Ligation of the Vertebral Artery.-The incision for the ligation of the vertebral artery is three or three and a half inches in length, parallel with the anterior edge of the sterno-cleido-mastoid muscle, ending an inch above 
the clavicle (Fig. 351, D). The anterior edge of the sterno-cleido-mastoid being exposed, the middle cervical fascia is divided and the carotid artery and jugular vein are exposed and drawn inward. The gap between the longus colli muscle and the scalenus anticus muscle is next felt for about an inch below the carotid tubercle; the fascia covering it is next torn through and the muscles are separated and the vertebral vein comes into view. When this vein is held aside the vertebral artery is exposed, and the ligature is then passed around it.

Ligation of the Inferior Thyroid Artery.-The incision for the inferior thyroid artery is the same as that for the vertebral artery (Fig. 351, D). The anterior edge of the sterno-cleido-mastoid muscle being exposed, it is drawn outward, the middle cervical fascia is next divided, and the carotid artery and internal jugular vein are drawn outward with a retractor. The head heing flexed slightly, the surgeon feels for the carotid tubercle, and then separates the cellular tissue with a director, and the artery should be found below the carotid tubercle. The needle should be passed between the artery and vein.

Ligation of the Internal Mammary Artery.-The incision, a vertical one, two and a half inches in length, commences at the lower border of the clavicle, paralle] with and three lines external to the margin of the sternum. Divide the skin and superficial fascia and expose the fibres of the great pectoral musele, the external intercostal aponeurosis, and the muscular fibres of the internal intercostal muscle. Raise the fasciculi of the latter muscle upon a director and divide them, and the vessel will be exposed. The internal mammary artery is not often tied below the fourth intercostal space.

Ligation of the Common Carotid Artery.-The point of election for the ligation of the common carotid artery is just above the ono-hyoid muscle, about three-quarters of an inch below the bifurcation of the vessel, which takes place at a point on a line with the upper border of the thyroid cartilage.

The incision for the common cartoid artery is three 
FIG. 353.

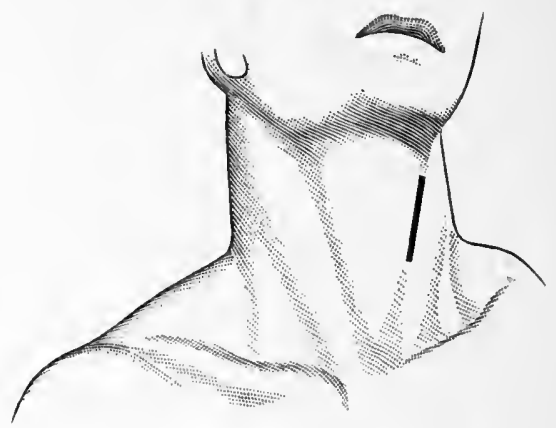

Line of incision for common carotid artery at point of election. (STIMson.) FIG. 354.

เ

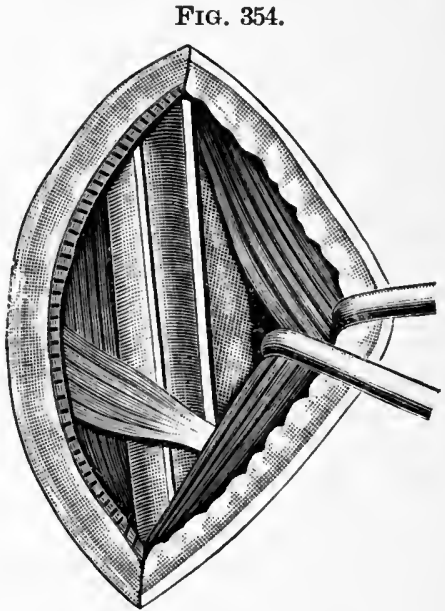

Relations of the left common carotid artery above the omo-hyoid muscle. (EsMa RCh.)

inches in length along the anterior border of the sternocleido-mastoid muscle, the centre of which corresponds with the crico-thyroid space (Fig. 353).

Divide the skin, platysma, cellular tissue, and aponeurosis, avoiding the superficial veins, and expose the anterior edge of the sterno-cleido-mastoid; seek for the inter- 
space between this muscle and the sterno-hyoid and sterno-thyroid muscles, draw the latter muscles inward, and the artery will be exposed with the jugular vein external to it; the descendens noni nerve lying upon its sheath should be displaced outward. The sheath is next picked up and opened and the artery is separated from it with a director; the artery lies internally, the internal jugular vein externally and somewhat more superficial, and the pneumogastric nerve lies between the two, and is more deeply placed. The sympathetic nerve is posterior to the vessel external to the sheath. The needle is passed from without inward, eare being taken to avoid injury of the vein and nerve (Fig. 354).

Ligation of the External Carotid Artery.-The incision for the ligation of the external carotid artery is over

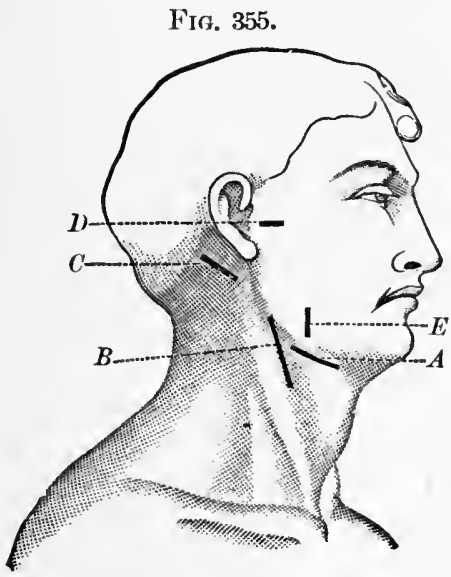

Lines of ineision for $-A$, lingual artery ; $B$, external and internal carotid arte: ries; $C$, oceipital artery ; $D$, temporal artery; $E$, facial artery. (STIMson.)

the inner edge of the sterno-cleido-mastoid muscle from the angle of the jaw to a point corresponding to the middle of the thyroid cartilage (Fig. $355, B$ ). The skin, platysma, and cellular tissue being divided, the external 
jugular vein is drawn aside when encountered ; the deep fascia being opened, the facial and lingual veins will be exposed, which should be drawn to one side; the artery is next exposed, covered by the hypoglossal nerve and the stylo-hyoid and digastric muscles. The vessel should next be isolated from the internal carotid artery and internal jugular vein, both of which lie along its outer side. The needle should be passed from without inward.

Ligation of the Internal Carotid Artery.-The incision is the same as for the external carotid artery (Fig. $355, B)$; the vessel is external to the external carotid artery, and in passing the needle the point should be directed away from the internal jugular vein-that is, from without inward.

Ligation of the Superior Thyroid Artery.-The incision is about three inches in length along the anterior border of the sterno-cleido-mastoid muscle, starting a little lower down than that for the external carotid artery. The skin, superficial fascia, platysma, and deep fascia being divided, the cellular tissue in the sulcus between the upper portion of the larynx and the great vessels of the neck should be broken up with the director and the vessel exposed. The needle should be passed around the vessel from above downward.

Ligation of the Lingual Artery.-The incision is a curved one two inches long, its concavity directed upward from the anterior edge of the sterno-cleido-mastoid muscle, half an inch above the great horn of the hyoid bone, to a point one inch within the median line of the neck (Fig. $355, A)$. Divide the skin and platysma, displacing the superficial veius, and open the deep fascia, when the submaxillary gland will be exposed; this is displaced upward with the handle of the knife, when the tendon of the digastric muscle attached to the hyoid bone, and the hypoglossal nerve will be exposed ; next divide the fibres of the hyoglossus muscle midway between the hypoglossal nerve and the hyoid bone, and the lingual artery will be exposed (Fig. 356). The needle should be passed around the 
vessel from above downward, in order to avoid the nerve. FIG. 356.

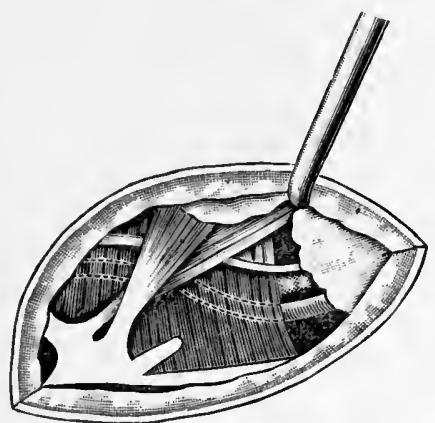

Relations of the lingual artery. (Esмarch.)

Ligation of the Facial Artery.-The facial artery passes over the inferior maxilla just in front of the anterior edge of the masseter musele, and is aceompanied by the facial vein, which lies nearer to the muscle.

The incision is either a horizontal one along the lower border of the maxilla or a vertical one an inch in length (Fig. 355, $E$ ). The skin, subeutaneons tissue, and fascia being divided, the artery is exposed; the needle should be passed around the vessel away from the vein.

Ligation of the Occipital Artery.--The incision is two inches in length, starting from a point half an inch below and in front of the apex of the mastoid process, and earried obliquely backward, parallel to the border of this proeess (Fig. 355,C). Divide the skin and faseia and expose the insertion of the sterno-eleido-mastoid muscle, which is also divided, and the aponeurosis of the splenius is exposed; this is also opened and the digastrie groove is felt for, and when the belly of the digastrie muscle is exposed the artery is brought into view by separating the cellular tissue in the anterior angle of the wond with a director (Fig. 357).

Ligation of the Temporal Artery.-The incision is a 
transverse one, one inch in length, starting from the tragus of the ear forward over the zygomatic arch (Fig. 355, D), or a vertical one of the same length a little in front of the tragus of the ear.

Divide the skin and expose the subcutaneous cellular tissue, which in this region is very dense and fibrous. This tissue should be broken up with a director, and the artery should be found in it about a quarter of an inch in front of the ear (Fig. 358). The temporal vein accompanies the artery and lies nearer to the ear, and in some - cases the auriculo-temporal nerve is in close relation to

Fig. 357.

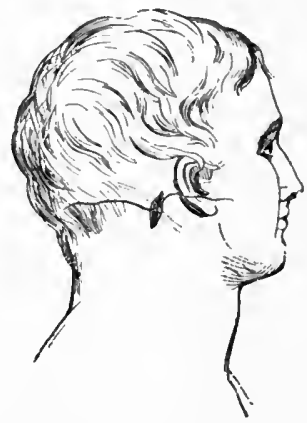

Ligation of the occipital artery. (SKEY.)
Fig. 358.

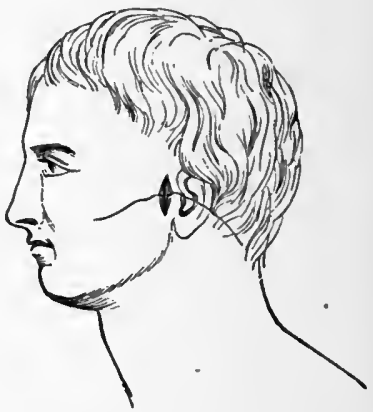

Ligation of the temporal artery. (SKEY.)

the artery. The needle should be passed from behind forward.

Ligation of the Axillary Artery.-The axillary artery extends from the middle of the clavicle to the insertion of the teres major into the humerus; the axillary vein lies upon the inner side and in front of the artery. The axillary artery is tied either in its upper portion, just below the clavicle, or at its lower portion in the axilla.

Axillary Artery below the Clavicle.-'The incision is four inches in length from the summit of the coracoid process inward a short distance below the clavicle (Fig. 351, E), or an incision three inches in length, commencing at a 
point one-half an inch from the sterno-clavicular articulation, and carried obliquely downward toward the axilla.

The skin and subeutaneous tissue having been divided, the deep fascia is exposed and opened, and the axillary artery niay be reached by following the intermuscular space between the sternal and clavicular fibres of the pectoralis major which leads upward toward the clavicle and to the pectoralis minor; or the fibres of the pectoralis major being exposed, are cut through and the costo-coracoid membrane is next torn through with a director, eare being taken to avoid injury of the cephalic vein at the outer portion of the wound; the pectoralis minor is now seen, and after separating the cellular tissue with a direetor the axillary vein is seen crossing from the upper edge of the muscle to the clavicle; the vein almost conpletely covers the artery, which is exposed by drawing the vein inward. The needle is passed around the artery from within outward.

Axillary Artery in the Axilla.-The incision is two and a half inches long, started at the upper part of the axilla

FIG. 359.

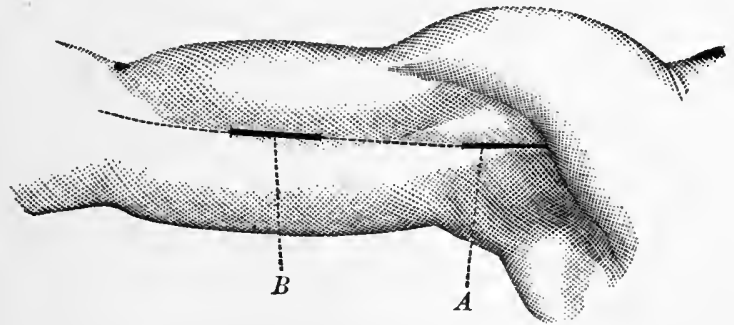

$A$. Incision for axillary artery in axilla. B. Incision for brachial artery. (STIMSON.)

and carried down the arm at the edge of the coracobrachialis muscle (Fig. 359, A). The skin only is divided in the first incision. The deep fascia is then picked up and divided upon a director. As soon as the fibres of the inner border of the coraco-brachialis muscle are exposed and held aside by a retractor, the operator will see the 
median nerve, the musculo-s ataneous nerve, and the axillary artery. To the inner side of the artery are the axil-

FIG. 360.

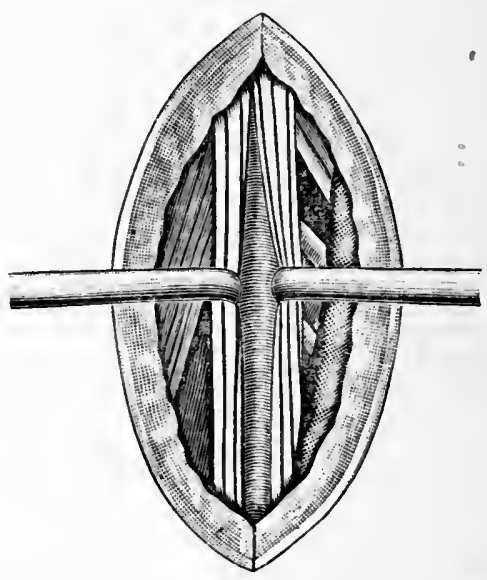

Relations of right axillary artery in axilla. (EsMarCH.)

FIg. 361.

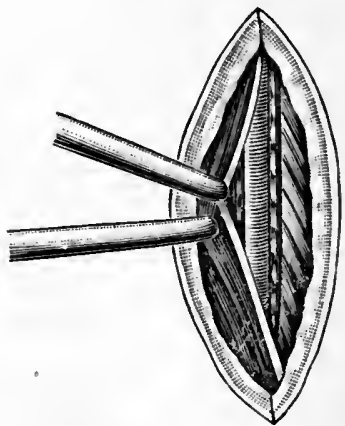

Relations of right brachial artery at middle of arm. (EsMarcH.)

lary vein, ulnar and internal cutaneous nerves (Fig. 360). The needle should be passed around the artery from the vein toward the coraco-brachialis muscle. 
Ligation of the Brachial Artery.-The incision is three inches long at the middle of the arm, on a line corresponding to the inner edge of the biceps muscle (Fig. $359, B)$. The skin and cellular tissue having been divided, care being taken not to injure the basilic vein, which should be displaced posteriorly, the deep fascia is next cut through and the fibres of the biceps muscle are exposed (Fig. 361); this muscle should be drawn forward and the sheath of the vessels enclosing the artery, veins, and median nerve exposed; the sheath having been opened, the median nerve is pressed aside and the artery is separated from its veins, and the needle is passed from the side of the nerve around the vessel. In ligating the brachial artery the occasional high division of the vessel must be borne in mind.

Brachial Artery at Bend of the Elbow.-The incision is two inches in length, along the inner border of the tendon

FIG. 362.

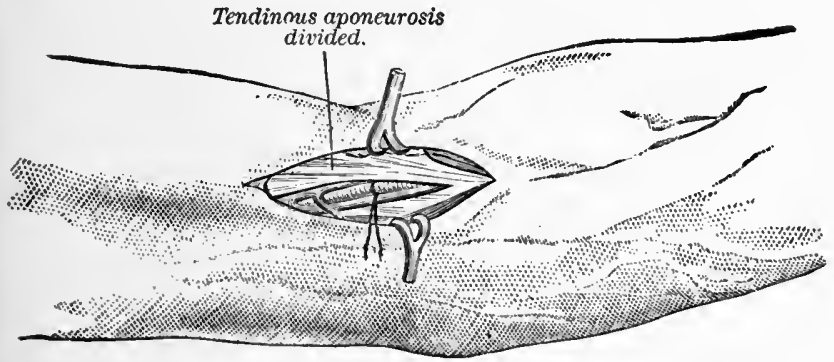

Ligation of the brachial artery at the bend of the elbow. (BRYANT.)

of the biceps muscle. Divide the skin, superficial fascia, and the bicipital aponeurosis, under which the artery will be exposed, resting upon the brachialis anticus muscle (Fig. 362). The median nerve is to the inner side and some distance from the artery. The needle should be passed around the vessel, after isolating the veins, from within outward.

Ligation of the Radial Artery.-The radial artery extends in a straight line from a point half an inch below 
the centre of the fold of the elbow to the inner side of the styloid process of the radius.

The radial artery may be tied at its upper, middle, or lower third, or at the root of the thumb.

FIG. 363.

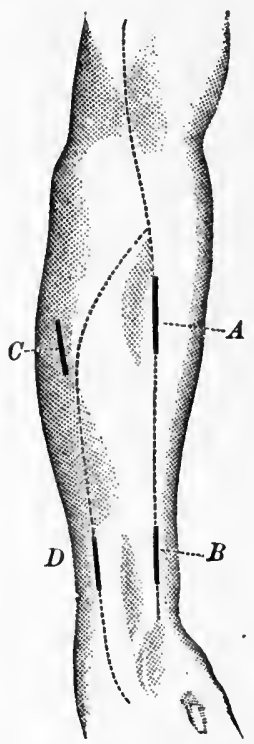

Line of incision for $-A$. Radial artery in upper third. $B$. Radial artery in lower third. $C$. Ulnar artery in upper third. $D$. Ulnar artery in lower third. Relations of right radial artery above (STIMSON.)
FIG. 364.

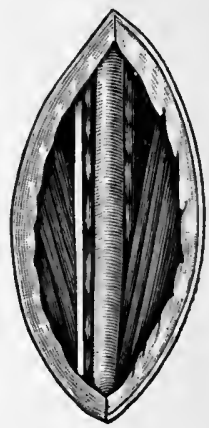

Relations of right radial artery in the upper

FIG. 365.

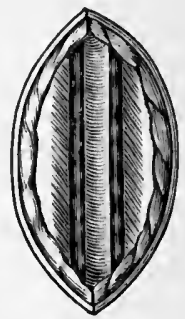

the wrist. (ESMARCH.) third of the forearm. (ESMARCK.)

Radial Artery in the Upper Third of the Forearm.-The incision for the radial artery at its upper third is two and a half inches in length on a line drawn from the middle of the bend of the elbow to the ulnar side of the styloid process of the radius; the incision should begin one and a half inches below the bend of the elbow (Fig. 363, A). Divide the skin and superficial fascia, avoiding the super- 
ficial veins. When the deep fascia is exposel, find the edge of the supinator longus muscle and divide the aponeurosis along its ulnar side, and expose the fibres of the pronator radii teres muscle. The vessel lies in the interspace between these muscles surrounded by adipose tissue, and upon being exposed the veins should be isolated and the needle passed from without inward. The radial nerve lies so far external to the artery that it is not often exposed in the operation (Fig. 364).

Radial Artery in the Middle Third of the Forearm.-The incision is two inches in length, following the same line as that for the upper third of the artery. After dividing the skin, superficial and deep fascia, the artery is found in the interspace between the flexor carpi radialis on the inner side and the supinator longus on the outer side; the radial nerve at this part of the arm is in close relation with the vessel to the radial side, and the needle should be passed around the artery from without inward.

Radial Artery in the Lower Third of the Forearm.-The incision is two inches in length, following the same line (Fig. $363, B$ ), ending one inch above the wrist. The skin, superficial and deep fascia being divided, the artery will be found between the tendon of the flexor earpi radialis on the inner side and the tendon of the supinator longus on the outer side (Fig. 365). The veins being separated, the needle may be passed in either direction.

Radial Artery at the Root of the Thumb. - The radial artery may also be tied at the root of the thumb. The incision is one inch in length between the tendons of the extensor ossis metacarpi pollicis and extensor primi internodii pollicis on the outer side, and the tendon of the extensor secundi internodii pollicis on the inner side. The skin and superficial fascia being divided and the radial vein being displaced, the deep fascia is opened and the artery is exposed at the bottom of the wound; the needle may be passed in either direction.

Ligation of the Ulnar Artery.-The ulnar artery is tied at the junction of the upper and middle thirds of the forearm and at the lower third. 
Ulnar Artery at the Junction of the Upper and Middle Thirds of the Forearm.-The incision is three inches in length, starting four inches below the internal condyle of the humerus on a line passing from the internal condyle of the humerus to the outer border of the pisiform bone (Fig. 363, $C$ ). Divide the skin and superficial fascia, and when the deep fascia has been exposed and the interspace between the flexor carpi ulnaris and the flexor sublimis digitorum appears, enter this interspace and raise the flexor sublimis digitorum and work transversely across

FIG. 366.

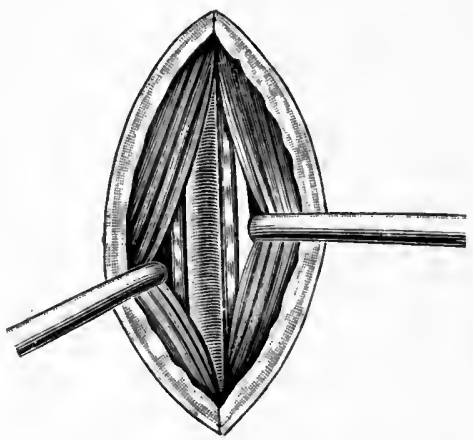

Relations of the right ulnar artery at upper third of the forearm. (EsMA RCH.) the arm. The artery will be found resting upon the deep flexor, with theulnar

FIG. 367.

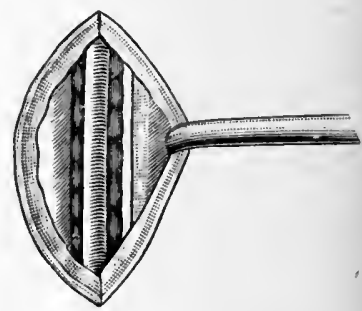

Relations of the right ulnar artery above the wrist. (ESMARCH.)

nerve to the ulnar side. The needle should be passed from the nerve around the artery (Fig. 366).

Ulnar Artery in the Lower Third of the Forearm.-The incision is two inches in length, a little to the radial side of the tendon of the flexor carpi ulnaris, which is attached to the pisiform bone, ending an inch above the wrist (Fig. $363, D)$. Divide the skin and superficial fascia and open the deep fascia; the artery will be exposed with its accompanying veins, between the tendons of the flexor carpi ulnaris and flexor sublimis digitorum, the ulnar nerve being to the ulnar side of the vessel. The needle should be passed from within outward to avoid the nerve (Fig. 367). 
Ligation of the Interosseous Artery.-The incision is similar to that employed in the ligation of the nlnar artery in its upper third.

Ligation of the Abdominal Aorta.-The incision is in the linea alba from a point three inches above the umbilicus to a point three inches below it. The superficial structures being divided, the peritoneum is opened upon a director, and the intestines are pressed aside and the aorta is exposed, covered by peritoneum, with the filaments of the sympathetic nerve resting upon it and the vena cava to the right side. Tear through the peritoneum and pass the needle from right to left around the vessel. After tying the ligature the ends should be cut short and the external wound should be closed as in the ordinary laparotomy wound.

The vessel may also be exposed by an incision along the anterior border of the quadratus lumborum muscle, from the last rib to the crest of the ilium. The skin, lumbar muscles, and fascia transversalis being divided, the wound is held open with blunt hooks, so that the retroperitoneal space is exposed and the aorta brought into view. The vessel being separated from the vena cava and nerves, the needle is passed around it and the ligature applied.

Ligation of the Common Iliac Artery.-The aorta divides into the two common iliac arteries on the left side of the fourth lumbar vertebra, and these arteries are usually about two inches in length, and bifurcate opposite the sacro-iliac synehondrosis to form the internal and external iliae arteries; the length of the common iliac artery, however, may vary considerably, being three or fonr inches in some cases.

The incision for ligation of the common iliac artery is four to six inches in length, beginning one-half inch above the middle of Poupart's ligament, and is carried outward, curving upward after passing the anterior superior spine of the ilium (Fig. 368, A).

Divide the skin, superficial fascia, and aponeurosis of the external oblique muscle, and then divide the fibres 
of the internal oblique and transversalis muscles upon a director and expose the transversalis fascia. This is opened at the lower part of the wound, and the finger is introduced and the peritoneum pressed back; the opening in the transversalis fascia is next enlarged, and the peritoneum is earefully drawn inward and upward with the fingers toward the inner edge of the wound. The operator next feels for the external iliac artery, and passes the finger along this until the common iliac artery

FIG. 368.

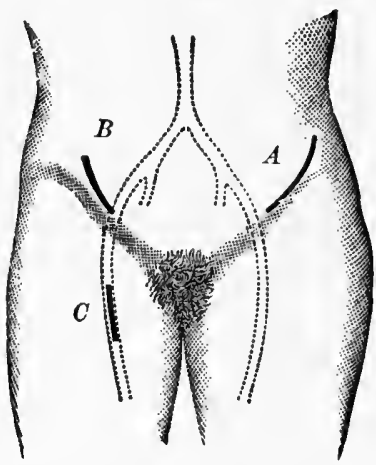

Lines of ineision for $-A$. Common iliae artery. B. External iliae artery. C. Femoral artery in Scarpa's triangle. (STIMsox.) is reached. The loose cellular tissue in which it is imbedded is next separated, and the needle is passed from within outward, to avoid the common iliac vein (Fig. 369), which on the left side lies on the inner side of the artery, and on the right side lies behind the artery. The ureter generally remains attaehed to the peritoneum ; if not, it is seen crossing the bifureation of the common iliae with the gen itoerural nerve; care should be taken to avoid injury of these structures.

Transperitoneal Method.-The common iliac artery may also be exposed and tied by an incision made over the artery through the abdominal wall opening the peritoneal eavity : the vessel being tied, the ends of the ligature are cut short, and the external wound is closed in the same manner as that resulting from exposure of the abdominal aorta by incision through the peritoneum.

Ligation of the Internal Iliac Artery.-The incision is in the same line as for the common iliac artery, but it need not be quite so long (Fig. $368, A$ ). The peritoneum being exposed, it is pushed upward and inward, and the internal iliac artery is exposed. The vessel is carefully isolated from the vein, which lies behind and 
on the inner side, and the needle is passed from within outward.

'The transperitoneal method may also be employed in exposing and ligating this vessel.

Ligation of the External Iliac Artery.-The incision is three or four inches in length, half an inch above the middle of Poupart's ligament, made at first parallel to it I

FIG. 369.

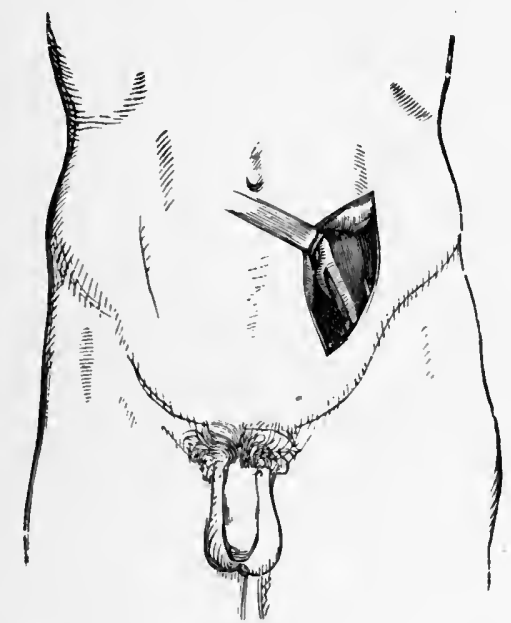

Ligation of the common iliac artery (LIstoN.)

and then eurved upward (Fig. 368, B). The tissnes of the abdominal wall being divided and the peritoneum exposed, it is pushed npward and inward in the same manner as for exposure of the eommon iliac artery. The artery lies at the inner border of the psoas muscle, the vein on its inner side and the anterior crural nerve covered by the iliae fascia on the onter side; the genito-erural nerve passes obliquely across the artery (Fig. 370). The needle should be passed from within outward.

The transperitoneal method may also be employed in ligating this vessel. 
Ligation of the Gluteal Artery. The incision is three or four inches in length, from the posterior superior spinous process of the ilium to a point midway between the tuber ischii and the great trochanter (Fig. 371, A). After division of the skin and fascia, the fibres of the gluteus maximus muscle are separated and held apart, the deep faseia is divided, and the artery should then be sought for above the pyriformis muscle at the upper border of the

FIg. 370.

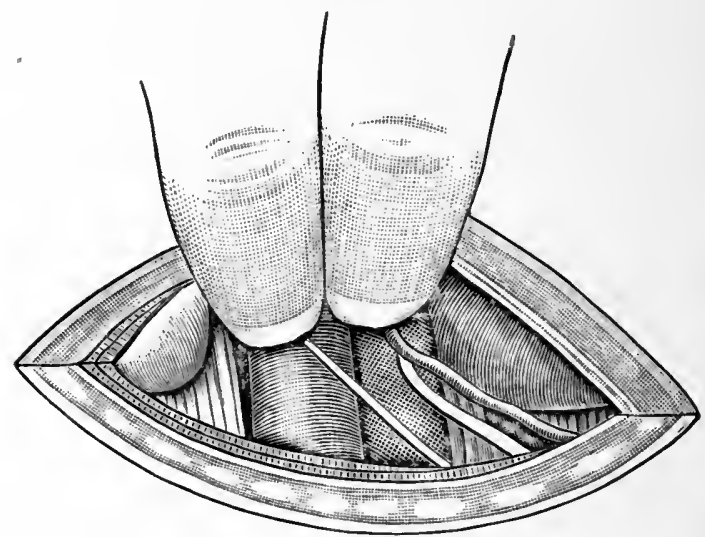

Relations of the right external iliac artery. (EsMarch.)

great sacro-sciatic notch. It is accompanied by large veins, injury to which should be avoided in exposing the artery and passing the needle.

\section{Ligation of the Sciatic and Internal Pudic Arteries.} -The incision is three or four inches in length, a little lower than that emplored for exposure of the gluteal artery (Fig. $371 ; B)$. Divide the skin, superficial fascia, and fibres of the glnteus maximus muscle and deep fascia, and search for the vessels as they leave the great sciatic notch at the lower edge of the pyriformis muscle. The internal pudic artery enters the pelvis through the lesser seiatic noteh, lying on the inner side of the sciatic artery during its pas- 
sage over the spine of the ischium. The vessels are isolated and the needle is passed so as to avoid injury of the veins.

Ligation of the Femoral Artery.-The femoral artery may be ligated just below Poupart's ligament, at the apex of Scarpa's triangle, at the middle of the thigh, or in Hunter's canal.

Femoral Artery below Poupart's Ligament.-The incision begins midway between the anterior superior spinous

FIG. 371.

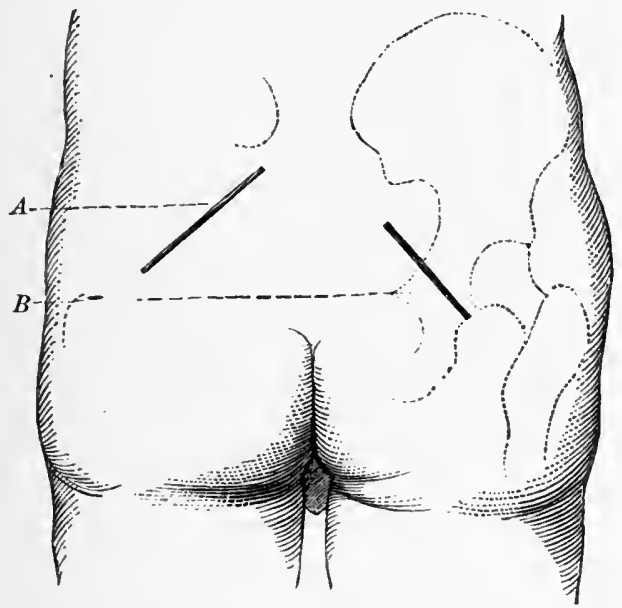

Lines for-A. Gluteal artery. $B$. Seiatic and internal pudic arteries. (STIMson.)

process of the ilinm and the symphysis pubis, one-fourth of an inch above Poupart's ligament, and extends two inches downward. Divide the skin and superficial fascia and the deep fascia so as to expose the sheath of the vessels; open this one-half an inch below Poupart's ligament and isolate the femoral artery from the femoral vein which lies to the inner side; the anterior crural nerve lies to the outer side. Pass the needle from within outward (Fig. 372).

Femoral Artery at the Apex of Scarpa's Triangle.-The incision is three inches long, the centre of which should be 
a little above the point where the sartorius muscle crosses a line drawn from the middle of Poupart's ligament to the inner condyle of the femur (Fig. 373). Divide the skin, superficial and deep fascia, avoiding the internal saphenous vein, and expose the edge of the sartorius muscle, which may be recognized by the direction of its fibres. This muscle is drawn outward and the sheath of the vessels is exposed and opened; the vein lies on the inner side and somewhat behind the artery, and the long

FIG. 372.

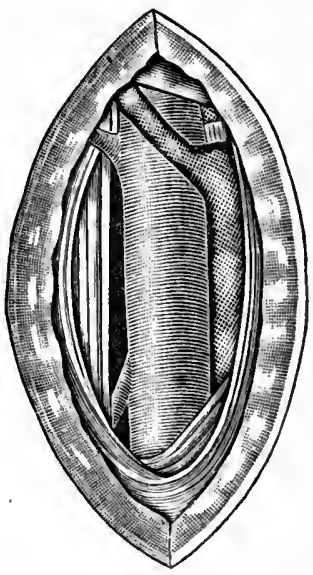

Relations of the right femoral artery below Poupart's ligament. (ESMARCH.) saphenous nerve is on the outer side (Fig. 374). Pass the needle from within outward.

Femoral Artery in the Middle of the Thigh.-The incision is in the line above mentioned, its centre being a little above the middle of the thigh. Divide the skin, superfieial and deep fascia, and expose the sartorius muscle, which is drawn outward after the leg has been flexed; the sheath of the vessels is exposed and opened; the long saphenous nerve lies upon the artery and the femoral vein lies behind the artery; the saphenous vein lies more superficially and internal to the vessel. Pass the needle from within ontward (Fig. 375).

Femoral Artery in Hunter's Canal. -The incision is three inches in length along the tendon of the adductor magnus, the centre of which is at the junction of the lower and middle thirds of the thigh (Fig. 373). Divide the skin, superficial and deep fascia, care being taken not to injure the internal saphenous vein, which should be displaced, and expose the sartorius muscle, which should be displaced downward, and expose the aponeurosis which forms the anterior wall of the vaseular canal; this should be opened upon a director, and the artery uncovered and separated 
from the vein which lies upon the outer side. The needle is passed from within outward.

Ligation of the Popliteal Artery.-The incision is

Frg. 373.

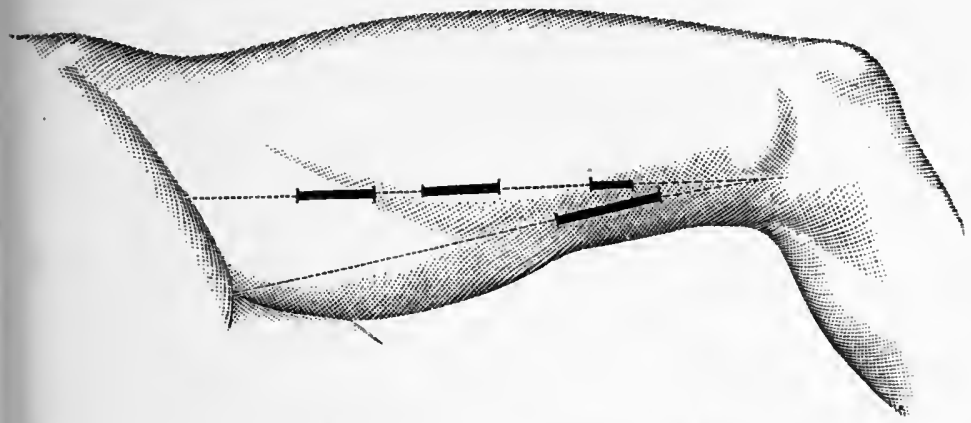

Lines of incision for the femoral artery. (STIMsoN.)

Fra. 374.

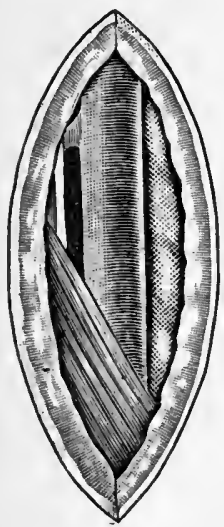

Relations of the right femoral artery at the apex of Searpa's triangle. (EsMa RCH.)
FIG. 375.

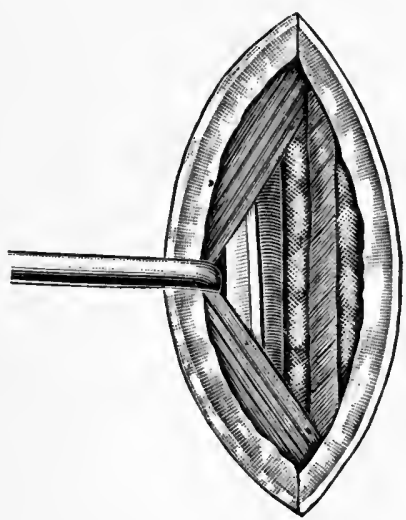

Relations of the right femoral artery in the midale of the thigh. (ESMA RCH.)

three or four inches in length, along the external border of the semi-membranosus muscle. Divide the skin and 
superficial fascia, taking care not to injure the saphenous vein, and open the deep fascia. The edges of the wound being held apart, the adipose tissue is broken up with a director, and the internal popliteal nerve will first be exposed, and the vein next-both external to the artery (Fig. 376). The artery is isolated and the needle passed from without inward.

FIG. 376.

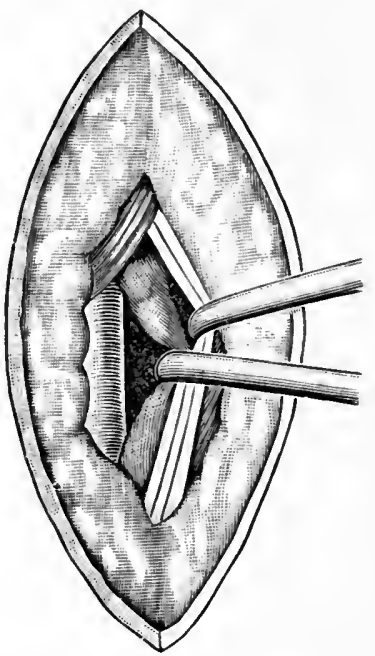

Relations of the right popliteal artery. (EsMARCH.)
Fig. 37.

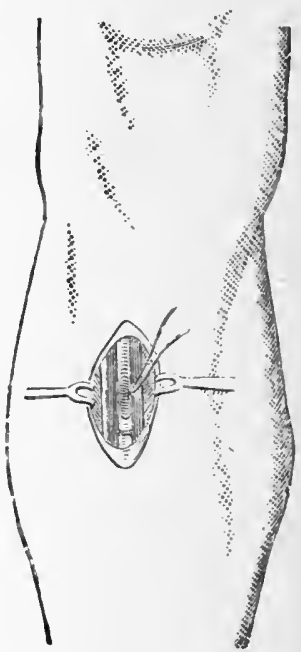

Ligation of the anterior tibial artery at its upper third. (STIMSON.)

Ligation of the Anterior Tibial Artery.-The anterior tibial artery may be tied in the upper, middle, and lower thirds of the leg; the general direction of the artery corresponds with a line drawn from the middle of the space between the head of the fibula and the tuberele of the tibia to the middle of the anterior intermalleolar space.

Anterior Tibial Artery in the Upper Third of the Leg.The incision is two and a half to three inches in length, one and one-fourth inches external to the spine of the tibia. Divide the skin and superticial fascia, and when 
the deep fascia is exposed, open it on a line corresponding to the intermuseular space between the tibialis anticus and the extensor longus digitorum muscles. Separate the museles and work down in this interspace until the artery is found with a vein on either side of it, and the anterior tibial nerve exterially (Fig. 377). The needle should be passed from without inward after isolating the veins.

Anterior Tibial Artery at its Middle Third.-The incision is three inches in length in the same line as that for the upper portion of the vessel. After dividing the skin, superficial and deep fascia, the interspace betwcen the tibialis anticus and the extensor longus digitorum muscles is opened, when a third muscle comes into view, the extensor proprius pollicis. The artery lies between the extensor proprius pollicis and the tibialis anticus muscles; and the anterior tibial nerve is to the outer side. The veins should be isolated and the needle passed from without inward.

Anterior Tibial Artery in its Lower Third.-The incision is two inches in length, beginning three inches above the ankle-joint on the line of the artery. Divide the skin, superfieial and deep fascia, and seek for the tendon of the extensor proprius pollicis muscle, the seeond tendon from the tibia. 'The artery is found in the interspace between this tendon and the tendon of the extensor longus digitorum muscle, the nerve being to the outer side. 'The veins are isolated from the artery, and the needle is passed from without inward.

Ligation of the Dorsalis Pedis Artery.-The incision is one ineh in length on a line drawn from the middle of the anterior intermalleolar space to a point midway between the extremities of the first two metatarsal bones or along the outer border of the tendon of the extensor proprius pollicis. Divide the skin, superficial and deep fascia, and the artery will be found lying next to the inner tendon of the short extensor muscle of the toes (Fig. 378). The nerve is to the outer side. After separating the veins the needle is passed from without inward.

Ligation of the Posterior Tibial Artery.-The course of the posterior tibial artery is indieated by a line drawn 
from the middle of the popliteal space to a point midway between the tendo-Achillis and the internal malleolus of the tibia.

The posterior tibial artery may be ligated in its upper, middle, and lower thirds.

Posterior Tibial Artery at its Upper Third.-The incision is three and a half inches in length, one-half inch from the inner edge of the tibia, beginning two inches from the upper edge of the bone (Fig. 379). Divide the skin and

FIG. 378.

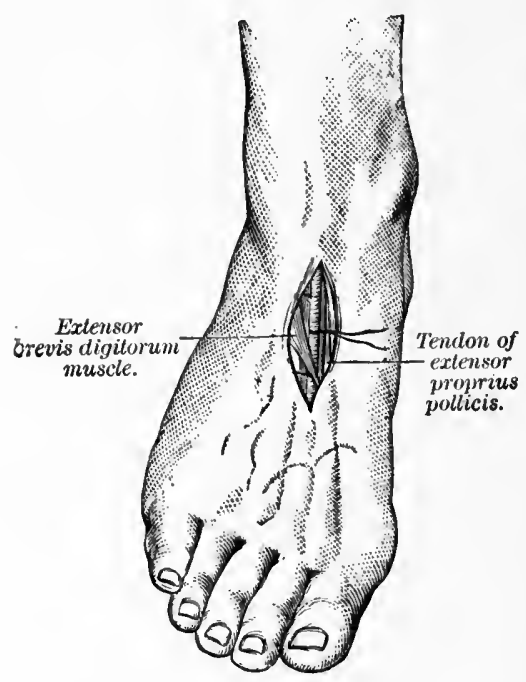

Ligation of the dorsalis pedis artery. (BRYANT.)
FIG. 379.

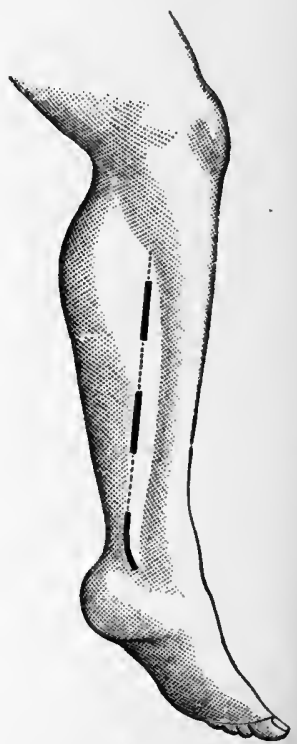

Lines of incision for the posterior tibial artery.

superficial fascia, avoiding large superficial veins; next open the deep fascia and detach the origin of the solens muscle from the tibia, and on raising it the under surface will present a white, shining sheath of tendinous material, beneath which will be seen a layer of fascia covering the 
tibialis posticus muscle. If search is made toward the middle of the leg the artery will be found covered by the intermuscular fascia, the nerve being to the outer side. The needle is passed from without inward after the veins have been separated from the artery (Fig. 380).

Fig. 380.

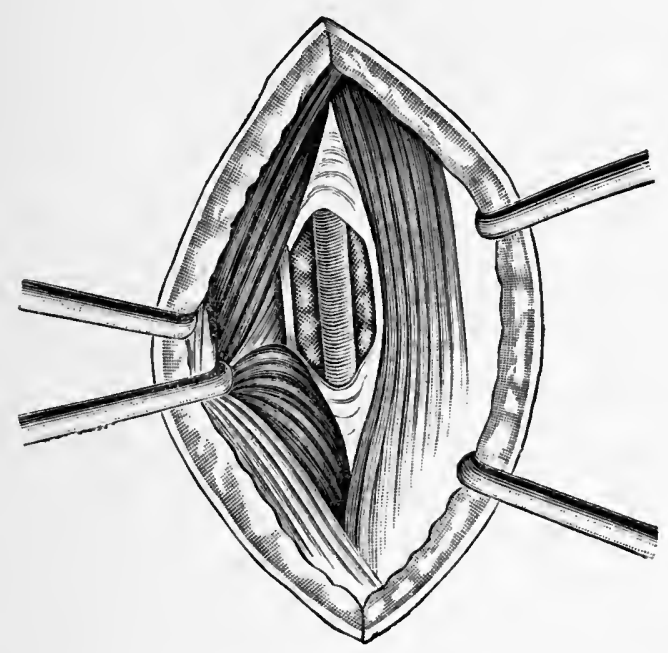

Relations of the right posterior tibial artery in its upper third. (EsMarch.)

Posterior Tibial Artery at its Middle Third.-The incision is two and a balf inches in length, parallel with the inner edge of the tibia and half an inch from its border. Divide the skin, superficial and deep fascia, and the inner edge of the soleus will be exposed; press this outward, when the artery with its veins will be exposed, also the posterior tibial nerve to the onter side. Pass the needle from without inward after separating the veins.

Posterior Tibial Artery behind the Inner Malleolus.-The incision is a curved one two inches in length, midway between the tendo-Achillis and the internal malleolus (Fig. 
379). Divide the skin and superficial fascia, then lift the deep fascia upon a director and open it freely, when the artery will be exposed, with the tendons of the tibialis posticus and flexor longus digitorum muscles on the inner side and the posterior tibial nerve and the tendon of the

\section{FIG. 381.}

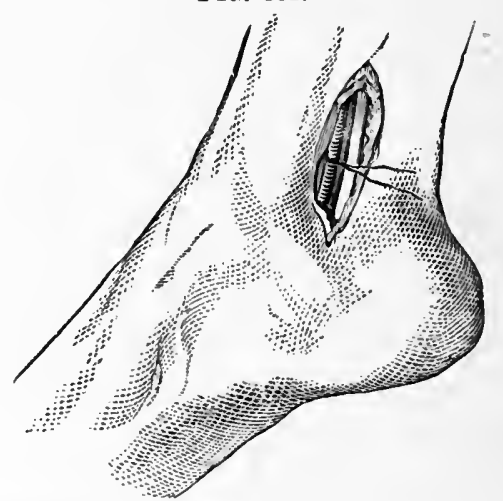

Ligation of the posterior tibial artery behind the inner malleolus. (BRYANT.)

flexor longus pollicis muscle on the outer side (Fig. 381). After separating the veins from the artery the needle should be passed from without inward. 


\section{PART VII. A M P UTATIONS.}

ThE term amputation is now generally applied to the removal of a limb, and this may be effected through the bones, when the operation is spoken of as an amputation in the continuity of the limb; or it may be removed through its joints, when it is known as an amputation in the contiguity or a disarticulation.

\section{Methods of Amputating.}

Amputations may be performed by the circular, modified circular or oval, elliptical, and transfixion methods. Teale's method by rectangular flaps is also employed.

Circular Method.-In performing an amputation by this method the incision of the skin is made at a distance below the point where the bone is to be divided. An assistant grasps the limb and draws the skin evenly and firmly toward the root of the part, and the surgeon passes the heel of the knife well into the tissues and makes a circular sweep around the limb, completing the division of the skin and cellular tissue with one motion of the knife (Fig. 382).

The second incision in an amputation by the circular method consists, after retraction of the skin, in making a circular cut through all of the tissues down to the bone (Fig. 383).

'The third step in an amputation by this method consists, 
FIG. 382.

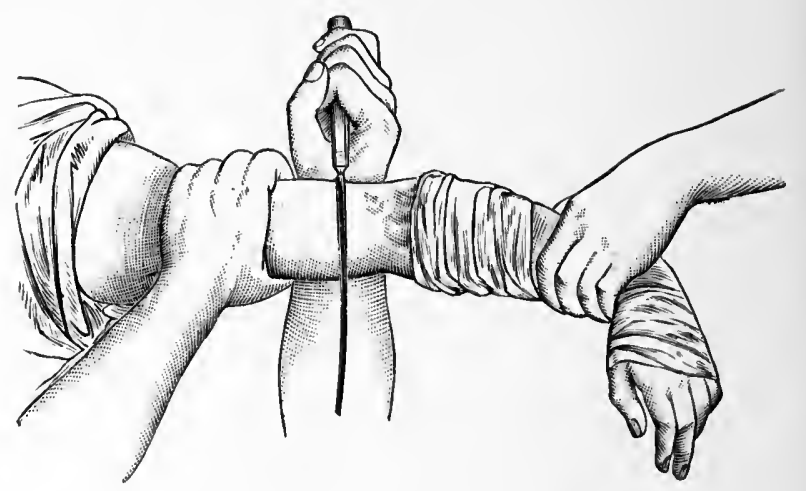

Amputation by circular method. (DruITT.)

after retracting the skin and muscles and holding them back by a retractor, in the division of the bone with a saw.

Fig. 383.

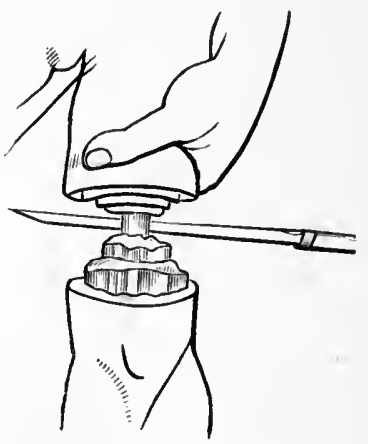

Division of muscles in circular amputation. (SмIтH.)

Transfixion Method.-This is a variety of the flap method, the flaps being cut from within outward ; they may be lateral or antero-posterior. In amputating by this method the surgeon grasps the limb and enters the point of a long knife into the tissues at the side nearest himself, and push- 
ing it across and round the bone or bones brings its point out through the skin at a point diametrically opposite its point of entrance. He then sliapes the flap by cutting downward with a rapid sawing motion, and then cuts obliquely forward until all the tissues are divided. The flap being turned up, he re-enters his knife at the same point and passes it on the other side of the bone or bones and euts the second flap in the same manner (Fig. 384). A retractor is next applied and the bone is divided with a saw.

Fig. 384.

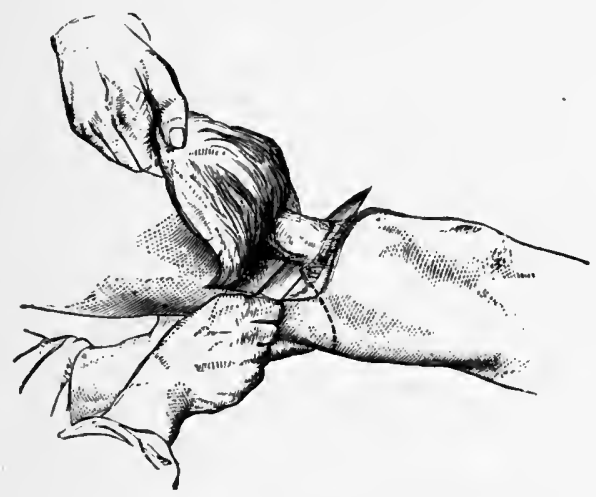

Amputation by antero-posterior flaps. (BRYANT.)

Modified Circular or Oval Method.-In this form of amputation two oval antero-posterior or lateral flaps of skin are marked out by incisions and dissected up to the point at which the museles and bone are to be divided. The museles are then divided elose to the base of the flaps by a circular sweep of the knife, and the operation is completed by sawing the bones. This form of amputation is at present widely employed, and is especially to be recommended in museular limbs (Fig. 385).

Elliptical Method.-This is a form of the oval method of amputation which is employed in amputations at the knee-joint and elbow-joint, the incision forming an ellipse, 
Fra. 385.

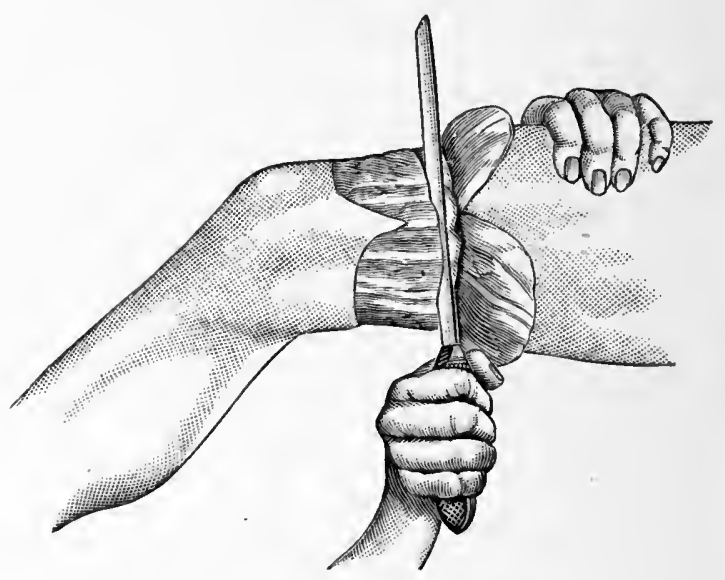

Modified circular amputation. (SKey.)

coming below the joint on the front or outside of the limb, the resulting flap being folded upon itself.

FIG. 386.
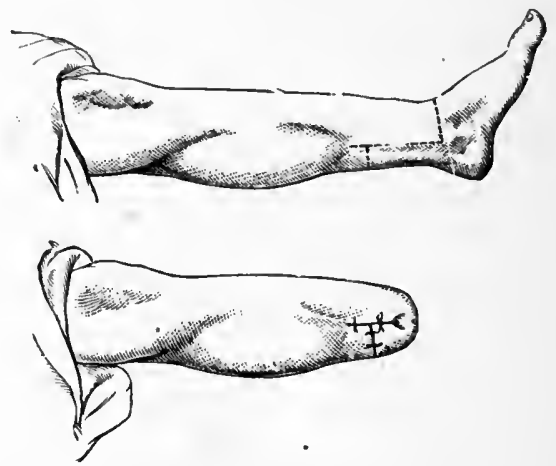

Teale's method of amputation. (BRYANT.)

Teale's Method by Rectangular Flaps.-In this method of amputation two flaps are made of unequal length ; the incisions are so planned that the shorter flap contains the main vessel or vessels. The flaps are cut of 
equal width and the length of the long flap should be onehalf of the circumference of the limb at the point where the bone is to be divided; the length of the short flap should be one-eighth of the circumference of the limb. The flaps are cut from without inward, and embrace all of the tissues of the limb down to the bone. After the flaps have been dissected up the bone is divided with a saw, and the long flap is folded over and sutured to the short flap (Fig. 386). The disadvantage of this method of amputation is that in muscular limbs it requires the bone to be divided at a higher point than would otherwise be necessary.

Bier's Osteoplastic Method.-To provide a better bearing surface for stumps, Bier has recommended an osteoplastic amputation. It may be employed in primary amputation or in cases of re-amputation. In amputating the leg

FIG. 387.

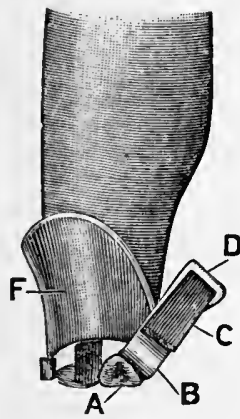

Bier's osteoplastic amputation of the leg.
Fic. 388.

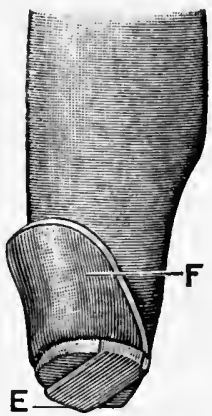

Bier's osteoplastic amputation of the leg, with osteoperiosteal flap in position.

by this method, an oval flap, composed of the skin and cellular tissue of one-half of the width of the leg, is dissected to the point where the bones are to be divided, care being taken not to injure the periosteum. A rectangular flap of the periosteum, large enough to cover the sawn surface of the tibia and fibula, is next marked out by incisions, the longitudinal incisions extending a little beyond the anterior edge of the tibia. The flap is then reflected 
about one-half a centimeter from the transverse incision, and a thin lamella of bone is next sawed in an upward direction with a fine saw, the saw being turned toward the periosteum at its upper part to complete the bone flap. This flap is turned so as to cover the sawn surface of the bones and secured by a few sutures (Figs. 387, 388). The amputation is completed by making a circular incision of the tissues on the posterior aspect of the leg and sawing the bones close to the border of the inverted bone flap.

Periosteal Flaps.-In any of the methods of amputation previously described the periosteum may be dissected up in two flaps attached to the muscles, or pushed up as a sleeve by means of a director or periosteotome before the bone is sawed. This procedure is most easily accomplished in young subjects. When these flaps are made and are brought together, the periosteum covers the cut surface of the bone, to which it soon forms adhesions.

Instruments Required for Amputations.-The instru. ments required for amputations are knives of various shapes and sizes, saws, dissecting forceps, bone-forceps, artery forceps, tenacula, hæmostatic forceps, scissors, periosteotomes, tourniquets, Esmarch's bandage and strap, retractors, ligatures, sutures, and needles.

Amputating Knives.-The knives required for amputations vary according to the method of amputation and the

FIG. 389.

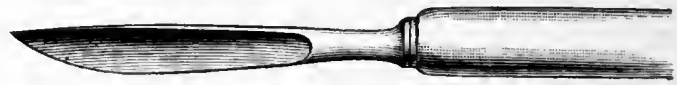

Scalpel.

part to be amputated. In certain amputations a scalpel (Fig. 389) or straight bistoury (Fig. 390) may be used, while in other cases the employment of amputating knives of various sizes will be found more satisfactory. For amputations of the thigh a knife with a blade of eight or nine inches is generally employed, and for smaller limbs a knife with a blade of six or seven inches in length ; doubleedged catlins are employed in amputations of the leg and 
forearm, to divide the interosseous tissues before applying the saw. The amputating knives now employed are con-

FIG. 390.

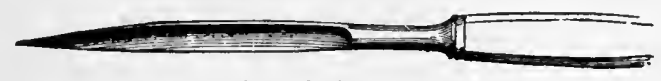

Straight bistoury.

structed with solid metal handles, so that they may be rendered thoroughly aseptic by immersion in boiling water before being used (Fig. 391).

FIa. 391.

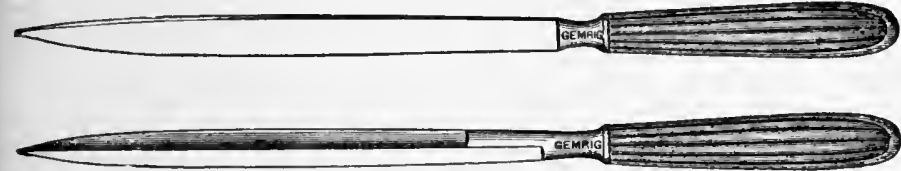

Amputating knife and catlin.

Amputating Saws.-Several kinds of amputating saws are in general use; one with a blade ten inches long by two and a half inches wide, with a heavy back to give it additional firmness, is a very good variety of saw (Fig. 392). For amputations about the foot or hand a narrow

Fra. 392.

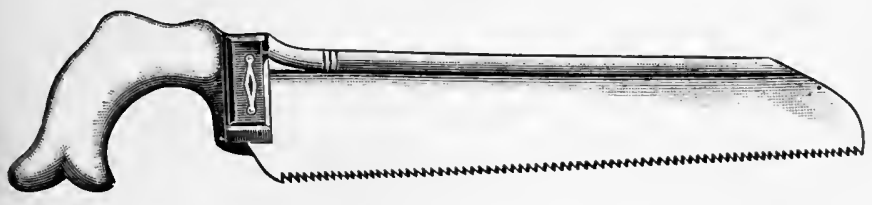

Amputating saw.

saw with a movable back will be found very convenient (Fig. 393). A bow saw with a metallic handle and a reversible blade is a very useful variety of saw, as it can be used either in amputations or in excisions, and, being con- 
FIG. 393.

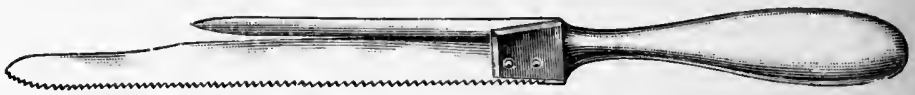

Small amputating saw.

structed entirely of metal, it can be easily rendered aseptic (Fig. 394).

FIG. 394.
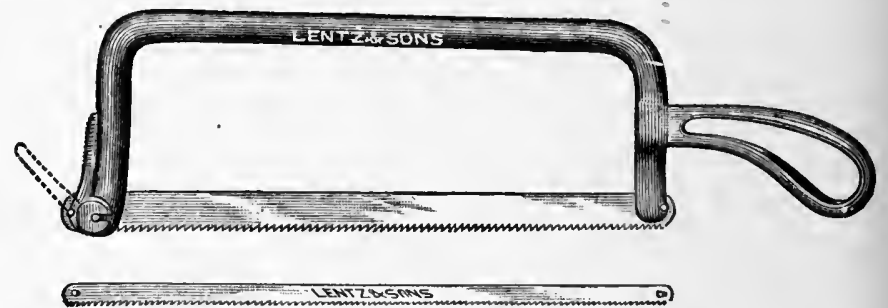

Amputating saw with reversible blade.

Bone-forceps, or Cutting Pliers.-These instruments are used in smoothing off any rough edges of bone left after the use of the saw, or for the division of the small bones in amputations of the fingers and toes. The forceps should be from ten to twelve inches in length, with blades from one to one and a half inches in length (Fig. 395).

Fíg. 395.

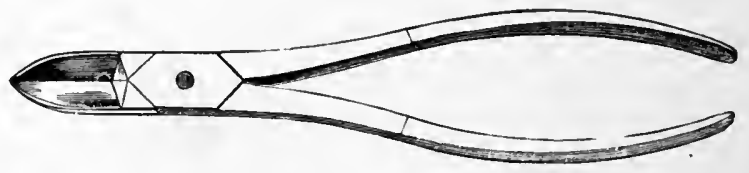

Bone-forceps, or cutting pliers.

Periosteotome.-The periosteotome, or raspatory, is employed for dissecting up a flap of periosteum, which, after sawing the bone, is drawn down over the sawed end of the bone (Fig. 396).

Artery Forceps and Tenacula.-These instruments are used for taking up the vessels, and one of the best forms 
FIG. 396.

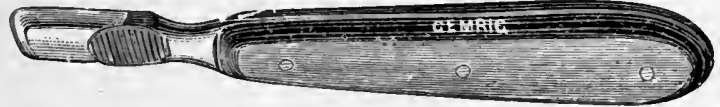

Periosteotome.

of artery forceps is that known as the double-spring artery forceps (Fig. 222). Tenacula are also employed for the same purpose (Fig. 223). Hcrmostatic forceps will also be found most useful in cases of amputation, for the rapid control of hemorrhage from small vessels after the tourniquet has been removed, the vessels being secured by torsion or by ligatures before the hæmostatic forceps are removed.

Retractors. - These consist of pieces of sterilized muslin six or eight inches in width, one end of which is split

FIG. 397.

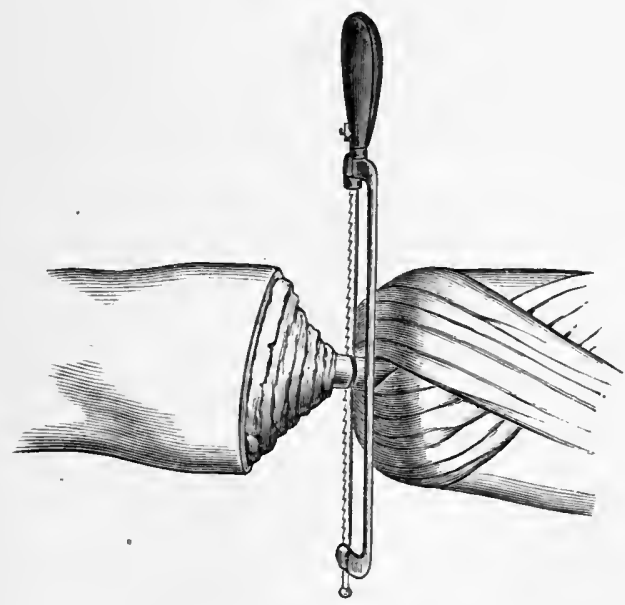

Retractor applied. (EsmaRch.)

into two or three tails; the former variety of retractor is employed where one bone is divided, as in amputations 
of the arm and thigh ( $\mathrm{Fig} .397$ ), and the latter in cases where two bones are divided, as in amputations of the forearm and leg.

Ligatures.--The best material to employ for the ligature of vessels is plain or ehromicized eatgut or sterilized silk, the preparation of which has been described (page 138).

Sutures.-The materials employed for sutures in cases of amputation may be silkworm-gut, catgut, silk, or silver wire; deep or buried sutures of eatgut, bringing together the edges of the periosteal flaps, muscles, and fascia, are often employed with advantage in amputations (Fig. 398), the skin flaps being brought together with interrupted or

FIG. 398.

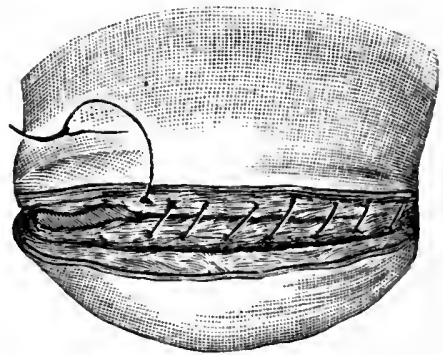

Deep or buried sutures of musclas. (EsMarch.).
FIG. 399.

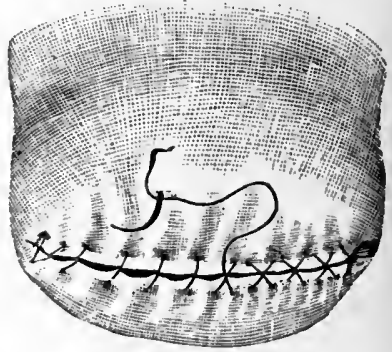

Sutures of the skin.

(ESMARCI.)

eontinnous sutures of silk, eatgut, silkworm-gut, or silver wire (Fig. 399).

Tourniquets.-For the eontrol of hemorrhage during amputation the Esmarch apparatus (Fig. 221) or Petit's tourniquet (Fig. 215) is employed ; or the employment of both at the same time will often be found most satisfactory. The Esmareh bandage and tube being applied, after removal of the bandage, the tourniquet of Petit is loosely applied at a higher point, and after the main vessels have been secured the elastic strap is removed, and the tourniquet is screwed down and controls the bleeding until the smaller vessels have been secured by ligatures. Wyeth's 
pins may be used in conjunction with the elastic strap in amputations at the hip-joint and shoulder-joint.

Details of an Amputation.-The following are the steps of an amputation of the lower part of the thigh:

The skin is first thoroughly cleansed by scrubbing it with turpentine, soap and water, and alcohol. It is then washed with a solution of bichloride of mercury, $1: 2000$. Provision is next made to prevent the loss of blood during the operation by the application of Esmarch's bandage and tube; the bandage being removed a tourniquet is placed over the femoral artery in Scarpa's triangle and loosely secured. The limb is again washed with bichloride solution. The instruments having been previously thoroughly sterilized, a rubber cloth covered with sterilized towels wrung out in a bichloride solution is placed under the limb. The variety of amputation having been decided upon, the flaps are cut and the muscles are divided down to the bone; the periosteum being dissected up, a two-tailed retractor is applied, and the tissues are held back by an assistant while the surgeon divides the bone with the saw. When the bone has been divided the retractor is removed. Irrigation of the surface of the wound is not necessary if the operation has been an aseptic one. Sterilized gauze pads may be employed to wipe away the blood. The femoral artery and vein are next found and secured with ligatures, and any muscular branches which can be found are also secured. The elastic strap is removed after screwing down the tourniquet; and by gradually letting up the pressure on the smaller vessels which bleed, they are picked up with artery forceps or hæmostatic forceps and secured. After all bleeding has been controlled the tourniquet is removed. If there is much oozing from the smaller vessels, irrigation with hot saline solution or sterilized water may be employed; the fluid should be as hot as the hands of the operator can comfortably stand, which will act promptly in controlling this variety of bleeding. The periosteal flaps, if they have been made, are brought together by two or three catgut sutures, and a long drainage-tube is next introduced, or two short tubes are intro- 
duced at either extremity of the wound and secured by sutures or safety-pins. Drainage may be omitted, but I consider it wise to employ it in major amputations. The muscles and tendons should next be brought together over the face of the stump by a few deep or buried sutures of eatgut, thereby making a good cushion and tending to lessen the subsequent muscular atrophy, and the skin Haps should then be brought into apposition by a number of interrupted sutures (Fig. 400). The surface of the stump is next washed with salt solution or sterilized water, and a

\section{FIG. 400.}

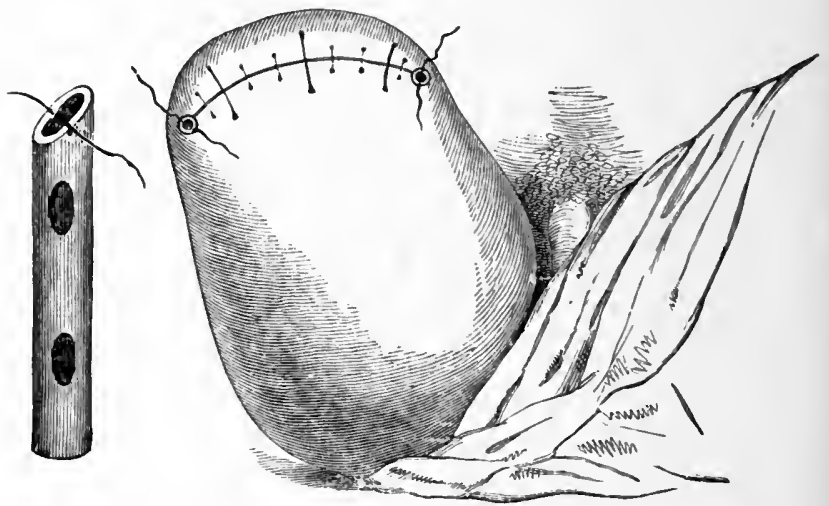

Stump showing application of sutures and drainage-tubes. (SMrт.)

number of layers of dry sterilized gauze are applied over its surface and over the gauze dressing a number of layers of sterilized cotton are placed, and the whole dressing is held in place by a recurrent bandage of the stump.

If the antiseptic method is employed, the surface of the wound is irrigated at intervals during the operation with a $1: 2000$ bichloride solution, and the stump is dressed with moist or dry biehloride gauze and biehloride cotton.

Re-dressing of Amputations.-The first dressing of au amputation, if strict antiseptic precautions have been 
observed at the time of operation, need not, as a rule, be made for a week or ten days, except in eases where the oozing is so profuse as to soak the dressings, or where consecutive hemorrhage has occurred, or the patient's condition shows that the wound is not rumning an aseptic course. The re-dressing of a stump can be accomplished without pain to the patient if the surgeon and his assistants are careful in their manipulations.

The dressings to be applied, the solutions for irrigation, and the instruments required should be prepared and at hand before the stump is exposed. The surgeon and his assistants should wash their hands carefully, and then soak them in a $1: 2000$ bichloride solution. 'The bandage retaining the dressings to the stump should be divided with bandage scissors without lifting the stump from the pillow upon which it rests. After the bandage has been divided and turned aside, the gauze dressing is next unfolded and turned down; an assistant now slips his hands under the stump and gently raises it from the dressings, and at the same time a rubber cloth covered with sterilized towels or towels which have been wrung out in a $1: 2000$ bichloride solution is slipped under the stump and the soiled dressings are removed. If the dressings are adherent at the line of incision, irrigation with saline solution or distilled water may be employed to soften them and facilitate their removal.

If the wound is aseptic and there seems to be no further indication for the use of the drainage-tubes, they may be removed. The sutures are next examined, and if the wound is firmly healed alternate sutures may be removed; if catgut or silkworm-gut sutures have been used, they need not be disturbed at this dressing, and their removal may be postponed until a subsequent dressing.

The wound should next be covered with a sterilized gauze dressing consisting of a number of layers, and over this several layers of sterilized cotton, and the dressings should be held in place by a recurrent bandage of the stump. If the antiseptic method has been employed the stump may be irrigated with a 1:2000 bichloride solution 
and a dressing of bichloride gauze and cotton used to cover the stump. The assistant should hold the stump firmly, to prevent museular spasm, and after the dressings have been secured it should be placed upon a elean pillow prepared for its reeeption. The same procedures are adopted at subsequent dressings; and if the wound has run an aseptic course, two or three dressings, at most, will be required.

\section{AMPUTATIONS OF THE HAND.}

Amputations of the Fingers.-The fingers may be amputated in the continuity of the phalanges or in their eontiguity, and, as a rule, as it is important to save as much

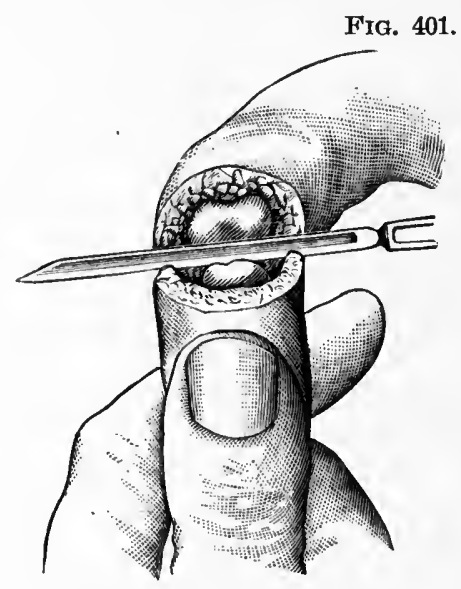

Amputation of a finger by the long palmar flap

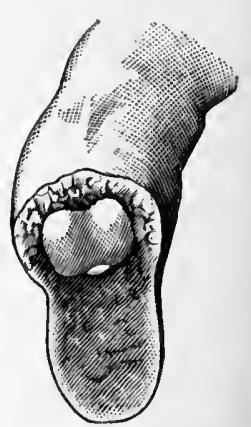

(After Esmarch.)

as possible of the finger, the former method is generally to be employed instead of disarticulation at a higher point. The incision should be so planned that the eieatrix does not oecupy the palmar surface; the larger flap should, therefore, be taken from the palmar aspeet of the finger. In amputating the phalanges of the fingers in their continuity, the eireular method (Fig. 405, B) or a short 
dorsal flap and a long palmar flap may be employed. In disarticulating a phalanx it is best to enter the joint with a narrow knife from the dorsal side, and after having carried it through the joint, to cut a long palmar flap, keeping close to the bone (Fig. 401). In locating the position of the phalangeal joints, it is well to remember that the prominence of the knuckle when the finger is flexed is formed entirely of the head of the proximal and not of the

FIG. 402.

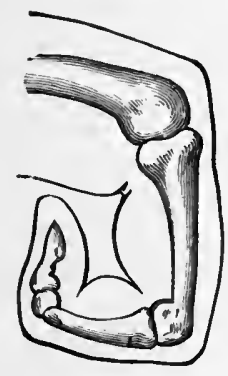

Phalanges flexed.
FIG. 403.

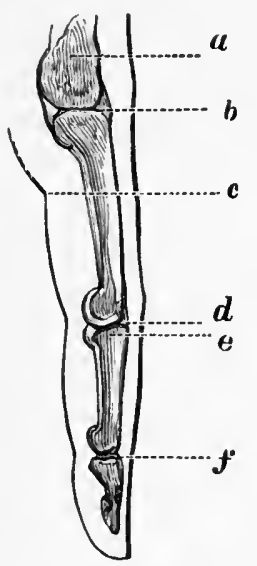

Guides to articulations of the fingers. $a$, head of metaearpal bone; $b$, metacarpo-phalangeal articulation ; $c$, relation of palmar fold to artieulation; $d, e$, interphalangeal articulation ; $f$, articulation of distal phalanx. (SMrri.)

base of the distal phalanx (Fig. 402), and also that the folds on the palmar surface of the finger do not correspond exaetly to the joints (Fig. 403).

\section{Amputation of the Finger through the Metacarpo-} phalangeal Articulation.-In this variety of amputation an incision is made from a point on the dorsal surface of the metacarpal bone a quarter of an inch above the articulation, which is earried through the interdigital web and back upon the palmar surface to a point a quarter of an inch above the flexor fold (Fig. 405, $C$ ). A similar incision be- 
ginning and ending at the same points is made upon the opposite side of the finger. The flaps are dissected back, and the lateral ligaments, tendons, and remainder of the capsule are divided (Fig. 404). The finger may also be amputated at the metacarpo-phalangeal joint by making an incision on one side and dissecting the flap back to the joint, then dividing the lateral ligament, opening the joint

Fig. 404.

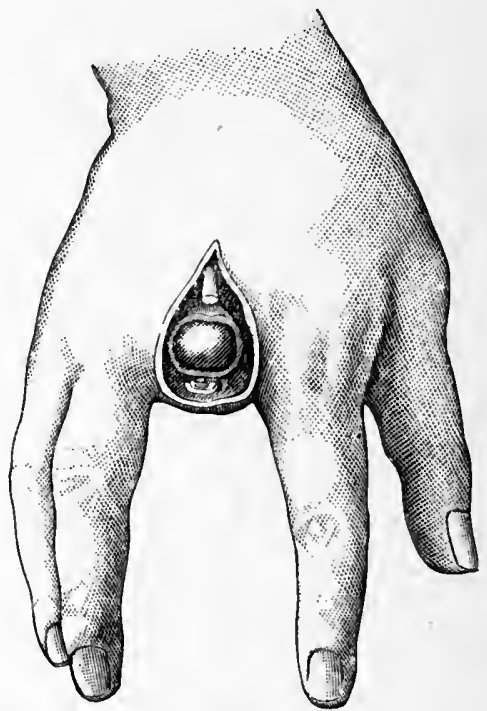

Racket-shaped incision for amputation of the finger at the metacarpophalangeal joint. (After RoTTER.)

and carrying the knife across this, dividing the tendons and lateral ligament on the other side and cutting a flap; from within outward.

Removal of the head of the metacarpal bone if desired may be accomplished by the use of cutting pliers (Fig. $406)$; but, as a rule, this procedure is not to be recommencled, for, although the deformity is lessened, the strength of the hand is diminished. 
In amputating the little and index fingers a full lateral flap may be ent on the free side, and an ineision is next carried across the palmar surface to the angle of the web, and thence back to the joint, which is opened and the disarticulation effeeted (Fig. 405, E).

Fig. 405.

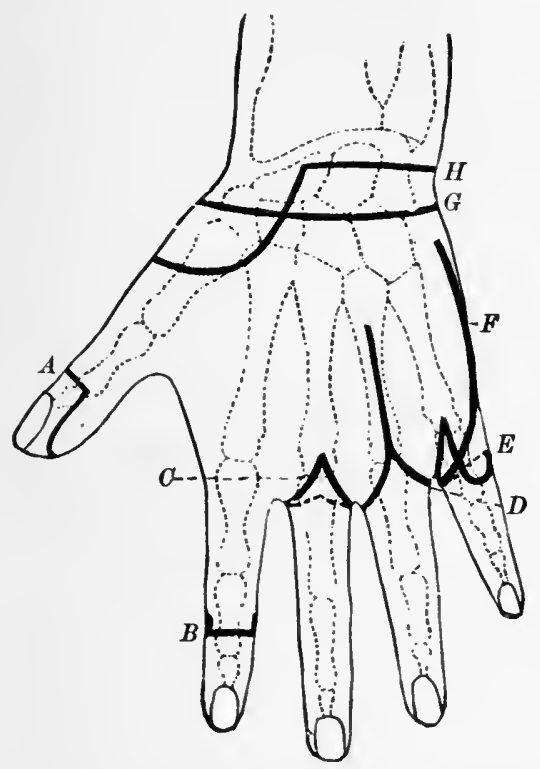

A. Disarticulation of distal phalanx; palmar fiap. B. Amputation in con. tinuity by a circular flap. C. Metacarpo-phalangeal disarticulation. $D$. Ampu* tation of metacarpal bone in continuity. $E$. Disarticulation of little finger. $F$. Disarticulation of fifth metacarpal bone. $G$. Amputation at the wrist, circular. $H$. Amputation at the wrist, lateral. (STimsoN.)

Amputations of the Metacarpal Bones.-In amputating the metacarpal bones it is advisable to leave the carpal ends of the bones to avoid opening the wrist-joint, except in the case of the first and fifth metaearpal bones, which do not eommunieate with the others and with the synovial sacs.

The incisions for the removal of the metacarpal bones 
are the same as for the removal of a finger at the metacarpo-phalangeal joint, the incision being prolonged backward as far as necessary over the dorsal surface of the bone (Fig. 405, D.) After the metacarpal bone has been bared for a sufficient distance, it is eut through with bonepliers or disarticulated, and the distal end is raised from its bed and earefully separated from the soft parts, care being taken to avoid injury of the structures of the palm of the hand.

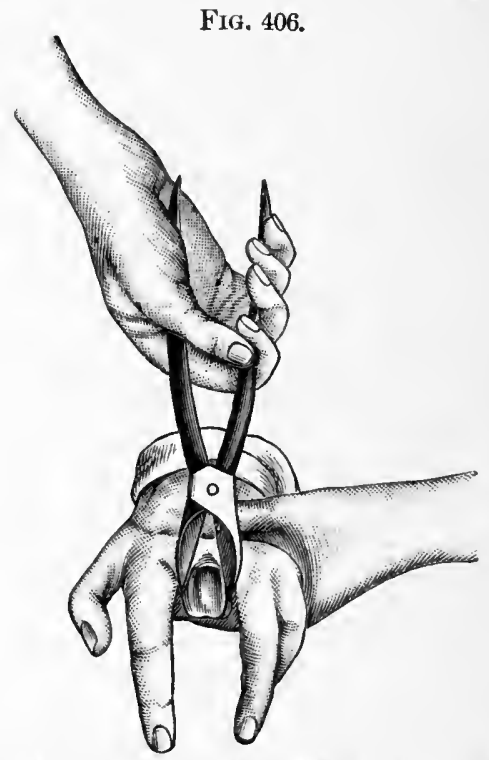

Removal of the head of a metacarpal bone. (SKEY.)

In amputating the fifth metacarpal bone, the incision should be made along the inner border of the hand and carried down to the bone between the skin and the abductor minimi digiti muscle (Fig. 407). The lower end of the incision passes over the knuckle to the web of the finger, and backward under the palmar surface to join the first incision.

Amputation of the entire thumb with its metacarpal bone is effected by making an oval flap from the palmar 
surface; in the case of the left thumb, the joint may be opened by an oblique ineision on the dorsal surface of the hand, beginning a little in front of the joint and being carried down to the web between the thumb and forefinger; the palmar flap is then made by thrusting the knife upward to its point of entrance and cutting downward and outward. In amputating the right thumb with its metacarpal bone, it is better to make the palmar flap first by transfixion, the dorsal flap being made subsequently.

FIG. 407.

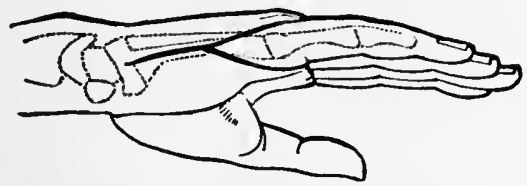

Incision for removal of the fifth metacarpal bone. (SMith.)

Amputation of the hand at the carpo-metacarpal joint is occasionally performed, or between the rows of carpal bones; but is not, as a rule, to be recommended, as the carpal bones are apt subsequently to become diseased and require removal; it is, therefore, better to amputate at the radio-carpal joint.

\section{AMPUTATIONS AT THE WRIST.}

Circular Method.-The skin of the forearm near the wrist being retracted by an assistant, a circular incision of the skin and cellular tissue is made half an inch below the point of the styloid process of the radius (Fig. 405, $G$ ). The skin and cellular tissue are next dissected back as far as the joint, which is opened and the disarticulation completed.

Antero-posterior Flap Method.-This method is also employed in amputations at the wrist-joint; an incision curved downward is made on the back of the hand from one styloid process to the other; the hand being flexed, the tendons are divided and the joint opened, and the 
palmar flap, which should extend as far as the base of the metacarpal bones, is ent from within outward (Fig. 408). Amputation at the wrist is sometimes performed by cutting a single flap from the palm, the joint being opened by a transverse incision on the back of the hand from one styloid process to the other.

FIG. 408.

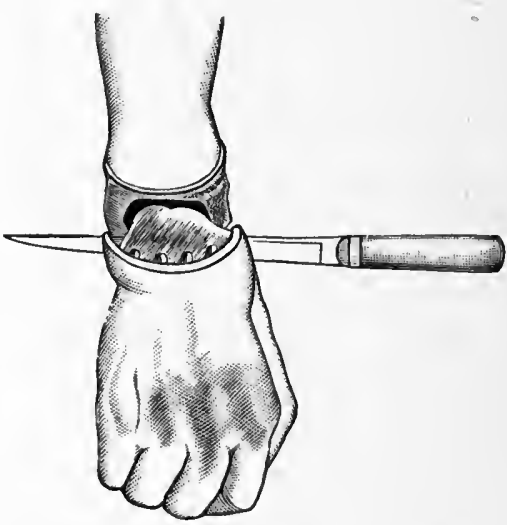

Amputation at the wrist. (ERICHSEN.)

Lateral Flap Method.-This method (Fig. 405, $H$ ) is also sometimes employed in amputation at the wrist, and may be employed with advantage in cases of laceration of the hand, in which the injury to the tissues is so great as to prevent the formation of the flaps used in the other methods of amputation.

\section{AMPUTATIONS OF THE FOREARM.}

The forearm may be amputated by the cireular or flap method, or by making rectangular flaps ('Teale's method).

Circular Method.-At the lower portion of the forearm the circular method of amputation is to be preferred. A circular incision of the skin and cellular tissue is made and a cuff is dissected up, the muscles and interosseous mem- 
brane being cut through; a three-tailed retractor is next applied and the bones are divided with a saw.

Modified Circular Method-Amputation of the forearm by the oval or mixed method, whieh eonsists in first dissecting up two antero-posterior oval flaps of skin and cellular tissue and then dividing the museles by a cireular incision, is also a satisfactory operation (Fig. 409).

FIG. 409.

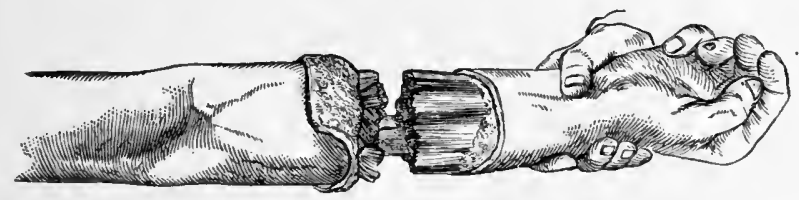

Amputation of the forearm by the modified circular method. (BRYANT.)

In amputation at the upper portion of the forearm, antero-posterior or lateral flaps, cut from without inward or by transfixion, or rectangular flaps, may be made with advantage.

\section{AMPUTATIONS AT THE ELBOW.}

The methods of amputation employed at the elbow are the anterior flap, lateral flap, cirenlar, and elliptical.

Anterior Flap Method.-A flap three inches in length, with its base parallel to and half an ineh below the condyles of the humerus, is cut either by transfixion or from without inward. The joint is next opened and the lateral ligaments divided. The olecranon is then exposed, the attachment of the triceps muscle separated, and a posterior flap cut from without inward, or from within outward, a little below the line of the condyles (Fig. 410, A).

Lateral Flap Method.-In anıutation at the elbowjoint lateral flaps may be employed, cut either from without inward or by transfixion (Fig. 410,B). A flap three inches in length is made on the outer side of the forearm, starting from a point a finger's breadth below the bend 
FIG. 411.
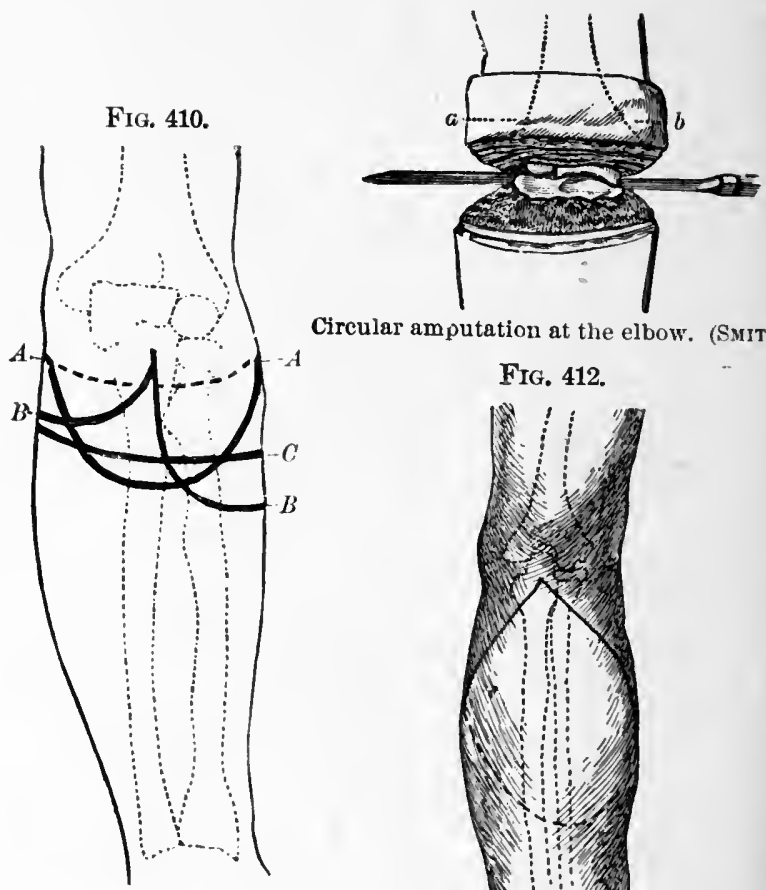

Circular amputation at the elbow. (SMrth.) FIG. 412.

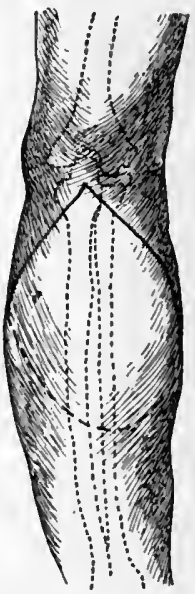

An putation at the elbow-joint. A. Anterior flap method. $B$. External flap method. C. Circular method. (STimson.)
Incision for elliptical amputation at the elbow. (After TrEvEs.)

of the elbow, by transfixion or by cutting from withont inward; a shorter internal flap is next cut in the same manner, and the joint is opened and the disarticulation effected.

Circular Method.-An incision dividing the skin and cellular tissue is made aronnd the limb three inches below the line of the condyles of the humerus (Fig. 410, $C$ ), the skin is dissected up and a circular incision made 
through the muscles, the joint is opened and the disarticulation effected (Fig. 411).

Elliptical Method.-In this method of amputating at the elbow an incision is carried from the olecranon process downward and forward to a point a little above the middle of the forearm; it is then continued across the anterior aspect of the limb, and is carried back to the olecranon process (Fig. 412). The incision includes only the skin and the cellular tissue. The flap having been dissected up for a short distance, the soft parts close to the joint are transfixed; the muscles are cut obliquely, so that an anterior flap is formed. This flap is held up, the bones are disartieulated, the attachment of the triceps tendon to the olecranon is divided, and any tissues which lave escaped division along the posterior aspect of the limb are severed. After the vessels have been secured, the flap is turned over and sutnred, and a curved cieatrix on the posterior aspect of the limb results.

\section{AMPUTATIONS OF THE ARM.}

The arm may be removed at any point below the attachment of the muscles at the axilla, by either the cireular, flap, oval, or modified eircular method.

Circular Method.-This amputation is usually employed in removing the arm in its lower third. A eireular incision of the skin and subentaneous tissue is first made, and when the euff has been dissected up, a circular division of the muscles is made; after applying the retractor the bone is sawed through (Fig. 413).

Transfixion Method.-From the central position of the bone in the arm the flap method in FIG. 413.

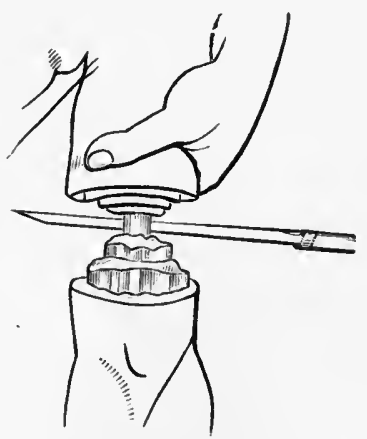

Circular amputation of the arm. (SMITH.) amputating the arm is preferred by many operators. The 
arm being grasped by the hand, the point of a mediumsized amputating-knife is thrust through the arm so as to pass over the humerus and make its exit at a corresponding point in the skin on the opposite side; a flap of sufficient length is eut from within outward. The knife is next passed behind the bone and a posterior flap is eut in the same manner (Fig. 414); the bone is next cleared of muscular tissue, the flaps are retraeted, and it is divided with a saw.

Lateral flaps may be made in this amputation instead of the antero-posterior flaps, and they may be cut from within outward in the same manner.

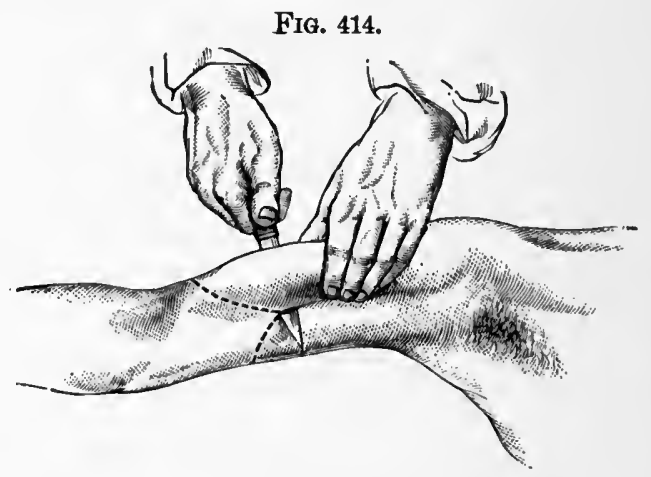

Amputation of the arm by transfixion. (BRYANT.)

Modified Circular Method.-This method of amputating the arm is also employed with advantage. Two oval flaps of skin and eellular tissue are dissected up, and the muscles divided by a circular sweep of the knife.

In high amputations of the arm there is sometimes not sufficient room to apply Esmarch's strap or a tourniquet to the arm itself to control the hemorrhage during the operation; in such cases the strap may be passed from the axilla around the outer end of the clavicle, as is done to control the bleeding during amputation at the shoulderjoint (Fig. 415), or Wyeth's pins may be employed. 
FIG. 415.

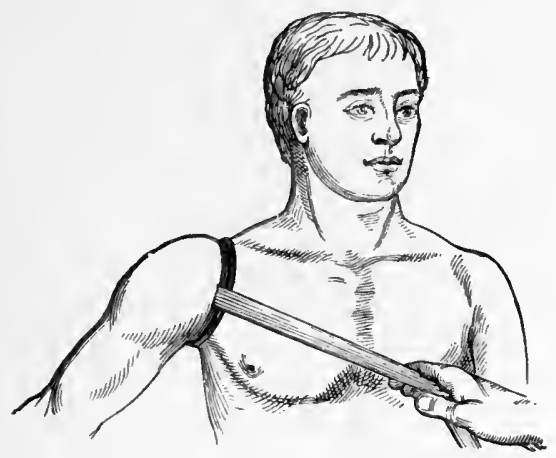

Esmarch's strap applied in high amputation of the arm. (S.ITH.)

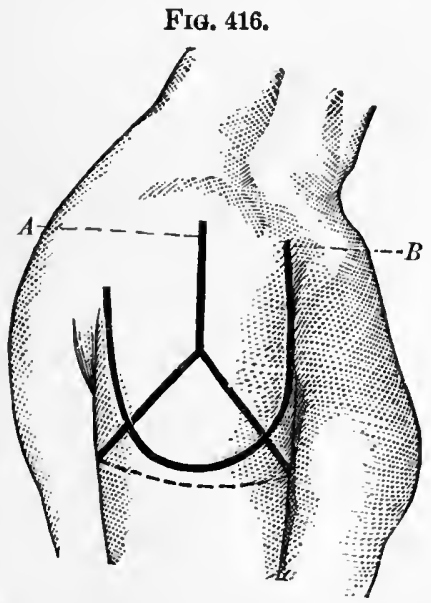

Amputation at the shoulder-joint. A. Oval, or Larrey's method. B. Double. flap, or Dupuytren's method. (STisison.)

\section{AMPOTATIONS AT THE SHOULDER-JOINT.}

Several methods of operation are employed in amputating at the shoulder-joint, sueh as Larrey's method, Lisfranc's and Dupuytren's methods, and Spence's methoo 
(Fig. 416). The control of the bleeding from the axillary artery during the operation is a matter of the first importance; it may be arrested by pressure made upon the subclavian artery, as it erosses the first rib, with the thumb, or the padded handle of a large key, or by the fingers of an assistant grasping the axillary flap and compressing the vessel after the head of the bone has been disarticulated, or by the use of an elastic strap applied around the axilla and shoulder (Fig. 415).

Wyeth's pins may also be employed with an elastic tube or strap to control bleeding during amputation at the

FIG. 417.

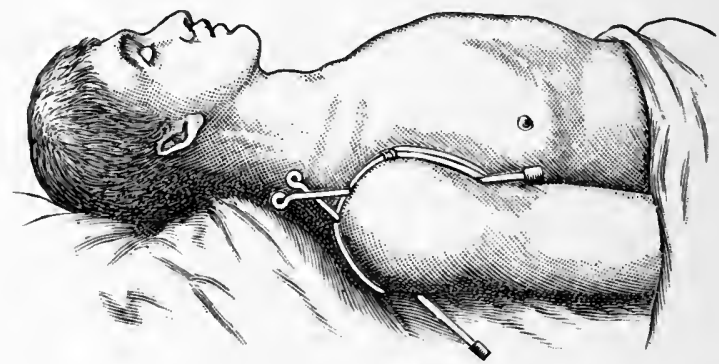

Method of applying Wyeth's pins.

shoulder-joint. The anterior pin is passed through the anterior fold of the axilla, and is brought out in front of the acromion, the posterior pin is passed through the posterior fold of the axilla and is bronght ont behind the aeromion, the rubber strap or tube is then wrapped around the shoulder behind the pins and controls the hemorrhage during the operation (Fig. 417).

Larrey's Method.-In this method of amputation the point of the knife is entered just below the acromion process, and a deep incision three inches in length is made down to the head of the bone along the axis of the arm; from the middle of this incision two others are made obliquely downward to the points where the anterior and posterior folds of the axilla end in the tissues of the arm; 
the latter incisions should be only sufficiently deep to divide the skin and superficial fascia (Fig. 416, A). The flaps are then dissected up until the head of the bone is well exposed, and, after opening the capsule and dividing the

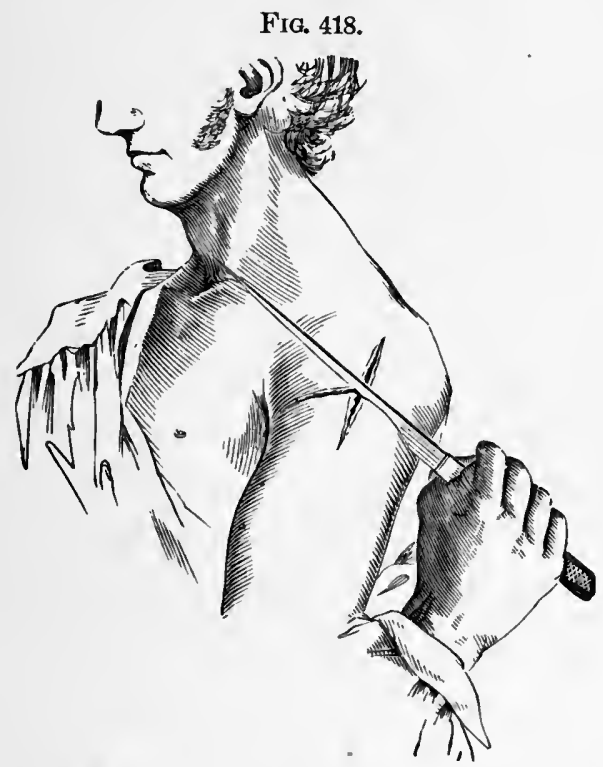

Amputation at the shoulder-joint by Larrey's method.

muscles inserted into the neck and tuberosities of the humerus, which division may be facilitated by rotating the head of the bone outward and inward, the disarticulation is effected by adducting the elbow; the knife is next passed downward behind the bone and made to cut out ward in the line of the cutaneous incisions, an assistant controlling the artery before it is divided by grasping the axillary tissues behind the knife with his fingers if Wyeth's pins have not been employed.

Dupuytren's Method.-In this method of amputation at the shoulder-joint the flaps may be cut either by transfixion or from without inward; the large flap embraces 
the greater part of the deltoid musele (Fig. 416, B), and the smaller or short flap is cut from the inside of the arm after the head of the bone has been disarticulated. When cut by transfixion, the point of the knife should be entered an ineh in front of the acromion process and pushed across the onter aspect of the head of the humerus, and brought out at the posterior fold of the axilla; the knife is made to ent downward until a large deltoid flap is formed, This flap is turned up and the head of the bone is dis. articulated; the knife being placed behind it, a short flap

FIG. 419.

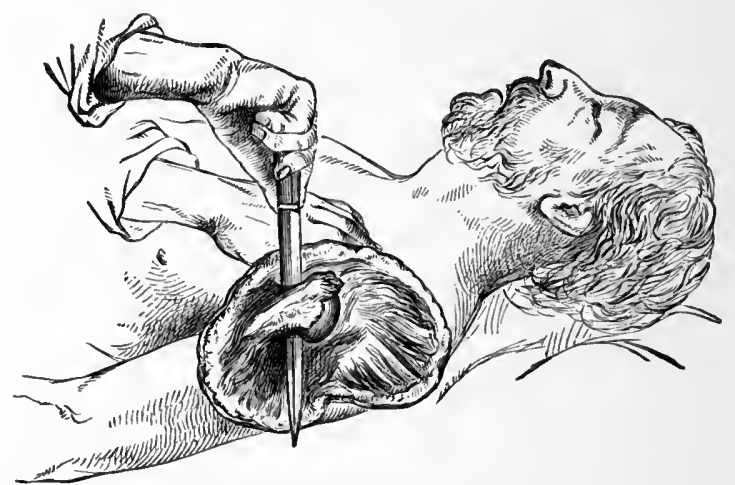

Amputation at the shoulder-joint. Dupuytren s method. (BRYANT.)

is formed, keeping close to the bone, so that the vessels are divided with the last ent of the knife (Fig. 419). An assistant should control the vessel by grasping the axillary tissues with his fingers behind the knife.

Lisfranc's Method.-In this method of amputation at the shoulder-joint the point of the knife is entered at the outer side of the coracoid process, and is carried across the outer aspeet of the head of the humerus and brought out a little below the posterior border of the aeromion process, and a long flap is cut downward. This flap is turned up and the attachments of the head of the bone are divided and it is disarticulated. The knife is again entered behind 
the bone, and a long posterior flap cut from within outward.

Spence's Method.-In this method of amputation at the shoulder-joint an incision is made down to the head of the humerus immediately in front of the coracoid process, and is continued downward through the clavicular fibres of the deltoid and the pectoralis major muscles until the attachment of the latter to the humerus is reached (Fig. 420). The incision is now earried backward to the posterior fold of the axilla. A second incision, including only the skin and cellular tissue, is next made from the anterior portion of the first incision across the inside of the arm to meet the incision on the outer part. The outer flap thus formed is turned up and the head of the bone is disarticulated. The operation is compieted by dividing the remaining tissues on the axillary aspect.

Amputation above the Shoulderjoint.-This form of amputation consists in removal of the arm with a part

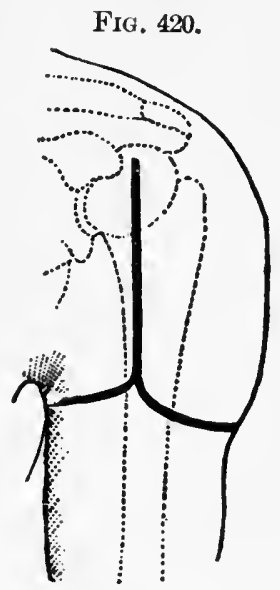

Amputation at the shoulder-joint. Spence's method. (STIMsoN.) or the whole of the scapula, and a portion or whole of the clavicle.

As this form of amputation is required in cases in which the laceration of the parts has extended beyond the shoulder-joint, or in cases of growths involving the tissues beyond the joint, no definite rule can be laid down for the ineisions; the only rule being as far as possible to make them in such a manner that the smallest amount of skin is sacrificed, so that a sufficient covering for the wound may be obtained. Treves recommends the following method: The patient should be placed on his back close to the edge of the operating-table. An incision should be made over the clavicle, extending from the inner extremity outward to a point a little beyond the acromio-clavicular articulation, which should be carried down to the bone; the clavicle 
being exposed, it should be divided in its middle third or disartieulated from the sternum, and, its onter portion being lifted up, it is disarticulated at its acromial extremity. The subelavian ressels are thus exposed, and should be tied by two ligatures, about an inch apart, and the vessels finally divided between the ligatures. The axillary plexus of nerves should next be divided. The second incision is made at the centre of the first incision, and the knife is carried directly across the anterior part of the axilla and inner border of the arm to the inferior angle of the scapula; from the outer extremity of the first incision over the elavicle a third incision should be made posteriorly, across the dorsum of the scapula to its inferior angle, joining the termination of the second incision (Fig. 421). Upon turning back the posterior flap

FIG. 421.

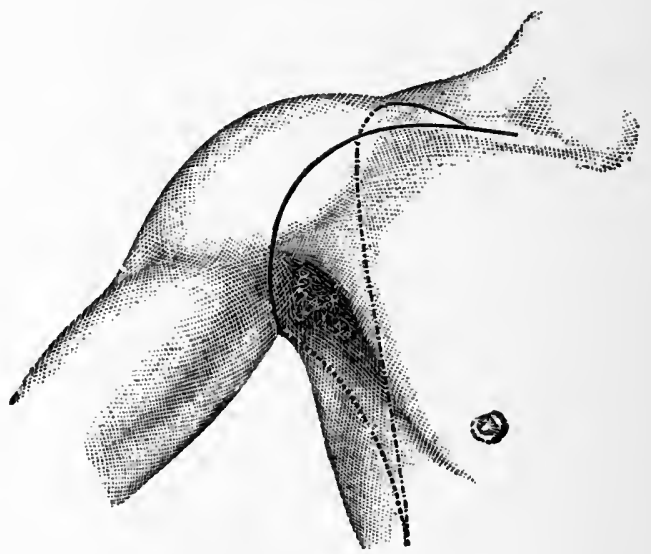

Amputation of arm, scapula, and clavicle, the dotted line representing the posterior incision. (Treves.)

thus formed and severing the connections of the scapula with the trunk and the muscular attachments which remain anteriorly, the upper extremity will be entirely freed from the trunk. 'The wound, when closed, forms an oblique line running from above downward, outward, and backward. 


\section{AMPUTATIONS OF THE FOOT.}

Amputations of the Toes.-The phalanges of the toes may be removed in the same manner as those of the fingers. It is better to amputate at the metatarso-phalangeal

FIG. 422.

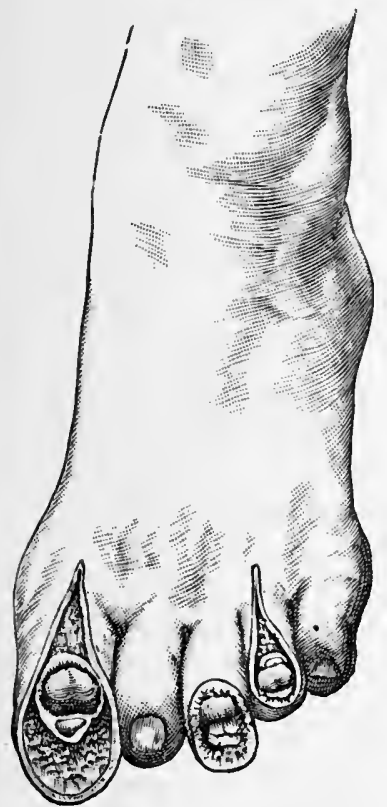

Amputation of the toes by the racket-shaped incision and flap method. (After RoTTER.)
Fig. 423.

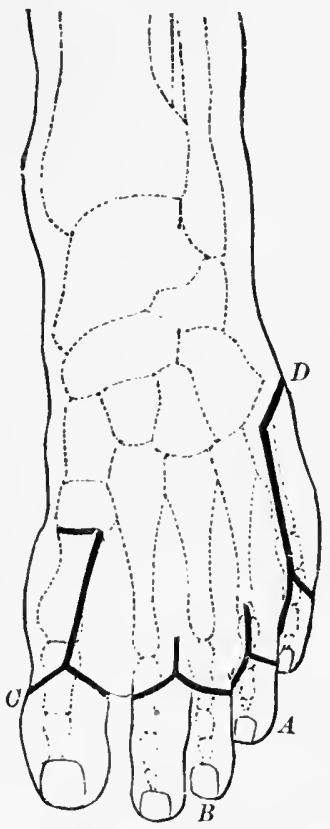

Incisions for amputation of toes and metatarsal bones. (STIMSON.)

articulations than to attempt to remove them at the joints in front of this articulation, except in the case of the great toe, as the preservation of a portion of a toe is rather a discomfort than an advantage, except in the instance mentioned. All incisions should be made so that the resulting cicatrix does not occupy the plantar surface, and it is well 
to remember that the web of the toes is considerably below the position of the metatarso-phalangeal joint.

The toes are usually remored by an incision on the dorsal surface a little above the joint, which is carried down to the bone for about an inch and then diverges into the web, and is carried under the toe and back on the other side to the point of divergence (Figs. 422, 423).

Amputation of Two Adjoining Toes.--The dorsal incision should be made in the inter-metatarsal space just above the level of the joint (Fig. 423, $B$ ) and carried down to the beginning of the web; then over the toes to the beginning of the adjoining web; then under the plantar surface of both toes in the line of the digito-plantar fold, through the web, and back to the point of divergence.

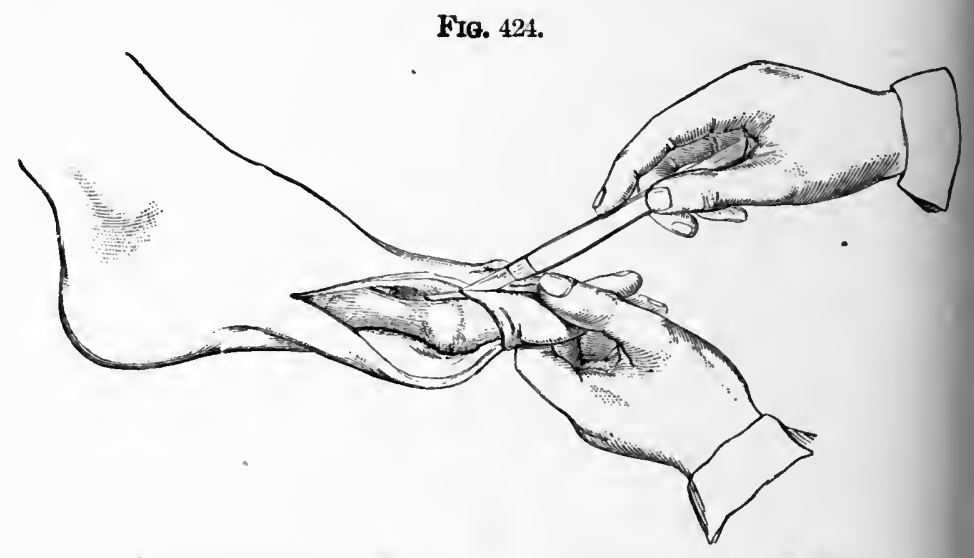

Amputation of the great toe and first metatarsal bone. (Surra.)

Amputation of the Great Toe.-This may be accomplished by means of the racket-shaped incision employed in amputation of the other toes (Fig. 422), or by means of a lateral flap. In the latter case the knife is made to enter the joint by cutting through the commissure, and the operation is completed by carrying the knife through the joint and along the outer side of the bone, forming a flap of the required size. In this amputation a snort dorsal 
flap and a long plantar flap may be employed, or a large internal flap may be used.

Amputation of the Great Toe with its Metatarsal Bone.The incision begins upon the dorsal surface of the metatarsal bone, a little below the point at which the bone is to be divided, and is carried down below the metatarsophalangeal joint, then diverges and passes under the toe, and comes back again to the point of divergence (Fig. 423, C), The bone is exposed and eut throngh with cutting foreeps, and is then lifted up and disseeted loose from the tissues (Fig. 424).

Amputation of All the Toes.-To amputate all the toes, make a dorsal incision from the head of the fifth to the head of the first metatarsal bone; the incision should be a curved one passing just in front of the joints (Fig. 425). Dissect up the flap and open the joints, dividing the lateral ligaments, and pass the knife behind the phalanges and cut a flap from the plintar surface.

\section{Amputations of the Metatarsal} Bones.--It is better in these amputations to leave the tarsal head of the metatarsal bone in place and divide the bone, or, in other words, to do an amputation in continuity to prevent opening up the tarsal articulations.

Amputation of the Little Toe and the Fifth Metatarsal Bone.-The incision for the removal of the little toe and the Incisiun for amputation fifth metatarsal bone is made over the FIfi. 425.

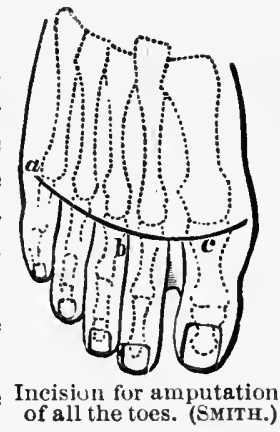
bone a little below the metatarso-tarsal articulation, and is earried down and curved around the toe (Fig. 423,D), and after the bone is exposed by dissecting back the flaps it is divided, or the joint is opened and it is dissected out.

Amputation through all the Metatarsal Bones.-In performing this amputation an incision is made across the dorsum of the foot, and a short dorsal flap is dissected up ; tine metatarsal bones are next divided with a saw and a 
long plantar flap is cut from within outward by entering the knife behind the ends of the bones.

Tarso-metatarsal Amputations.-In all amputations of the foot involving the tarsus the surgeon should be thoroughly familiar with the anatorny of the foot and the surgical landmarks of the different articulations. I shall

FIG. 426.

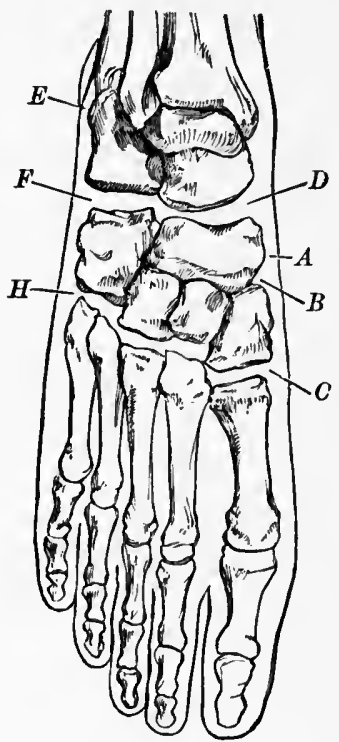

Surgical guides to the foot as expressed by anatomy. (BRYANT.)
FIG. 427.

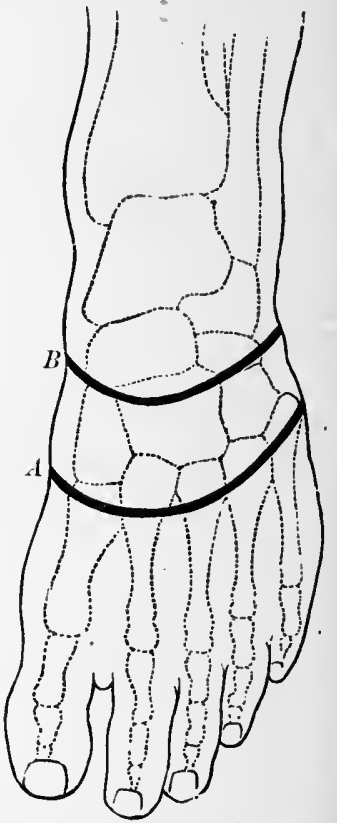

Incisions for $-A$. Lisfranc's amputation. B. Chopart's amputation. (STimsox.)

refer to those laid down by Mr. Bryant, which are as follows:

"On the inner side of the foot, not far from the inner malleolus, the tubercle of the scaphoid (Fig. $426, A$ ) is to be felt as a marked prominenee; about one-half an inch 
in front of this will be found the articulation with the cuneiform bone $(B)$, and one inch in front of this the joint which the surgeon will have to open in Lisfrane's or Hey's operation $(C)$; just above the tubercle of the scaphoid will be found the articulation with the astragalus, the line of Chopart's amputation $(D)$. On the outer side of the foot, one inch below the exterual malleolus, a sharply defined projection will always be felt, which is the peroneal tubercle $(E)$; one-half an inch in front of this will be

FIG. 428.

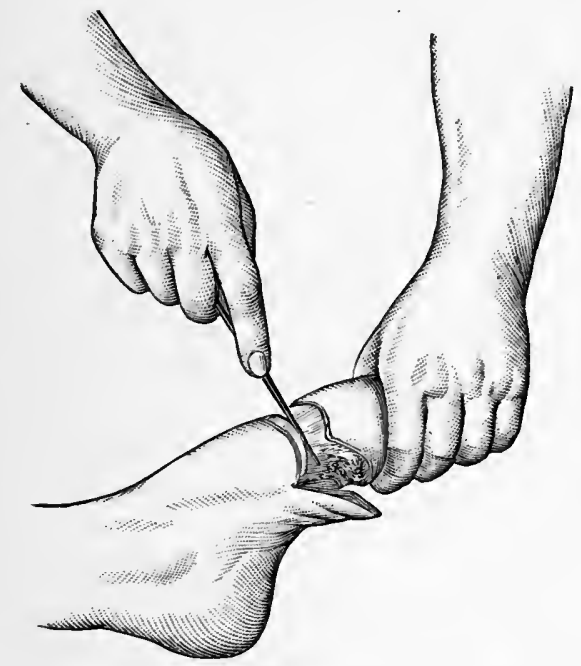

Amputation at the tarso-metatarsal joint. (Lisfranc's.)

found the joint which separates the os calcis from the cuboid $(F)$, this joint forming the onter circle to Chopart's amputation. Half an inch in front again, or one inch from the tubercle, the prominence of the fiftli metatarsal bone is always to be felt $(H)$, the line above this prominence indicating the articulation with the cuboid bone, which forms the outer boundary of the incision for Hey's or Lisfranc's operation." 
Tarso-metatarsal Amputation (Lisfranc's). - The incision for this amputation is a curved one earried across the dorsum. of the foot from the base of the fifth to the base of the first metatarsal bone (Fig. 427, A). The incision should involve the skin only, its centre lying half an inch or more below the centre of the line of the articu. lations, and it should begin and end at the sides of the foot at their junction with the sole. A plantar flap should be marked out by a curved incision erossing the sole of the foot near the origin of the toes, starting and ending at the same points as the dorsal incision.

The dorsal flap is next dissected baek to the line of the articulations; the tendons, museular fibres, and faseia being divided, the joints between the tarsal and metatarsal bones are opened with a stout, narrow-bladed knife (Fig.

\section{FIG. 429.}

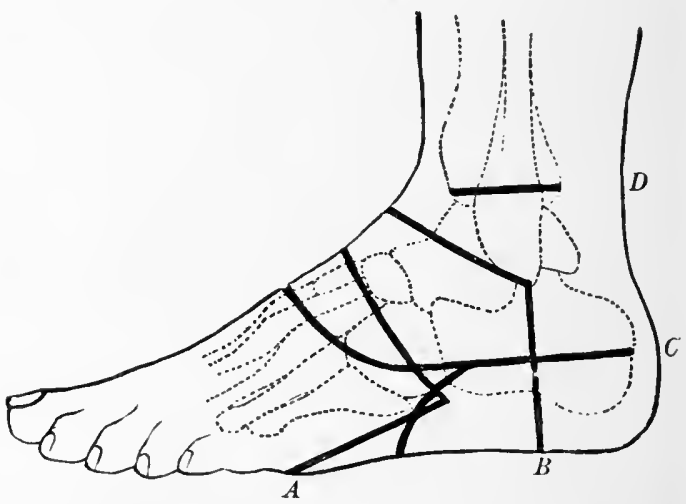

Lines of incision for- $A$. Chopart's amputation. $B$. Syme's amputation. $D$. Section of bone in syme's amputation. $C$. Subastragaloid amputation. (STIMSON.)

428). Difficulty is sometimes experienced in opening the joint between the head of the second metatarsal bone and the second euneiform bone, which oceupies a position higher on the foot than the other articulations. 'The disartieulation may also be faeilitated by forcibly depressing the 
anterior portion of the foot. After all the joints have been opened, the knife is passed behind the ends of the metatarsal bones, and a plantar flap is eut from within outward, following the line of the incision previously marked out. The plantar flap may be cut from without inward if preferred.

Tarso-metatarsal Amputation (Hey's).- The line of incision and the steps of this operation are similar to those in Lisfranc's amputation, with the exception that Hey sawed off the projecting portion of the internal euneiform bone after disarticulating the metatarsal bones. This modification, although it improves the appearance of the stump, possesses no advantages over the latter procedure.

Medio-tarsal Amputation (Chopart's). - In this amputation the disarticulation is through the joints formed by

FIG. 430.

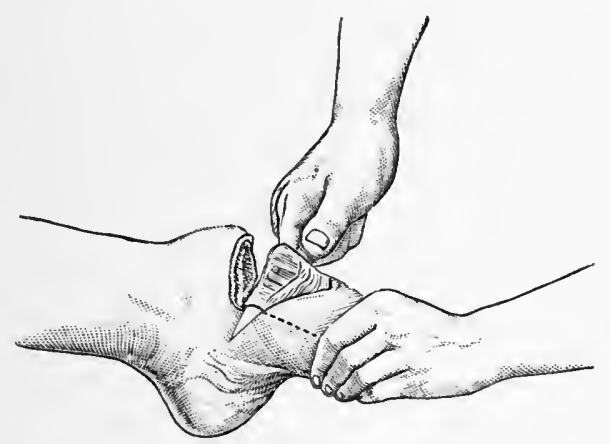

Chopart's amputation. (BRYANT.)

the astragalus and calcaneum behind and the scaphoid and cuboid in front. An incision is made from the tubercle of the scaphoid across the dorsum of the foot an inch in front of the head of the astragalus to the lower and outer border of the cuboid (Fig. 427,B). The plantar flap is next marked out by an incision beginning and ending at the same points as the first incision and crossing the sole of the foot four or five finger-breadths nearer the toes 
(Fig. 429, A). The dorsal flap is next dissected up, and after the tendons and fascia have been divided the joint is opened and a plantar flap is cut from within outward, following the line of the previously marked-out plantar incision (Fig. 430).

Subastragaloid Amputation.-In this amputation all the bones of the foot are removed except the astragalus. An incision is made, beginning an inch below the tip of the external malleolus, which is earried forward to the base of the fifth metatarsal bone; it is then carried over the dorsum of the foot to the calcaneo-cuboid articulation (Fig. $429, C$ ). The joints between the scaphoid and

FIG. 431.

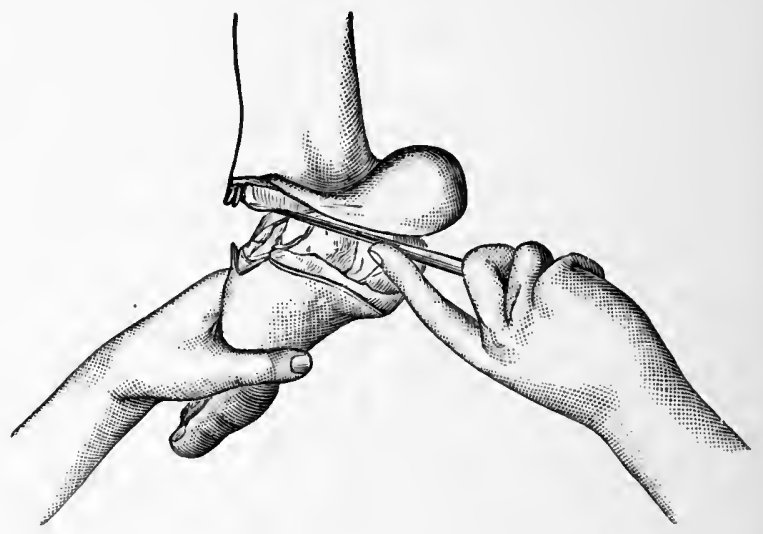

Syme's amputation at the ankle-joint. (SкEY.)

astragalus and between the astragalus and os calcis are opened, and the latter bone is carefully dissected ont; the ligaments are divided and the astragalus only is allowed to remain in place.

\section{AMPUTATIONS AT THE ANKLE-JOINT.}

Syme's Amputation at the Ankle-joint.-In this amputation, the foot being at a right angle to the leg, an 
incision is made from the centre of one malleolus direetly across the sole of the foot to the centre of the opposite malleolus (Fig. 429, B). The tissues of the heel are next earefully dissected from the bone by keeping the knife close to the osseous surface until the tuberosity of the os caleis is fairly turned (Fig. 431). The two extremities of the first ineision are then joined by a transverse one across the instep, and, the joint being opened, the

Fia. 432.

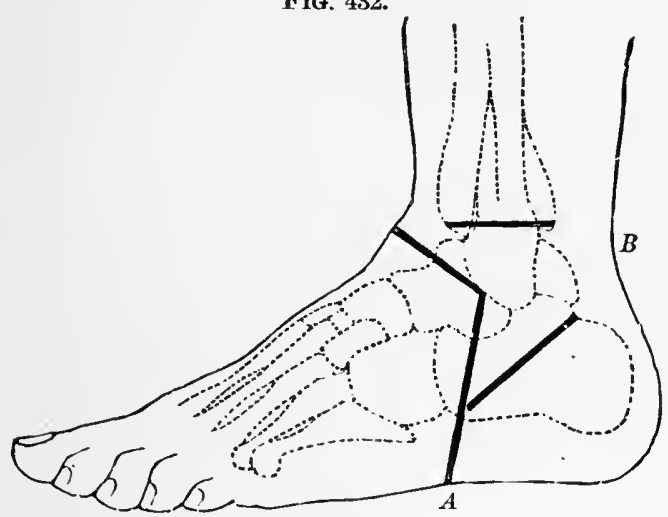

Pirogoff's amputation. A. Cutaneous incision. $B$, Line of section of bones. (STIMSON.)

lateral ligaments are divided to complete the disarticulation. The knife is next used to clear the malleoli, and they are next removed by the saw in the line indicated (Fig. $429, D$ ).

Pirogoff's Amputation at the Ankle-joint.-In this amputation the posterior portion of the os calcis is retained. The incision is earried from the tip of the inner malleolus, over the instep, half an ineh in front of the anterior edge of the tibia, to a point half an inch in front of the tip of the outer malleolus; a seeond incision, crossing the sole of the foot and carried down to the bone, is next made (Fig. 432, A). The plantar flap is dissected back for a quarter of an inch, the joint is opened by 
dividing the lateral ligaments, the astragalus is disartieulated, and the malleoli are exposed. A narrow saw is next applied to the upper and posterior part of the calcanemu behind the astragalus, and the former is divided obliquely downward in the line of the plantar ineision (Fig. 433). The malleoli and a thin sliee of the tibia are next removed with the saw, as in Syme's amputation (Fig. 432, B). Some surgeons do not remove the malleoli, but press the sawed surface of the os caleis between them when it is possible to do so. The position of the os calcis in relation to the tibia after union has oeeurred is shown in Fig. 434.

\section{FIG. 433.}

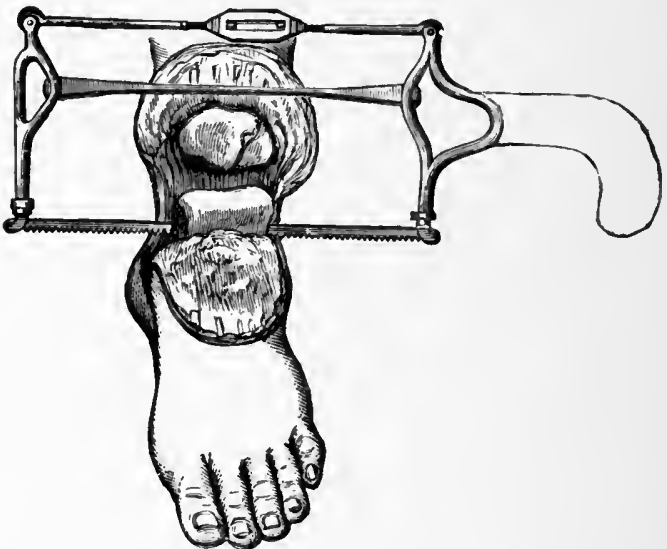

Application of saw to os calcis in Pirogoff's amputation. (ERICusEN,)

Roux's Amputation at the Ankle-joint.-In this method of ampntation an incision is made at the onter edge of the tendo-Achillis, a little above its insertion, whieh is earried forward under the onter malleolus, and aeross the instep half an inch in front of the anterior edge of the tibia, and back to a point just in front of the inner malleolus; the incision is carried from this point downward and partly across the sole of the foot, and then back to the point of origin of the original in- 
cision (Fig. 435). The flaps are dissected up for a short distanee, the ankle-joint is opened, disarticulation is effeeted, and the internal flap is carefully dissected from the bones.

Other methods of amputation of the foot are sometimes employed, such, for instance, as that advocated by Hancock, who has combined Pirogoff's amputation with the subastragaloid method, bringing the sawed surface of the os calcis in contact with a transverse section of the astragalus.

Hancock has advocated the propriety of amputating the foot without regard to the position of the tarsal joints, cutting the flaps of sufficient length and dividing the bones with a saw.

Tripier has also modified the subastragaloid amputation by leaving the upper part of the calcancum, which he saws through on a level with the sustentaculum tali, and at right angles to the axis of the leg; the cxternal incisions are made as in Chopart's amputation.

In the method advocated by Mikulicz the astragalus and caleaneum are removed, the ends of the tibia and fibula are sawed off, and the sawed surfaces of the seaphoid and enboid are approximated to these, the stump resulting resembling the foot of pes equinus.

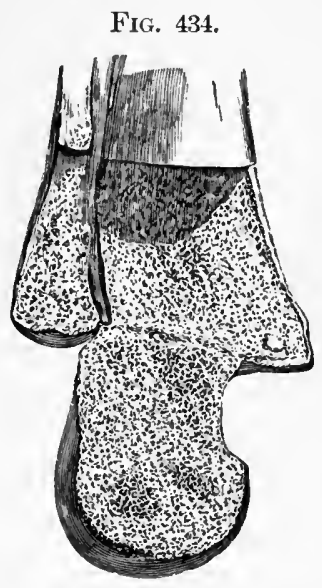

Union between calcaneum and tibia in Pirogoff's amputation. (HEwsoN.)

\section{AMPUTATIONS OF THE LEG.}

The leg may be amputated at its lower, middle, or upper third, the rule being to save as much of the limb as possible; but as regards the application of prosthetic apparatus, I think the stumps resulting from amputations in the middle and upper thirds will be found more satisfactory. It is well also in sawing the bones to divide the 
fibula at a slightly higher point than the tibia. The circular, modified circular, oval, elliptical, long anterior flap, rectangular flap, antero-posterior flap, lateral flap, or external flap method may be employed.

Circular Method.-A circular incision is made through the skin and connective tissue just above the malleoli, the cuff is dissected up for a sufficient distance, a circular incision of the tendons and muscles is next made, and the tissnes being retracted, the bones are divided with a saw.

Modified Circular Method.-In this method of ampu-

FIG. 435.

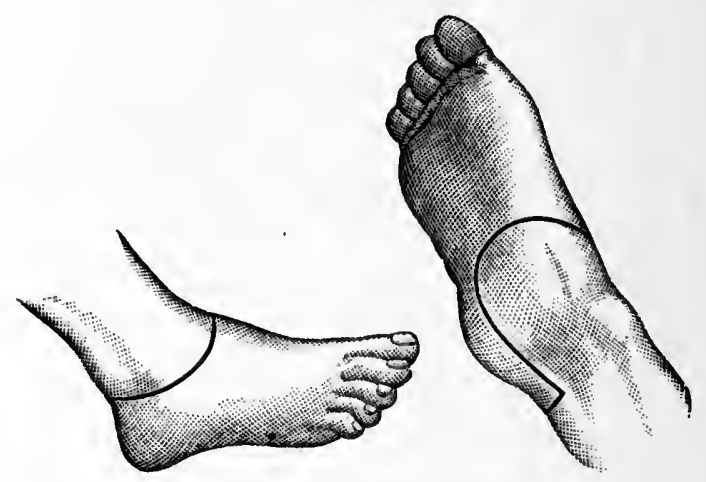

Incision in Roux's amputation.

tation of the leg two oval flaps, either antero-posterior or lateral, of the skin and connective tissue are marked out by incisions. The flaps are then dissected up to the ends of the incisions, and a circular division of the muscles is made (Fig. 436, $A$ ).

Elliptical Method.-In this method of amputation the incision is in the form of an ellipse; its lower end crosses the heel below the insertion of the tendo-Achillis, and the upper end of the incision is about an inch above the anterior articular edge of the tibia (Fig. 437, $B$ ).

Long Anterior Flap Method.-An anterior flap equal in length to the diameter of the leg at its base is marked 
FIT. 436.

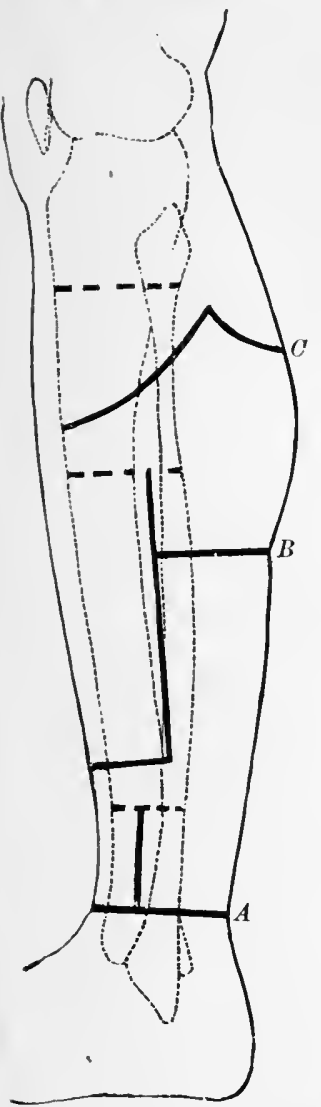

FIG. 437.

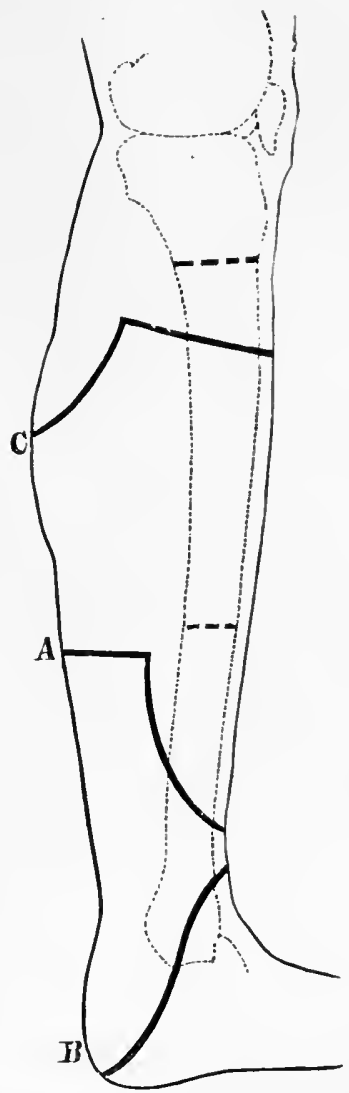

FIG. 436. - Amputation of the leg. A. Modified circular method. B. Rec. tangular flap. C: Antero-posterior flap. The dotted lines indicate the levels at which the bones are to be sawn through. (STInson.)

FIG. 437,-Amputation of the leg. A. Long anterior flap. $B$. Elliptical flap. C. At upper third. (STIMsov.)

out by a curved incision through the skin beginning at the posterior edge of the tibia on the inner side, a little below the point at which the bones are to be divided, and is carried over the leg to a point directly opposite over the 
fibula (Fig. 437, A). The anterior muscles are divided transversely balf an inch above the lower end of the flap, and are dissected from the hone to the base of the flap. The posterior flap is then made by entering the knife behind the bones at the point of the original incision and cutting directly outward.

Rectangular Flap Method (Teale).-In this method of amputation of the leg an incision equal in length to half of the circumference of the leg is made from the point at which the bones are to be divided on one side

Fig. 438.

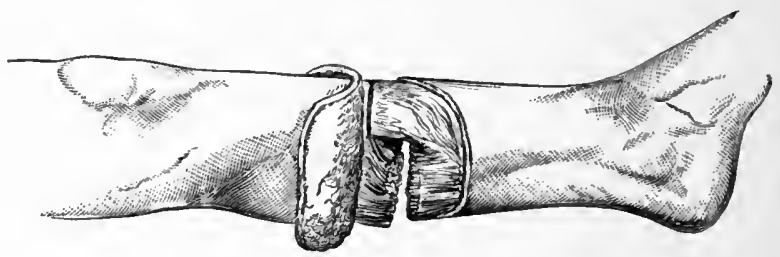

Amputation of the leg by lateral flaps. (BRyANT.)

of the leg, and is carried across the limb and baek upon the opposite side to a point opposite the point of starting. The flap thus marked ont is dissected up to its base, and a posterior flap of one-fourth the length is next cut by a transverse ineision down to the bones, and is dissected back to the line of the origin of the first incision (Fig. 436, B). The long flap is next doubled back and its edges secured to the posterior flap, or the long flap may be cut from the posterior surface of the leg and the short flap from the anterior surface.

Antero-posterior Flap Method.-A long anterior flap, including half of the circumference of the limb, may be cut from without inward, composed of skin, connective tissue, and muscles; and a short posterior flap, cut from within outward, may also be employed. This method is often employed in amputations in the upper portion of the leg (Fig. 436,C).

Lateral Flap Method.-In the lower and middle thirds 
of the leg the method of amputation by means of lateral skin flaps may be employed with advantage. In this method an incision is made over the spine of the tibia, and an oval flap, embracing one-half of the circumference of the leg, composed of the skin and connective tissue, is dissected up ; starting from the same point, a similar flap is formed on the opposite side of the leg and dissected up ; the muscles at the upper extremity of the flaps are next divided by a circular incision and the bones are divided with a saw (Fig. 438).

External Flap Method (Sedillot). - In this method of amputation of the leg the point of the knife is entered a finger's breadth external to the spine of the tibia and carried outward, grazing the fibula, and is brought ont as far as possible to the inner side; a flap three or four inches in length is then cut from within outward; the extremities of the incision are next united by an incision across the inner side of the limb, involving the skin only; any remaining muscular tissue is next divided and the bones are sawed. The long external flap is then brought over the ends of the bones and fastened to the edges of the incision on the inner side of the limb. Professor Ashhurst modified this operation by cutting the long external flap from without inward, and made also a short internal flap in the same manner. By either method the resulting stump is a good one, with the ends of the bones covered by the tissues of the external flap.

\section{AMPUTATIONS AT THE KNEE-JOINT.}

Amputations at the knee-joint may be done either by the circular or elliptical incision, or by means of flaps, and may consist in sinple disarticulations or sections through the condyles of the femur.

Elliptical Method.-In this operation an incision crossing the spine of the tibia, five finger-breadths below the lower extrenity of the patella, is carried around the back of the leg three finger-breadths higher than in front; the tissues on the front of the leg are dissected up until 
the tendon of the patella is exposed; the leg is then flexed and the ligament of the patclla is divided; the capsular ligament and the lateral and crucial ligaments are next

FIG, 439.

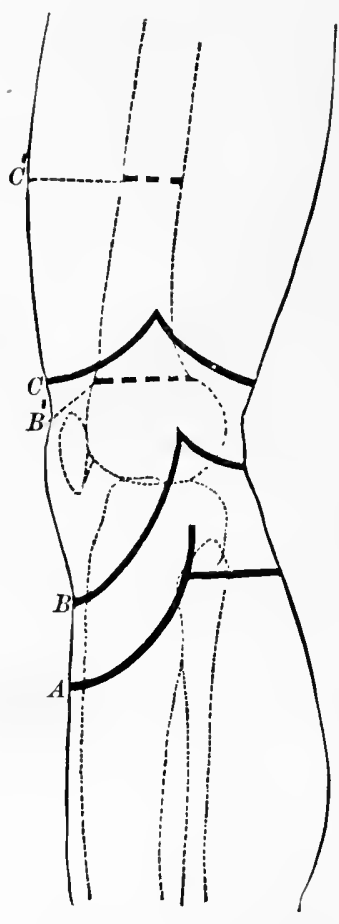

Amputations at the kneejoint and lower third of the thigh. A. Long anterior flap. $B$. Amputation through condyles. $B^{\prime}$. Line of section of the eondyles of the femur. $C$. Modificd flap at the lower third of thigh. (STInson.) parts are divided, the patella re-
moved, and the condyles next sawed through just above the edge of the articular eartilage (Fig. $439, B$ ). injure the popliteal vessels with the point of the knife. The tibia is next drawn forward, the knife is passed behind its posterior border, and the remaining soft parts are divided from within outward.

Anterior Flap Method.-In this method of amputation a long cutaneous flap is formed. The incision, beginning half an inch below the articulation, is carried five inches below the patella; crossing the anterior surface of the leg, it is carried back to the condyle of the femur on the opposide side. This flap is dissected $u_{2}$, and the ligament of the patella divided and the disarticulation effected. A short posterior flap, uniting the anterior incision one inch below its extremities, is next cut by transfixion or from without inward (Fig. 439, $A$ ). The patella is not removed.

\section{Amputation through the Con-} dyles of the Femur.-In this amputation, which is known as Carden's amputation, an anterior flap, whose lower extremity is three finger-breadths. below the patella, is cut and the disarticulation effected; the posterior soft parts are divided, the patella re-
moved, and the condyles next sawed through just above 'severed, care being taken not to 
Lateral Flap Method.-In this operation an ineision is made just below the patella, and is carried down the spine of the tibia for three inches, and is then carried backward to the middle of the leg at a point opposite the beginning of the incision; a similar flap is cut on the opposite side of the leg, and the flaps dissected up to the line of the articulation. When this point is reached, the joint is opened and the disarticulation effected. The patella is not renoved (Fig. 440).

\section{Gritti's Amputation at the} Knee-joint.-In this operation a long rectangular anterior flap is first eut and dissected up, and after the disarticulation has been effected the skin covering the posterior surface of the knee is eut from within outward. The condyles of the femur are next removed by a saw above the edge of the articular cartilage, and the artic-

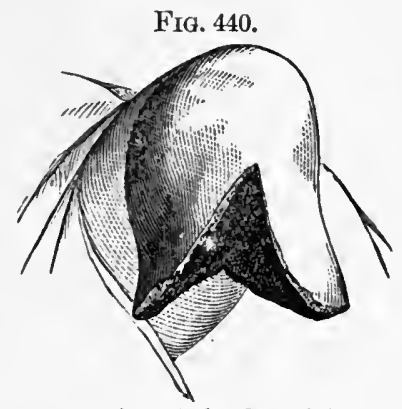

Amputation at the knee-joint by lateral flaps. (SMITH.)

ular surface of the patella is removed by the saw or cutting forceps. The patella is next brought down, so that its sawed surface is in contact with the sawed surface of the eondyles, and the flaps are brought together (Fig. $441, A)$.

\section{AMPUTATIONS OF THE THIGH.}

Modified Circular Method.-Two oval flaps of skin and connective tissue, the upper extremities of which are several inches above the condyles of the femur, are marked out by ineisions and dissected up, the muscles are next divided by a cireular ineision, and the bone is divided with a saw.

Long Anterior Flap Method.-In this operation an incision is made on the anterior aspect of the thigh, marking out a flap whose length is equal to one-third, 
FrG. 441.

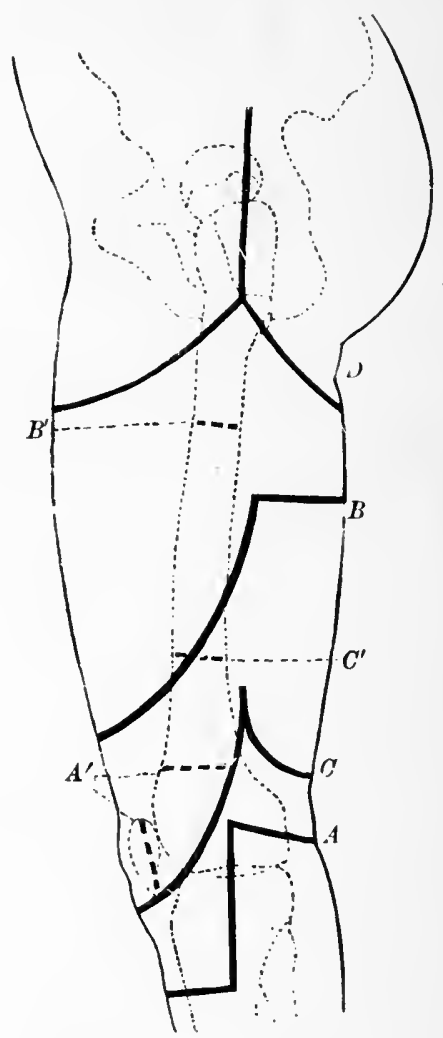

$A$. Gritti's amputation at the knee. $A^{\prime}$. Lines of division of the bones, $B$. Amputation of the thigh, long anterior flap. $B^{\prime}$. Division of the bone. C. Amputation at the lower third of the thigh. $C$. Division of the bone. $D$. Disarticulation at the hip-joint. (STIMson.)

and whose width at its base is equal to two-thirds, of the circumference of the limb. The anterior muscles are next divided obliquely upward and backward, so that the flap shall not be too thick, and the posterior muscles are cut transversely and the bone divided with a saw (Fig. 441, $B$ ).

Amputation in the lower third of the thigh may also be 
effected by employing a long anterior and a short posterior flap. The anterior flap is cut, its lower extremity extending down to the lower edge of the patella, and after dissecting up the skin and cellular tissue to the upper extremity of the patella the muscles are cut obliquely up to the point at which the bone is to be divided. A short posterior flap is next cut, and, the soft parts being retracted, the bone is sawed through (Fig.441, C).

FIg. 442.

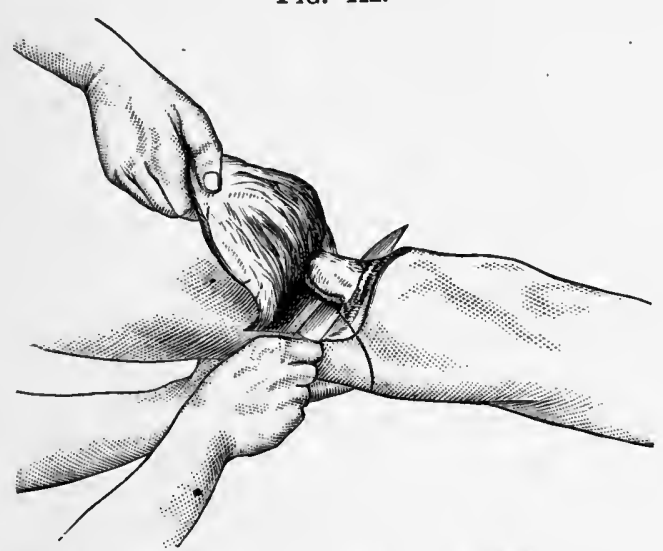

Amputation of thigh by flaps cut by transfixion.

Transfixion Method-In amputations of the thigh the flaps may also be cut by transfixion. either lateral or antero-posterior flaps being employed (Fig. 442).

Amputation of the Thigh through the Trochanters. -When, for any reason, it is inadvisable to amputate at the hip-joint, an amputation may be made through the trochanters, a long anterior and a short posterior flap being employed, with circular division of the muscles.

\section{AMPUTATIONS AT THE HIP-JOINT.}

In amputations at the hip-joint, it is important that provision be made for the control of hemorrhage during 
the operation, and this is accomplished by compression of the femoral artery by the fingers of an assistant, or by the

FIG. 443.

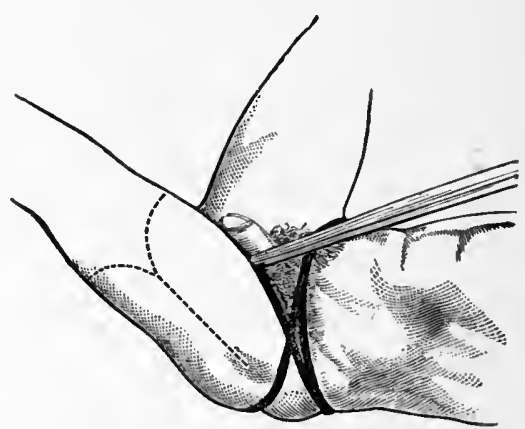

Esmarch's elastic strap applied to control hemorrhage during amputation at the hip-joint.

preliminary ligation of the femoral artery just below Poupart's ligament. Esmarch's elastic strap may also be employed for the control of bleeding during amputation at the hip-joint, the strap being applied in such a manner that it occupies the position of the turns of a spica-bandage of the groin (Fig. 443).

Dieffenbach and Wyeth, to avoid hemorrhage, make first a circular amputation in the continuity of the thigh, and after controlling the hemorrhage disarticulate the head of the femur and remove it; Jordan and Senn disarticulate the head of the bone first throngh an external incision, and control the bleeding before the amputation is completed by passing an elastic tourniquet around the soft parts above the point where they are to be divided.

The methods of amputation at the hip-joint are the oval, antero-posterior flap and lateral flap, and modified circular methods.

Transfixion Method.-In this method the point of a long amputating knife is thrust into the tissues about two finger-breadths below the anterior superior spinous process 
of the ilium; and is pushed through the tissues, grazing the hip-joint, and brought out on the opposite side of the thigh close to the junction of the scrotum. The knife is next carried downward close to the bone, and an anterior flap of sufficient length is cut from within outward. This -flap is held up by an assistant and the head of the bone disarticulated, and, the knife being passed behind the

FIG. 444.

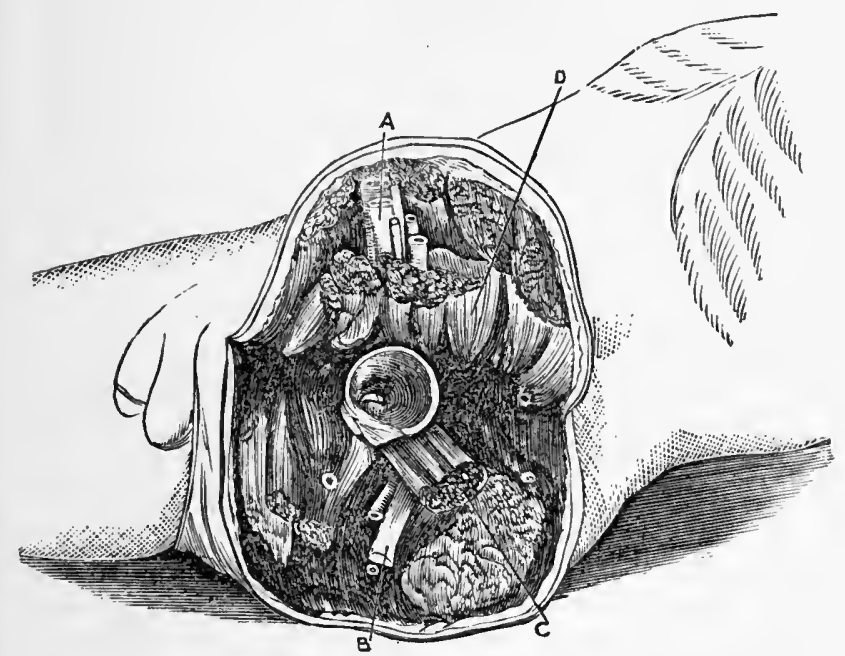

Amputation at the hip-joint by antero-posterior flaps. (HoLMEs.)

bone, a posterior flap of equal length is cut from within outward (Fig. 444).

Guthrie's method of amputation at the hip-joint consists in eutting the flaps from without inward, a smaller knife being used for this purpose and the posterior flap being cut first.

Modified Circular Method.-In this operation short antero-posterior flaps of skin and connective tissue are cut and dissected up, the muscles are divided by a circular incision at the level of the joint, and disarticulation of the head of the femur is next effected. 
Lateral Flap Method.-In this operation two flaps are cut from the inner and outer side of the thigh by transfixing, or by cutting from without inward and exposing the joint, which is opened and disarticulation of the head

FI't. 445.

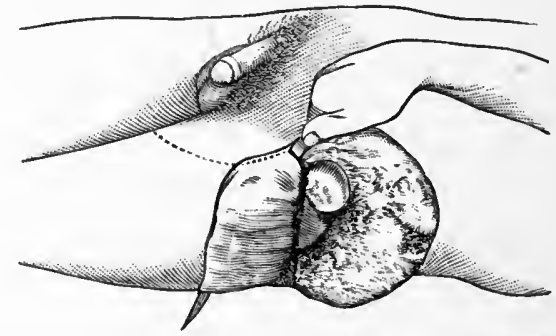

Amputation at the hip-jolnt by external and internal flaps. (BRYANr.)

of the femur effected as in the preceding methods (Fig. $445)$.

Wyeth's Method of Controlling Hemorrhage in Amputating at the Hip-joint.-In amputating at the hip-joint by this method the hip to be operated upon is brought well over the edge of the table and the Esmarch bandage applied to the limb. Two stout steel pins, twelve or fourteen inches in length, are required: the point of one of these pins is passed through the skin one and a half inches below and slightly to the inner side of the anterior superior spine of the ilium and carried through the tissues about half-way between the great trochanter and the spine of the ilium external to the neck of the femur, and its point is made to emerge just behind the trochanter; the second pin is made to enter the skin an inch below the croteh, internal to the saphenous opening, and its point is marle to emerge about an inch and a half in front of the tuber isehii. The points of the pins are next protected with corks, and a long piece of rubber tubing or an Esmarch elastic strap is wound tightly five or six times about the limb above the fixation pins (Fig. 446). The Esmarch bandage should then be removed and a circular incision of the skin and cellular tissue made five 
inches below the constricting band; this cellulo-cutaneous cuff should next be reflected to the level of the trochanter minor; a circular division of all the muscles should next be made at this point and the bone divided with a saw (Fig. 447). The large vessels should next be secured, and after this has been done the rubber tube should be removed, and any vessels which bleed should then be

FIG. 446.

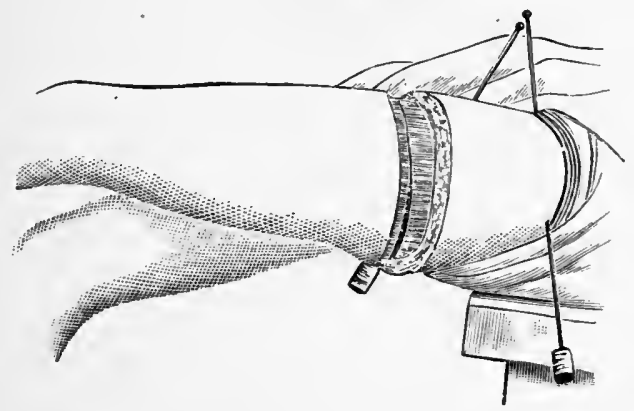

Pins inserted and tube applied.

tied. The exposed end of the femur is then grasped with bone forceps, and an incision is next made upon the outer side through the skin and muscles until the neck and head of the bone are exposed, and the disarticulation is accomplished.

This method is now practised without first sawing the femur. The pins and elastic constrictor are applied as previously described and a circular incision, including the skin and cellular tissue, is made around the thigh six inches below the tip of the great trochanter and a cuff of skin and cellular tissue is dissected up (Fig. 448). A vertical incision is then made down to the bone from the outer side of the cutaneous incision to a point one inch above the great trochanter. The flaps thus formed are retracted and the bone is dissected out of its muscular bed. The capsule of the joint is then exposed and opened and the disarticulation is effected by adduction and rotatory move- 
ments of the limb. As soon as the head of the bone has

Fig. 447.

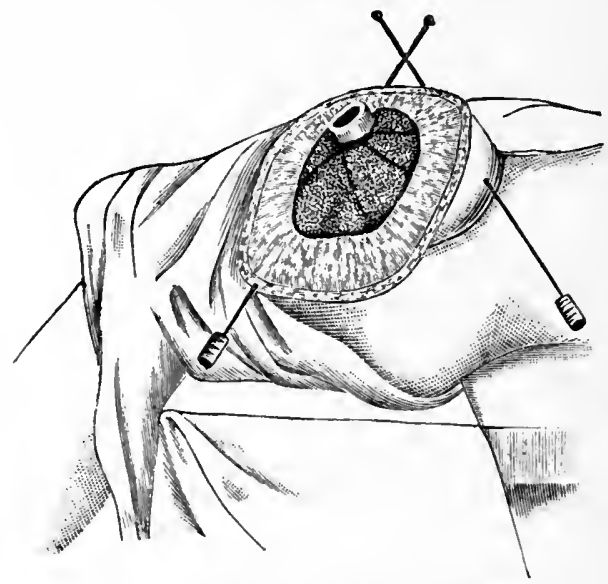

Limb amputated and bone sawed. (WYETH). been disarticulated the posterior muscular attachments'are severed and the limb is removed. The femoral artery and vein, the profunda, and decending branch of the external circumflex should be ligated before the elastic constrictor is removed. A number of muscular branches will after this require ligation.

Drainage should be introduced and the flags approximated

Fia. 448.

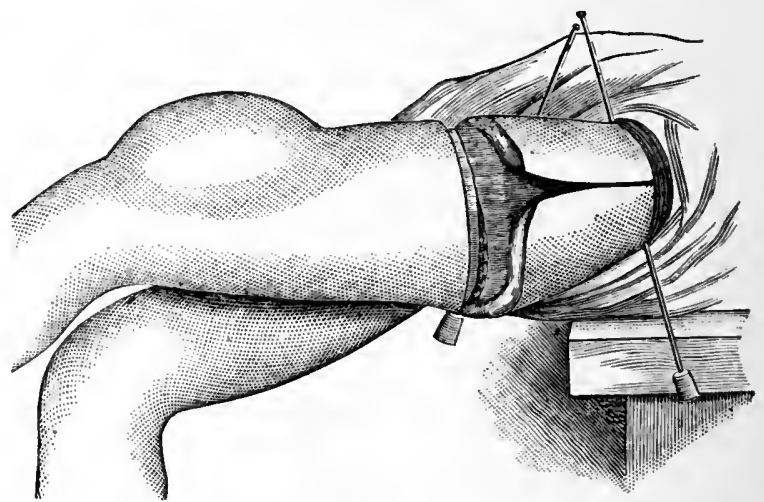

The needles and constrictor applied: cireular and longitudinal incisions for skin flap. (WYETH.)

with sutures, and a copious gauze dressing should be applied. 


\section{PART VIII. \\ EXCISIONS OR RESECTIONS.}

\section{EXCISION OF THE JOINTS.}

THIs operation consists in the partial or emplete removal of the articular surface of the bones making up the joint. The term resection is sometimes used as synonymous with excision, but it is usually employed to indicate the rernoval of a portion or the whole of the shaft of one of the long bones. Excisions or resections of joints and bones may be required on account of injury, disease, or anchylosis of a joint in faulty position. In the operation of excision of the joint the incision should be sufficiently free to permit of an inspection of the diseased portions of the joint, and it is preferable to remove the diseased articular surface of the bone with a saw; small areas of diseased bone may be removed with the curette or gonge forceps. In performing excisions of joints in young subjects care should be taken to see that the epiphyseal cartilage is not encroached upon, for if this is remover the subsequent growth of the limb may be interfered with. The result desired in cases of excision of joints, in addition to removal of the diseased tissue, varies somewhat with the joint involved ; for instance, in a knee-joint anchylosis is desired; in the shoulder, hip, elbow, and wrist, we wish to obtain a movable false joint; when the latter condition is desired after excision, care should be exercised not to divide muscles or tendons, and as far as possible not to interfere with their attachments. When anchylosis is 
desired, the division of muscles or tendons is not a serious consideration; any injury to the principal arteries, veins, and nerves should always be avoided.

Fig. 449.

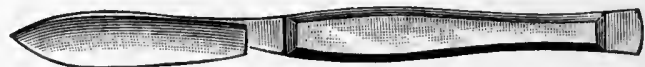

Heavy scalpel.

Fig. 450.

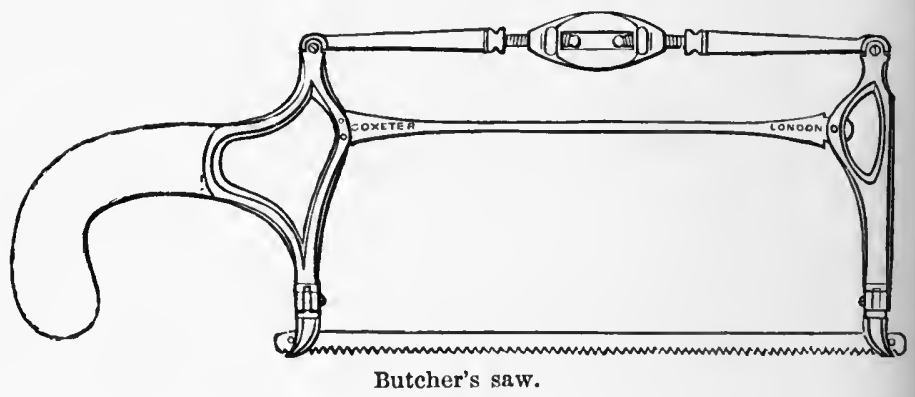

Fie. 451.

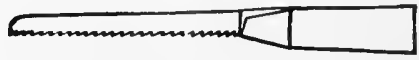

Narrow-bladed saw.

Fig. 452.

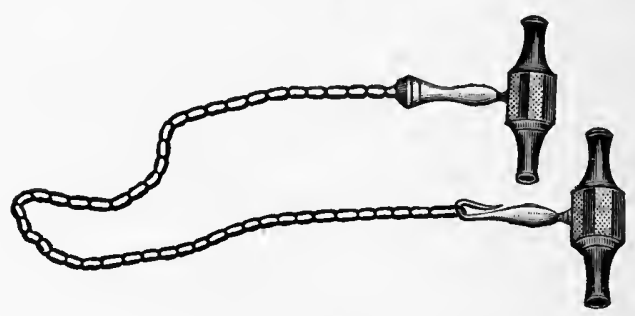

Chain-saw.

The instruments required for the excision of joints are a stout scalpel (Fig. 449), probe-pointed knife, and ex- 
FIG. 453.

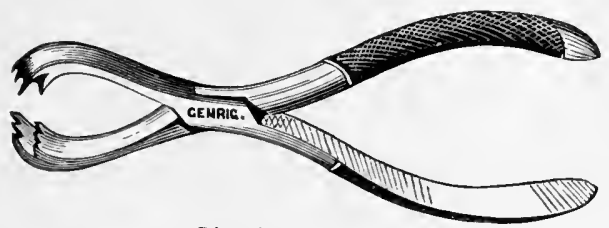

Lion-jawed forceps.

F1G. 454.

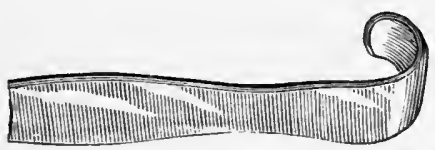

Retractor.

FrG. 455.

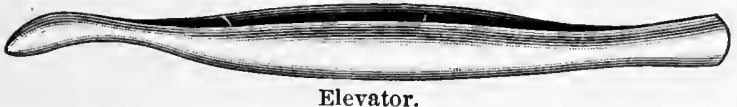

Elevator.

FIG. 456.

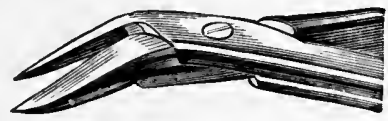

Bone-cutting pliers.

FIG. 457.

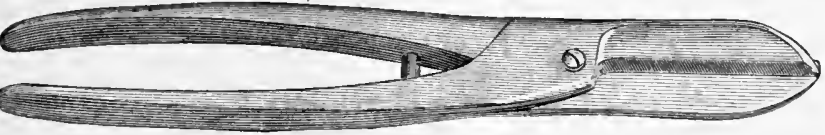

Knife-bladed forceps.

Frg. 458.

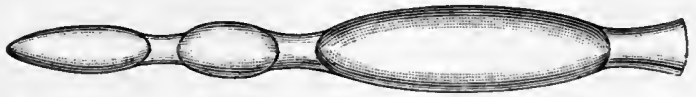

Periosteotome. 
cision saw with reversible blade (Fig. 450), narrow-bladed saw (Fig. 451) or chain-saw (Fig. 452), strong lion-jawed forceps (Fig. 453), retractors (Fig. 454), an elevator (Fig. 455), heavy bone-cutting pliers (Fig. 456), knife-bladed forceps (Fig. 457), and a periosteotome (Fig. 458).

Excision of the Shoulder-joint.-In excising this joint, the arm is abducted and rotated inward, and a straight incision is made extending from the beak of the coracoid process down the arm in the line of the bicipital groove; this incision may be supplemented by a short, transverse incision from the upper edge of the first inci-

FIG. 459.

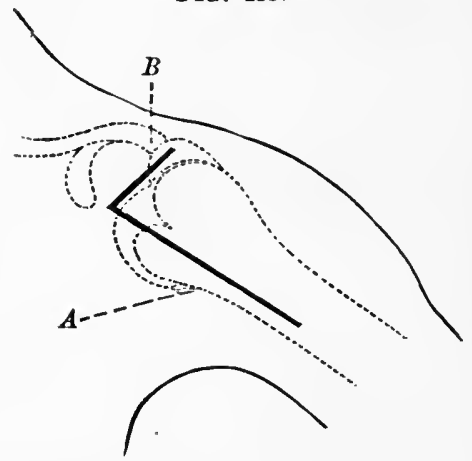

Excision of the shoulder-joint. $A$. Regular incision. B. Supplementary incision. (STMSON.) sion to the acromion process (Fig. 459). As the incision is deepened the fibres of the deltoid muscle are divided in this line, and the capsule of the joint is exposed and divided along the outer edge of the tendon of the long head of the biceps muscle; this tendon is held to one side, the capsule of the joint is freely opened, and the muscles inserted into the tuberosities of the humerus are divided with a probe-pointed knife and freed with an elevator; the head of the bone may then he removed by sawing across the surgical neck of the bone with a narrow metacarpal saw or chain-saw, and the sawed surface of the humerus rounded off with bone forceps. The end of the bone is then replaced in the glenoid cavity and the wound drained and closed.

Resection of the Humerus.-A portion of the humerus may require resection for injury or disease. The incision should be made upon the outer side of the bone and carried down in the muscular interspaces on a line with the shaft, care being taken to avoid injury of the musculo- 
spiral nerve, which, as it passes around the posterior surface of the humerus, lies elose to the bone between the humeral heads of the triceps muscle at a point corresponding to the deltoid insertion anteriorly $-i$. e., about the centre of the shaft of the humerus. This nerve should be isolated and held aside, and the bone exposed. After separating the periosteum as completely as possible, if the shaft of the bone is diseased, it should be removed by dividing it in the middle with a saw or forceps, and removing each fragment as far as the upper and lower epiphyses, or the upper or lower portion only may require removal.

Excision of the Elbow-joint.-In excising this joint, the forearm is slightly flexed and a longitudinal incision is begun about two inches above the olecranon process and a little to its inner side, and carried about three or four inches down in the line of the ulna (Fig. 460); the tissues are then divided down to the bones, and the ulnar nerve is dissected from its groove behind the inner condyle of the humerus and held aside by a retractor; the tendon of the triceps is divided, and its attachment to the fascia and periosteum over the olecranon process is separated with an elevator or periosteotome and turned downward; the joint is next opened and the lateral ligaments divided as the forearm is flexed upon the arm. The upper part of the ulna and the head of the radius are freed witl a probe-pointed knife and removed with a narrow-bladed saw, eare being taken in making the section of the radius to divide its neck so that the attachment of the biceps muscle is not interfered with. The condyles of the humerus are next freed and removed with a saw. In freeing the bones at the anterior portion of the joint, great care should be used to avoid injury of the brachial artery and vein and the median nerve.

Resection of the Radius or Ulna.-The radius or uhna may be resected, either entirely or partially, by making an incision upon the back of the forearm over the bone to be removed; the bone being exposed, the periosteum is separated with an elevator and the bone divided 
with a saw, and each fragment lifted and separated from its muscular attachments up to the point where it is desired to remove it (Fig. 461). If the articular surface of the bone is to be removed, the disarticulation should be made carefully with a strong scalpel or a probe-pointed knife, care being taken to avoid injury of the vessels and nerves lying upon its palmar surface.

Excision of the Wrist.-The wrist is covered on its posterior and lateral aspects with skin, fascia, and tendons; the relative position of the

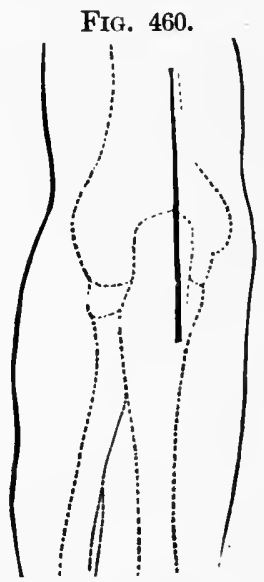
bones entering into the articulation may be seen in the accompanying figure (Fig. 462).

FIIG. 461.

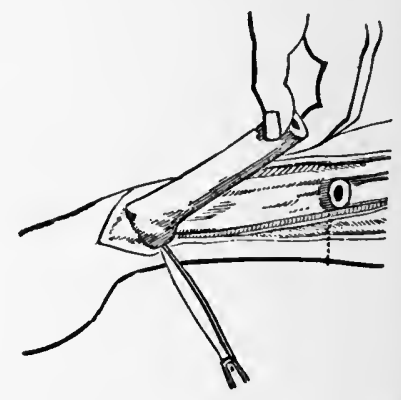

Incision for excision of the elbow- Resection of the lower end of the radius. joint. (STIMSON.)

The wrist-joint may be excised by making a dorsal incision, beginning at the middle of the ulnar border of the second metacarpal bone, and carried upward about four inches, crossing the ulnar edge of the tendon of the extensor carpi radialis brevior, and splitting the dorsal ligaments of the wrist between the tendons of the extencor secondi internodii and the extensor of the forefinger (Fig. 463). The incision should be carried down to the bone and the soft parts and tendons dissected loose with an elevator. By flexing the hand, the first row of the carpus is made to present in the wound, and the scaphoid is sepa- 
rated from the trapezium and removed; the semilunar and euneiform should next be removed; the trapezium and pisiform should be left if possible. In removing the second row of carpal bones, the knife shonld be passed between the trapezium and the trapezoid and then into the earpo-metacarpal joint, and by cutting the ligaments on the dorsal side of the ends of the metacarpal bones the

Frg. 462.

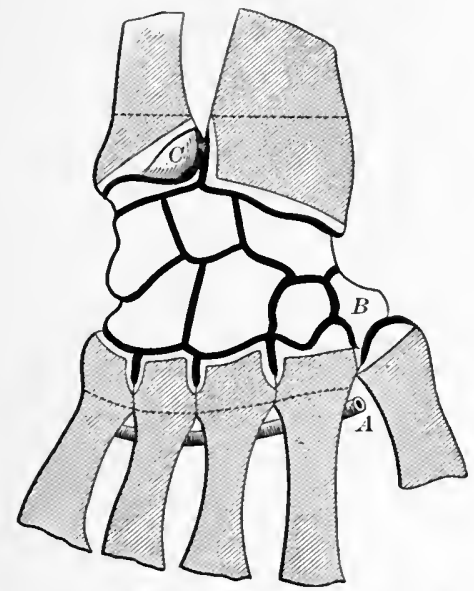

Articulations of the wrist-joint. (LISTER.)

trapezoid, os magnum, and uneiform may then be removed. The lateral ligaments are next earefully divided, and the articular ends of the radius and ulna removed with a saw; the ends of the metacarpal bones should next be removed with a saw or bone-forceps.

Mynter's Method.-This eonsists in making an ineision on the dorsum of the hand extending from the radius downward between the second and third metaearpal bones and through the palm, splitting the hand as high as the superficial palmar arch, thus entering the wrist between the trapezoid and os magnum and between the seaphoid and semilunar bones. By this method it is possible to excise the 
carpus without injury to the palmar arches and palmar bursæ.

Resection of a Metacarpal Bone.-A metacarpal bone may be resected by making a longitudinal incision on the back of the hand over the bone to be removed. The incision should extend Fig. 463. from one articular end

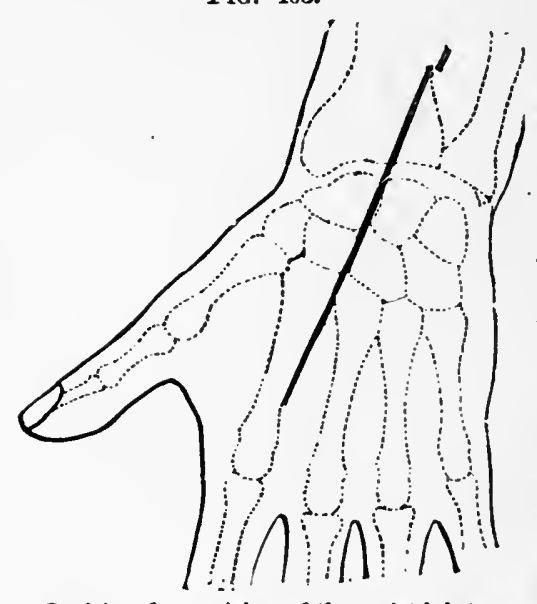

Incision for excision of the wrist-joint. (Stimson.) to the other, and the extensor tendon when exposed should be held to one side by retractors; the periosteum should next be separated as far as possible, and when the bone has been fully exposed it may be removed by dividing it in the mildle with bone-forceps and then disarticulating each fragment; or the articular ends may be freed and the bone removed in one piece (Fig. 464).

\section{Excision of Meta-} carpo-phalangeal Joints or Interphalangeal Joints.The metacarpo-phalangeal joint is exposed by a longitudinal incision over the dorsal surface of the knuckle; the extensor tendon being exposed and held to one side, the lateral ligaments are divided. The articular ends of the bones are then exposed and removed with a metacarpal saw or with bonc-forceps (Fig. 465). In excising the interphalangeal joints, the incision is usually made upon the side of the joint, and when the articular surfaces of the bone have been exposed they are removed with a small saw or forceps.

Excision of the Clavicle.-The clavicle is excised by making an incision over the bone from one articulation to the other, which is carried directly down to the bone; the 
periosteum is then separated, and the shaft of the bone may be divided at the middle and each fragment raised and disarticulated; or the bone may be disarticulated at one extremity, then raised up and freed from its adherent tissues, and disarticulated at the other extremity. In disarticulating the sternal articulation of the clavicle (Fig. 466), a probe-pointed knife should be used, and great care should be exercised to avoid injury of the important vessels and nerves which lie in close proximity to it.

Resection of the Ribs.-In this operation the incision should correspond in length

FIG. 464.

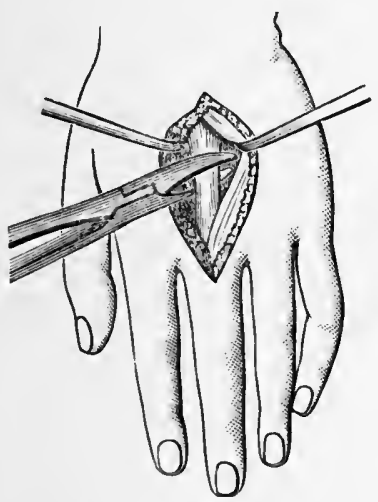

Resection of a metacarpal bone. and direction with the portion of bone to be removed, and may be crossed at each

FIG. 465.

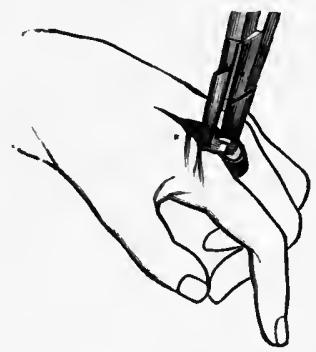

Excision of a metacarpo-phalangeal joint.

end by a short transverse incision. The tissues overlying the rib are then dissected loose, the periosteum is separated as far as possible, the rib is divided with bone-forceps at two points, the fragment is grasped with forceps, and the attachments to the under surface of the rib are separated with an elevator. Care should be taken to avoid opening the pleural cavity.

Estlander's Operation.-This operation is employed in cases of empyema, and consists in resecting the portions 
of several adjoining ribs to allow the chest-wall to sink inward and unite with the pulmonary pleura. A rectangular or oval flap is marked out by an incision, corresponding to the portion of the ribs to be removed, including all of the tissues external to the ribs. The flap is dissected up, and portions of several ribs are divided with bone-forceps or a saw, and removed with forceps. If the costal pleura is very thick, to expose the cavity so as to permit of free drainage and allow the ehest-wall to sink in, it may be cut away over a part of the area from which

FIG. 466.

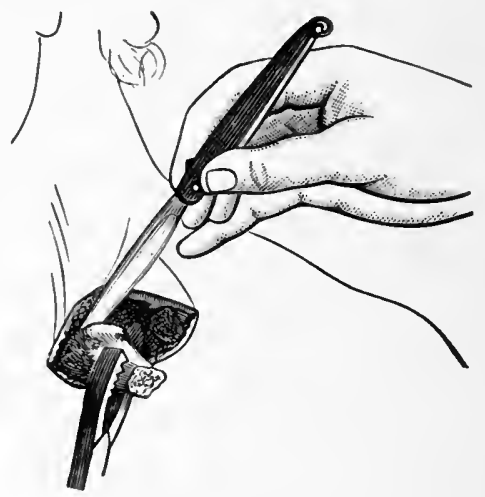

Resection of the sternal end of the clavicle.

the ribs have been resected; one to four inches of three to six adjoining ribs may be removed.

Resection of the Sternum.-This operation is performed by making a longitudinal incision over the portion of the bone to be removed; the periosteum is separated, and the diseased portion of the sternum is then carefully freed with an elevator and removed.

Excision of the Scapula.-To excise this bone, an incision should be made along the whole length of the spine of the scapula, and from its posterior extremity two other incisions should be made, one running about an 
inch or two above, and the other passing down the posterior border of the bone to its inferior angle (Fig. 467); the flaps thus made are loosened by separating the muscles attached to the outer surface of the bone. The attachments of the deltoid and trapezius muscles to the acromion and spine of the scapula are separated, and the lower angle is freed by detaching the teres major and serratus magnus; the bone is then raised and the subscapularis muscle detached from below upward. 'The neck of the scapula should be divided with a chain-saw or bone-forceps; the acromion is next separated from the clavicle, and the scapula turned upward, the joint being opened from below. The coracoid process should be separated from its muscular and ligamentous attachments, or may be divided with a saw and left in place. In clearing the supraspinous fossa, care should be taken not to injure the suprascapular nerve in the suprascapular notch; it should be raised with the periosteum and its fibrous sheath.

Excision of the Hip.In excising the hip-joint, a curved or angular incision is made from a point about three inches below the erest of the ilium and about the

FIG. 467.

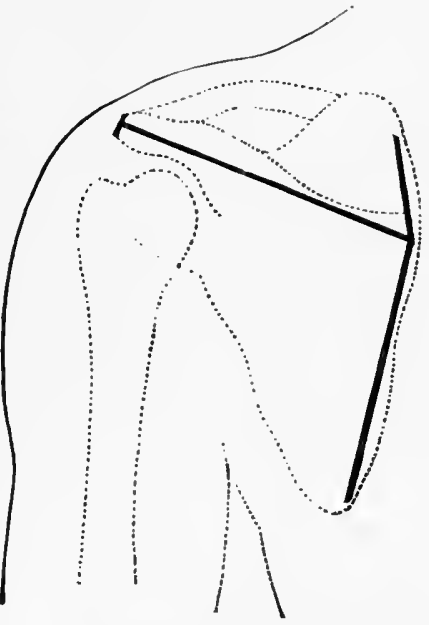

Incision for excision of the scapula. (STIMsox.)

same distance behind the anterior superior spine of the ilinm, which should be carried downward over the great trochanter in the line of the femur for about five or six inches (Fig. 468); the soft parts are dissected from the great trochanter and upper part of the shaft of the femur, and the capsule of the joint opened. While an assistant rotates the thigh inward and ontward, the muscles attached 
to the trochanters are shaved off close to the bone; the neck of the femur is next freed by the use of a knife and elevator; the thigh is adducted and pushed upward, and the head and neck of the bone are made to project from

FIG. 468.

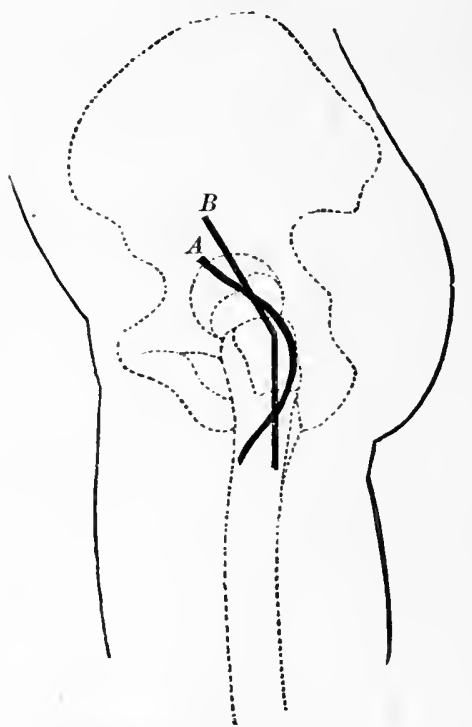

Incision for excision of the hip-joint. (STIMSON.) the wound. A transverse section of the bone is then made with a saw just below the great

\section{Fig. 469.}

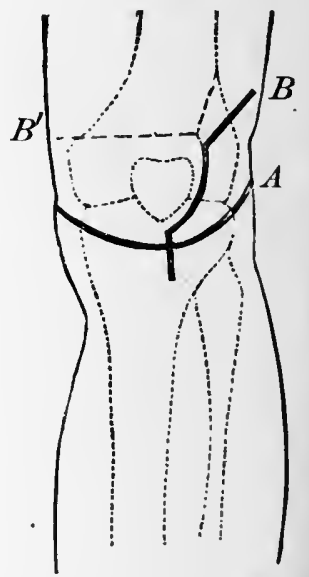

Incision for excision of the knee-joint. A. Curved incision. $B$. Angular incision. (STIMsoN.)

trochanter. In some cases it is difficult to remove the head of the bone, which may be anchylosed firmly to the acetabulum; here the bone may first be divided with a ehain-saw passed around the femur just below the great trochanter, or may be divided with a chisel, the head and neck of the bone afterward being removed with a gouge or bone-forceps.

Anterior Excision of the Hip.-In this method of excising the hip-joint, an incision is made upon the front of the thigh over the joint, beginuing half an inch, below the anterior superior spine of the ilium, and is carried three 
or four inches downward and a little inward ; as the ineision is deepened the tensor vaginæ femoris and the glutei museles are exposed, and should be drawn to the outer side, the sartorius and rectus muscles are held to the inner side and the neck of the femur exposed; the neck of the bone is then divided with a metacarpal saw or Adams' saw, the diseased portion of the bone is next grasped with strong sequestrum forceps, and by the use of these and an elevator the head of the bone is removel.

Excision of the Knee-joint.-The knee-joint is excised by making an incision which begins at the inner side of the limb over the inner condyle of the femur, and is carried over the front of the knee just below the patella to a corresponding point upon the external condyle of the femur (Fig. 469, A), or by an angular ineision (Fig. 469, B). The flap thus formed is dissected up to a point corresponding with the upper edge of the patella, the ligamentum patellæ is then eut through, the leg is slightly flexed, and the joint is opened; the lateral ligaments are then divided, and by flexing the leg upon the thigh the joint is freely exposed. The semilunar cartilages are next removed and the eondyles of the femur are freed; a narrow-bladed saw is placed under the condyles and a transverse seetion. of the condyles is removed; the head of the tibia is next eleared, and a transverse seetion of this bone is also removed with a saw. The patella may be removed before exeising the ends of the bone, or, if anchylosed to the condyles, may be removed with the scetion of bone which removes a portion of the condyles. In excising the knee-joint in young persons, care should be taken to remove only so much bone as may be done without encroaching upon the lines of epiphyseal eartilages, as removal of the epiphyseal cartilage would interfere with the subsequent growth of the bones.

Arthrectomy of the Knee-joint.-This operation is employed as a substitute for the operation of excision in discase of the knee-joint, and is performed by exposing the joint by an incision similar to that employed in excision. The ligamentum patellæ is divided and the patella 
is reflected with the skin flap. When the joint lias been freely exposed, the diseased articular cartilages, semilunar cartilages, crucial ligaments, and synovial pouches are removed by the use of the knife or scissors and the curette; if the surface of the bone is found to be carious, it is removed by the curette or gouge. After the joint has been thoroughly cleared of diseased tissue it is irrigated, the divided ligamentum patellæ is sutured with

\section{FIG. 470.}

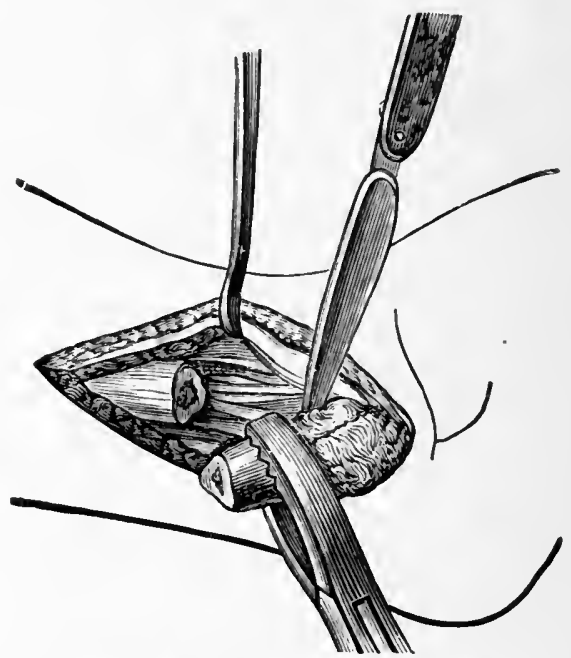

Resection of lower end of the fibula.

several strands of chromicized catgut or silk, and the wound is drained and closed.

Excision of the Patella.-The patella may be excised by making a longitudinal or crucial incision; the periosteum is carefully separated from the bone, and the latter is grasped with strong forceps and dissected free from its attachments upon the under surface. The knee-joint is generally opened in removing the patella, unless removal of the bone be undertaken for necrosis or caries, when it 
is possible to accomplish its complete removal without opening the joint.

Resection of the Tibia or Fibula.-In resecting the tibia or fibula, the bones may be exposed by a longitudinal incision over the bone to be removed, and after the shaft of the bone has been exposed and the periosteum separated as completely as possible, the shaft of the bone may be divided at its middle, and each fragment grasped with forceps and dissected up, and removed at its epiphyseal junction (Fig. 470).

Excision of the Ankle-joint.In excising the ankle-joint, an incision is made at a point two inches above the external malleolus, and carried downward over the fibula to the tip of the external malleolus; it is then curved slightly upward toward the dorsum of the foot (Fig. 471 ), care being taken that the incision does not extend so far forward as to endanger the extensor tendons or the dorsal artery. The bone is exposed in this incision and the periosteum is separated and turned aside; the peroneal tendons are next exposed and held to one side by re-

FIG. 471.

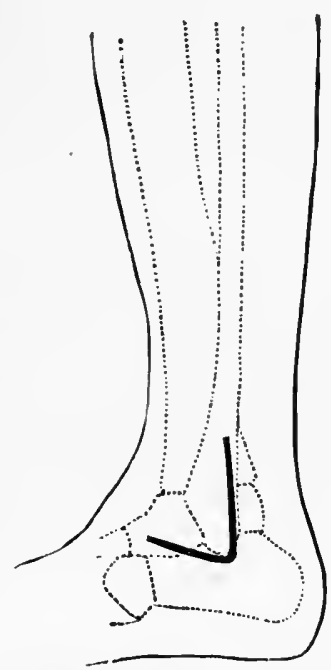

Incision for excision of the ankle-joint. (STrMsoN.) tractors; the external malleolus is next divided by bone-forceps and removed, and the astragalus exposed. The upper articulating surface of the astragalus is next removed with bone-forceps or a saw, or the whole bone may be removed. The foot is next inverted and the end of the tibia cleared with a probepointed knife, eare being taken not to injure the posterior tibial artery, nerve, or vein; and when the articular surface has been freed, it is removed with a saw or bone-forceps. The articular end of the tibia may be exposed by making an additional incision upon the 
inner side of the ankle over the internal malleolus if desired.

Excision of the Astragalus.-In excising the astragalus, a semilunar incision is made upon the outside of the ankle-joint, very similar to that employed in excising the ankle ; the external lateral ligaments are divided with a probe-pointed knife, and the astragalus is exposed by foreibly inverting the foot; the bone is then seized with strong forceps, its ligamentous attachments are divided with a probe-pointed knife, and it is removed.

Excision of the Os Calcis.-An ineision is made at the level of the upper part of the bone, beginning at the inner border of the tendo-Achilles, dividing this tendon and passing around the back and outer surfaee of the foot to the base of the fifth metatarsal bone; a short ineision is

FIG. 472.

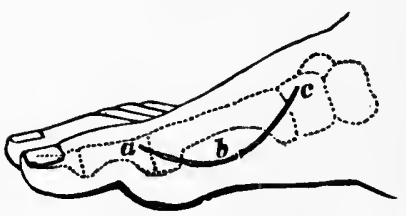

Incision for resection of the metatarsal bone of the great toe. (SMITH.) then made at the anterior end of the first incision and carried down to the sole of the foot; the bone is exposed and lield by forceps; the flap thus formed, whieh includes the peronei tendons, is then separated from the bone, and the enboid ligaments are cut and also the interosseous ligament between the os calcis and the astragalus, and the bone is removed with foreeps.

Resection of the Metatarsal Bones.-Any of the metatarsal bones may be resected by an ineision on the dorsum of the foot over the bone to be removed; the bone is exposed, the extensor tendons being held aside by retraetors; the bone is disarticulated at either end or is cut in its middle, and each fragment disseeted up and removed at its artienlation. The metatarsal bone of the great toe is exposed by making a curved incision over that bone on the inner side of the foot (Fig. 472).

Excision of the Coccyx.--In excising the coceyx, the finger is passed into the rectum and the position of the 
bone determined; a longitudinal incision through the skin and fibrous tissues covering the coeeyx is made from a point ahout a quarter of an inch above its upper limit, and is earried down to a little below its lower extremity. This ineision may be supplemented with a transverse ineision. The sacro-coecygeal artieulation is then opened ; an elevator is next introduced into the articulation and the bone is raised up and grasped with foreeps. It should then be freed from its lateral attachments, and those upon its anterior surface, with the knife and elevator.

Excision of the Upper Jaw.-In exeising one-half of the upper jaw, the ineision is begun half an inch below the

FIG. 473.

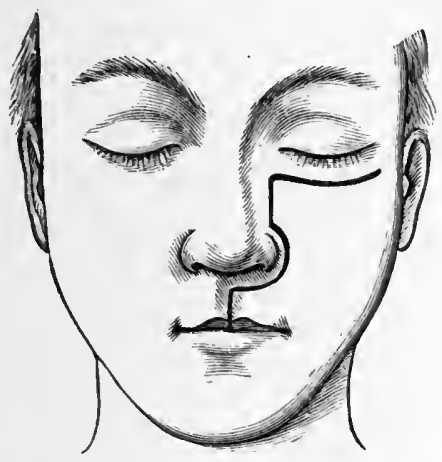

Incision for excision of the upper jaw.
FIG. 474.

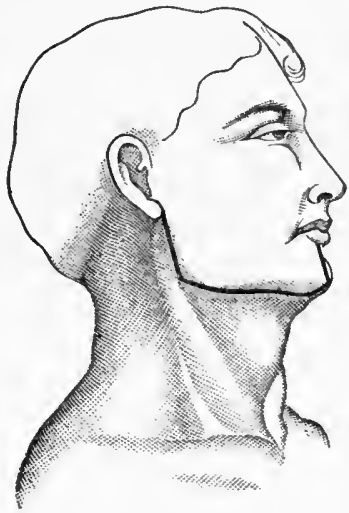

Incision for excision of the lower jaw.

inner eanthus of the eye, and is earried downward along the line of junction of the nose and cheek, along the conrse which linits the alæe nasi, and longitudinally to the septum, and then down through the free border of the lip; it is also advisable to carry the incision along the lower edge of the orbit outward over the malar bone (Fig. 473); the flap being dissected away from the surface of the bone, a small, narrow metacarpal saw is then applied to the floor of the nostril until a deep groove is made; the soft palate 
and the tissue covering the hard palate are next divided from within the month with a strong knife; one or two incisor teeth should be removed, and one blade of a pair of strong bone-forceps introduced into the floor of the nose in the line of the saw incision, the other blade is introdnced into the month in the line of the division of the structures of the palate, and the bone divided. The malar bone is next divided with a saw or forceps, and, finally, the blades of a strong pair of bone-cutting forceps are introduced, one into the nostril and the other at the edge of the orbit, the important struetures of the orbit being held upward with a retractor, and the inner angle of the orbit is eut aeross; the superior maxillary bone is then grasped with strong, lion-jawed foreeps and twisted out, any band of tissues which holds it being divided with the knife or seissors.

Excision of the Lower Jaw.-Partial or complete excision of the lower jaw may be practised.

Excision of the Ramus and Half of the Body of the Lower Jaw.-The incision shonld be made from a point just below the free border of the lip over the symphysis, and carried down to the lower border of the jaw, and from this point it is carried along the ramus to the lobe of the ear (Fig. $474)$; the flap is then dissected up, sejarating the masseter musele from the bone as far as possible without opening the eavity of the mouth; an incisor tooth is next drawn and the bone is sawed through near the symphysis ; the jaw is then seized with foreeps and drawn downward and forward and denuded upon its inner surface. The insertion of the temporal muscle into the coronoid process is divided, the condyle of the jaw is disarticulated from the glenoid cavity, and the remaining soft parts carefully detacherl with a knife or elevator. The facial artery and the inferior dental nerve and artery are necessarily diviled in removing this portion of the jaw.

Partial Excision of the Lower Jaw or Alveolus.-The removal of a portion of the alveolar process of the jaw may often be accomplished through the mouth without the aid of a cutaneous incision. The condyle of the jaw may be 
exeised by making an incision close in front of the temporal artery and carrying it forward along the zygoma for an inch and a half; the tissues being divided and the bone exposed, a seeond incision involving only the skin is then carried from the centre of the first directly downward for about an inch; the soft parts are next carefully separated with a knife and elevator from the margin of the zygoma and outer surface of the joint and drawn downward with a retractor, to prevent injury of the parotid gland, nerves, and vessels. The neek of the condyle is then cleared by working around it in front and behind with a director, keeping close to the bone to avoid injury of the internal maxillary artery. A chain-saw is then passed around the neek of the bone, which is divided, and the condyle is seized with forceps and removed with an elevator or gouge.

\section{OSTEOTOMY.}

This operation consists in dividing the bones with a saw or osteotome, and is employed to eorreet deformities of the bones or joints.

FIG. 475.

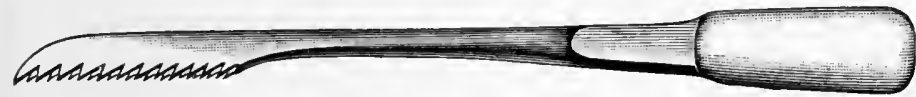

Adams's saw.

FIG. 476.

\section{GFNERT: \& 50 N}

Macewen's osteotome.

The instruments employed are a saw with short eutting surface, Adams's saw (Fig. 475), or osteotomes (Fig. 476); a heavy mallet is used to drive the osteotome through the 
bone. Osteotomy is often employed to correct deformities of the hip following coxalgia, and here the femur is divided either at the neck, Adams's operation, or just below the trochanters, Gant's operation.

Osteotomy of the Femur below the Trochanters. A puncture is made with a bistoury on the outer side of the femur just below the great trochanter, and is carried down to the bone; the blade of the saw is then introduced and the femur divided from before backward. The femur may also be divided in this position with an osteotome.

\section{Osteotomy for Knock-knee.-}

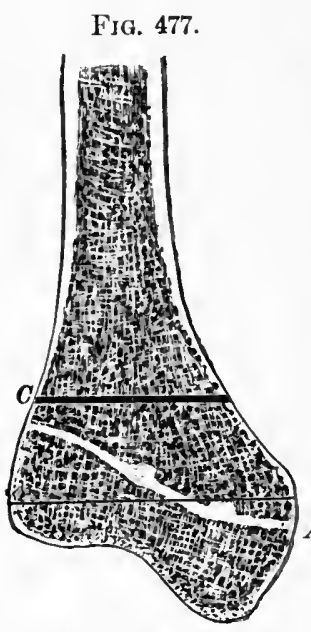

A. Epiphyseal line. $C$. Line of bone section in supracondyloid osteotomy.
The operation employed to correct this deformity is a transverse section of the femur above the condyles (Fig. 477). In the operation of supracondyloid osteotomy the lnee is flexed and supported on a sandbag. A longitudinal incision one inch in length is made half an inch anterior to the tendon of the adductor magnus and a finger-breadth above the internal condyle; the knife is earried down to the bone, and before it is withdrawn an osteotome is introduced and its edge turned so as to divide the bone transversely. The section of the bone is accomplished by the use of the osteotome and mallet. After the bone has been divided, the deformity is corrected, the wound closed, and the limb put up in a plaster-of-Paris dressing in the corrected position.

Osteotomy for Bowlegs.- To correct this deformity, the tibia and fibula are divided at the point of greatest bowing with an osteotome. The fibula is divided first with an osteotome entered through a puncture over the fibula, and next the tibia is divided in the same manner. The bones being divided, the deformity is corrected and the limb put up in a plaster-of-Paris dressing in the cor- 
rected position. Osteotomy may also be employed to eorreet deformities in other bones, or for the deformity resulting from fractures united in faulty position.

\section{TREPHINING THE SKULL.}

This is an operation in which a dist of bone of the skull is removed by a circular saw or trephine to expose the membranes and the brain. If a wound is already present in the scalp, exposing the skull, as in the case of compound fracture of the skull, it is exposed and bared, so that the crown of the trephine may be placed fairly on the bone; if no wound exists, a U-shaped flap is made, including all the structures down to the bone. The base of the flap should be so situated as to contain a sufficient blood-supply, and the flap should be so planned as to favor drainage from the wound. When the bone has been exposed, the trephine is placed with the centre pin projecting about one-sixteenth of an inch, and the instrument is turned from right to left until a groove is made in the bone; the trephine is then removed, and the centre pin is raised so that as the teeth of the trephine approach the inner table of the FIa. 478. skull the point of the centre pin will not injure the membranes or brain. The instrument is then reapplied and worked cautiously as the groove in the bone is deepened. When the diploë is reached, there is usually some bleeding from the wound, and as the trephine approaches the inner table of the skull it should be manipulated with great care, and when the resistance is felt to diminish at any one part of the bone the trephine is made to cut at other points of the bone where resistance is still apparent. When the disk is completely cut through, it may be lifted ont in the crown of the trephine or may be removed with forceps or an elevator. If the opening in the skull has to 
be enlarged to obtain greater exposure of the membranes or brain, it may be done very satisfactorily with a form of rongeur forceps.

A portion of the skull may also be removed by the use of the gouge and mallet; the gouge is generally preferred to the trephine in opening the mastoid cells.

FIG. 479.

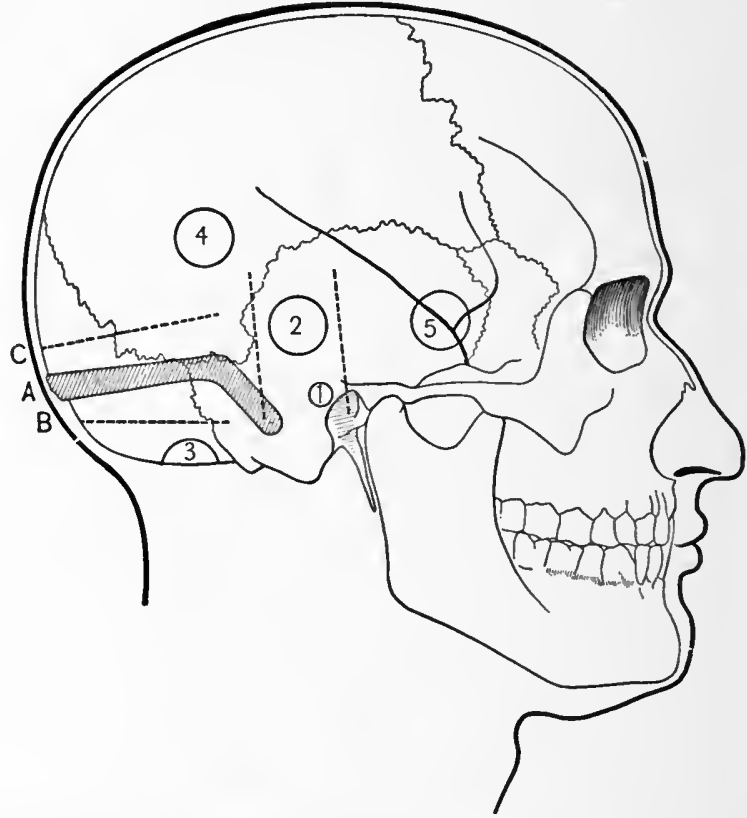

1. Trephine opening for mastoid antrum. 2. For abscess from otitis media. 3. To expose cerebellum. 4,5. For middle meningeal hemorrhage. A, Lateral sinus. $B$ and $C$. Limit of up and down variation. (STimsos.)

When the trephine is applied to expose the seat of hemorrhage from the middle meningeal artery, or hemorrhage from the lateral sinus, or an abscess from middle-ear disease, or to open the mastoid antrum, the positions for the application of the trephine are indicated in Fig. 479. 
Osteoplastic Resection of the Skull.-In this operation for exposing the membranes of the brain, a portion of the skull having the soft parts attached is turned aside, so that it may subsequently be replaced and sutured in its original position. The operation is frequently employed to expose the ganglia at the base of the brain and in the removal of tumors of the brain. A horseshoeshaped incision is made, and the edges are allowed to retract (Fig. 480). A groove is next cut through the bone, following the line to which the skin flap has retracted, with a chisel or with a circular saw run by a dental engine or electric motor. The line of division of the bone should be oblique, so that the outer table of the flap rests upon the inner table of the skull when the bone flap is turned back into place. The base of the bone flap is then partly divided with the chisel, with as little disturbance of the soft parts as possible, and the remaining bone in the base of the flap is broken and the flap turned back, the scalp acting as a hinge (Fig. 481).

Gigli's wire saw may be used in operating upon the skull. Two small trephine openings are made, and a flat director passed into one of the openings, to separate the dura on a line between them, and the wire saw drawn through this space by a thread attached to a flexible silver probe. The bridge of bone is then divided by the saw. Any desired amount of bone can be removed by making three or four trephine openings and sawing between them.

If the osteoplastic flap method is employed, the skin is left undivided on one side and adherent to the bone flap, and the saw is made to cut the bridge of bone between the trephine openings obliquely, so as to bevel the edges of the flap.

An instrument for osteoplastic resection or trephining of the skull, which accomplishes the object more rapidly, has recently been introduced by Dr. T. C. Stellwagen, Jr.

Trephining the Antrum of Highmore.-The antrum may be opened by extracting the first or second molar tooth and deepening its socket with a small gouge or bone drill.

The antrum may; also be opened through the mouth, to 
FIG. 480.

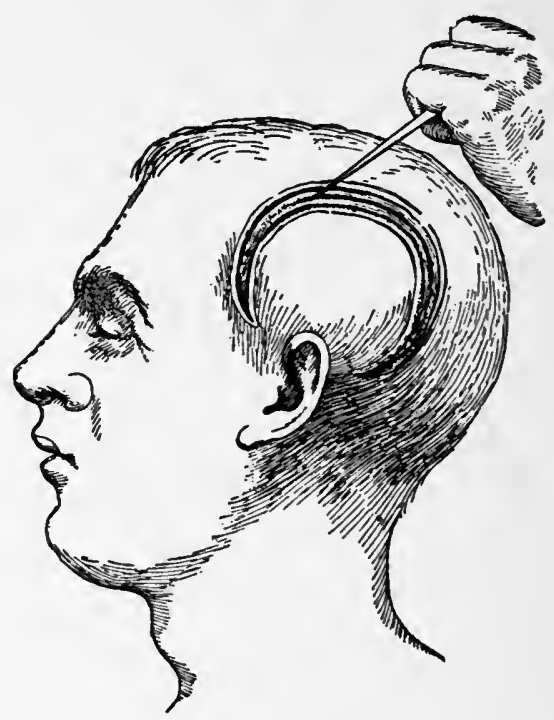

FIG. 481.

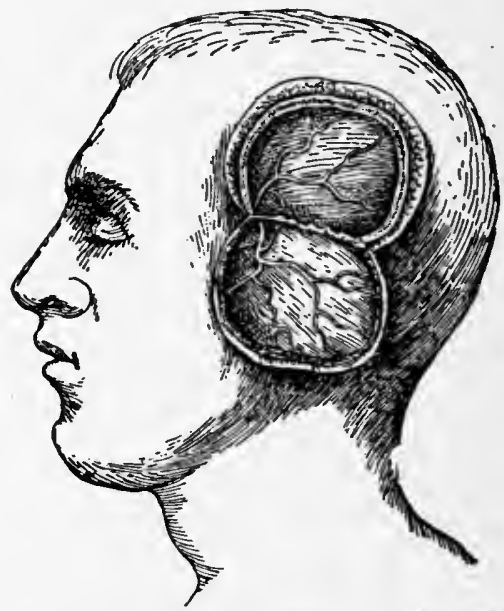

Osteoplastic resection of the skull. (After Treves.) 
avoid a sear upon the face, by the use of a small trephine or bone-gonge; the gingivo-labial fold is divided up to a point just below the infra-orbital foramen, the trephine is placed here, and a disk of bone removed, opening the antrum.

Trephining the Frontal Sinus.-This sinus may be opened by a trephine or bone-gouge. An ineision is made from the centre of the supra-orbital ridge to the median line above the root of the nose. 'The tissues are divided down to the periosteum; this is incised and turned aside, the trephine or gouge is placed at the centre of the incision near the inner edge of the supra-orbital ridge and a disk of bone is removed, exposing the frontal sinus.

Lumbar Puncture.-This procedure is frequently employed for diagnostic and therapeutic purposes.

The skin of the lumbar region is carefully sterilized and may be anæsthetized by injecting a few drops of Schlesich's solution. The patient is next made to bend forward and the tip of the spinous process of the fourth lumbar vertebre is located by the left index finger. The needie attached to an empty syringe is made to penetrate the skin one half an inch to the right and just below the tip of this process and is pushed forward, inward (toward the median line) and slightly upward into the intraspinous space between the fourth and fifth lumbar vertebræ (Fig. 482). The entrance of the needle into the subarachnoid space is shown by the sense of lessened resistance. The distance penetrated is from two-and-half to three inches. If the needle is in the subarachnoid space

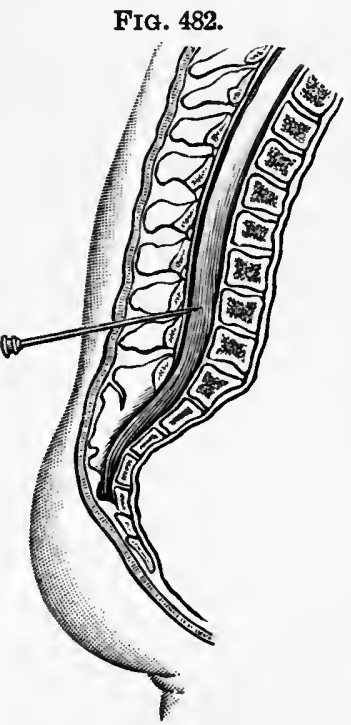

Lumbar puncture showing position of needle. (After position of 
upon drawing out the piston of the syringe, clear cerebrospinal fluid will appear in the syringe.

After removing a sufficient quantity of fluid the needle is withdrawn and the small wound is sealed with gauze and collodion.

Laminectomy. - This operation, which consists in exposing and cutting away the arches of the vertebræ, to secure a free exposure of the spinal canal and cord, is resorted to in cases of fracture of the verteira, tumors of the spinal cord, and in cases of tubercnlosis of the spine in which there is marked deformity with paralysis, the object being, as a rule, to relieve the spinal cord from pressure. A straight incision, four or five inches in length, is made over the point at which the arches of the vertebra are to be removed, and the skin, museles, and fascia are divided, and the spinous processes and arches of the vertebræ are laid bare. 'Then with strong boneforceps the arches of the vertebræ on each side are divided, care being taken to avoid injuring the dura. A better method is the formation of a lateral flap by an incision over the arches upon one sicle, the periosteum and muscles being reflected to the base of the spinous processes, the latter then being divided with bone-forceps or chisel and lifted up in the flap, the dissection of which is continued toward the other side until the arehes are exposed from end to end. The latter are then cut away. It is often neeessiry to remove several lamina if any considerable amount of the spinal eord or canal is to be exposed.

\section{OPERATIONS UPON NERVES.}

Neurotomy.-Neurotomy is an operation in which the nerve-trunk is exposed and a section made through the nerve. As in the case of ligation of vessels, it is most important that the operator should lave an accurate knowledge of the anatomical relations of the nerves and the surrounding structures. The nerve is exposed by an incision similar to that for the exposure of an artery for the application of a ligature. 
Nerve-stretching, or Neurectasy.-In the operation of neurectasy, or stretching of nerves, the nerve is exposed and isolated and is lifted upon a blunt hook or retractor; or, in case of the larger nerves, is hooked out of the wound by the finger, and is thoronghly stretched and replaced in the wound, and the latter closed with sutures.

Neurectomy.-In this operation the nerve is exposed and a portion of the nerve is excised.

Suture of Nerves, or Neurorrhaphy.-In bringing into apposition the ends of divided nerves, primary or secondary sutures may be employed. The material employed for sutures should be fine silk or fine ehromicized eatgut. In using primary sutures, the suture in the case of the smaller nerves should be passed through the sheath and substance of the nerve, and in the larger nerves two sets of sutures may be used, one passing through the substance of the nerve, the other through the sheath.

Nerve-grafting.-In employing secondary sutures to unite the divided ends of nerves when there has been a

Fra. 483.

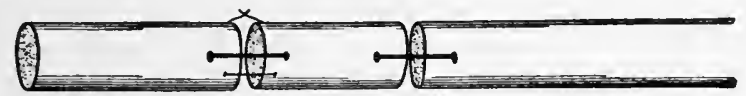

Nerve-grafting. (WiLlaRD.)

loss of substance in the nerve, or there has been so much retraction of the nerve that it is impossible to bring the ends together, nerve-grafting may be made use of; the ends of the nerve being freshened, a section of a fresh nerve from an amputated limb or animal is sutured to the ends of the divided nerve to fill up the gap, as seen in Fig. 483.

Nerve-implantation.-This operation consists in suturing a healthy merve, either in whole or in part, to a paralyzed one. Implantation is praetised by inserting the cut end of a nerve into an incision made into the sheath of an adjacent nerve and securing it in this position by sutures. Both the upper and lower ends of a nerve may be implanted into a neighboring nerve in this manner. 
Neuroplasty.-Another method of lengthening the ends of a divided nerve, known as neuroplasty, may be employed where the ends cannot be brought into apposition by the ordinary method; in this method flaps are made for the nerve in the same way as in the lengthening of shortened tendons, and the ends of the flaps are sutured

FIG. 484.

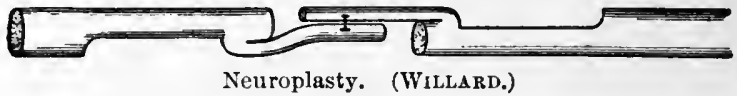

together, as seen in Fig. 484. Sutures a distancé may also be employed, as in the case of the separated ends of tendons.

The following incisions are given to expose the nerves for some of these various operations :

The Supra-orbital Nerve.-This nerve is exposed at the supra-orbital notch at the junction of the middle and inner thirds of the supra-orbital arch. An incision is made one and a half inches in length, parallel to the eyebrow (Fig. 485, $A$ and $B$ ), and is carried down to the bone; the nerve is exposed and grasped with forceps, and resected or stretehed as may be desired.

The Superior Maxillary Nerve.-A vertical incision is made along the inner side of the nose from the bony ridge of the nasal process of the superior maxillary bone to the ala of the nose; a second incision is begun at the upper part of this incision and carried outward along the lower margin of the orbit beyond its centre (Fig. 485, $C$ ); the lower flap is dissected up and the infra-orbital nerve exposed. The upper flap is next lifted up - with the lower eyelid and eyeball, exposing the floor of the orbit, and the infra-orbital canal may be recognized running backward and inward; the canal is opened with a knife or chisel, and the nerve separated from the artery and cut off as far lack as may be neeessary. The nerve may also be reached by exposing the anterior wall of the antrum, and trephining this and the posterior wall, and, when found, may be cut off close to the exit of the main trunk from the round foramen in the sphenoid bone. 
The Inferior Dental Nerve.-To expose this nerve, an incision is made along the lower jaw, from a point just behind the angle, and carried forward to a point just in front of the edge of the masseter muscle; the periosteum and masseter muscle are then separated from the bone with an elevator, and the inferior dental canal opened with a small trephine or chisel; the exposed nerve is then raised upon a hook and resected.

The Lingual Nerve.-The lingual nerve may be felt just behind the attachment of the pterygo-maxillary ligament, on the inner side of the lower jaw, close to the bone, below the last molar tooth; the tongue should be drawn to one

FIG. 485.

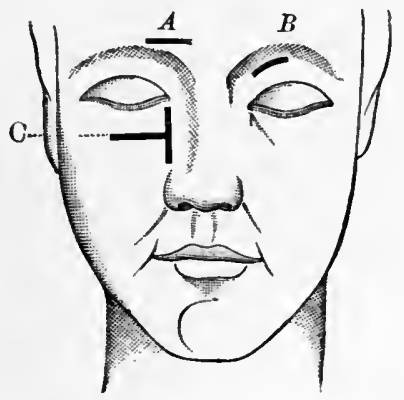

$A$ and $B$. Incisions for resection of supra-orbital nerve. $C$. Incision for resection of the superior maxillary nerve.

side and the mucous membrane divided for an inch, parallel to the alveolar process, beginning at the last molar tooth; the nerve is then found in the submucous tissue.

The Facial Nerve.- This nerve may be exposed at the posterior border of the ramus of the jaw by an incision extending from just in front of the tragus of the ear to the angle of the jaw. The parotid fascia is divided, the cervicofacial branch is exposed first, and may be followed back to its junction with the temporo-facial branch.

The Brachial Plexus.-'The brachial plexus consists of the four lower cervical nerves and the greater part of the 
first dorsal ; it lies between the anterior and middle scaleni muscles and crosses the floor of the subclavian triangle at the base of the neck. To expose the brachial plexus, the neck and head are extended and the face turned toward the opposite side; an incision is made half an inch above the clavicle, between the sterno-cleido-mastoid and trapezius muscles, and carried forward for about three inclies parallel to the anterior border of the trapezius. The skin

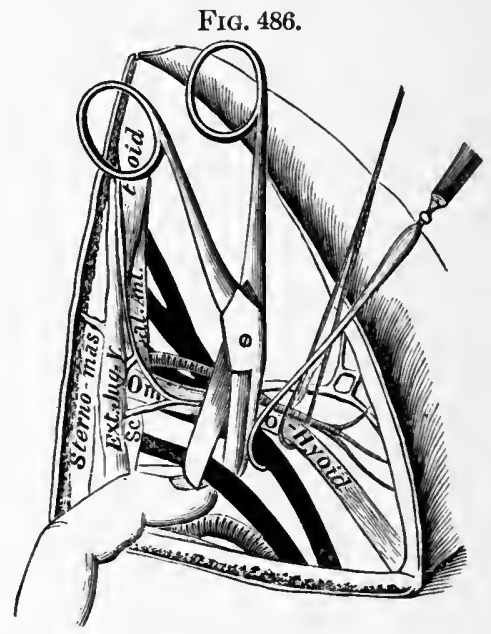

Resection of the brachial plexus.

and platysma are divided, and the external jugular veiis is either cut and ligated or held to one side; the deep cervical fascia is next opened in the line of the external incision, and the outer border of the anterior scalene muscle felt for; the brachial plexus is found just outside the latter, and is exposed by careful dissection (Fig. 486).

The Spinal Accessory Nerve.-To expose the spinal accessory nerve, an incision about three inches in length is made downward from the tip of the mastoid process along the anterior border of the sterno-mastoid muscle; the cervical fascia should be divided and the muscle strongly retracted, 
to put the nerve on the stretch. The nerve should be found external to the jugular vein, about an inch and a half below the tip of the mastoid process, on the fascia covering the rectus capitis anticus major.

The Median Nerve. - The median nerve may be exposed at the bend of the elbow or just above the wrist. To expose the median nerve at the bend of the elbow, an incision is made about an inch and a half in length upon the inner edge of the biceps tendon; the bicipital fascia is divided and the nerve exposed at the inner side of the brachial artery. The median nerve may also be exposed above the wrist by an incision two inches in length along the inner border of the tendon of the palmaris longus muscle.

The Ulnar and Radial Nerves.-These nerves may be exposed by an incision similar to that employed for ligation of the ulnar or radial artery.

The Musculo-spiral Nerve.-The musculo-spiral nerve is exposed by an incision on the outer side of the arm above the elbow, from the upper part of the supinator groove; the fascia being divided, the nerve is sought for at the bottom of this groove.

The Great Sciatic Nerve.-To expose the great sciatic nerve, an incision three or four inches in length is made vertically downward from the gluteal fold at a point midway between the tuberosity of the ischium and the great trochanter; the skin and fascia being divided, the lower border of the gluteus maximus and the hamstring muscles are exposed; the nerve rests on the external rotators of the thigh just in front of the outer side of the hamstring muscles.

The Internal Popliteal Nerve.-This nerve is exposed by an incision two inches in length in the middle of the popliteal space. The nerve is slightly external to the vein and artery, and is more superficially placed.

The External Popliteal Nerve.-This nerve is exposed by an incision two inches in length, parallel and close to the inner side of the biceps tendon, and lies close behind and to the inner side of this tendon. 
The Anterior Crural Nerve.-This nerve is exposed by an incision about two inches in length, extending from Poupart's ligament downward, and about an inch to the outer side of the femoral artery.

\section{OPERATIONS UPON TENDONS.}

Tenotomy.-This operation consists in the division of a tendon, and it may be done subcutaneously or by an open operation. The former method of tenotomy is to be preferred in most cases, but in certain tendons which lie in close proximity to important vessels and nerves it is safer to employ the open operation. In dividing tendons, the parts should be placed in such a position as to render

FIG. 487.

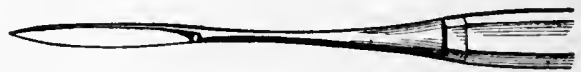

Sharp-pointed tenotome.

the tendons tense. The instruments required are a sharpand a blunt-pointed tenotome. The sharp-pointed tenotome (Fig. 487) is used to make a puneture down to the edge of the tendon, being entered flatwise ; it is then withdrawn and a blunt-pointed tenotome (Fig. 488) introduced through the puncture, passed under the tendon, and turned so that the tendon rests upon its cutting edge; by a gentle rocking motion the tendon is then divided, and the tenotome turned flatwise and withdrawn.

FIG. 488.

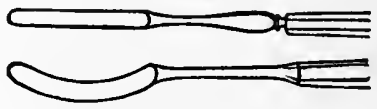

Blunt-pointed tenotomes.

The Tendo-Achillis.-In dividing this tendon, a sharppointed tenotome should be entered at the inner border 
of the tendon about an inch above its attachment to the calcaneum (Fig. 489); the heel should be depressed as

Fig. 489.

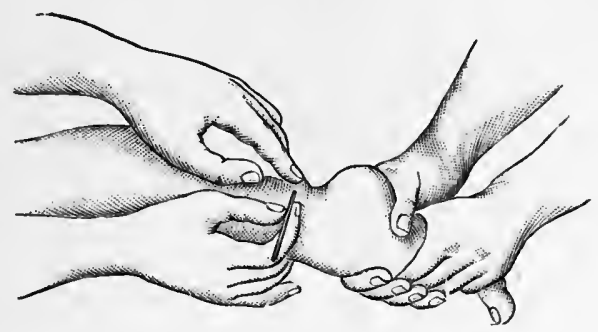

Tenotomy of tendo-Achillis.

much as possible, so as to make the tendon prominent, and a sharp-pointed tenotome passed through the skin and behind the tendon; it is next withdrawn and a bluntpointed tenotome introduced and the tendon divided. The posterior tibial artery, nerve, and vein lie to the inner side, and are not likely to be injured if the tendon is divided at this point.

The Posterior Tibial Tendon.-This tendon may be divided above the inner malleolus. The musele is made tense by everting the foot, and the tenotome is entered at the inner side of the tendon and passed behind it. The posterior tibial tendon may also be divided upon the side of the foot; for this operation the foot is everted, and the tenotome is passed from above downward and under the upper border of the tendon at a point half an inch below and in front of the tip of the internal malleolus. To avoid injury of the posterior tibial artery and nerve the open operation is generally practised upon this tendon.

The Anterior Tibial Tendon.-This tendon is divided upon the dorsal surface of the foot, just below the annular ligament of the ankle, midway between the two malleoli.

The Peroneal Tendons.-The peroneal tendons may be 
divided about an inch above the external malleolus, the tenotome being passed from before backward between the fibula and the tendons, or the tendons may be divided at a point midway between the end of the external malleolus and the tubercle of the cuboid.

The Hamstring Tendons.-The inner hamstring consists of the tendons of the semi-tendinosus, semi-membranosus, gracilis, and sartorius. The external hamstring consists of the tendon of the biceps. To divide either of these tendons, the knife is entered at the inner side of the tendon. In dividing the external hamstring, care should be taken to keep close to the tendon of the biceps, as the external popliteal nerve lies close to its inner border. For this reason the open operation is advisable upon this tendon.

The Adductor Longus.-To divide this tendon, abduct the thigh and make the muscle prominent near its insertion; then pass the tenotome from without downward and inward.

The Flexor Longus Pollicis.--This tendon may be divided on the first phalanx or near the inner edge of the foot, where it may be made prominent by strong extension of the great toe, the tenotome being passed close to the border of the tendon.

The Extensor Longus Digitorum.-These tendons are divided upon the dorsal surface of the metatarsal bones, where they are quite prominent. They may also be divided near the ankle.

The Extensor Proprius Pollicis.-This tendon may be divided in the same incision used for division of the long extensor of the toes, the point of the knife being carried inward.

The Sterno-cleido-mastoid Muscle.-In tenotomy of this muscle, the sternal and clavicular attachments are divided about an inch above the sternum and clavicle. A puncture is made to the outer side of the muscle with a sharp tenotome, and when the tendinous expansion of the muscle is reached it is withdrawn, a blunt tenotome substituted, and 
the structure divided (Fig. 490). The sternal attachment is in like manner divided through a separate puncture. The external jugular vein at the outer border of the muscle is to be avoided. The division of the muscle, or its tendinous expausion by an open operation, is a better operation, as there is less risk of injuring the vein by this procedure.

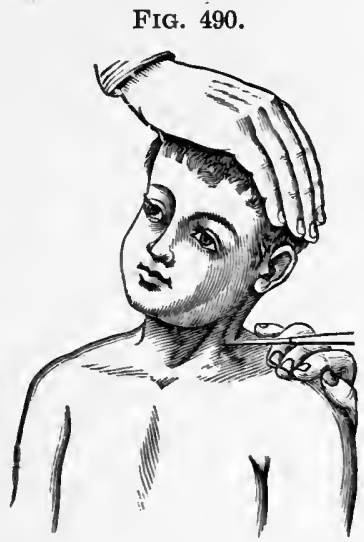

Tenotomy of sterno-mastoid.

Suture of Tendons.-In bringing together the divided ends of tendons, primary or secondary sutures are employed ; primary sutures are those introduced immediately after the injury, and secondary sutures are those intrcduced after retraction of the ends has occurred and the wound has healed.

Primary Suture of Tendons. - The material employed for sutures may be silk, silkworm-gut, catgut, or kangarootendon, and one or more sutures should be used, being passed through the substance of the ends of the tendon and secured by tying; the divided sheath of the tendon, if possible, should be brought together by fine silk sutures (Fig. 491). Very marked retraction of the ends of the 
tendon is liable to occur, and a considerable dissection is often required to bring them into view.

Fig. 491.

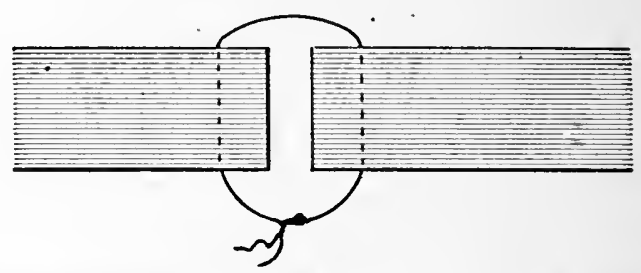

Suture passed through the substance of the ends of a divided tendon.

When there is diffienlty in bringing the ends of the tendon together, and the sutures are apt to cut out, the form of suture shown in Fig. 492 may be employed.

FiG. 492.

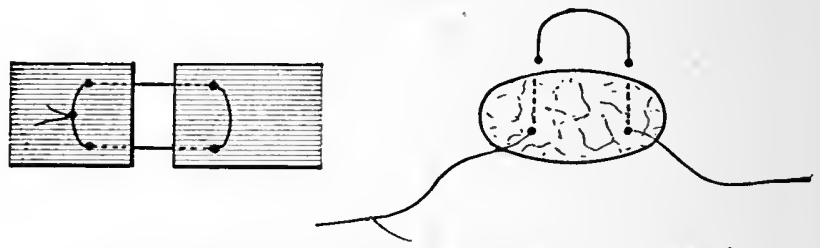

Tendon-suture which does not easily tear out. (STIMson.)

Secondary Suture of Tendons.-In applying secondary sutures to tendons, the principal difficulty is often encountered in bringing the ends of the tendon in contact and in holding them successfully in this position. The ends of the tendon have first to be freshened, and this may be done by cutting them obliquely and introducing a suture as shown in Fig. 493. This method of section presents a large raw surface of the tendon for union.

Lengthening of Tendons. - When so large a gap exists between the ends of the tendon that they cannot be brought into apposition, a plastic operation may be performed upon their ends, which often overcomes the difficulty. This 
consists in making a seetion halfway through the tendons, at some distance from their ends, and splitting them toward their divided extremities, and then turning out these flaps and securing their ends by means of sutures (Fig. 494).

FIG. 493.

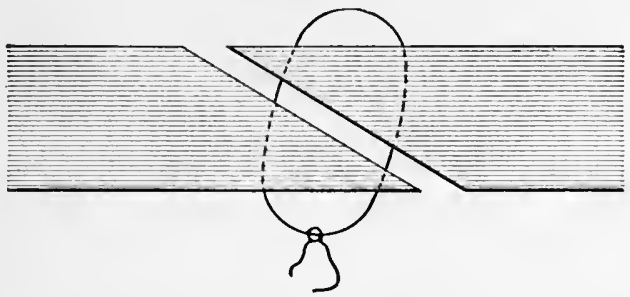

Oblique section of ends of tendon to increase surface of contact. (STIMson.)

When the ends of the tendon are so widely separated that they cannot be approximated, sutures a distancé may be employed. These consist of sutures of sterilized silk or ehromicized catgut passed between the ends of the tendon and tied, the sutures acting as a scaffolding upon which

FIG 494.
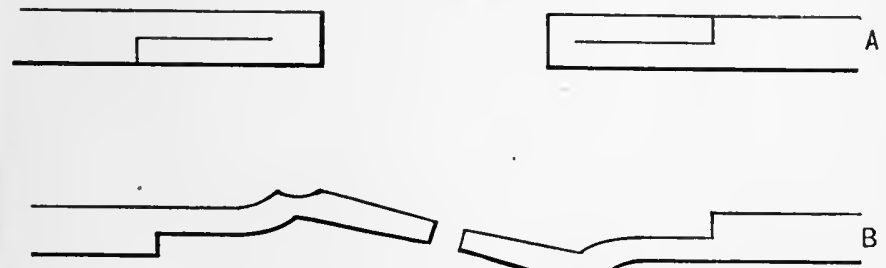

Lengthening of retracted tendon by flaps. (STrmsor.)

reparative material forms between the separated ends of the tendon.

Transplantation of Tendons.-This operation consists in altering the attachments of the tendons of healthy muscles so as to have them fulfil the functions of those 
which are paralyzed. Four methods of transplantation are practised : first, the tendon of the healthy muscle may be completely divided and the upper end sutured to the paralyzed tendon; second, the tendon of the paralyzed muscle may be divided and the lower end sutured to the healthy one; third, the tendon of the sound muscle may be split, one end remaining attached to its normal insertion, and the other sutured to the paralyzed tendon; fourth, a portion or the whole of the healthy tendon may be implanted subperiosteally at the desired point, instead of stitching it to the paralyzed tendon.

\section{ORDINARY EXCISION OF THE MAMMARY GLAND.}

The region of the neek, shoulder, upper arm, breast, and side-wall of the ehest should be rendered aseptic by the usual preparation, the axilla being thoroughly shaved. The arm should be held at a right angle with the body.

An elliptical incision should be made, with its long axis parallel with the anterior axillary fold, its centre corresponding with the nipple. The incision is continued into the axilla, over the upper point of the ellipse, and one also made extending a short distance below its lower portion. The skin and superficial and connective tissue are then divided, bleeding vessels being clamped with hæmostatic forceps, and the fibres of the pectoral musele are exposed. Drawing the breast away from the thoracie wall, the surgeon frees it from the peetoralis major muscle. The breast is then drawn upward, and the surgeon divides the tissues down to the thoracic museles along the lower line of the ellipse. The connective tissue of the breast is now severed towards its axillary aspeet, and the breast is freed from the chest. If it is desired to explore the axilla, the incision is carried further upward, and enlarged glands are felt for and removed. After hemorrhage has been controlled, the edges of the wound are brought together with silkworm-gut sutures. A drainage-tube may be introduced if it is considered desirable. 


\section{OPERATION FOR RADICAL REMOVAL OF THE BREAST.}

The arm should be held at a right angle to the body and an incision made over the insertion of the pectoralis major muscle into the humerus, over the point of the shoulder, and then downward on the chest-wall to the inner side of the nipple to a point two or three inches below the lower margin of the gland. From the upper portion of this incision a second incision is carried downward along the edge of the pectoral muscle, and curved inward, to meet the lower extremity of the first incision. These incisions are carried through the skin and superficial fascia, which are dissected, so as to expose the pectoral muscle throughout its entire length. The tendon of the muscle is next separated from its attachment to the humerus and retracted downwards, and the attachment of the pectoralis minor muscle exposed and severed; the clavicular fibres of the pectoralis major may be left. The axillary space is now exposed, which is next cleared, from above downward, of all fascia, lymphaties, and connective tissue, care being taken to preserve, if possible, at the lower portion, the subscapular and posterior thoracic nerves. After clearing the axillary space, the costal and sternal attachments of the pectoralis major muscle are divided, as well as those of the pectoralis minor. 'The entire mass, consisting of the mammary gland, both muscles, the axillary glands, and the connective tissue, is then removed. If it is desired to remove the connective tissue and glands from the supraclavicular space, a curved or straight incision several inches in length is made above the clavicle, and the lymphatics and connective tissue along the subclavian vessels and behind the sterno-mastoid muscle are removed. Before bringing together the edges of the wound a small opening is usually made for drainage through the axillary flap, and a cigarette drain or rubber drainagetube introduced. The incisions are then brought together 
with silkworm-gut sutures, and a large gauze dressing is applied over the entire wound, and the arm is secured to the side of the chest by circular turns of a bandage.

\section{TRACHEOTOMY.}

This operation consists in dividing the tissues over the trachea in the median line of the neck, and after the trachea has been exposed it is opened by dividing two or three of the tracheal rings.

Under certain circumstances the operation may be performed with very few instruments; but if the surgeon has the choice, he will find it convenient to have at hand two small scalpels, one short grooved director, a tenaculum, two aneurism needles (which may be used as retractors), one pair of artery forceps, hæmostatic forceps, two pairs of dissecting-forceps, a pair of scissors, a sharp-pointed tenotome, a pair of tracheal forceps, a tracheal dilator, tracheotomy tubes, tapes, ligatures, sponges, a flexible catheter, and feathers. The director should be short; the ordinary grooved director is too long to use with satisfaction in operating upon the short necks of children; so that I use a shorter and somewhat broader one, having a bevelled extremity, which allows it to be passed with ease between the different layers of the tissues (Fig. 495).

FIG. 495.

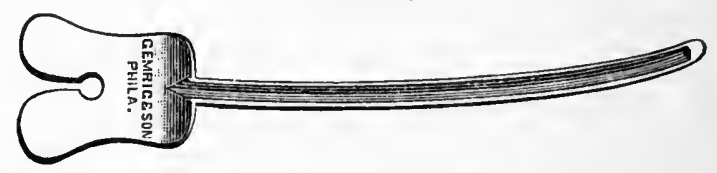

Author's tracheotomy director.

Hcemostatic forceps are also useful in controlling hemorrhage during the operation in case of the division of vessels which bleed freely, when the operator from the urgency of the case does not think it justifiable to ligate 
them at the time of their division. They may also be employed under similar cireumstances to elamp the isthmus of the thyroid gland on either side of the trachea when it beeomes necessary to divide it to expose the trachea.

A sharp-pointed tenotome is the instrument I prefer to employ in opening the trachea, as its sharp point enables it to be easily thrust into the trachea.

Tracheal dilators of various kinds are employed, but the most satisfactory tracheal dilator which I have employed is that of Golding-Bird (Fig. 496), which is a self-retaining instrument; the blades are slipped through the tracheal incision and are then expanded by turning the screw to which they are attached. Trousseau's tracheal dilator, the blades of which are introduced through the incision in the trachea and are expanded by bringing together the handles, is also a satisfaetory instrument (Fig. 497 ), but it is not so useful as Golding-Bird's dilator, as it has to be retained in position by the hand. Tracheal dilators may be improvised from bent hair-pius or pieces of wire, which will often serve a useful purpose where ordinary dilators cannot be obtained.

Fia. 496.

FIG. 497.

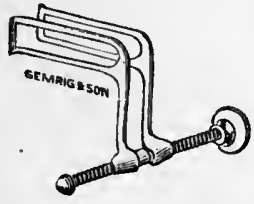

Golding-Bird's tracheal dilator.

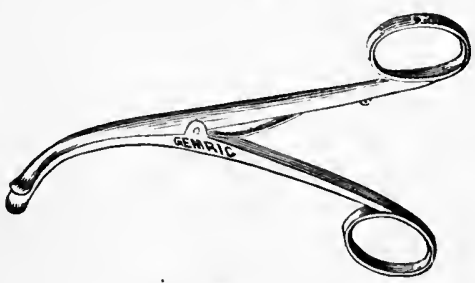

Trousseau's tracheal dilator.

It is also well to have at hand a number of pliable feathers, to be used in clearing the trachea or larynx of mueus or membrane after it has been opened; by their use this object may be aceomplished with little risk of injury to the mueous membrane. 
Tracheal forceps, which are eonstrueted with a double spring and curved blades, are also useful in removing

FIG. 498.

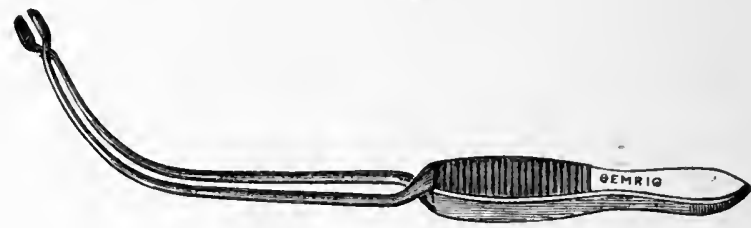

Tracheal forceps.

membrane or foreign bodies from the larynx above the wound or from the tracliea below the tracheal incision (Fig. 498).

Tracheotomy-tubes of various shapes are made of silver,

FIG. 499.

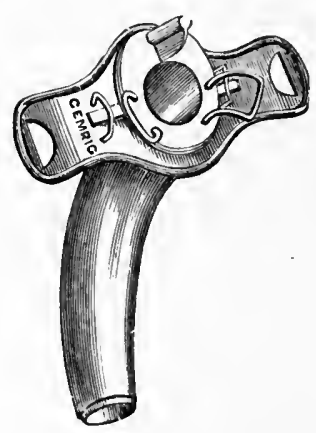

Silver tracheotomy-tube.
FIG. 500.

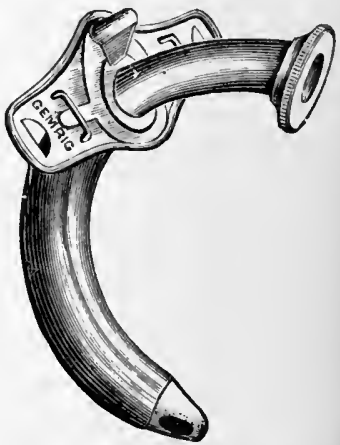

Silver tracheotomy-tube with fenestrated guide.

aluminum, hard and soft rubber, but the tube which $\mathbf{I}$ consider the most satisfactory for general use is a silver quarter-circle tube with a movable collar (Fig. 499), and provided with a fenestrated guide (Fig. 500). A satisfaetory tracheotomy-tube is one which inflicts the least possible 
injury upon the mucous membrane of the trachea, and to insure this object the part of the tube within the trachea should lie exactly in its axis, and its free extremity should be capable of as little movement as possible. The tracheotomy-tube is held in position, after being introduced, by means of tapes attached to the shield of the tube and tied around the neck.

Position of Patient for Tracheotomy.-The best position in which to place the patient for this operation is one which brings the neck into the greatest prominence, and this may best be obtained by laying the patient upon his back upon a firm table and placing under the shoulders a round eushion; or an empty wine-bottle or a roller-pin wrapped in towels, will answer the same purpose (Fig. $501)$; or the head may be held over the edge of the table. If an anæsthetic is not used, the arms should be held by an assistant, which is better than securing them by a binder around the chest, which restricts respiratory movements.

FIG. 501.

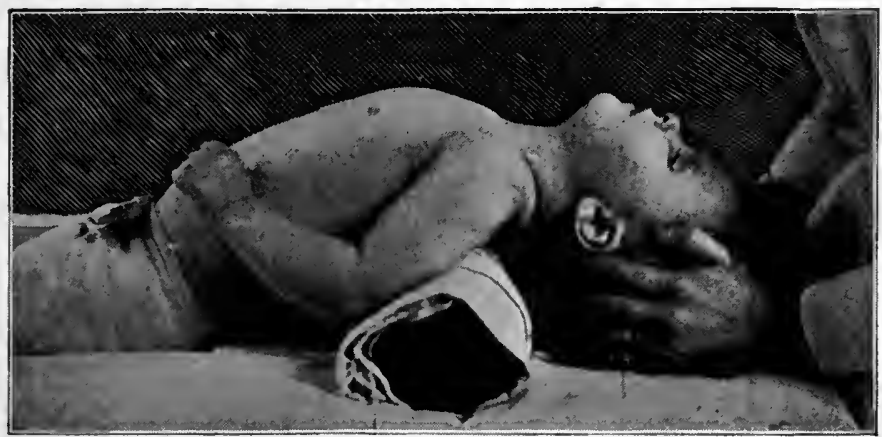

Position of patient for tracheotomy.

Operation of Tracheotomy.-The trachea may be opened above the isthmus of the thyroid gland or below it, and these operations constitute respectively the high and the low operation. 
The high operation is generally seleeted, because at this point the trachea is more superficial and is more easily exposed, whereas in the low operation the trachea is more difficult to expose by reason of its relatively greater depth, the large size and number of veins, and its proximity to the large arterial trunks.

High Operation.-The patient being placed in position, the operator stands at the head of the patient; this position I prefer, as it is easicr from this point to keep the incisions exactly in the median line of the neck. The operator next makes himself familiar with the landmarks of the neck; locating the position of the cricoid cartilage, he makes an incision through the skin in the median line of the neck from one and a half to two inches in length, the position of the erieoid cartilage being the middle point. There is no disadvantage in making a longer ineision if a freer exposure of the parts is required. Having divided the skin, the operator will often see a large vein lying in the superficial fascia-the superficial anterior jugular; this should be displaced and the fascia divided upon a director.

The surgeon should keep his incisions strietly in the median line of the neek, for this is the line of safety ; and he should be eareful, as the wound increases in depth, not to make the ineisions too short, so that the wound becomes funnel-shaped. When the deep faseia is exposed, it should be picked up and divided upon a director; any large veins in the line of the wound should be earefully displaced, or, if this is impossible, they should be ligated on each side and then divided between the ligatures.

The operator next looks for the intermuseular space between the sterno-hyoid and the sterno-thyroid muscles, which may generally be found without diffieulty; the museles are now separated in this line, with the handle of the knife or with a director, and the isthmus of the thyroid gland exposed. The muscles should now be held aside by retraetors placed on either side. He should earefully explore the wound with the finger, to locate exactly the position of the trachea, and to ascertain, if possible, the presence of anomalous arteries. 
The isthmus of the thyroid gland having been exposed, which generally occupies a position over the first three tracheal rings, the gland will be found surronnded by a plexus of veins, which should be displaced with the director; or, if this is impossible, they should be ligated on each side and divided between the ligatures. 'The thyroid isthmus is next displaced upward or downward, according as the surgeon desires to open the trachea below or above this body. This is often done without difficulty, especially its upward displacement; but when there is difficulty in displacing it downward, a procedure recommended by Bose may be employed, which consists in making a transverse incision across the cricoid cartilage to divide the layer of fascia by which the isthmus is bound down; the director is then passed into this incision and the isthmus is depressed without difficulty.

Having displaced the isthmus of the thyroid gland downward, the trachea, yellowish white in appearance, covered by the tracheal fascia, will be exposed; this fascia should next be thoroughly broken up with a director or the handle of the knife, so as to bare the trachea, and in doing this the operator may feel it crepitate under the finger from the suction of air drawn in with inspiration. The trachea is next fixed with a tenaculum, introduced into it a little to one side of the median line; an incision is made into it with a narrow knife from below upward, from onehalf to three-fourths of an inch in length (Fig. 502), care being taken to see that this incision is in the median line, for if the trachea be opened by a lateral incision the wound does not heal so promptly and the tracheotomytube does not fit well, and its lower extremity may cause injury to the mucous membrane of the trachea. If the wound be a deep one, after fixing the trachea with the tenaculum the operator may lift it slightly from its bed, thereby bringing it more prominently into view and making it more superficial in the wound, thus facilitating its opening. As soon as the incision is made into the trachea, air mixed with blood and mucus escapes from the incision. A tracheal dilator should next be introduced and the 
trachea cleared of membrane, if it is present in the region of the wound, with a feather or with forceps. The tracheotomy-tube is next introduced, and is secured in position by tapes tied around the neck. If respiration has ceased, artificial respiration should be resorted to.

FIG. 502.

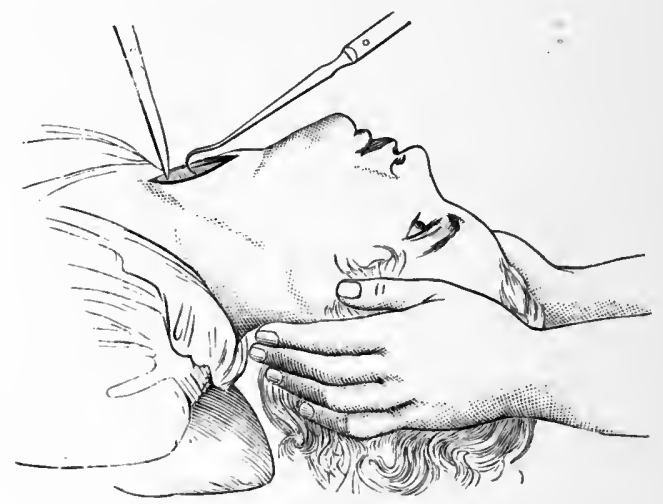

Opening of the trachea. (Liston.)

Laryngotomy.-In this operation an opening is made into the air-passages through the crico-thyroid membrane. It is a simple operation, and one which is practically free from risk, and can, therefore, be performed much more rapidly and safely in urgent cases than tracheotomy.

The patient being placed in the recumbent posture, with the shoulders slightly elevated and the head thrown back, to make the neck as prominent as possible, the surgeon feels for the prominence of the thyroid cartilage, and steadying the larynx between the finger and thumb of the left hand, he makes an incision in the median line over the centre of the thyroid cartilage and extending downward for an inch or an inch and a half. The skin and superficial fascia being divided, the fascia between the sterno-hyoid muscles and the areolar tissue is exposed and divided, and the crico-thyroid membrane is exposed. The 
knife is then passed transversely through the membrane into the larynx, care being taken that both that membrane and the mueous membrane which covers its inner surface are divided at the same time. As soon as the knife enters the cavity of the larynx blood and mueus will be forcibly expelled.

The wound should be carefully enlarged and a tube introduced, which differs from the ordinary tracheotomytube in being slightly flattened ; this is secured in position by tapes tied aromnd the neck. as in the case of the ordinary tracheal tube. The only bleeding which is likely to oceur is from the crieo-thyroid arteries or veins, and if these cannot be avoided, and are divided in the operation, they should be temporarily seeured by hæmostatic foreeps or ligated ; if the ease is not extremely urgent, all bleeding should be arrested before the erieo-thyroid membrane is ineised.

Laryngo-tracheotomy.-This operation consists in making an incision into the air-passages by dividing one or two of the upper rings of the trachea, the erico-tracheal membrane, the cricoid cartilage, and the crico-thyroid membrane. This operation is employed in eases where, from the age of the patient, the erico-thyroid space is too small to admit of a sufficient opening, or in those in which, for any reason, the surgeon does not deem it advisable to attempt to open the trachea lower down. The incision in the skin and superficial fascia of the neck is made in the same manner as in the operation of laryngotomy, but is carried a little further downward. It may be necessary to displace the isthmus of the thyroid gland downward to expose the upper portion of the trachea, and when the trachea is exposed the incision should be made through this and the erieoid cartilage from below upward. A tracheotomy-tube is introduced through the wound and seeured by tapes tied around the neck.

\section{INTUBATION OF THE LARYNX.}

This procedure, at the present time, is widely employed as a substitute for tracheotomy in the treatment of dyspnoa due to inflammatory affections of the larynx or trachea, or 
stenosis of the larynx; it consists in the introduction of a metallic or hard-rubber tube into the larynx, which is allowed to remain in place for a few days. This nperation has been reintroduced to the profession by the late Dr. O'Dwyer, of New York, who devised a set of ingenious instruments for the purpose of laryngeal intubation.

The instruments required are a mouth-gag (Fig. 503),

FIG. 503.

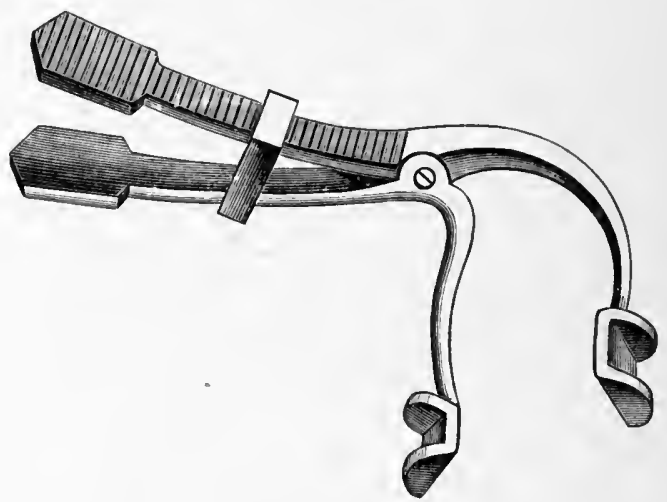

Mouth-gag.

FIG. 504.

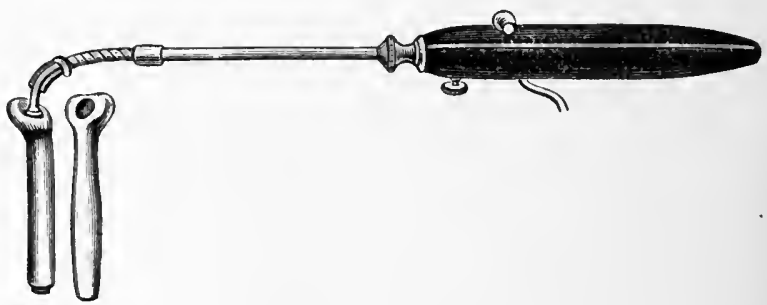

Intubation-tube and introducer.

with which the jaws are separated and hell open ; an instrument for the introduction of the tube, which is fastened 
Fig. 505.

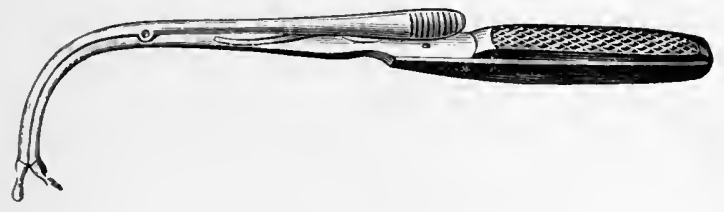

Intubation-tube extractor.

to the obturator, which fills the cavity of the tube (Fig. 504); and an instrument for extracting the Fig. 506. tube after it has been placed in the larynx

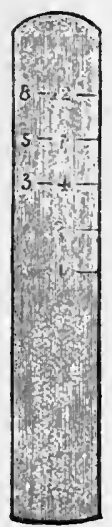

Scale of intubation-tubes. (Fig. 505). The tubes are of metal or hard rubber, and have a collar which rests upon the false cords, and bulge slightly toward their middle and again taper toward their lower extremity ; at the collar of the tube there is a perforation through which a strand of silk is passed which is made into a loop ; this is used to enable the operator to remove the tube if on its introduction it is found to have passed into the œsophagus instead of the larynx, and is also useful in removing the tube if it becomes occhnded with membrane while in the larynx. The intubation set now in common use is provided with a scale of seven tubes, ranging in size from such as are suited for a child of one year or less up to the age of twelve or fourteen years (Fig. 506). Special tubes are required for intubation in adults.

Operation of Intubation of the Larynx.-In performing the operation of intubation, the child is placed upon the lap of the nurse or assistant, wrapped in a blanket, and the arms secured by the nurse holding the elbows so as not to interfere with the respiratory movements. The patient's head is next held by an assistant. The position of the head, neck, and body should be as if the child were hung from the top of the head, and this position should be maintained during the insertion of the 
tube. An intubation tube can be introdueed without difficulty with the patient in a recumbent posture. This position should be utilized when it is not desirable to place him in the sitting posture. The month-gag is next inserted upon the left side, and the blades dilated so as to open the jaws widely, and as the gag is self-retaining, this position is easily maintained. The jaws being thus held open, the operator, sitting on a chair facing the patient (Fig. 507), next introduces the index finger of the left

FIG. 507.

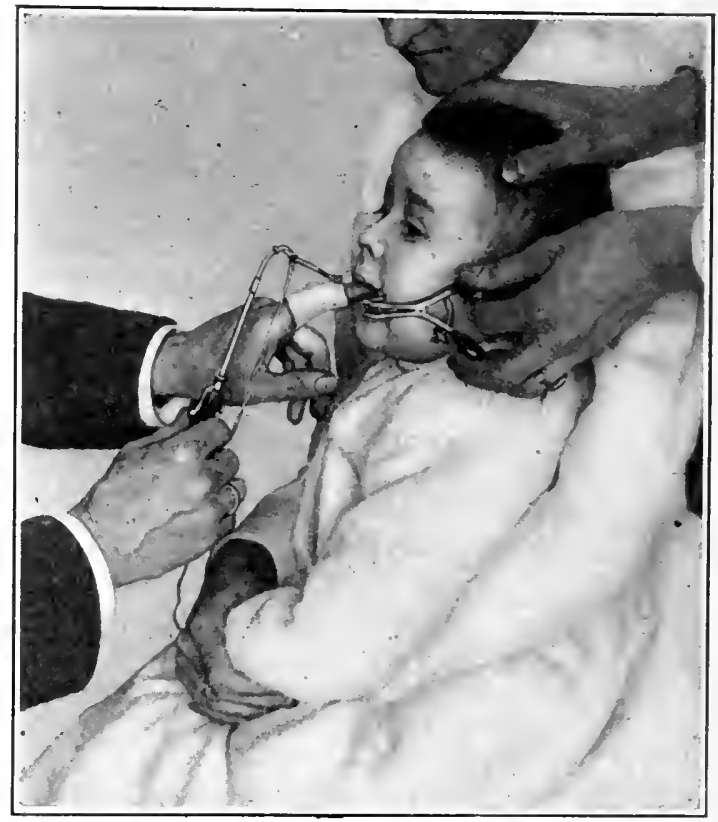

Intubation of the larynx.

hand, protected by a strip of adhesive plaster or a metal shield, into the mouth and passes it over the tongue until he feels the epiglottis. The introdueing-instrument, to which the tube is attaehed, is held in the right hand, 
and introduced iuto the mouth, after observing that the silken loop is free; it is swept over the tongue and passed down until it touches the epiglottis; this is hooked up by the index finger of the left hand and the tube passed into the larynx; the index finger of the left hand is then transferred to the edge of the tube, and by pressing upon the trigger of the instrument with the thumb of the right hand the obturator is detached and the instrument is withdrawn, and before removing the finger it is well to place it upon the head of the tube and to sink it well into the larynx. As soon as the obturator is removed, there is usually a violent expiratory effort, which is accompanied by a gush of mucus, mucopurulent matter, or membrane from the tube, and after this escapes the breathing is usually satisfactorily established. If the operator has passed the tube into the osophagus and has detached it from the introducing-instrument, no improvement in the respiration takes place; it should then be withdrawn by the silk loop attached to the tube, and another attempt made to introduce it into the larynx.

The mistake, which inexperienced operators make in attempting to introduee the tube is in not hugging the posterior surface of the tongue closely, thereby passing the tube over the epiglottis into the osophagus.

The silken loop may be brought out at one side of the mouth and adjusted around the ear or fastened to the side of the face by strips of adhesive plaster for a few hours, so that by drawing upon it the nurse or attendant can withdraw the tube instantly if it should become obstructed with membrane; or, if it is coughed up, by this means it may be withdrawn from the oesophagus if it has not been expelled from the mouth. Some operators keep the loop attached to the tube during the time it is retained in the larynx. I prefer to remove it after the tube is securely placed in the larynx, and to withdraw the tube by means of the extracting-instrument when required.

Extubation or the removal of the tube is, as a rule, more diffieult than its introduction. This is done with the patient either in the sitting position or prone, the jaws are 
held open by the mouth-gag and the operator passes his finger into the mouth and locates the epiglottis and head of the tube, the extracting instrument is next introduced and the closed blades are passed into the opening in the tube and the blades expanded and the instrument withdrawn with the tube attached. If there is difficulty in grasping the tube, it may be loosened and pushed upwards by pressure and manipulation on the neek over the upper part of the trachea and larynx, and when it protrudes from the larynx it may be grasped with forceps and removed.

FIG. 508.

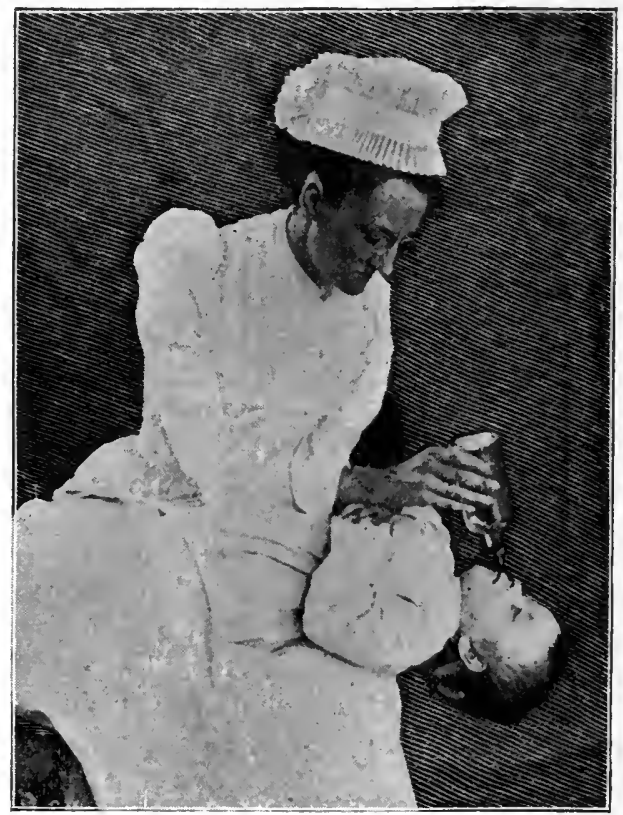

Feeding a case of intuhation of the larynx.

The tube should be removed at the end of the second or third day, and if the child can breathe comfortably for an hour or two it need not be reintroduced; if, however, the 
dyspncea returns, it should be reintroduced, and allowed to remain one or two days longer; several attempts may have to be made before the tube can be permanently removed; it is usually dispensed with from the third to the eighth day.

The most serious complication which is apt to occur during the introduction of the intubation-tube is the detachment and pushing of a mass of membrane in front of the tube into the trachea; if the mass is too large to be expelled through the tube, the breathing is suddenly arrested. The tube should be removed at once, and if the mass of membrane does not escape with the expiratory efforts of the patient, the trachea should be rapidly opened as the only means of re-establishing the respiratory function. So much do I dread this accident, which has oceurred in a few cases, that I never introduce the intubation-tube without having at hand the necessary instruments to do a tracheotomy if it should be suddenly required, and, if possible, obtain the consent of the parents or friends to perform tracheotomy if it should be indicated.

Feeding after Intubation.-One of the greatest difficulties after intubation of the larynx is the satisfactory feeding of the patient; liquids, as a rule, are not swallowed well, a portion escaping into the tube, causing coughing and difficulty in breathing. The diet I usually prefer is semisolid, such as corn-starch, soft-boiled eggs, and mush; and if these are not well swallowed, it may be necessary to resort to nutritions enemata or the use of a stomachtube to introduce food. Some patients swallow liquids and semisolids quite well if the head is placed a little lower than the body during the act of deglutition (Fig. 508).

Paracentisis Thoracis. - This may be done with an ordinary troear and canula, but it is better to employ an aspirator. For purposes of diagnosis a hypodermic syringe with a large needle may be used. The instrument and the skin over the area in which the tapping is to be made are sterilized and the patient is placed in a semirecumbent posture. The one usually selected is an intercostal space between the sixth and eighth rib in the midaxillary line. A puncture is made throngh the skin over 
the upper edge of the rib. This position is selected to prevent injury of the intercostal vessels, and the skin is drawn upward and the aspirating trocar is passed through the puncture into the chest near the upper edge of the rib, the trocar is removed, and the stopcock is turned, which allows the fluid to enter the vacuum bottle. After the fluid has been removed, the aspirating canula is removed and the small wound is sealed with pledget of gauze and collodion.

Paracentisis Abdominalis.-This operation may be required for the withdrawal of extravasated serum. It may be done with trocar and canula, or with an aspirator. The latter is better, as it prevents the admission of air. The patient should be in the sitting posture and the bladder should be empty.

The point usually selected is in the medium line, from 2 to 3 inches below the umbilicus. This is the thinnest part of the abdominal wall and is free from large vessels.

The skin and instrument being sterilized, a puncture is made through the skin with a sharp-pointed knife, and the aspirating trocar is made to enter this puncture and gently pushed through the abdominal wall. The surgeon should watch to note the depth to which the trocar is passed. The trocar is next removed and the stop-cock turned, which allows the fluid to escape in the vacuum bottle. If the fluid fails to escape, either the instrument has not been introduced sufficiently far enough or the omentum may have occluded the canula; by further introduction or changing the position of the canula the difficulty may be removed and the fluid escapes.

Sometimes, on account of the condition of the patient, it is not advisable to remove all of the fluid at one sitting. Some surgeons apply a many-tailed bandage to the abdomen with the tails secured on the back. This is gradually tightened and secured with pins as the abdomen becomes emptied of fluid.

When the fluid has been removed the canula is removed and the puncture sealed with a pledget of gauze 
and collodion. If serum continues to escape from the puncture, as often occurs, a sterilized gauze pad should be secured over the wound. This leakage usually stops within a few hours.

A firm, many-tailed bandage should next be applied and is a comfortable dressing for the patient.

Paracentisis Pericardii. - This operation may be required to remove serum or purulent fluid from the pericardium. The aspirating trocar is introduced through the skin in the fifth left intercostal space two inches to the left border of the sternum, external to the internal mammary artery. A moderate sized aspirating needle should be used, as it is advisable to have the fluid escape slowly. The needle is removed and the wound sealed with gauze and collodion. The pericardium may also be reached by a puncture in the fourth or fifth left interspace, near the border of the sternum, which avoids the pleura and internal mammary artery.

Paracentisis Vesicæ.-In cases of impassible stricture, as a temporary procedure, a distended bladder is sometimes emptied by aspiration. There is always risk of infection of the tissues in the line of puncture by the urine; so this procedure is to be avoided if possible. A trocar and canula or an aspirating trocar is introduced through the tissues in the middle line just above the pubes; the urine is withdrawn, and, after removing the canula, the puncture is sealed with a pledget of gauze and collodion.

Ingrown Toenail.-In this condition the edge of the nail usually of the great toe, by pressure upon the flesh beneath it, causes ulceration or suppuration. The condition is painful and causes marked disability. It may be treated by carefully introducing a little wisp of cotton under the edge of the nail and raising ont of the ulcer, and apply to the cotton a few drops of collodion and tincture of benzoin, which saturates the cotton and becomes dry from a scab on which the edge of the nail rests. This dressing should be repeated at intervals of a few days, and will often result in relief of the condition. 
In aggravated cases operative treatment is 'often required. The essential point is to remove both the edge of the nail and that portion of the matrix from which it grows. The following operation will be found satisfactory : Sterilize the toe as completely as possible and tie a rubber band around its base; inject a few drops of a eucaine or stovaine solution along the edge of the nail and beneath it as far back as the second phalanx. Make an incision through the nail and overlying skin and matrix in about

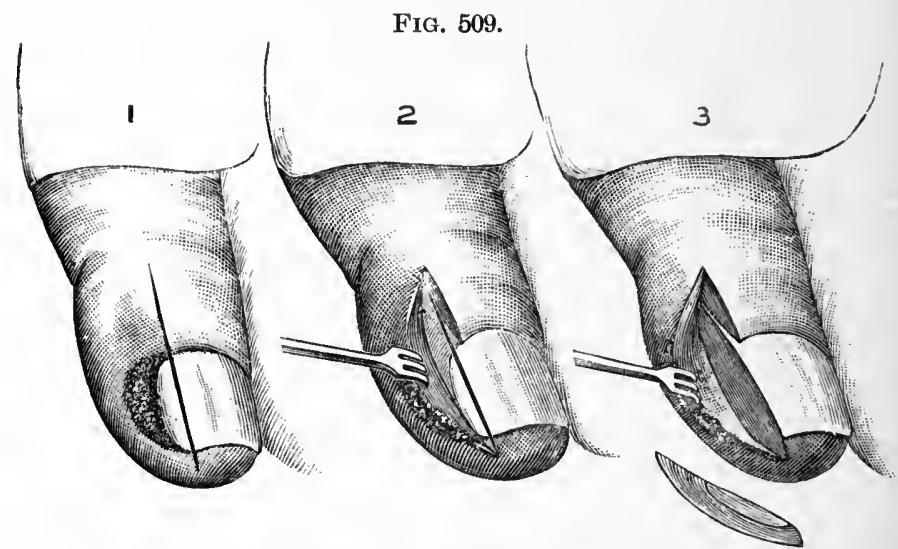

Operation for ingrown toenall. 1. Line of incision. 2. Skin flaps reflected. 3. Section of nail and matrlx removed. (After FooTe.)

one-fourth of an inch from the edge of the nail (Fig. 509, 1). The overlying skin is next dissected free from the edge of the nail and its matrix (Fig. 509, 2).

The portion of the nail marked out by the first incision is then dissected out with its matrix, care being taken that all of the matrix corresponding to the nail is removed (Fig. 509, 3). The wound is irrigated with a 1 to 2000 bichloride solution and closed by wrapping a gauze dressing around the toe and securing it by a bandage firmly applied. The rubber bandage is next removed. The wet dressing should be renewed daily for a few days, and after this time a dry dressing may be employed. 


\section{OPERATIONS UPON THE KIDNEY.}

Nephrotomy.-In this operation the kidney is incised for drainage or for exploration of the organ. The incision for exposure of the kidney is four inches in length, and should be made from a point two and a half inches from the spine, half an inch below the last rib and parallel with it. The latissimus dorsi, external and internal oblique, and transversalis muscles are divided, and the lumbar fascia is opened, exposing the perinephric fat; by displacing this the kidney is reached.

Lumbar Nephrectomy.-The incision is the same as for nephrotomy ; the wound may be enlarged by another incision at right angles to the first if more space is required. After the kidney is exposed, its capsule is incised and the finger passed around the organ to separate it freely from the capsule. When the ureter is recognized, it is brought into view, ligated, and cut off. The pedicle containing the vessels is next tied, and divided

FIG. 510.

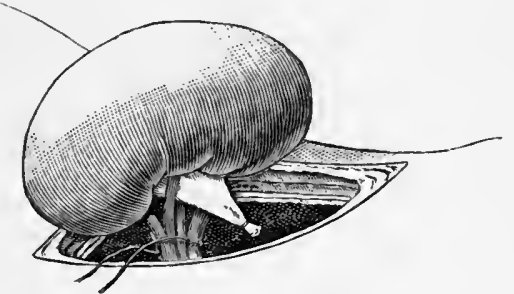

Delivery of kidney and ligation of its vessels and ureter. (HARTMANN.) in advance of the ligature with scissors, and the kidney removed (Fig. 510).

Abdominal Nephrectomy.-To reach the kidney by abdominal incision, an incision four inches long is made at the outer border of the rectus muscle; the abdomen is opened and the viscera turned aside; the kidney is exposed and the capsule opened; the ureter and the renal vessels are ligated and divided, and the organ removed; a drainage-tube may be introduced or the wound in the abdominal walls may be closed without drainage.

Nephrorrhaphy or Nephropexy.-Nephrorrhaphy is an operation in which the kidney is exposed through a similar incision to that for nephrotomy, with the object of suturing a movable kidney fast in its normal position 
in the back; when the kidney has been reached, a number of sutures are introduced into the capsule of the kidney or the kidney itself, and secured to the fibrous and muscular tissue of the incision (Fig. 511). Many surgeons prefer to omit the introduction of sutures, and simply scarify the capsule of the kidney or dissect off a portion of the capsule, and then pack the wound with strips of gauze and allow it to heal by granulation.

FIG. 511.

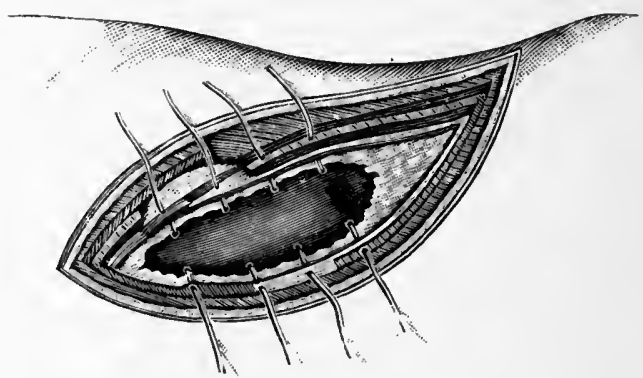

Sutures passed through both capsule and kidney. (HARTMANN.)

Other methods of fixing a movable kidney consist in dissecting a flap from the capsule of the kidney and suturing it to the muscular tissues of the external wound before elosing the wound. Some surgeons prefer to introduce no sutures, but to pass two gauze loops around the kidney at different points, and pack the wound with gauze. The loops are not removed for a few days, and the wound is allowed to heal by granulation.

Decapsulation of the Kidneys. - This operation consists in exposing the kidneys by the operation previously described, and when a free exposure of the kidney has been obtained, the fibrous capsule is incised for its whole length, and is then dissected loose from the organ to a point near the pelvis of the organ. When this has been accomplished the kidney is dropped back into its place and the 
wound is closed without damage. This operation has been practised in chronic nephritis in some cases with good results.

\section{OPERATIONS UPON THE URETER.}

Ureterotomy.-This operation consists in the exposure of the ureter by an oblique lumbar incision, and its incision for the removal of a calculus. The pelvic portion of the ureter may be exposed by an extension downward of the oblique lumbar incision to the iliac region, and retraction of the peritoneum from the ilio-psoas muscle and side-wall of the pelvis. In the female the pelvic portion of the ureter may be exposed by the extraperitoneal route or by vaginal incision.

FIG. 512.

Ureterectomy.-This consists in exposing and resecting a portion of the ureter, and the operation may be required for stricture or growths of the urethra. When the ureter is resected, the separated ends of the ureter should be united or the free end should be implanted into the bladder.

Wounds of the ureter, if longitudinal or oblique and not too extensive, should be closed by sutures of fine silk. A round needle should be used and the sutures placed closely together (Fig. 512). If the ureter is partially or completely divided uretro-ureterostomy should be practiced.

Uretero-ureterostomy.-This consists in uniting the ends of a divided ureter to secure patency of the canal. The lower segment of the ureter is ligated with silk, and longitudinal incision is made into the ureter just below the ligature. A loop of catgut threaded with double needles is passed through the tip of the upper end of the ureter, and this is drawn through the incision by means of the catgut loop, the needles passing out of the ureter a little below the 
lower limb of the incision; the catgut ligature is then tied and a few additional sutures are applied (Fig. 513).

FIG. 513.
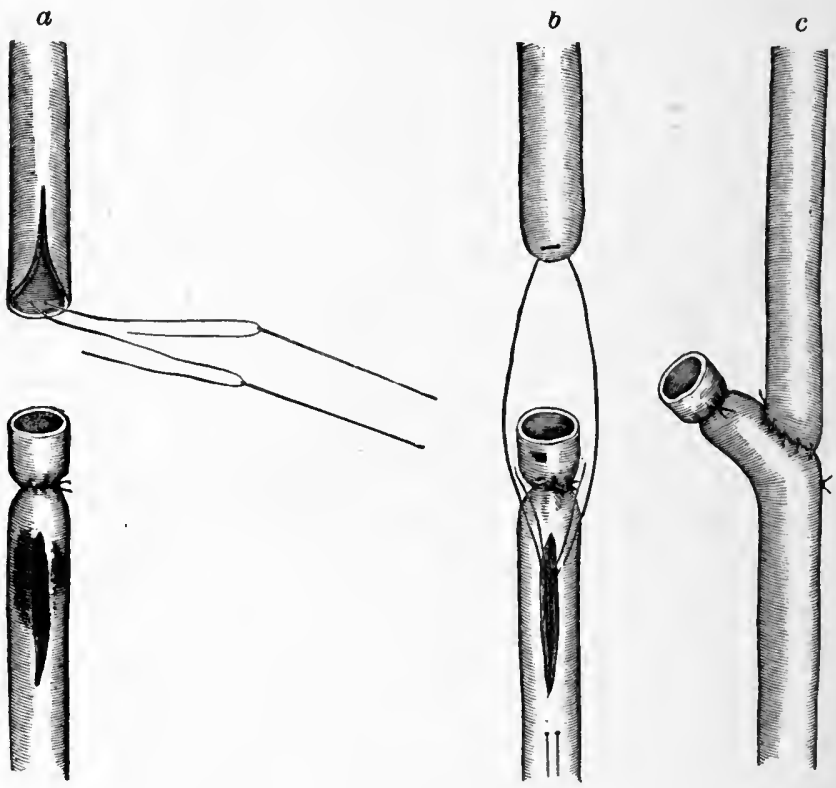

Uretero-ureteral anastomosis, end-in-side implantation (Van Hook's method): $a$, first step; $b$, seeond step; $c$, completed operation. (Bryaxt.)

Implantation of Ureter into Bladder.-When the distal end of the ureter is too short to admit of an anastomosis the ureter may be implanted into the bladder. The distal portion of the ureter should be ligated and an incision made into the bladder near the ureteral orifice. The upper end of the ureter is drawn down by a loop of catgut as in the method of Van Hook and is secured by tying the ends a little below the wound. A few interrupted sutures of catgut or fine silk are next introduced at the line of union. Additional security may be obtained 
by suturing over the wound a layer of peritoneum or omentum.

\section{OPERATIONS UPON THE COLON.}

Lumbar Colostomy.-In performing lumbar colotomy, or colostomy, on the left side, the patient should be placed

FIG. 514.

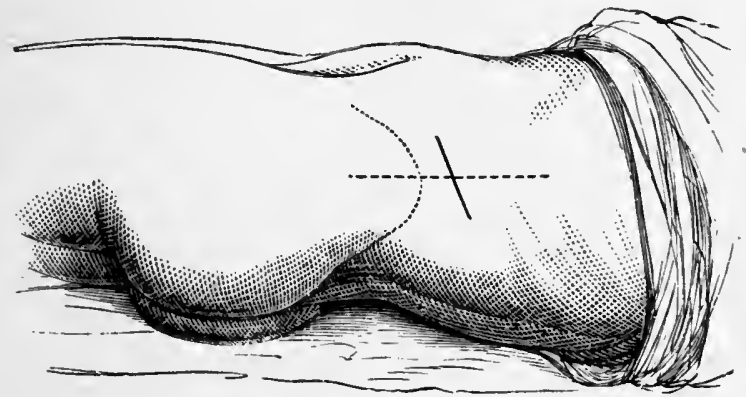

Incision in lumbar colostomy-dotted line shows the situation of the colon. (BRYANT.)

upon the right side, and a pillow placed under the loin to make the left side more prominent. An incision four inches in length is made midway between the last rib and the crest of the ilium, the centre of the incision corresponding to a point midway between the anterior superior and posterior superior spines of the ilium; the tissues are divicled to the full extent of the wound until the lumbar fascia and edge of the quadratus lumborum muscle have been reached; the forner being cut through and the edge of the muscle divided, the bowel is exposed, when it is brought to the surface and fastened by sutures to the skin and subjacent tissues, and opened (Fig. 514).

Inguinal Colostomy.- In this operation an incision three inches in length is made on the left side parallel to and one inch above Poupart's ligament, with its centre on a level with the anterior superior spine of the ilium, or a 
little lower; or, as practised by Ball, the colon may be exposed by an incision two and a half inches in length, following the line of the linea semilunaris, stopping just short of Poupart's ligament; the tissues are divided layer by layer and the peritoneum opened ; the skin and parietal peritoneum may be united by a few sutures; the gut is then brought out at the wound, fastened to its margins by fine sutures, and opened.

Maydl's Operation.- In this operation the colon is exposed as in the preceding operation, and then drawn out

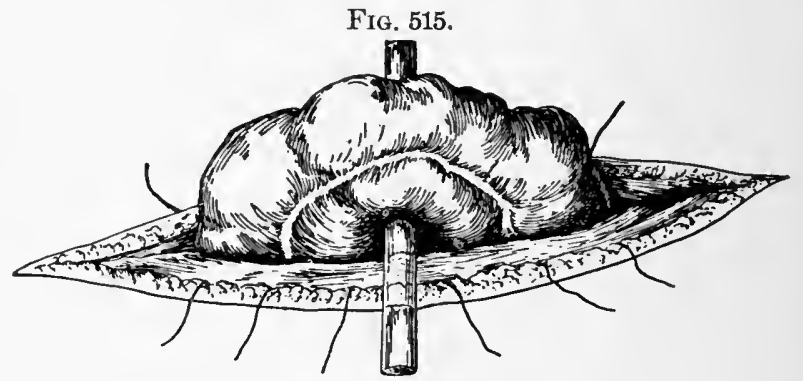

Colon held in wound by glass rod. (PILCHER.)

of the wound until its mesenteric attachment is on a level with the external incision. A sterilized glass rod or piece of catheter, or a roll of gauze three inches in length, is slipped through a slit in the mesocolon close to the gut (Fig. 515). This holds the intestine in the wound and prevents its return to the abdominal cavity until adhesions have formed. The two limbs of the flexure of the gut exposed in the wound should be united by sutures beneath the rod. If the gut is to be opened immediately, it should be stitched to the parietal peritoneum of the abdominal incision. If the opening of the bowel can be postponed for twenty-four or forty-eight hours, the introduction of sutures is not required. The bowel may be opened by a transverse incision with a knife, or by the thermo-cautery, to avoid bleeding. 


\section{OPERATION FOR ACUTE APPENDICITIS.}

An incision three inches in length should be made over the outer portion of the rectus muscle about half an inch from its external border. The skin and connective tissue are divided, and the sheath is exposed and opened in the direction of the cutaneous incision, and the muscular fibres are retracted inward. This incision exposes the posterior wall of the sheath, and the epigastric vessels, which may be retracted or divided. The posterior layer of the sheath of the rectus is then opened, exposing the peritoneum and subconnective tissue, which is grasped with two pairs of forceps, carefully drawn up and divided.

If a tumor exists in the right iliac fossa, indicating an inflammatory exudate or appendicular abscess, the incision should be made over the most prominent portion of the mass.

After exposing the peritoneal cavity, if no abscess is present, the finger is introduced and the appendix sought for, or the wound may be dilated with retractors, and the search for the appendix aided by inspection of the parts. If the appendix is freely movable, it may be drawn through the wound, the meso-appendix ligated in two or three portions, and the base of the appendix securely ligated with a catgut or silk ligature, and the appendix divided in advance of the ligature. The eut surface of the appendix should then be touched with a drop of pure carbolic acid or the actual cautery. If, however, the appendix is bound down by adhesions, these should be carefully separated and the meso-appendix ligated, and after the base of the appendix has been tied off, it should be removed as described above. After removing the appendix in such a case, a cigarette drain or a rubber tube should be introduced, and the wound of the abdominal walls should be partially closed with layer sutures, one set elosing the peritoneum, a second the muscular fibres, a third the fascia, and another the skin and connective tissue.

If, on opening the abdomen, inflammatory exudate is found, strips of sterilized ganze packing should be intro- 
duced to shut off the adherent intestines from the general peritoneal cavity, and by introducing the finger the structures around the appendix are gently separated in their lines of natural cleavage. As soon as pus escapes, the opening should be enlarged and the pus gently wiped away with pads or sponges, and the wound dilated so as to expose the appendix as freely as possible. When the appendix is found it should be removed. A cigarette drain or rubber tube should be introduced into the cavity, and it should also be loosely packed with gauze. The gauze packing may be then taken out and replaced with clean packing, and the wound should be partially closed by sutures or layer sutures. If there is free fluid in the peritoneal cavity in addition to the localized abscess, or if the abscess ruptures and pus escapes into the pelvis, this should be cleansed by sponges or by irrigation with warm salt solution or sterile water, thoroughly dried with gauze pads, and drainagetubes or cigarette drains introduced and carried down to the bottom of Douglass's pouch. If, on opening the peritoneal cavity, it is found that there is a diffused peritonitis, the entire peritoneal cavity should be flushed with an abundance of hot sterile water or salt solution, rubber, cigarette, or gauze drains should be introduced, and the wound partially closed and dressed.

Murphy's Method.-Murphy, in cases of appendicitis complicated by diffused peritonitis, recommends that the abdomen be opened and that no extensive search be made for the appendix, but that free drainage be secured by rubber or gauze drains passed to the lower portion of the pelvis and the patient be placed in the Fowler position tc drain fluids into the pelvis - that is, a sitting or semi-sitting posture, and that he be given saline solution by the rectum continuously, the vessel containing the fluid being raised slightly above the body and the tube so arranged as the fluid passes slowly into the rectum, the object being to have only so much fluid enter the rectum as will be absorbed. The temperature of the solution should be about $100^{\circ} \mathrm{F}$. 


\section{OPERATION FOR CHRONIC APPENDICITIS.}

In removing the appendix in the interval between attacks, two methods may be employed-either McBurney's method or by an incision through the outer edge of the rectus muscle.

McBurney's Operation.-This consists in making an incision two or three inches in length parallel with the fibres of the external oblique aponeurosis, the centre of which would lie a little to the outer side of McBurney's point. The skin and subcutaneous tissues are then divided down to the aponeurosis, the latter is then split in the direction of its fibres and well retracted, exposing the internal oblique, the fibres of which are next separated by a blunt dissection and retracted in the opposite direction. The transversalis fibres are then separated in the same manner, which exposes the transversalis faseia and subperitoneal fat. These structures are next divided, and the peritoneum drawn upward in the transverse fold by two pairs of hæmostatic forceps and opened. The wound is next carefully enlarged, and the colon is usually found directly beneath the opening, and may be recognized by its longitudinal band. The finger is introduced and the appendix is sought for, and when found, if no adhesions exist, both the appendix and the exeum can be drawn out through the wound, the peritoneum being proteeted by one or two gauze pads. The meso-appendix is then ligated by passing a eatgut ligature through its folds, near the junetion of the appendix with the excum, and tying it well below the attachment of the meso-appendix to the appendix. If the meso-appendix is large, it should be tied off in sections. The appendix is next separated from its mesentery and held upward by an assistant, while the surgeon surrounds its base by a silk or a fine ehromieized catgut pnise-string suture. When this is in place the end should be knotted; the knot tied and held by an assistant, while the surgeon divides the appendix about a quarter of 
an inch from the cæcum. If the canal is pateut, the openıng is dilated by means of a small pair of fine-bladed forceps, and the stump inverted into the cæcum by another pair of fine forcejs grasping the cut margins and pushing them inward. An assistant then tightens the purse-string suture, and the small forceps is withdrawn. By this manipulation the stump is inverted and buried. In many cases there is so much infiltration of the cæcum or appen-

\section{FIG. 516.}

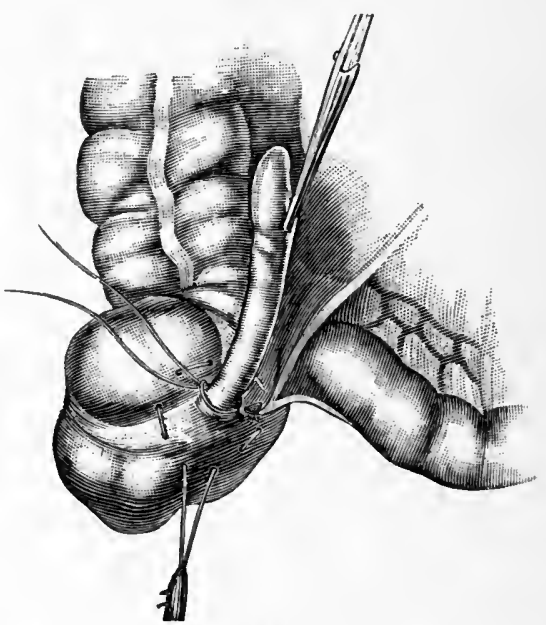

Base of appendix surrounded by ligature, purse-string suture in place. (GOSSET.)

dix that it is difficult to accomplish a satisfactory inversion of the stump. In such cases the base of the appendix should be ligated and the appendix removed, and the exposed mucous surface should be touched with the actual cautery or with a drop of pure carbolic acid. A cuff of peritoneum may be dissected from the appendix before it is ligated, and after this has been accomplished the cuff may be sutured over the surface of the stump by two or three sutures of fine silk, or it may be buried by a purse string suture applied as shown in Fig. 516. After removal of the 
appendix the cecum shonld be carefully sponged with gauze and returned into the abdominal cavity. The peritoneum is next closed with continuous suture of catgut or fine silk, and the muscular layers are next allowed to fall together, and are held in contact by a few interrupted catgut sutures. The aponeurosis is then brought together with a few interrupted or continuous sutures, and the skin and connective tissue closed by sutures of silkworm-gut or catgut. If there is any question as to the contamination of the wound, a small cigarette drain should be introduced down to the stump of the appendix, and may be allowed to remain for twenty-four or forty-eight hours and then removed.

Incision Through the Outer Edge of the Rectus Muscle.-This consists of an incision two and a half or three inches in length, over the onter portion of the rectus muscle, about half an inch from its external border. The skin and cellular tissue are divided, and the sheath of the muscle is exposed and opened in the direction of the cutaneous incision, and the muscular fibres are retracted inward. This exposes the posterior wall of the sheath and the epigastric vessels, which may be retracted or divided. The posterior portion of the sheath is then opened, exposing the peritoneum and subserous connective tissue, which is picked up with two pairs of hæmostatic forceps and carefully divided, opening the peritoneal cavity. The appendix is then sought for, and the steps of its removal are the same as previously described.

\section{LITHOTOMY.}

Left Lateral Lithotomy.--In performing this operation, the patient is placed upon his back, the hands and feet are secured together, and the bladder is injected with a few ounces of boric acid solution. A grooved staff is introduced into the bladder, the operator first passing one finger into the rectum to locate the position of the staff as regards the prostate. An incision is then made a little to the left of the raphe of the perineum, a quarter to half 
an inch in front of the anus, and is carried downward by careful strokes of the knife until the staff" is reached, about half an inch in front of the prostate. When the point of the knife enters the groove in the staff; it is pushed backward, keeping it well in the groove until the prostate is incised and a gush of fluid escapes along the knife, when it is removed and the index finger introduced and the stone located; lithotomy forceps are next introduced and the stone removed (Fig. 517).

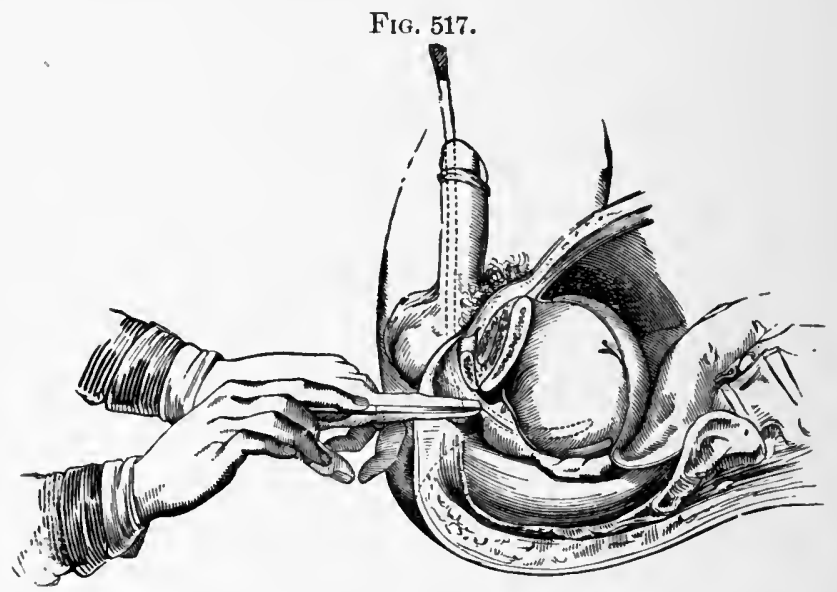

Deep incision in lateral lithotomy. (FERGcsson.)

Suprapubic Lithotomy.-The operation of opening the bladder above the pubes may be performed for the removal of stone from the bladder, for the extirpation of growths, or for drainage of the bladder. The hair on the pubes should be shaved off, the bladder injected with a few ounces of saline solution, and a rubber band tied around the penis; a small rubber bag is then introduced into the rectum empty and flled with air or water. An incision two or three inches in length is made in the median line of the abdomen just above the symphysis pubis, and is leepened gradually until the deep fascia is reached; this is divided, exposing the prevesical fat; when this is dis- 
placed, the wall of the bladder is exposed to view. A tenaculum is next introduced into the highest part of the vesical wall, to fix it, and a knife thrust through the wall of the bladder, the incision being carried downward about an inch. After the bladder is opened, forceps are introduced and the calculus removed. If opened for calculus and the bladder-walls are healthy, the wound may be sutured with stitches which do not pass through the micous coat. The external wound is then sutured and the bladder drained by a soft catheter passed by the urethra. If the bladder-walls are much diseased, the wound is left open and drainage effected by a rubber tube passed through the suprapubic wound into the bladder.

\section{CIRCUMCISION.}

Circumcision is performed by drawing the prepuce forward and then enclosing it in a pair of clamp-forceps

FIG. 518.

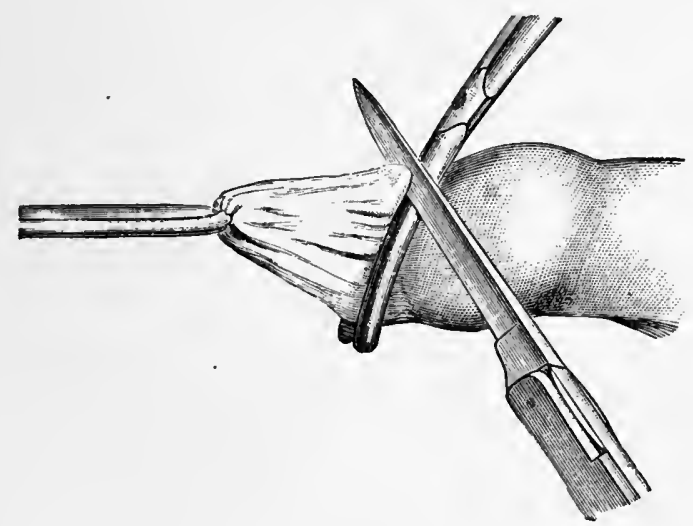

Circumcision.

placed obliquely just in front of the glans (Fig. 518). The prepnce is next divided with a straight bistoury, and the 
forceps removed, when the skin and mucous membrane retract. The mucous membrane, if adherent, is dissected loose from the glans; if redundant, it is trimmed with scissors to make it correspond to the line of skin incision; the cut edge of the mucous membrane is next fastened to the out edge of the skin by a few sutures of silk or catgut.

\section{REMOVAL OF THE TESTICLE.}

In removing the testicle, a longitudinal incision is made over the upper part of the gland and spermatic cord and the envelopes of the testicle and cord divided; the cord is then exposed and ligated, or the different components of the cord may be separated and tied independently; the cord is divided in advance of the ligatures and the gland removed.

\section{OPERATION FOR VARICOCELE.}

In operating for varicocele the veins of the spermatic cord may be exposed by an incision an inch and a half or two inches in length, at the upper part of the scrotum, over the cord, or the cord may be exposed by an incision as it emerges from the inguinal canal. The veins being exposed, the larger portion of them are isolated, and two ligatures are passed around the mass of veins about an inch or an inch and a half apart and firmly tied, care being taken that the vas deferens is not included with the veins. The portion of the cord between the ligatures is excised and the divided ends of the veins brought in contact by tying together the ends of the ligatures upon the proximal and distal ends of the veins; the wound is then closed with sutures.

\section{TREATMENT OF HYDROCELE.}

The Palliative Treatment.-The skin of the scrotum should be sterilized and the tumor rendered tense by grasping it with the hand; a sterile trocar is then introduced through the anterior wall of the scrotum into the 
eyst, being directed upward and backward to avoid wounding the testicle. After the fluid has escaped the canula is removed and the puncture sealed with a sterile piece of gauze or cotton and collodion.

The Radical Treatment.-This consists in emptying the cyst by means of a trocar and injecting from 1 to 2 drachms of tincture of iodine. Another method consists in first introducing the needle of a hy podermic syringe, charged with 10 to 12 drops of pure carbolic acid, into the upper part of the sac-this is held by an assistant. The sac is next emptied by introducing a trocar at a lower portion of the cyst. After removing the fluid the carbolic acid is injected into the cyst and evenly distributed by rubbing the walls together. The instruments are removed and the punctures sealed with gauze and collodion. Pain and swelling follow each of these operations, but usually subside in forty-eight hours.

Eversion of Sac.-An incision is made exposing the sac for several inches, it is then incised and emptied and the testicle is brought out through the wound.

The gubernaculum testis is next Operation for hydrocle by everligated and divided, then fold the

Fra. 519.

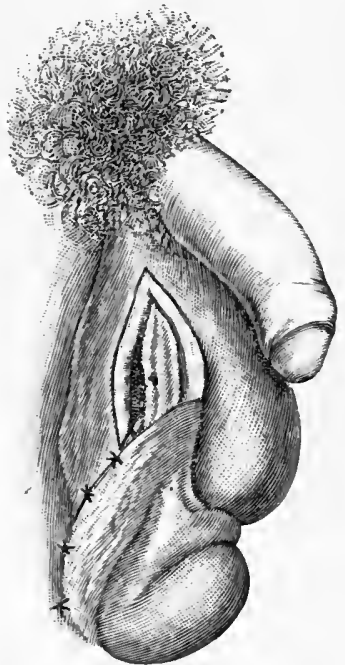

two sides of the divided sac behind the testicle and fix them by a few sutures, one of which should intersect the superficial tissue of the cord. The testicle is then replaced in the scrotum and the wound closed with sutures without drainage.

Excision of the Sac.-This eonsists in making an incision two to three inches in length over the anterior surface of the scrotal tumor, and dividing the tissues until 
the sac is exposed. This is next incised, and the parietal layer is dissected out as far as possible; a strip of rubber tissue or a gauze pack is introduced and allowed to remain for forty-eight hours and is then removed. The wound usually heals in from ten days to two weeks.

\section{CHOLECYSTOTOMY.}

This operation consists in opening the gall-bladder for exploration or for the removal of gall-stones.

An incision three or four inches in length is made vertically downward along the outer edge of the right rectus musele from the lower border of the eighth rib to a point opposite the umbilicus; the tissues are divided and the peritoneum opened. The gall-bladder is then exposed and drawn upward in the wound and the peritoneum protected by gauze pads. An incision is then made in the fundus and the stone removed, after which the opening in the gallbladder is elosed with two layers of Lembert sutures, the parts disinfected, and the organ returued to the abdominal cavity; the latter is next closed by sutures, or the gallbladder may be sutured to the subcutaneous tissues of the external wound and then opened and the stone removed, the wound being left open, a drainage-tube or gauze drain being introduced. If the gall-duct is to be explored, this is done with the finger from without or by a probe introduced into it through the gall-bladder.

Cholecystostomy.-This operation is employed for drainage of the gall-bladder, which is exposed as in the operation of cholecystotomy. Two circular purse-string sutures are introluced into the peritoneal covering of the fundus of the gall-bladder ; the first suture is placed so as to include a circle about one-third of an inch in diameter, and the second is placed so as to secure the first about one-third of an inch from it. An incision is then made within the inner circle and a rubber drainage-tube passed into it. As the tube is introduced the first suture is tightened and tied, and as the tube is pushed further inward it carries with it a funnel-like depression of the fundus, when 
it is tied. The gall-bladder is then attached to the peritoneum by one or two eatgut sutures and the abdominal wound is partly closed by sutures. This method secures good drainage, and prevents leakage and the possibility of a permanent fistula after the drainage-tube has been removed.

Cholecystectomy.-The gall-bladder is exposed as in the previous operation, the cystic duct is located, and two ligatures of silk or chromicized catgut are passed around it. The peritoneal covering of the gall-bladder is incised at its junction with the liver, and the gall-bladder is separated from the under surface of the liver by a blunt dissection. When entirely separated from the liver, the cystic duct is divided between the two ligatures and the organ is removed. Hemorrhage is controlled by ligatures or by gauze paeking. The abdominal wound is next partly closed, a gauze packing being allowed to remain in the wound in contact with the raw surface of the liver.

Choledochotomy.-This operation consists in opening the bile-dúct for the removal of a stone or for drainage. A long incision, extending as high as possible, is made through the right rectus muscle, and the gall-bladder region is freely exposed. The intestines are next walled off with gauze packing. The operator introduces the left hand into the wound and carries the index finger through the foramen of Winslow into the lesser peritoneal sac. With the finger in the foramen, the eystic, hepatic, and common ducts can be examined, and if a stone is felt the duet is raised upon the finger and brought near the surface of the wound. When the stone is located the duct is exposed by the removal of the peritoneal covering and incised longitudinally, and the stone removed. A probe is next passed into the duct in both directions to insure its patency, and if there is no reason to suspect infection of the duct, the wound is closed by a few fine silk sutures introduced with a round, curvẹd needle. A small gauze drain or cigarette drain is then carried down to the wound, and after removing the ganze packing the abdominal wound is partly closed by sutures. If the duct is found infected, a smail 
drainage-tube is introduced into the duct and secured by a single catgut suture, the gauze packing is removed and replaced by new packing, and the abdominal wound is partially closed.

Cholecystenterostomy.-This operation is employed in cases of complete and permanent obstruction of the common duct, and consists in anastomozing the fundus of the gall-bladder to the second portion of the duodenum. Sometimes from misplacement or diminished size of the gall-bladder or adhesions, it may be necessary to make the communication with the jejunum or hepatic flexure of the colon. The gall-bladder is exposed by the ordinary incision, and the anastomosis is effected by means of a small Murphy button. The line of union, after the anastomosis has been made, may be strengthened by wrapping a mass of omentum around it and securing it in place by a few catgut sutures.

\section{EXTERNAL GESOPHAGOTOMY.}

A sound is passed through the mouth into the osophagus until its point comes in contact with the stricture of the oosphagus or the foreign body which requires removal. An incision is then made from a point one inch above the sternum to the line of the upper border of the thyroid cartilage on the inner side of the sterno-cleido-mastoid muscle; the anterior jugular vein is displaced, the fascia is divided, the omohyoid muscle is drawn aside, the sternomastoid muscle and the vessels are drawn to the outer side with blunt hooks, and by dissecting down with the finger the resophagus is exposed; the sound which has been passed into the csophagus may easily be felt, and the œesophagus incised upon the point of this sound. If a permanent opening is desired, the edges of the osophagus are sutured to the skin. 


\section{OPERATIONS UPON THE STOMACH AND INTESTINES.}

Gastrotomy.-This operation consists in opening the stomach for purposes of diagnosis, the removal of a foreign body or for the dilatation of the pyloric orifice. The stomach is exposed by an incision through the left rectus muscle and is drawn into the wound and the peritoneal cavity is protected by gauze pads. An incision is

FIG. 520.

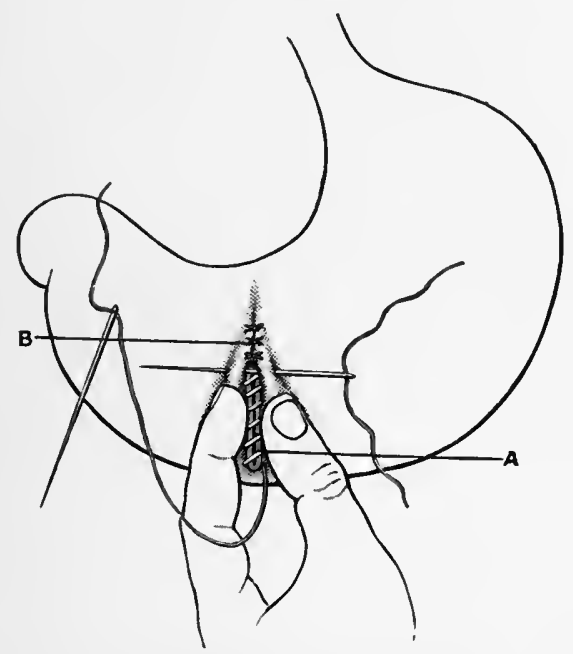

Gastrorraphy. A. Continuous suture through all the coats. B. Interrupted Lembert sutures through outer coats. (After BECKHAM.)

next made in the anterior wall of the stomach parallel with its longitudinal axis, which exposes the cavity of the organ. Through this wound exploration of the organ is made or a foreign body is removed. The wound of the stomach is closed by two layers of sutures. The deep layer of cat-gut sutures is applied to the mucous coat and the superficial layer of silk or celluloid thread sutures including the serous and muscular coats is introduced by the 
Lambert method. The abdominal incision is closed in the ordinary manner.

Gastrorrhaphy.-This procedure is employed in closing accidental wounds of the stomach or in approximating the wounds resulting from plastic operations upon the stomach. In penetrating wounds a double layer of sutures should be used, the mucosa being part closed by a layer of fine catgut sutures and a second layer of silk or celluloid thread sutures being applied to approximate the serous and muscular coats. Lambert sutures are usually applied (Fig. 520 ).

Gastrostomy.-An incision one and a half to two inches in length is made paiallel to and a finger's breadth from

FIG. 521.

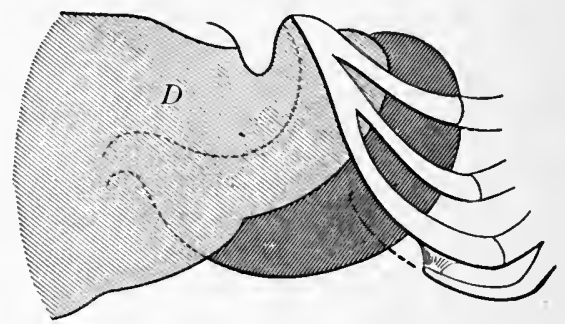

Anatomical relations of the stomach. (STIMson.)

the border of the left costal cartilage, ending opposite the border of the tenth rib; the tissues are divided layer by layer until the peritoneum is reached (Fig. 521). The latter membrane should be pinched up and opened; the stomach is recognized and brought out of the wound; the parietal peritoneum is stitched to the skin around the wound, and a fold of the unopened stomach is brought out of the wound and sutured to the parietal peritoneum and the abdominal wall. The opening of the stomach is delayed for twenty-four hours, if possible, to allow of the formation of adhesions between its surface and the parietal peritoneum. 
Ssabanajew-Frank Method.-A curved incision, three or four inches in length, is made at the margin of the costal cartilages of the left side, and the surface of the stomach is exposed. A cone of the stomach-wall is then grasped with forceps, pulled out of the wound (Fig. 522), and passed under a bridge of skin and connective tissue and made to project from a separate wound made about one and a half inches above the original wound (Fig. 523). 'The wall of

FIG. 522.

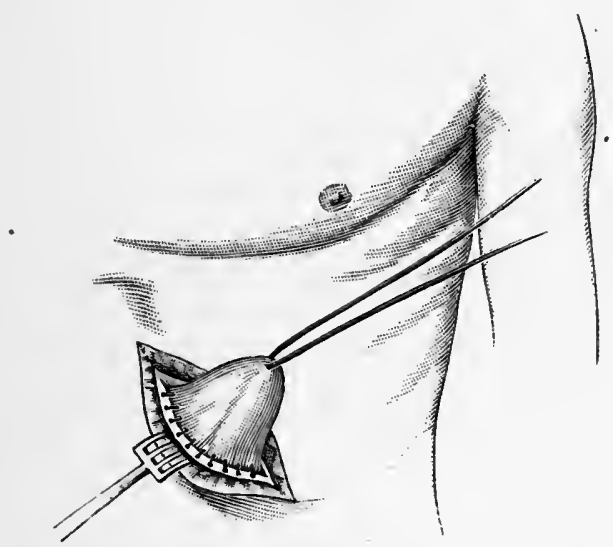

Ssabanajew-Frank method ; first stage. (RICHAkDson.)

the stomach is fastened in the original wound by sutures and the wound closed, the projecting portion of the stomaeh in the upper wound being seeured by sutures. The stomach may be opened at any time.

Witzel's Method.-This method of gastrostomy also prevents leakage, and is accomplished by making an incision and exposing the wall of the stomach. A small incision is made in the wall of the stomach and a rubber tube or eatheter introduced; the portion of the tube in contact with the stomach external to the wound is then infolded by peritoneal approximation, as shown in Fig. 524. The stomach is then stitched to the abdominal wall and the 
FIG. 523.

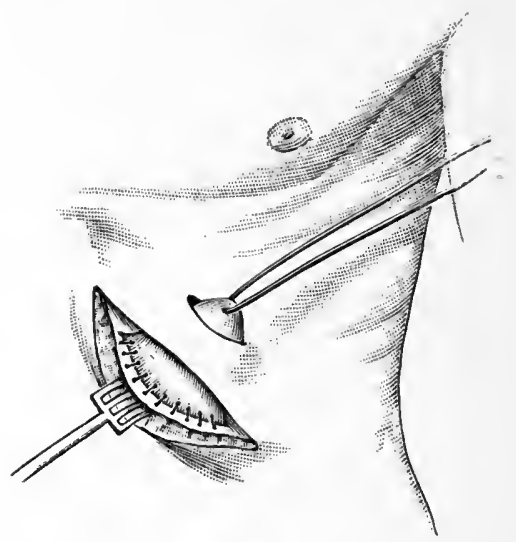

Ssabanajew-Frank method; second stage. (RICHARDson.)

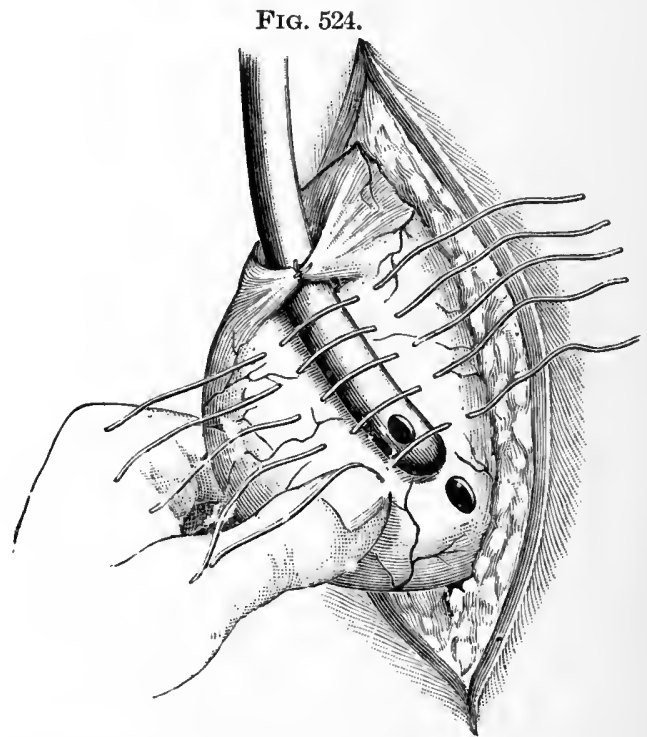

Witzel method of infolding the tube. (RICHARDSoN.) 
external wound closed (Fig. 525). The tube should not be removed for a week; feeding may be begun through the tube immediately. Contraction of the fistula may be

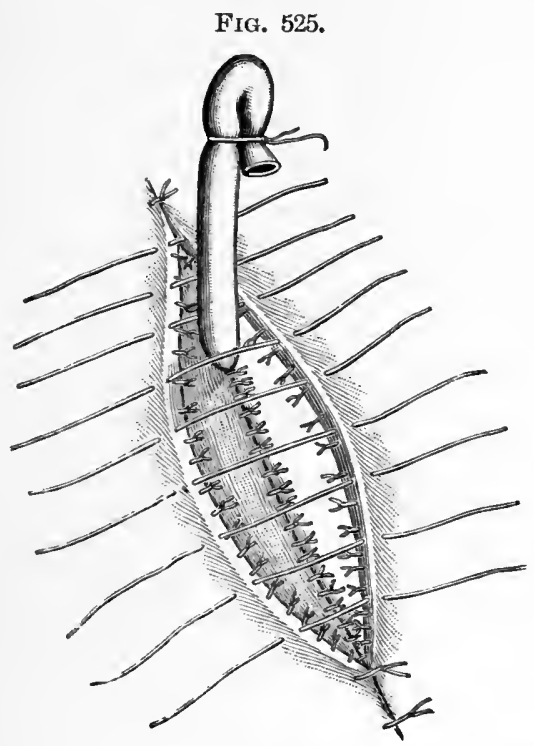

Witzel method; tube infolded and sutures introduced to close the wound.

(RICHA RDSON.)

prevented by the occasional introduction of the tube or catheter.

Pyloroplasty.-This operation is practised in non-malignant strietures of the pylorus. The pyloric extremity of the stomach is exposed by a median incision, and a longitudinal incision is made through the auterior surface of the constricted pylorus (Fig. 526), and the incision closed by sutures introduced transversely, as shown in Fig. 527.

Mikulicz makes the incision on the inferior surface of the pylorus and continues it into the stomach and duodenum. The posterior lips of the incision are united by 
sutures, and then the anterior lips, this enlarges the pyloric opening and lowers the outlet of the stomach.

Frg. 526.

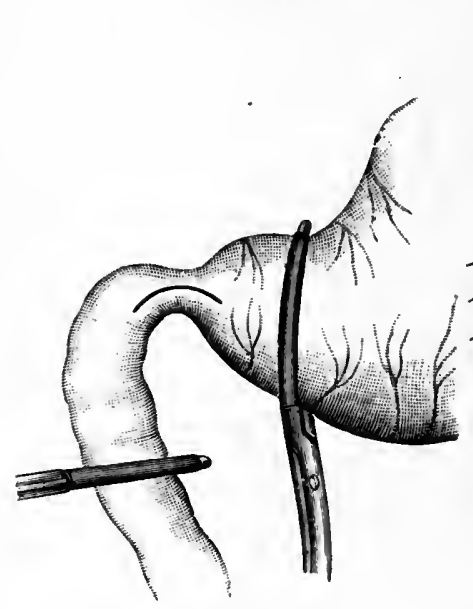

FIG. 527.

Incision in pyloroplasty. (After Вескнам.)
Incision retracted in opposite direction and sutures applied. (After Вескнам).

Finney's Method.-In this method the region of the pylorus is freed from all adhesions, and a heavy silk ligature is introduced at the summit of the pylorus to act as a retractor, and another through the anterior wall of the stomach about three inches from the first one, and a third ligature is passed through the anterior wall of the duodenum at the same distance from the pylorus. By making traction upon the first ligature upwards and upon the two others downwards, the anterior surface of the duodenum and stomach are brought into contact and united by a row of fine silk sutures passed through the peritoneal coats of the viscera (Fig. 528). An inverted U-shaped incision is next made through the walls of the stomach, pylorus, and duodenum about half an inch from the line of sutures. The adjacent mucous and muscular coats of the duodenum and 
stomach are united by a continuous suture of catgut and the opening is next closed by a layer of mattress sutures (Fig. 529).

FIG. 528.

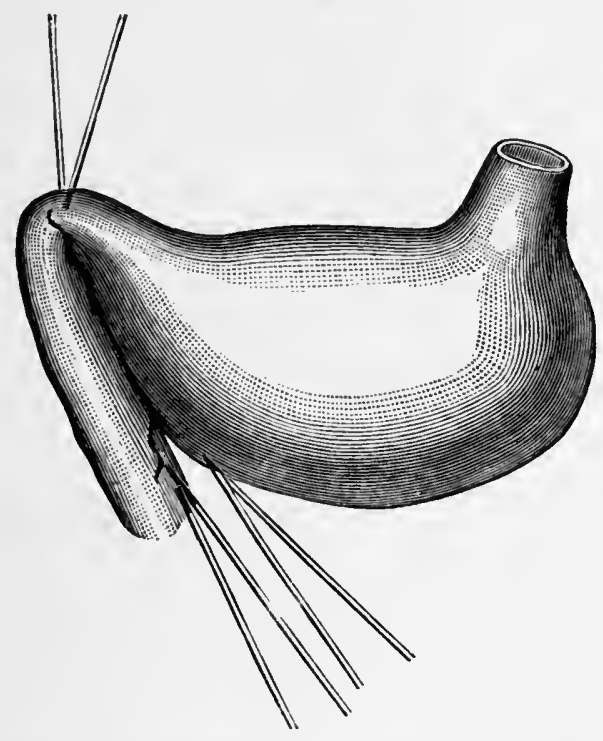

Finney's operation: position of ligatures. (BREWER.)

Pylorectomy and Gastro-Duodenostomy.-This operation is practised in malignant strictures of the pylorus. It consists in exposing the stomach and duodenum by a median abdominal incision ; the upper portion of the duodenum and the stomach are drawn through the incision, and resection of the diseased portion accomplished. The opening in the stomach being much larger than that resulting from resection of the duodenum, the wound in the stomach should be partially elosed by Lembert sutures (Fig. 530); and when it has been reduced to a proper size to fit the free end of the duodenum, they are fitted together and held in position by the introduction of a circular row of elosely. applied Lembert sutures (Fig. 531). 
Fra. 529.

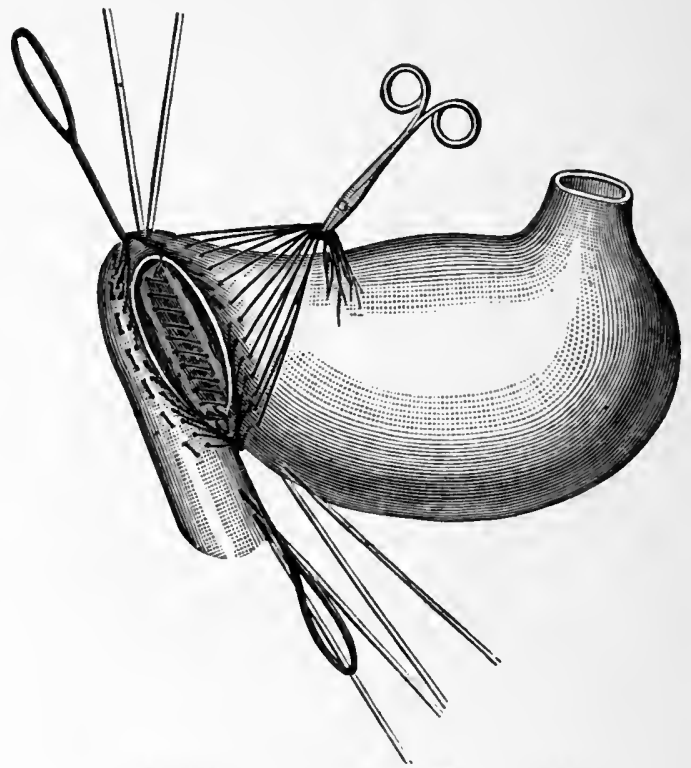

Finney's operation: sutures in place. (Brewer.)

FIG. 530 .

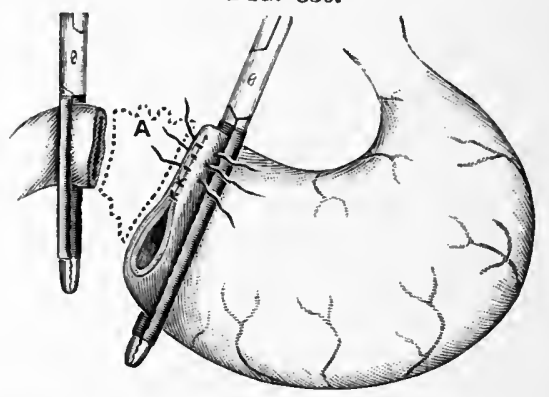

Pylorus excised and opening into the stomach partially closed.

Gastro-Enterostomy.-This operation is practised, in cases where it is inadvisable to resect the pylorus, a lateral anastomosis between the stomach and a coil of small intes- 
tine near the stomach, being made, so that the contents of the stomach may find their way into the intestines through this artificial opening.

The operation consists in establishing an anastomostic opening between the stomach and an adjacent loop of small intestine. The anterior operation eonsists in uniting the highest aceessible loop of small intestine to the anterior wall of the stomach. The posterior operation consists in anastomosing a high portion of the jejunum with the posterior

Fra. 531.

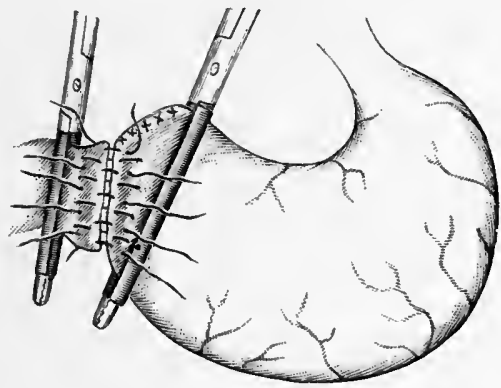

Gastro-duodenostomy completed.

wall of the stomach near the pyloric extremity. The latter operation is the one now most frequently employed.

The operation is employed to relieve pyloric obstruction and in cases of gastric ulcer.

Posterior gastro-enterostomy is done as follows: An incision opening the abdomen is made a little to the right of the median line; the transverse colon is exposed and drawn to the right and upwards, and the mesocolon made to follow it until the jejunum is exposed. The latter is grasped at a distance of three or four inches from its origin. When drawn tight the mesocolon should be torn through at a non-vaseular part to expose the posterior wall of the stomach. The posterior wall of the stomach is then drawn through the mesocolic opening to expose that portion of the organ about one inch above the greater curvature and 
two and a half inches to the left of the pylorus. This is held in the grasp of a Doyen's or Moynehan's or Mayo's clamp. The jejunum is next secnred with clamps similarly placed at a point from one and a half to three and a half inches of its origin (Fig. 532). If properly

FIG. 532.

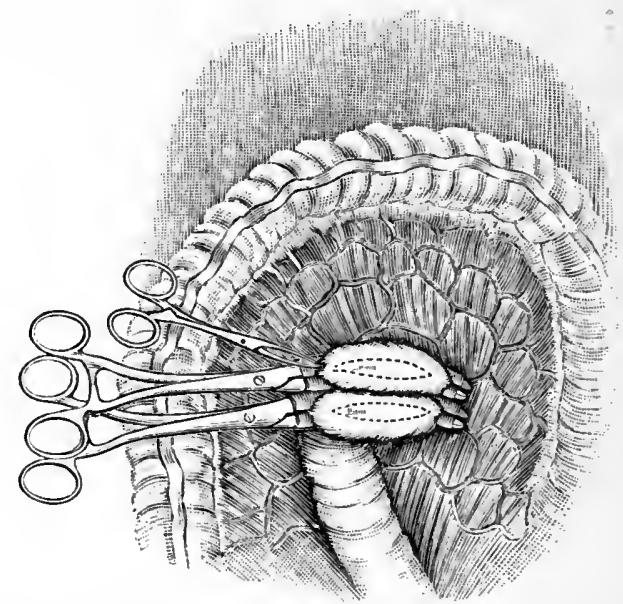

Clamps applied to stomach and jejunum, lines of Incision indicated. (Mayo).

placed, a low portion of the stomach is grasped by one clamp and the first portion of the duodenum is held by the other clamp, and the two portions should be easily brought into contact. A ganze pad is placed behind the clamps to prevent soiling in case of leakage of the intestinal contents. The clamps are bronght together and the gastric and intestinal surfaces are first united by a row of silk or celluloid thread sutures, including the serous and muscular coats, for a length of two inches. An incision is then made with a scalpel a quarter of an inch from and parallel to the line of sutures, through the serous and muscular coats of the stomach or jejunum. The incision should be a little shorter than the line of sutures. 
If vessels bleed they should be secured by ligatures.

Through the opening the mucous membrane prolapses, and it is grasped with forceps and a narrow elliptical portion is exeised with scissors, exposing the eavity of the stomach and gut. A row of silk or celluloid thread sutures is next applied as a continuous suture, bending together all the coats of the intestine and stomach, and should be closely placed so as to prevent hemorrhage when the clamps are removed. The lower side having been first closed the sutures are continued, applied in the same manner until the upper margin of the opening is completely closed. A fresh layer of sutures is next applied which secures accurate apposition of the serous and muscular coats and prevents leakage. Two or three extra sutures may be applied at either end of the line of sutures for additional security. The clamps and gauze pads are removed and the margin of the mesocolic opening is attached to the posterior wall of the stomach by two or three sutures, and the parts are replaced within the abdomen and the abdominal incision closed.

Gastro-Enterostomy with Elastic Ligature.-This procedure, devised by McGraw, which has been employed in a considerable number of cases with success, may be used in gastro-enterostomy or entero-enterostomy. A round rubber ligature $2 \mathrm{~mm}$. in diameter is employed. The walls of the stomach and of the intestine are first united by a posterior row of Lembert sutures. A long straight needle armed with the rubber ligature is passed into the lumen of the bowel and brought out at a point 5 to $10 \mathrm{~cm}$. distant from the site of its introduction.

An assistant holds the intestine, and the surgeon stretehes the ligature in the needle and when it is quite thin draws it through the intestine; the same procedure is repeated in passing the ligature through the walls of the stomach. A strong silk ligature is next placed across and beneath the rubber ligature, between the latter and the point where the stomach and intestine come in contact. The rubber liga- 
ture is then drawn tight and tied with a single knot; the silk ligature is passed around the ends of the ligature where they cross and is firmly tied with three knots, and the ends of the elastic ligature are released and cut off. An anterior row of Lembert sutures are then applied to make the junetion between the stomach and intestine complete. The operator should be careful not to tie the rubber ligature too far backward and thus place it behind the posterior row of Lembert sutures.

Gastro-Enterostomy with Murphy Button.-The viseera are exposed by the usual ineision, and the jejumum is found and carried in front of the great omentum and transverse colon and bronght in contact with a non-vascular part of the greater curvature of the stomach. The wall of the stomach is incised and the male half of the button is inserted and seeured by a purse-string suture. For this operation a button with halves of different size and weight has been devised, the larger half being placed in the intestine to favor the button escaping into the intestine rather than into the stomach when it becomes detached. The female half of the button is next inserted into the jejunum opposite the mesenteric attachment and secured in the same manner. The two halves of the button are approximated in the usual manner; the button-anastomosis may be reinforced by a few Lembert tissues.

Gastroplication.-This operation consists in taking a number of "tucks" in the stomach wall to reduce its capacity. The operation is employed in cases of dilated stomach, but should not be employed when the dilatation is dne to pylorie stenosis, as the relief of the latter condition will cause the stomach to return to its normal size.

Gastropexy.-This operation is employed to fix a displaced stomach in its normal position. The stomach is exposed by the usual abdominal incision, and is secured to the anterior abdominal wall by sutures passed through the serous and muscular walls of the stomach and the parietal peritoneuni and fascia. The method of applying sutures 
is shown in Fig. 533. The stomach is also held in its normal position by shortening the gastro-hepatie and gastrophrenie ligaments by the introduetion of several layers of sutures.

FIG. 533.

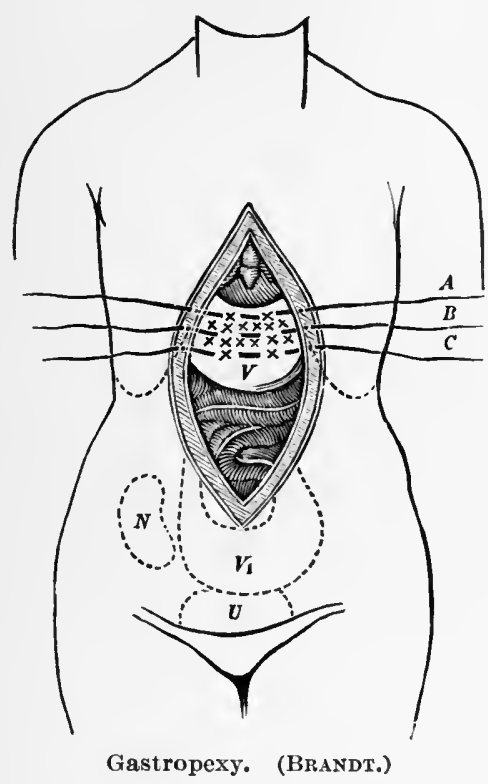

\section{INTESTINAL SUTURES.}

Sutures employed in wounds and operations upon the intestines and hollow viseera are either of fine eatgut, silk, or celluloid thread. A round needle such as the ordinary sewing needle is used in preference to the bayonet-pointed needle, as it does not eut the tissues and there results less bleeding from the punctures. 'Two layers of sutures should be used when it is possible, the deep layer of sutures including the mucosa and the superficial layer of the serous and muscular coats. 
Aceurate apposition of the edges of the bowel should be obtained to prevent leakage. This is especially important in wounds of the small intestine.

Care should be taken that the lumen of the bowel is not reduced by the sutures.

Lembert's Suture.-Lembert's suture is used in wounds of the viscera covered by the peritoneum, with the objeet of bringing in contact the peritoneal surfaces. This form of suture is usually employed in closing wounds of the intestine, bladder, and stomach.

FIG. 534.

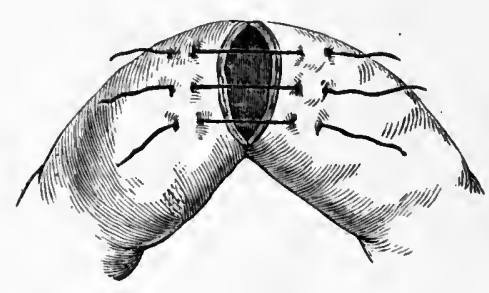

Lembert's suture. (BRYANT.)
FIG. 535.

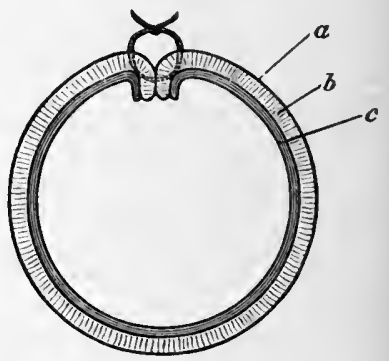

Lembert's suture. $a$, serous : $b$, museular; and, $c$, mueous coat. (SмiтH.)

A needle armed with a fine catgut, silk, or celluloid thread is first carried through the peritoneal and muscular coats of the intestine a short distance from the wound, and it is then carried across the wound and passed through the same portions of the intestine a short distance from the edge of the wound on the opposite side (Fig. 534), and when the suture is tightened the peritoneal surfaces of the intestine are inverted and brought into contact with each other (Fig. 535); the interrupted or continued suture nay be enployed in making this form of suture.

Halstead's Mattress or Quilt Suture.-This is a modification of Lembert's suture. The needle penetrates the peritoneal and muscular coats of the gut, including a 
small portion of the submucosa, twice on each side of the wound and is then tied (Fig. 536).

Purse-string Suture.-This form of suture is frequently used in operations upon the intestines and other abdominal viscera for covering in the stump of an appendix and in operations upon the gall-bladder, and in securing the two sections of the Murphy button. It is applied by introdneing a continuous suture of silk or eatgut in a cireular manner at a little distance from the part to be covered in, the stitehes pick up the peritoneal and muscular coats at intervals, and when the part has been circumseribed by tying the ends of the suture, the parts are puckered and bring the serous surface in contact over the part to be included (Fig. 537).

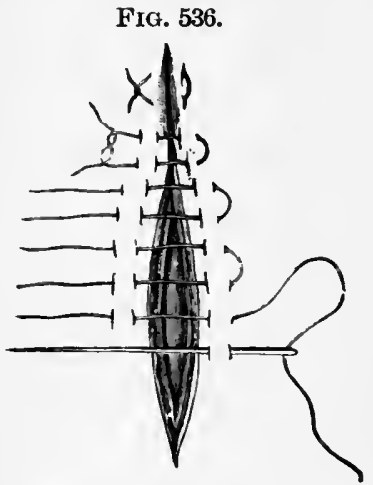

Halsted's quilt suture for intestine.

Czerny-Lembert Suture.-This suture is a combination of the Czerny suture, which is a suture passed through all the coats of the bowel, and a Lembert suture, which includes only the serous and muscular coats of the bowel in its grasp. For the deep suture, which includes all the coats of the bowel, catgut should be used and the knots should be placed within the lumen of the bowel. Either of these sutures may be interrupted or continuous. This suture may be employed in wounds or in endto-end approximation or lateral anastomosis of the intestines.

Enterotomy.- This consists in an incision into the large or small intestine for the purpose of diagnosis or for the removal of tumors or foreign bodies. The abdomen is opened by the ordinary incision and the bowel is drawn into the wound, gauze pads being packed around it to protect the peritoneal cavity. The bowel is incised in the 
longitudinal direction and the wound is elosed with fine silk Lembert sutures.

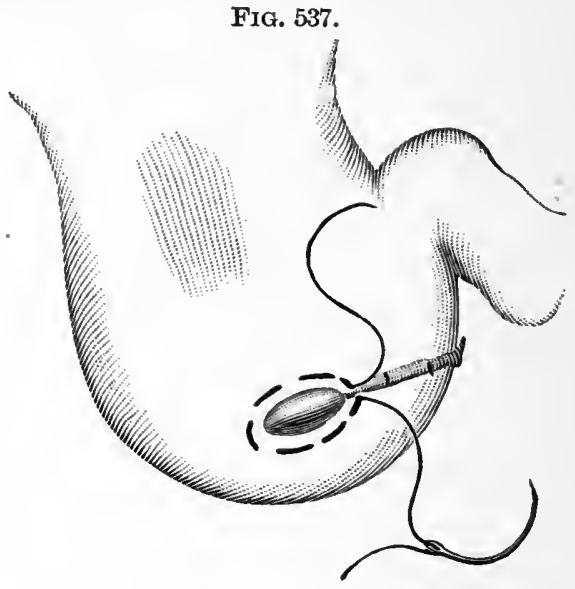

Purse-string suture applied to cover in stump of appendix.

Enterectomy.-This consists in removing a section of the intestine for extensive wounds or gangrene of the gut, FIG. 538 .

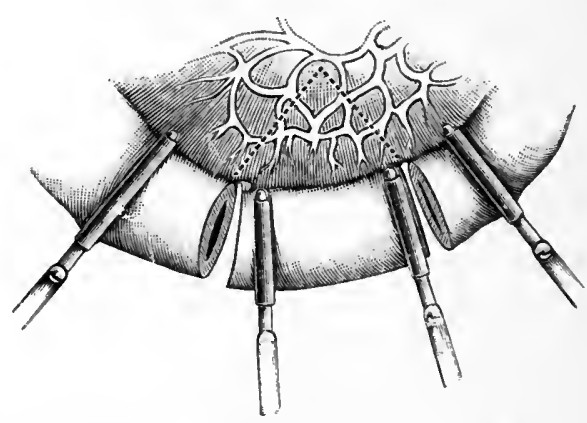

Enterectomy, application of clam ps, dotted line shows section of mesentery. (After BINNiE.)

for the removal of a growth, for stricture, or for the treatment of a fecal fistula. The bowel is exposed by the ordi- 
nary abdominal incision and the portion of the bowel to be removed is drawn out of the abdomen; the peritoneal cavity being protected by gauze pads. The lumen of the bowel, above and below the area to be removed, is closed by clamps protected by rubber or by gauze ligatures. (Fig. 538). An incision, $V$-shaped, including the intestine and a portion of the mesentery, is next made, and after ligating any bleeding vessels the mucous edges of the gut are disinfected by bichloride solution. The edges of the mesenteric wound and the ends of the gut are finally approximated by sutures, circular enterorrhapliy, or by the Murphy button.

FIG, 539.

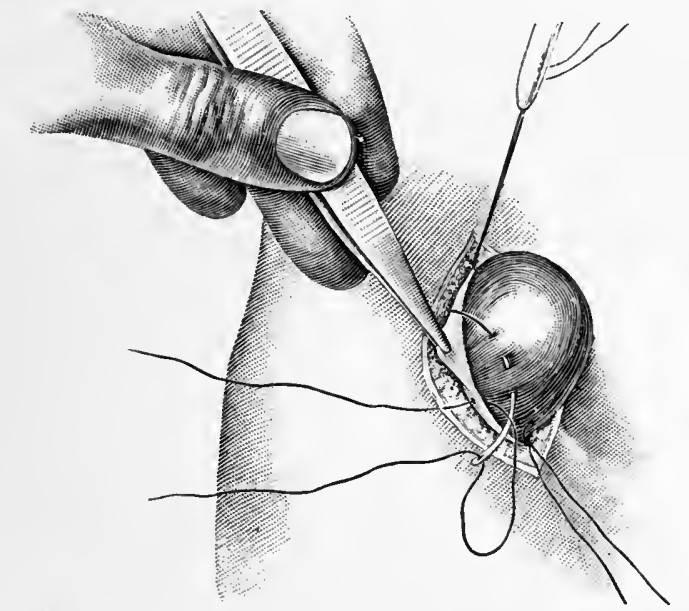

Fixation of a loop of bowel to the peritoneum. (LEJARS.)

Enterostomy.-This operation consists in attaching some portion of the bowel to the parietal peritoneum to establish a fecal fistula or artificial anus. The portion of bowel selected should be above the disease or constriction.

The abdomen is opened by the usual incision, and a 41 
distended loop of gut is seized and traced to the point of obstruction.

The parietal peritoneum is grasped with forceps and the distended loop of gut is attached to it by a few fine sutures passed with a round needle (Fig. 539). If the emergency is great the bowel may be opened immediately; if not, the opening of the bowel may be postponed for twelve hours to permit of the formation of adhesions shutting off the abdominal cavity.

A glass or rubber tube may be secured in the opening to carry off the fecal discharge.

\section{METHODS OF INTESTINAL ANASTAMOSIS.}

End-to-end Entero-enterostomy.-After division or resection of the intestine the ends may be united by sutures. Interrupted or continuous sutures may be employed. The sutures should be first applied at the mesenteric border, and great eare should be exercised to make the apposition close at this point. The ends of the bowel should then be brought together with closely applied Lembert sutures, including the serous and muscular coats. A second layer of Lembert sutures is next applied covering the first layer. If the mesentery has been divided, it should also be approximated by sutures (Fig. 540).

Circular Enterorraphy.- This consists in an end-toend union of a divided bowel. The ends of the intestine are brought into apposition and sutures of silk or catgut in a curved needle held by a needle-holder are passed as interrupted sutures through all the coats of the bowel, and are tied so that the knots are within the lumen of the bowel. (Fig. 541).

When the circular enterorraphy is almost completed, the last two or three sutures are difficult to place, owing to the tendency of the knots to remain outside of the bowel. This can be overcome by invaginating them with a probe within the second layer of interrupted Lembert sutures. 
The second layer of interrupted Lembert sutures are next passed by a straight needle armed with silk so as to include the serous and muscular coats of the bowel and completely cover the first layer of sutures. For additional security it is well to insert one or two extra sutures at the mesenteric border.

FiG. 540.

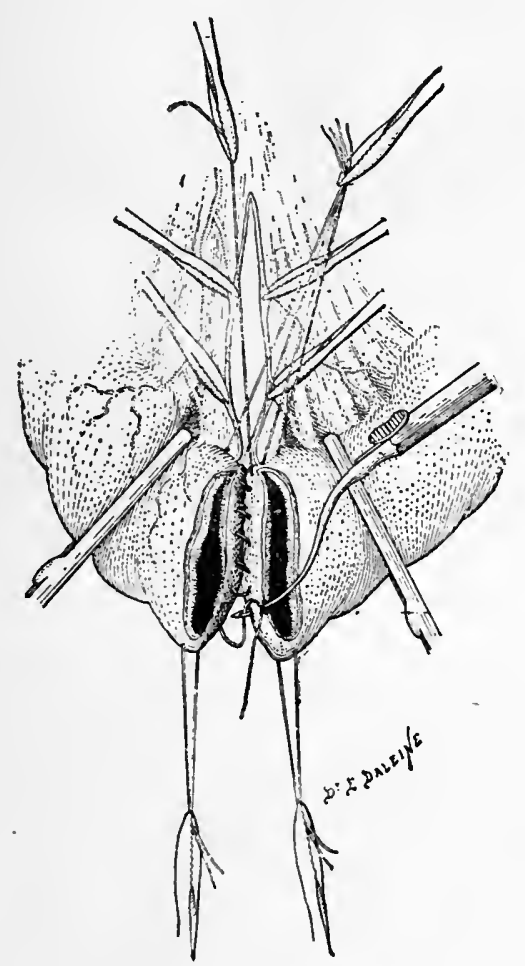

End-to-end or circular anastomosis. (LEJARs).

The Murphy Button.-This is a mechanical contrivance which may be employed to secure end-to-end-apposition of the divided intestine, or may be used to form a lateral 
anastomosis between the intestines or hollow viscera. The construction of the button is shown in Fig. 542. This

FIG. 541.

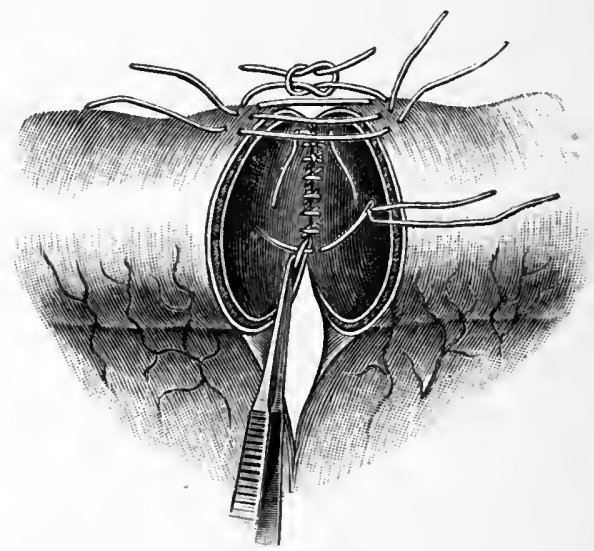

Entero-enterostomy with Czerny-Lembert interrupted sutures. (After BЕСКнАН.)

method of end-to-end approximation or anastomosis can be accomplished with accuracy and with great rapidity.

FIG. 542.

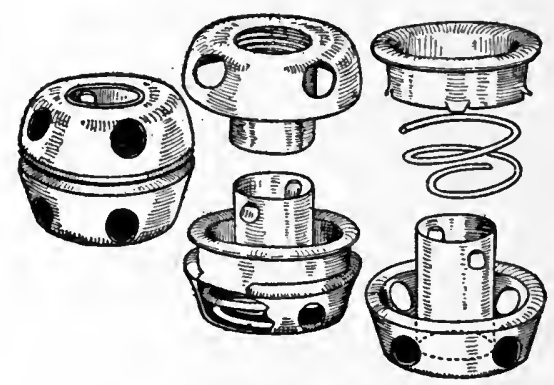

The Murphy button.

In employing the button for these purposes, it is separated into its two parts, and each part is slipped into the divided 
end of the intestine and secured by a purse-string suture (Fig. 543), and the parts are approximated by fastening the two portions of the button together (Fig. 544). After

FIG. 543.
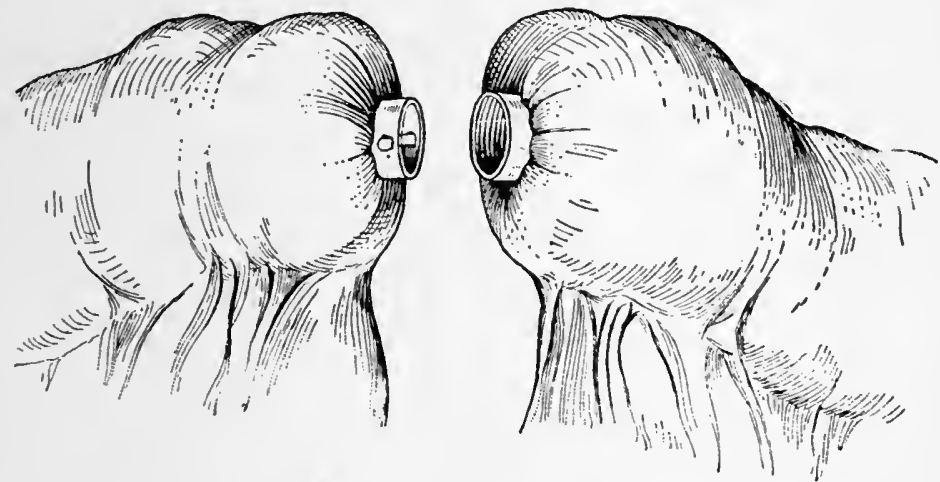

The two portions of the Murphy button held in place by purse-string sutures. (RICHARDSON.)

securing the button, for additional security it is well to bring the gut together over the line of apposition by a layer of interrupted or continuous Lembert sutures.

Fig. 544.

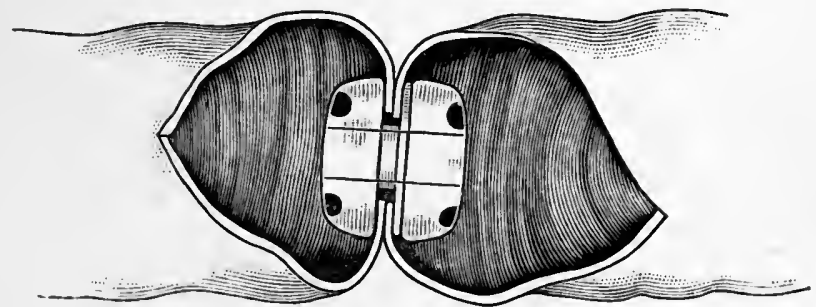

End-to-end union of intestine with Murphy's button. (RICHaRdon.)

Where lateral anastomosis between the intestines, or between the intestine and another hollow viscus, is desired, an incision is made in each organ, and half of the button is slipped into each opening and secured by a purse-string 
suture, and the portions of the button aire then fastened together (Fig. 545). Union of the peritoneal surfuces results, and the button is usually released in from ten to twelve days by sloughing of the included tissues, and is passed by the anus.

FIG. 545.

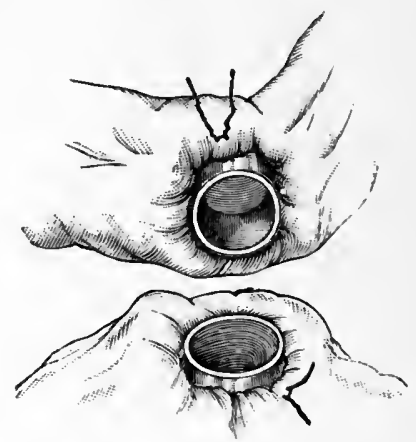

Lateral anastomosis with Murphy button, each half the button belng secured in the gut ready for approximation. (BERGMANN.)

\section{Abbie's Method of Lateral Anastomosis.-Portions} of the intestinal tract more or less distant, or the intestine and the stomach, may be united by this procedure, thus permitting the contents to pass through the new opening. The bowel upon each side of the constricted portion is manipulated, so that both portions lie side by side; or, in case a portion of the bowel has been removed, the ends are inverted and closed by Lembert's sutures. The two portions of the bowel are brought side by side, and a longitudinal cut three inches in length, opposite the mesenteric attachment, is made through the coils to be united. The posterior edges of the incision should first be brought together by continuous interrupted sutures (Fig. 546).

The margins of the incision may be hemmed before uniting them. The anterior edges of the incision are next united by another continuous stitch, and for additional security a second line of interrupted or continuous sutures 
FIG. 546.

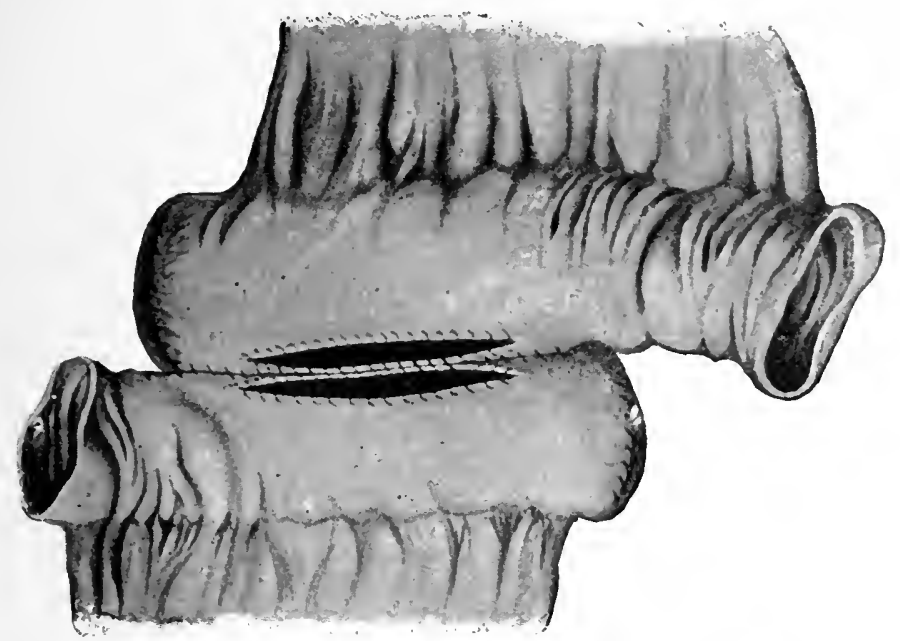

Lateral anastomosis. First stage of operation. (RICHARDSON.)

Fra. 547.

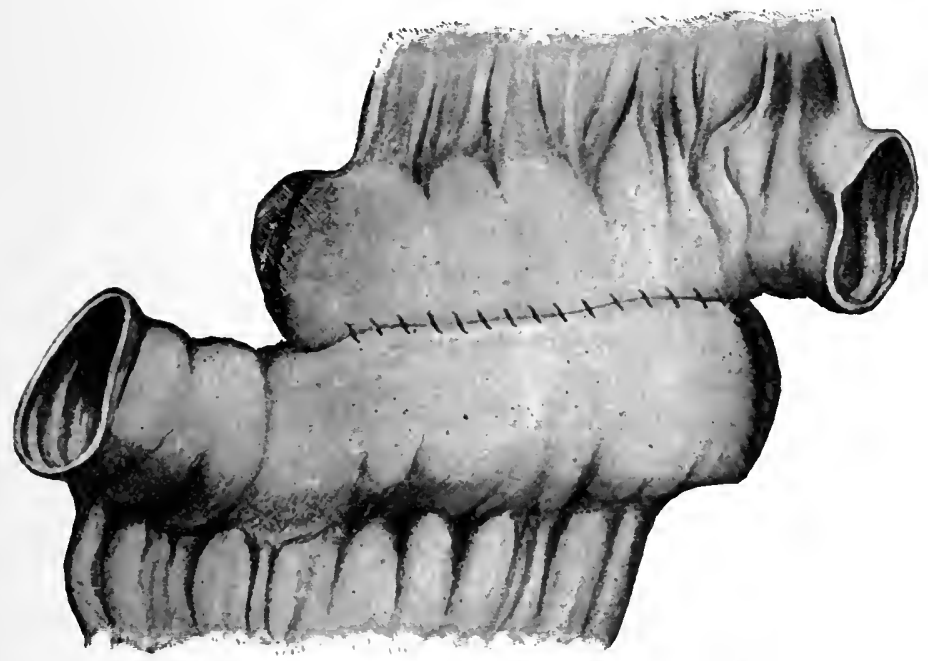

Lateral anastomosis; operation completcd. (RICHARDSON.) 
may be applied (Fig. 547). The time required for the application of the sutures is one disadvantage of this operation.

Lateral anastomosis may be employed instead of the circular suture in wounds completely dividing the intestine, and after resection of the intestine for the removal of growths or for stricture.

Harrington's Segmented Ring.-This device, which is employed for end-to-end anastomosis, lateral anastomosis, and gastro-enterostomy, consists of aluminum rings made of different sizes, the largest less than an inch in diameter, in four sections, whieh are held together by a serew at the end of a handle (Fig. 548).

FIG. 548.

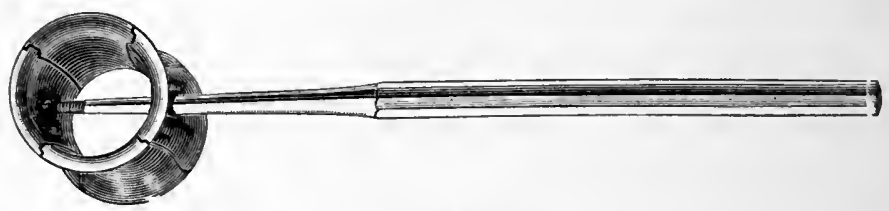

Harrington's segmented ring.

Purse-string sutures of silk or catgut are introduced into the ends of the intestine to be united after introducing the ring into each end of the intestine. These sutures are firmly tied in the groove of the ring and the peritoneal surface of the gut is approximated by a layer of Lembert sutures. After applying the sutures the handle of the ring is carefully removed by turning the screw, and a sulture is introduced into the gut at the part where the handle is removed. The purse-string sutures hold the ring in place until the sutures soften; or if it is desired to immediately separate the segements of the ring, it may be done by gentle manipulation upon the ring through the walls of the intestine.

Anastomosis or End-to-end Approximation by Bone Plates, Forceps, Absorbable Bobbins.-Senn devised a method of anastomosis by the use of decalcified bone plates, these were employed for a long time but have been replaced 
by other methods. Special forceps have been clevised by Laplace, O'Hara and other surgeons to accomplish the same purpose. Absorbable bobbins of vegetable tissue have also been employed at times but nost of these devices have fallen into disuse, as the technique of circular suture and lateral anastomosis have been perfected.

\section{OPERATION FOR STRANGULATED HERNIA.}

This consists in an incision over the hernial swelling, with careful dissection of the various layers until the sac is reached. The sac should then be freely opened and its contents examined. If the bowel is dark and without lustre, gangrene is present, but if the gut is dark and retains its lustre, it may be restored by the relief of the strangulation. If the sac contains a fair amount of bloodstained serum, the bowel is usually found in good condition as regards viability. The surgeon should next locate the point of constriction of the bowel and divide it, either from within by means of a small, blunt-pointed hernial knife, or better, by a careful dissection from without, dividing all overlying tissues. When the constriction has been relieved, the bowel should be drawn well down into the wound. If the color of the bowel improves after division of the stricture, it should be irrigated with warm salt solution, dried and returned into the abdominal cavity, the wound closed, and a gauze dressing applied. If the bowel is gangrenous, the gangrenous portion should be resected, and an end-to-end anastamosis should be made with sultures or by the use of Murphy's button. If the condition of the patient is such that this procedure is not advisable, the bowels should be incised and the wound allowed to remain open for the formation of a fecal fistula. In many cases the operation for radical cure may be performed immediately after the relief of a strangulated hernia. 


\section{OPERATION FOR THE RADICAL CURE OF FEMORAL HERNIA.}

Bassini's method consists in making an incision parallel with, and just below Poupart's ligament, with the centre over the saphenous opening. The skin and superficial fascia are incised and the edges of the saphenous opening

Fia. 549.

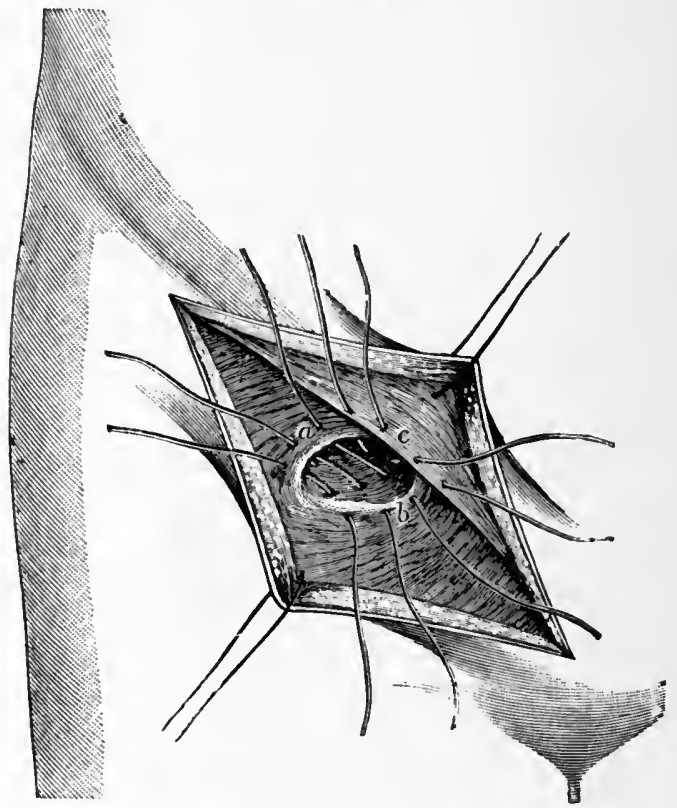

Operation for the radical cure of femoral hernia (Bassini's method): $\boldsymbol{a}$, falciform process; $b$, pubic portion of fascia lata; $c$, Poupart's ligament. (BRYANT.)

exposed. The hernial sac is then exposed and dissected out of its canal to as high a point as possible. The sac is next opened and its contents returned to the abdominal cavity; it is then drawn well downward and its neck transfixed with a double ligature of chromicized catgut; the ends of the ligature are crossed, and the neck of the sac is ligated 
and eut off below the ligatures. The stump is then returned into the abdomen. Sutures of kangaroo tendon or ehromicized catgut, earried by a curved needle, are introdueed to approximate Poupart's ligament to the peetineal faseia, the first suture near the pubic spine, the seeond a quarter of an inch to the outer side of the first, and the third about three-quarters of an inch to the inner side of

FIG. 550.

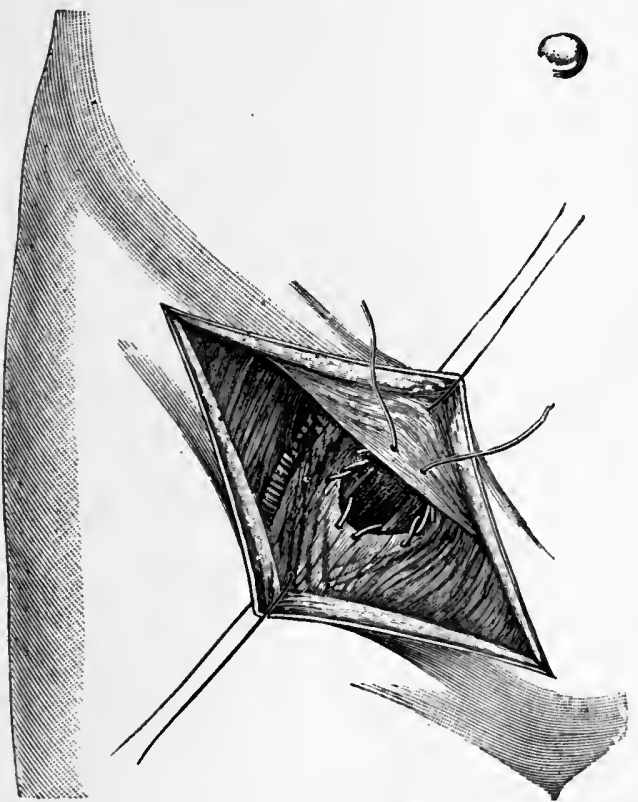

Operation for the radical cure of femoral hernia: purse-string suturc (Cushing's method.) (BRYANT.)

the femoral vein; these sutures passing through the peetineal fascia below. These sutures are left untied. 'Three or four sutures are next passed through the faleiform process of the iliac portion of the fascia lata externally and the peetineal fascia; the lowest suture passes just above the saphenous vein (Fig. 551). The three upper sutures are next tightened and tied, and Poupart's ligament drawn 
FIG. 551.

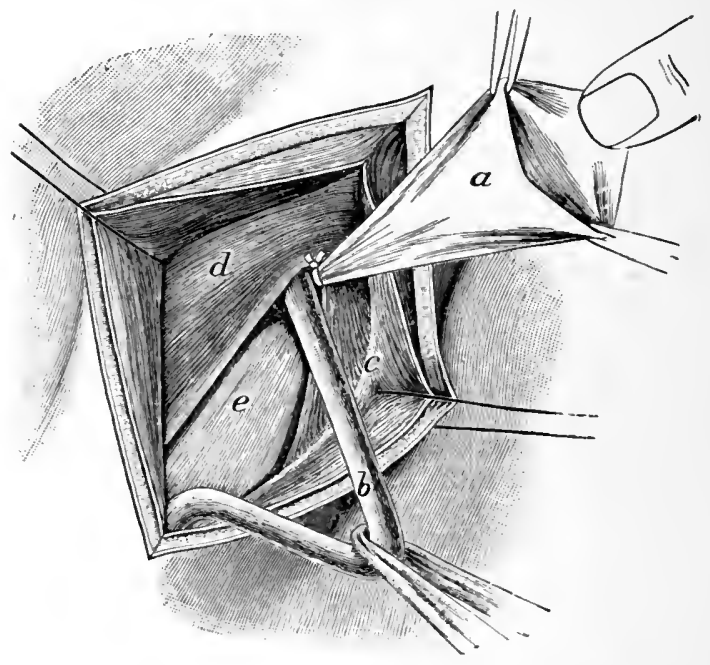

Operation for the radical cure of inguinal hernia (Bassini's method): $a$, sac dissected from the cord and neck ligated : $b$, cord : $c$. Poupart's ligament; $d$, arched fibres of internal oblique muscle; $e$, transversalis fascia. (BRYANT.)

FIG. 552 .

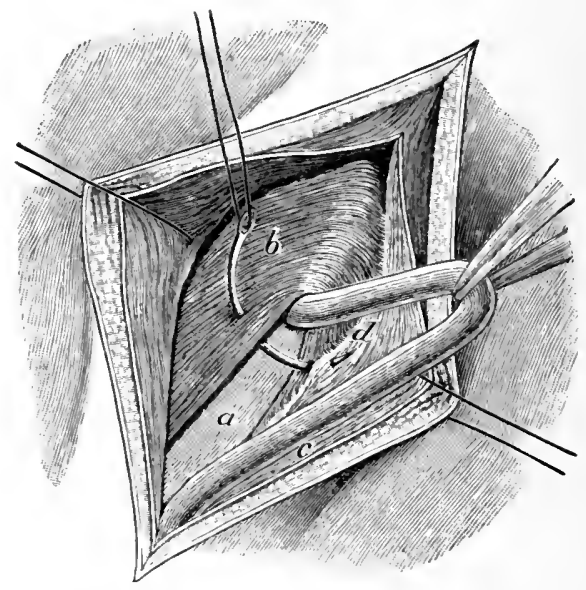

Operation for the radical cure of inguinal hernia (Bassini's method) : sac removed $(c)$, cord drawn aside, and stitching of lower fibres of the internal oblique and transversalis muscles $(b)$ to Poupart's ligament $(d)$ from without inward; $a$, transversalis fascia. (BRYANT.) 
Fra. 553.

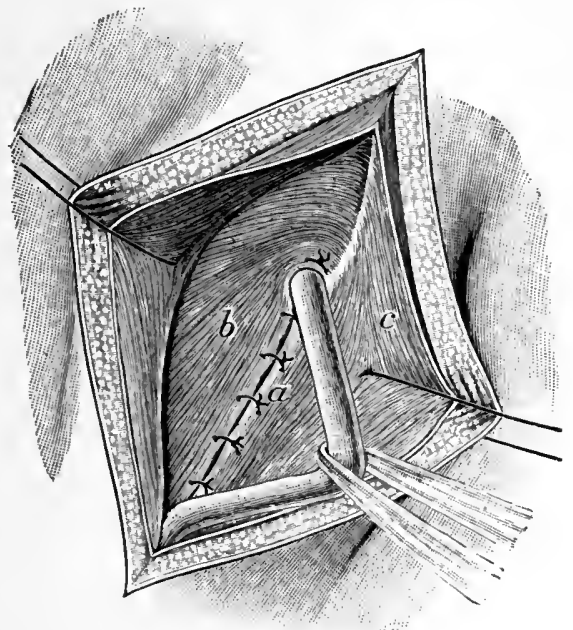

Operation for the radical cure of inguinal hernia (Bassini's method): arched muscular fibres and conjoined tendon $(b)$ sewed to Poupart' ligament (a); c, aponeurosis of external oblique muscle. (BRYANT.)

Fig. 554 .

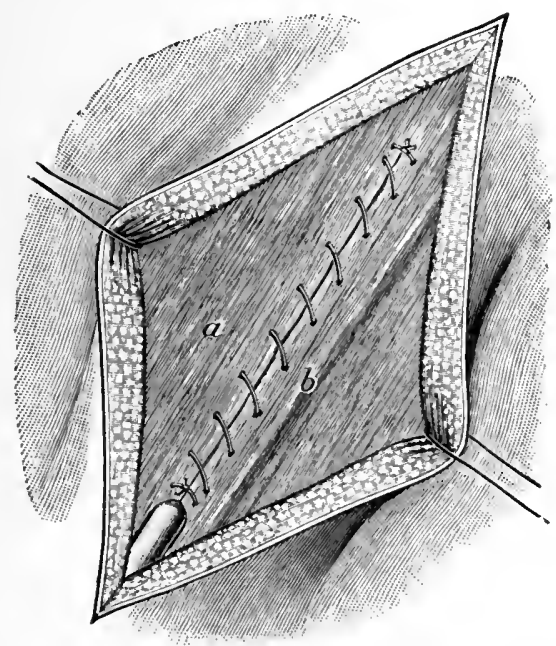

Operation for the radical cure of inguinal hernia (Bassini's method): aponeurosis of external oblique $(a)$ sewed with continuous sutures to Poupart's ligament (b). (BRYANT.) 
backward to the linea pectinea. The lower sutures are next tightened and seeured, and approximate the anterior and posterior walls of the femoral canal. The skin aurl superficial faseia are then closed with catgut or silkwormgut without drainage. A gauze dressing is applied over the wound and held in position by a spica bandage.

Another method is that of Cushing, which consists in the introduetion of a purse-string suture around the margin of the saphenous opening, beginning and ending through the dense tissue of Poupart's ligament (Fig. 550).

\section{OPERATION FOR THE RADICAL CURE OF INGUINAL HERNIA.}

The Bassini operation is the one most usually employed, and consists in making an incision over the inguinal canal, parallel with Poupart's ligament, extending from a point just above the outer side of the internal ring to a point one inch below and to the inner side of the spine of the pubis. The tissues are divided down to the aponeurosis of the external oblique muscle, and the external abdominal ring is exposed. A director is next introduced into the canal through the external ring, and the aponeurosis is divided in the direction of its fibres to the upper limit of the incision. The lower flap is everted and the strueture separated from its inner surface, exposing Poupart's ligament. The spermatic cord and the sac of the hernia are then separated by division and removal of the various tunies, and the cord is retracted. After the sac has been isolated from the surrounding tissues and opened, to insure that it contains no intestine or omentum, its neek is drawn outward and ligated with a transfixion suture of catgut, and the distal portion is excised (Fig. 551). The stump is allowed to fall back into the abdominal cavity. The cord is held up out of the wound by a retractor or loop of gauze, and a number of deep sutures of kangaroo tendon or chromieizod catgut are next passed through the lower border of the internal oblique musele, and the portion of Poupart's liga- 
ment which is exposed by inverting the inferior flap of the aponeurosis of the external oblique (Fig. 552). After the sutures have been tightened and secured, reconstructing the floor of the inguinal canal, the cord is allowed to drop into place (Fig. 553). The roof of the canal is reconstructed by uniting the divided external oblique aponeurosis. This may be done with a continuous or interrupted suture of fine catgut, and the closure should be complete enough to leave only the external ring open sufficiently to allow the cord to pass outward without causing constriction. (Fig. 554). The skiu and connective tissue should then be closed with catgut or silkworm-gut sutures, and a gauze dressing applied and held in position by a spica bandage.

\section{OPERATION FOR THE RADICAL CURE OF UMBILICAL HERNIA.}

An elliptical incision is made over the hernia, with upper and lower ends in the median line, the wilth of the ellipse depending upon the size of the hernia. The incision is first carried through the skin and fascia only, then deepened until the abdominal aponeurosis is reached, just outside of the hernial neck. The hernial sac is then exposed and opened, and if it contains a mass of omentum, this is ligated, and the bowel is returned into the abdomen and kept in place by a gauze pad. The entire sac, with the umbilicus and the coverings, is then excised, dividing the peritoneum in an elliptical manner about the neck of the sac. The edges of the peritoneum and the transversalis fascia are next sutured with interrupted or continuous catgut sutures; the borders of the abdominal ring, which consist of the sheath and margins of the recti muscles, are then freshened and the edges brought together with interrupted sutures of kangaroo tendon or chromicized catgut. The skin and fascia are then approximated with interrupted silkworm-gut sutures. A gauze dressing is then placed over the wound and held in place by a firmly applied abdominal bandage.

Blake's Method.-This consists in making a large elliptical incision around the hernia and removing the connective tissue so as to expose a large area of the aponeurosis. 
The sac is excised and the edges are brought together with sutures. The linea alba is next divided to the upper and lower limits of the skin incision. The peritoneum is next separated from the abdominal wall on one side, and the muscular and aponeurotic flap thus formed is drawn to the opposite rectus muscle and secured by the layer of mattress sutures of chromicized catgut. The first layer of sutures unites the free border of the overlapped flap to the overlapping wall by throngh-and-through sutures, the second layer unites the free borders of the superficial to the deeper

FIG. 555 .

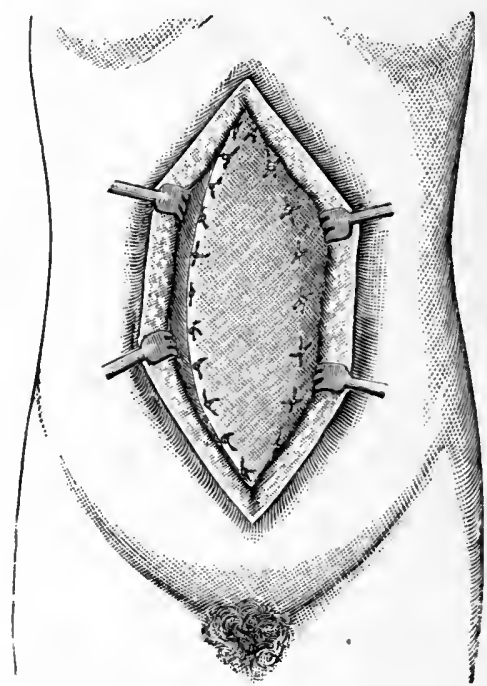

Blake's operation for the radical cure of umbilical hernia. (BREWER.)

wall by sutures which do not penetrate the peritoneal cavity. The skin wound is finally closed without draiuage. Mayo's Operation.-This consists in making a transverse elliptical incision arcund the umbilicus and the hernia and deepening it until the base of the hernial protrusion is exposed. Then expose the aponeurotic structures for an inch and a half in all directions from the neck of the 
sac. Next divide the peritoneal and fibrous coverings of the hernia at the neck of the sac in a circular direction, and expose the hernial contents. If viscera are present separate the adhesions and reduce them. Ligate the contained omentum and remove it and the entire sac of the hernia.

Grasp the margins of the ring with forceps and approximate them in whatever direction overlaping proves easiest. Next incise the aponeurotic and peritoneal structures of the ring for a distance of one inch or more transversely to each side and separate the peritoneum from the under surface of the upper of the two flaps thus formed.

Starting one inch or more above the margin of the upper flap introduce three or four mattress sutures the loops of which should grasp firmly the upper margin of the lower flap. Sufficient traction in these sutures should be made to permit closure of the peritoneum with a continuous catgut suture.

Slide the entire lower aponeurotic flap into the space between the peritoneum and aponeurosis above and secure it in this position by tying the mattress sutures. The lower edge of the upper aponeurotic flap should next be sutured with eatgut to the aponeurosis below.

The superficial wound should then be sutured. 



\section{INDEX.}

A

ABBIE's intestinal anastomosis, 646

Abdomen, bandage of, manytailed, 32

Abdominal aorta, ligation of, 485

bandage, 32

nephrectomy, 607

Abscess, acute, treatment of, 268

chronic, treatment of, 269

Hilton's method in, 268

palmar, 272

treatment of, $26 \mathrm{~S}$

tuberculous, aspiration in, 270

incision in, 271

injection in, 270

iodoform emulsion in, 270

puncture in, 270

treatment of, 269

Absorbent cotton, 12:

Acetanilid, 322

Acetate of aluminum, 322

Acid, boric, 323

carbolic, 319

picric, dressing in burns, 284

salicylic, 324

Acromion process of seapula, fracture of, $38 s$

Actinomyces, 311

Active hyperæmia, 187

Actual cautery, 144

in venous hemorrhage, 263

Acupressure in arterial hemorrhage, 260
Acute abscess, treatment of, $26 \mathrm{~S}$

Adductor longus tendon, tenotomy of, 584

Adhesive plaster, 124

Adrenalin chloride in arterial hemorrhage, 254

Aluminum acetate, 322

Amputating knives, 502 saws, 503

Amputation or Amputations, 497

above shoulder-joint, 525

at ankle-joint, 534

Amputation at ankle-joint, Pirogoff's 535

Roux's 536

of arm, 519

circular, 519

by lateral flaps, 520

modified circular, 520

by transfixion, 519

Bier's osteoplastic method, 501

carpo-metacarpal, 515

circular, 497

details of, 507

at elbow, 517

anterior flap, 517

circular, 518

elliptical, 519

lateral flaps, 517

elliptical, 499

of fingers, 510

of foot, 527

Chopart's, 533

Hancock's, 537

Hey's, 533

Lisfranc's, 532 
Amputation of foot, Mikulicz's, 537

subastragaloid, 534

Tripier's, 5877

of forearm, 516

circular, 516

modified circular, 517

of hand, 510

at hip-joint, 545

by lateral flaps, 548

modified circular, 547

by transfixion, $5+6$

Wyeth's method, 548

instruments for, 502

at knee-joint, 541

anterior flap, 542

Carden's 542

elliptical flaps, 541

Gritti's, 543

lateral flaps, 543

through condyles of femur, 542

of leg, 537

circular, 53S

elliptical, 538

flap method, anterior, 538

antero-posterior, 540

external, 541

lateral, 540

rectangular, 540

modified circular, 53s.

Sedillot's 541

Teale's 540

ligatures in, 506

medio-tarsal, 533

of metacarpal bones, 513

metacarpo-phalangeal, 511

of metatarsal bones, 529

modified circular, 499

oval, 499

periosteal flaps in, 502

redressing of, 508

retractors in, 505

at shoulder-joint, 521

Dupuytren's, 523

Larrey's, 522

Lisfranc's, 524

Spence's, 525

Wyeth's pins in, 522

sutures in, 506
Amputation, tarso-metatarsal, 530

Teale's method in, 500

of thigh, 543 et seq.

by anterior flap, 543

modified circular, 543

through the trochanters, 545

by transfixion, 545

of toes, 527

tourniquets in, 506

transfixion method in, 498

at wrist, 515

circular, 515

flap method, 515

lateral flaps, 516

Anæsthesia from chloride of ethyl, 193

from cocaine, 194

from cold, 193

general, 193

Anæsthesia from kelene, 193

local, 193

from rapid respiration, 194 regional, 193

from scopolamine hydrobromate, 215

spinal, 195

Anæestheties, 193

in fracture, 362

Anastomosis forceps, 649 intestinal, 642

Ankle, dislocation of, 462

Ankle-joint, amputation at, 534

excision of, 565

strapping of, 131

Anterior crural nerve, exposure of, $5 \mathrm{~S} 2$

figure-of-eight bandage of chest, 72

tibial artery, ligation of, 492 tendon, tenotomy of, 583

Antipyrin in arterial hemorrhage, 254

Antisepsis, 297

Antiseptic dressings, improvised, 335

operation, details of, 348

poultice, 134 
Antitoxin, 304

injection of, 170

Antrum of Highmore, trephining of, 573

Aorta, abdominal, ligation of, 485

Appendicitis, acute, operation for, 613

chronic, operation for, 615

incision through rectus muscle, 617

McBurney's operation for, 615

suppurative, Murphy.'s method in, 614

Approximation sutures, 231

Aqua ammonia as a rubefacient, 142

as a vesicant, 144

Aristol, 324

Arm, amputation of, 519

and chest bandage, 70

spiral reversed bandage of, 59

Arterial hemorrhage. Sce under Hemorrhage, arterial, 246

ligature in, 257

hyperæmia, 187

transf usion, 154

Arteriorraphy, 25s

Arteriotomy, 153

Artery forceps, 256, 504

Artery or Arteries, ligation of, 467. See under each artery.

temporary, 257

suture of, 258

wounded, ligation of, 261

Arthrectomy of knee-joint, 563

Artificial respiration, 156

direct, 158

Laborde's method, 161

Silvester's method, 160

Asepis, 297

agents to secure, 317

from heat, 317

Aseptic dressings, improvised, 335
Asceptic method, 316

operation, details of, 347

materials in, 325 et seq.

preparation for, 339

of patient for, 339

of room for, 339

Aspiration, 162

in tuberculous abscess, 270

Aspirator, Potain's, 163

Astragalus, excision of, 566

Anto-transfusion, 154

Axillary artery, ligation of, $47 \mathrm{~S}$

\section{B}

Bacrilus, aerogenes capsulatus, 311

anthracis, 309

coli communis, 308

of malignant œdema, 310

mallei, 309

of tetanus, 309

tubercle, $30 \mathrm{~s}$

Back, strapping of, 132

Bacteria, 297

cultivation of, 300

elimination of, 302

entrance into tissues, 301

infection by, 302

inoculation by, 300

intoxication by, 302

pathogenic action of, 302

resistance of tissues to, 303

staining of, 300

of suppuration, 307

varieties of, 307 et seq.

Bacteriology, surgical, 297

Bandage or Bandages, 17

abdominal, 32

many-tailed, 32

arm and chest, 70

Barton's, 42

modified, 42

black muslin, 100

Borsch's, 94

circular, 23

compound, 27

dimensions of, 20

demi-gauntlet, 57

Desault's, 67 
Bandage, elastic webbing, 102 Esmarch's, 251

figure-of-eight, 26,61

of knee, 82,84

of leg, 90

of neck and axilla, 65

flannel, 98

of foot, American, 86

complete, 85

French, 87

in fractures, 368

gauntlet, 56

gauze, 95 et scq.

Gibson's, 45

handkerchief, 33

hardening, 103

of hand, complete, 58

of head, 42

and neck, 50

of heel, 88

Liebreich's, 93

of lower extremity, 76

many-tailed, 31

oblique, 23

of head, 53

occipito-facial, 53

occipito-frontal, 54

paraffin, 118

of perineum, 91

plaster-of-Paris, application of, 105

preparation of, 104

for thigh and pelvis, 106 recurrent, 27

of stump, 91

removal of, 22

roller, 18

rubber, 100

scissors, 22

of Scultetus, 94

for securing hands and feet, 93

silicate of potassium, 117 of sodium, 117

spica, 26

of buttock, 81

of foot, 85

of groin, ascending, 76 descending, 78 double, 79
Bandage, spica, of shoulder, ascending, 62 descending, 64 of thumb, 59

spiral, 23

of chest, 72

of finger, 55

reversed, 24

of arm, 59

of fingers, 56

of leg, 88

of lower extremity, 88

of penis, 91

of thigh, 89

of upper extremity, 60 starched, 116

sterilized, 335

suspensory and compressor, of breast, 74

of trunk, 72

of upper extremity, 55

varieties of, 23

Velpeau's, 65

Bandaging, rules for, 21

Barton's bandage, 42

modified, 42

Bavarian dressing, 111

Beck's bismuth emulsion, 272

Bedsores, 288

Bellocq's canula, 265

Beta-naphthol, 321

Biceps flexor cruris tendon, tenotomy of, 584

Bichloride cotton, 335

gauze, 334

of mercury, 318

Bier's hyperæmic treatment, 183

osteoplastic method in amputation, 501

Binder's board splints, 119,336

Bis-axiliary cravat, 36

Bismuth emulsion, 272

Black muslin bandage, 100

Bladder, foreign bodies in, 291

hemorrhage from, treatment of, 267

implantation of ureter into, 610 
Bladder, irrigation of, 229 sccuring catheter in, 228 sterilization of, 341

Blood, transfusion of, 153 direct, 154

Bloodletting, 147

Bobbins, absorbable for intestinal anastomosis, 649

Bone chips, decalcified, 175 forceps, 504 hyoid, fracture of, 377 malar, fracture of, 374 metacarpal, amputation of, 513

nasal, fracture of, 372

plates, decalcified, 175

in intestinal anastomosis, 649

wax, 175

Bone-grafting, 174

Boric acid, 323

Borosalicylic powder, 324

Borsch's eye bandage, 94

Bougies, 224

œsophageal, 166

rectal, 167

sterilization of , 221,345

Bow-legs, osteotomy for, 570

Brachial artery, ligation of, 481

plexus, exposure of, 579

Breast, excision of, 585 radical, 589

suspensory bandage of, $3 \mathrm{~S}$ and compressor bandage of, 74

Bronchi, foreign bodies in, 294

Bronchoscope, 180

Bruises, 283

Brush-burn, 283

Buried suture, 236

Burns, 283

picric acid dressing in, 284

white lead dressing in, 284 $x$-ray, 288

Buttock, spica bandage of, 81

Button suture, 240
Cantharidal collodion, 143

Capillary hemorrhage, treatment of, 263

Capsicum as a rubefacient, 142

Carbolic acid, 319

Carbolized gauze, 335

Carden's amputation at kneejoint, 542

Cargile membrane, 333

Carotid artery, common, ligation of, 473

external, ligation of, 475

internal, ligation of, $4 \bar{i} 6$

Carpal bones, dislocation of, 453

fracture of, 407

Carpo-metacarpal amputation, 515

Carpus, dislocation of, 453

Cartilages, costal, dislocation of, 439

fracture of, $37 \mathrm{~s}$

semilunar, dislocations of, 462

Catgut, boiled, $32 \mathrm{~s}$

chromic acid, 330

chromicized, 330

cumol, 329

drainage, 332

dry sterilized. $32 \mathrm{~S}$

Elsenberg's, 329

formalin, $32 \mathrm{~s}$

iodine, 329

alcohol, 330

ligatures, 327

silverized, 330

Von Bergmann's, 32t

Catheter, female, introduction of, 227

introduction of, 224

male, securing of, in bladder, 228

metallic, 222

flexible, 222

sterilization of, 221,345

Catheterization, permanent, 227 
Catheterization of ureters in female, 227

Cauterization in arterial hemorrhage, 256

Cautery, actual, 144

in venous hemorrhage, 263

irons, 145

Celluloid thread, 330

C-E mixture, 214

Chain-stitch suture, 237

Chalk and gum splints in fiacture, 367

Chest, figure-of-eight bandage of, anterior, 72 spiral bandage of, 72 strapping of, 128 T-bandage of, 31

Chin, four-tailed bandage of, 32

Chloride of ethyl, anxsthesia from, 193

of lime and sodium for sterilizing hands, 344

of sodium, 325

of zinc, 322

Chloroform, administration of, 212

preparation of, patient for, 212

as a rubefacient, 141

as a vesicant, 144

Cholecystectomy, 623

Cholecystenterostomy, 624

Cholecystotomy, 622

Choledochotomy, 623

Chopart's amputation of foot, 533

Chromic acid catgut, 330

Chromicized catgut, 330

Chronic abscess, treatment of, 269

Cigarette drain, 332

Circular amputations, 497

Circular bandage, 23 enterorraphy, 642

Circumcision, 619

Clavicle, dislocation of, 440 acromial end of, 441 sterral end of, 441
Clavicle, excision of, 558 fracture of, 382

in children, 387

Closed fracture, 359

Clove-hitch knot, 437

Coaptation sutures, 231

Cocaine, 194

Coccyx, dislocation of, 438

excision of, 566

fracture of, 380

Cold, anæsthesia from, 193

in arterial hemorrhage, 254

Cold-water dressings, 138

Colles's fracture of radius, 402

reversed, 406

Colon, operations on, 611

Colostomy, inguinal, 611

lumbar, 611

Maydl's, 612

Comminuted fracture, 359

Common carotid artery, ligation of, 473

iliac artery, ligation of, 485

ligation of, transperitoneal, 486

Complete dislocation, 435

fracture, 357

Complicated dislocation, 435

treatment of, 466

fracture, 359

Compound bandage, 27

dislocation, 435

treatment of, 465

dorso-bis-axillary cravat, 37

fracture, 359,428

treatment of, 428 et seq.

Compresses, 123

in arterial hemorrhage, 248

in fracture, 368

Compression, 181

digital, in arterial hemorrhage, 247

in venous hemorrhage, 263

Congenital dislocations, treatment of, 466

Constriction, elastic, in arterial hemorrhage, 249

Continued suture, 236 
Contused wounds, treatment of, 279

Contusions, 283

Coracoid process of scapula, fracture of, $38 s$

Coronoid of ulna, fracture of, 400

Corrosive sublimate gauze, 334

Costal cartilages, dislocation of, 439

fracture of, 378

Cotton, 122

absorbent, 122

bichloride, 335

gloves, 346

sterilized, 336

Counterirritation, 140

Seguin's method, 144

Crossed bandage of eye, 51

Cumol catgut, 329

Cupping, 148

dry, 148

wet, 149

Cutting pliers, 504

Cystoscope, 178

Czerny-Lembert suture, 639

\section{D}

DeCalcified bone plates, 175

Decapsulation of the kidneys, 608

Decubitus, 288

Deep incisions, 148

sutures in arterial hemorrhage, 258

Demi-gauntlet bandage, 57

Desault's bandage, 67

Diffuse suppuration, 271

Digital compression in arterial hemorrhage, 247

Dislocation or Dislocations, 435

of acromial end of clavicle, 441

of ankle, 462

of carpal bones, 453
Dislocation of carpus, 453

of clavicle, 440

of coccyx, 438

complete, 435

complicated, 435

treatment of, 466

compound, 435

treatment of, 465

congenital, treatment of, 466

of costal cartilages, 439

of elbow, 449

of femur, 455

anomalous, 460

backward, 455

dorsal, 455

downward, 457

forward, 457

and upward, 459

pubic, 459

of fibula, 462

of fingers, 453

habitual, 436

treatment of, 466

of head of humerus, 443

of radius, 451

of hip, 455

of humerus, Kocher's method in, 447

Mothe's method in, 447

subclavicular, 443

subcoracoid, 443

subglenoid, 443

of hyoid bone, 439

of inferior angle of scapula, 442

of knee, 461

of lower jaw, 438

of metacarpal bones, 453

of metatarsal bones, 464

old, 436,465

reduction of, 465

partial, 435

of patella, 460

pathological, 466

of pelvis, 440

of phalanges of fingers, 453

of toes, 464

recent, 436

of ribs, 439 
Dislocation of scapula, 442 of semilunar cartilages, 462

of shoulder, 443

reduction of, 444 et seq. simple, 435

of sternal end of clavicle, 441

of sternum, 440

subspinous, of humerus, 444

of tarsal bones, 463

of toes, 464

treatment of, 436

of upper end of ulna, 451

of vertebræ, 437

of wrist, 452

Dorsal dislocation of femur, 305

Dorsalis pedis artery, ligation of, 493

Dorso-axillary cravat, 37

Double ligature, 243 roller-bandage, 20

T-bandage, 30

Drainage, catgut, 332 gauze, 332

horsehair, 332

Drainage-tubes, 331

Dressings, antiseptic, improvised, 335

aseptic, improvised, 335

Bavarian, 111

cold-water, 138

fixed, 103

gauze, $\mathbf{3 3 4}$

moist method, 351

plaster-of-Paris, 103

provisional, in fracture, 363

Velpeau's, 385

white lead, in burns, 284

of wounds, 277

Dry cupping, 148

method of dressing wounds, 338

sterilized gauze dressings, 336

Dupuytren's amputation at shoulder-joint, 523 splint, 426

\section{$\mathbf{E}$}

EAR, foreign bodies in, 295

Elastic constriction in arterial hemorrhage, 249

ligature, 245 gastro-enterostomy with, 635

Elastic-webbing bandage, 102

Elbow, amputation at, 517

dislocation of, 449

figure-of-eight bandage of, 61

Elbow-joint, excision of, 555 Electricity, injuries from, 286 treatment of, 287

Electrolysis, 176

Elimination of bacteria, 302 Elliptical method in amputation, 499

Emulsion, Beck's bismuth, 273

Enema, glycerine, 168 nutritious, 168

Enemata, 167

Enterectomy, 640

Entero-enterostomy, 642

Enterorraphy, circular, 642 with Murphy button, 643

Enterostomy, 641

Enterotomy, 639

Epiphyses, separation of, $\mathbf{3 7 0}$ diagnosis of, 371 symptoms of, 370 treatment of, 372

Epiphysis of humerus, upper, separation of, 390

Epistaxis, treatment of, 264

Esmarch's bandage, 251

Estlander's operation on ribs, 559

Ethyl chloride, anæsthesia from, 193

Excision or Excisions, 551 of ankle-joint, 565

of astragalus, 566

of breast, 588

of clavicle, 558

of coccyx, 566

of elbow-joint, 555

of hip, anterior, 562 
Excision of hip-joint, 561 of interphalangeal joints, 558

of joints, 551

of knee-joint, 563

of lower jaw, 568

of mammary gland, 588

of metacarpo-phalangeal joints, 558

of os calcis, 566

of patella, 564

of scapula, 560

of shoulder-joint, 554

of testicle, 620

of upper jaw, 567

of wrist-joint, 556

Mynter's method, 557

Exploring-needle, 172

Extensor longus digitorum tendon, tenotomy of, 5S4 proprius pollicis tendon, tenotomy of, 584

External carotid artery, ligation of, 475

iliac artery, ligation of, 487 œsophagotomy, 624

popliteal nerve, exposure of, 581

Extubation of larynx, 601

Eye, crossed bandage of, 51 foreign bodies in, 294

\section{F}

Facral artery, ligation of, 477 nerve, exposure of, 579

Faradization, 177

Feet, sterilization of, 341

Felon, 272.

Felt splints, 120, 366

Femoral artery, ligation of, 489

hernia, radical cure of, 650 truss for, 220

Femur, dislocation of, 455

anomalous, 460

backward, 455

dorsal, 455

downward, 457

forward and upward, 459 iliac, 455
Femur, dislocation of, pubic, 459

thyroid, 457

fracture of, 409

ambulatory treatment in, 417

incomplete, 416

shaft of, 412

in childden, 415

upper extremity of, 409

osteotomy of, 570

Fermenting poultice, 134

Fibula, dislocations of, 462

fracture of, 425

Pott's fracture of, 425

resection of, 565

Figure-of-eight bandage, 26

of chest, anterior, 72

posterior, 73

of elbow, 61

of knee, 82,84

of leg, 90

of neck and axilla, 65

Fingers, amputation of, 510

dislocation of, 453

fractures of, 407

spiral bandage of, 55

reversed bandage of, 56

Fissured fracture, 358

Fixed dressings, 103

Flannel bandage, 98

Flat knot, 234

Flaxseed poultice, 133

Flexible catheters, 222

Flexor longus pollicis tendon, tenotomy of, 584

Fluoroscope, 187

Fly blister, 143

Fomentations, hot, 134

Foot, amputation of, 528, 530

bandage of, American, 86 complete, 85

French, 87

spica bandage of, 85

Forced respiration, 162

Forceps, anastomosis, 649

artery, 256, 504

bone, 504

hæmostatic, 251 
Forearm, amputation of, 516 fracture of both bones of, 400

Foreign bodies in bladder, 291

in bronchi, 294

in ear, 295

in eye, 294

in intestines, 293

in larynx, 294

in nose, 295

in œesophagus, 293

in pharynx, 293

in rectum, 292

in stomach, 293

in trachea, 294

in urethra, 291

in vagina, 292

Formaldehyde, 321

Formalin, 321

catgut, 328

Four-tailed bandage of chin, 52

of head, 32

Fracture or Fractures, 357

of acromion process of scapula, 388

anesthetics in, 362

bandage in, $36 s$

bed, 365

of body of scapula, $3 s s$

of bones of leg, 419

ambulatory treatment in, 424

of both bones of forearm, 400

box, 367

of carpal kones, 407

of clavicle, 382 et seq.

in children, 387

Sayre's dressing in, 385

temporary dressing in, 383

Velpeau's dressing in, 385 closed, 359

of coccyx, 380

comminuted, 359

complete, 357

complicated, 359

compound, 359, 428
Fracture, compound, plasterof-Paris, dressing in, 430

treatment of, 425 et seq. compresses in, 368

of condyles of humerus, 393 of coracoid process of scapula, 388

of coronoid process of ulna, 400

of costal cartilages, 378 cradle, 368

deformity in, 361

dressing of, 372

evaporating lotions in, 368

examination of, 361

of femur, 409

ambulatory treatment in, 417

greenstick, 416

of fibula, 425

of fingers, 407

fissured, 358

greenstick, 357

of ulna and radius, 402

gunshot, 358

of head of radius, 400

of humerus, 388

of hyoid bone, 377

impacted, 350

incomplete, 357

of ulna and radius, 402

indented, 358

of inferior maxillary bone, 375

of jaw, lower, 375

upper, 374

of larynx, 377

longitudinal, 360

of lower end of radius, 402

extremity of humerus, 393

treatment by acute flexion, 395

of malar bone, 374

massage in, 369

of metacarpal bones, 407

of metatarsal bones, 427

multiple, 359

of nasal bones, 372 
Fracture of neck of radius, 400

of scapula, $3 \mathrm{Ss}$

oblique, 359

of olecranon process of ulna, 398

open, 359, 428

partial, 357

of patella, 417

of pelvis, 379

perforating, 358

of phalanges, 407

of toes, 427

plaster-of-Paris splints in, 367

provisional dressings in, 363

punctured, 358

rack, 368

reduction of, 364

of ribs, 377

of sacrum, 380

of scapula, 3॰7

setting of, 364

of shaft of femur, 412

in children, 415 incomplete, 416

of humerus, 391

silicate of potassium splints in, 367

of sodium splints in, 367 simple, 359

of skull, 382

splints in, 365

starched splints in, 367

of sternum, 379

subperiosteal, 358

of superior maxillary bone, 374

symptoms of, 360

of tarsal bones, 426

of tibia and fibula, 419

of trachea, 377

transverse, 359

of ulna and radius, 400

incomplete, 402

ununited, 432

of upper extremity of femur, 409

of humerus, 388
Fracture of upper jaw, 374 varieties of, 357

of vertebræ, 380

$x$-ray examination in, 363 of zygoma, 374

Franklinization, $17 \mathrm{~s}$

Frontal sinus, trephining of, 575

\section{G}

Galvano-Cautery, 177

Gastro-duodenostomy, 631

Gastro-enterostomy, 633

by elastic ligature, 635

with Murphy button, 636

Gastropexy, 636

Gastroplication, 636

Gastrorrhaphy, 626

Gastrostomy, 626

Ssabanajew-Frank's, 627

Witzel's, 627

Gastrotomy, 625

Gauntlet bandage, 56

Gauze bandages, 95

bichloride, 334

carbolized, 335

corrosive sublimate, 334

drainage, 332

dressings, 334

dry sterilized, 336

moist sterilized, 336

iodoform, 334

pads, 326

pledgets, 326

sterilized, 336

Gelatin in arterial hemorrhage, 254

General anæsthesia, 193

Gibson's bandage, 45

Gloves, cotton, 346

rubber, 345

silk, 346

Gluteal artery, ligation of, 488

Gluteo-femoral triangle, 39

Gluteo-inguinal cravat, 40

Glycerin enema, 168

tampon, 123

Gonococcus, 308

Granny knot, 234 
Greenstick fracture, 357 of femur, 416

of ulna and radius, 402

Gritti's amputation at kneejoint, 543

Groin, spica bandage of, ascending, 76

descending, $7 \mathrm{~S}$

double, 79

T-bandage of, 29

Gunshot fracture, 358

wounds, treatment of, 281

Gunstock deformity in fracture of condyles of humerus, 394

Gutta-percha splints, 366

\section{H}

Habitual dislocation, 436

Hæmostatic forceps, 251

Halsted's mattress suture, 638

Hamstring tendons, tenotomy of, 584

Hancock's amputation of foot, 537

Hand or Hands, amputation of, 510

bandage of, complete, $5 \mathrm{~S}$

removal of plaster-of-Paris from, 113

sterilization of, 342

chloride of lime and sodium, 344

Harrington's method, 343 by permanganate of potassium and oxalic acid, 343

Handkerchief bandages, 33 varieties of, 33,34

Hardening bandages, 103

Hatter's felt splints, 120

Head, bandages of, 42 four-tailed bandage of, 32 and neck bandage, 50 oblique bandage of, 53 recurrent bandage of, 47 transverse, 49

$\mathrm{V}$-bandage of, 49

Heat, asepsis from, 317
Heel, bandage of, SS

Hemorrhage, arterial, acupressure in, 260

adrenalin chloride in, 254

antipyrin in, 254

cauterization in, 256

compresses in, 248

deep sutures in, 258

digital compression in, 247

elastic constriction in, 249

gelatin in, 254

hot water in, 254

ligation in, 257

permanent control of,

253 et seq.

plaster tape in, 260

position in, 253

pressure in, 255

styptics in, 254

temporary control of, 247 et seq.

torsion in, 256

tourniquets in, 248

capillary, treatment of, 263

parenchymatous, treatment of, 263

from bladder, treatment of, 267

from rectum, treatment of, 267

from urethra, treatment of, 267

secondary, treatment of, 264

treatment of, 246

constitutional, 246

Iocal, 246

venous, actual cautery in, 263

compression in, 263

lateral ligature in, 262

treatment of, 262 et seq.

Hernia, femoral, radical cure of, 650

truss for, 220

inguinal, radical cure of, 654

truss for, 219 
Hernia, irreducible, truss for, 221

strangulated, operation for, 649

umbilical, radical cure for, 655

Blake's method of, 655

Mayo's method of, 656 truss for, 221

Hey's amputation of foot, 533

Hilton's method in opening abscess, 268

Hip, dislocation of, 455

Hip-joint, amputation at, 545 et seq.

excision of, 561

Horsehair drainage, 332 sutures, 327

Hot air, application of, 181 fomentations, 134

water in arterial hemorrhage, 254

as rubefacient, 140

Howard's method of artificial respiration, 158

Humerus, dislocation of head of, 443

epiphysis of, upper, separation of, 390

excision of, 554

fracture of, $3 s s$

of acromion process of, 388

of condyles of, 393

gunstock deformity in, 394

Jones's method in, 395

lower extremity of, 393

shaft of, 391

subclavicular dislocation of, 443

subcoracoid dislocation of, 443

subglenoid dislocation of, 443

subspinous dislocation of, 444

Hydrocele, eversion of sac in, 621

excision of sac in, 621
Hydrocele, injection treatment, 621

operations for, 620

radical treatment of, 621

Hydrogen peroxide, 323

Hyoid bone, dislocation of, 439

fracture of, 377

Hyperæmia, active, 187

arterial, 187

congestive, 183

treatment, Bier's, 183

Hypodermic injections, 169

of mercury, 171

IIypodermoclysis, 156

\section{I}

ICE-BAG, 139

Ichthyol plaster, 125 poultice, 136

Iliac artery, common, ligation of, 485

external, ligation of, 487

internal, ligation of, 486

ligation of, transperitoneal, 487

dislocation of femur, 455

Immediate irrigation, 136

Immunity, 303

Impacted fracture, 359

Implantation of nerves, 577

of ureter into bladder, 610

Incised wounds, treatment of, 277

Incisions, deep, 148

in tuberculous abseess, 271

Incomplete fracture, 357

of ulna and radius, 402

Indented fracture, 35s

Infected wounds, aseptic treatment of, 353

antiseptic treatment of, 353

Infection by bacteria, 302

Inferior dental nerve, exposure of, 579

maxillary bone, excision of, 568 
Inferior maxillary bone, fracture of, 375

thyroid artery, ligation of, 473

Inflation, mouth to mouth, 157

Infusion of saline solution, 156

Ingrown toenail, 605

Inguinal colostomy, 611 hernia, radical cure of, 654 truss for, 219

Injection of antitoxin, 170 hypodermic, 169

of mercury, hypodermic, 171

in tuberculous abscess, 270 urethral, 230

Injuries from electricity, 286 Innominate artery, ligation of, 469

Inoculation by bacteria, 300

Instruments for amputation, 502

sterilization of, $3+4$

Internal carotid artery, ligation of, 476

iliac artery, ligation of, 486

mammary artery, ligation of, 473

popliteal nerve, exposure of, 581

pudic artery, ligation of, 488

Interosseous artery, ligation of, 485

Interphalangeal joints, excision of, 558

Interrupted plaster-of-Paris dressing, 107 suture, 235

Intestinal anastomosis, 642

Abbe's method, 646

end-to-end, entero-enterostomy, 642

by forceps, 649

by Harrington's ring, 648 lateral, 646

by segmented ring, 648
Intestinal anastomosis, with absorbable bobbins, 649 with bone plate, 649

with Murphy button, 643 sutures, 637

Intestine, foreign bodies in, 293

Intoxication by bacteria, 302 Intravenous injection of saline solution, 155

Intubation of larynx, 597 et scq.

Iodine alcohol catgut, 330 catgut, 329

as a rubefacient, 141

Iodoform, 320

collodion, 320

emulsion, 270,320

in tuberculous abscess, 270

gauze, 334

Irreducible hernia, truss for, 221

Irrigation, 136

of bladder, 229

immediate, 136

mediate, 137

urethral, 231

Isinglass plaster, 126

J

$\mathrm{J}_{\mathrm{Aw}}$, lower, dislocation of, 438

excision of, 568

fracture of, 375

oblique bandage of, 46

upper, excision of, 567

fracture of, 374

Joints, excision of, 551 et seq. strapping of, 131

Jones' method in fracture of lower extremity of humerus, 395

Junk bags, 367

\section{$\mathbf{K}$}

Kelene, 193

Kidney, decapsulation of, 608 operations on, 607 
Knee, dislocation of, 461 figure-of-eight bandage of, 82,84

Knee-joint amputation at, 541 et seq. excision of, 563 arthrectomy of, 563

Knives, amputating, 502

Knock-knee, osteotomy for, 570

Knots for securing sutures and ligatures, 234

Koch's law, 301

Kocher's method in dislocations of humerus, 447

Krause's method of skingrafting, 174

Kreolin, 323

\section{$\mathbf{L}$}

LABORDE's method of artificial respiration, 161

Lacerated wounds, treatment of, 278

Laminectomy, 576

Larrey's amputation at shoulder-joint, 522

Laryngo-tracheotomy, 597

Laryngotomy, 596

Larynx, extubation of, 601 foreign bodies in, 294

-fracture of, 377 intubation of, 597 et seq. feeding after, 603 operation of, 599

Lateral ligature in venous hemorrhage, 262 lithotomy, 617

Lavage, 165

Lead water and laudanum, 135

Leather splints, 118, 366

Leech, mechanical, 151

Leeching, 150

Leg, amputation of, 537 et seq. figure-of-eight bandage of, 90

fracture of bones of, 419 ambulatory treatment in, 424
Leg, spiral reversed bandage of, 88

Lembert's suture, 638

Lengthening of tendons, 586

Leucocytosis, 306

Liebreich's eye bandage, 93

Ligation or Ligations, 467

of abdominal aorta, 485

of anterior tibial artery, 492

of arteries provisional, 257

temporary, 257

of axillary artery, 478

of brachial artery, 481

of common carotid artery, 473

iliac artery, 485

of dorsalis pedis artery, 493

of external carotid artery, 475

iliac artery, 457

of facial artery, 477

of femoral artery, 489

of gluteal artery, 488

of inferior thyroid artery, 473

of innominate artery, 469

of internal carotid artery, 476

iliac artery, 486

mammary artery, 473

pudic artery, 488

of interosseous artery, 485

of lingual artery, 476

of occipital artery, 477

of posterior tibial artery, 493

of radial artery, 481

of sciatic artery, 488

of subclavian artery, 471

of superior thyroid artery, 476

of temporal artery, 477

of ulnar artery, 483

of vertebral artery, 472

of wounded arteries, 261

Ligatures in amputations, 506

in arterial hemorrhage, 257

catgut, 327

double, 243 
Ligatures, elastic, $2 \dot{45}$

en masse, 258

lateral, 262

quadruple, 244

securing of, 233

silk, 326

single, with pin, 242

subcutaneous, 244

in vascular growths, 242

Lightning-stroke, 287

Lingual artery, ligation of, 478

nerve, exposure of, 579

Lint, 121

Lisfranc's amputation of foot, 532

of shoulder-joint, 524

Lister's aorta compressor, 250

Lithotomy, 617

lateral, 617

suprapubic, 618

Local anæsthesia, 193

Longitudinal fracture, 360

Lower extremity, bandages of, 76

spiral reversed bandage of, 88

jaw, dislocation of, 438

fracture of, 375

Lumbar colostomy, 611

nephrectomy, 607

puncture, 575

\section{MI}

AcBurney's hooks, 174

operation in appendicitis, 615

Magnesium sulphate, 197 solution, 135

Malar bone, fracture of, 374

Malignant œdema, bacillus of, 310

Mammary artery, internal, ligation of, 473 gland, excision of, 588

radical operation in, 589

Many-tailed bandages, 31 of abdomen, 32

Massage, 180

in fractures, 369
Maxillary bone, inferior, dislocation of, 438 excision of, 568 fracture of, 375 superior, excision of, 567 fracture of, 374

Mayo's operation for umbilical hernia, 656

Mechanical leech, 151

Median nerve, exposure of, 581

Mediate irrigation, 137

Medio-tarsal amputation, 533

Mento-vertico-occipital cravat, 35

modified, 36

Mercury bichloride, $\mathbf{3 1 8}$

hypodermic injection of, 171

Metacarpal bones, amputation of, 513

dislocation of, 453

fracture of, 407

resection of, $\cdot 558$

Metacarpo-phalangeal amputation, 511

joints, excision of, 558

Metallic catheters, 222

Metatarsal bones, amputation of, 529

dislocations of, 464

fracture of, 427

resection of, 566

Mikulicz's amputation of foot, 537

Modified circular amputation, 499

Moist dressing in wounds, $\mathbf{3 3 8}$ sterilized gauze dressings, 336

Moulded plaster splints, 112 splints, 118

Mouth, sterilization of, 342

Mouth-to-mouth inflation, 157

Multiple fracture, 359

Murphy button, 643

gastro-enterostomy with, 636

method in appendicitis, 614 
Muscle-grafting, 176

Musculo-spiral nerve, exposure of, $5 \$ 1$

Muslin, oiled, 122

Mustard papers, 142 plaster, 141

as a rubefacient, 141

\section{$\mathbf{N}$}

NASAL bones, fracture of, 372 cavities, sterilization of, 342

Neck and axilla, figure-ofeight bandage of, 65

Needle-holder, 233

mounted, 233

surgical, 232

Nephrectomy, abdominal, 607 lumbar, 607

Nephropexy, 607

Nephrorrhaphy, 607

Nephrotomy, 607

Nerve or Nerves, anterior crural, exposure of, 582 external popliteal, exposure of, 581

facial, exposure of, 579

grafting, 176, 577

implantation, $5 \pi 7$

inferior dental, exposure of, 579

internal popliteal, exposure of, 581

lingual, exposure of, 579

median, exposure of, 581

musculo-spiral, exposure of, 581

operations on, 576 et seq. popliteal, external, exposure of, 581

internal, exposure of, 581 radial, exposure of, 581 sciatic, exposure of, 581 spinal accessory, exposure of, 580

stretching, 577

superior maxillary, exposure of, $57 \mathrm{~S}$

supra-orbital, exposure of, 578

suture of, 577
Nerve ulnar, exposure of, 581

Neurectasy, 577

Neurectomy, 577

Neuroplasty, 578

Neurorrhaphy, 577

Neurotomy, 576

Nose, foreign bodies in, 295

T-bandage of, 31

Novocaine, 196

Nutritious enema, 168

\section{$\mathbf{O}$}

OAKUM, 121

Oblique bandage, 23

of head, 53

of jaw, 46

fracture, 359

Occipital artery, ligation of, 477

Occipito-facial bandage, 53

Occipito-frontal bandage, 54 triangle, 35

Oesophageal bougie, 166

Oesophagotomy, external, 624

Oesophagus, foreign bodies in, 293

Oiled muslin, 122 silk, 122

Old dislocations, 436,465

reduction of, 465

Olecranon process of ulna, fracture of, 398

Open fracture, 359,428

Operating-bag, surgical, 337

Operation or Operations, 467 antiseptic, details of, 348

for appendicitis, 613

aseptic, clothing of surgeon for, 346

details of, 347

material in, 325 et seq.

preparation for, 339

of patient for. 339

of room for, 339

on colon, 611

for hernia, 649 et seq.

for hydroccle. 620

on kidney, 607

on nerves, 576 
Operation on stomach, 625 on tendons, 582

on ureter, 609

for varicocele, 620

Opsonic treatment, 305

Os calcis, excision of, 566

Osteoplastic resection of skull, 573

Osteotomy, 569

for bowlegs, 570

of femur, 570

for knock-knee, 570

of tibia, 570

Oval method in amputation, 499

\section{$\mathbf{P}$}

PaDs, gauze, 326

Palmar abscess, 272

Panelectroscope, 179

Paper-lint, 121 splints, 367

Paquelin's thermo-cautery, 146

Paracentisis, abdominalis, 604 pericardii, 605

thoracis, 603

vesicæ, 605

Paraffin bandage, 118 paper, 122

Parchment-paper, 123

Parenchymatous hemorrhage, treatment of, 263

Partial dislocation, 435 fracture, 357

Passive motion, 181

Pasteboard splints, 119

Patella, dislocations of, 460 excision of, 564

fracture of, 417

Pathogenic action of bacteria, 302

Pathological dislocations, 466

Pelvic supporter for application of plaster bandage, 106

Pelvis, dislocation of, 440 fracture of, 379

Penis, spiral reversed bandage of, 91
Perforating fracture, 358

Perineum, T-bandage of, 82 , 91

Periosteal flaps in amputation, 502

Periosteotome, 504

Permanganate of potassium, 324

Peroneal tendons, tenotomy of, 583

Peroxide of hydrogen, 323

Petit's tourniquet, 249

Phalanges of fingers, dislocation of, 453

fracture of, 407

of toes, dislocation of, 464 fracture of, 427

Pharynx, foreign bodies in, 293

Picric acid dressing in burns, 284

Pirogoff's amputation at ankle-joint, 535

Plaster or Plasters, 124

adhesive, 124

ichthyol, 125

isinglass, 126

mustard, 141

resin, 125

rubber adhesive, 125

soap, 126

swansdown, 125

tape in arterial hemorrhage, 260

zinc oxide, adhesive, 126

Plaster-of-Paris bandage, application of, 105

preparation of, 104

removal of, 114

saw for, 115

shears for, 116

for thigh and pelvis, 106 trapping of, 113

dressings, 103

application of, 103

in compound fractures, 430

interrupted, 107

jacket, application of, $10 \mathrm{~S}$

frame for applying, 105 
Plaster-of-Paris, removal of, from hands, 113 splints in fracture, 367

Plate suture, 240

Pledgets, gauze, 326

Pliers, cutting, 504

Poisoned wounds, treatment of, 281

Popliteal artery, ligation of, 491

nerve, exposure of, 581

Position in arterial hemorrhage, 253

Posterior figure-of-eight bandage of chest, 73

tibial artery, ligation of, 493

tendon, tenotomy of, $5 \$ 3$

Potassium permanganate, 324 and oxalic acid for sterilizing the hands, 343

Pott's fracture of fibula, 425

Poultices, 133 antiseptic, 134

fermenting, 134

flaxseed, 133

soap, 134

stareh, 134

Powder-burns, 282

Pressure in arterial hemorrhage, 255

Pressure-sores, 288

Primary suture of tendons, 585

Protective, 332

Provisional ligation of arteries, 257

Pubic dislocation of femur, 459

Pudic artery, internal, ligation of, 4S8

Puncturation, 145

Punctured fracture, 358 wounds, treatment of. 280

Purse-string suture, 639

Pylorectomy, 631

Pyloroplasty, 629

Finney's, 630

Pyrozone, 323

\section{$\mathbf{Q}$}

QUADRUPLE ligature, 244

Quilled suture, 239

Quilt suture, 638

\section{$\mathbf{R}$}

RADIAL artery, ligation of, 481

nerve, exposure of, 581

Radium, 191

Radius, Colles's fracture of, 402

reversed, 406

dislocation of head of, 451

fracture of head of, 400

lower end of, 402

neck of, 400

resection of, 555

Rapid respiration, anæsthesia from, 194

Raw-hide splints, 118

Ray fungus, 311

Recent dislocation, 436

Rectal bougies, 167 tube, $\mathbf{1 6 6}$

Rectum, foreign bodies in, 292 hemorrhage from, treatment of, 267

sterilization of, 342

Recurrent bandage, 27

of head, 47

transverse, 49

of stump, 91

Reduction of fracture, 364

Reef knot, 234

Regional anæsthesia, 193

Relaxation sutures, 231

Removal of sutures, 241

Resection or Resections, 551 of fibula, 565

of humerus, 554

of metacarpal bones, $55 \mathrm{~S}$

of metatarsal bone, 566

of radius, 555

of ribs, 559

of skull, osteoplastic, 573

of sternum, 560

of tibia, 565

of ulna, 555 
Resin plaster, 125

Resistance of tissues to bacteria, 303

Respiration, artificial, 156

direct, 158

Laborde's method, 161

Silvester's method, 160

forced, 162

rapid, anæsthesia from, 194

Retractors, 124

in amputations, 505

Reversed Colles's fracture, 406

Ribs, dislocation of, 439

Estlander's operation on, 559 .

fracture of, 377

resection of, 559

Roller-bandage, 18

Rontgen rays, 187

Roux's amputation at anklejoint, 536

Rubber adhesive plaster, 125

bandage, 100

gloves, 345

tissue, 123, 333

Rubber-dam, 333

Rubcfacients, 140

$\mathbf{S}$

SACRUM, fracture of, 380

Salicylic acid, 324

Saline solution, 325

infusion of, 156

intravenous injection of, 155

Sand bags, 367

Saws, amputating, 503

for removal of plaster-of-

Paris bandages, 115

Scalds, 283

Scalp, sterilization of, 342

Seapula, dislocation of, 442

inferior angle of, 442

excision of, 560

fracture of, 387

acromion process of, 388

body of, 388

coracoid process of, 388

neck of, 388
Searification, 147

Sciatic artery, ligation of, 488 nerre, exposure of, 581

Scopolamine hydrobromate, 215

Scultetus bandage, 94

Secondary hemorrhage, treatment of, 264

sutures, 231

of tendons, 586

Sedillot's amputation of leg, 541.

Segregation of urine, 227

Seguin's method of. counterirritation, 144

Semilunar cartilages, dislocation of, 462

Semi-membranosus tendon, tenotomy of, 584

Semi-tendenosus tendon, tenotomy of, 584

Separation of epiphysis, 370

Setting of fracture, 364

Shears for removal of plaster-of-Paris 'bandage, 116

Shock, 274

diagnosis of, 274

prophylaxis of, 275

treatment of, 276

Shotted suture, 240

Shoulder, dislocation of, 443 reduction of, 444 et seq. spica bandage of, ascending, 62

descending, 64

Shoulder-joint, amputation at, 521

above, 525

excision of, 554

Signorini's tourniquet, 251

Silicate of potassium balldage, 117

splints in fracture, 367

of sodium bandage, 117

splints in fracture, 367

Silk gloves, 346

ligatures, 326

oiled, 122

sutures, 326

Silkworm-gut, 327 
Silver foil, 333

salts, 322

Silverized catgut, 330

wire sutures, 327

Silvester's method of artificial respiration, 160

Simple dislocation, 435 fracture, 359

Sinapism, 141

Single ligature with pin, 242 roller-bandage, 20

T-bandage, 28

Sinus, frontal trephining of, 575

Sinuses, 271

Skiagraphy, 187 et seq.

Skin-grafting, .172

Krause's method, 174

Thiersch's method, 173

Skull, fracture of, 382 osteoplastic, resection of, 573

trephining of, 571

Slings, 31

Soap plaster, 126 poultice, 134

Sodium chloride, 325

Sounds, 224

Spanish windlass, 249

Spence's amputation at shoulder-joint, 525

Spica bandage, 26

of buttock, 81

of foot, 85

of groin, ascending, 76

descending, 78 double, 79

of shoulder, ascending, 62 descending, 64

of thumb, 59

Spinal accessory nerve, exposure of, 580

anæesthesia, 198

puncture, 575

Spiral bandage, 23

of chest, 72

of finger, 55

reversed bandage, 24

of $\mathrm{arm}, 59$

of fingers, 56
Spiral reversed bandage, of lower extremity; ss of penis, 91

of thigh, 89

of upper extremity, 60

Splints, binder's board, 119 , 366

chalk and gum in fracture, 367

Dupuytren's, 426

felt, 120,366

in fractụre, 365

gutta-percha, 366

hatter's relt, 120

leather, 118, 360

moulded, 118

paper, 367

pasteboard, 119

plaster, 112

plaster-of-Paris, in fracture, 367

raw-hide, 118

silicate of potassium, in fracture, 367

of sodium in fracture, 367

starched, 367

Volkmann's, 423

wooden, 366

Sponges, 323

Spores, 298

Sprain-fracture, 290

strapping of, 290

Sprains, 289

Staffordshire knot, 234

Staphylococcus pyogenes aureus, 307

Starch poultice, 134

splints in fracture. 367

Starched bandage, 116

Sterilization of bladder, 341

of bougies, 221, 345

of catheters, 221, 345

of feet, 341

of hands, 342

Harrington's method, 343

of instruments, 344

of mouth, 342

of nasal cavities, $3+2$

of rectum, 342 
Sterilization of scalp, 342

of stomach, 341

of urethra, 341

of vagina, 341

Sterilized bandages, 335

catgut, $32 \mathrm{~S}$

cotton, 336

gauze, 336

dressings, moist, 336

water, 325

Stcrno-cleido-mastoid tendon, tenotomy of, 5S4

Sternum, dislocation of, 440 fracture of, 379

resection of, 560

Stomach, foreign bodies in, 293

operation upon, 625

sterilization of, 341

Stomach-pump, 165

Stomach-tube, 164

Storaine hydrochloride, 197

Strains of fascia, 290 of muscles, 290

Strangulated hernia, operation for, 649

Strapping, 127

the ankle-joint, 131

the back, 132

the chest, 128

of joints, 131

the testicle, 127

of ulcers, $12 \mathrm{~S}$

Streptococcus pyogenes, $30 \mathrm{~S}$

Stump, recurrent bandage of, 91

Stupe, turpentine, 141

Styptics in arterial hemorrhage, 254

Subastragaloid amputation of foot, 534

Subclavian artery, ligation of. 471

Subclavicular dislocation of humerus, 443

Subcoracoid dislocation of humerus, 443

Subcutaneous ligature, 244

Subcuticular suture, 237
Subglenoid dislocation of humerus, 443

Subperiosteal fracture, 358

Subspinous dislocation of humerus, 444

Sulphocarbolate of zinc, 322

Superior maxillary bone, excision of, 567

nerve, exposure of, 578

Superior thyroid artery, ligation of, 476

Suppuration, bacteria of, 307 diffuse, 271

Suppurative thecitis, 272

Supraorbital nerve, exposure of, 578

Suprapubic lithotomy, 618

Surgeon's clothing for operations, 346

knot, 234

Surgical bacteriology, 297 needles, 232

operating bag, 337

Suspensory bandage of breast, 38

and compressor bandage of breasts, 74,75

Suture or Sutures, 231

in amputations, 506

of approximation, 231

in arterial hemorrhage, 258

of arteries, 258

buried, 236

button, 240

chain-stitch, 237

of coaptation, 231

continued, 236

Czerny-Lembert, 639

deep, 236

in hemorrhage, 258

Halstead's mattress, 635

horsehair, 327

interrupted, 235

intestinal, 637

Lembert's, 63S

mattress, Halsted's, 638

of nerves, 577

plate, 240

purse string, 639

quilled, 239 
Suture or Sntures, quilt, 635 of relaxation, 231 removal of, 241 secondary, 231 securing of, 233 shotted, 240 silk, 326 silver wire, 327 subeuticular, 237 of tendons, 585 primary, 585 secondary, $5 \& 6$ of veins. 262

Swan's-down plaster, 125 Srme's amputation of anklejoint, 534

\section{$\mathbf{T}$}

TAMPON, 123 glycerin, 123

Tarsal bones, dislocation of, 463

fracture of, 426

Tarso - metatarsal amputations, 530

T-bandage, $2 \mathrm{~S}$

of chest, 31

double, 30

of groin, 29

of nose, 31

of perineum, $\$ 2,91$

single, 25

Teale's amputation of leg, 540 method in amputation, 500

Temporal artery, ligation of, 475

Temporary ligation of arteries, 257

Tenaculum, 257

Tendo Achillis, tenotomy of, ¿\$2

Tendon, adductor longus, tenotomy of, 5s4

anterior tibial, tenotony of. 583

biceps flexor cruris, tenotony of, 5\$4

extensor longus digitorum, tenotomy of, $5 \$ 4$
Tendon, extensor proprits pollicis, tenotomy of, $5 \$ 4$ flexor longus pollicis, tenotomy of, 584

hamstring, tenotomy of, $5 \& 4$

lengtheming of, 586

operations on, $5 \mathrm{~S} 2$

peroneal, tenotomy of, $5 \& 3$

posterior tibial, tenotomy of. 583

semi-membranosus, tenotomy of, 584

semi-tendinosus, tenotomy of, 584

sterno-cleido-mastoid, tenotomy of, 584

suture of, 585

primary, 585

secondary. 586

transplantation of, 587

Tenotomy, 5s2 ct scq.

Tent. 123

Testicle, excision of, 620 strapping of, 127

Tetanus, bacillus of, 309

Thecitis, suppurative, 272

Thiersch's method of skingrafting, 173

Thigh, amputation of, 543 ct ser.

spiral reversed bandage of, 89

Thread, celluloid, 330

Thumb, spica bandage of, is

Thyroid artery, superior. ligation of. $4 \bar{i} 6$

inferior, ligation of. 473

dislocation of femur, $45 \mathrm{~s}$

Tibia and fibula. fracture of, 419

osteotomy of, 570

resection of. $5 f 5$

Toes, amputation of, 527

dislocation of, 464

fracture of phalanges of, 427

Torsion in arterial hemorrhage, 256 
Tourniquets, 248

in amputations, 506

Petit's, 249

Signorini's, 251

'Toxins, 303

Trachea, foreign bodies in, 294

fracture of. 377

Tracheotomy, 590 et seq.

high operation. 594

operation of, 593

tube, 592

Transfixion method in amputations, 498

Transfusion, arterial, 154

of blood. 153

direct, 154

Tramsperitoneal ligation of common iliac artery, $\$ 56$

Transplantation of tendons, is 7

Transverse fracture, 359

recurrent bandage of head, 49

Trapping plaster - of - Paris bandage, 113

Trephining of antrum of Highmore, 563

frontal sinus, 575

:kull, $5 \pi 1$

Triangular cap of breast, 38 'Tripier's amputation of foot, 537

Trunk, bandages of, 72

Truss for femoral hernia, 220

Ilood's, 220

for inguinal hernia, 219

for umbilical hernia, 221

worsted, 219

Tubercle bacillus, $30 \mathrm{~s}$

Tuberculous abscess, incision in, 271

injection in. 270

puncture, 270

treatment of. 269

Turpentine as rubefacient. 140

stupe, 141
$\mathbf{U}$

ULCERS, strapping of, 128

Ulna, dislocation of upper end of, 451

fracture of coronoid process of, 400

of olecranou process of, 398

and radius, fracture of, 400

resection of, $55 \overline{5}$

Ulnar artery, ligation of, 483 nerve, exposure of, 581

Umbilical hernia, Mayo's operation for, 6.5.

radical cure of. 655

truss for, 221

Ununited fracture, 432

Upper epiphysis of humerus, separation of. 330

extremity, bandages of, 5 spiral reversed bandage of, 60

jaw, fracture of, 374

Ureter, eatheterization of, 227 implanation of, in bladder, 610

operations on, 609

Ureterectomy, 609

Ureterotomy, 609

Uretero-ureterostomy, 609

Urethra, foreign bodies in, 291

hemorrnage from, treatnent of. 267

sterilization of, 341

Urethral injections, 230

irrigation, 231

Urethroscope, 179

Urine, segregation of, 227

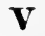

Vaccination, 168

Vagina, foreign bodies in, 292 sterilization of, 341

Varicocele, operation for, 620

Vascular growths, ligatures for, 242

V-bandage of head, 49 
Veins, suture of, 202

Velpeau's bandage, 65 dressing, 385

Venesection, 151

Venous hemorrhage. Hemorrhage, venous. treatment of, 262

Vertebræ, dislocation of, 437

Vertebre, fracture of, $3 \$ 0$

Vertebral artery, ligation of, $47 \cdot 2$

Vesicants, 143

Volkmann's splint, 423

\section{WV}

WAter, sterilized, 325

Waxed paper, 122

Wet cupping, 14!

White lead dressing in burns, 284

Whitlow, 272

Wood-wool. 122

Wooden splints, 366

Worsted truss, 219

Wounded arteries, ligation of. 261

Wounds, contused, treatment of, 279

dressings of 277

aseptic, $33 \mathrm{~s}$

dry method of dressing, 33s drving and chemieal sterilization in, 338

gunshot, treatment of, $2 \$ 1$
Wounds, incised, treatment of, 277

infected, antiseptic treatment of, 353

aseptic treatment of, 353 lacerated, treatment of, $27 s$ modified moist dressings in, 339

moist dressing in, 338

poisoned, treatment of, 281

pumctured, treatment of, 280

redressing of 351

Wrist-joint, amputation at, 515

dislocation of, 452

excision of, 556

Myuter's method, 557

Wyeth's method of amputa-

tion at hip-joint. 548

pins in amputation at shoulder-joint, 522

\section{$\mathbf{X}$}

Xeroform, 324

I-rays, 187

examination in fracture, 303

burns, 2ss

\section{$\mathbf{Z}$}

ZINc chloride, 322

oxide adhesive plaster, 126

sulphocarbolate, 322

Zygoma, fracture of, 374 



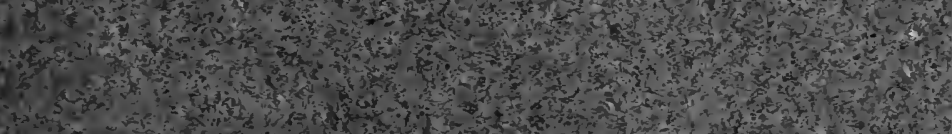

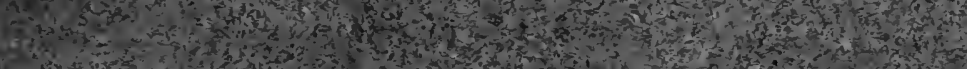
(1)

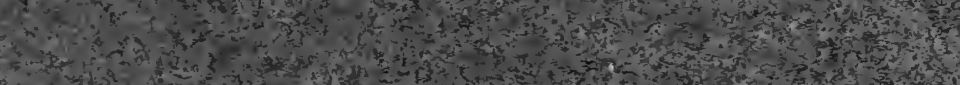

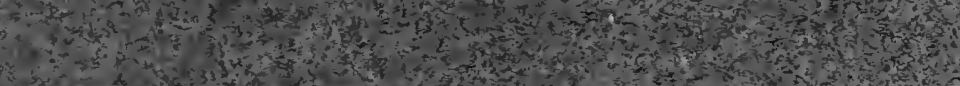

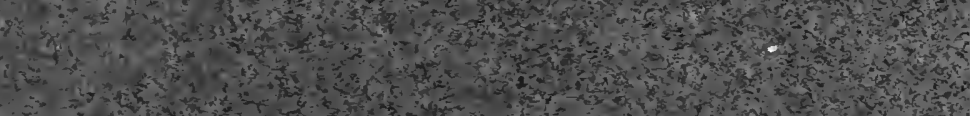

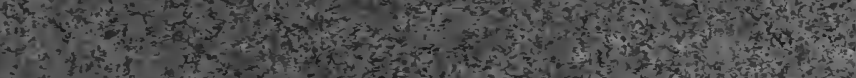

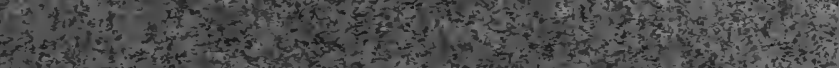

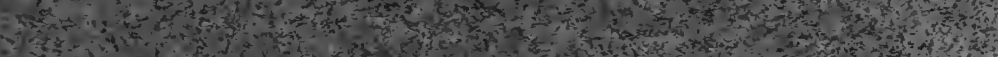
of

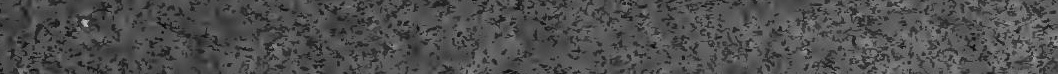

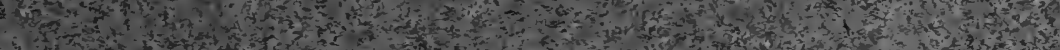

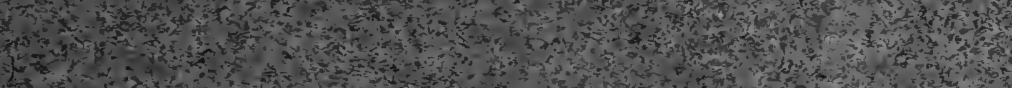

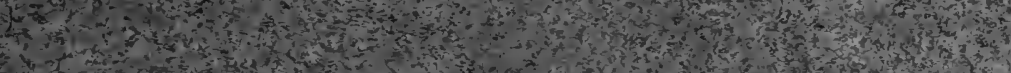

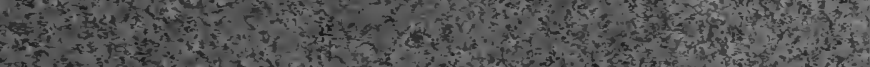

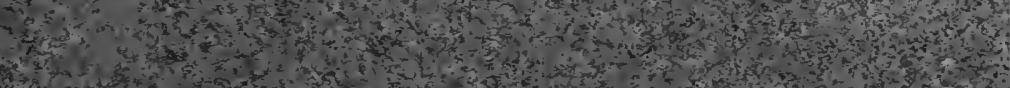
(n)

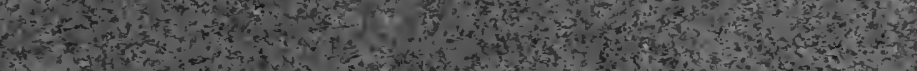

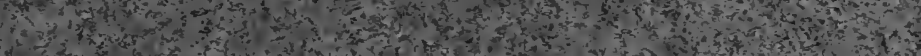

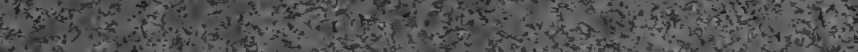
and we

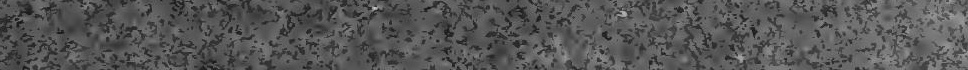

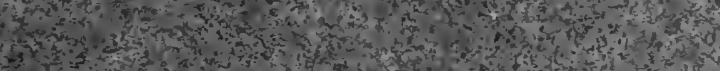
We

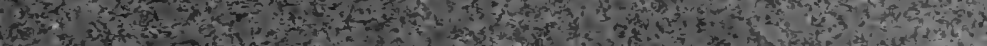
(5) ard 



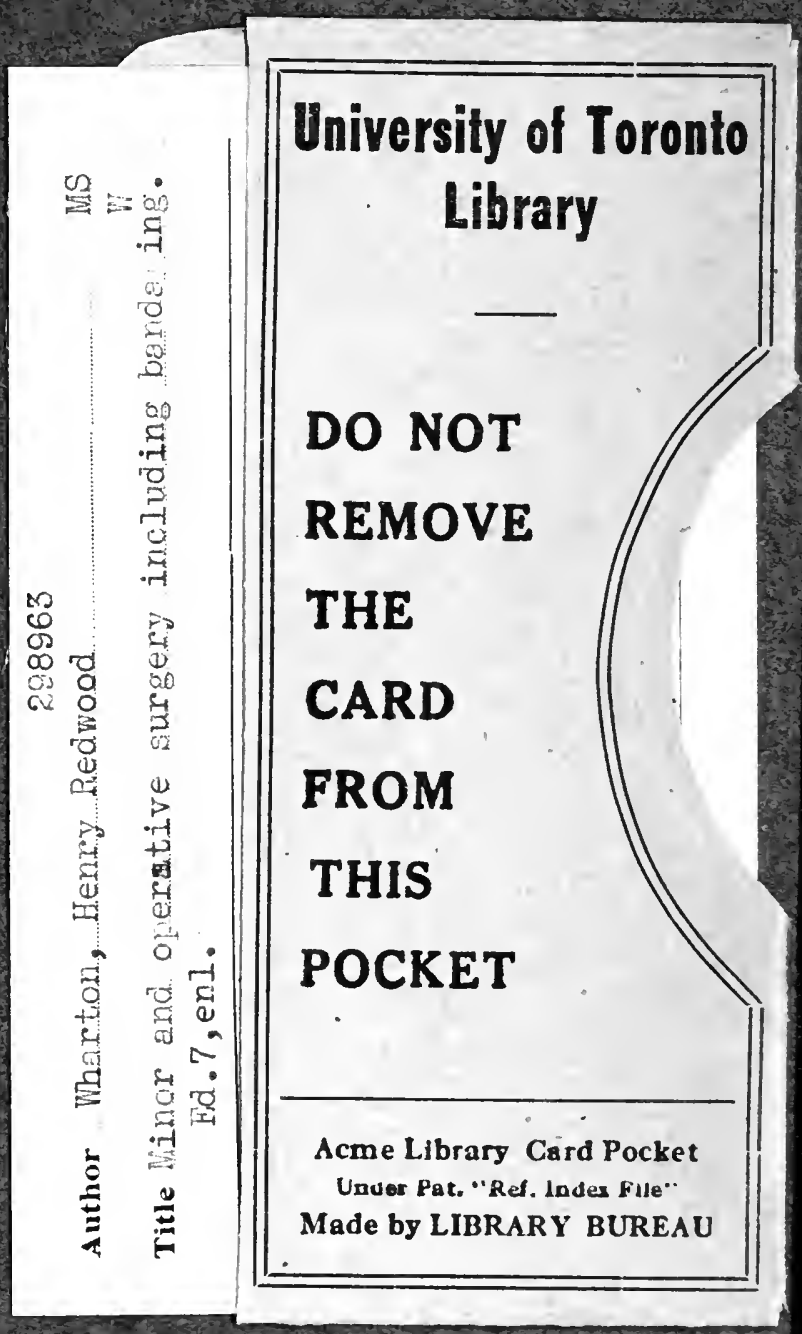


3

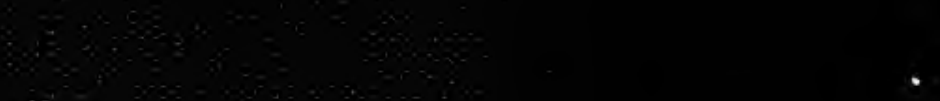

